\title{
SELF-HEALING CEMENTITIOUS COMPOSITES INCORPORATING INNOVATIVE MATERIALS
}

By

Mohamed A A Sherir

MASc Ryerson University, 2012

B.Sc. Islamic University of Gaza, Gaza Strip 2001

\author{
A Dissertation \\ Presented to Ryerson University \\ In partial fulfillment of the requirements for degree of \\ Doctoral of Philosophy \\ in the Program of \\ Civil Engineering
}

Toronto, Ontario, Canada 2016

(C) Mohamed A A Sherir 2016 


\begin{abstract}
AUTHOR'S DECLARATION
I hereby declare that I am the sole author of this dissertation. This is a true copy of the dissertation, including any required final revisions, as accepted by my examiners.
\end{abstract}

I authorize Ryerson University to lend this dissertation to other institutions or individuals for the purpose of scholarly research.

I further authorize Ryerson University to reproduce this dissertation by photocopying or by other means, in total or in part, at the request of other institutions or individuals for the purpose of scholarly research.

I understand that my dissertation may be made electronically available to the public. 


\title{
SELF-HEALING CEMENTITIOUS COMPOSITES INCORPORATING INNOVATIVE MATERIALS
}

\author{
Mohamed A A Sherir \\ Doctor of Philosophy, 2016 \\ Civil Engineering, Ryerson University
}

\begin{abstract}
Discovering new cement based materials characterized by higher durability and longer service life is crucial for sustainable infrastructure. Engineered Cementitious Composite (ECC) with high potential of micro-crack healing can enhance ductility/durability of concrete structures. However, low water-to-cement ratio in ECC matrix in addition to the lack of cementitious properties of newly formed healing-products in micro-cracks could be an obstacle to implementing continuous hydration to stimulate self-healing and stop re-opening of old healed micro-cracks during reloading. MgO-type expansive agent (MEA), widely used to compensate effectively autogenous shrinkage in mass concrete, can be used to eliminate such obstacles and to induce self-healing. This research studied self-healing capability of MEA in ECC through an extensive experimental investigation and developed/proposed an ECC-MgO system. Test results indicated " $900^{\circ} \mathrm{C}-2$ hours of holding time-45 $\mu \mathrm{m}$ particle size" as the best calcination system based on higher MEA hydration in powder state. Additionally, 5\% lightly burnt $\mathrm{MgO}$ combined with low-calcium Class-F-fly ash (as 55\% cement replacement) was found to be a better choice in designing self-healing-ECC-MgO system in terms of lower expansion effect of MEA.
\end{abstract}

Further, self-healing property of ECC-MgO system under different environmental exposures (laboratory/water/natural(field)/autoclave curing) was investigated based on mechanical/durability properties of control/pre-cracked specimens compared with their ECC counterparts. The ECC$\mathrm{MgO}$ system exhibited remarkable self-healing property in multiple-cracked/damaged specimens when test results were analyzed based on development/recovery of compressive/flexural 
strength/load resistance, ultrasonic pulse velocity, heat of hydration, expansion/drying shrinkage, rapid chloride permeability, sorptivity/water absorption and freeze/thaw resistance in addition to crack healing/cracking characteristics and microstructural characterizations through Thermogravimetric Analysis (TGA)/Differential Scanning Calorimetry (DSC) and Scanning Electron Microscopy (SEM). Finally, the self-healing ability of ECC-MgO system was evaluated through structural performance (regarding strength/stiffness/energy absorbing/crack-healing capacity recovery) of damaged and subsequently healed water/field cured link slabs used for jointfree bridge deck construction. Superior self-healing ability of ECC-MgO system was attributed to low MEA water demand coupled with its delayed hydration characteristics which lead to the formation of more cohesive/strong cementitious $\mathrm{MgO}$ crystals within crack walls (at later ages) supplementing $\mathrm{CaCO}_{3}$ precipitations (formed earlier) leading to more effective crack-healing and developing post-healed cracks at new locations upon reloading. 


\section{Acknowledgments}

Above all, I am thankful to Almighty God for giving me the vision, control, spirit and strength to complete this interesting research.

I wish to express my deepest gratitude to my supervisor Dr. Mohamed Lachemi for providing support, inspiration, motivation and continued encouragement for completing my PhD. I thank him for assisting me generously during my years at Ryerson and he has been there for me without hesitation.

I would like to express my highest appreciation and gratitude to the person who believed in me, my supervisor Dr. Khandaker M. Anwar Hossain for his willingness to always help along with patience, kind words and advice during this research for the past four years. Without his motivation, countless proof readings, corrections, and support this document would not have been possible.

I am also deeply grateful to my teacher and examiner Dr. Medhat Shehata who was always available with invaluable suggestions and expert opinions during my research despite his busy schedule. I also extend my special thanks to Dr. Daman Panesar, Dr. Dontaus Oguamanam and Dr. Abdurrahmaan Lofty for their constructive feedback and for being examiners of my dissertation. I also would like to thank Mr. Nidal Jaalouk, Mr. Min Yao and Mr. Domenic Valle for assisting me in casting and testing in the concrete, NDT and structural laboratories. Also, the support provided by the lab/research assistants Mr. Houshmand, Mr. Mattes, Mr. Batista, Mr. Anwar, Mr. Grewal, Mr. Temor and Mr. Ghadimi are acknowledged.

Financial support from Natural Science and Engineering Research Council (NSERC) of Canada is gratefully acknowledged. Thanks and appreciation also to our industrial partners, Lafarge Canada and St. Marys Cement, for their support and in-kind donations throughout the duration of this research project.

Finally, I would like to thank my parents for their support, care and love during my entire study period. I am also deeply thankful to my family, especially my four children and my beloved wife, Heba. Without Heba's constant love and sacrifice, my success would not be possible. 


\title{
Dedicated To My Wife Heba \& My Children
}

\author{
Iyada, Ahmed, Mohanad, Malak
}

And

\section{To those who supported me towards success}




\section{Table of Contents}

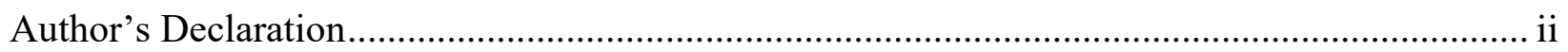

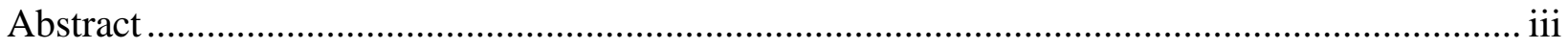

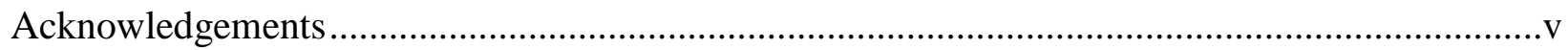

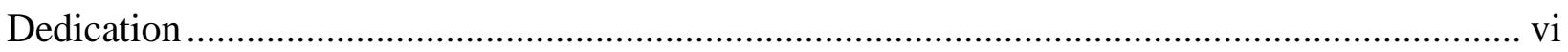

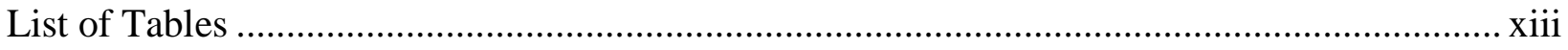

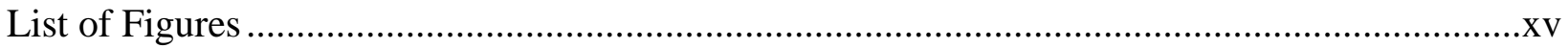

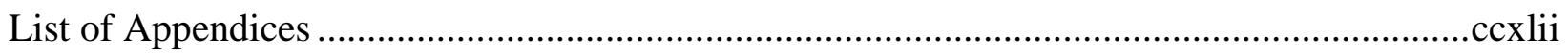

1. INTRODUCTION..........................................................................................................................1

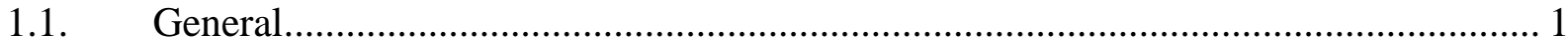

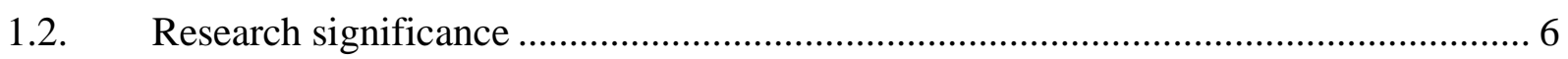

1.3. Research Objectives and Scope ........................................................................... 7

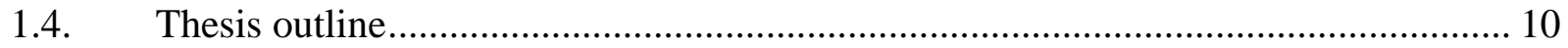

2. LITERATURE REVIEW AND BACKGROUND .................................................11

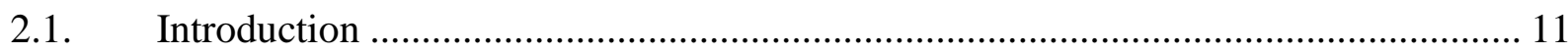

2.2. Early studies of self-healing ………………….................................................. 12

2.3. Engineered Cementitious Composites (ECC) ……….............................................. 13

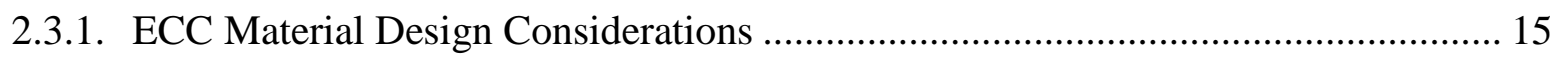

2.4. Autogenous (intrinsic) self-healing property ......................................................... 17

2.4.1. Hydration effect on self-healing .............................................................................. 17

2.4.2. Calcium carbonate effect on self-healing (Chemical ions and $\mathrm{pH}$ value) ................. 18

2.4.3. Water temperature effect on self-healing ............................................................... 19

2.4.4. Crack width effect on self-healing .................................................................. 19

2.4.4.1. Restriction of crack width under chloride environment............................................... 19

2.4.4.2. Restriction of crack width under natural environment................................................20

2.4.4.3. Restriction of crack width by using synthetic fibers .................................................21

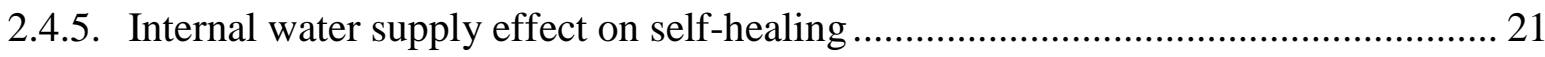

2.4.5.1. Superabsorbent Polymers (SAPs) ........................................................................ 21

2.4.5.2. Restriction of crack width by using internal curing ..................................................2 23 
2.4.6. Supplementary cementing materials effect on self-healing …………………....... 24

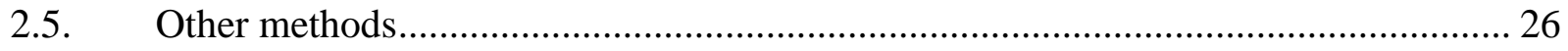

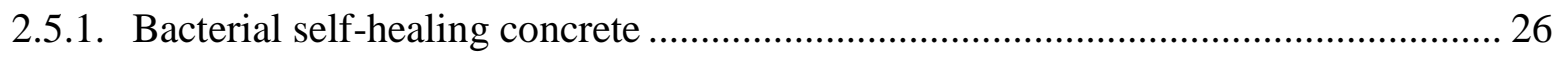

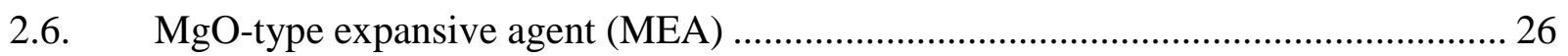

2.6.1. $\mathrm{MgO}$ concrete ………………................................................................................ 27

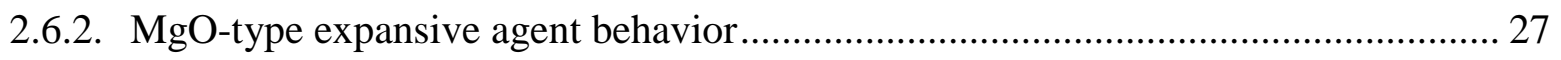

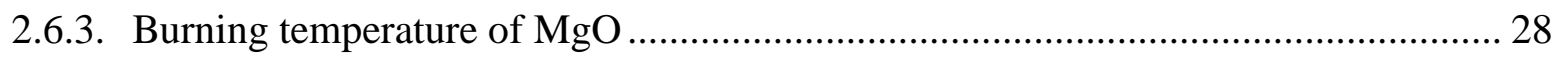

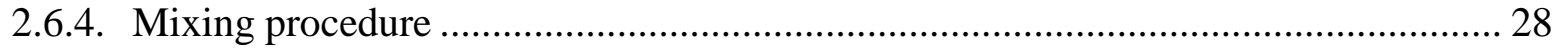

2.6.5. Mechanical and durability properties of $\mathrm{MgO}$ concrete ............................................ 29

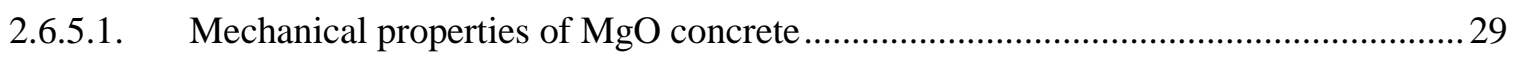

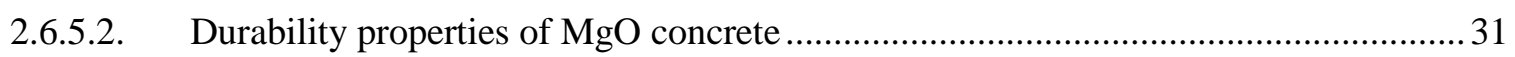

2.6.6. Supplementary cementing materials effect on $\mathrm{MgO}$ concrete ………….................. 32

2.6.7. Curing conditions and $\mathrm{w} / \mathrm{c}$ ratio effect on $\mathrm{MgO}$ concrete …..................................... 34

2.7. ECC for bridge deck link slab ............................................................................. 34

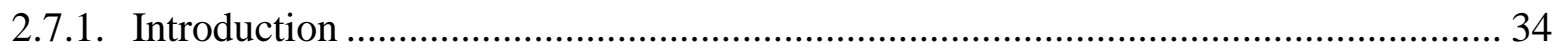

2.8. Previous Research on Link Slab for Joint-free Bridge Construction ......................... 36

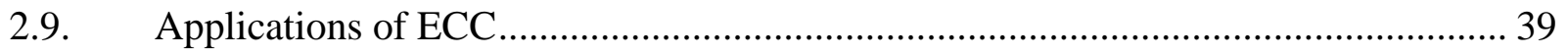

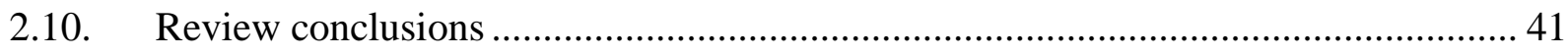

3. EXPERIMENTAL PROGRAM..............................................................................................42

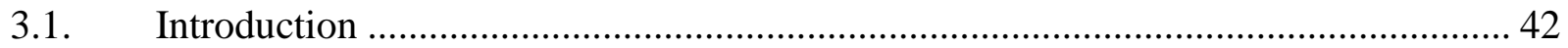

3.2. Research program and methodology ………................................................... 43

3.2.1. Task I: Selection of materials ............................................................................. 44

3.2.2. Task II: Determination of optimum dosages of MgO-type expansive additive for ECC-

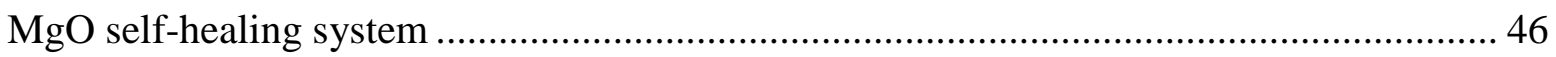

3.2.2.1. Sub-Task II-I: Finding best calcination system for $\mathrm{MgO}$ agent................................. 47

3.2.2.2. Sub-Task II-II: Finding the best percentage of $\mathrm{MgO}$ additive ..................................47

3.2.3. Task III: Selection of ECC-MgO Mixtures ............................................................ 48

3.2.4. Task IV: Self-Healing evaluation of proposed ECC-MgO system ........................... 48

3.2.4.1. Sub-Task IV-I: Self-healing evaluation through different environmental exposures

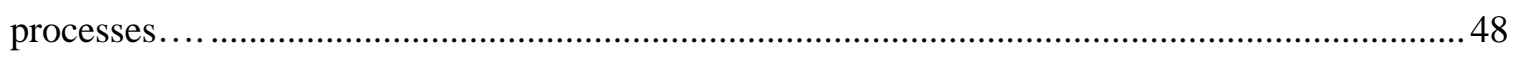

3.2.4.2. Sub-Task IV-II: Self-healing evaluation through autoclave process .........................50 viii 
3.2.5. Task V: Self-Healing Performance Evaluation of ECC in Structural Elements ....... 51

3.3. Mixing Sequences and Production of ECC and ECC-MgO................................ 51

3.4. Crack creation methods in different types of specimens ...................................... 52

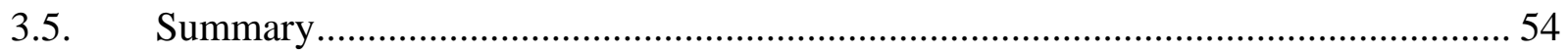

4. CALCINATION AND EXPANSION CHARACTERISTICS OF MGO-TYPE EXPANSIVE AGENT WHEN INCORPORATED AS SELF-HEALING AGENT IN ENGINEERED CEMENTITIOUS COMPOSITES ....................................................55

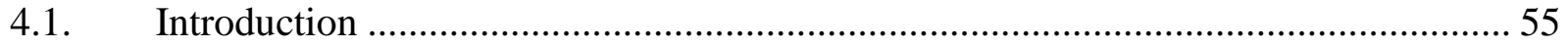

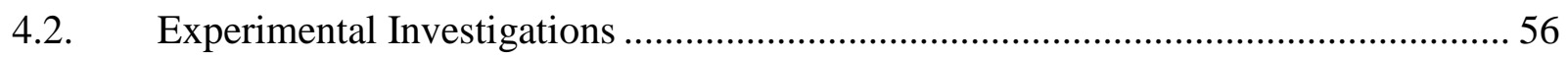

4.2.1. Preparation of powder MgO-type expansive agent .......................................... 56

4.2.2. ECC-MgO Mixture Proportions .................................................................. 58

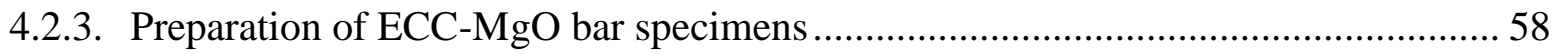

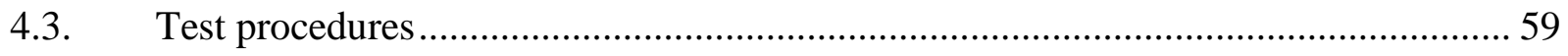

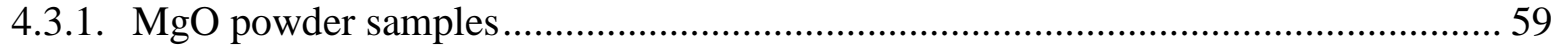

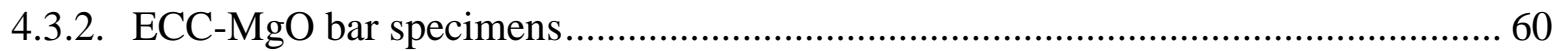

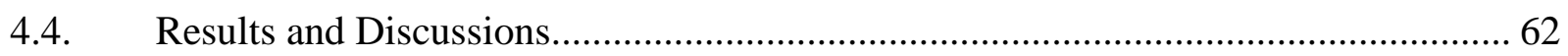

4.4.1. Phase I: Finding Best Calcination System for MgO Additive ............................... 62

4.4.1.1. Best Calcination based on weight loss of $\mathrm{MgCO}_{3}$ after kiln burning process.............62

4.4.1.2. Best Calcination based on degree of hydration of previously burned $\mathrm{MgO}$ samples by

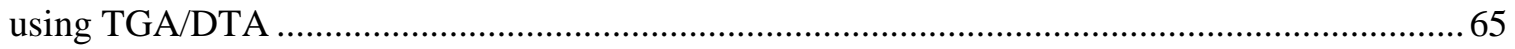

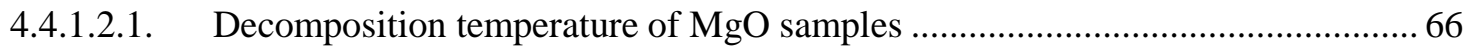

4.4.1.2.2. Difference in weight loss of $\mathrm{MgO}$ samples (TGA/Kiln burning processes) ........ 68

4.4.2. Phase II: Linear expansion effect of $\mathrm{MgO}$ expansive additive .............................. 71

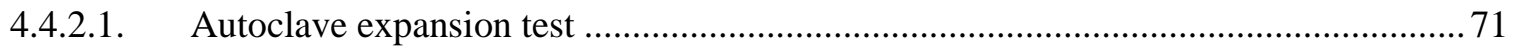

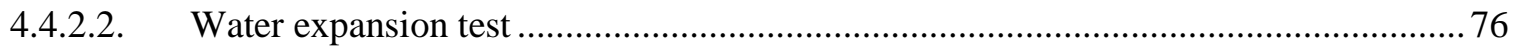

4.4.3. Comparison between autoclave and water cured ECC-MgO bar specimens ........... 81

4.4.4. Microstructure of ECC-MgO self-healing system ........................................... 87

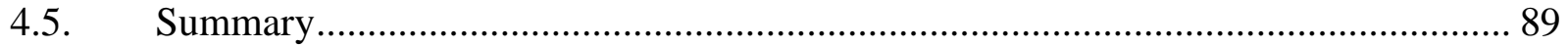




\section{SELF-HEALING AND EXPANSION CHARACTERISTICS OF ENGINEERED CEMENTITIOUS COMPOSITES WITH HIGH VOLUME FLY ASH AND MGO-TYPE}

EXPANSIVE AGENT .....................................................................................................................90

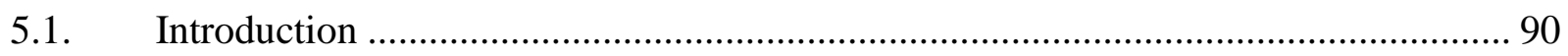

5.2. Experimental Investigations ……………............................................................. 91

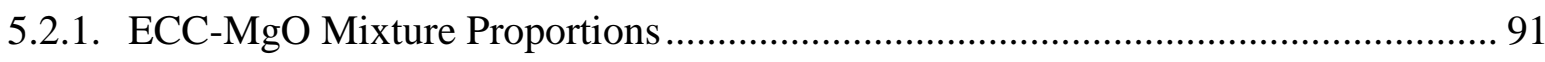

5.2.2. Preparation of ECC-MgO bar specimens ............................................................... 92

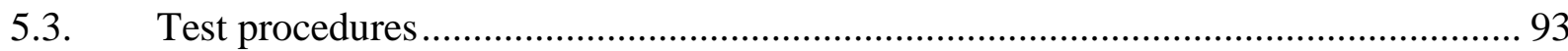

5.3.1. Water and autoclave expansion tests ...................................................................... 93

5.3.2. Compressive strength development and recovery tests ........................................... 94

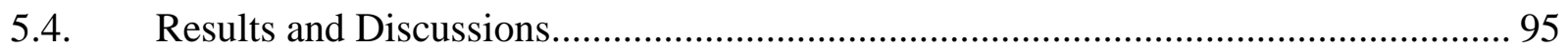

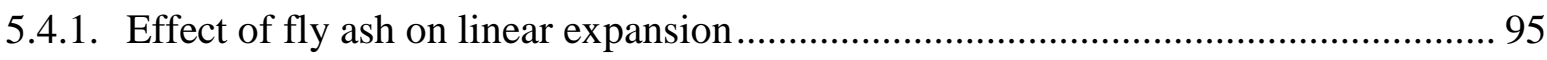

5.4.1.1. Autoclave expansion .......................................................................................... 95

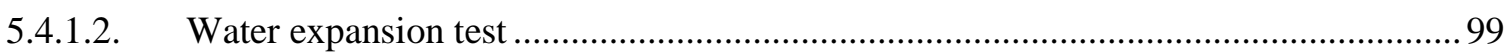

5.4.2. Comparison of water cured and autoclave ECC-MgO bar specimens ..................... 102

5.4.3. Effect of $\mathrm{MgO}$ content on ECC-MgO self-healing system ...................................... 103

5.4.4. SEM analysis of ECC-MgO self-healing system .................................................. 106

5.4.5. Compressive strength development/recovery of ECC-MgO self-healing system... 111

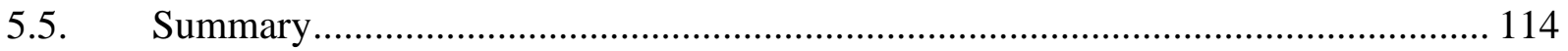

\section{MECHANICAL PROPERTIES OF SELF-HEALED CEMENTITIOUS}

COMPOSITES WITH MGO-TYPE EXPANSIVE AGENT …...........................................115

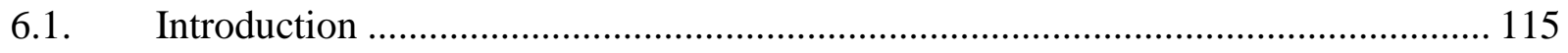

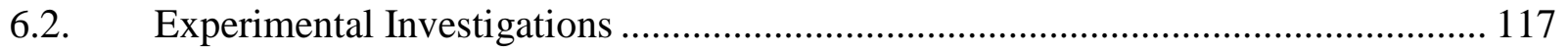

6.2.1. ECC-MgO Mixture Proportions ........................................................................... 117

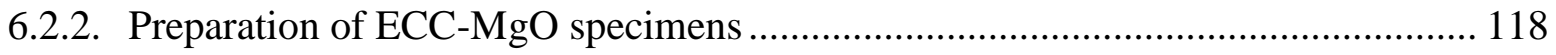

6.2.2.1. Specimens for compressive strength .................................................................... 118

6.2.2.2. Specimens for the study of flexural strength characteristics.....................................119

6.3. Test procedures ............................................................................................. 122

6.3.1. Compressive strength cube specimens preparation............................................. 122

6.3.2. Flexural strength (simulating load-displacement) evolution/recovery and UPV .... 124 


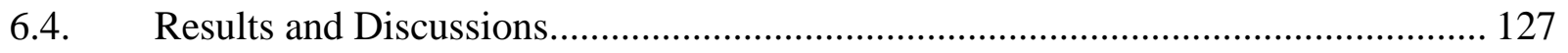

6.5. Compressive strength development and recovery ................................................. 127

6.5.1. Compressive strength recovery of self-healed ECC \& ECC-MgO specimens ....... 130

6.5.2. Effect of multiple damage (repeatability of reloading) on compressive strength recovery of self-healed ECC \& ECC-MgO mixtures .......................................................... 133

6.6. Flexural load/strength development/recovery and UPV measurements................... 135

6.6.1. Flexural load/strength recovery of self-healed ECC \& ECC-MgO specimens....... 139 6.6.2. Long-term study of strength recovery of self-healed ECC \& ECC-MgO

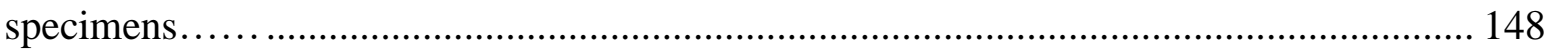

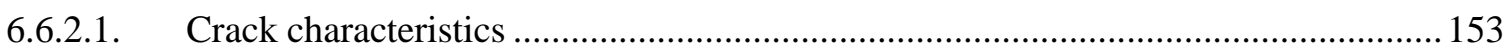

6.6.3. Effect of multiple damages under repeated reloading on load/strength recovery of self-

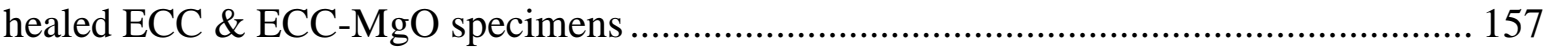

6.6.4. Strength recovery of ECC-MgO self-healing system under autoclave curing ........ 162

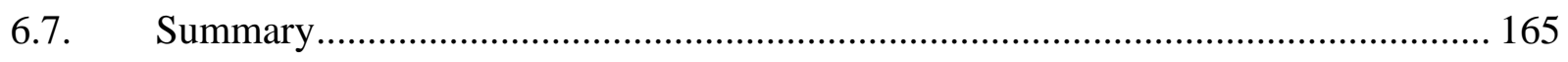

\section{MECHANICAL AND DURABILITY PROPERTIES OF SELF-HEALED} CEMENTITIOUS COMPOSITES WITH MGO-TYPE EXPANSIVE AGENT .................166

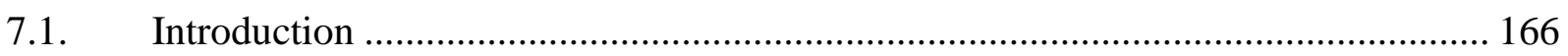

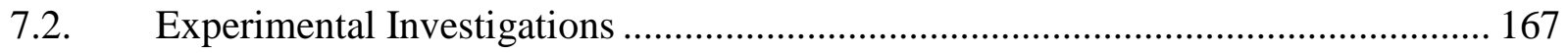

7.2.1. ECC-MgO Mixture Proportions ................................................................................ 167

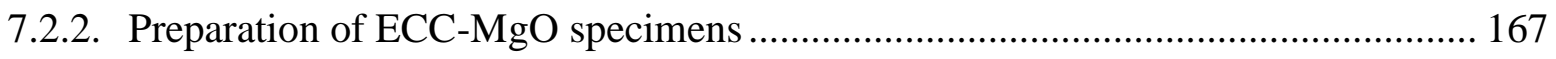

7.2.3. Heat of hydration .......................................................................................... 168

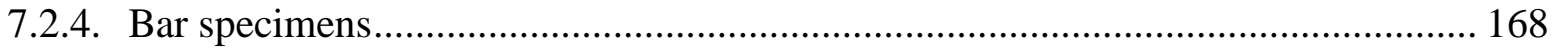

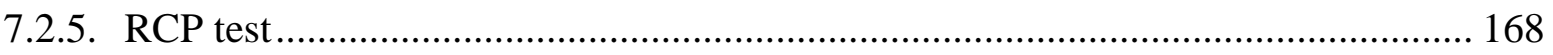

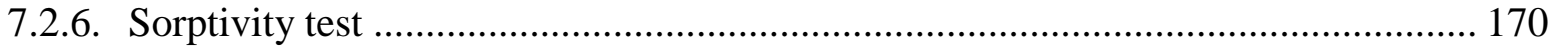

7.2.7. Autoclave cured sorptivity properties of ECC and ECC-MgO specimens ............. 172

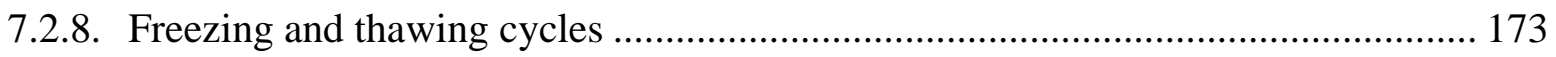

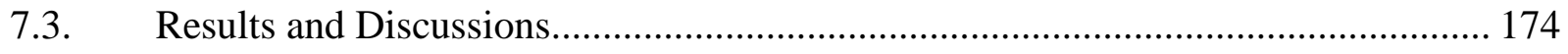

7.3.1. Heat of Hydration of ECC and ECC-MgO ..................................................... 174

7.3.2. Drying shrinkage and expansion of ECC and ECC-MgO bar specimens ............... 177

7.3.3. Rapid Chloride Permeability (RCP) of ECC and ECC-MgO mixtures .................. 179 
7.3.4. Sorptivity properties 182

7.3.5. RCP \& Sorptivity Properties of ECC \& ECC-MgO specimens under Autoclave Curing Condition 186

7.3.6. Freeze and Thaw resistance of ECC and ECC-MgO prisms and self-healing against frost damage 188

7.3.7. TGA/DSC and SEM analysis of ECC-MgO self-healing system ........................... 196

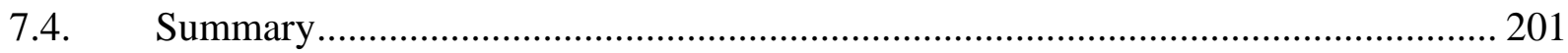

\section{STRUCTURAL PERFORMANCE OF WATER AND NATURAL CURED SELF-} HEALED LINK SLABS UNDER MONOTONIC LOADING ..........................................202

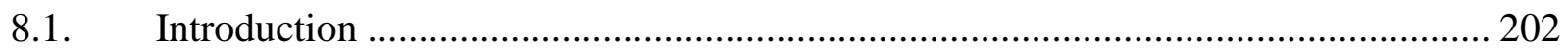

8.2. Experimental program using one-fourth scale ECC link slabs................................. 203

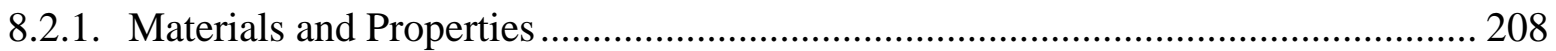

8.2.2. Casting of link slab specimens ............................................................................ 209

8.2.3. Test Set-Ups, Instrumentation and Testing Procedure ........................................... 210

8.2.4. Self-healing curing methods, testing scheme and post-healing performance

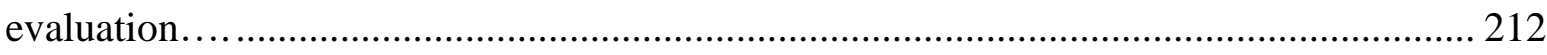

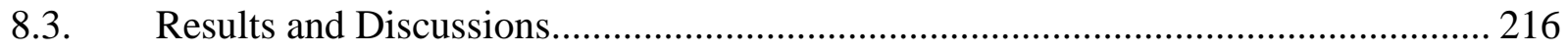

8.3.1. Structural performance of ECC and ECC-MgO link slabs subjected to monotonic loading and evaluation of self-healing potential ......................................................... 216 8.3.2. Comparative structural performance and self-healing characterization using load-

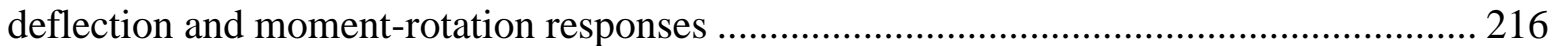

8.3.3. Comparative structural performance and self-healing characterization using strain

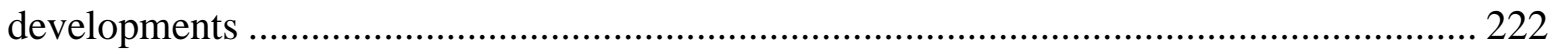

8.3.4. Comparative performance and self-healing characterization using crack

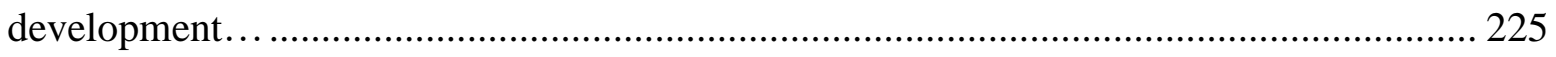

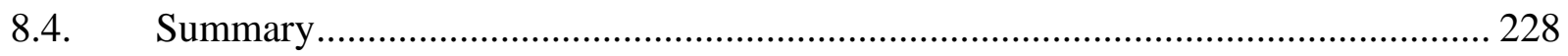

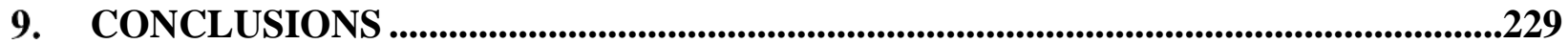

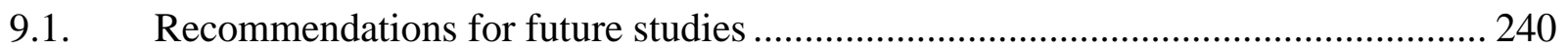

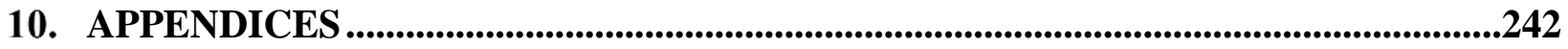

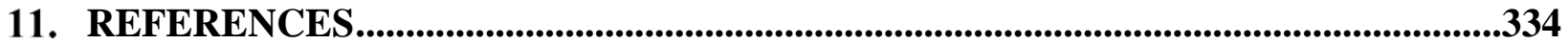




\section{List of Tables}

Table 2-1: Typical mix design proportion of ECC by weight (Li, 2003) 14

Table 3-1: Chemical composition and physical properties of Portland cement, Class-F \& CI fly ashes

Table 3-2: ECC mixture proportions 48

Table 4-1: ECC mixture proportions with the addition of $\mathrm{MgO}$ 58

Table 4-2: TGA decomposition temperature for already burned MgO powder samples by kiln . 66 Table 4-3: $\mathrm{MgO}$ powder behavior before* and after* utilizing them into ECC-MgO self-healing system 84

Table 5-1: ECC mixture proportions produced from different combinations of fly ash and $\mathrm{MgO}$ contents 92

Table 5-2: Compressive strength of pre-cracked ECCs with and without MEA after autoclave test at 28 days 111

Table 6-1: Typical ECC mixture proportions with and without the addition of $\mathrm{MgO}$ 118

Table 6-2: Preparation of the compressive strength cubic specimens 119

Table 6-3: Preparation of the load strength prismatic specimens 121

Table 6-4: Compressive strength of ECC and ECC-MgO controls . 128

Table 6-5: Compressive strength of pre-cracked ECC mixtures 131

Table 6-6: Maximum load/strength and ultimate deflection of ECC and ECC-MgO controls at different ages and curing conditions 135

Table 6-7: Maximum load/strength and ultimate deflection of self-healed ECC and ECC-MgO specimens over 10 months of water and natural curing conditions 140

Table 6-8: Differences in UPVs between first and last day of water and natural cured MC/Failed ECC and ECC-MgO specimens 148 
Table 6-9: Maximum load/strength and ultimate deflection of water and natural cured self-healed ECC and ECC-MgO specimens over long term

Table 6-10: Crack characteristics of ECC and ECC-MgO specimens before and after failure cured under water and natural conditions. 154

Table 6-11: Maximum load strength and ultimate deflection of multiple damaged ECC and ECC$\mathrm{MgO}$ specimens over long term of water and natural curing conditions

Table 7-1: RCP property of water and natural cured ECC and ECC-MgO specimens .....

Table 7-2: Sorptivity index, $\mathrm{S}_{\mathrm{o}}$ of ECC and ECC-MgO specimens cured under water and natural conditions 183

Table 8-1: Link Slab Specimens configurations, loading and curing conditions 205

Table 8-2: ECC and ECC-MgO mixture proportions 208

Table 8-3: Compressive strength of ECC mixtures 209

Table 8-4: Flexural and deformation properties of ECC mixture. 209

Table 8-5: Summary of load-deflection and moment-rotation responses 219

Table 8-6: Peak load/deflection/energy absorption/stiffness capacity of link slabs w.r.to control 220

Table 8-7: Maximum strain in reinforcing steel/ECC and load at steel yielding ...... 225

Table 8-8: Crack characterization of ECC and ECC-MgO link slabs 226 


\section{List of Figures}

Figure 2-1: Tensile stress-strain curve and crack width development (Weimann and Li, 2003)...

Figure 2-2: Ductile response of ECC under flexural loading (Li, 2011) ............................... 16

Figure 2-3: Joint free bridge deck with link slab (Hossain and Anwar, 2014) ......................... 35

Figure 2-4: Schematic of link slab showing components ............................................... 36

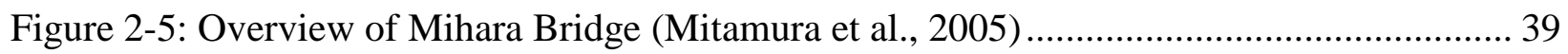

Figure 2-6: (a) Patch repair on a bridge deck. (b) Crack width development in concrete patch and

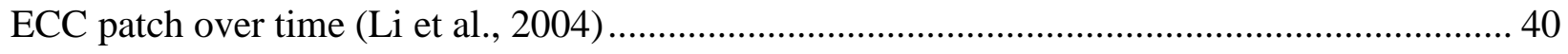

Figure 2-7: ECC link slab on Grove Street Bridge, Michigan, USA (Lepech and Li, 2008) ....... 41

Figure 3-1: Experimental methodology flowchart ....................................................... 43

Figure 3-2: X-ray diffraction of pure $\mathrm{MgCO}_{3}$ used to prepare $\mathrm{MgO}$-type expansive agent......... 45

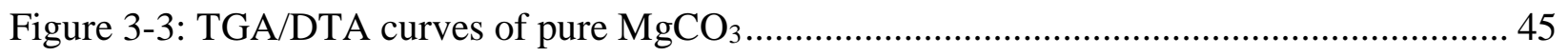

Figure 3-4: Grain size distribution of Portland cement, Class-F fly ash and silica sand (SS)..... 46

Figure 3-5: (A) High-pressure steam vessel - autoclave test and (B) special autoclave racks made

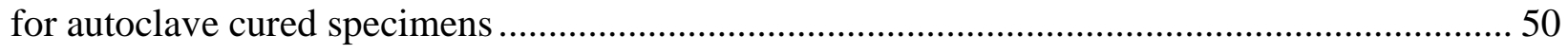

Figure 3-6: Mixing sequences of ECC mixtures (Sherir, 2012) ......................................... 52

Figure 3-7: (A) Compressive strength test set up and (B) Tensile splitting test set up to induce

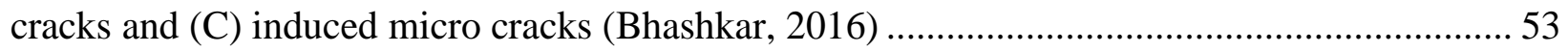

Figure 3-8: (A) Four-point bending test setup and to induce cracks and (B) induced micro cracks 53

Figure 4-1: Cooling down MgO samples suddenly at room temperature at the end of calcination

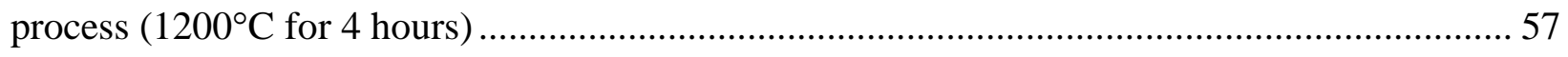

Figure 4-2: Weight loss of $\mathrm{MgCO}_{3}$ material after burning by kiln represented by $\%$ of $\mathrm{CO}_{2} \operatorname{loss} 63$ 
Figure 4-3: Effect of calcination temperature and 2 hours of holding time on grain size of MEA 63

Figure 4-4: Weight loss of $\mathrm{MgCO} 3$ material after burning by kiln represented by $\%$ of $\mathrm{CO}_{2}$ loss

Figure 4-5: Decomposition temperature in terms of holding times of already burned $\mathrm{MgO}$ powder samples represented by $\%$ of remaining hydration acquired by TGA test

Figure 4-6: Decomposition temperature in terms of calcined temperature of already burned $\mathrm{MgO}$ powder samples represented by $\%$ of remaining hydration acquired by TGA test 68

Figure 4-7: Weight loss of $\mathrm{MgO}$ samples by kiln and TGA burning process represented by $\mathrm{CO}_{2}$ loss 70

Figure 4-8: $\mathrm{MgO}$ expansions for autoclave cured ECC-MgO bar specimens in terms of temperature 73

Figure 4-9: $\mathrm{MgO}$ expansion readings for autoclave cured ECC-MgO bar specimens in terms of holding times.. 75

Figure 4-10: Similar behavior of MEA in Powder state and after utilized in the production of autoclave cured ECC-MgO bar specimens in terms of temperatures and holding times 76

Figure 4-11: ECC-MgO bar specimens made of Class-F fly ash cured at $20^{\circ} \mathrm{C}$ in water for 500 days (A \& D: 1 hour holding time, B \& E: 2 hours holding time and C \& E: 4 hours of holding time) 77

Figure 4-12: ECC-MgO bar specimens made of Class-CI fly ash cured at $20^{\circ} \mathrm{C}$ in water for 500 days (A \& D: 1 hour holding time, B \& E: 2 hours holding time and C \& E: 4 hours of holding time) 80

Figure 4-13: Water cured ECC-MgO bar specimens made of Class-F fly ash for different holding times. 82

Figure 4-14: Relationship between all MgO expansion readings for both autoclave and water ECC$\mathrm{MgO}$ bar specimens as a function of temperature (A: Class-F fly ash \& B: Class-CI fly ash)..... 86 
Figure 4-15: Self-healing of ECC-MgO concrete at different ages..................................... 88

Figure 5-1: Expansion of autoclaved ECC-MgO bar specimens with two types of fly ash ......... 96

Figure 5-2: Expansions for autoclaved ECC-MgO bar specimens with cement replacement of fly

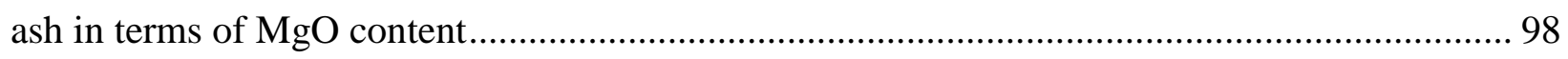

Figure 5-3: Expansion of water cured ECC-MgO specimens made of different fly ash and $\mathrm{MgO}$

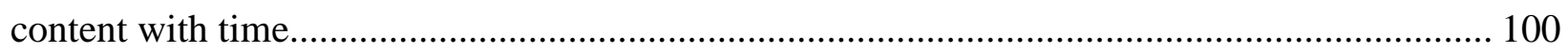

Figure 5-4: Expansion of ECC-MgO specimens made of different FA and $\mathrm{MgO}$ contents as a function of time (part A \& B: 150 days, and part C \& D: 50 days) ...................................... 101

Figure 5-5: Comparison of water and autoclave ECC-MgO expansions as a function of $\mathrm{MgO}$ contents, (part A: last readings at 150 days, part B: highest readings within first 50 days) ....... 103

Figure 5-6: Best $\mathrm{MgO}$ content utilized for ECC-MgO self-healing system based on water and

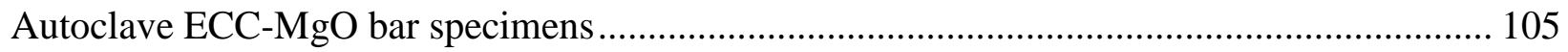

Figure 5-7: Effect of high volumes of $\mathrm{MgO}$ content on water cured ECC-MgO bar specimens with different cement replacements as a function of time ....................................................... 106

Figure 5-8: Microstructure of ECC-control without the addition of MEA at different ages (7, 14 and 28 days), cured in water at $20^{\circ} \mathrm{C}$

Figure 5-9: Microstructure of ECC-MgO matrix with the addition of MEA at different ages (7, 14 and 28 days), cured in water at $20^{\circ} \mathrm{C}$

Figure 5-10: Compressive strength development and recovery ECC-MgO specimens before and after autoclave test at the age of 28 days 112

Figure 6-1: Natural curing condition 123

Figure 6-2: Four-point bending test setup for sideway specimens (Sherir, 2012)..... 124 Figure 6-3: Compressive strength of ECC and ECC-MgO controls as a function of time and curing condition. 129

Figure 6-4: (A); The evaluation of self-healing property of ECC mixtures and (B); ECC-MgO mixtures based on compressive strength as a function of time. 132 xvii 
Figure 6-5: Reloading of pre-cracked water and natural cured ECC \& ECC-MgO specimens, 50\% (left) and $80 \%$ (right)

Figure 6-6: Self-healing assessment of multiple re-loading of ECC \& ECC-MgO specimens through compressive strength recovery under water and natural curing 134

Figure 6-7: Maximum flexural strength and ultimate deflection of ECC and ECC-MgO controls as a function of time cured under water and natural conditions 136

Figure 6-8: Trend development of maximum load of ECC and ECC-MgO controls as a function of time 137

Figure 6-9: (A) Trend of development of UPV of ECC \& ECC-MgO controls as a function of time and (B) differences in UPVs between first and last day of curing. 138

Figure 6-10: Load vs deflection curves of water and natural cured control and self-healed ECC specimens over different periods

Figure 6-11: Load vs deflection curves of water and natural cured control and self-healed ECC$\mathrm{MgO}$ specimens over different periods

Figure 6-12: Recovery loads of water and natural cured ECC and ECC-MgO specimens loaded at different stage loadings as a function of time

Figure 6-13: Comparison between load recovery of multiple cracked (left) and failed (right) ECC and ECC-MgO specimens cured under water and natural conditions as a function of time ...... 146 Figure 6-14: (A) Trend of UPV development with time for water and natural cured MC/Failed ECC and (B) MC/Failed ECC-MgO specimens compared to ECC-controls

Figure 6-15: Maximum load vs ultimate deflection curves of self-healed ECC and ECC-MgO specimens over 15 months of curing under water and natural conditions 150 Figure 6-16: Strength recovery (part-A), deflection development (part-B), Energy absorption recovery (part-C) and UPV development (part-D) of water \& natural cured ECC and ECC-MgO specimens over 15 months of curing 152 
Figure 6-17: Formation of new cracks after reloading to failure of self-healed natural or water cured ECC and ECC-MgO specimens: old cracks in green formed at 7 days (after pre-loading case), new cracks in blue formed after failure loading applied after 450 days of self-healing....

Figure 6-18: Surface observation of self-healed ECC and ECC-MgO specimens under water and natural curing conditions over 450 days - cracks were observed before loading to failure at 450 days 156

Figure 6-19: Load vs ultimate deflection curves of multiple damaged long term water and natural cured ECC and ECC-MgO specimens 158

Figure 6-20: Strength recovery (part-A \& B) and UPV development (part-C \& D) of multiple damaged ECC and ECC-MgO specimens over long term of water and natural curing conditions 160

Figure 6-21: Deflection development (A \& B) and energy absorption (C \& D) of multiple damaged ECC and ECC-MgO specimens over long-term water and natural curing 161

Figure 6-22: (A) Load vs. ultimate deflection curves of ECC and ECC-MgO controls and (B) precracked ECC and ECC-MgO specimens before and after autoclave curing ...... 163

Figure 6-23: (A) UPV development of ECC and ECC-MgO controls and (B) pre-cracked ECC and ECC-MgO specimens before and after autoclave curing (AC) 164

Figure 7-1: Natural curing condition 169

Figure 7-2: Test setup for heat of hydration (A) and rapid chloride permeability test (B)........ 170

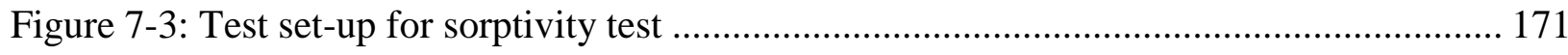

Figure 7-4: Test setup for freezing and thawing cycles test ......................................... 174

Figure 7-5: Heat of hydration of ECC and ECC-MgO mixes ........................................... 176

Figure 7-6: Expansion/drying shrinkage of water and laboratory (air) cured ECC and ECC-MgO specimens 177 
Figure 7-7: RCP property of water and natural cured ECC and ECC-MgO specimens as a function of time 181

Figure 7-8: Sorptivity index $\left(\mathrm{S}_{\mathrm{o}}\right)$ as percentages for water and natural cured ECC and ECC-MgO controls and pre-loaded specimens as a function of time 184

Figure 7-9: Sorptivity index $\left(\mathrm{S}_{\mathrm{o}}\right)$ as percentages for autoclave cured ECC and ECC-MgO controls and pre-loaded specimens 186

Figure 7-10: RCP property of autoclave cured ECC and ECC-MgO sorptivity specimens at 21+180 days 188

Figure 7-11: Relative dynamic modulus of ECC and ECC-MgO prisms based on UPV change after 300 and 600 freeze/thaw cycles 190

Figure 7-12: Mass gain changes of ECC and ECC-MgO prisms with freezing and thawing cycles (left); surface cracking (right)

Figure 7-13: Residual strength and maximum deflection of ECC and ECC-MgO controls at the end of 300 freeze/thaw cycles. 194

Figure 7-14: Residual strength and maximum deflection evolution of pre-loaded ECC and ECC$\mathrm{MgO}$ prisms at 300 and 600 freeze/thaw cycles 195

Figure 7-15: TGA/DSC analyses of ECC/ECC-MgO materials collected from inside the paste $(\mathrm{A} / \mathrm{C})$ and from crack wall $(\mathrm{B} / \mathrm{D})$ at the age of 450 days 196

Figure 7-16: white crystals within the crack walls of ECC and ECC-MgO prisms 197

Figure 7-17: Self-healing of ECC-MgO concrete at the age of 450 days - SEM analyses ........ 200

Figure 8-1: Two-span bridge deformation with focus on link slab section (Kim et al., 2004)... 204

Figure 8-2: Representative full-scale prototype section of link slab, dimensions in mm (Kim et al., 2004) 206

Figure 8-3: Geometry and reinforcement details of model link slabs (dimensions in mm) ....... 207 
Figure 8-4: Casting Process of 1/4 ${ }^{\text {th }}$ Scale link slab specimens; A) mold preparation and casting SCC bridge deck portion; B) casting ECC or ECC-MgO Link slab segment; C) finished link slab specimen 210

Figure 8-5: Schematic representation of test set-up for $1 / 4^{\text {th }}$ scale link slab specimens showing instrumentation (dimensions in $\mathrm{mm}$ )

Figure 8-6: Testing and instrumentation of link slab specimen showing cracking and deformation

Figure 8-7: Link slab natural curing; a) specimens cured under harsh environment for 120 days; b) natural cured specimens at the day of testing after bringing them to the lab.

Figure 8-8: Link slab water curing steps; A) placing the link slab specimens above hot water; B) securing firmly the strain gauges; C) sealing the water container; D) curing link slab specimens for 120 .

Figure 8-9: Load-deflection responses of link slabs 217

Figure 8-10: Moment-rotation responses of link slabs 218

Figure 8-11: Strain development in reinforcing steel for link slab specimens 223

Figure 8-12: A) Crack propagation and failure of pre-loaded link slab specimen; B \& C) Forming cracks in new locations after 28 days +4 months of self-healing 227 


\section{CHAPTER ONE}

\section{INTRODUCTION}

\subsection{General}

Concrete is a very important material that has been used widely in the construction of different infrastructures. The annual global production of concrete is estimated at 5.30 billion $\mathrm{m}^{3}(\mathrm{Ng}$ and Justnes, 2016). To meet the high demand for concrete, cement is worldwide produced with an annual estimate of 3.5 billion tonnes in 2011 (Gao et al., 2016). Among others, the excellent resistance to aggressive waters, ability to be cast into different shapes and low cost made concrete a predominant material for construction. However, low tensile strength and ductility make concrete a brittle material and limits its use in certain applications. Concrete cracks are one of the consequences of this brittleness, and they can dramatically affect the service life of structural elements. The damages in concrete structure augmented and the cost of maintenance increased once the service life of structural element deteriorated with time. Therefore, the need for new technology in cement-based materials accompanied with higher durability is essential. Different studies and practical experiences have observed that the hydration of concrete can heal its inherent cracks by itself. Consequently, material scientists started to inspire by the idea of "self-healing" which has often come from biomimicry such as the natural process of blood clotting or healing fractured bones. However, using the same concept in engineering materials was challenging due to the complicated nature of healing processes (Ghosh, 2009; Mehta and Monteiro, 2006; Mindess et al., 2003).

Early studies have proven that concrete cracks can be healed by themselves when concrete exposed to water over time (Mihashi and Nishiwaki, 2012). Among others, subsequent crack-healing studies can be divided into two self-healing techniques: the first is "intrinsic healing" which has indicated that the crack-healing phenomenon in concrete might be due to several factors such as 
(a) further hydration of un-hydrated cementitious particles (Heide and Schlangen, 2007; Şahmaran et al., 2008; Schlangen et al., 2006); (b) swelling of matrix (Calcium Silicate Hydration) products (C-S-H) (Tittelboom and De Belie, 2013); (c) subsequent carbonation of $\mathrm{Ca}(\mathrm{OH})_{2}$ to produce $\mathrm{CaCO}_{3}$ precipitation; and (d) closing the cracks by water impurities or by concrete loose particles due to concrete cracking may also result in self-healing (Ghosh, 2009). It has been concluded by Edvardsen (1999) that the precipitation of $\mathrm{CaCO}_{3}$ is considered as the main reason of autogenous crack healing (Edvardsen, 1999). The second technique is divided into two sub-groups; which are vascular and capsules based healing that can sense the damage with or without human intervention, respectively. Both techniques depend on embedding adhesive reservoirs within the matrix for selfrepair. However, some technical defects had occurred when the chemical agents did not present simultaneously at the damaged regions. Further, permanent cavities were formed inside concrete after consuming the whole reservoir leading to durability reduction. Among all promising techniques, it was concluded that only intrinsic healing studies were culminated by achieving a significant reduction in crack width, less water permeability and improvement/recovery in mechanical properties (Dry, 1996, 1994, 2001; Ghosh, 2009; Mihashi and Nishiwaki, 2012; Wu et al., 2012).

Over the last decades, new types of cement and concrete based materials such as fiber reinforced concrete and shrinkage compensated concrete have been developed with tensile strain greater than 1\%. Recently, Engineered Cementitious Composite (ECC) technology has emerged from laboratory testing to field applications. ECC is a special type of High Performance Fiber Reinforced Cementitious Composite (HPFRCC) tailored to achieve multiple cracking and tensile strain capacity of 300 to 500 times higher than normal concrete by using only $2 \%$ of fiber content ( $\mathrm{Li}, 2003,1997)$. The formation of closely spaced multiple micro-cracks in ECC is necessary to achieve higher ductility even under ultimate loadings. By controlling the ECC's crack width less than $60 \mu \mathrm{m}$, autogenous crack healing can be stimulated, and cracks might heal completely and thus, improving the durability and mechanical properties of structural elements (Herbert and Li, 2013).

Supplementary Cementing Materials (SCMs) such as fly ash (FA) have been widely used to produce effective FA-ECC mixtures. Further to economic reasons and reducing the environmental 
burdens, the addition of fly ash has improved the fresh state workability of ECC which facilitated well fiber dispersion (Mehta, 1985; Mindess et al., 2003). Both spherical shape and smaller size of fly ash particles reduced the demand for water and enhanced the density of fiber/matrix interfacial zones in ECC, respectively (Mindess et al., 2003; Wang and Li, 2007). Once unhydrated fly ash particles acted as filler, the frictional bond within ECC matrix improved leading to reduce the steady-state crack openings and thus, enhanced the durability against aggressive waters. The use of high volume FA (HVFA) in concrete matrix reduces the heat of hydration and thermal stresses significantly, provides less cracking and enhances the structural integrity in mass concrete (Bisaillon et al., 1994; Langley et al., 1992; Mavani, 2012; Thomas et al., 1995). Also, the use of HVFA can reduce the matrix toughness which allowed for more multiple-cracking formation (Lepech and Li, 2009a; Wang and Li, 2007; Yang et al., 2007).

Different to conventional curing methods, new curing strategies have been developed to mitigate the concern of autogenous shrinkage in mass concrete. Internal curing agents such as saturated lightweight aggregates and Superabsorbent Polymers (SAP's) were used as additives to compensate the shrinkage behavior of mass concrete. Recently, SAP was used as a self-healing agent in ECCs and served as a water reservoir for internal curing (Kim and Schlangen, 2011; Sahmaran et al., 2009; Snoeck et al., 2012a, 2012b). Moreover, Mo et al. (2012) revealed that the shrinkage of cement pastes can be compensated effectively with the use of MgO-type expansive additive (MEA) compared to expansive conventional additive of sulfo-aluminate (ettringite) (Mo et al., 2012). Lower water demand of MEA compared to ettringite can form chemically stable "magnesium hydroxide" $\mathrm{Mg}(\mathrm{OH})_{2}$ which is considered the dominant part to compensate concrete autogenous shrinkage.

Because of high $\mathrm{MgO}$ content in cement clinker, a number of bridges in France failed in 1884 due to concrete expansion two years after construction. Around the same time, a German hall was demolished and rebuild due to cracking related to expansions. The $\mathrm{MgO}$ content in German cement was $27 \%$ while it was up to $30 \%$ in French cement. Accordingly, it was agreed that $\mathrm{MgO}$ content should be limited to $\leq 5 \%$ in cement. Accordingly, (ASTM C150, 2016) approved that the amount of $\mathrm{MgO}$ content should be limited to $6 \%$ in cement along with a maximum expansion of $0.50 \%$ (currently $0.80 \%$ ). Typical autoclave expansion test, adopted in 1940, is currently the standard test 
method to evaluate the expansion effect of $\mathrm{MgO}$ (Helmuth and West, 1998). However, limiting the $\mathrm{MgO}$ content alone was not enough to protect concrete from expansion. It was discovered that high contents of $\mathrm{C}_{3} \mathrm{~A}$ and free $\mathrm{CaO}$ can also lead to unsoundness in cement (Mehta, 1978). However, Gonnerman et al. (reported by Helmuth \& West (Helmuth and West, 1998)) revealed earlier that suddenly cooled clinkers with low free $\mathrm{CaO}$ content will not show disruptive expansions with cement containing up to $6 \%$ of $\mathrm{MgO}$ content.

During the construction of Baishan dam in China (1966-1976), less attention paid for controlling temperatures in such mass concrete. However, no cracking was developed in concrete dam foundation even though the concrete was exposed for consecutive seasons of very hot and cold weathers. The amazing ability of dam's concrete to sustain severe weather was related to the existence of $5 \%$ of $\mathrm{MgO}$ content in cement. The effect of delayed $\mathrm{MgO}$ expansion at later ages compensated effectively the thermal shrinkage in mass concrete (Du, 2005; Mo et al., 2014).

It should be noted that most previous studies have focused on the expansion effect of "heavily burnt" $\mathrm{MgO}$ already contained in cement clinker (burning temperature is around $1450^{\circ} \mathrm{C}$ ) which will cause harmful expansions in concrete structures at later ages. Another approach that used "lightly burnt" $\mathrm{MgO}$ with burning temperature ranges between $850^{\circ} \mathrm{C} \sim 1200^{\circ} \mathrm{C}$ was adopted to prepare $\mathrm{MgO}$ separately and use it as concrete additive based on calcination temperature and holding time (Neville, 1995). This approach was used in China in the 1970s and reported by Mehta as well in 1980. However, the research idea of Mehta was only performed in the laboratory while the novel concrete technology of using $\mathrm{MgO}$ expansive agent has been used effectively in China and up to date over 30 dams have been constructed (Mo et al., 2014).

The existence of multiple cracks with tight widths, in addition to the presence of un-hydrated cementitious particles due to low w/c ratio can stimulate the self-healing property in ECC. The accumulation of moisture in ECCs around the un-hydrated cementitious particles within micro cracks can activate the chemical cement hydration to form C-S-H products (Şahmaran et al., 2008). In addition, the presence of carbon dioxide $\left(\mathrm{CO}_{2}\right)$ in moisture which flows within the crack cavities can lead to more diffusion process of calcium ions located at both crack surfaces and in deeper locations inside hardened cement pastes; leading to form $\mathrm{CaCO}_{3}$ precipitations and stimulate 
autogenous crack healing (Edvardsen, 1999). Moreover, the negatively charged hydroxide ions at Polyvinyl Alcohol (PVA) fibers in ECCs can help to attract more calcium ions from hardened cement pastes to form $\mathrm{Ca}(\mathrm{OH})_{2}$ to be consumed by pozzolanic reactions to form newly hydration products (Homma et al., 2009; Nishiwaki et al., 2012). With the help of all aforementioned factors, ECC can promote the crack self-healing property.

However, it was revealed that high demand of water is essential in any self-healing study to trigger the autogenous healing property; which is not always available during the lifetime of concrete structural members (Qian et al., 2010). Hence, low w/c ratio in impermeable ECC matrix could be an obstacle to implement long-term and continuous hydration which is among other factors considered to stimulate self-healing property. In addition, one of the most challenges in the studies of self-healing is the weakness in cohesion force of newly formed self-healing materials within the crack walls compared to primary hydration products (Tittelboom and De Belie, 2013). Low cementitious properties of newly formed fillers within the cracks of ECC have motivated old cracks to reopen again when reloading applied at same damaged regions (Qian et al., 2010). In order to overcome these obstacles, the MgO-type expansive additive was used as self-healing agent due to less water demand than other expansive additives to form chemically stable "magnesium hydroxide" $\operatorname{Mg}(\mathrm{OH})_{2}$. This will help to compensate the drying shrinkage in concrete featured with low w/c ratio and thereby might sealing the cracks effectively (Mo et al., 2012). Further, due to the delayed formation of $\mathrm{MgO}$ hydration which is characterized as cementitious based materials, MEA can provide more cohesive forces within the faces of crack walls at a later age and supplement the early formation of C-S-H products. Therefore, the cementitious behavior of MEA might help to form cracks in new locations even after concrete samples reloaded again at same damaged areas.

Since FA-ECC mixtures enhanced the strain hardening and multiple-cracking behaviors featured with tight crack width less than $60 \mu \mathrm{m}$, while MEA enhanced effectively concrete microstructure by providing cohesive cementitious based materials at later ages featured with low water demand, upon the validation of this concept, ECC-MgO self-healing system can be successfully implemented to promote self-healing phenomenon in structural applications. 


\subsection{Research significance}

For decades, extensive studies have been done to improve the brittle failure of high strength concrete materials which caused non-controlled cracking behavior in such materials; leading to premature deterioration of concrete structures. The discovery of emerging ductile materials, such as ECC, has been giving hope to construct structures with smaller crack widths that resist the ingress of different aggressive agents and reduces corrosion and spalling.

The aim of this research is to develop new techniques/methods to induce self-healing capabilities in ECC materials featured with tight crack width by using $\mathrm{MgO}$ as cement self-healing additive. The successful development of self-healing techniques based on ECC-MgO system can revolutionize the concrete industry and improve infrastructure durability through maintenance free, sustainable and cost effective construction. Consequently, the self-healing development of ECC-MgO materials could be utilized successfully in different engineering applications; such as expansion joints in bridges.

Rehabilitation of bridge structures damaged by continuous wear, as well as time-dependent and environmental effects is a major concern for a large number of reinforced concrete bridge structures. Every year, in North America, billions of dollars are spent to repair and maintain bridges. As bridge structures ages, the cost of repairs and maintenance magnifies. The poor durability of concrete bridges throughout Canada is an increasingly large concern for highway transportation authorities. With decreasing budget allocations for infrastructure maintenance, rehabilitation, and replacement, the need for greater durability is apparent. The leaking expansion joints, which are considered as a major source of deterioration of multi-span bridges in Canada, allow salt water to contact the steel girder and rebar, causing corrosion and eventually leading to structural failure. Therefore, expansion joints could be replaced by flexible link slabs made with ECC-MgO materials forming a jointless multi-span bridge with self-healing ability and hence, solving permanently the problem of premature deterioration (Caner and Zia, 1998; Hossain et al., 2012). 
Upon the validation of this concept, ECC-MgO self-healing system can open a new door for future research in self-healing processes; which is compatible with the aspirations of U.S. Congress to the scientific research priorities that self-healing process is one of them.

\subsection{Research Objectives and Scope}

The main objective of the proposed research was to develop new techniques to induce self-healing phenomena in ECC that can stop further deterioration and recover most of the original characteristics of concrete structures. So far, no research has been done on producing ECC with the combination of MEA as self-healing agent and SCMs. This research explored experimental methods and testing procedures to assess the effectiveness of $\mathrm{MgO}$-type expansive agent incorporated in ECC to promote self-healing function under different environmental exposures for the short and long-term duration. Also, the effects of mix design parameters such as amount/type of SCMs (in combination with MEA) on self-healing were analyzed based on an extensive experimental investigation on ECC and $\mathrm{ECC}-\mathrm{MgO}$ specimens. This lead to the identification of best self-healing techniques and the development of ECC-MgO mixtures with optimized selfhealing capabilities (measured based on crack closing, strength recovery and micro-structural characteristics) for structural applications.

To achieve the objective, the research had been conducted on four following major themes to study the performance of proposed self-healing ECC-MgO system extensively.

Firstly, material exploration was conducted to:

- Find out the desired calcination system for MEA by exposing two different particle sizes $\leq 45 \mu \mathrm{m}$ and $45 \sim 100 \mu \mathrm{m}$ of $\mathrm{Mg}(\mathrm{CO})_{3}$ raw material under $750^{\circ} \mathrm{C}, 900^{\circ} \mathrm{C}, 1050^{\circ} \mathrm{C}$ and $1200^{\circ} \mathrm{C}$ for 1,2 and 4 hours, respectively.

- Find out the best MEA amount (studied from 0\% up to 30\%) and best types/amounts of SCMs (30\% and 55\% of Class-F or Class-CI as cement replacements) to be used to fit the essential requirements of sealing/filling micro-cracks without jeopardizing ECC durability. 
The material exploration was introduced through investigating the hydration activity of MEA in powder state before and after utilizing them in extensive water and autoclave cured linear expansion tests on bar specimens; in addition to studying the densification effect of MEA on ECC microstructure through Scanning Electron Microscopy (SEM) analysis and compressive strength development/recovery of autoclave cured cube specimens.

Secondly- after finding the desired calcination system, MEA amount and best SCM perfectly fit for producing self-healing ECC-MgO system, a broad research was conducted to investigate the mechanical properties of virgin (un-cracked/undamaged)/pre-cracked (damaged/multiple damaged under repeated loading events) specimens made of proposed ECC-MgO self-healing system and control ECC (without MEA) under the different environmental exposures (water and natural/field curing up to 450 days as well as laboratory and autoclave curing conditions). The self-healing capability of ECC-MgO specimens was analysed compared with their ECC counterparts based on development/recovery of compressive/flexural strength/load resistance, ultrasonic pulse velocity, heat of hydration and expansion/drying shrinkage in addition to crack healing/cracking characteristics and micro-structural characterizations through Thermogravimetric Analysis (TGA)/Differential Scanning Calorimetry (DSC) and Scanning Electron Microscopy (SEM).

Thirdly, wide-ranging durability tests were conducted on virgin (un-cracked/undamaged)/precracked (damaged/multiple damaged under repeated loading events) specimens to investigate the self-healing performance of ECC-MgO system (compared to control ECC) through development/recovery of rapid chloride ion permeability (up to 156-days), water absorption/sorptivity (up to 180-days), freeze/thaw resistance (up to 300 cycles and 600 cycles) and load (strength)/maximum deflection (after 300 or 600 cycles for freeze-thaw) in addition to micro-structural characterization by TGA, DSC and SEM analyses.

Fourthly, self-healing ECC-MgO mixture was employed to construction link slab used for jointfree bridge deck construction. The self-healing ability of ECC-MgO system was evaluated through structural performance (regarding strength/stiffness/energy absorbing/crack-healing capacity recovery) of damaged and subsequently healed water/field cured (for four months) link slab 
specimens. Such test results will facilitate to create guidelines for newly developed ECC-MgO mixtures that can be used with confident in the local concrete industry to construct sustainable, cost effective and self-healed concrete structures in general, and for link slab application in particular.

Overall, this research provided guidelines for the optimization of ECC-MgO mix proportioning and illustrated the performance of proposed ECC-MgO system based on the analyses of test results to ensure satisfactory mechanical, durability and self-healing properties. This research will significantly contribute to the implementation of ECC-MgO system with self-healing characteristics in future sustainable construction with enhanced service life. 


\subsection{Thesis outline}

Chapter 1 introduced self-healing ECC-MgO system in general and described objectives, scope and significance of the research.

Chapter 2 presented the background of early self-healing studies in addition to ECC, a literature review of intrinsic self-healing property in ECC, other methods of self-healing, $\mathrm{MgO}$ concrete and a brief summary of bridge deck link slab applications.

In Chapter 3, research program and methodology, materials used and crack creation methods in different types of ECC specimens.

"Due to the high number and types of specimens, test procedures and test preparations used in this research, experimental investigations and test procedures are presented before results and discussions sections for each technical chapter."

In Chapter 4, the results and discussions of exploring the calcination system which fit the requirements of self-healing ECC-MgO system are presented (material investigation - part 1).

In Chapter 5, results and discussions of obtaining the best combination of SCM and $\mathrm{MgO}$ dosages for self-healing ECC-MgO system are presented (material investigation - part 2).

In Chapter 6, the performance of ECC-MgO self-healing system was tested and discussed based on the mechanical properties.

In Chapter 7, the development of ECC-MgO system was tested and discussed based on fresh state and mechanical properties while the self-healing property of the system was evaluated based on durability properties.

In Chapter 8, the structural performance of self-healing bridge deck link slabs subjected to monotonic static loading was discussed and tested.

The conclusions of the research and recommendations for future research studies are presented in Chapter 9. Also, Chapter 10 introduced appendices. 


\section{CHAPTER TWO}

\section{LITERATURE REVIEW AND BACKGROUND}

\subsection{Introduction}

Cracking in concrete structures can occur due to many different reasons. Among others, errors in structural design, poor construction practices or undesirable interactions with the surrounding environment can cause concrete cracks in structural elements. Concrete cracks can be very small microcracks connected to each other throughout the concrete microstructure causing considerable internal damages before becoming visible at the exterior surfaces. In many cases, the presence of concrete cracks might not dramatically affect the load carrying capability of structural elements. However, the long-term durability of the concrete structure can be affected directly by allowing easy ingress of aggressive agents which might accelerate the concrete deterioration (Mindess et al., 2003). Therefore, the development of new concrete technologies such as self-repairing is essential to solving autogenously the problems related to both reliability and durability of concrete elements.

Self-repairing, autonomic-healing or self-healing are terms commonly used to describe this property in materials. Self-healing can be defined as "the ability of a material to heal (recover/repair) damages automatically and autonomously, that is, without any external intervention" (Ghosh, 2009). However, repairing concrete cracking usually needs external human intervention to initiate self-healing.

This chapter covers the general idea of early self-healing studies and their function, what is ECC and its material design consideration, a literature review of self-healing property in ECC, the brief literature of other methods of self-healing, properties of $\mathrm{MgO}$ concrete and a brief summary of jointless bridge deck link slab and ECC structural applications. 


\subsection{Early studies of self-healing}

Crack healing studies which have been done so far can be divided into two groups. The first group is "self-healing studies" which possess an idea that depends on the objective of the crack healing itself; the objective could be either to recover the structural element strength for safety performance or just to fill the cracks of the structural element for durability performance. Another idea for this kind of studies is to investigate the intrinsic self-healing potential of cementitious materials themselves; for instance, studying the continuous reaction of un-hydrated particles and the ability of $\mathrm{CaCO}_{3}$ precipitation (Mihashi and Nishiwaki, 2012; Wu et al., 2012). The second group is “selfrepairing studies" which depends on the embedment of adhesive hollow reservoirs inside the concrete matrix. This group can be divided into two sub-groups. The first sub-group is "passive mode self-repairing" which can sense the damage within the cementitious matrix without any human intervention. Once cracks are formed, the hollow glass fibers will break, and the available chemical agents are released to fill the crack. During the crack filling process, the observed main problem was that the chemical agents inside the broken tubes did not present simultaneously at the same crack location; or they already hardened inside the tubes before flowing inside the cracks. The second sub-group is "active mode self-repairing" which can sense the damage as well but with human intervention. After crack formation, a sensor will actuate a device to vacuum external healing agents through punched tube walls into the matrix. During concrete casting, extra care must be paid to avoid tube breakages which is hard to be ensured during concrete casting process (Dry, 1996, 1994, 2001; Ghosh, 2009; Mihashi and Nishiwaki, 2012; Wu et al., 2012). As a result, it was indicated that the second group "self-repairing mechanisms" cannot work easily in normal concrete due to higher intrinsic crack widths of such composites ( $\mathrm{Li}$ et al., 1998). The chemical healing agents in such concrete matrices will be consumed rapidly once larger cracks occurred. To overcome this problem, crack width has to be controlled otherwise very large hollow tubes are needed to be filled up with enough volumes of chemical agents, leading to a significant reduction in mechanical properties of concrete composites. To do so, Li and fellows have embedded hollow glass fibers filled with glue agents into controlled crack ECCs (Li et al., 1998). The presence of Polyethylene (PE) fibers in ECCs limited the crack widths to the extent that healing agents will be discharged deliberately from broken hollow tubes towards these cracks. Moreover, it was proposed 
that the presence of capillary suction pressures between micro cracks and hollow tubes will draw out most of the healing agents to fill the cracks. However, only a small number of cracks was healed in the first loading cycle while no crack healing at all observed in the second cycle. This inferior performance was attributed to the multiple-cracking behavior of ECC which disturbed the effective distribution of the healing agent (Ghosh, 2009). It was concluded that self-repairing mechanisms had limited the ability of crack healing not only in normal concrete but also in crackcontrolled ECC composites.

Among the aforementioned promising techniques, it was confirmed by many researchers that concrete has a natural ability to heal the inherent cracks by itself. Therefore, the first group of "self-healing studies" was culminated. The autogenous self-healing in concrete cracks is a phenomenon that was discovered by the French Academy of Science in 1836, as observed by (Edvardsen, 1999). By achieving a significant reduction in crack width, less water permeability and improvement/recovery in mechanical properties, the healed cracks will reduce the ingress of aggressive ions into concrete and lower concrete deterioration. As a result, many subsequent experimental studies and practical experiences focused on intrinsic self-healing techniques fall under "self-healing studies", which will be discussed thoroughly in the following sections. However, it was pointed out that attaining relatively small crack width in normal concrete is the most serious challenge to heal the concrete cracks completely (Sahmaran et al., 2013). Therefore, the emerging ECC technology was utilized in this study. Due to the ability to control the crack width to less than $60 \mu \mathrm{m}$ in ECCs, autogenous crack healing could be stimulated easily and inherent cracks might be successfully healed completely and thereby improving long-term mechanical and durability properties of structural elements (Herbert and Li, 2013).

\subsection{Engineered Cementitious Composites (ECC)}

As a special type of HPFRCC, ECC is a fiber reinforced cement-based composite material that has been systematically engineered to achieve high ductility under tensile and shear loading (Li and Kanda, 1998; Li, 2003, 1997; Li et al., 2001). High tensile strain capacity of 3 to $5 \%$ is an important material property that can distinguish ECC from other types of fiber reinforced concrete (FRC). Strain capacity of ECC is realized through forming many closely spaced micro cracks that allow 
strain capacity of over 300 times that of normal concrete. Although ECC has similar components to FRC, the unique ECC characteristic of strain hardening and multiple cracking behaviors are achieved through tailoring the interfacial properties of micromechanical components $(\mathrm{Li}, 1997$, 1993; Lin et al., 1999). In comparison to other HPFRC, only $2 \%$ volume of short discontinuous fiber is used in ECC mix. The typical mix proportions of ECC using a polyvinyl alcohol (PVA) fiber are given in Table 2-1. All proportions are given with materials in the dry state.

Table 2-1: Typical mix design proportion of ECC by weight (Li, 2003)

\begin{tabular}{|c|c|c|c|c|c|}
\hline Cement & Fly Ash & Sand & Water & HRWRA & Fiber (\%) \\
\hline 1 & 1.2 & 0.8 & 0.58 & 0.013 & 2 \\
\hline
\end{tabular}

Different types of fibers have been utilized with ECCs. However, PVA fibers were concluded to be the most effective fibers to achieve the strain hardening and multiple cracking behaviors after forming the first cracking (Weimann and $\mathrm{Li}, 2003$ ). The typical uniaxial tensile stress-strain curve and cracking development of the ECC at 2\% PVA fiber is presented in Figure 2-1.

Even under ultimate loading, the crack width of ECC remained less than $60 \sim 80 \mu \mathrm{m}$ with developing more ECC's tensile stress due to unique strain hardening after first cracking formation. The steady-state cracking behavior is independent of the type of the loading and the amount of fiber reinforcement (Özbay et al., 2013a; Ranade et al., 2014). 


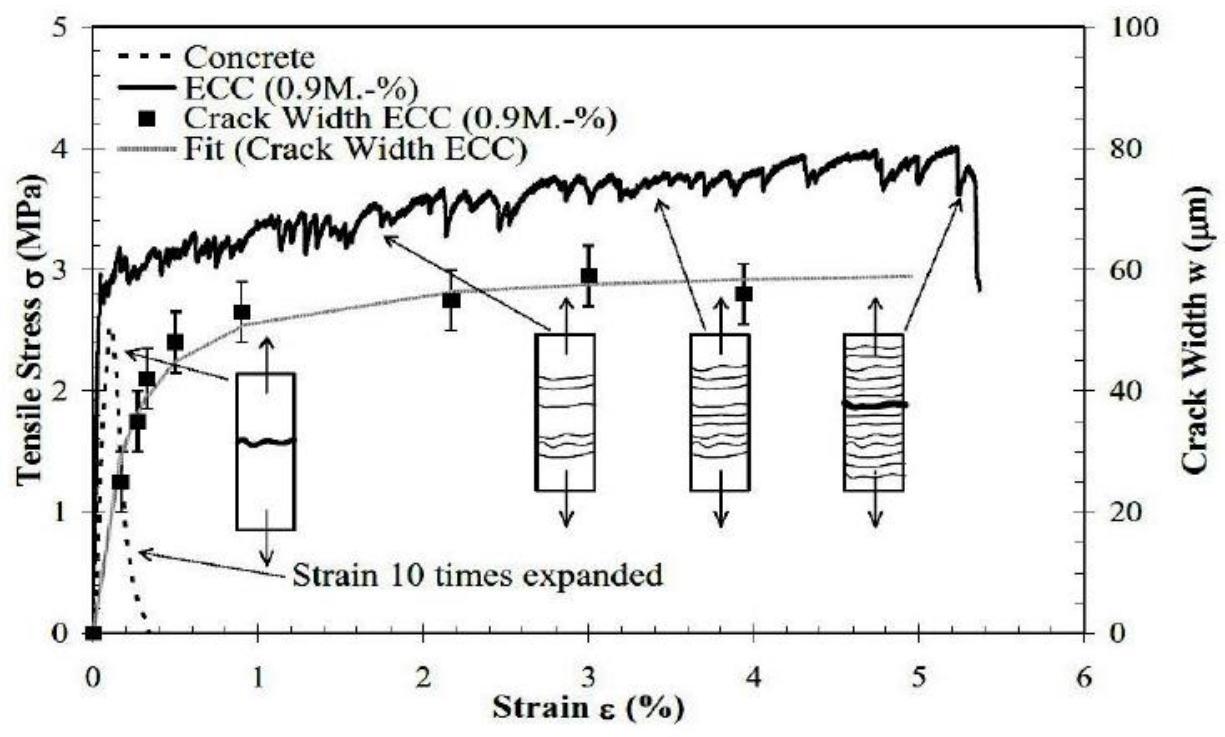

Figure 2-1: Tensile stress-strain curve and crack width development (Weimann and Li, 2003)

\subsubsection{ECC Material Design Considerations}

To achieve strain-hardening behavior in ECC, Kanda \& Li suggested two conditions; the first condition is first crack stress defined as strength criterion and the second is steady-state cracking defined as energy criterion (Kanda and Li, 1998). Both conditions are important to induce multiple-cracking behavior in ECC.

Strength criterion is responsible for initiating micro cracks in the composite and then ensuring the applied tensile loads that created the micro cracks are always less than the maximum capacity of fiber bridging to form additional cracks in the new crack planes. Otherwise, multiple-cracking behavior terminates earlier. Once crack is initiated, energy criterion prescribes switching the Griffith-type crack (the opening of the crack increases while the crack length extends) as in the case of tension-softening behaviour of FRC to steady-state flat-crack propagation mode (the opening of the crack remains constant while the crack length extends) as in the case of strainhardening behaviour of ECC. The crack-type switching is done by ensuring the energy from the applied loads is always less than or equal to the energy absorbed by fiber bridging process (fibers located in the crack opening and near to the crack tip only) by sufficient margin during flat-crack 
propagation. This is done to keep the crack opening constant regardless of the extended crack length. Otherwise, localized crack formation occurs leading to early termination of multiple cracking behavior (Nawy, 2008; Sherir et al., 2014).

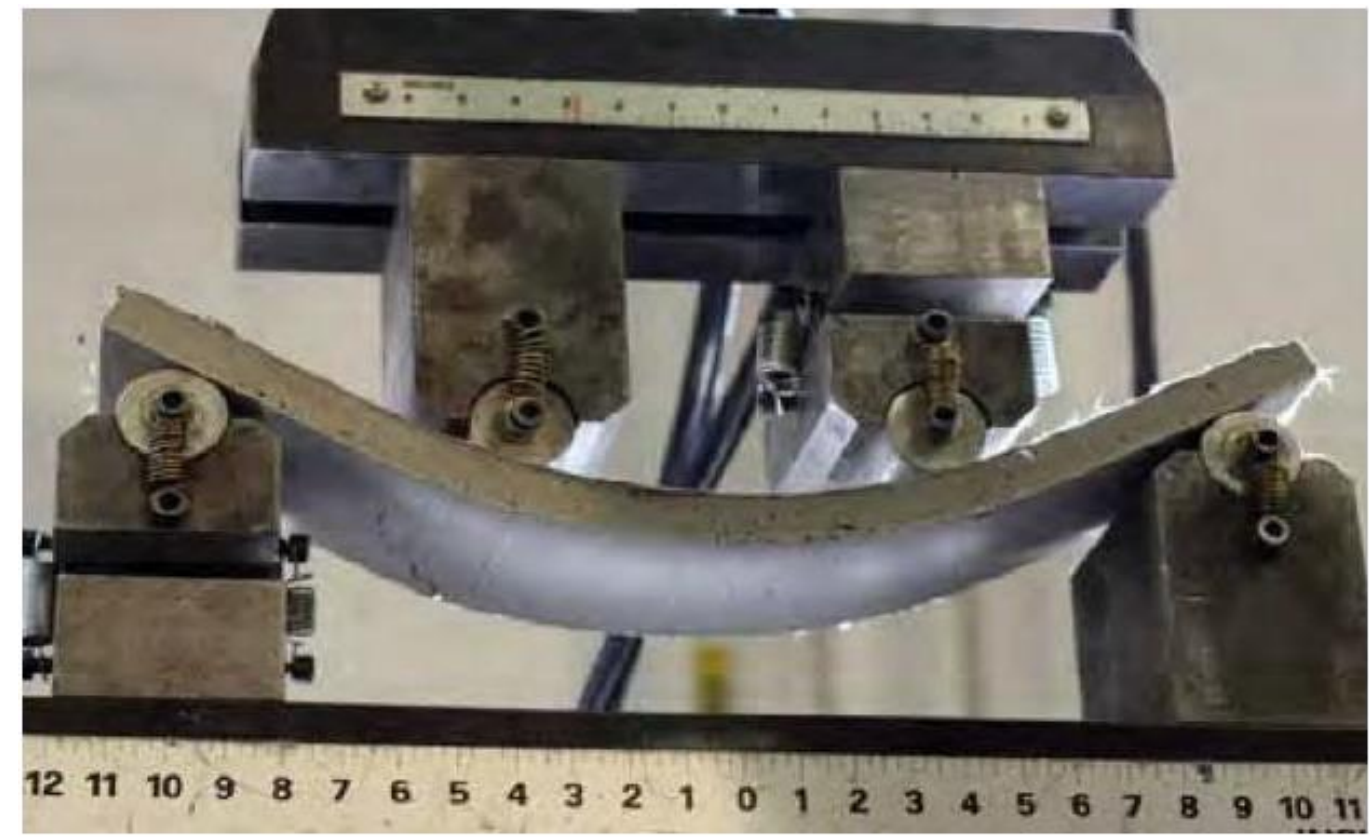

Figure 2-2: Ductile response of ECC under flexural loading (Li, 2011)

It could be concluded that both strength and energy criteria must be considered to ensure adequate initiation of multiple cracks. And once the cracks are initiated, they propagate in a steady-state flat-cracking mode to achieve saturated multiple cracking so that the ductility of the composite is assured (Kanda and Li, 1998; Yang and Li, 2006). The use of these micromechanical models for tailoring ECC material ensures the strain hardening characteristic and large bending capacity (Figure 2-2) similar to a ductile metal plate at its plastic deformation phase (Li, 2011; Nawy, 2008).

Using the same strength and energy criteria, greener ECC was further developed by incorporating locally available crushed/mortar sand aggregates and fly ash/volcanic materials as a replacement for cement (Hossain and Anwar, 2014; Şahmaran et al., 2009; Sherir et al., 2015a, 2014). Although each material influenced the mechanical and durability properties of the hardened ECC, but similar responses were attained as observed in experimental results. 


\subsection{Autogenous (intrinsic) self-healing property}

\subsubsection{Hydration effect on self-healing}

Şahmaran et al. (2008) studied the effect of self-healing on Self-Consolidating Concrete (SCC) incorporating high volume fly ash content of up to $0 \%, 35 \%$ and $55 \%$. It is well known that SCC characterized by lower permeability and dense matrix due to higher amount of fine packed particles content than normal concrete. Although SCC shows early autogenous shrinkage due to lower w/c ratio, it is difficult to crack in early ages compared to normal concrete. But once cracks develop, the permeability in SCC will increase significantly. SCC cylindrical specimens were used and preloaded up to $70 \%$ and $90 \%$ of the maximum compressive load to form micro cracks at the age of 28 days. After pre-loading, the pre-cracked specimens stored in water for one month, and then the loss in the mechanical properties and the increase in permeation properties were monitored every two weeks. Experimental results indicated that specimens with 55\% fly ash content cured in water for one month had only $7 \%$ compressive strength reduction; while specimens without water curing had $27 \%$ compressive strength reduction compared to the virgins. Further, specimens without fly ash content cured in water for one month had only $13 \%$ of compressive strength reduction; while others without water curing had $19 \%$ of compressive strength reduction when related to the virgins. The presence of fly ash in SCC specimens reduced the permeable properties compared to the samples without fly ash. The higher residual strength and the improvement in permeation properties may be attributed to the presence of un-hydrated fly ash particles within the crack walls which can hydrate in the presence of water to promote crack healing. This is signified by the formation of new C-S-H products due to pozzolanic reactions (Şahmaran et al., 2008). Additionally, Heide and Schlangen (2007) and Schlangen et al. (2006) studied the autogenous healing behavior of the concrete cracks when compressive stresses are applied to close the cracks and make their sides contact each other. It was concluded that the un-hydrated cement particles will open the way for further C-S-H products to fill the cracks (Heide and Schlangen, 2007; Schlangen et al., 2006). It should be noted that in the above-mentioned studies, crack healing was stimulated more through un-hydrated cementitious particles when pre-cracked specimens were stored in water. 


\subsubsection{Calcium carbonate effect on self-healing (Chemical ions and pH value)}

Edvardsen (1999) and Mihashi and Nishiwaki (2012) mentioned several important factors that affect the formation of $\mathrm{CaCO}_{3}$ such as high $\mathrm{pH}$ value of water, the presence of chemical ions such as carbon dioxide and calcium ions $\mathrm{Ca}^{2+}$, high water temperature and small crack width (Edvardsen, 1999; Mihashi and Nishiwaki, 2012). The precipitation of $\mathrm{CaCO}_{3}$ will form at $9.8 \mathrm{pH}$ value which lies between $13.5 \mathrm{pH}$ of pore water in hardened cement paste and $5.5-7.5 \mathrm{pH}$ of normal water flow in cracks. The blend of both types of water (pore water and water flow in cracks) has the value of $\mathrm{pH} \approx 9.8$ which is the foundation to trigger the precipitation of $\mathrm{CaCO}_{3}$. Moreover, the formation of $\mathrm{CaCO}_{3}$ crystals was separated into two phases. The first phase is surface controlled crystal growth which is faster than the subsequent second phase which is diffusion controlled crystal growth (Edvardsen, 1999). In surface controlled phase, the normal water $\left(\mathrm{H}_{2} \mathrm{O}\right)$ flow inside the cracks ( $\mathrm{pH}$ value $=5.5$ to 7.5 ) is usually loaded with $\mathrm{CO}_{2}$ from surrounded air which facilitates to form carbonates $\left(\mathrm{CO}_{3}\right)$. Simultaneously, $\mathrm{CO}_{2}$ will start to consume the available $\mathrm{Ca}^{2+}$ ions from both $\mathrm{Ca}(\mathrm{OH})_{2}$ and $\mathrm{C}-\mathrm{S}-\mathrm{H}$ found at shallower locations of hardened cement paste near the crack walls. As a result, $\mathrm{CO}_{3}$ and $\mathrm{Ca}^{2+}$ ions do combine together to form $\mathrm{CaCO}_{3}$ crystals and hence stimulate autogenous crack healing. After the completion of the first phase and once $\mathrm{Ca}^{2+}$ ions "located at shallower locations" are already consumed by $\mathrm{CO}_{2}$, the subsequent diffusion controlled phase starts. In this phase, the low speed of water flows inside the cracks will give $\mathrm{CO}_{2}$ more chance to diffuse more $\mathrm{Ca}^{2+}$ ions from deeper locations of hardened cement paste; leading to more calcite precipitation. It was concluded that the major formation of $\mathrm{CaCO}_{3}$ crystals was completed in the surface controlled phase within the first 3 to 5 days of hydration; which was signified by the significant reduction in crack width and water flow rate. Thereafter, only up to $20 \%$ of $\mathrm{CaCO}_{3}$ crystal was formed in the diffusion controlled phase. This explains why the surface controlled phase is faster than the diffusion controlled phase. As a result, it was pointed out that the dormant cracks (cracks that the repair material will not be affected) with $0.2 \mathrm{~mm}$ crack width healed completely within 7 weeks while active cracks with same crack width healed completely within 16 weeks under water exposure (Edvardsen, 1999). 


\subsubsection{Water temperature effect on self-healing}

The self-healing behavior of cracked samples was studied through permeability tests with fixed temperature of 20,50 and $80^{\circ} \mathrm{C}$ (Reinhardt and Jooss, 2003). When cracks reduced from $0.15 \mathrm{~mm}$ to $0.05 \mathrm{~mm}$ at constant $20^{\circ} \mathrm{C}$, the water flow rate dropped from $75 \%$ to $45 \%$ of the original flow rate. Moreover, when temperature increased from $20^{\circ} \mathrm{C}$ to $80^{\circ} \mathrm{C}$, water flow rate dropped from $45 \%$ to $10 \%$ only when related to original flow rate. It was concluded that the decrease in water flow rate depends on both crack width and water temperature. Higher water temperatures stimulate faster crack healing and smaller cracks to heal faster than bigger cracks as well.

\subsubsection{Crack width effect on self-healing}

\subsubsection{Restriction of crack width under chloride environment}

It was mentioned that the permissible crack width is $150 \mu \mathrm{m}$ at the tensile face of reinforced concrete structures for exposure conditions of seawater, seawater spray, wetting and drying; and $180 \mu \mathrm{m}$ for de-icing chemical exposure according to JSCE and ACI 224R (Li and Li, 2011). In their study, ECC, which was characterized by a tight crack width, was exposed and allowed to heal under aggressive chloride environment. The ECC samples were preloaded by $0.5 \%, 1.0 \%$ and $1.5 \%$ tensile strain levels then immersed in 3\% chloride solution $(\mathrm{NaCl})$ for 30, 60 and 90 days, respectively and finally reloaded to failure. Test results revealed that even under severe marine environment conditions, ECC still exhibits tensile strain capacity greater than $2.5 \%$ and multiple cracking behavior while the average crack width increased from $50 \mu \mathrm{m}$ to $100 \mu \mathrm{m}$ only. The increment in crack width was related to the leaching of calcium hydroxide ions leading to the reduction in fiber/matrix interfacial bonds and thus reducing the fiber bridging capacity. Similar finding was observed by Christophe and Raoul who indicated that the presence of chloride ions accelerated the leaching of calcium hydroxide ions and increased the matrix porosity which can harm the fiber/matrix interfacial bonds (Christophe and Raoul, 1997). However, $100 \mu \mathrm{m}$ crack width is still below the recommended crack width allowed by different design codes for marine structures. It was concluded that tight crack width and dependable tensile ductility in ECC mixtures can promote self-healing in ECC within the crack width even under chloride exposure 
environment. In addition to the tight crack width, the self-healing in ECC can be attributed to the presence of high amount of un-hydrated fly ash particles due to the lower w/c ratio within ECC mixtures and due to the pozzolanic reaction of fly ash as well.

\subsubsection{Restriction of crack width under natural environment}

The self-healing property of ECC was monitored under natural environment exposure (allowing them to heal outdoor) for one year (Herbert and Li, 2013). ECC samples with 70\% fly ash cement replacement were preloaded to tensile strains equal to $0.5 \%$ and $1.0 \%$ of the original values. The average crack width was allowed to reach $30 \mu \mathrm{m}$ only at the age of 7 days then exposed to the natural environment conditions. Consequently, the samples reloaded to the same tensile strain levels at the exposure times of 3, 6, 9 and 12 months, respectively. Resonant frequency (RF) and mechanical re-loadings (such as stiffness and first cracking strength recovery) were measured before and after every preloading to assess the quality of self-healing for ECC samples. Results indicated that RF dropped drastically after a preloading event and then after one week, fluctuating recovery of $95 \%$ to $105 \%$ was observed compared to the virgins. Samples that underwent repeatable damages even after three re-loadings still recovered up to $84 \%$ of original RF values. RF dropped severely after every reloading event due to the reopening to the healed cracks in addition to new cracks formation. The fluctuation in RF (95\% to 105\%) was due to the exposure of $\mathrm{Ca}(\mathrm{OH})_{2}$ within the crack walls to different rainfall events. The concentration of $\mathrm{Ca}(\mathrm{OH})_{2}$ will be washed out easily during the rainfall and may be increased when there is enough rain water inside the crack to convert $\mathrm{Ca}(\mathrm{OH})_{2}$ to more stable C-S-H products. Although large amounts of fly ash in ECC mixtures will lead to more consumption of $\mathrm{Ca}(\mathrm{OH})_{2}$ and convert it to $\mathrm{C}-\mathrm{S}-\mathrm{H}$ products due to the pozzolanic reaction, still the kinetics of this reaction is slower than the dissolution of $\mathrm{Ca}(\mathrm{OH})_{2}$ during the rainfall event. Moreover, strength and first cracking recovery often exceeded $100 \%$ of original values after 6 months of exposure to the natural environment and all micro cracks healed in ECC samples. It was concluded that the self-healing of ECC depends on the weather temperature and the amount of rainfall during the natural environment exposure. Moreover, the self-healing property in ECC is repeatable. It should be emphasized that it was decided to use in this study an ECC mixtures with higher content of fly ash and tighter crack widths of no more than 
$30 \mu \mathrm{m}$ rather than $44 \mu \mathrm{m}$ which was obtained in a previous study for the same authors (Herbert and $\mathrm{Li}, 2013)$.

\subsubsection{Restriction of crack width by using synthetic fibers}

The effect of different types of synthetic fibers on the self-healing capacity of fiber reinforced cementitious composites (FRCC) was studied (Nishiwaki et al., 2012). FRCC Samples were cured in water and strained by applying uniaxial tension tests at the age of 6 days in order to form a crack width of $300 \mu \mathrm{m}$, then strained again at 28 days as well. After the first tension test, samples were subjected to subsequent water permeability tests until the 28 day. Test results revealed that PVA fibers had the best performance in self-healing of FRCC among other types of fibers. PVA has the highest polarity strength due to the higher presence of negatively charged hydroxide ions $\left(\mathrm{OH}^{-}\right)$ which can attract easily lots of positively charged $\mathrm{Ca}^{2+}$ ions within the crack walls and then precipitate $\mathrm{CaCO}_{3}$. It was concluded that the self-healing capability of PVA fibers in FRCC was able to heal concrete cracks up to $300 \mu \mathrm{m}$ in the presence of water. Homma et al. (2009) revealed the same behavior of self-healing in FRCC. The presence of PE fibers accelerated the self-healing phenomenon in FRCC due to the higher polarity of the fibers which can attract more $\mathrm{Ca}^{2+}$ ions within the crack walls and then precipitate $\mathrm{CaCO}_{3}$ (Homma et al. 2009).

\subsubsection{Internal water supply effect on self-healing}

\subsubsection{Superabsorbent Polymers (SAPs)}

The capacity of SAPs swelling depends on both the alkalinity and the ionic concentration $\left(\mathrm{K}^{+}, \mathrm{Na}^{+}, \mathrm{Mg}^{+}\right.$and $\left.\mathrm{Ca}^{2+}\right)$ of the solution. So when $\mathrm{pH}(\approx 13)$ of solution is high as in the case of fresh and hardened cement pastes, SAPs will show lower swelling, and when $\mathrm{pH}(\approx 5.5$ to 7.5$)$ of solution is low as in the case of water flows into the cracks, SAPs will show considerable swelling leading to a physical blocking effect of cracks. Similar to the alkalinity, the capacity of SAPs swelling was measured by investigating the entry of different types of fluids with different ionic concentrations into the concrete cracks. It was found that the swelling capacity of SAPs decreases in the presence of high positively charged ions in a fluid. This is due to the higher ionic attraction between positively charged ions in the fluid and the negatively charged acrylic groups in SAPs. 
For example, the absorption capacity of SAPs is lower in cement slurry than deionized water (pure $\mathrm{H}_{2} \mathrm{O}$ without ions). This is due to the presence of high positively charged cations $\left(\mathrm{K}^{+}, \mathrm{Na}^{+}, \mathrm{Mg}^{+}\right.$and $\left.\mathrm{Ca}^{2+}\right)$ in the slurry. Another reason for the decrease in the swelling capacity of SAP's is due to the presence of high ionic concentration outside SAP particles in hardened cement pastes leading to decrease osmotic pressure inside them. This will result in lower water absorption capability and therefore decrease the swelling capacity of SAP particles (Sherir et al., 2015b; Snoeck et al., 2012a, 2012b; Tittelboom and De Belie, 2013).

The effect of SAPs to promote self-healing phenomenon of ECC was studied (Kim and Schlangen, 2011). Four-point bending test was applied on ECC coupon samples which have two pre-cracking ages at 7 and 28 days up to $2 \mathrm{~mm}$ and then unloaded. It was found that the strength of wet/dry cured specimens with SAPs exceeded the strength of samples without SAPs, which signified that SAPs facilitated to keep the water within its structure and promoted the self-healing property. Moreover, air cured samples $\left(50 \% \mathrm{RH}\right.$ at $20^{\circ} \mathrm{C}$ ) for only 28 days did not show any strength recovery and therefore no sign for self-healing. It should be noted that cracks re-opened at the same locations in the second wet/dry cycle without showing any signs of healing. This was due to the lack of unhydrated cementitious particles in the cracked zone reacting with water because most of them already reacted with water in the first wet/dry cycle. Snoeck et al. studied the combined effect of both PVA fibers and SAPs on self-healing property of ECC under both water and air curing conditions with $\mathrm{RH}=90 \%$ and $\mathrm{RH}=60 \%$, respectively (Snoeck et al., 2012b). Test results showed that micro cracks smaller than $30 \mu \mathrm{m}$ in ECC samples were completely healed when cured under water condition with or without SAPs; while crack widths up to $138 \mu \mathrm{m}$ were healed in the presence of SAPs under air curing condition. Crack widths greater than $200 \mu \mathrm{m}$ showed no healing in air curing. It was observed that new crack locations were formed in samples with SAPs compared to the virgins when reloading mechanical tests were applied to assess the self-healing property of ECC samples. Moreover, the increase in the quantity of SAP particles up to $4 \%$ of mass (4 m\%) relative to the cement weight decreases the mechanical strength properties of ECC samples due to the formation of bigger empty macropores left behind the big water pockets. It was concluded that the use of ECC samples with only $1 \mathrm{~m} \%$ of SAPs under RH=60\% gave optimum self-healing capacity of ECC samples; which is similar to the original samples of ECC that can get superior 
self-healing capacity with strain hardening and multiple cracking behavior. The combination of PVA fibers and SAP particles in ECC samples served the precipitation of $\mathrm{CaCO}_{3}$ and therefore facilitated to form new C-S-H products to promote self-healing property of ECC. As mentioned before in (Homma et al., 2009; Nishiwaki et al., 2012), PVA fibers with higher negatively charged $\left(\mathrm{OH}^{-}\right)$ions can attract the positively charged $\mathrm{Ca}^{2+}$ ions from the hardened cement pastes within the crack walls to form $\mathrm{Ca}(\mathrm{OH})_{2}$ and then convert to $\mathrm{CaCO}_{3}$ precipitation; in addition, SAPs can release the saturated water with $\mathrm{CO}_{2}$ stored within their structure to consume the available $\mathrm{Ca}^{2+}$ ions from the deeper locations of hardened cement pastes inside the cracks and then precipitate $\mathrm{CaCO}_{3}$ as well (Edvardsen, 1999; Snoeck et al., 2012b).

\subsubsection{Restriction of crack width by using internal curing}

The dual effect of curing condition and pre-cracking time on the self-healing behavior of ECC mixtures was studied when blast furnace slag, limestone powder and nano clay were included (Qian et al., 2010). Four curing conditions were investigated: air curing, 3\% $\mathrm{CO}_{2}$ curing, cyclic wet/dry curing and water curing. In addition, three pre-cracking times (up to $1.5 \mathrm{~mm}$ and unloaded) were conducted at 14 days, 28 days and 56 days. Each pre-cracking time was followed by 28 days of curing time. It was pointed out that the presence of water in any self-healing study is very essential to trigger autogenous healing property in concrete, which is not always available during the lifetime of concrete structural members. To overcome this obstacle, an attempt at using nanoclay as water internal curing was utilized to promote the self-healing property of ECC microstructure without relying on poor external water curing. The experimental results indicated that both residual flexural strength and stiffness decreased with the increase of pre-cracking time. This is because the lack of un-hydrated cementitious particles which reacted already due to the continuous hydration. The recovery of deflection capacity was higher in mixtures that contained higher cementitious materials such as slag compared to the lower ones. Again, this is due to the presence of both higher quantities of un-hydrated cementitious materials and to the addition of nano-clay which worked as water internal curing and promoted the self-healing property in ECC mixtures. It was concluded that when applying reloading failure tests under air curing condition, the pre-existing healed cracks will reopen again in all pre-cracking times (14, 28 and 56 days) and never formed new cracks at new locations compared to other curing conditions $\left(\mathrm{CO}_{2}\right.$, wet/dry and 
water curing). This finding is contradicted by the finding of (Herbert and $\mathrm{Li}, 2013$ ) study which revealed that both mechanisms of reopening to the existed healed cracks and new cracks formation can be obtained within the specimen even under natural environment exposure. This was signified by the recovery for RF values up to $84 \%$ of virgins for samples underwent to repeatable damages even after three reloading events. It should be noted that the main difference between the two studies could be the study period itself; (Qian et al., 2010) cured their samples only for 28 days after pre-cracking events while (Herbert and $\mathrm{Li}, 2013$ ) cured their samples up to one year. In the

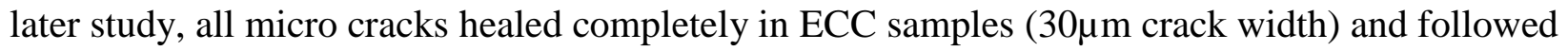
with strength recovery exceeded $100 \%$ of the virgins after 6 months of natural exposure.

\subsubsection{Supplementary cementing materials effect on self-healing}

The effect of water curing was studied under higher temperatures for long period of time on the self-healing performance of cement mixtures incorporating fly ash (Na et al., 2012). It was found that curing the samples for one week in water with $20^{\circ} \mathrm{C}$ was not enough to heal the deteriorated samples compared to 4 weeks in water with $40^{\circ} \mathrm{C}$. It was also found that lower w/c ratio in samples incorporating fly ash leads to smaller carbonation coefficient and better self-healing performance. Lower w/c ratio leads to smaller volume of empty pores and micro cracks; which can be filled quickly by cement hydration products and then improve the self-healing phenomenon. The number of cracks was also reduced when using fly ash in mortar samples than those with ordinary Portland cement for 4 weeks in water curing condition with $40^{\circ} \mathrm{C}$. It was concluded that using $30 \%$ cement replacement of fly ash cured in water under higher temperatures was an effective choice because it will lead to more consumption of $\mathrm{Ca}(\mathrm{OH})_{2}$ that comes from the cement and then produces more C-S-H products; which may heal the micro cracks and improve the self-healing ability. The effect of using high volumes of fly ash as a cement replacement was observed on ECC long-term mechanical properties (Yang et al., 2007). It was found that the use of Class-F fly ash up to 85\% cement replacement improved the tensile ductility of ECC up to $3 \%$ even at the age of 90 days. Moreover, both drying shrinkage property and micro crack widths were reduced significantly due to the microstructure densification caused by un-hydrated fly ash particles. It was concluded that the use of high volume fly ash particles improved the mechanical properties of ECC such as tighter crack width which can promote the self-healing property in ECC infrastructures. The effect of 
cement replacement by slag or fly ash and w/c ratio was studied on the autogenous crack healing (Tittelboom et al., 2012). Test results showed that increasing w/c ratio from 0.4 to $0.5 \%$ will lower the crack healing ability due to further hydration of cement grains which can lead to lower availability in un-hydrated particles. Moreover, increasing the cement replacement of slag and fly ash up to $50 \%$ and $85 \%$, respectively, reduces the cumulative heat production over longer period of time. The heat reduction is mainly due to replacing more cement by admixtures leading to lower $\mathrm{Ca}(\mathrm{OH})_{2}$ content in the cement, which is signified to be the main activator of the cement hydration; in addition to more consumption of $\mathrm{Ca}(\mathrm{OH})_{2}$ according to the hydraulic and pozzolanic effects of slag and fly ash, respectively. The reduction in un-hydrated particles, by increasing water/binder (w/b) ratio, and the reduction in $\mathrm{Ca}(\mathrm{OH})_{2}$ content, by increasing cement replacement ratio, will reduce the ability of crack healing. Among other mechanisms, it was confirmed in the literature that crack healing process could be caused by both continuous hydration and $\mathrm{CaCO}_{3}$ precipitation. According to their perspective, it was differentiated between the two mechanisms according to their functionality, so when the crack healing is required, it could be obtained from $\mathrm{CaCO}_{3}$ precipitation but when both strength recovery and crack healing mechanisms are required, they could be obtained from continuous hydration and $\mathrm{CaCO}_{3}$ precipitation as well. Consequently, it was concluded that there is no advantage to replace cement by slag or fly ash in terms of crack healing alone because the content of main activator $\mathrm{Ca}(\mathrm{OH})_{2}$ to cement hydration in cement will be reduced significantly, unless the continuous hydration of un-hydrated particles is the main mechanism. According to crack width healing, it was confirmed that crack width of $200 \mu \mathrm{m}$ was possible to heal completely within 42 days under water curing condition due to $\mathrm{CaCO}_{3}$ precipitation. 


\subsection{Other methods}

\subsubsection{Bacterial self-healing concrete}

A more advanced technology was introduced in the recent literature in which a biological concrete was being developed that uses specially selected bacteria of the genus Bacillus, along with a combination of nutrients to create a healing agent within the concrete (De Muynck et al., 2008a, 2008b; Jonkers and Schlangen, 2008; J. Wang et al., 2012; J. Y. Wang et al., 2012). With these bacteria mediated self-healing concrete it is possible to seal the crack width of more than $100 \mu \mu$. Therefore, this area of research appears to be promising an alternative to non-sustainable cement based healing systems. In this approach, the bacteria convert $\mathrm{CO}_{2}$ into carbonate ions under alkaline condition and subsequently these react with $\mathrm{Ca}$ ions from the concrete matrix leading to the formation of calcium carbonate crystals. Also, locally produced $\mathrm{CO}_{2}$ directly reacts with calcium hydroxide in the matrix which leads to the calcite precipitation (Jonkers and Schlangen, 2008; J. Y. Wang et al., 2012). This production of large sized calcium carbonate crystals in the bacteria incorporated self-healing concrete leads to a superior self-healing capacity compared to conventional or engineered non-sustainable self-healing cementitious systems (Jonkers and Schlangen, 2009). Recently, Sini (2015) and Sini et al, (2014, 2015) developed self-healing composites by using two types of bacteria with various nutrients (such as zeolite and pumice) and activating agents. The self-healing capability of such composites was assessed based on strength and durability properties and their recovery in damaged/cracked specimens supported by microstructural investigations (Bhashkar, 2016; Bhaskar et al., 2015, 2014).

\subsection{MgO-type expansive agent (MEA)}

Since most of the relevant literature and experience of $\mathrm{MgO}$ concrete was published and issued in Chinese and Korean languages, the authors used the available knowledge of $\mathrm{MgO}$ literature as much as possible. 


\subsubsection{MgO concrete}

Incidentally, $\mathrm{MgO}$ concrete was discovered during the construction of Baishan dam in China. The dam was constructed within the period of Chinese Cultural Revolution (1966-1976), which promoted the lack of attention for temperature control measures in such a bulk mass of concrete. However, no significant concrete cracking due to the heat shrinkage was observed in the dam concrete structure even though the concrete was exposed for consecutive seasons of very hot and cold weather. The amazing ability of dam's concrete to sustain severe weather conditions surprised the engineers and motivated them to investigate this phenomenon deeply. It was found that $\mathrm{MgO}$ content in the cement was higher than usual (up to 4.5\%) and the cement burning temperature was relatively low as well. It was concluded that the maximum amount of $\mathrm{MgO}$ powder should be limited to $5 \%$ of the cement weight with no more than $8 \%(\mathrm{MgO}$ additives to be added as additives,

$5 \%$, plus to the already contained in the cement) to get sound concrete otherwise harmful cracking occurs (Du, 2005). This reference value was derived from research and experience of about 30 constructed dams in China with no crack deleterious over the past three decades. However, it was approved that even if the limit of $\mathrm{MgO}$ in cement exceeded 5\% and reached almost $7.6 \%$ in total, still the concrete is sound (Gao et al., 2007). Moreover, lightly burnt $\mathrm{MgO}$ is a reactive material that can provide long-term expansive effect when concrete is hardened (starts at 7 to 1000 days) without causing concrete cracking (Du, 2005; Mo et al., 2012; Moradpour et al., 2013; Zheng et al., 1991).

\subsubsection{MgO-type expansive agent behavior}

When $\mathrm{MgO}$ concrete used in structural elements under restrained boundary conditions, it can create an autogenous expansion to compensate the shrinkage in concrete. Two effects of stresses can be generated in $\mathrm{MgO}$ concrete related to its expansion behavior. The first effect is the precompressive stresses at early ages which can compensate the tensile stresses partially when concrete starts to cool down. The pre-compressive stresses distribute uniformly in the concrete matrix to reduce the opposite tensile stresses caused by externally restrained boundary conditions. The second effect which called the long-term expansion effect is the reversible part of precompressive deformations to compensate the tensile stresses at later ages. The long-term expansion 
of $\mathrm{MgO}$ will improve creep property and relax both tensile and compressive stresses which reduce cracking in concrete (Du, 2005; Moradpour et al., 2013). No information is currently available in the literature describing the effect of $\mathrm{MgO}$ expansive additives on the performance of self-healing property in ECC mixtures.

\subsubsection{Burning temperature of $\mathrm{MgO}$}

Burning temperature is considered to be the most important factor to control the expansion effect of $\mathrm{MgO}$. Generally, the classification of burning temperature divided $\mathrm{MgO}$ into two classes, "lightly burnt" $\mathrm{MgO}$ with a burning temperature of $850^{\circ}$ to $1200^{\circ}$ and "heavily burnt" $\mathrm{MgO}$ with a burning temperature of $1500^{\circ}$ to $1800^{\circ}$ ( $\left.\mathrm{Du}, 2005\right)$. Only "heavily burnt" crystalline $\mathrm{MgO}$ has deleterious and harmful reactive while "lightly burnt" crystalline $\mathrm{MgO}$ has not (Neville, 1995). The rate of $\mathrm{MgO}$ hydration with water becomes very slow when the burning temperature increases; leading to deleterious expansion of concrete at very later ages ( $\mathrm{Du}, 2005$; Gao et al., 2007). Lack of control in such delayed expansion leading to poor compensating in concrete shrinkage and resulting for severely concrete cracking (Gao et al., 2007). Specifically, the burning temperature of "heavily burnt" $\mathrm{MgO}$, which is already contained in the cement, is about $1450^{\circ}$ and about $1100^{\circ}$

in "lightly burnt" $\mathrm{MgO}$, which is to be added to the concrete as additives (Du, 2005; Mo et al., 2012). In addition to the burning temperature effect, higher $\mathrm{MgO}$ quantity, fineness and higher curing temperature can accelerate the expansion behavior in $\mathrm{MgO}$ concrete (Du, 2005; Zheng et al., 1991).

\subsubsection{Mixing procedure}

To control the harmful expansion effect of $\mathrm{MgO}$ additives, a good distribution of $\mathrm{MgO}$ particles is a must while mixing with cement/sand dry particles. Failing to reach full dispersibility in $\mathrm{MgO}$ concrete will lead to severe concrete cracking and deterioration in the concrete structure. It was mentioned that dry $\mathrm{MgO}$ powder could be added as an additional material and the mixing of $\mathrm{MgO}$ could be done either in a cement factory, in situ or by adding both $\mathrm{MgO}$ and cement clinkers before grinding them together in the final stage of cement production ( $\mathrm{Du}, 2005)$. The mixing procedure of $\mathrm{MgO}$ particles might be similar to that of conventional concrete but with extra mixing time is 
needed. To ensure well dispersion of $\mathrm{MgO}$ particles during mixing procedure, extra caution should be given when weighing the $\mathrm{MgO}$ powder which can be done by using computerized electrical scales. Also, a particular sequence should be followed in mixing procedure and enough mixing time should be provided as well.

Recently, the nanoparticles started to be used in many fields and have received increasing attention due to their unique physical and chemical properties (Moradpour et al., 2013). It can improve the mechanical properties of concrete dramatically compared to the normal size with same chemical composition. Due to the difficulty of mixing nanoparticles in conventional ways, well-dispersed suspensions of $\mathrm{MgO}$ particles could be done by using the ultrasonic irradiation method to homogenize the suspensions for eliminating the nanoparticles agglomeration. In this method, the ultrasonic waves propagating through the suspension medium cause collision with the nanoparticles which leading to the splitting of the agglomerated $\mathrm{MgO}$ particles. In contrary to the mixing procedure of normal $\mathrm{MgO}$ particles, nano $\mathrm{MgO}$ particles will be added to the solid cement/sand mixture in the form of suspension, not in the form of solid powder.

\subsubsection{Mechanical and durability properties of $\mathrm{MgO}$ concrete}

\subsubsection{Mechanical properties of $\mathrm{MgO}$ concrete}

The volume of $\mathrm{Mg}(\mathrm{OH})_{2}$, which is the hydration product of $\mathrm{MgO}$ and water together, is larger than the volume of $\mathrm{MgO}$ alone explaining the autogenous expansion behavior in $\mathrm{MgO}$ concrete. Because the hydration of $\mathrm{MgO}$ to $\mathrm{Mg}(\mathrm{OH})_{2}$ is an irreversible process which means that $\mathrm{Mg}(\mathrm{OH})_{2}$ is stable and therefore its expansion is stable as well. This irreversible process will lead to stability in mechanical properties of $\mathrm{MgO}$ concrete.

Moradpour et al. (2013) studied the effect of nano-MgO particles on concrete mechanical properties. The nano $\mathrm{MgO}$ particles were added as additives to $1 \%, 3 \%$ and $5 \%$ to the already $\mathrm{MgO}$ content already present in Portland cement (4.25\%) to get an output of 5.25\%, $7.25 \%$ and 9.25\%, respectively. They concluded that $5.25 \%$ of combined value of $\mathrm{MgO}$ material was the optimum amount based on mechanical properties, permeability tests, sulfate attack resistance, Scanning Electron Microscope (SEM) and X-Ray Diffraction analysis (XRD). It should be noted that the 
limit of the optimum addition of nano-sized $\mathrm{MgO}$ material was only $5.25 \%$ of total $\mathrm{MgO}$ content rather than $8 \%$ as in the case of normal sized $\mathrm{MgO}$ additives. This confirms the significant improvement in concrete properties due to the effect of nano-grain sized $\mathrm{MgO}$ material rather than micro grain sized with the same chemical composition (Moradpour et al., 2013).

The addition of nano- $\mathrm{MgO}$ material to cement based composites causes the mechanical strength of such composites at different ages higher than those of conventional concrete. It was revealed that the use of $1 \%$ of nano- $\mathrm{MgO}$ particles as an additional material of binder by weight was the optimum amount that increased the compressive strength by $103 \% \& 80 \%$ and flexural strength by $95 \%$ \& $70 \%$ than conventional concrete at 7 and 28 days, respectively. The percentage of the expansion effect of $\mathrm{MgO}$ particles was higher at 7 days than 28 days due to higher matrix porosity at early ages. The improvement in the mechanical properties might be attributed to two reasons; first is due to well dispersibility of nano-MgO particles which can lead to less porous structure and second is due to the un-numbered of nano-MgO particles which can lead to tightly bond with hydrated products around the transition zones resulting in an improved microstructure. In addition to these reasons, the formation of magnesium crystals might be another reason to improve the mechanical properties of concrete. Through the hydration process of $\mathrm{C}-\mathrm{S}-\mathrm{H}$ in the presence of $\mathrm{MgO}$ and after a while, the left over $\mathrm{MgO}$ particles in the matrix will start to hydrate to form $\mathrm{Mg}(\mathrm{OH})_{2}$ within the ratio of $\mathrm{MgO} / \mathrm{SiO}_{2} \approx 1.5$ (Moradpour et al., 2013). Also, Fernandez et al. (2008 \& 2005) revealed that the incorporation of magnesium ions with cement can be significant when $\mathrm{CaO} / \mathrm{SiO}_{2}$ ratios > 1 while it has lower incorporation of magnesium ions for very low $\mathrm{MgO} / \mathrm{CaO}$ ratios in C-S-H gels (Fernandez et al., 2008, 2005). In Moradpour et al. (2013) study, the ratio of $\mathrm{CaO} / \mathrm{SiO}_{2}$ was greater than $1(142.4 / 49.82 \approx 2.85>1)$ and $\mathrm{MgO} / \mathrm{CaO}$ ratio was relatively high $(71.05 / 142.4 \approx 0.50)$ which leads to a considered interaction between magnesium ions and cement particles. This will lead to the significant formation of magnesium crystals in addition to the already formed C-S-H products and thereby, get higher mechanical properties. The study was also revealed clearly that adding more than this amount (1\% of nano-MgO particles) to the extent of $3 \%$ and $5 \%$ will lead to less mechanical properties due to the formation of higher micro cracks by the expansive effect of nano- $\mathrm{MgO}$ particles. Also, adding more $\mathrm{MgO}$ particles might lead to agglomeration effect and increasing the viscosity of mixing process which might 
reduce the workability significantly and hence severe concrete cracking may occur at later ages (Moradpour et al., 2013). Furthermore, there are other factors that can increase both compressive and tensile s of $\mathrm{MgO}$ concrete such as additional limited quantities of $\mathrm{MgO}$ powder, curing temperature, and age of concrete. Higher curing temperature increases the rate of hydration of $\mathrm{MgO}$ leading to denser microstructures (Du, 2005). Both studies (Du, 2005; Moradpour et al., 2013) revealed that modulus of elasticity (MOE) of MgO concrete is higher than that of normal concrete. It should be noted that increasing the value of MOE is not favored in terms of concrete crack formation which is required more carefulness when tailoring the compositions of $\mathrm{MgO}$ concrete.

It was indicated that the tensile strain capacity of $\mathrm{MgO}$ concrete with $4.5 \% \mathrm{MgO}$ content would be increased by $19 \%, 24 \%$ and $28 \%$ as the curing temperature increased from $20^{\circ} \mathrm{C}$ to $30^{\circ} \mathrm{C}, 40^{\circ} \mathrm{C}$ and $50^{\circ} \mathrm{C}$, respectively; and usually ranges between 80 to 150 micro-strains ( $\left.\mathrm{Du}, 2005\right)$. It is well known that increasing the tensile strain capacity of the structures is always prescribed to reduce concrete cracking. It was also revealed that 3.5 to $4.5 \% \mathrm{MgO}$ concrete has a greater value of creep than conventional concrete by 20 to $25 \%$ with same mixture proportions. The long term expansive effect of $\mathrm{MgO}$ material improves creep property and help to relax both tensile and compressive stresses resulting in concrete cracking reduction. The creep relaxation property is only promoted by the reversible part of pre-compressive deformations which compensating the tensile stresses at later ages.

\subsubsection{Durability properties of MgO concrete}

Composites consist of nano-MgO additives have more compacted microstructures, homogeneous and denser than the composites of conventional concrete due to the unique expansive effect of $\mathrm{MgO}$ hydration products and porosity filling effect of nanoparticles as well.

It was revealed that the addition of nano- $\mathrm{MgO}$ particles by $1 \%, 3 \%$ and $5 \%$ to the cement increased the permeability mean depth of the samples $(40 \mathrm{~mm} \times 40 \mathrm{~mm} \times 160 \mathrm{~mm})$ by $1.23 \mathrm{~cm}, 1.60 \mathrm{~cm}$ and $1.70 \mathrm{~cm}$, respectively compared to $1.83 \mathrm{~cm}$ in conventional concrete (Moradpour et al., 2013). The permeability of nano-MgO samples was found to be less than those of conventional concrete even

after adding $5 \%$ of nano- $\mathrm{MgO}$ particles. As a conclusion, the addition of $1 \% \mathrm{MgO}$ content was the 
optimum choice that confirmed the results of mechanical property tests. Du (2005) revealed that as the mechanical strength increased due to the addition of extra normal $\mathrm{MgO}$ powder, the permeability will decrease by 0.6 to 7 times ( $\mathrm{Du}, 2005)$. Further experimental works are needed to confirm the difference between the two types of particles, nano $\mathrm{MgO}$ particles and normal $\mathrm{MgO}$ particles, which may lead to different microstructure behavior.

SEM results indicated that the hydration of $\mathrm{MgO}$ particles when combined with water forms $\mathrm{Mg}(\mathrm{OH})_{2}$ crystals which can produce self-expansive stresses on the walls of the concrete pores. Any excessive amount of nano-MgO additives will result in higher expansive stresses pushing on the pore walls. Once the pores cannot locally restrain the stresses, macro cracks in the cement paste will occur (Mo et al., 2012).

The property of concrete porosity was utilized to articulate the sulfate resistance of $\mathrm{MgO}$ concrete (Moradpour et al., 2013). When the porosity of hardened cement paste was large, more risks occur to the concrete paste to be impregnated by higher sulfate solution. Consequently, more ettringite will form leading to more expansions. Similar to the inferior results of mechanical strength when adding extra $\mathrm{MgO}$ powder, the sulfate resistance in concrete will decrease when the amount of nano- $\mathrm{MgO}$ content increased from $1 \%$ to $5 \%$ due to the higher crack formation in the sample's microstructure. These cracks were formed after the concrete hardened due to the expansive effect of $\mathrm{MgO}$. Again, it was concluded that $1 \%$ of nano- $\mathrm{MgO}$ concrete had the larger sulfate resistance factor $\approx 0.915$ than both conventional concrete $\approx 0.90$ and $5 \%$ of nano-MgO concrete which was less than 0.90 .

\subsubsection{Supplementary cementing materials effect on MgO concrete}

SCMs can exhibit pozzolanic reaction when it is used as cement replacement materials. The function of such reactions is to consume $\mathrm{Ca}(\mathrm{OH})_{2}$ in the presence of alkaline solution to form C$\mathrm{S}-\mathrm{H}$ products. It was revealed that after the formation of $\mathrm{C}-\mathrm{S}-\mathrm{H}$ in the presence of $\mathrm{MgO}$ particles and after a long period, the leftover $\mathrm{MgO}$ particles will hydrate to form $\mathrm{Mg}(\mathrm{OH})_{2}$ (Moradpour et al., 2013). When using higher amounts of SCMs as cement replacement such as fly ash, this will limit the presence of $\mathrm{OH}$ ions in alkaline solution which will affect the formation of $\mathrm{Mg}(\mathrm{OH})_{2}$ crystals. Moreover, the use of SCMs as a cement replacement will lead to reducing concrete heat 
of hydration. By reducing the concrete temperature, the rate of $\mathrm{MgO}$ hydration will be reduced as well (Du, 2005). As a result, these factors can affect the performance of $\mathrm{MgO}$ expansion indirectly. Accordingly, it was confirmed from literature and engineering dam experience that no more than $30 \%$ of fly ash as cement replacement should be used in normal grain sized $\mathrm{MgO}$ concrete. This limited percentage of fly ash will not affect the $\mathrm{MgO}$ expansion performance (Du, 2005). In the study of (Gao et al., 2007), the soundness of MgO concrete was evaluated based on using different amounts of $\mathrm{MgO}$ as an expansive agent, $0 \%, 4 \%, 8 \%$ and $12 \%$. Then combined with different low calcium fly ash cement replacement ratios, $0 \%, 30 \%$ and $50 \%$ at different autoclave conditions. Generally, it was shown that the expansion value decreases significantly when the content of fly ash increases up to $50 \%$. No exact percentage of fly ash recommended to be used in $\mathrm{MgO}$ concrete was adopted in their study. Additionally, the presence of $20 \%$ fly ash as cement replacement was observed in cement paste samples with $6 \% \mathrm{MgO}$ content. The presence of fly ash consumed the available calcium ions in concrete to form C-S-H. This will leave the alkalinity in concrete with limited $\mathrm{OH}$ ions in later ages and therefore suppress the formation of $\mathrm{Mg}(\mathrm{OH})_{2}$. The early formation of $\mathrm{C}-\mathrm{S}-\mathrm{H}$ products improved and densified the concrete microstructure resulting in higher resistance to the expansion values (Ali and Mullick, 1998). Further, Mo et al. revealed that the autogenous deformations of cement pastes containing FA/C ratio of $30 \%$ with the presence of $\mathrm{MgO}$ expansive agent was compensated completely but was slower than Portland cement samples. This is due to the slower hydration of fly ash than Portland cement. The long-term observations for samples' deformations revealed that the presence of fly ash delayed the expansion of $\mathrm{MgO}$ rather than suppress it. At 540 days, the expansion of fly ash samples with $8 \%$ of $\mathrm{MgO}$ material was $1203 \times 10^{-6}$ strain which is larger than $1085 \times 10^{-6}$ strain of Portland cement samples. Moreover, the results showed a continuous progression of fly ash expansion even after 540 days. The delayed $\mathrm{MgO}$ expansion mentioned in their study is contradicted to some studies which revealed that fly ash can restrain the expansion of $\mathrm{MgO}$; the delay expansion is due to the limited alkalinity which will suppress the formation of $\mathrm{Mg}(\mathrm{OH})_{2}$ and hence reduce the expansion performance of $\mathrm{MgO}$ concrete (Mo et al., 2012). 


\subsubsection{Curing conditions and w/c ratio effect on $\mathrm{MgO}$ concrete}

All autoclave cured samples with w/c ratio of 0.25 contained $1 \%, 3 \%$ and $5 \%$ of nano-MgO particles showed sound expansion within the limit of (ASTM C150, 2016) standard which is $0.8 \%$ (Moradpour et al., 2013). In addition, expansion effect increased when MgO amount increased. Higher amount and denser crystals of $\mathrm{Mg}(\mathrm{OH})_{2}$ was formed when the curing temperature increased from $20^{\circ} \mathrm{C}$ to $50^{\circ} \mathrm{C}$ due to accelerated hydration of $\mathrm{MgO}$ material (Gao et al., 2008). Moreover, it was revealed that $\mathrm{CaO}$ will hydrate with water completely to form $\mathrm{Ca}(\mathrm{OH})_{2}$ within the first 3 days when the samples cured at $20^{\circ} \mathrm{C}$ which was similar to (Edvardsen, 1999) findings, while the hydration of $\mathrm{MgO}$ with water to form $\mathrm{Mg}(\mathrm{OH})_{2}$ is very slow and only about $57 \%$ of $\mathrm{MgO}$ formed $\mathrm{Mg}(\mathrm{OH})_{2}$ within 180 days when the samples cured at $20^{\circ} \mathrm{C}$ as well (Gao et al., 2008). The $\mathrm{CaO}$ and $\mathrm{MgO}$ reactions will cause expansion to compensate the concrete shrinkage in early and later ages, respectively. In addition, the expansion effect of both ettringite and $\mathrm{MgO}$-type expansive additives was studied under non-wet curing conditions with low water to cement ratio $(\mathrm{w} / \mathrm{c}=0.28)$. For ettringite expansive additives, larger amount of water was needed to form 32 water molecules in ettringite, $\mathrm{C}_{4} \mathrm{ASH}_{32}$, which is not available in mass concrete due to low w/c ratio. Therefore, continuous external curing method is always prescribed for concrete containing ettringite additives, which is again insufficient in mass concretes due to impermeable matrices. As a result, the ability of using ettringite additives to compensate concrete shrinkage is not effective in such concrete due to lower permeability and water content as well. Unlike ettringite, the $\mathrm{MgO}$ expansive additives can expand even under non-wet curing conditions due to lower demand for water to form $\mathrm{Mg}(\mathrm{OH})_{2}$ which is the dominant part of producing the expansion (Mo et al., 2012).

\subsection{ECC for bridge deck link slab}

\subsubsection{Introduction}

Many highway bridges are designed as multi-span simply supported (over piers or abutments) steel or pre-stressed concrete girders supporting concrete bridge deck. At the end of each simple span deck, a mechanical expansion joints are installed for allowing a free deformation caused by girder's deflection, shrinkage, temperature variation and creep effect (Figure 2-3 - A). Aside from 
a high installation cost of these expansion joints, constant maintenance is required due to their low durability performance. The leakage of chloride-containment water through these mechanical expansion joints leads to corrosion of the steels girders and deterioration of the substructures (Kim et al., 2004). The accumulative debris reduces the strength and functionality of the bridge structures and eventually leading to a catastrophic failure. In fact the mechanical expansion joints are a major source of bridge deterioration in Canada (Au et al., 2013). The possible approach for improving the durability performance of the bridge structures is the elimination of the mechanical expansion joints. Different design methodologies have been proposed by scholars and ministry of transportation authorities for substituting these mechanical expansion joints (Alampalli and Yannotti, 1998; Au et al., 2013; Gilani, 2001; Zia et al., 1995). The first approach is to use the haunched deck for developing a continuous deck system over the piers while keeping the girders as simply supported span. The second method is the construction of integral concept with continuity of the girders and the third approach is the implementation of the link slabs on the deck for developing a joint-free bridge deck with simply supporting spans (Figure 2-3 - B). The joint free-bridge deck is the most economical and efficient rehabilitation strategy in comparison to other proposed methods (Alampalli and Yannotti, 1998).

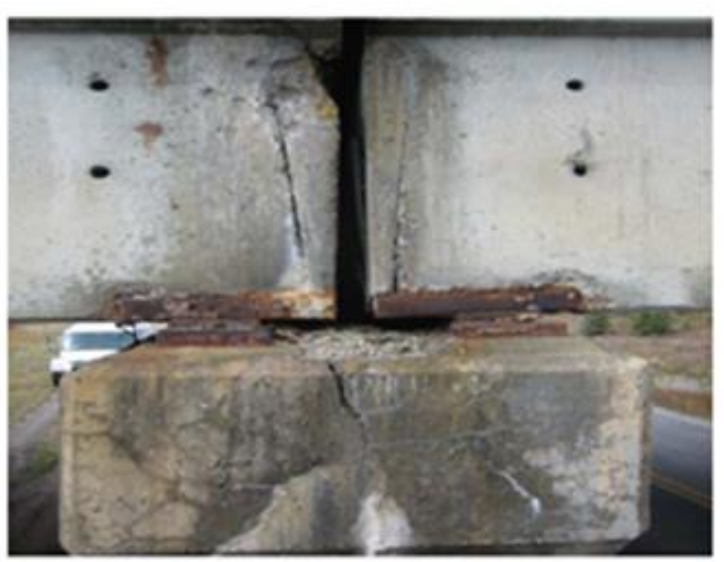

(A) Bridge deck with joint
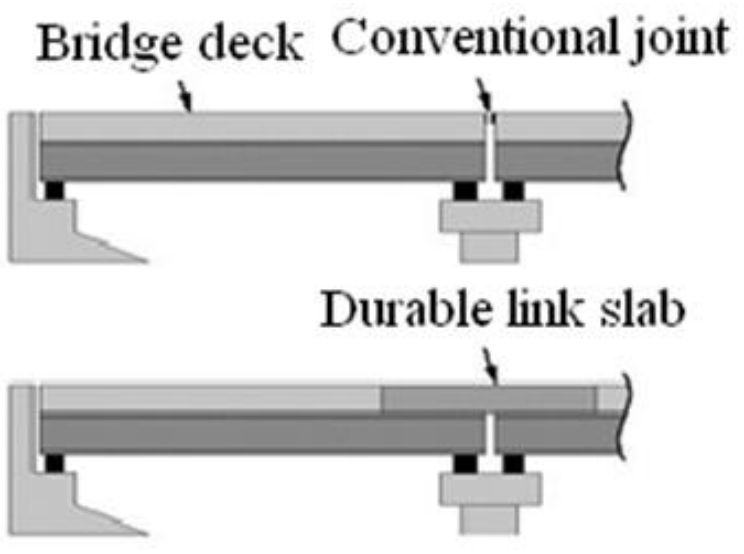

(B) Bridge deck with link slab

Figure 2-3: Joint free bridge deck with link slab (Hossain and Anwar, 2014)

The link slab is defined as a section of the deck connecting the two adjacent simple-span girders (Figure 2-4). The total length of the link slab consists of the debonding and transition zone. The 
length of the debonding zone (where all the shear connectors are removed and debonding mechanism is placed on top of the flange's girder) is limited to $5 \%$ of each adjacent bridge span. For reducing the stress concentration on the debonding zone, transition zone was introduced with additional design of 2.5\% increase in length and 50\% additional shear stud connectors than required by AASHTO design code (Caner and Zia, 1998; Qian et al., 2009). Caner and Zia (1998) experimentally analyzed the performance of the joint-free bridge decks and proposed a design method for the normal concrete link slab. These investigations revealed that the link slab was subjected to bending under typical traffic conditions rather than axial elongation. Tensile cracks were observed at the top of the link slab under service conditions due to a negative bending moment. For steel girders, the measured maximum crack width was $300 \mu \mathrm{m}$ at $40 \%$ of ultimate load and $750 \mu \mathrm{m}$ at $67 \%$ of ultimate load. It was pointed out that additional tensile stress may be imposed on the link slabs due to shrinkage, creep, and temperature loading, and that crack width must be carefully controlled. The recommendation was to use epoxy coated reinforcing bars in the link slab in order to avoid reinforcement corrosion.

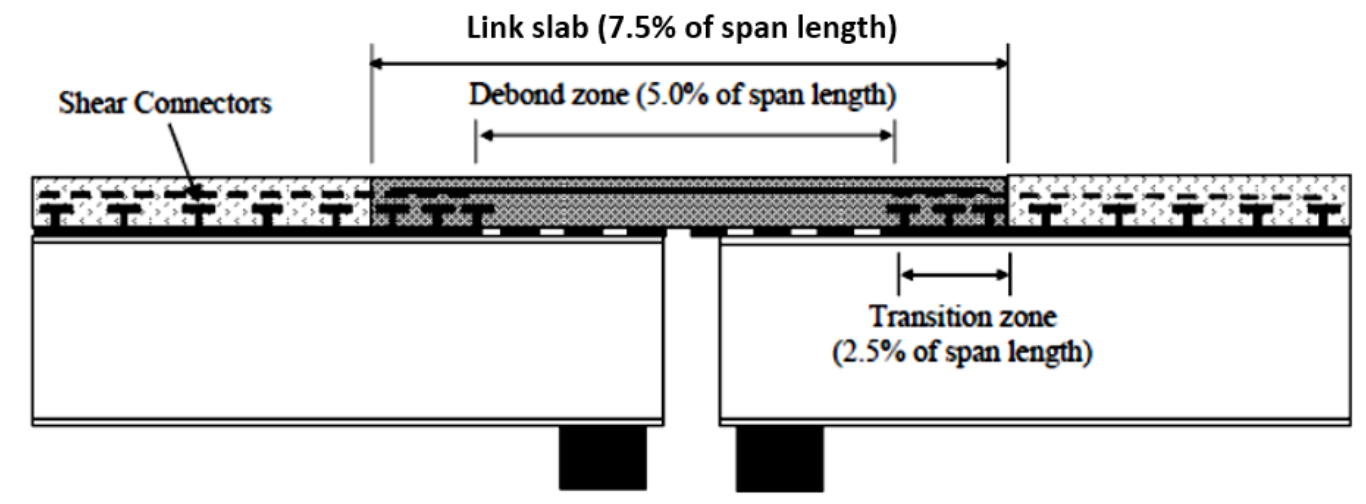

Figure 2-4: Schematic of link slab showing components

\subsection{Previous Research on Link Slab for Joint-free Bridge Construction}

Back in 1970's many bridge structures were designed as simply supported girders supporting a simply span concrete decks. A mechanical expansion joints were provided at the end of each deck spans for allowing a girders to deform freely under internal and external stresses (Lam et al., 2008). The low durability performance of mechanical expansion joints in the bridge deck led the engineers 
to develop an efficient design of jointless bridge structure known as link slab (Caner and Zia, 1998). Experimental research has been conducted on developing design guidelines for durability and structural performance of link slabs using different concretes (normal concrete and ECC), varying reinforcing ratio and debonding length (Caner and Zia, 1998; Gilani, 2001; Lepech and Li, 2009b; Zia et al., 1995). Limited research has also been conducted on the fatigue performance of link slab as well its implementation and performance in a real bridge structure (Hossain et al., 2015). Kim et al. (2004) investigated the durability performance of the traditional ECC link slab subjected to flexural fatigue loading only up to maximum allowable AASHTO's rotation of 0.00375 radian at 100000 fatigue cycles (Kim et al., 2004). Caner and Zia (1998) tested the performance of normal concrete link slab in reduced scale bridge structure and proposed some design guidelines.

Since the early 1980's, researchers have investigated the performance of joint-free bridge decks in a multiple simply supported girders. Zuk (1981) analyzed the effects of expansion and contraction joints in joint-free bridge deck, but due to lack of experimental results, his model was not implemented in an actual application (Zuk, 1981). Gastel and Zia (1989) developed a finite element model for joint-free bridge decks using a linear strain theory along the depth of the link slab, but once again the model was not validated due to lack of experimental results (Gastal and Zia, 1989).

The first experimental investigation of the joint-free bridge deck with connecting link slab was conducted by Caner and Zia (1998). Two reduced-scale bridge specimens with simply supported steel and pre-stressed girders and a connecting link slab were tested under monotonic loading. The first experimental investigation revealed that link slabs were subjected to flexural stresses under typical traffic loading rather than elongation like a mechanical expansion joints. Tensile cracks were formed on top of the link slab under service condition due to development of negative moment. It was pointed out that in addition to applied loading, additional tensile stresses were imposed on the link slab due to creep, shrinkage and temperature loading. For enhanced durability performance of link slab, they suggested to use epoxy coated reinforcement or carbon fiber reinforced polymer (FRP) bar to avoid corrosion. Further, it was proposed to reduce the stiffness of the link slab by limiting the debonding length to $5 \%$ of the adjacent bridge for maintaining the simply support performance of the girder with a continuous deck. 
Many problems were associated with the experiments conducted by Caner and Zia (1998). The major problem was the development of large tensile crack widths on top of the link slab due to the use of normal concrete. Although heavily steel reinforced link slab would have reduced the crack's width to the acceptable limits, but it would increase the stiffness of the link slabs. Weak interfaces were formed between the concrete bridge deck and link slab joint because the applied tensile stresses were resisted only by the link slab rather than a composite action of deck and girder. Also, there was a lack of experimental and analytical results on the fatigue performance of the link slabs.

Experimental investigations were conducted on the full-scale link slabs subjected to both monotonic and fatigue loading using ECC (Kim et al., 2004; Li et al., 2003). Experimental results of ECC based link slabs were compared to the conventional reinforced concrete (RC) link slab. While the previous research was focused on the reduced scale full bridge models using conventional RC, the new investigation focused on the full-scale link slab portion only. Therefore, the imposed end rotations of the link slabs caused by adjacent bridge spans were replicated in the laboratory. The reinforcing ratio for the ECC and RC link slabs was adopted from the guidelines proposed by Caner and Zia (1998) for satisfying the stress criterion of RC link slab at the end rotation of 0.0015 radians. Monotonic and fatigue test results revealed the suitability of ECC material for the link slab application developing a low structural stiffness for allowing the hinge formation while keeping the crack widths below the acceptable limits at the ultimate loading. The yielding of the reinforcing bar was delayed in comparison to the conventional RC because of high tensile strain capacity and ductility of ECC that allowed a compatible deformation of the composite with reinforcing bar. Based on the analysis, it was suggested to reduce the amount of the steel reinforcement further for the ECC link slab to maintaining the simply supporting behavior of the girders with lower structural stiffness. The stiffness of the ECC links slabs remained unchanged after cyclic testing at 100000 fatigue cycles. All ECC link slabs formed multiple micro-cracks (with width below $130 \mu \mathrm{m}$ ) within the debonding zone only. Unlike previous experiments, no cracking was observed at the interface of the concrete and the ECC link slab, since additional shear studs were provided only within the interface for reducing the stress concentration by the composite action of the girder and the deck. 


\subsection{Applications of ECC}

To illustrate the performance of ECC in real world applications, a number of recent/ongoing projects involving the use of ECC are briefly highlighted.

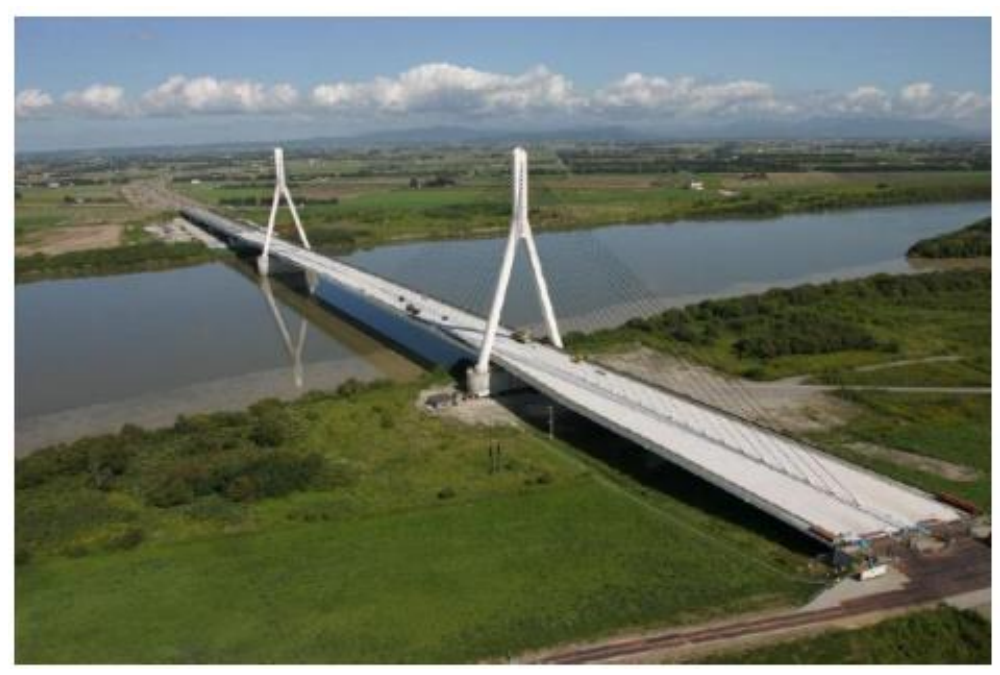

Figure 2-5: Overview of Mihara Bridge (Mitamura et al., 2005)

Members made of ECC in combination with steel plates provide higher flexural resistance with a thinner cross-section than normal steel-concrete members. Figure 2-5 shows the Mihara Bridge in Hokkaido, Japan with a bridge length of 972m and a central span of 340m (Mitamura et al., 2005). In 2004, half the depth of the asphalt overlay on the steel deck of this bridge was replaced with 40 $\mathrm{mm}$ thick ECC to increase the load bearing capacity and stiffness of the decks while reducing the stress generated, thereby improving the fatigue resistance of the stiffener for the steel deck. This became necessary because the requirements for fatigue resistance in the standard specifications were revised while the bridge was under construction. The bridge was opened to traffic in 2005 . The steel-reinforced road bed contains nearly $800 \mathrm{~m}^{3}$ of ECC material. The tensile ductility and tight crack control behavior of ECC led to a $40 \%$ reduction in material used during construction.

As one of the first field applications of ECC in the USA, an ECC patch repair Figure 2-6 - (A) placed on the deck of the Curtis Road Bridge in Michigan, the USA in 2002 (Li et al., 2004). A complete summary of this work has been outlined by authors. During this work, one small section of a deteriorated bridge deck was repaired with ECC while the remaining portion was repaired 
with a commercial concrete patching material commonly used by the Michigan Department of Transportation (MDOT). This repair scenario allowed for a unique ECC/concrete comparisonsubjected to identical environmental and traffic loads. As of the time of this writing, ECC patch and surrounding concrete have experienced almost three full winter freeze-thaw cycles. This bridge is traveled by heavily loaded 11-axle trucks. Figure 2-6 - B shows the monitored maximum crack width as a function of age. It reveals that the crack width in the ECC patch remains almost at a constantly low level, around $50 \mu \mathrm{m}$, while the maximum crack width in the surrounding concrete is significantly higher at the same age. The last data point at 780 days after repair indicates a maximum crack width of $3.8 \mathrm{~mm}$ in the concrete.

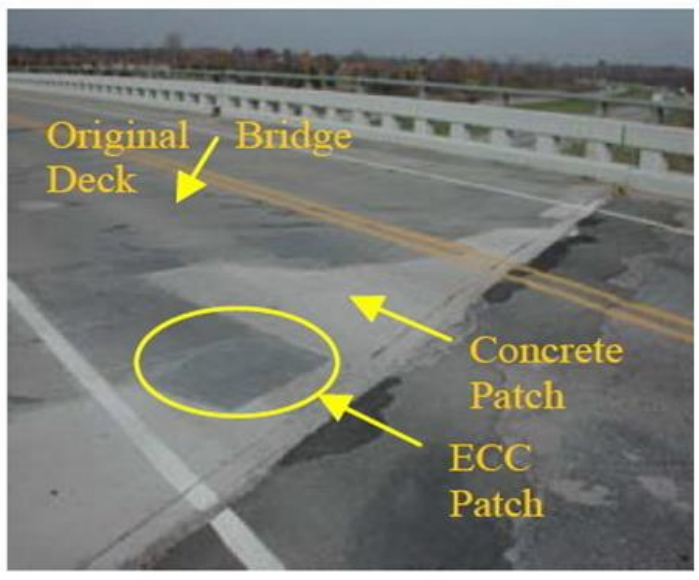

(a)

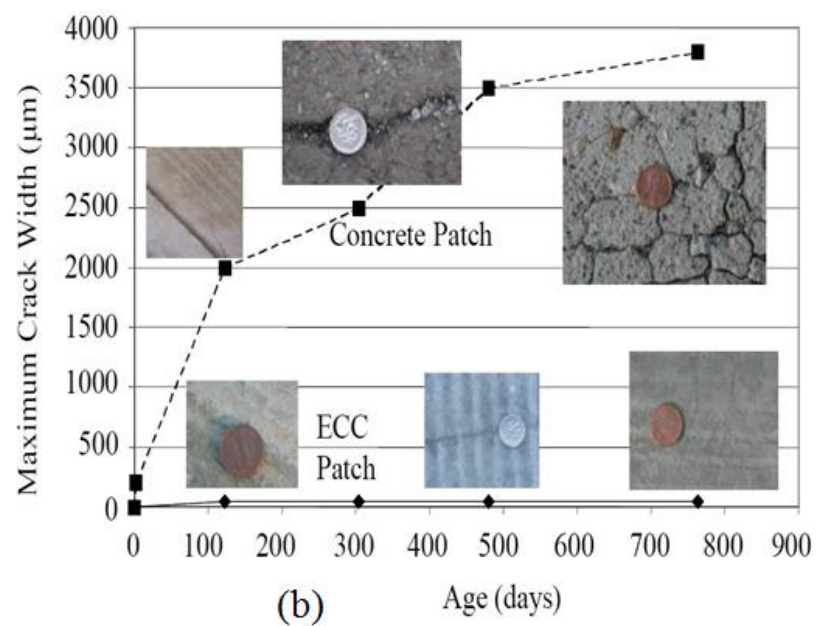

(b)

Figure 2-6: (a) Patch repair on a bridge deck. (b) Crack width development in concrete patch and ECC patch over time (Li et al., 2004)

In addition to bridge deck patching repairs, the most recent field application of ECC in the US is with a bridge "link slab" completed in cooperation with MDOT on Grove Street Bridge in Southeast Michigan in 2005 as shown in Figure 2-7 (Lepech and Li, 2008). The objective was to eliminate the maintenance requirements associated with typical bridge deck expansion joints. In this project, about $32 \mathrm{~m}^{3}$ of ECC were cast in place using standard ready-mix concrete trucks to build the first ECC link slab in the USA. With a strain capacity exceeding $2 \%$, these composites can be used to replace traditional steel expansion devices and can fully accommodate the thermal deformations of adjacent bridge spans. This ECC link slab design was adopted in 2006 in the highway segment that extends from Bolzano to the Austrian border bridge in north Italy. 


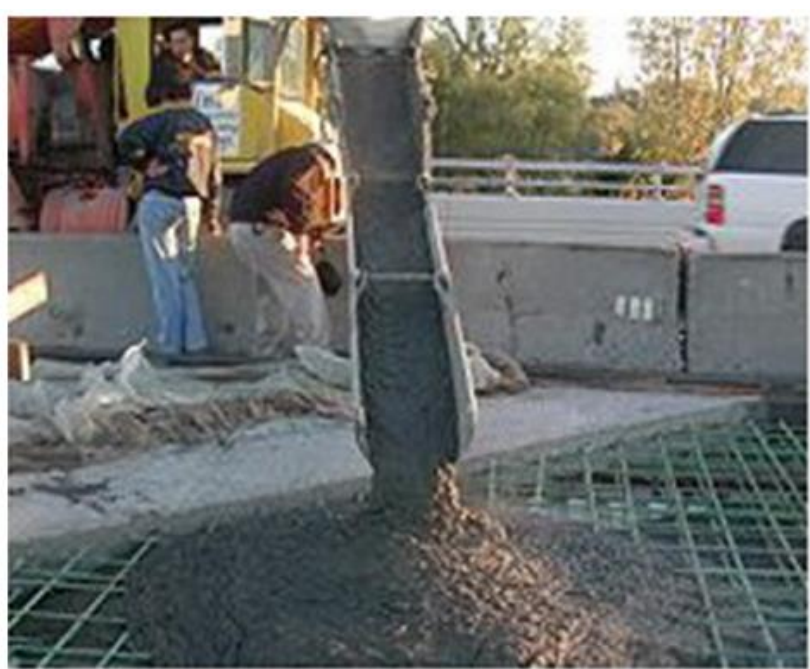

(a) During construction

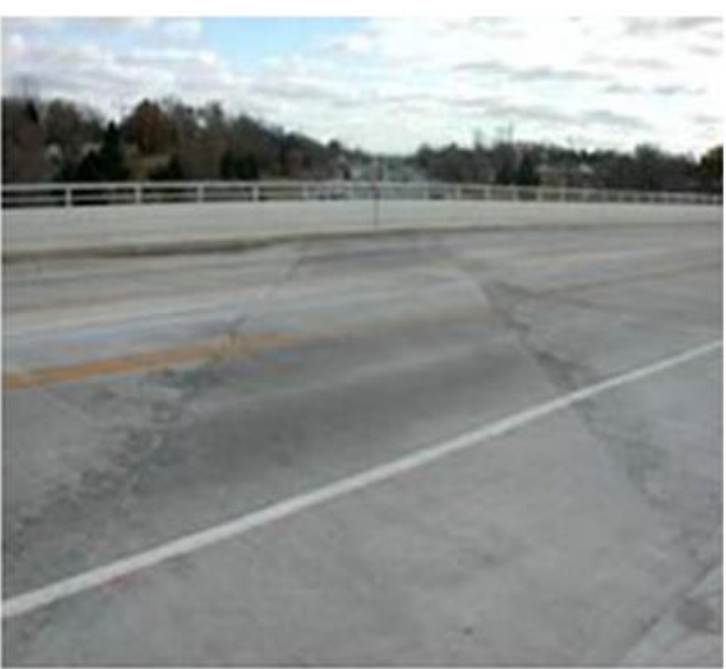

(b) After construction

Figure 2-7: ECC link slab on Grove Street Bridge, Michigan, USA (Lepech and Li, 2008)

\subsection{Review conclusions}

Researches have been conducted in the past to evaluate the self-healing ability of concrete including ECCs with different techniques. However, the effectiveness of those techniques/methods to induce self-healing is questionable due to many limitations as revealed from the previous research studies. Moreover, little research has been conducted to date on the self-healing potential ECC or other materials in real structural applications. This warrants further research to identify other mechanisms that could enhance the self-healing ability in combination with costeffectiveness and long-term durability. The use of $\mathrm{MgO}$ is found to be a promising/viable approach to improve self-healing capabilities of emerging ECCs. This research focusing on this approach of developing self-healing concrete materials and their performance in structural elements (such as link slabs in joint-free bridge construction) is a timely initiative and can contribute to the development of self-healing technology in concrete. 


\section{CHAPTER THREE}

\section{EXPERIMENTAL PROGRAM}

\subsection{Introduction}

This chapter presents all the materials used in the development/production of ECC and ECC-MgO mixtures with their properties, research program (describing various tasks) and methodology in general, mixing procedure and crack/damage creation methods in different types of specimens to induce the self-healing properties. The various methodologies of evaluating the self-healing capability of proposed ECC-MgO system are also described.

Due to high number/types of specimens and variable test procedures/test preparations involved in each of the tasks in this research, all mixture proportions, detailed research program, specimen preparations and test procedures will be presented before Results and Discussions sections of each of the subsequent chapters. 


\subsection{Research program and methodology}

A comprehensive investigation has been designed and selected to develop self-healing techniques/methods using green and cost-effective ECC mixtures by utilizing MgO-type expansive agent. The investigation consisted of five major tasks as shown in the following flowchart (Figure 3-1).

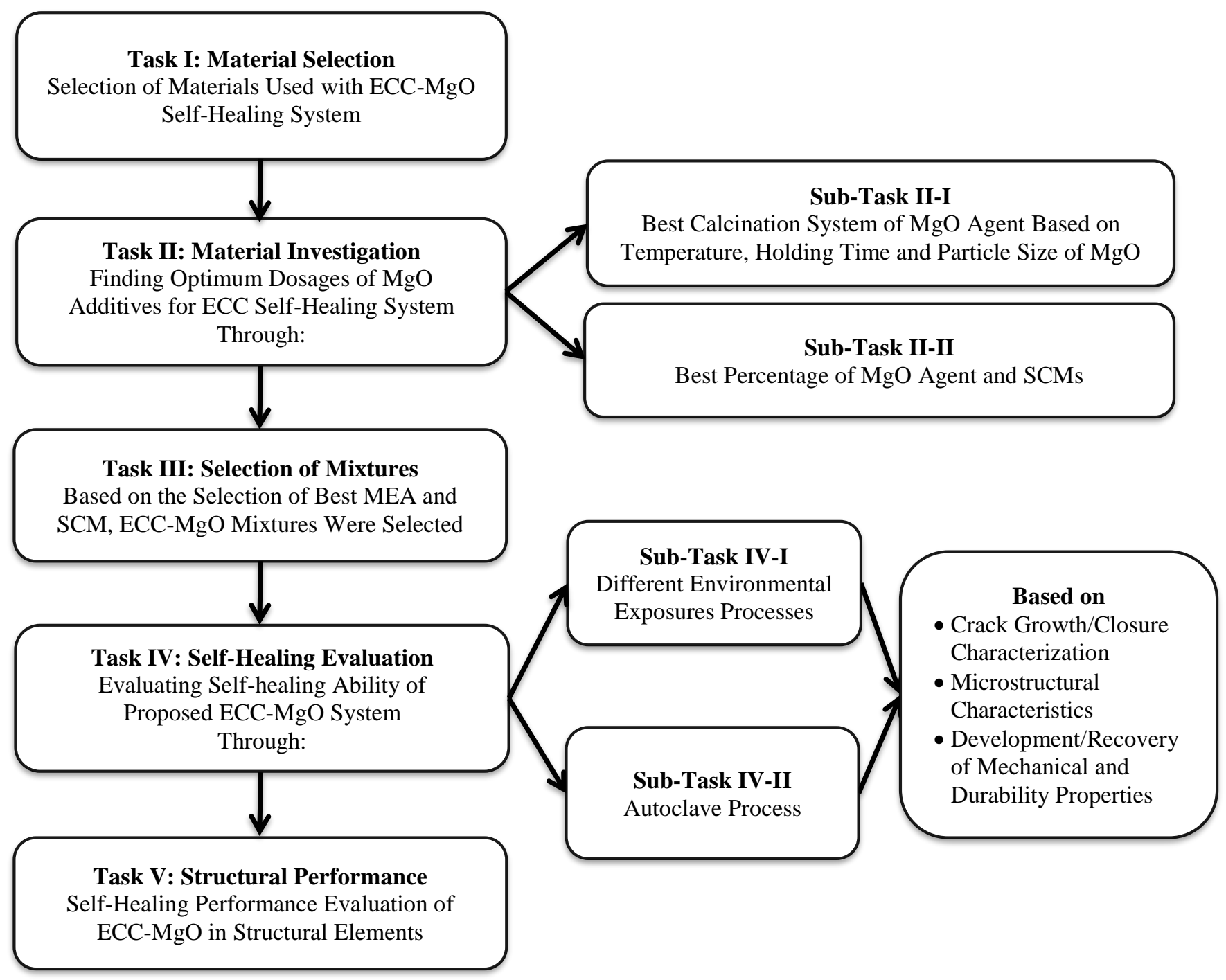

Figure 3-1: Experimental methodology flowchart 
It should be emphasized that the research program and methodology will be explained in general in this chapter while a full detailed explanation will be presented before the Results and Discussions sections in each subsequent chapter.

\subsubsection{Task I: Selection of materials}

The materials used to produce standard ECC mixtures were Type GU (general use), two different classes of fly ash (FA): Class-F fly ash with a low calcium content of $3.55 \%$ and Class-CI fly ash with a high calcium content of $17.28 \%$ obtained from Lafarge plant. The chemical composition and physical properties of Portland cement (PC), Class-F and Class-CI fly ash (FA) are presented in Table 3-1.

Table 3-1: Chemical composition and physical properties of Portland cement, Class-F \& CI fly ashes

\begin{tabular}{|c|c|c|c|}
\hline Chemical composition $(\%)$ & Cement $(\mathrm{C})$ & Class-F Fly Ash & Class-CI Fly Ash \\
\hline Calcium Oxide $\mathrm{CaO}$ & 62.70 & 3.55 & 17.28 \\
\hline Free $\mathrm{CaO}$ & 1.00 & - & - \\
\hline Silicon Dioxide $\mathrm{SiO}_{2}$ & 19.30 & 46.19 & 43.20 \\
\hline Aluminum Oxide $\mathrm{Al}_{2} \mathrm{O}_{3}$ & 4.70 & 23.39 & 21.46 \\
\hline Ferric Oxide $\mathrm{Fe} 2 \mathrm{O}_{3}$ & 3.00 & 21.81 & 8.31 \\
\hline Magnesium Oxide $\mathrm{MgO}$ & 3.00 & 0.82 & 3.28 \\
\hline Sulfur Trioxide $\mathrm{SO}_{3}$ & 3.47 & 1.13 & 1.38 \\
\hline Alkalis as $\mathrm{Na}_{2} \mathrm{O}$ & 0.22 & 0.51 & 0.97 \\
\hline Loss on ignition $\mathrm{LOI}$ & 2.40 & 3.00 & 0.50 \\
\hline Sum $\left(\mathrm{SiO}_{2}+\mathrm{Al} 2 \mathrm{O}_{3}+\mathrm{Fe} 2 \mathrm{O}_{3}\right)$ & 27.00 & 91.39 & 72.97 \\
\hline Physical properties & Cement $(\mathrm{C})$ & Class-F Fly Ash & Class-CI Fly Ash \\
\hline Residue $\left.45 \mu \mathrm{m}(\%)^{3}\right)$ & 5.10 & 18.00 & 15.00 \\
\hline Density $\left(\mathrm{g} / \mathrm{cm}^{3}\right)$ & - & 2.54 & 2.60 \\
\hline Blaine fineness $\left(\mathrm{m}^{2} / \mathrm{kg}\right)$ & 401 & 306 & - \\
\hline
\end{tabular}

In addition, MgO-type expansive agent (MEA) prepared from pure Magnesite (Magnesium Carbonate, $\mathrm{MgCO}_{3}$ ). The X-ray diffraction shown in Figure 3-2 revealed that MEA was prepared mainly from pure $\mathrm{MgCO}_{3}$. The decomposition temperature of $\mathrm{MgCO}_{3}$ started around $650^{\circ} \mathrm{C}$ and finished around $700^{\circ} \mathrm{C}$. The weight loss corresponding to the TGA/DTA curves was $46.82 \%$ as shown in Figure 3-3. 


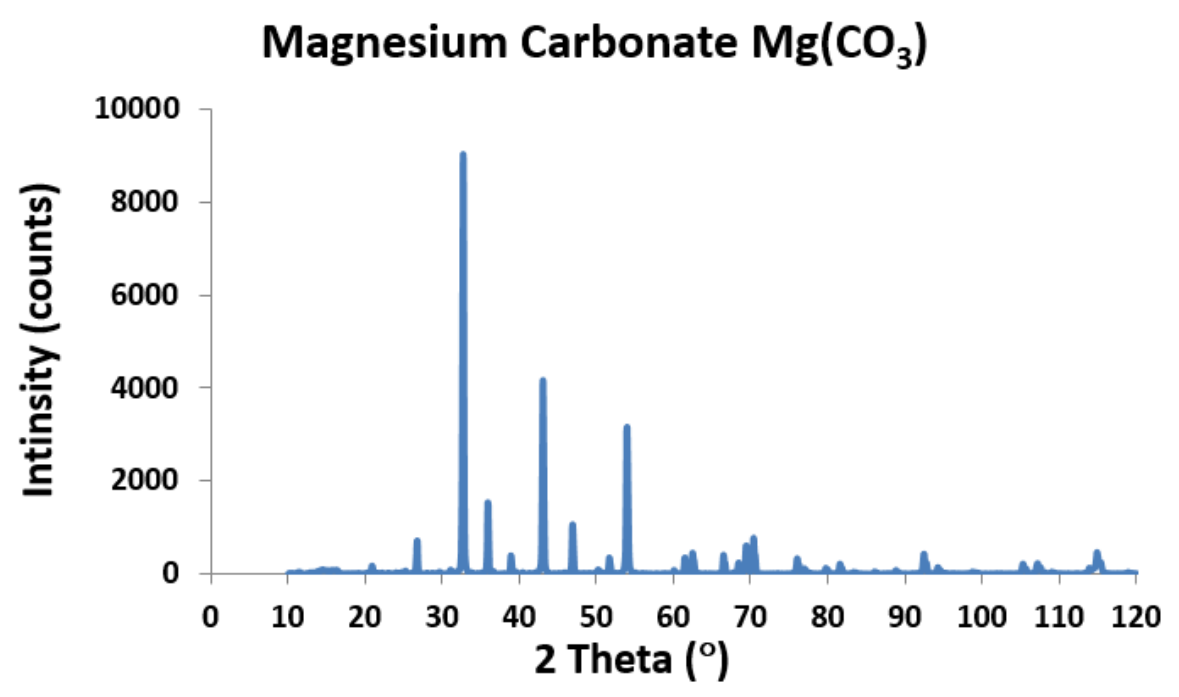

Figure 3-2: X-ray diffraction of pure $\mathrm{MgCO}_{3}$ used to prepare $\mathrm{MgO}$-type expansive agent

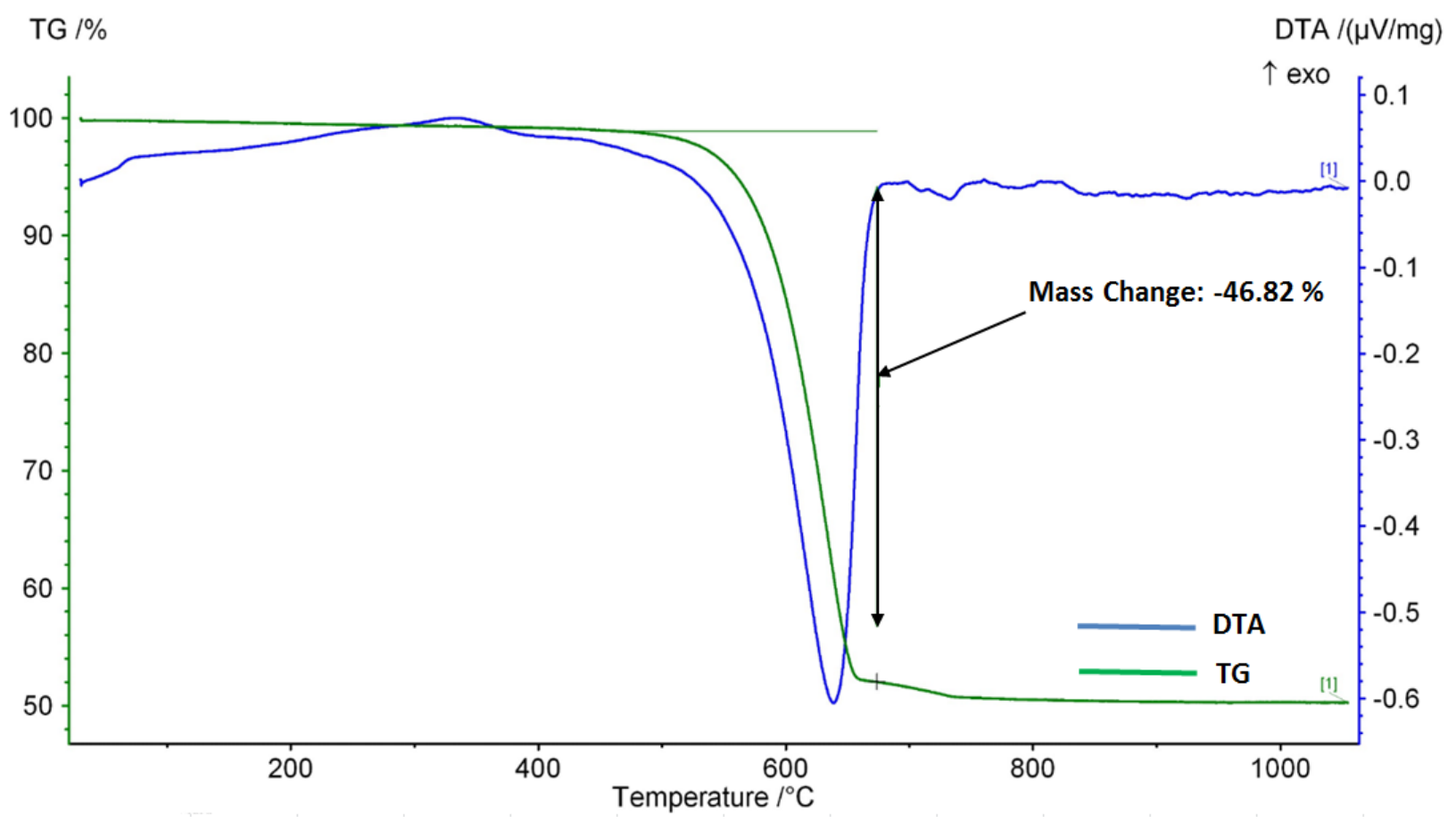

Figure 3-3: TGA/DTA curves of pure $\mathrm{MgCO}_{3}$ 
Silica sand (SS) with a maximum grain size of $0.40 \mathrm{~mm} / \mathrm{mean}$ size of $0.30 \mathrm{~mm}$. The grain size distribution of silica sand, Portland cement and Class-F fly ash are given in Figure 3-4.

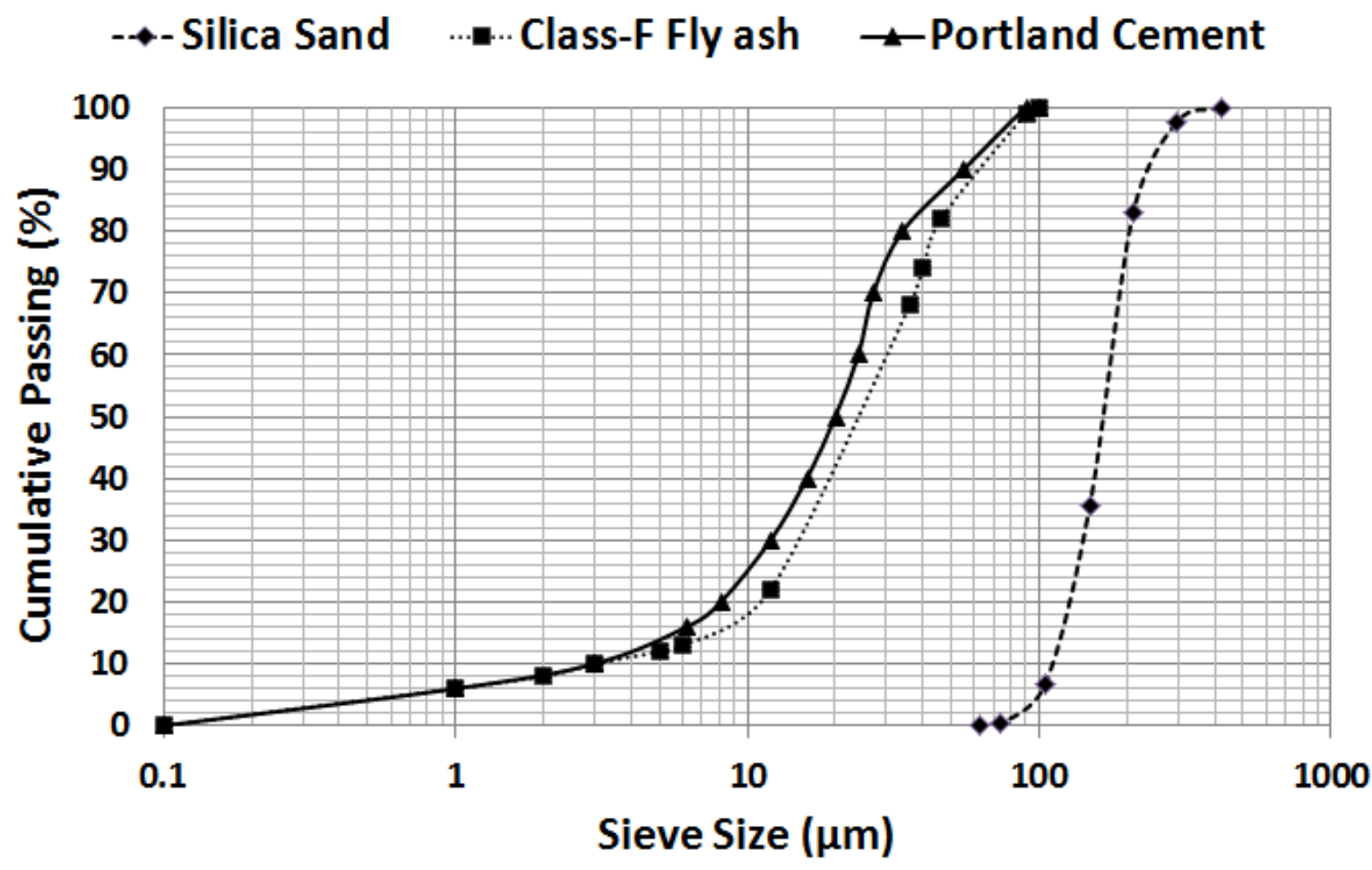

Figure 3-4: Grain size distribution of Portland cement, Class-F fly ash and silica sand (SS)

Polyvinyl alcohol (PVA) fiber with $8 \mathrm{~mm}$ length/39 $\mu \mathrm{m}$ diameter was used. The fiber surface is commercially coated with $1.2 \%$ oil by weight to reduce fiber/matrix frictional bond (Li et al., 2002). The tensile strength of the PVA fiber is $1600 \mathrm{MPa}$ and the density is $1,300 \mathrm{~kg} / \mathrm{m}^{3}$. To improve the workability for ECC mixtures, ADVA® cast 575 from Grace Construction Products was used as a high range water-reducing admixture (HRWRA).

\subsubsection{Task II: Determination of optimum dosages of MgO-type expansive additive for ECC-MgO self-healing system}

The objective of this task is to develop and assess the effectiveness of $\mathrm{MgO}$ expansive agent in ECC-MgO self-healing systems. The main variables of this task were calcination temperature $\left(750^{\circ} \mathrm{C}, 900^{\circ} \mathrm{C}, 1050^{\circ} \mathrm{C}\right.$ and $\left.1200^{\circ} \mathrm{C}\right)$, calcination time $(1,2$ and 4 hours $)$, particle diameter $(\leq$ 
$45 \mu \mathrm{m}$ and $45 \sim 100 \mu \mathrm{m}$ ) of $\mathrm{MgCO}_{3}$ raw material and the content (\%) of $\mathrm{MgO}$ additive. Consequently, the findings of the best calcination system and percentage of $\mathrm{MgO}$ additive in powder state was employed in all ECC mixtures to study the self-healing phenomena in Task III.

\subsubsection{Sub-Task II-I: Finding best calcination system for MgO agent}

Lightly burnt $\mathrm{MgO}$ expansive agent was prepared in this study from raw material $\mathrm{MgCO}_{3}$. In order to do so, $\mathrm{MgCO}_{3}$ was sieved at raw condition into two particle sizes ( $\leq 45 \mu \mathrm{m}$ and $45 \sim 100 \mu \mathrm{m}$ ). After sieving, the sieved $\mathrm{MgCO}_{3}$ material was calcined (burned) in an electric kiln at $750^{\circ} \mathrm{C}$, $900^{\circ} \mathrm{C}, 1050^{\circ} \mathrm{C}$ and $1200^{\circ} \mathrm{C}$ for 1,2 and 4 hours as holding time for each temperature, respectively.

After finding best calcination system based on (temperature/holding time/particle size), the behavior of MEA in powder state was investigated and compared with its behavior after it was used in the production of water and autoclave cured ECC-MgO bar specimens $(25 \times 25 \times 285 \mathrm{~mm})$. Two types of fly ashes (Class-F and Class-CI) were used in the production of ECC-MgO bar specimens in an attempt at selecting best supplementary cementing material that can fit the requirements of ECC-MgO self-healing techniques. The role of finding the desired calcination system was based on lower expansion effect of ECC-MgO bar specimens and higher remaining hydration of $\mathrm{MgO}$ in powder state. Further, the behavior of MEA in powder state was investigated and compared with its behavior after used in the production of ECC-MgO bar specimens.

\subsubsection{Sub-Task II-II: Finding the best percentage of $\mathrm{MgO}$ additive}

After finding the best calcination system (temperature/holding time/particle size), bar specimens $(25 \times 25 \times 285 \mathrm{~mm})$ made of two types of fly ash (Class-F and Class-CI) with cement replacements (30\% and 55\%) and different low dosages of MEA content (2\%, 4\%, 5\%, 6\% 8\% and 10\%) in addition to high dosages $(15 \%, 20 \%, 25 \%$ and $30 \%)$ were tested to find out best combination of SCM and MEA dosages used in ECC-MgO self-healing system based on lower expansion effect of bar specimens. The effectiveness of ECC-MgO self-healing system using the best combination of SCM and MEA dosages was assessed based on the compressive strength development/recovery through accelerated autoclave cured cube specimens. 


\subsubsection{Task III: Selection of ECC-MgO Mixtures}

After finding the best calcination system with the best combination of SCM and MEA dosages Task II, Class-F fly ash and 5\% of $\mathrm{MgO}$ powder amount were used in the production of ECC-MgO mixtures with respect to binder weight $(\mathrm{PC}+\mathrm{FA})$. The mixture proportions and designations for both ECC mixtures are given in Table 3-2. The water/binder ratio was kept in the range of 0.27 for both ECC mixes and the amount of aggregate/binder was held constant (0.36). It should be noted that fly ash content was reduced when $\mathrm{MgO}$ content was added to the $\mathrm{ECC}-\mathrm{MgO}$ mixes.

Table 3-2: ECC mixture proportions

\begin{tabular}{|c|c|c|c|c|c|c|c|c|}
\hline \multirow{2}{*}{ Mixture ID } & \multicolumn{7}{|c|}{ Ingredients per 1 part of binder measured by mass } \\
\cline { 2 - 10 } & $\begin{array}{c}\text { Water/ } \\
\text { Binder }\end{array}$ & $\begin{array}{c}\text { Binder } \\
(\mathrm{PC}+\mathrm{FA})\end{array}$ & $\mathrm{MgO}$ & $\begin{array}{c}\text { FA/Binder after } \\
\text { adding MgO }\end{array}$ & $\begin{array}{c}\text { Silica } \\
\text { Sand }\end{array}$ & $\begin{array}{c}\text { PVA } \\
\mathrm{kg} / \mathrm{m}^{3}\end{array}$ & $\begin{array}{c}\text { HRWRA } \\
\mathrm{kg} / \mathrm{m}^{3}\end{array}$ & FA/PC \\
\hline ECC & 0.27 & 1 & - & $55 \%$ & 0.36 & 26 & 5.4 & 1.20 \\
\hline ECC-MgO & 0.27 & 1 & $5 \%$ & $50 \%$ & 0.36 & 26 & 5.4 & 1.09 \\
\hline
\end{tabular}

\subsubsection{Task IV: Self-Healing evaluation of proposed ECC-MgO system}

The evaluation of the ability of crack closure/healing of proposed ECC-MgO self-healing system was done based on comprehensive experimental investigations under laboratory, water, natural and autoclave curing conditions. It should be noted that water curing means storing ECC and ECC$\mathrm{MgO}$ specimens continuously in water, natural curing means taking out the specimens to the outdoor site to cure them naturally and laboratory curing means curing the specimens inside sealed plastic bags until the day of testing. This task was divided into two sub-tasks:

\subsubsection{Sub-Task IV-I: Self-healing evaluation through different environmental exposures processes}

The assessment of the effectiveness of ECC-MgO self-healing system was evaluated by studying the mechanical and durability properties of cubic (50x50x50 mm), prismatic $(50 \times 76 \times 355 \mathrm{~mm})$, disk $(\varnothing 100 \mathrm{~mm}$ in diameter and $50 \mathrm{~mm}$ in thickness $)$ and bar $(25 \times 25 \times 285 \mathrm{~mm})$ specimens when MEA was combined with Class-F fly ash as self-healing system in ECC mixtures to heal micro cracks. Different pre-cracking events were conducted on specimens for getting ultimate 
loading/deformation capacities of the controls. Consequently, the pre-cracked specimens were exposed to different environmental exposures (water and natural curing) for different time durations to initiate the self-healing processes. It should be noted that the relative humidity (ranging between $13 \%$ and $98 \%$ ), temperature (ranging between $-26.3^{\circ} \mathrm{C}$ and $+34^{\circ} \mathrm{C}$ ), snow on ground (ranging between $1 \mathrm{~cm}$ and $21 \mathrm{~cm}$ ) and precipitation (ranging between $14.3 \mathrm{~mm}$ and $160.2 \mathrm{~mm}$ ) data is collected from Toronto weather website ("http://toronto.weatherstats.ca/," 2016) for the period from January 2015 to May 2016. All data are provided in detail in Appendix A.

The evaluation/recording of the recovery of pre-cracked specimens was assessed after each environmental exposure and compared with the controls. The mechanical and durability tests used in this research to evaluate development/recovery of self-healing ECC and ECC-MgO specimens included: compressive strength (ASTM C39, 2016) and flexure/load strength tests (ASTM C78-b, 2015), Ultrasonic Pulse Velocity (UPV) test (ASTM C597, 2016), drying shrinkage and expansion length change tests (ASTM C1038-b, 2014), water permeability through Rapid Chloride Ion Penetration (RCP) (ASTM C1202, 2012) and sorptivity tests (ASTM C1585, 2013), frost resistance (freeze-thaw resistance - (ASTM C666, 2015) - "Procedure A") and heat of hydration test under fresh state (ASTM C1679, 2014). The different evaluation tests were conducted on water and natural cured specimens and compared with the controls cured under similar conditions. Furthermore, surface crack observations (macro cracks characteristics), microstructure characteristics by conducting Thermogravimetric Analysis (TGA), Differential Scanning Calorimetry (DSC) and Scanning Electron Microscope (SEM) analyses were also studied. 


\subsubsection{Sub-Task IV-II: Self-healing evaluation through autoclave process}

To confirm the self-healing property of proposed ECC-MgO system, of specimens (cubes, prisms, bars and disks) were cured under autoclave curing condition and evaluated through development/recovery of compressive and flexure/load strength, UPV, RCP and sorptivity tests.

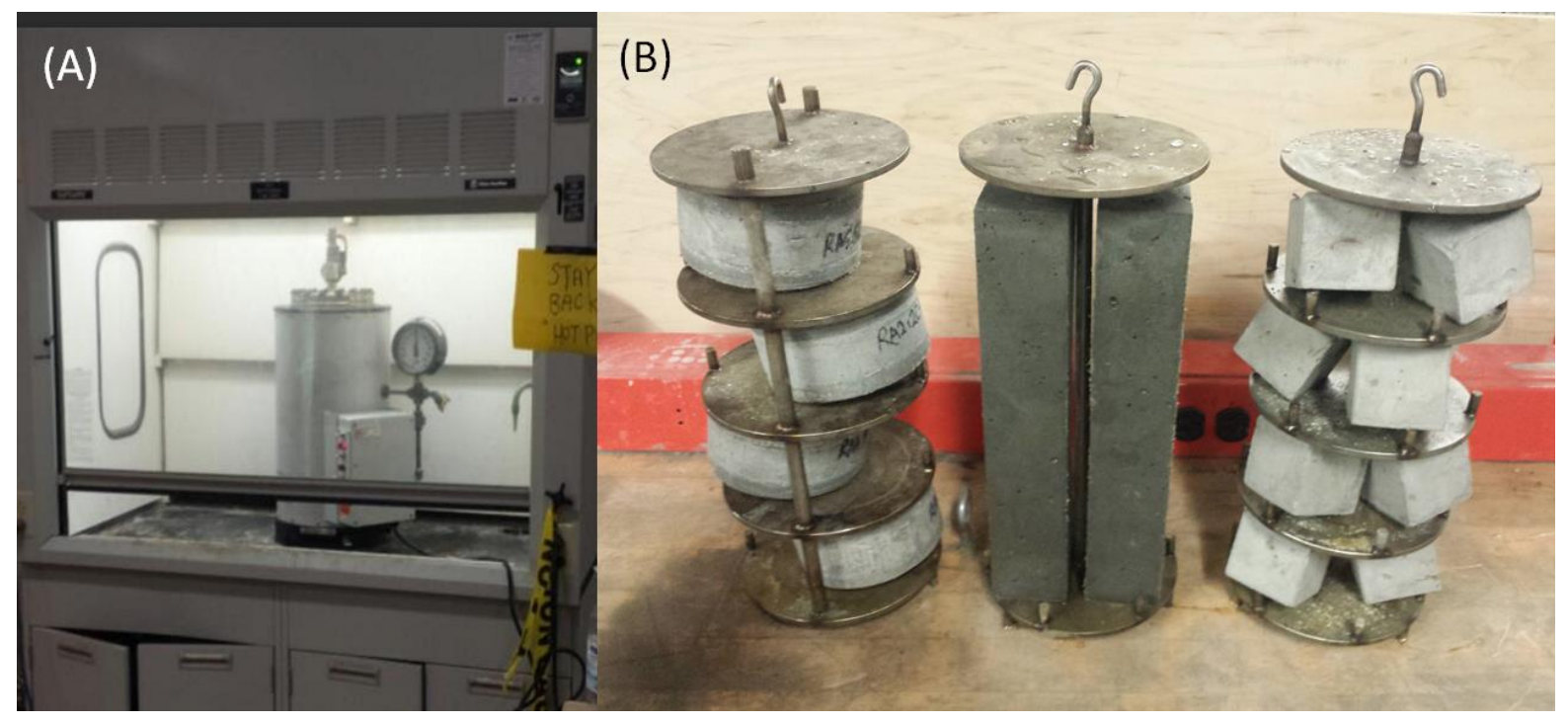

Figure 3-5: (A) High-pressure steam vessel - autoclave test and (B) special autoclave racks made for autoclave cured specimens

Autoclave process is the only standard test used to evaluate the expansion of magnesia in concrete by using bar specimens. During autoclave testing, the hydration of ECC-MgO bar specimens was expedited to reveal the delayed expansion caused by the hydration of $\mathrm{MgO}$ crystals present in cementitious materials. Therefore, to evaluate the mechanical and durability properties through autoclave test, special autoclave racks were made for cube, prism (with modified size of $50 \times 76 \times 285 \mathrm{~mm}$ ) and disk specimens to fit the autoclave vessel (see Figure 3-5). 


\subsubsection{Task V: Self-Healing Performance Evaluation of ECC in Structural Elements}

Self-healing property of ECC-MgO system was evaluated based on the structural performance of $1 / 4^{\text {th }}$ small-scale model link slab specimens. Controls were tested under flexural loading at the age of 28 days to determine load-deformation response, failure modes, crack development/propagation and crack width. The remaining specimens were pre-loaded up to $65 \%$ of the ultimate load of controls and then subjected to water and natural curing conditions. After 28 days +4 months, the precracked link slab specimens were post loaded to failure and compared with controls to evaluate the self-healing ability of ECC-MgO based link slab specimens. The development/recovery of precracked specimens in addition to crack development/closure/width measured before and after every exposure period was assessed as well and compared with the controls. It should be noted that the dimensions and other details of $1 / 4^{\text {th }}$ scale model link slab specimens follow ongoing research studies at Ryerson University (Gahtrehsamani, 2015; Hossain and Lachemi, 2014; Mavani, 2012; Sherir, 2012).

\subsection{Mixing Sequences and Production of ECC and ECC-MgO}

All mixtures used in the experimental program were produced by using a Hobart Type mixer with 20-liter capacity but for PVA fibers, the weighted solid materials were placed into the mixture and mixed for 30 seconds. Then $90 \%$ of the water was slowly added to the mixer and mixed at normal speed for additional 2 minutes. 


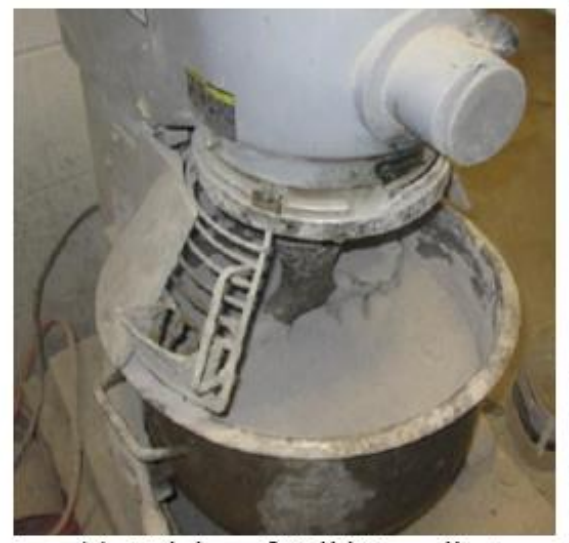

(a) Mixing of Solid Ingredients

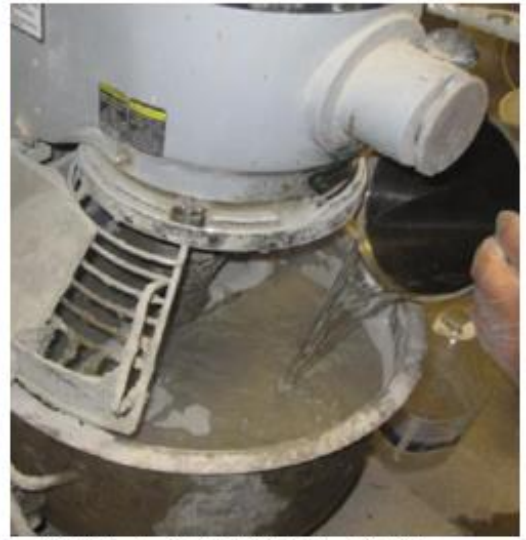

(b) Water\& HRWRA Addition

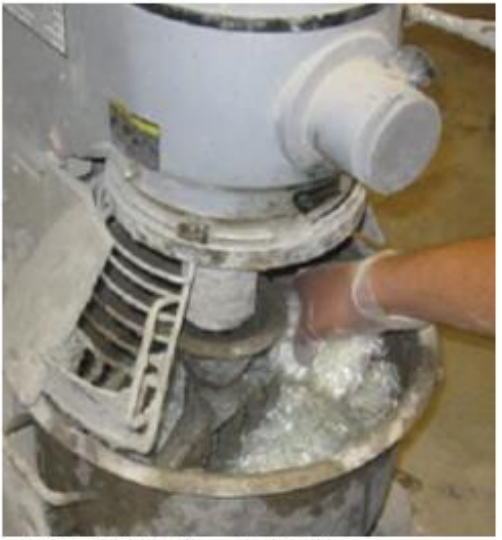

(c) PVA Fiber Addition

Figure 3-6: Mixing sequences of ECC mixtures (Sherir, 2012)

After, the remaining water and HRWRA were introduced to the mix (mixed for 1 minute) until a uniform consistent mortar mix is developed. Lastly, the PVA fibers were slowly added to the mortar for another 3 minutes of stirring until all fibers were dispersed completely within the mortar mixture. The absence of fiber-lumps at final mixing stage indicated good dispersion of fiber which is essential for better strain hardening and multiple cracking behaviors of ECC. The schematic representation of the mixing sequence is presented in Figure 3-6.

\subsection{Crack creation methods in different types of specimens}

Several approaches have been used in the literature to induce cracking in concrete specimens. Most of these approaches have a similar concept which is evaluating the development/recovery of the mechanical and durability properties of self-healed pre-cracked specimens when cured under different environmental exposures over different periods of time (Bhashkar, 2016). In this research, compressive strength and tensile splitting tests were used to induce concrete cracking in ECC and ECC-MgO specimens as shown in Figure 3-7. 


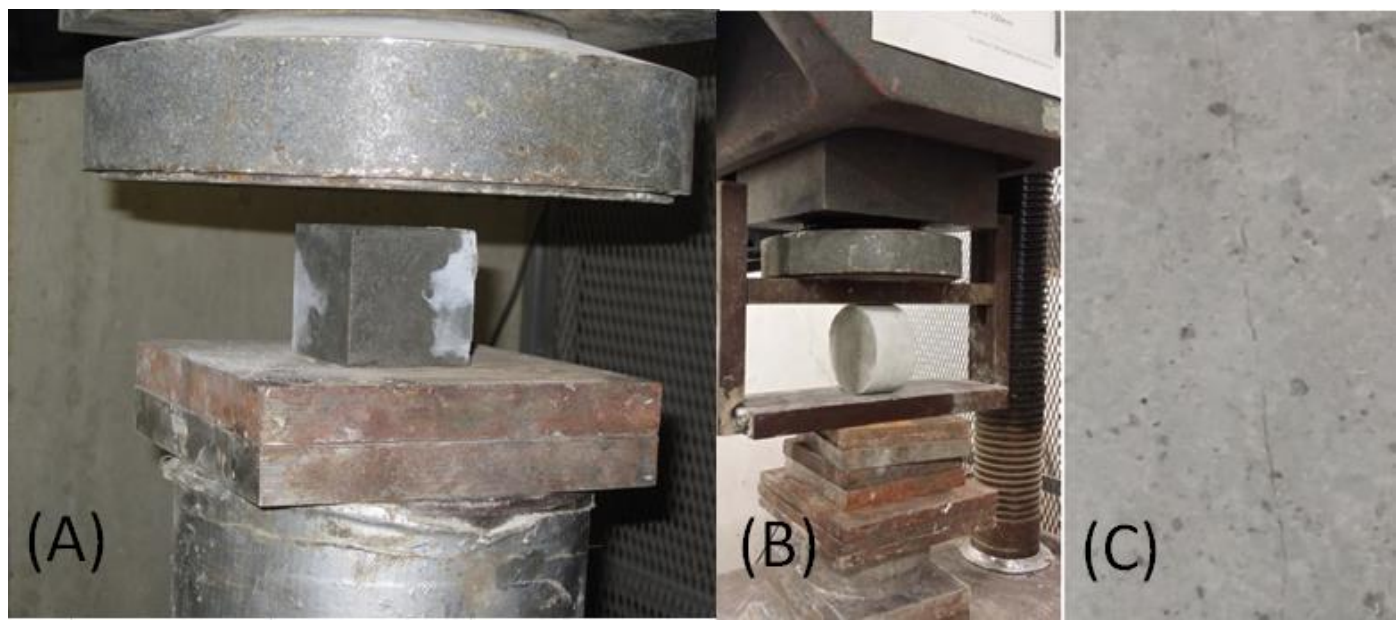

Figure 3-7: (A) Compressive strength test set up and (B) Tensile splitting test set up to induce cracks and (C) induced micro cracks (Bhashkar, 2016)

Further, as shown in Figure 3-8, the crack width controlled four-point bending test was also utilized in this research to induce cracking in self-healing ECC-MgO specimens which was considered one of the most widely used important tests for this purpose (Tittelboom and De Belie, 2013).

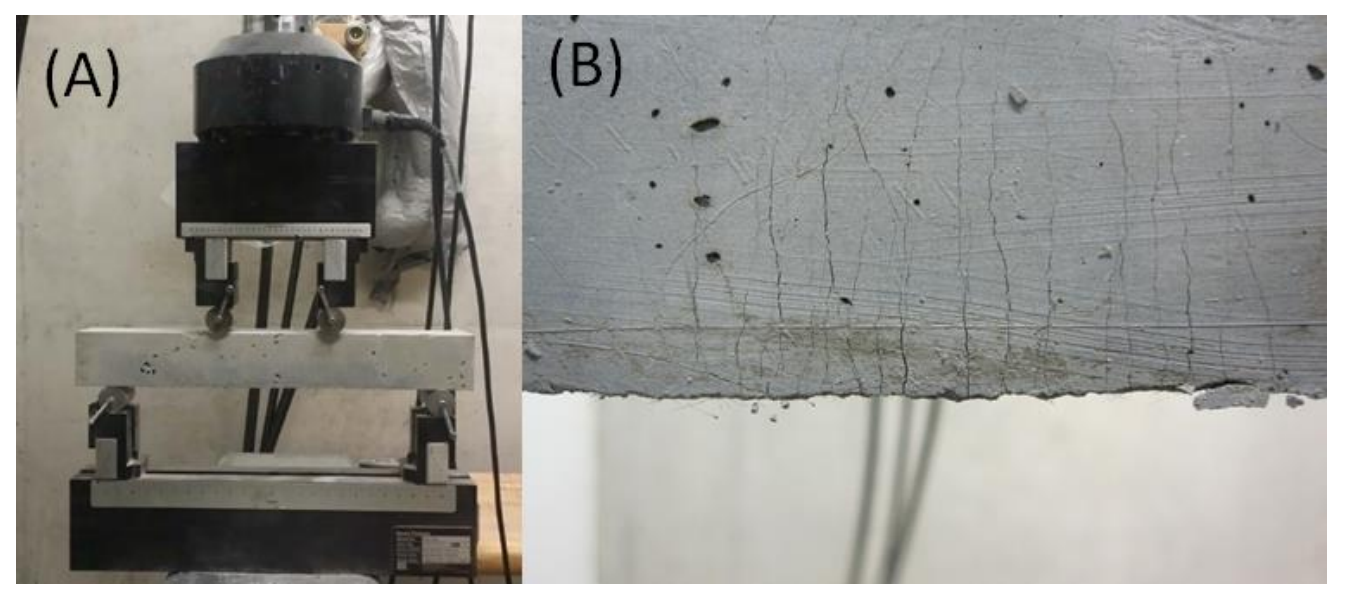

Figure 3-8: (A) Four-point bending test setup and to induce cracks and (B) induced micro cracks 


\subsection{Summary}

Chapter 3 presented the mixing procedure for ECC and ECC-MgO mixtures. It should be noted that the proposed ECC-MgO self-healing mixtures showed similar workability to standard ECC mixtures and the amount of HRWRA was kept fixed in both mixtures. In addition, all ECC mixtures have been cast following the same casting procedure and kept in same curing conditions.

In this chapter, the experimental program for the present research was introduced in general. A complete/detailed explanation of the experimental program, specimen preparations and procedures is presented for each task separately in the following chapters before the test results sections. This is because of the high number/types of specimens and test procedures/preparation for each task in this research. 


\section{CHAPTER FOUR}

\section{CALCINATION AND EXPANSION CHARACTERISTICS OF MGO-TYPE EXPANSIVE AGENT WHEN INCORPORATED AS SELF-HEALING AGENT IN ENGINEERED CEMENTITIOUS COMPOSITES}

\subsection{Introduction}

The aim of this chapter was to find out the best calcination system perfectly fit for ECC-MgO selfhealing technique based on the hydration activity of $\mathrm{MgO}$ type expansive agent (MEA) in powder state and expansion effect of MEA when involved in the production of ECC-MgO concrete through employing kiln burning process, accelerated autoclave/water linear expansion tests, scanning electron microscope (SEM) and thermogravimetric analysis (TGA). In addition, new measures were adopted when examining the performance of ECC-MgO self-healing system based on autoclave test results to simulate concrete state in an actual field environment. Eventually, a comprehensive comparison has been made to study the compatibility between the behaviors of $\mathrm{MgO}$ powder samples before and after use in ECC. 


\subsection{Experimental Investigations}

Two phases of investigations were conducted to study the efficiency of MEA before and after utilizing them in the production of ECC-MgO self-healing system. The efficiency of MEA was evaluated based on higher remaining hydration of $\mathrm{MgO}$ powder samples and lower expansion effect of ECC-MgO bar specimens.

In Phase I, MEA was used to find out the best calcination system that fits the requirements of ECC$\mathrm{MgO}$ self-healing system. The main variables of calcination system in this phase were calcination temperature, holding time and the particle diameter of MEA. MgO powder samples were prepared from pure Magnesite (Magnesium Carbonate, $\mathrm{MgCO}_{3}$ ) by exposing the later under four calcination temperatures inside the kiln $\left(750,900,1050\right.$ and $\left.1200^{\circ} \mathrm{C}\right)$. For every exposure, each temperature was applied for three holding times (1,2 and 4 hours), respectively. This burning process was conducted on two particle sizes of $\mathrm{MgCO}_{3}$ raw material $(\leq 45 \mu \mathrm{m}$ and $45 \sim 100 \mu \mathrm{m})$ to end up with twenty-four $\mathrm{MgO}$ powder samples. The key factor of finding the desired calcination system was based on higher remaining hydration capability of $\mathrm{MgO}$ powder samples.

Phase II: After finding the best calcination system (higher remaining hydration based on temperature/holding time/particle size) in the first phase, lower expansion effect of MEA was examined based on ECC-MgO bar specimens $(25 \times 25 \times 285 \mathrm{~mm})$ prepared for both water and autoclave linear expansion tests. In the attempt of selecting best supplementary cementing material that can fit the needs of ECC-MgO self-healing system, two types of fly ashes (Class-F and ClassCI) were used in the production of ECC-MgO bar specimens.

Finally, the behavior of MEA in powder state was investigated and compared with its behavior when it was used in the production of ECC-MgO system.

\subsubsection{Preparation of powder MgO-type expansive agent}

Pure Magnesite (Magnesium Carbonate, $\mathrm{MgCO}_{3}$ ) was sieved at the raw condition into two particle sizes. The sieving process was done by sieving the $\mathrm{MgCO}_{3}$ material through the standard $45 \mu \mathrm{m}$ sieve. The passed $\mathrm{MgCO}_{3}$ was considered as the material of particle size less than $45 \mu \mathrm{m}$ while the 
retained $\mathrm{MgCO}_{3}$ was considered as the material of particle size between 45 to $100 \mu \mathrm{m}$. At the end, two particle sizes of $\mathrm{MgCO}_{3}$ material $(\leq 45 \mu \mathrm{m}$ and $>45$ to $100 \mu \mathrm{m})$ were used.

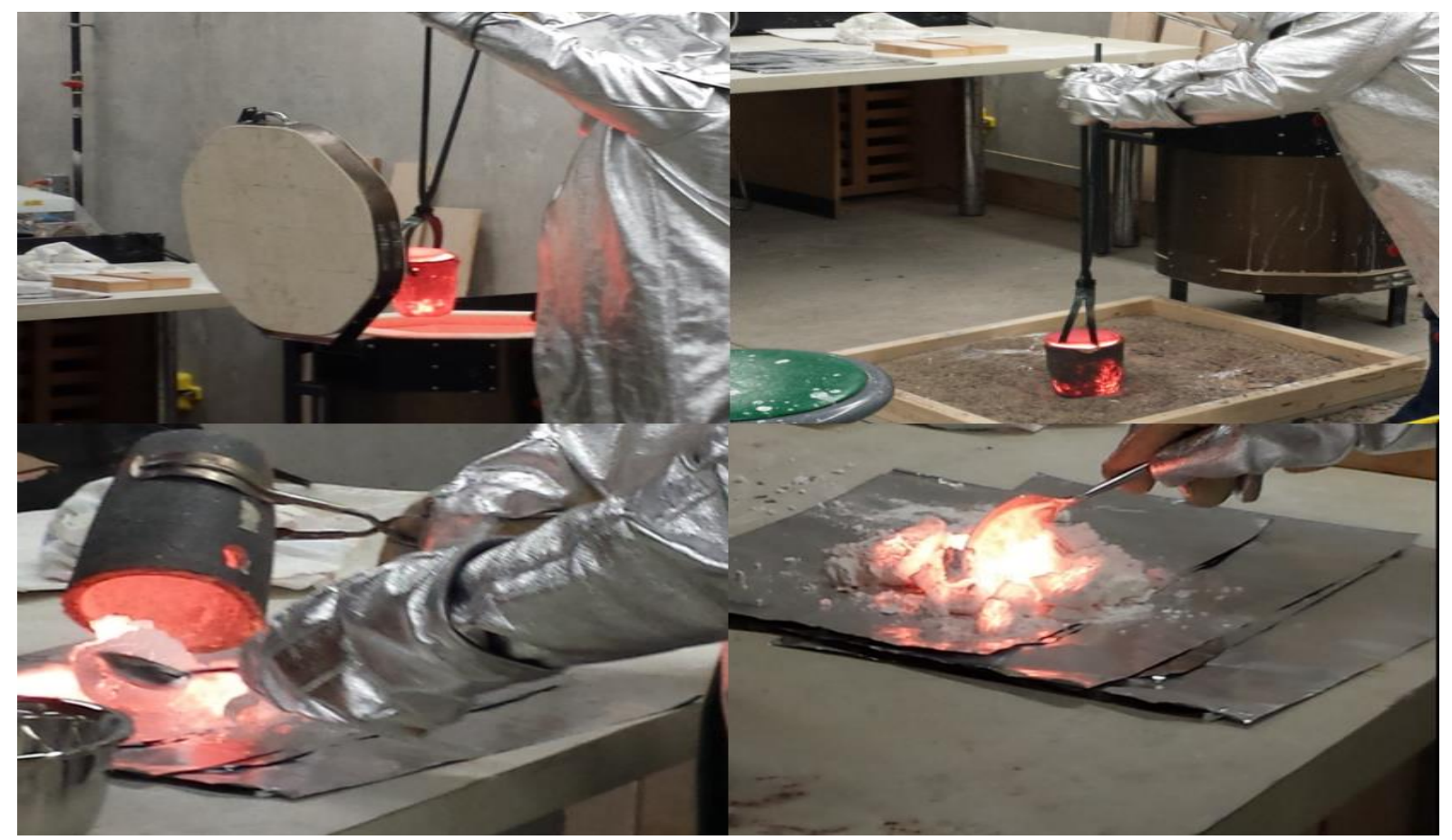

Figure 4-1: Cooling down $\mathrm{MgO}$ samples suddenly at room temperature at the end of calcination process $\left(1200^{\circ} \mathrm{C}\right.$ for 4 hours $)$

After sieving process, the sieved $\mathrm{MgCO}_{3}$ material was burnt in an electric kiln at 750, 900, 1050 and $1200^{\circ} \mathrm{C}$ for 1,2 and 4 hours of holding time at each temperature to obtain twenty-four $\mathrm{MgO}$ samples. After the completion of the calcination process, the very hot $\mathrm{MgO}$ powder samples were taken out of the kiln (right after the end of the assigned holding time) and dispersed on an aluminum sheet to allow higher possible contact with air in order to cool down the samples suddenly at room temperature (see Figure 4-1). This process was adopted to protect $\mathrm{MgO}$ from recomposing to the previous condition $\left(\mathrm{MgCO}_{3}\right)$. The $\mathrm{MgO}$ was the final outcome material after the completion of kiln burning process decomposed from the $\mathrm{MgCO}_{3}$ raw material after most of the $\mathrm{CO}_{2}$ content had been driven away.

After cooling down, the $\mathrm{MgO}$ (MEA) samples were stored inside sealed containers filled with silica gel and soda lime to mitigate the effect of both moisture and $\mathrm{CO}_{2}$. This was done as an extra 
caution to save the $\mathrm{MgO}$ stored in the sealed containers from recomposing again to the $\mathrm{MgCO}_{3}$ condition.

\subsubsection{ECC-MgO Mixture Proportions}

To develop and assess the effectiveness of MEA in ECC-MgO self-healing system, two ECC mixtures have been designed and selected. The first ECC mix was produced with Class-F fly ash and the second one was produced with Class-CI fly ash. Both ECC mixes were produced by using micro-silica sand. The ratio used for SCM as cement replacement was FA/PC $=1.2(\mathrm{FA} / \mathrm{Binder}=$ $55 \%$ ) for both types of fly ashes. The amount of $\mathrm{MgO}$ (MEA) powder used in the production of standard ECC bar specimens was $6 \%$ by binder weight $(\mathrm{PC}+\mathrm{FA})$. The mixture proportions and designations for both ECC mixtures are given in Table 4-1. The water/binder (W/B) ratio was kept in the neighborhood of 0.27 for both Class-F and CI fly ash. In both mixes, the ratio of aggregate and SCMs to binder ratio were held constant.

As shown in Table 4-1, both ECC mixtures are labeled in a way that the ingredients are identifiable from their Mix IDs. The first letter in the mixture designations indicates the SCM type (F = ClassF fly ash, CI = Class-CI fly ash). The numbers after the letter indicate the SCM (F/CI) - PC ratio and last letters represent aggregate type (SS).

Table 4-1: ECC mixture proportions with the addition of $\mathrm{MgO}$

\begin{tabular}{|c|c|c|c|c|c|c|c|c|}
\hline \multirow{2}{*}{ Mixture ID } & \multicolumn{7}{|c|}{ Ingredients per 1 part of binder measured by mass } \\
\cline { 2 - 9 } & $\begin{array}{c}\text { Water/ } \\
\text { Binder }\end{array}$ & $\begin{array}{c}\text { Binder } \\
(\mathrm{PC}+\mathrm{FA})\end{array}$ & $\mathrm{MgO}$ & $\begin{array}{c}\text { FA/Binder after } \\
\text { adding MgO }\end{array}$ & $\begin{array}{c}\text { Sand/B } \\
\text { inder }\end{array}$ & $\begin{array}{c}\text { PVA } \\
\mathrm{kg} / \mathrm{m}^{3}\end{array}$ & $\begin{array}{c}\mathrm{HRWRA} \\
\mathrm{kg} / \mathrm{m}^{3}\end{array}$ & FA/PC \\
\hline F_1.2_SS & 0.27 & 1 & $6 \%$ & $49 \%$ & 0.36 & 26 & 5.4 & 1.07 \\
\hline CI_1.2_SS & 0.27 & 1 & $6 \%$ & $49 \%$ & 0.36 & 26 & 5.4 & 1.07 \\
\hline
\end{tabular}

\subsubsection{Preparation of ECC-MgO bar specimens}

After the completion of kiln burning process, twenty-four MgO (MEA) samples with different calcination temperature $\left(750,900,1050\right.$ and $\left.1200^{\circ} \mathrm{C}\right)$, holding time $(1,2$ and 4 hours $)$ and two particle sizes $(\leq 45 \mu \mathrm{m}$ and $45 \sim 100 \mu \mathrm{m})$ were prepared and used in the production of bar specimens to investigate the behavior of ECC-MgO self-healing system. The two ECC-MgO mixtures were 
made of two types of fly ashes, the first one with class-F fly ash with a low $\mathrm{CaO}$ content of $3.55 \%$ and the second one with class-CI fly ash with a high $\mathrm{CaO}$ content of $17.28 \%$ (Table 4-1). For the purpose of identifying the optimum calcination temperature, as shown in Table 4-1, fixed amount of $6 \%$ of $\mathrm{MgO}$ powder sample measured by binder mass was employed in the production of standard bar specimens in accordance with (ASTM C1038-b, 2014). At least three $25 \times 25 \times 285 \mathrm{~mm}$ bar specimens were cast from each of the twenty-four MgO (MEA) samples to monitor linear expansion readings under water curing conditions. The total number of bar specimens prepared for water expansion test was 144. On the other hand, only two bar specimens (total 96) were prepared for autoclaved cured expansion tests. After casting, bar specimens were cured in plastic bags at 95 $\pm 5 \%$ relative humidity $(\mathrm{RH}), 23 \pm 2{ }^{\circ} \mathrm{C}$. All bar specimens were demolded after 24 hours and initial length measure was taken right after demolding. After obtaining the initial length comparator readings, the water cured ECC-MgO bar specimens were kept in water at $20^{\circ} \mathrm{C}$ until the next length measure while autoclave ECC-MgO bar specimens were placed in autoclave machine for testing.

\subsection{Test procedures}

\subsubsection{MgO powder samples}

To find out the best calcination system of ECC-MgO self-healing technique, hydration activity of $\mathrm{MgO}$ powder samples was studied based on two test methods. The first method was calculating the weight loss of raw $\mathrm{MgCO}_{3}$ (= original weight of $\mathrm{MgCO}_{3}$ raw material - the weight of $\mathrm{MgO}$ ) after subjected to different temperatures and holding time inside the electric kiln. The weight loss occurred due to the decomposition of $\mathrm{MgCO}_{3}$ into $\mathrm{CO}_{2}$ and $\mathrm{MgO}$ and subsequent loss of $\mathrm{CO}_{2}$.

In the second method, TGA/DTA machine was used to measure both weight loss and decomposition temperature of every previously burnt $\mathrm{MgO}$ powder samples which produced by electric kiln and compare them with TGA control. The control, in this case, was a sample of raw $\mathrm{MgCO}_{3}$ material burnt and tested by TGA test for comparison purposes. During the TGA test, the previously burnt $\mathrm{MgO}$ samples by kiln were exposed again to a higher temperature $\left(1200^{\circ} \mathrm{C}\right)$ for around 2.5 hours. Finally, the weight loss of TGA results of all twenty $\mathrm{MgO}$ samples was compared with the weight loss of kiln results to find out the difference between them. It should be emphasized 
that in this research the weight loss of $\mathrm{MgO}$ samples was represented by the loss of $\mathrm{CO}_{2}$ content and the decomposition temperature was represented by the remaining hydration of $\mathrm{MgO}$ samples.

\subsubsection{ECC-MgO bar specimens}

In addition to analyzing the best calcination system of ECC-MgO self-healing system through higher hydration activity, expansion characteristics of water and autoclave cured $25 \times 25 \times 285 \mathrm{~mm}$ ECC-MgO bar specimens were monitored. For water curing, all bar specimens were stored in water until the following length measure in accordance with (ASTM C1038-b, 2014) and the readings were taken up to 500 days by using length comparator in accordance with (ASTM C490, 2011). For autoclave cured (using high-pressure steam vessel in accordance with ASTM C151-15 (ASTM C151, 2015), Figure 3-5) ECC-MgO bar specimens, the length measure readings were taken right after the autoclaving. During autoclave testing, the hydration of ECC-MgO bar specimens was expedited to reveal the delayed expansion caused by the hydration of $\mathrm{MgO}$ crystals. All ECC-MgO bar specimens placed inside the autoclave in a special rack were exposed to saturated hot steam from all sides. The pressure generated by autoclave machine was $2 \pm 0.07 \mathrm{MPa}$ corresponding to a temperature of $216 \pm 2^{\circ} \mathrm{C}$ for at least 3 hours.

Due to the rapid acceleration of the hydration of $\mathrm{MgO}$ crystals, autoclave expansion test was

criticized by Mehta (1978) through different aspects. Firstly, elevating the temperature up to 216 $\pm 2^{\circ} \mathrm{C}$ can accelerate not only $\mathrm{MgO}$ hydration but also the hydration of $\mathrm{C}-\mathrm{S}-\mathrm{H}$ leading to destroying the cohesive forces present in cement pastes, reducing the expansion resistance and exaggerating the expansion values dramatically. Secondly, the nature of cement paste matrix is totally different than concrete matrix. It was reported in Mehta's research that concrete specimens cured in normal conditions (water curing) can show excessive expansions only when comparable cement pastes show more than $15 \%$ expansion cured in autoclaved conditions. Therefore, it was thought that the autoclave expansion limit might be safe even if it reached $10 \%$. The presence of aggregates in concrete or PVA fibers in ECC can improve the volume stability leading to lower expansion in concrete compared to pastes. Also, $\mathrm{MgO}$ content can be less per unit volume in concrete microstructures than pastes. Thirdly, only length measure has been used mainly to evaluate the correlation between the autoclave test and the soundness of cement whereas other mechanical and 
durability properties were still absent from the scene of autoclave test (Mehta, 1978; Mo et al., 2014). To make the accelerated autoclave test results closer to the concrete in the field, Mo et al. (2014) mentioned that many researchers were taking different measures to link autoclave test with the performance of $\mathrm{MgO}$ concrete in the field. For instance, mortar, concrete or even large scale concrete specimens were used instead of cement pastes and also lowering the autoclave test temperature from $216^{\circ} \mathrm{C}$ to $200^{\circ} \mathrm{C}, 150^{\circ} \mathrm{C}$ or $80^{\circ} \mathrm{C}$.

In this research, different measures were taken to analyze the results of accelerated autoclave test to assess the performance of ECC-MgO self-healing system effectively and compare them with water curing condition. Furthermore, based on the findings of Mehta (1978) which revealed that increasing the autoclave expansion limit in concrete specimens up to $10 \%$ instead of $0.80 \%$ in cement pastes might be perfectly safe, authors in this research used 6\% $\mathrm{MgO}$ by binder (PC+FA) weight instead of cement weight. This is equivalent to about $13 \% \mathrm{MgO}$ by weight of cement. Also, a comprehensive study of ECC-MgO self-healing systems used $\mathrm{MgO}$ content has been done based on mechanical and durability properties of ECC mixtures and is presented in the following chapters.

It should be emphasized that during autoclave test, care should be taken to ensure safety due to the emission of sharp fumes which could be toxic due to the presence of PVA fibers and chemical admixtures in ECC-MgO specimens when exposed to very high pressure and temperature. Consequently, a proper ventilation system such as fume hood and complete respiratory protection with ambient air pump must be used. 


\subsection{Results and Discussions}

All expansion data of water and natural cured prism/bar specimens are provided in Appendix B.

\subsubsection{Phase I: Finding Best Calcination System for MgO Additive}

\subsubsection{Best Calcination based on weight loss of $\mathrm{MgCO}_{3}$ after kiln burning process}

Most studies in the literature have revealed that calcination system which consists of calcination temperature, holding time and fineness to be the most important factor affecting the expansive behavior and the hydration of MgO-type expansion agent (MEA). The proper calcination temperature and holding time for MEA were identified by different researchers. Zhang et al. (2012) revealed that the decomposition rate and activity of $\mathrm{MgO}$ were significant at calcination temperature of $666.99^{\circ} \mathrm{C}$ and calcination time of 2.12 hours (Zhang et al., 2012). Gao et al. (2008) prepared MEA at a temperature of $1150^{\circ} \mathrm{C}$ with a holding time of 1 hour (Gao et al., 2008). Moreover, $\mathrm{Du}$ (2005) reported that the hydration rate of $\mathrm{MgO}$ powder was $100 \%$ within the first 3 days when the burning temperature was $800^{\circ} \mathrm{C}$ in the production of MEA while the hydration rate of $\mathrm{MgO}$ powder was $97.6 \%$ after 360 days when the burning temperature increased up to $1200^{\circ} \mathrm{C}$ (Du, 2005). Xian Li et al. (2010) mentioned that the best calcination system for producing MEA occurred when burning the raw $\mathrm{MgCO}_{3}$ at the temperature of $1000^{\circ} \mathrm{C}$ for 1 hour and using particle diameter of 45 150 $\mu \mathrm{m}$ (Xian Li et al., 2010). To find out the proper calcination system in this research, "lightly burnt" MEA was prepared by exposing the raw $\mathrm{MgCO}_{3}$ material to four calcination temperatures $\left(750,900,1050\right.$ and $\left.1200^{\circ} \mathrm{C}\right)$ for three holding times $(1,2$ and 4 hours), respectively. Also, two different particle sizes of $\mathrm{MgCO}_{3}$ material were used in this research $(\leq 45$ $\mu \mathrm{m}$ and $>100$ to $45 \mu \mathrm{m})$.

As shown in Figure 4-2, the loss of $\mathrm{CO}_{2}$ content increased with the increase of calcination temperature and holding time. The effect of $\mathrm{CO}_{2}$ loss was observed clearly at lower temperatures as in the case of $750^{\circ} \mathrm{C}$ for both particle sizes $(100$ and $45 \mu \mathrm{m})$. At $750^{\circ} \mathrm{C}$, the loss of $\mathrm{CO}_{2}$ content based on MEA mass loss was $23 \%$ after 1 hour of holding time. 

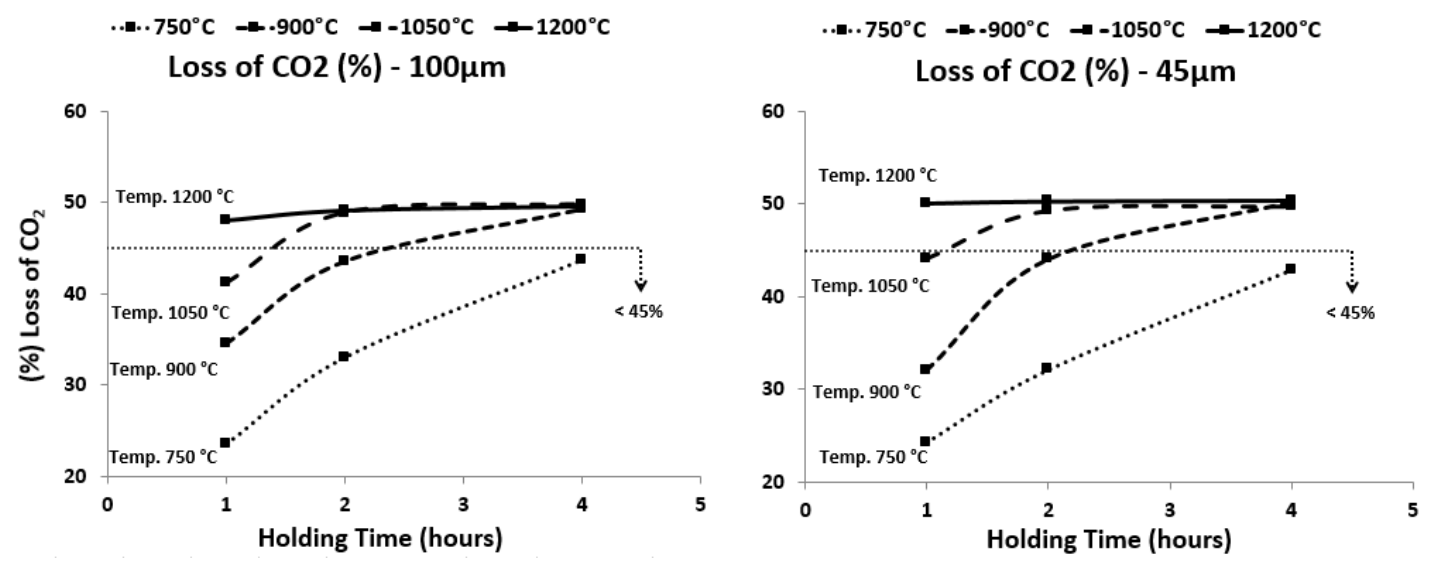

Figure 4-2: Weight loss of $\mathrm{MgCO}_{3}$ material after burning by kiln represented by $\%$ of $\mathrm{CO}_{2} \operatorname{loss}$
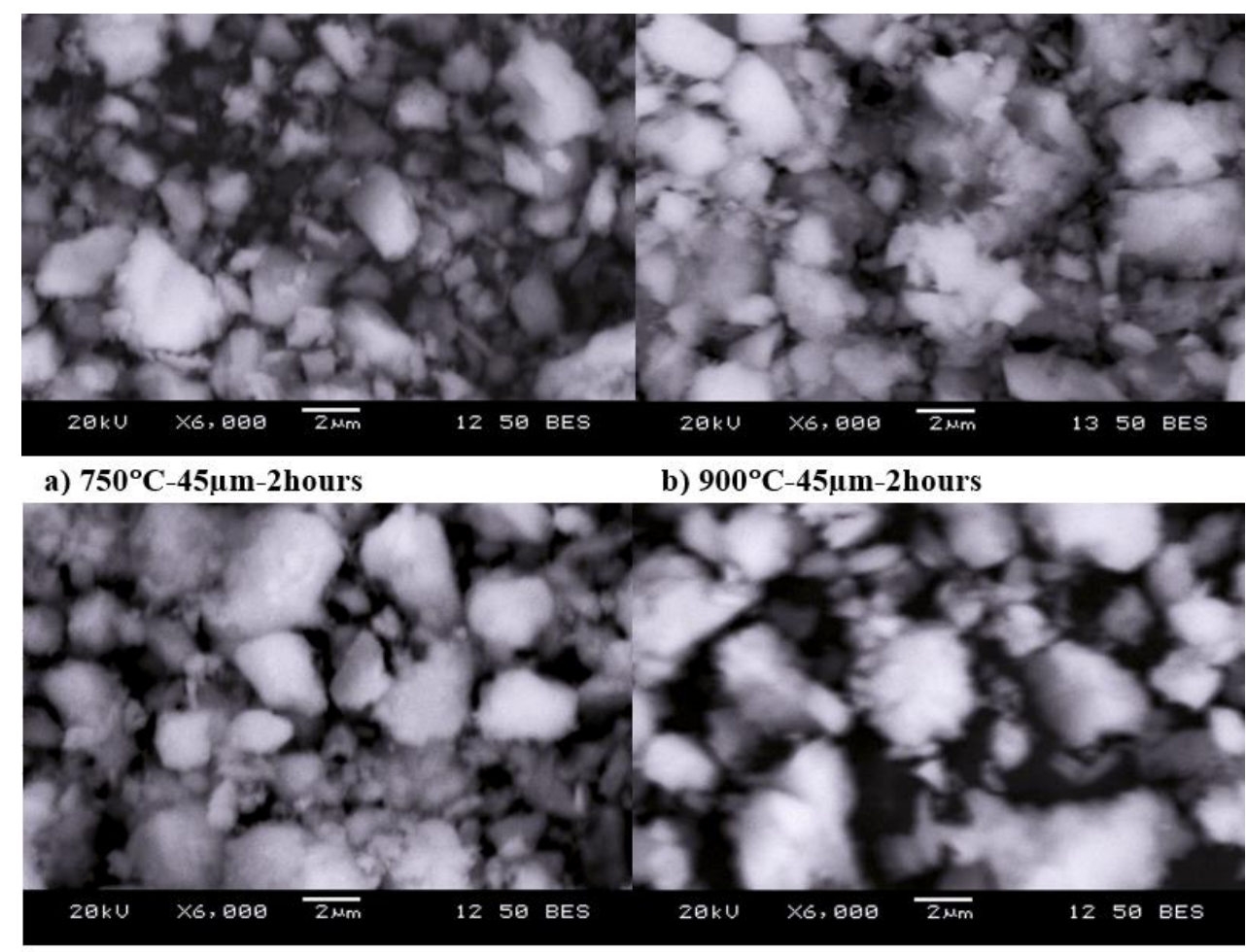

c) $1050^{\circ} \mathrm{C}-45 \mu \mathrm{m}-2 \mathrm{hours}$

d) $1200^{\circ} \mathrm{C}-45 \mu \mathrm{m}-2 \mathrm{hours}$

Figure 4-3: Effect of calcination temperature and 2 hours of holding time on grain size of MEA

Figure 4-3 shows the morphology of MEA when prepared at different temperatures and under fixed holding time. Again, the grain size of MEA increased with the increase of temperature. The average particle size of MEA was $1.56 \mu \mathrm{m}, 2.90 \mu \mathrm{m}, 3.25 \mu \mathrm{m}$ and $4.16 \mu \mathrm{m}$ when prepared at the temperatures of $750^{\circ} \mathrm{C}, 900^{\circ} \mathrm{C}, 1050^{\circ} \mathrm{C}$ and $1200^{\circ} \mathrm{C}$, respectively. Therefore, increased calcination 
temperature in the preparation of MEA will increase the loss of $\mathrm{CO}_{2}$ and grain particle size. Mo et al. (2010) revealed that when the calcination process starts, $\mathrm{MgO}$ crystals starts to form initially after $\mathrm{MgCO}_{3}$ decomposes due to the loss of $\mathrm{CO}_{2}$ content (Mo et al., 2010). With the proceeding of calcination process at higher temperatures and longer holding times, an agglomeration of $\mathrm{MgO}$ crystals will initiate a growth in $\mathrm{MgO}$ grain particle size and then lowering of the specific surface areas.

The progress of losing $\mathrm{CO}_{2}$ content (as shown in Figure 4-2) was negligible with a very minimal change especially at higher temperatures such as $1200^{\circ} \mathrm{C}$ for both particle sizes $(100$ and $45 \mu \mathrm{m})$. For instance, $1200^{\circ} \mathrm{C}_{-} 100 \mu \mathrm{m}$ curve lost around $48 \%$ of $\mathrm{CO}_{2}$ content at 1 hour of holding time while it was around $50 \%$ after 4 hours of holding time. Similar behavior was observed from $1200^{\circ} \mathrm{C} \_45 \mu \mathrm{m}$ curve but still the loss of $\mathrm{CO}_{2}$ content was fixed at $50 \%$ for both 1 hour and up to 4 hours of holding times. The slight difference between $1200^{\circ} \mathrm{C}$ curves for the two particle sizes $(100$ and $45 \mu \mathrm{m})$ at all holding times may be attributed to higher temperature exposure $\left(1200^{\circ} \mathrm{C}\right)$ which can aggravate the loss of $\mathrm{CO}_{2}$ content at the beginning of the burning process (first hour of holding time) and reaching a plateau curve earlier as shown in Figure 4-2. As a result, both particle sizes of $\mathrm{MgCO}_{3}$ material $(100$ and $45 \mu \mathrm{m})$ revealed similar behavior of the loss of $\mathrm{CO}_{2}$ content even though smaller particle size $(45 \mu \mathrm{m})$ has higher fineness than $100 \mu \mathrm{m}$. However, the researcher preferred to use both particle sizes $(100$ and $45 \mu \mathrm{m})$ of $\mathrm{MgCO}_{3}$ raw material in all experiments to study the particle size effect on MEA.

Similar to Figure 4-2, Figure 4-4 shows the behavior of the loss of $\mathrm{CO}_{2}$ content in terms of calcination temperatures $\left(750,900,1050\right.$ and $\left.1200^{\circ} \mathrm{C}\right)$. The higher the calcination temperature and holding time the higher the loss of $\mathrm{CO}_{2}$ content. As shown in Figure 4-4, the effect of $\mathrm{CO}_{2}$ loss was observed clearly at the curve of 1 hour of holding time. At this curve, the loss of $\mathrm{CO}_{2}$ content was around $23 \%$ at $750^{\circ} \mathrm{C}$ and increased gradually until it reached $50 \%$ at $1200^{\circ} \mathrm{C}$. All plateaus in Figure 4-2 \& Figure 4-4 for both particle sizes were started at 50\% loss of $\mathrm{CO}_{2}$ content. For instance, the progress of losing $\mathrm{CO}_{2}$ content started to form a plateau after $900^{\circ} \mathrm{C}$ under the condition of 4 hours of holding time and after $1050^{\circ} \mathrm{C}$ under 2 and 4 hours of holding times as well. Under all holding times studied, $1200^{\circ} \mathrm{C}$ had the highest loss of $\mathrm{CO}_{2}$ content for both particle sizes which was around $50 \%$. This means that the loss of $\mathrm{CO}_{2}$ content at $1200^{\circ} \mathrm{C}$ was affected 
directly by increasing the temperature no matter what was the holding time, or it was affected by cooking the $\mathrm{MgCO}_{3}$ raw material for a longer period of times even under lower temperatures (as in $900^{\circ} \mathrm{C}$ and $1050^{\circ} \mathrm{C}$ ). This finding was almost identical with $\mathrm{Du}$ (2005) and Mo et al. (2012) who revealed that the limit of burning temperature of "heavily burnt" $\mathrm{MgO}$ is about $1450^{\circ} \mathrm{C}$ (which is already contained in the cement) and the limit of burning temperature of "lightly burnt" $\mathrm{MgO}$ is about $1100^{\circ} \mathrm{C}$ which is to be added to the concrete as additives. Only "heavily burnt" crystalline $\mathrm{MgO}$ has deleterious and harmful reactivity on concrete structures at later ages (Neville, 1995). Conservatively, all temperatures that reached the plateau will be omitted from the goal of finding best calcination system. These temperatures were $900^{\circ} \mathrm{C} \_4,1050^{\circ} \mathrm{C} \_2 \& 4$, and finally $1200^{\circ} \mathrm{C}$ under all holding times. Less percentages of $\mathrm{CO}_{2}$ loss in this research was chosen to be less than $45 \%$ as shown in Figure 4-2 \& Figure 4-4.
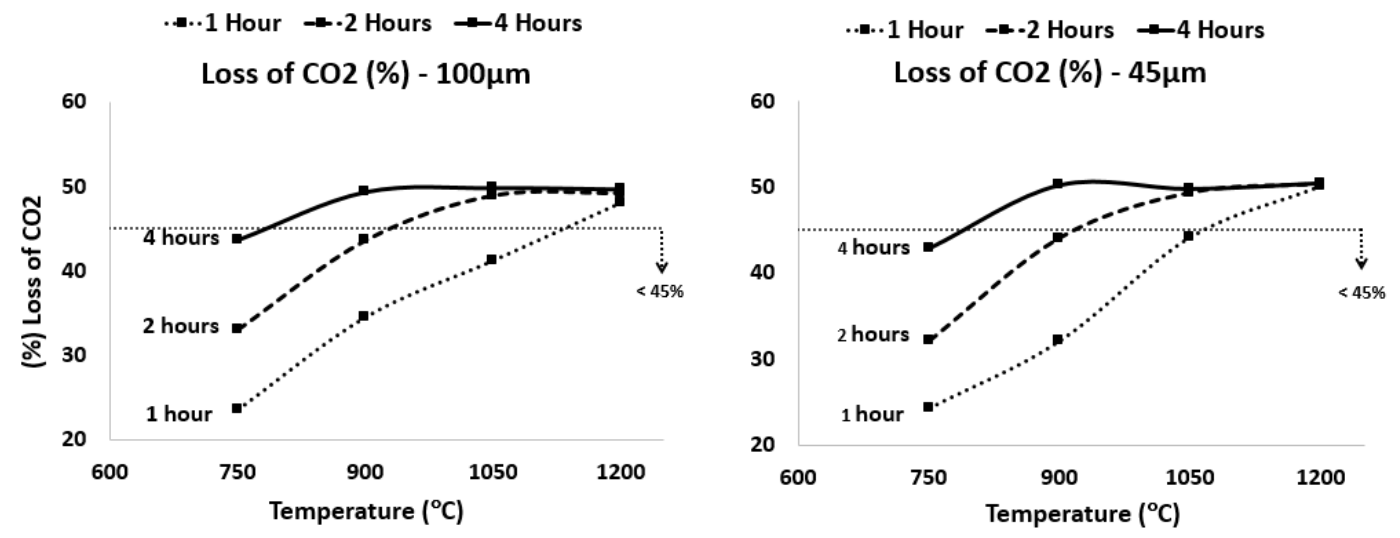

Figure 4-4: Weight loss of $\mathrm{MgCO} 3$ material after burning by kiln represented by $\%$ of $\mathrm{CO}_{2}$ loss

Referring to Figure 4-4 and as shown in Figure 4-2, both particle sizes of $\mathrm{MgCO}_{3}$ material $(100 \mu \mathrm{m}$ and $45 \mu \mathrm{m}$ ) revealed comparable behavior of the loss of $\mathrm{CO}_{2}$ content.

\subsubsection{Best Calcination based on degree of hydration of previously burned MgO samples by using TGA/DTA}

After the completion of kiln burning process, all $\mathrm{MgO}$ powder samples were cooled down suddenly to room temperature and then saved inside sealed box filled with silica gel and soda lime to prevent the re-composition to the first state $\left(\mathrm{MgCO}_{3}\right)$. The best calcination system for ECC-MgO self- 
healing system was selected at this stage based on higher remaining hydration. Therefore, before utilizing the previously burnt $\mathrm{MgO}$ samples (in the electric kiln) into specimen casting, more investigation was needed to predict the efficiency of the remaining hydration activity of all $\mathrm{MgO}$ powder samples. The degree of hydration (remaining hydration activity) of all $\mathrm{MgO}$ samples was analyzed based on the decomposition temperature of TGA tests and the difference in weight loss of $\mathrm{MgO}$ powder samples acquired by kiln and TGA tests.

\subsection{Decomposition temperature of MgO samples}

The TGA test results in terms of decomposing temperature for all previously burnt $\mathrm{MgO}$ samples are given in Table 4-2 and Figure 4-5 for both particle sizes $(100 \mu \mathrm{m}$ and $45 \mu \mathrm{m})$. As shown in Table $4-2,650^{\circ} \mathrm{C}$ is the control of decomposition temperature for the raw $\mathrm{MgCO}_{3}$ material burnt by TGA test for comparison purposes (Figure 3-3). Also, $630^{\circ} \mathrm{C}$ was the decomposition temperature which belongs to the kiln sample that initially burnt by kiln burning process at a temperature of $750^{\circ} \mathrm{C} \_2 \_100 \mu \mathrm{m}$; then it was burnt again at a temperature of $1200^{\circ} \mathrm{C}$ for around 2.5 hours through TGA test. The $96.92 \%(=630 \times 100 / 692)$ was the remaining hydration of the same sample measured by relating its new decomposition temperature $\left(630^{\circ} \mathrm{C}\right)$ to the decomposition temperature of the TGA control sample $\left(650^{\circ} \mathrm{C}\right)$.

Table 4-2: TGA decomposition temperature for already burned $\mathrm{MgO}$ powder samples by kiln

\begin{tabular}{|c|c|c|c|c|c|c|}
\hline \multirow{2}{*}{$\begin{array}{c}\text { Control } \\
\mathbf{6 5 0} 0^{\circ} \mathrm{C}\end{array}$} & \multicolumn{3}{|c|}{ Particle size $100 \mu \mathrm{m}$} & \multicolumn{3}{c|}{ Particle size $45 \mu \mathrm{m}$} \\
\cline { 2 - 7 } & 1 hour & 2 hours & 4 hours & 1 hour & 2 hours & 4 hours \\
\hline \multirow{3}{*}{$750^{\circ} \mathrm{C}$} & \multicolumn{3}{|c|}{ TGA decomposition temperature \& remaining hydration (\%) } \\
\cline { 2 - 7 } & $640^{\circ} \mathrm{C}$ & $630^{\circ} \mathrm{C}$ & $600^{\circ} \mathrm{C}$ & $630^{\circ} \mathrm{C}$ & $625^{\circ} \mathrm{C}$ & $600^{\circ} \mathrm{C}$ \\
\cline { 2 - 7 } & $98.46 \%$ & $96.92 \%$ & $92.31 \%$ & $96.92 \%$ & $96.15 \%$ & $92.31 \%$ \\
\hline \multirow{2}{*}{$900^{\circ} \mathrm{C}$} & $625^{\circ} \mathrm{C}$ & $635^{\circ} \mathrm{C}$ & $380^{\circ} \mathrm{C}$ & $635^{\circ} \mathrm{C}$ & $615^{\circ} \mathrm{C}$ & $355^{\circ} \mathrm{C}$ \\
\cline { 2 - 7 } & $96.15 \%$ & $97.69 \%$ & $58.46 \%$ & $97.69 \%$ & $94.62 \%$ & $54.62 \%$ \\
\hline \multirow{2}{*}{$1050^{\circ} \mathrm{C}$} & $610^{\circ} \mathrm{C}$ & $400^{\circ} \mathrm{C}$ & $395^{\circ} \mathrm{C}$ & $590^{\circ} \mathrm{C}$ & $390^{\circ} \mathrm{C}$ & $385^{\circ} \mathrm{C}$ \\
\cline { 2 - 7 } & $93.85 \%$ & $61.54 \%$ & $60.77 \%$ & $90.77 \%$ & $60.00 \%$ & $59.23 \%$ \\
\hline \multirow{2}{*}{$1200^{\circ} \mathrm{C}$} & $395^{\circ} \mathrm{C}$ & $395^{\circ} \mathrm{C}$ & $370^{\circ} \mathrm{C}$ & $400^{\circ} \mathrm{C}$ & $395^{\circ} \mathrm{C}$ & $375^{\circ} \mathrm{C}$ \\
\cline { 2 - 7 } & $60.77 \%$ & $60.77 \%$ & $56.92 \%$ & $61.54 \%$ & $60.77 \%$ & $57.69 \%$ \\
\hline
\end{tabular}


From Table 4-2 \& Figure 4-5, it can be noted that the remaining hydration decreased with the increase of holding time and the calcination temperature. Similar behavior between the two particle sizes was observed. In general, Table 4-2 revealed that $45 \mu \mathrm{m} \mathrm{MgO}$ particles had slightly lower decomposition temperatures than $100 \mu \mathrm{m} \mathrm{MgO}$ particles. This might be attributed to the fact that when particle size decreased (as in the case of $45 \mu \mathrm{m}$ ), the surface area of $\mathrm{MgO}$ particles increased leading to more heat exposure and subsequently decrease the remaining hydration. However, due to similar observed behavior, the influence of grain size was considered not significant on remaining hydration.
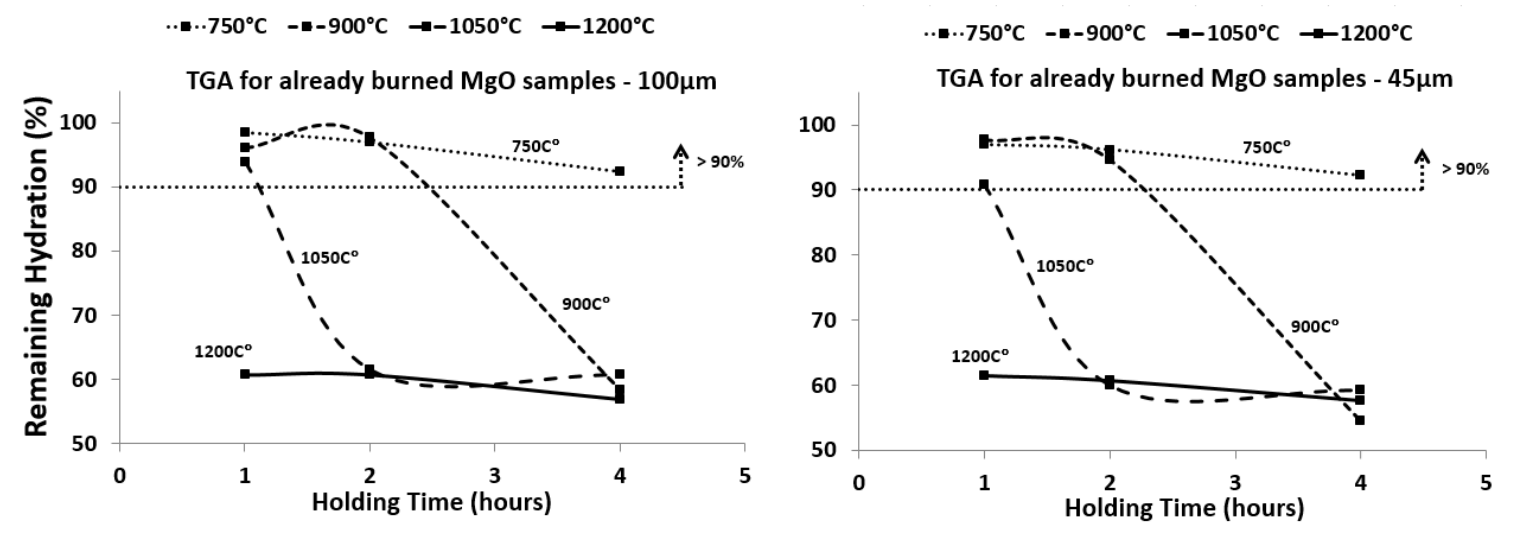

Figure 4-5: Decomposition temperature in terms of holding times of already burned $\mathrm{MgO}$ powder samples represented by $\%$ of remaining hydration acquired by TGA test

Based on kiln results (Figure 4-2 \& Figure 4-4), all $\mathrm{MgO}$ samples having loss of $\mathrm{CO}_{2}$ content greater than $45 \%$ were omitted from the goal of finding best calcination temperatures. As shown in Figure 4-5, all canceled temperatures after applying TGA test on them revealed the lowest remaining hydration within the temperatures studied. For convenience, these temperatures were $900^{\circ} \mathrm{C} \_4,1050^{\circ} \mathrm{C} \_2 \& 4$ and $1200^{\circ} \mathrm{C}$ for all holding times. This confirmed the compatibility with kiln results and opened the door to choose any temperature that had higher remaining hydration than the omitted ones. As a consequence, the focus was provided on the temperatures that had $90 \%$ and greater of remaining hydration such as $1050^{\circ} \mathrm{C} \_1,900^{\circ} \mathrm{C} \_1 \& 2$, and finally $750^{\circ} \mathrm{C}$ under all holding times for both particle sizes. More investigation had been conducted on these new calcined temperatures to choose the best one of them. 
Similar to Figure 4-5, Figure 4-6 shows the remaining hydration in terms of calcination temperatures $\left(750,900,1050\right.$ and $\left.1200^{\circ} \mathrm{C}\right)$. The higher the calcination temperature and holding time, the lower the remaining hydration of previously burnt $\mathrm{MgO}$ samples. Figure 4-6 confirms that any temperature with proportion greater than $90 \%$ had the highest remaining hydration.
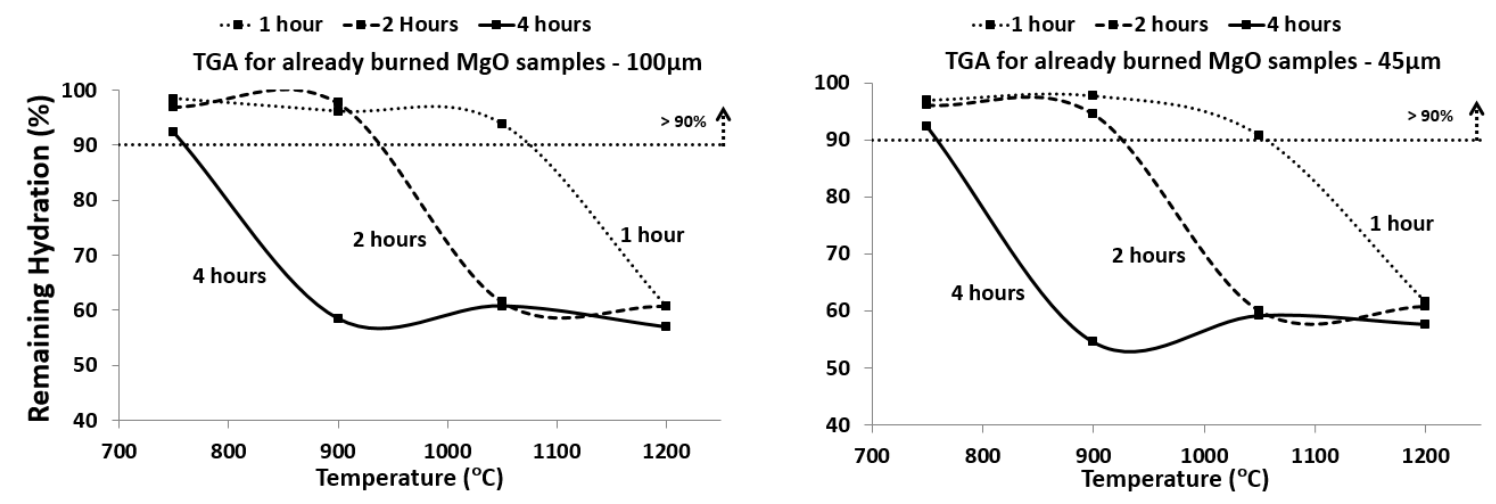

Figure 4-6: Decomposition temperature in terms of calcined temperature of already burned $\mathrm{MgO}$ powder samples represented by $\%$ of remaining hydration acquired by TGA test

\subsection{Difference in weight loss of MgO samples (TGA/Kiln burning processes)}

The weight loss of $\mathrm{MgO}$ from TGA and kiln burning are shown in Figure 4-7 for both particle sizes $(100 \mu \mathrm{m}$ and $45 \mu \mathrm{m})$. In this section, results of weight loss from both kiln and TGA burning processes are combined together in the same graph to observe the difference in weight loss between them. From Figure 4-7, it is observed that the difference in the loss of $\mathrm{CO}_{2}$ content between kiln and TGA samples increased when calcined temperature and holding time increased. Also, the loss of $\mathrm{CO}_{2}$ content increased for kiln samples and decreased for TGA samples (which already burnt by kiln burning process) when calcined temperature and holding time increased. As mentioned before, weight loss was represented by the loss of $\mathrm{CO}_{2}$ content.

In Figure 4-7, the higher value in the difference of the loss of $\mathrm{CO}_{2}$ content between the two burning processes will lead to lower potential in the degree of hydration in $\mathrm{MgO}$ samples. For instance, the difference in the mass loss of $\mathrm{CO}_{2}$ content of $900^{\circ} \mathrm{C} \_2 \_45 \mu \mathrm{m}$ was $26.30 \%$ while it was $38.70 \%$ for $1200^{\circ} \mathrm{C} \_2 \_45 \mu \mathrm{m}$. Referring to Table $4-2$, the remaining hydration of $900^{\circ} \mathrm{C} \_2 \_45 \mu \mathrm{m}$ was 
$94.62 \%$ while it was $60.77 \%$ for $1200^{\circ} \mathrm{C} \_2 \_45 \mu \mathrm{m}$. This means that the sample of $26.30 \%$ had not burnt enough to drive away that much of $\mathrm{CO}_{2}$ after kiln burning process (kiln loss was 44.06\%) leading to higher loss of $\mathrm{CO}_{2}$ when the sample was exposed again to the calcination process in TGA test (TGA loss was 17.77\%). Therefore, based on the morphology of MEA presented in Figure 4-3, the sample of $900^{\circ} \mathrm{C} \_2 \_45 \mu \mathrm{m}$ had higher reactivity due to higher fineness created at a lower temperature in kiln burning process compared to $1200^{\circ} \mathrm{C} \_2 \_45 \mu \mathrm{m}$. Unlike $900^{\circ} \mathrm{C} \_2 \_45 \mu \mathrm{m}$, the remaining hydration of $1200^{\circ} \mathrm{C} \_2 \_45 \mu \mathrm{m}$ was $60.77 \%$ which means that it was burnt enough after kiln burning process to drive away much higher $\mathrm{CO}_{2}$ (kiln loss was $50.32 \%$ ) leading to much lower $\mathrm{CO}_{2}$ content when the sample was exposed again to calcination process in TGA test (TGA loss: $11.62 \%$ ). Obviously, losing too much $\mathrm{CO}_{2}$ by burning process at higher temperatures is not a desirable situation because this will shift the burning level from "lightly burnt" of MgO samples to "heavily burnt" which has a harmful effect on concrete structure at later ages. As a result, the use of lower temperatures in kiln burning process within the range studied is much better to get a higher degree of hydration, especially for ECC-MgO self-healing system.

To determine the best calcination temperature, it was concluded in TGA results in terms of decomposition temperatures that the focus should be on desired temperatures that have remaining hydration greater than $90 \%$. These temperatures were $1050^{\circ} \mathrm{C} \_1,900^{\circ} \mathrm{C} \_1 \& 2$, and $750^{\circ} \mathrm{C}$ under all holding times for both particle sizes. To explore these temperatures closely, Figure 4-7 shows negative values in the difference of the loss of $\mathrm{CO}_{2}$ content at $750^{\circ} \mathrm{C}_{-} 1$ for both $45 \& 100 \mu \mathrm{m}$ samples. It also shows small values in the difference of the loss of $\mathrm{CO}_{2}$ content at $750^{\circ} \mathrm{C} \_2$ and $900^{\circ} \mathrm{C} \_1$ for both $45 \& 100 \mu \mathrm{m}$ samples. Again, calcining the raw material of $\mathrm{MgCO}_{3}$ at lower temperatures and shorter holding times led to the highest remaining hydration within the range of temperatures studied. This was also confirmed by Zhang et al. (2012) who revealed that MEA had the highest activity when calcined at $666.99{ }^{\circ} \mathrm{C}$ and burnt for 2.12 hours (Zhang et al., 2012). Accordingly, when previously burnt $\mathrm{MgO}$ samples were exposed again to $1200^{\circ} \mathrm{C}$ in $\mathrm{TGA}$ test, the TGA loss of $\mathrm{CO}_{2}$ content was aggravated leading to negative or lower values in the difference of the loss of $\mathrm{CO}_{2}$ content between kiln and TGA results. This may produce unbalanced reactivity at later ages for $\mathrm{MgO}$ powder samples when utilized in concrete samples. Conservatively, all temperatures that produced negative and small values in the difference of the loss of $\mathrm{CO}_{2}$ content 
between the two burning processes will be omitted from the goal of finding best calcination temperature. This will leave the choice opened only among the three following temperatures: $750^{\circ} \mathrm{C} \_4,900^{\circ} \mathrm{C} \_2$ and $1050^{\circ} \mathrm{C} \_1$.
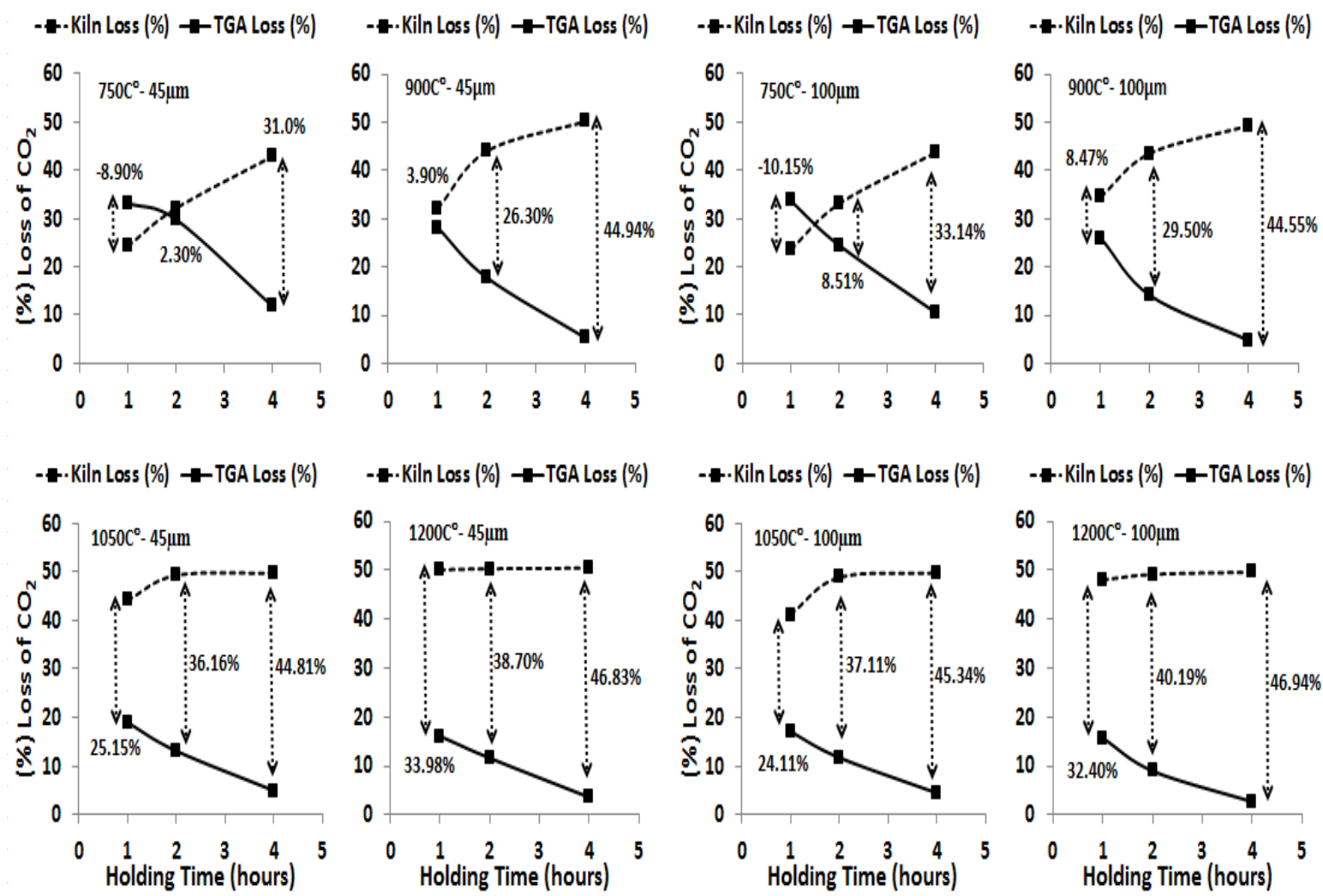

Figure 4-7: Weight loss of $\mathrm{MgO}$ samples by kiln and TGA burning process represented by $\mathrm{CO}_{2}$ loss

To review the characteristics of these desired temperatures, the findings are analyzed. Figure 4-2 \& Figure 4-4 showed almost identical percentages of the loss of $\mathrm{CO}_{2}$ content after kiln burning process (ranged between $40 \%$ and 45\%). Figure 4-5 \& Figure 4-6 showed almost the same remaining hydration of TGA burning process (ranged between $88 \%$ and 98\%). Finally, Figure 4-7 showed almost same difference in the loss of $\mathrm{CO}_{2}$ content between the kiln and TGA burning processes (ranged between 25 and 31\%). It can be concluded that using any of these temperatures will serve as best calcination temperature for $\mathrm{MgO}$ samples. However, the researcher preferred to choose the best calcination temperature based on economic reasons. For example, at $750^{\circ} \mathrm{C}_{-} 4$, the duration needed to heat up the kiln up to $750^{\circ} \mathrm{C}$ was around 45 minutes including 4 hours as holding time leading to almost 5 hours of kiln operation. For $900^{\circ} \mathrm{C} \_2$, the duration to operate the kiln 
including the holding time was around 3 hours while $1050^{\circ} \mathrm{C} \_1$ needed around 4 hours of kiln operation. It was also noticed that when using a higher temperature, more time was needed to reach the assigned temperature leading to more energy consumed by the electric kiln. Accordingly, $900^{\circ} \mathrm{C} \_2$ hours of holding time_45 $\mu \mathrm{m}$ particle size was considered to be the best choice.

From Figure 4-7, a slight increase in the difference of the loss of $\mathrm{CO}_{2}$ content was observed when $45 \mu \mathrm{m}$ particle size was used compared to $100 \mu \mathrm{m}$ particle size. However, no major influence was observed when the size of $\mathrm{MgO}$ samples changed from $100 \mu \mathrm{m}$ to $45 \mu \mathrm{m}$.

\subsubsection{Phase II: Linear expansion effect of MgO expansive additive}

A Higher degree of MEA hydration was considered to be the base of finding best calcination temperature for ECC-MgO self-healing system. The assessment of the effectiveness of higher degree of hydration of $\mathrm{MgO}$ powder samples has led to the selection of " $900^{\circ} \mathrm{C} \_2$ hours of holding time_45 $\mu \mathrm{m}$ particle size" as the best calcination system within the range of temperatures studied. In this section, the effectiveness of MEA in ECC-MgO self-healing system was evaluated based on lower $\mathrm{MgO}$ expansion effect conducted on both water and autoclave cured linear expansion tests on bar specimens. Further, best SCM that can fit the needs of ECC-MgO self-healing techniques was chosen as well. Again, all the $24 \mathrm{MgO}$ powder samples were employed in the production of FA-ECC-MgO bar specimens. Also, the compatibility between the behaviors of $\mathrm{MgO}$ powder samples before and after utilizing them in bar specimens was investigated.

\subsubsection{Autoclave expansion test}

Figure 4-8 shows expansion of autoclave cured ECC-MgO bar specimens produced by utilizing two different fly ashes (Class-F and Class-CI) with two different particle sizes of $\mathrm{MgO}$ powder samples (100 and $45 \mu \mathrm{m})$. Autoclaved expansion increased with the increase of holding time and calcination temperature. It was also observed that the addition of $55 \%$ of fly ash as cement replacement to ECC-MgO bar specimens produced very low $\mathrm{MgO}$ expansion readings. According to (ASTM C150, 2016), the maximum autoclave expansion should be limited to $0.80 \%$. From Figure 4-8 the maximum expansion of autoclave cured fly ash Class-CI bar specimens was $0.494 \%$ and $0.350 \%$ for their fly ash Class-F counterparts. Reshi (1983) reported that the addition of $30 \%$ 
fly ash to high magnesium cement made from clinker with $10 \% \mathrm{MgO}$ produced expansions less than $0.20 \%$ in bar specimens water cured for 7 years (Rehsi, 1983). In autoclave cured specimens, expansion reduced from $17 \%$ to $0.75 \%$ when $30 \%$ fly ash was added. Ali and Mullick (1998) revealed that linear expansions for autoclaved specimens were reduced from $10 \%$ to $0.13 \%$ after the addition of $20 \%$ low calcium fly ash to the clinker of high $\mathrm{MgO}$ cement $(\sim 6 \% \mathrm{MgO})$. For specimens water cured for 1 year, expansions were reduced after adding $20 \%$ fly ash from $0.14 \%$ to $0.11 \%$ (Ali and Mullick, 1998). Generally, the literature revealed that autoclaved specimens involved with MEA had higher porosity and larger pore size distribution compared to specimens cured in water conditions. During autoclave curing, the rapid rate of $\mathrm{MgO}$ hydration accelerates the formation of $\mathrm{Mg}(\mathrm{OH})_{2}$ crystallization by combining the available $\mathrm{OH}^{-}$ions in pore solution with $\mathrm{Mg}^{2+}$ ions. In addition to $\mathrm{MgO}$ hydration, $\mathrm{C}-\mathrm{S}-\mathrm{H}$ hydration will be accelerated leading to the destruction of the cohesive forces in cement paste microstructure. The quick growth of $\mathrm{Mg}(\mathrm{OH})_{2}$ crystals produces considerable expansive stresses on the surrounding pores with the help of destroyed cohesive forces and leads to bigger pores and weaker autoclaved microstructures causing higher linear expansion. Due to lower porosity in water cured specimens and very slow reactivity of $\mathrm{MgO}$ hydration in water conditions, lower expansions were recorded in water curing compared to autoclave conditions (Figure 4-8). By adding fly ash to cement pastes, pozzolanic reaction will consume $\mathrm{Ca}(\mathrm{OH})_{2}$ in the presence of both alkaline solution and silicon ions existed from fly ash to form stronger C-S-H products. Further to the pozzolanic reaction, the addition of fly ash will limit the presence of hydroxide and silicon ions in alkaline solution leading to slower formation of $\mathrm{Mg}(\mathrm{OH})_{2}$. The formation of stronger $\mathrm{C}-\mathrm{S}-\mathrm{H}$ products in the surrounding microstructures and the reduction of expansion stresses on pore walls by limiting $\operatorname{Mg}(\mathrm{OH})_{2}$ formation definitely will strengthen the microstructure of cement pastes leading to the increase in counteracting forces against $\mathrm{MgO}$ expansions. Therefore, lower expansion readings were observed for both water and autoclaved specimens due to the addition of fly ash as shown in Figure 4-8 and also in previous research studies (Ali and Mullick, 1998; Du, 2005; Gao et al., 2007; Helmuth and West, 1998; Moradpour et al., 2013). 

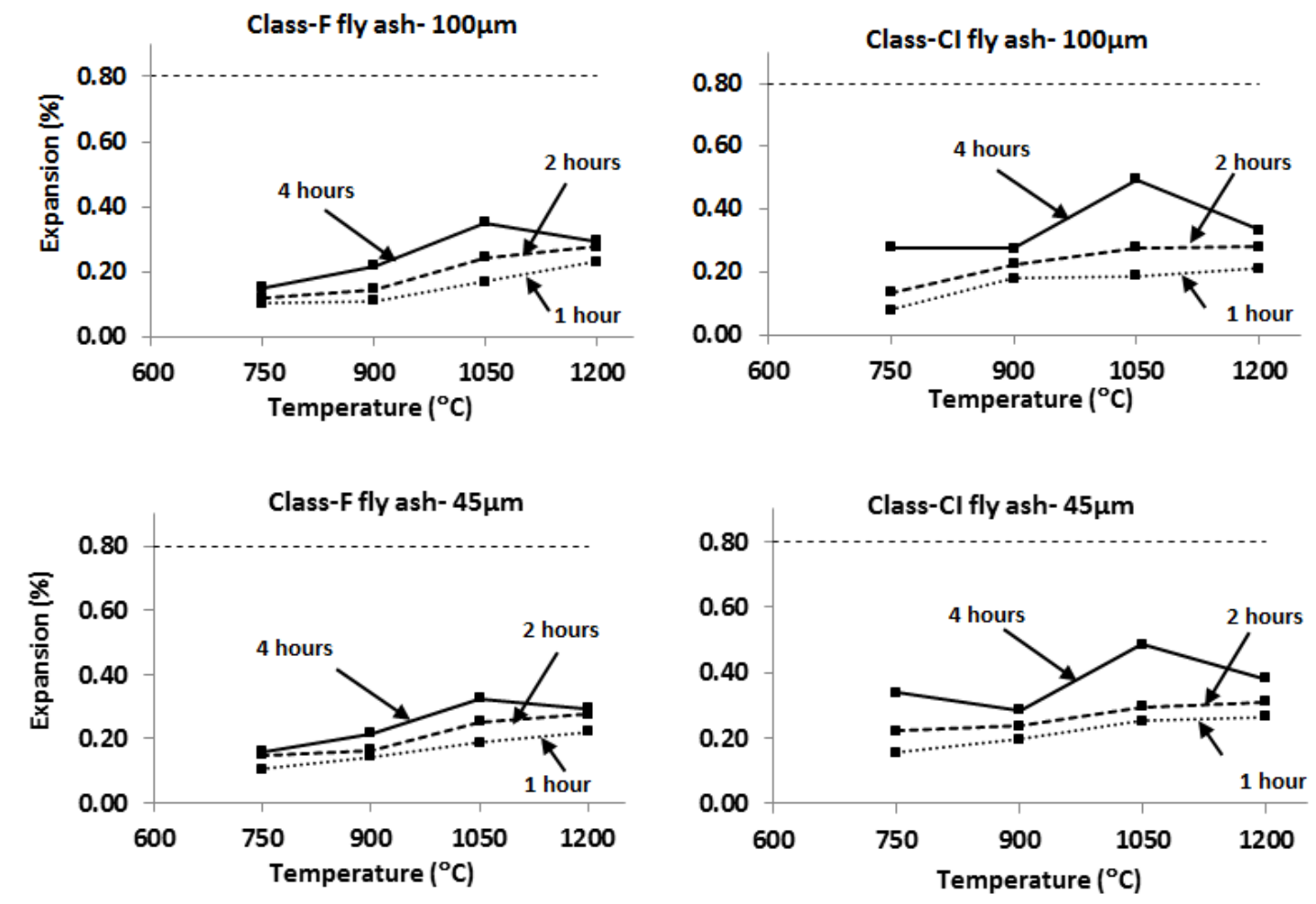

Figure 4-8: $\mathrm{MgO}$ expansions for autoclave cured ECC-MgO bar specimens in terms of temperature

Moreover, it was observed from Figure 4-8 that the autoclaved cured specimens made of Class-F fly ash had lower expansions than those made of Class-CI fly ash. The expansion of autoclaved Class-F bar specimens ranged between $0.102 \%$ and $0.350 \%$ and between $0.078 \%$ and $0.494 \%$ for their Class-CI counterparts. This might be attributed to the high $\mathrm{CaO}$ content of $17.28 \%$ in ClassCI fly ash compared to only $3.55 \%$ in Class-F fly ash (Table $3-1)$. The more $\mathrm{CaO}$ content in ClassCI fly ash, the more heat of hydration liberation than Class-F fly ash (Bisaillon et al., 1994; Langley et al., 1992; Mavani, 2012; Thomas et al., 1995). By reducing the concrete temperature, the rate of $\mathrm{MgO}$ hydration will be reduced as well leading to lower autoclaved expansion readings in ClassF fly ash (Du, 2005).

Additional to $\mathrm{CaO}$ content and as seen in Table 3-1, the $\mathrm{MgO}$ content which already presented in Class-CI fly ash was $3.28 \%$ while it was $0.82 \%$ in Class-F fly ash. Higher MgO content already present in fly ash may accelerate the rate of $\mathrm{MgO}$ hydration. In this research, the total $\mathrm{MgO}$ content (in total means the sum of all $\mathrm{MgO}$ content present in fly ash, cement and $\mathrm{MgO}$ additive) in ECC- 
$\mathrm{MgO}$ bar specimens produced by Class-CI fly ash was $11.67 \%$ by binder weight or $18.67 \%$ by cement weight. The $11.67 \%$ or $18.67 \%$ was distributed as follows: $3.28 \%$ already presented in fly ash, $6 \% \mathrm{MgO}$ measured by binder weight or $13 \% \mathrm{MgO}$ measured by cement weight to be added as an additive and 3.0\% already presented in Portland cement. While it was around 9.19\% in those produced with Class-F fly ash measured by binder weight or $16.19 \%$ by cement weight. In order for concrete to be sound, Du (2005) concluded that the maximum amount of $\mathrm{MgO}$ powder to be added as an additive to cement paste should be limited to $5 \%$ measured by cement weight with no more than $8 \%$ in total ( $\mathrm{Du}, 2005)$. In addition, Gao et al. (2007) confirmed that concrete is still sound even the limit of $\mathrm{MgO}$ additive in cement exceeded 5\% and reached up to $7.6 \%$ in total (Gao et al., 2007). But Nikiforov and Zosoulia (1980) stated that in order to produce sound cement, the total safe amount of $\mathrm{MgO}$ content in commercial Portland cement should be $6 \%$ and could reach in total up to $8 \%$ or even $11 \%$ if well dispersed and uniformly distributed (Nikiforov and Zosoulia, 1980). However, Joshi (1997) revealed that higher MgO content already presented in high calcium fly ash has no harmful effect on the soundness of concrete (Joshi, 1997). Therefore, the limitation of the use of only 5\% MgO in fly ash has been currently dropped from (ASTM C618, 2015). Consequently, as long as the proportion of $\mathrm{MgO}$ additive used in concrete showed expansions less than $0.80 \%$ according to ASTM standards, the $\mathrm{MgO}$ content already presented in fly ash may not affect the expansion and only higher $\mathrm{CaO}$ content plays a significant role in increasing the expansion in ECC-MgO bar specimens produced by Class-CI fly ash compared to Class-F fly ash as shown in Figure 4-8. Although when total $\mathrm{MgO}$ content is related to cement weight instead of binder weight which increased $\mathrm{MgO}$ content presented in ECC-MgO bar specimens up to $13 \%$, still both autoclave expansion results produced by Class-CI or Class-F fly ashes were within the $0.80 \%$ permissible limit of ASTM standards (ASTM C150, 2016).

From Figure 4-8, , similar expansion behavior between the two MgO particle sizes (100 and 45 $\mu \mathrm{m})$ was observed through autoclaved cured ECC-MgO specimens with $45 \mu \mathrm{m} \mathrm{MgO}$ particle size (using both fly ashes) showed slightly higher expansion than their $100 \mu \mathrm{m}$ counterparts. 

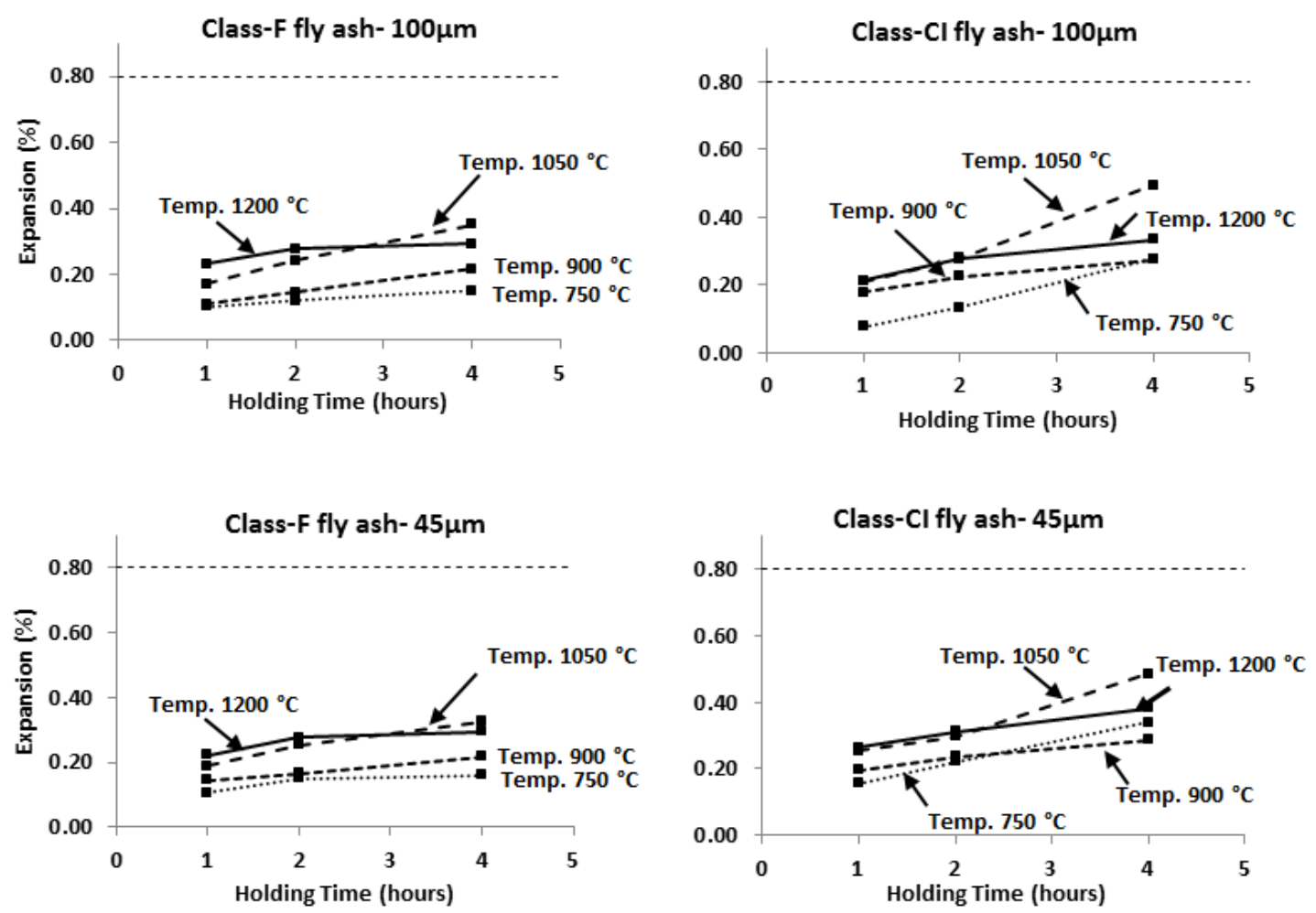

Figure 4-9: $\mathrm{MgO}$ expansion readings for autoclave cured ECC-MgO bar specimens in terms of holding times

Similar to Figure 4-8, Figure 4-9 showed that expansion in autoclave cured ECC-MgO bar specimens increased when holding time and calcination temperature increased. This fact was confirmed by Gao et al. (2007) who revealed that longer holding time and higher the autoclave temperatures increased the expansion of autoclaved cured $\mathrm{MgO}$ concrete specimens ( $\mathrm{GaO}$ et al., 2007).

In addition, Figure 4-10 showed similar expansion and $\mathrm{CO}_{2}$ loss behavior, respectively between autoclaved cured ECC-MgO bar specimens for both types of fly ashes and kiln burning $\mathrm{MgO}$ powder samples before utilizing them in ECC mixtures. For convenience, expansions of autoclave cured fly ash Class-F bar specimens (of $45 \mu \mathrm{m} \mathrm{MgO}$ grain size) and $\mathrm{CO}_{2}$ content loss of $\mathrm{MgO}$ powder samples after the completion of kiln burning process were combined together in one graph to investigate/examine the similarity between the two behaviors (Figure 4-10). Both expansion and 
$\mathrm{CO}_{2}$ loss increased with the increase of holding time (from 1 hour to 4 hours) and calcination temperature from $700^{\circ} \mathrm{C}$ to $1200^{\circ} \mathrm{C}$.
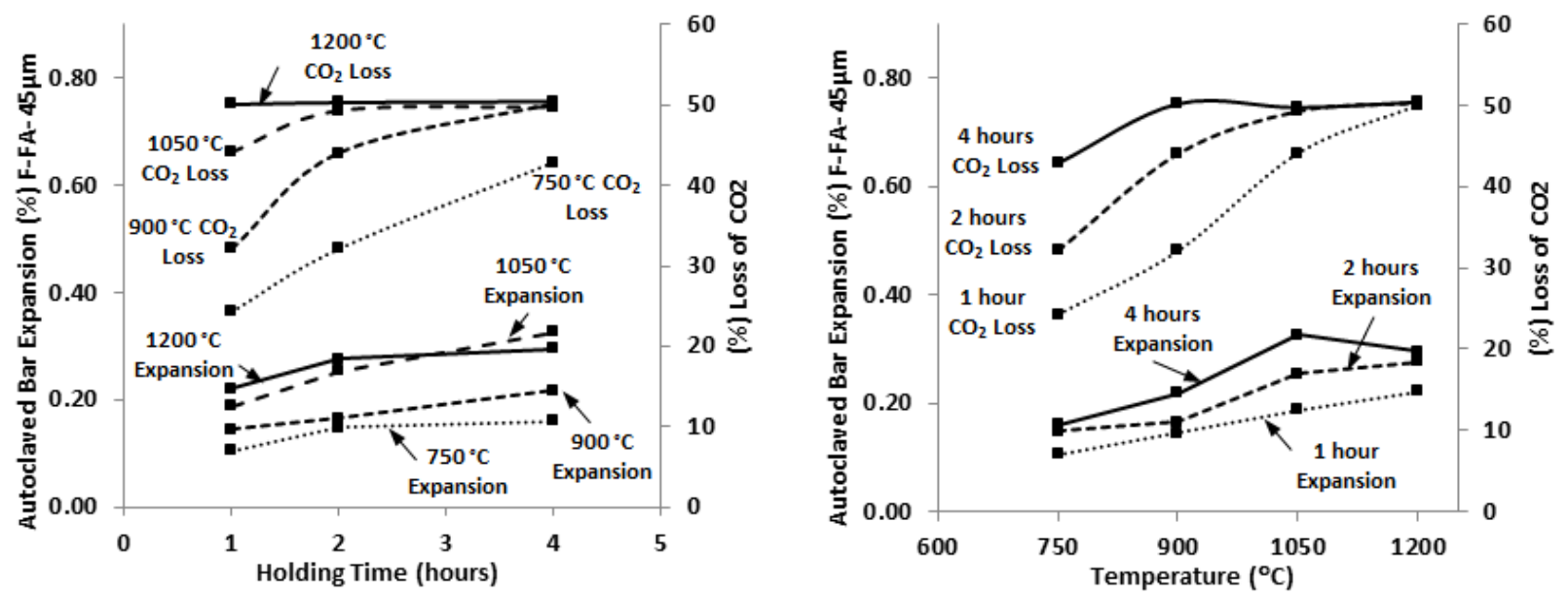

Figure 4-10: Similar behavior of MEA in Powder state and after utilized in the production of autoclave cured $\mathrm{ECC}-\mathrm{MgO}$ bar specimens in terms of temperatures and holding times

\subsubsection{Water expansion test}

In water expansion test, ECC-MgO bar specimens were cured in water at $20^{\circ} \mathrm{C}$ (termed as normal condition) for 500 days to monitor the expansion effect of MEA. The bar specimens were produced in accordance with (ASTM C1038-b, 2014) by using two kinds of fly ash (Class-F \& Class-CI). The cement replacement ratio of fly ash was $55 \%$ for both of them. Same as autoclave testing, twenty-four "lightly burnt" MEA (MgO) samples were employed in the production of water ECC$\mathrm{MgO}$ bar specimens at four calcination temperatures $\left(750,900,1050\right.$ and $1200^{\circ} \mathrm{C}$ ) for three holding times (1, 2 and 4 hours), respectively. Two different particle sizes of $\mathrm{MgO}$ powder samples (100 and $45 \mu \mathrm{m})$ were used as well.

It is noted from Figure 4-11 (part A-E) that when holding time and calcination temperature increased, the expansion of water cured ECC-MgO specimens increased except for the $1200^{\circ} \mathrm{C}$ at 4 hours of holding time. These results were opposite to the results of normal cement pastes (without fly ash) reported by Mo et al. (2010) and Mehta et al. (1980). It was reported that at 1 hour of holding time, the expansion of cement paste at $1300^{\circ} \mathrm{C}$ was lower than $900^{\circ} \mathrm{C}$. This meant that 
burning $\mathrm{MgO}$ particles at $900^{\circ} \mathrm{C}$ created highly reactive MEA than the one made at $1300^{\circ} \mathrm{C}$ (Mo et al., 2010). Similar findings were reported by Mehta et al. (1980) where $900^{\circ} \mathrm{C}$ calcinated $\mathrm{MgO}$ reacted much more rapidly than those treated at higher temperatures $\left(1300^{\circ} \mathrm{C}\right)$ (Mehta et al., 1980).

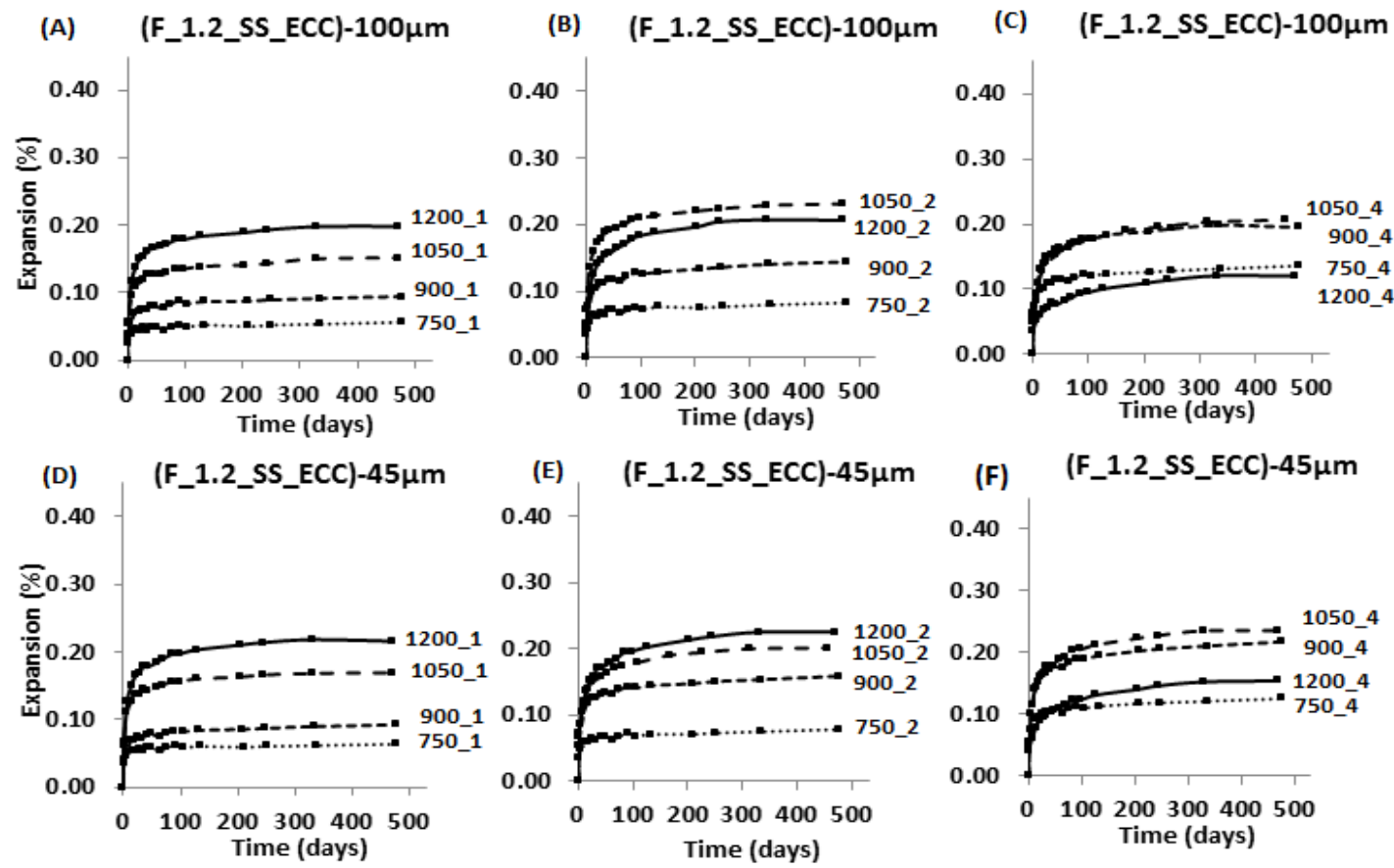

Figure 4-11: ECC-MgO bar specimens made of Class-F fly ash cured at $20^{\circ} \mathrm{C}$ in water for 500 days (A \& D: 1 hour holding time, B \& E: 2 hours holding time and C \& E: 4 hours of holding time)

According to Mo et al. (2010), lower calcination temperature and shorter holding time will lead to higher fineness and large number of interior pores with smaller size within $\mathrm{MgO}$ microstructures. The expansion model of Mo et al. (2010) for normal cement pastes revealed that the existence of $\mathrm{Mg}^{2+}$ ions inside the plenty of smaller pores in highly reactive MEA microstructure (prepared at $900^{\circ} \mathrm{C}$ ) facilitates saturated $\mathrm{OH}^{-}$ions in pore solution to diffuse into the interior pores to form $\mathrm{Mg}(\mathrm{OH})_{2}$. In addition, $\mathrm{Mg}(\mathrm{OH})_{2}$ crystals are also formed at the surface of grain boundaries of MEA particles and confined regions near $\mathrm{MgO}$ sites. The growth of $\mathrm{Mg}(\mathrm{OH})_{2}$ crystals increases the expansion pressure on the walls of interior pores leading to more expansions. Opposite to highly reactive MEA, the diffusion of pore solution into interior pores at low reactive MEA (prepared at $1300^{\circ} \mathrm{C}$ ) will be limited due to less interior pores and larger pore size leading to reduce the formation of $\mathrm{Mg}(\mathrm{OH})_{2}$ crystals in low reactive MEA. Therefore, the hydration of $\mathrm{MgO}$ will 
take place in longer time periods of leading to slower reactivity at early ages. However, at later ages, the formation of $\mathrm{Mg}(\mathrm{OH})_{2}$ crystals at the boundary of bigger $\mathrm{MgO}$ grains due to lower fineness can generate enough expansions to break apart the sintered $\mathrm{MgO}$ grains producing faster/higher expansions (Mo et al., 2010).

In this dissertation, the addition of high volume fly ash (as 55\% cement replacement) to water cured ECC-MgO bar specimens increased the presence of alkaline solution and silicon ions inside the matrix. Based on the expansion model presented by Mo et al. (2010), the burning temperature of $750^{\circ} \mathrm{C}$ used in this research has produced highly reactive MEA which with large number of interior pores with smaller pore size in $750^{\circ} \mathrm{C}$-ECC-MgO microstructures. Therefore, with the addition of high volumes of FA (HVFA), stronger C-S-H products are formed inside the interior pores of $\mathrm{MgO}$ structures at early ages instead of expansive $\mathrm{Mg}(\mathrm{OH})_{2}$ crystals. Accordingly, $750^{\circ} \mathrm{C}-$ ECC-MgO bar specimens revealed the lowest expansion in this research. Gao et al. (2008) noted that the hydration of $\mathrm{CaO}$ to form $\mathrm{Ca}(\mathrm{OH})_{2}$ was completed within the first 3 days when cured in water at $20^{\circ} \mathrm{C}$, while the hydration of $\mathrm{MgO}$ to form $\mathrm{Mg}(\mathrm{OH})_{2}$ was very slow and only about $57 \%$ of $\mathrm{MgO}$ formed $\mathrm{Mg}(\mathrm{OH})_{2}$ within 180 days when the specimens were cured at the same conditions (Gao et al., 2008). The presence of stronger C-S-H products within the interior pores of $\mathrm{MgO}$ structures in the presence of HVFA had created stronger pressure that counteracted the $\mathrm{MgO}$ expansion pressure. As a result, the $\mathrm{MgO}$ expansion pressure has suppressed or even may have stopped the expansion effect as revealed in autoclaved ECC-MgO expansion results (Figure 4-8). Unlike $750^{\circ} \mathrm{C}$, the burning temperature of $1200^{\circ} \mathrm{C}$ in this research had produced the highest expansions at 1 and 2 hours of holding time. This may be attributed to less formation of stronger $\mathrm{C}-\mathrm{S}-\mathrm{H}$ products within the smaller number of interior pores of $\mathrm{MgO}$ microstructures. In this condition, still the formation of $\mathrm{Mg}(\mathrm{OH})_{2}$ crystals at the boundaries of $\mathrm{MgO}$ grains played a significant role in creating enough expansions to crack the sintered $\mathrm{MgO}$ grains and promoted higher expansions. It can be concluded that the amount of formed $\mathrm{C}-\mathrm{S}-\mathrm{H}$ products in $\mathrm{MgO}$ porous structure depends on the calcination temperature and holding time. The lower the calcination temperature and shorter the holding time, the more the interior pores and the smaller the pore size, the more formation of $\mathrm{C}-\mathrm{S}-\mathrm{H}$ products inside $\mathrm{MgO}$ microstructures in the presence of HVFA and finally, the more counteracting forces against the expansion pressures created in the ECC-MgO 
self-healing system leading to lower expansions. Accordingly, the increase in the expansion of water cured ECC-MgO bar specimens started from $750^{\circ} \mathrm{C}$ up to $1200^{\circ} \mathrm{C}$ as shown in Figure $4-11$ (part A-E).

Referring to Figure 4-11 (C \& F), the expansion of water cured ECC-MgO bar specimens prepared at $1200^{\circ} \mathrm{C} \_4$ was less than those at $1200^{\circ} \mathrm{C}_{-} 1 \& 2$ in both particle sizes $(100$ and $45 \mu \mathrm{m})$. Although Mo et al. (2010) made a differentiation between high and low reactivity by stating that slower reactive MEA structure (were created at higher temperatures and longer holding times) has less interior pores with larger pore size, still the formation of $\mathrm{Mg}(\mathrm{OH})_{2}$ crystals at the boundaries of $\mathrm{MgO}$ grains did not contribute to the expansions in the case of $1200^{\circ} \mathrm{C} \_4$. This may be attributed to the fact that the higher calcination temperature with longer holding times pushes the burning level of $\mathrm{MgO}$ particles to "heavily burnt" level featured with very slow reactivity and harmful effect on concrete structures at later ages.

In order to comment on "harmful effect of MEA at later ages", White (1928) reported the addition of $4 \%$ of "hard burnt" $\mathrm{MgO}$ to low $\mathrm{MgO}$ content cement had not completely hydrated when stored in water for 40 years and had stopped expanding after 8 years (White, 1928). Moreover, Helmuth \& West (1998) summarized that when 6\% of $\mathrm{MgO}$ was added to Portland cement, there was no disruptive expansions for mortars stored in water for 10 to 15 years under normal conditions (above $50 \%$ of $\mathrm{RH}$ ). Free $\mathrm{MgO}$ additive can induce harmful effect to concrete structures at later ages without showing any large expansions as confirmed from the autoclave test conducted on paste and mortar bars already cured for 5 years. The autoclave expansion of 5 year water cured specimens was higher than 5 years of storage in water. Although many researchers reported that there was no disruptive effect of free $\mathrm{MgO}$ when added to cement even after 40 years of storage in water conditions, it was easy for them not to take any risk and looking for wide margin of safety during the industrial boom of cement, especially in 1940's and hence limited the usage of free $\mathrm{MgO}$ below the ASTM standard limits (Helmuth and West, 1998). In this research, the addition of $13 \% \mathrm{MgO}$ measured by cement weight with the presence of 55\% HVFA in ECC-MgO mortar bar specimens did not show any disruptive expansions and they were less than the expansion limit of $0.8 \%$ approved by ASTM standards. 

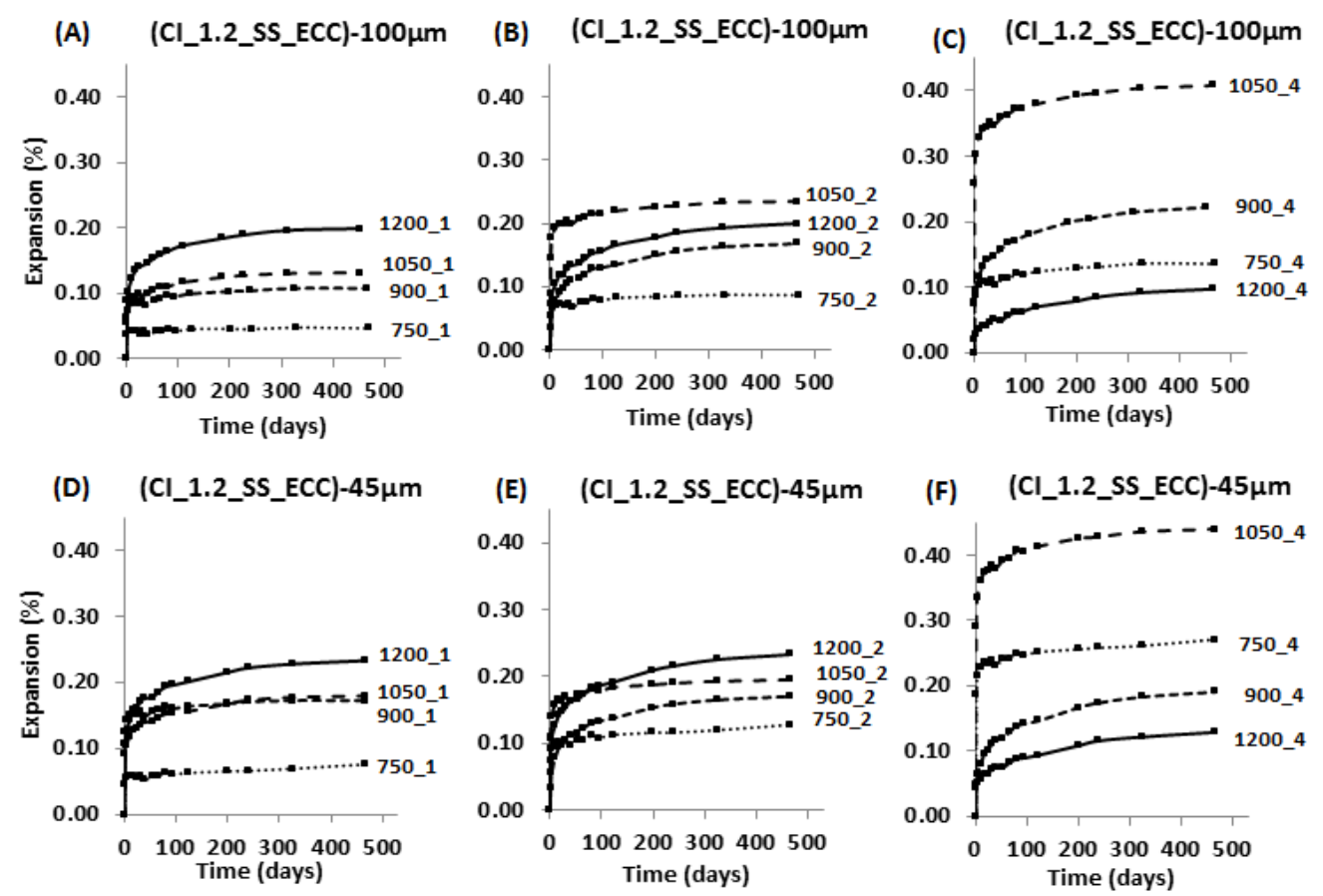

Figure 4-12: ECC-MgO bar specimens made of Class-CI fly ash cured at $20^{\circ} \mathrm{C}$ in water for 500 days (A

\& D: 1 hour holding time, B \& E: 2 hours holding time and C \& E: 4 hours of holding time)

As shown in Figure 4-12, water cured ECC-MgO bar specimens made of Class-CI fly ash exhibited higher expansions than their Class-F fly ash counterparts (Figure 4-11). Again, the increased rate of $\mathrm{MgO}$ hydration might be attributed to the presence of high $\mathrm{CaO}$ content in Class-CI fly ash compared to Class-F which increase the concrete temperature (Bisaillon et al., 1994; Du, 2005; Langley et al., 1992; Mavani, 2012; Thomas et al., 1995). However, both types of fly ashes still have produced expansion readings less than ASTM expansion limit of $0.80 \%$. The expansion readings for Class-CI fly ash ranged between $0.046 \%$ and $0.440 \%$ and ranged between $0.056 \%$ and $0.231 \%$ for Class-F fly ash (Figure 4-11). For Class-F fly ash, it was also observed that when holding time and calcination temperature increased the water expansion also increased for both particle sizes of $\mathrm{MgO}$ powder samples (100 and $45 \mu \mathrm{m}$ ). Similar to Class-F fly ash, Figure 4-12 (C \& F) show identical behavior for $1200^{\circ} \mathrm{C}_{-} 4$ in Class-CI fly ash. According to Figure 4-12 (F), the expansion of $750^{\circ} \mathrm{C} \_4$ was higher than $900^{\circ} \mathrm{C} \_4$. Even autoclave results shown in Figure 4-8 revealed higher expansion for $750^{\circ} \mathrm{C}_{-} 4$ compared to $900^{\circ} \mathrm{C} \_4$. Perhaps, the counteracting forces formed by C-S-H inside the interior pores of $\mathrm{MgO}$ structures were not enough to suppress the 
formation of expansion pressures of $\mathrm{Mg}(\mathrm{OH})_{2}$ crystals due to higher heat of hydration generated by high proportions of $\mathrm{CaO}$ content in Class-CI fly ash. This may apply to water and autoclave cured specimens made of $1050^{\circ} \mathrm{C}_{-} 4$ calcinated MEA (Figure 4-8 \& Figure 4-12).

\subsubsection{Comparison between autoclave and water cured ECC-MgO bar specimens}

For comparison between water and autoclave cured ECC-MgO bar specimens, the last expansion reading of water cured specimens at 500 days was taken and analyzed in terms of calcination temperature as shown in Figure 4-13. It can be seen clearly that both Class-F and Class-CI water cured ECC-MgO bar specimens showed almost similar expansion behavior compared to their autoclave cured counterparts as shown in Figure 4-8 except for the calcination temperature of $1200^{\circ} \mathrm{C}$ at 4 hours of holding time. Again, this is because $1200^{\circ} \mathrm{C}_{-} 4$ has reached the "heavily burnt" level of $\mathrm{MgO}$ characterized by sluggish reactivity at later ages.

Similar to Figure 4-8, water cured bar specimens made of Class-F fly ash revealed lower expansion readings than those made of Class-CI fly ash. Consequently, the assessment of the effectiveness of $\mathrm{MgO}$ expansive additive in water and autoclave cured bar specimens based on lower expansion readings has led to the selection of low calcium Class-F fly ash as best supplementary cementing material to be employed in the production of ECC-MgO self-healing system. Moreover, 55\% fly ash as cement replacement is believed to satisfy the requirements of ECC-MgO self-healing system. Although Du (2005) reviewed that research and engineering practice have limited the use of fly ash to $30 \%$ as cement replacement in normal grain sized $\mathrm{MgO}$ concrete in order to satisfy the requirements of shrinkage compensation, 55\% of fly ash as cement replacement was used in this dissertation. The intention of using HVFA in ECC-MgO self-healing system was because of two reasons. The first was to achieve smaller expansions as much as possible just to heal the microcracks in ECC-MgO self-healing system without affecting the durability. The second was to simulate the performance of ECC-MgO system in field conditions through accelerated autoclave test using bar specimens. In addition to the effect of HVFA on the performance of MEA, the presence of PVA fibers was also believed to play a significant role to achieve high volume stability in ECC-MgO self-healing system. 

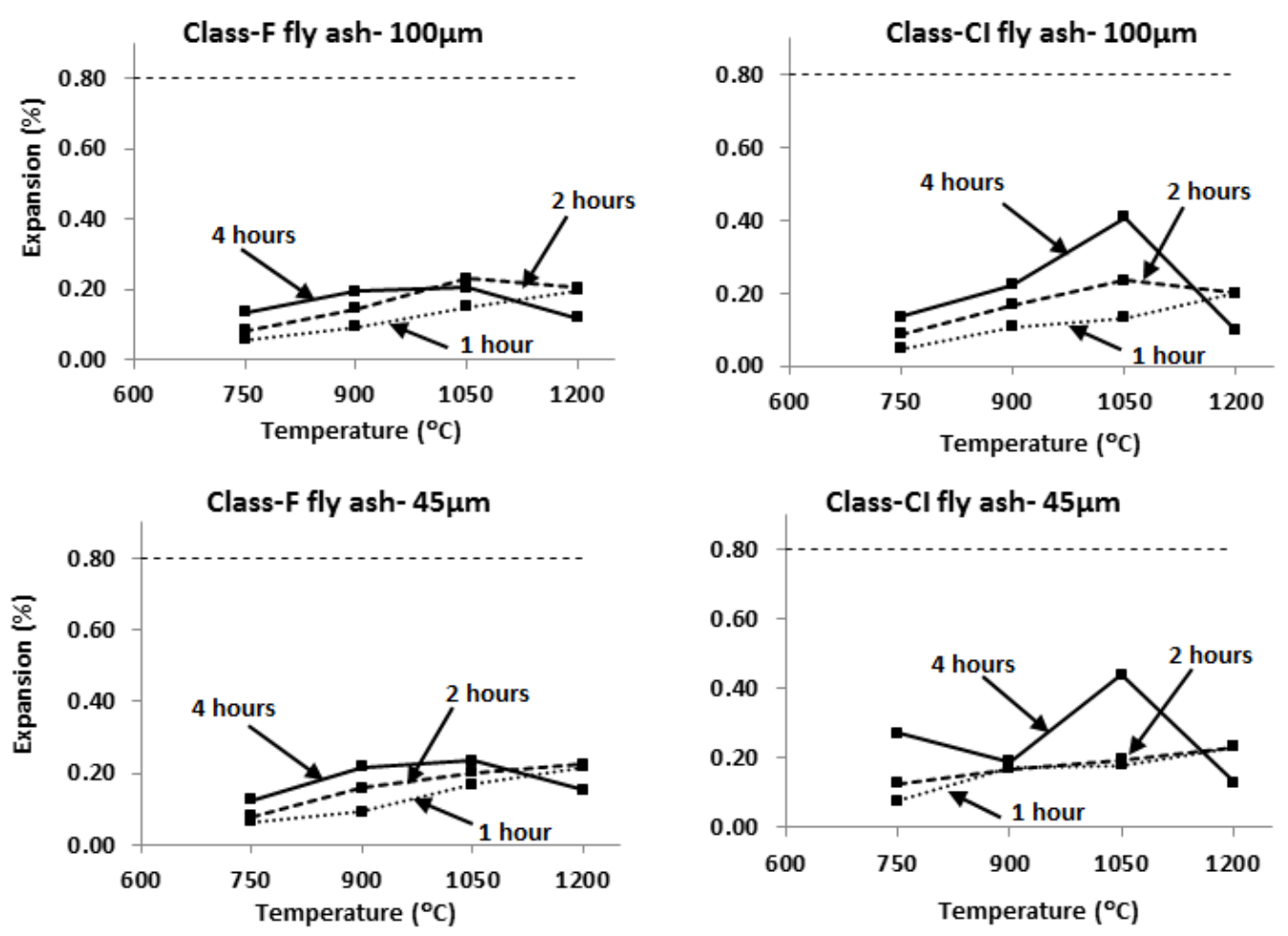

Figure 4-13: Water cured ECC-MgO bar specimens made of Class-F fly ash for different holding times

Table 4-3 presented a comprehensive review in order to examine the compatibility between the behaviors of $\mathrm{MgO}$ powder samples before and after utilizing them in ECC-MgO system. According to Table 4-3, less than $45 \%$ of the loss of $\mathrm{CO}_{2}$ content at kiln burning process was considered initially to produce best calcination temperature/holding time within the range studied in this dissertation. This was justified because the creation of higher porous $\mathrm{MgO}$ microstructure at lower calcined temperatures and shorter period of holding times $\left(750^{\circ} \mathrm{C} \_1,2 \& 4,900^{\circ} \mathrm{C} \_1 \&\right.$ 2 and $1050^{\circ} \mathrm{C}_{-} 1$ ). With higher porosity of MEA for these temperatures, higher remaining hydration (beyond 90\%) was produced when tested by TGA test based on decomposition temperatures for previously burnt $\mathrm{MgO}$ powder samples. Higher remaining hydration aggravated the loss of $\mathrm{CO}_{2}$ content for powder samples prepared at these temperatures when tested under TGA burning process due to higher potential of energy stored within MEA structures. Addition of HVFA to ECC-MgO self-healing system and the presence of plenty of smaller pores within the highly reactive $\mathrm{MgO}$ microstructures promoted the formation of stronger $\mathrm{C}-\mathrm{S}-\mathrm{H}$ products at early ages within these pores due to pozzolanic reaction and formation of expansion pressures produced by 
$\mathrm{Mg}(\mathrm{OH})_{2}$ crystals at later ages. As a whole, counteracting forces against the expansion of ECC$\mathrm{MgO}$ bar specimens were developed. This situation was favored in ECC-MgO system due to considerable lower expansion which was the base of finding best calcination temperature.

Although getting very low expansion readings for ECC-MgO bar specimens was desired just to block the cracks, there was a concern that the use of highly reactive MEA produced by $750^{\circ} \mathrm{C} \_1$ \& 2 and $900^{\circ} \mathrm{C}_{-} 1$ (as shown in Table 4-3) which produced very low expansion effect in ECC$\mathrm{MgO}$ self-healing system might not work effectively and not contribute with enough crystals of $\mathrm{Mg}(\mathrm{OH})_{2}$ to supplement the early formation of $\mathrm{CaCO}_{3}$ for sealing the cracks. Therefore, the use of moderately reactive MEA to produce reasonable expansions was believed to be a better choice for the sake of sealing the cracks in ECC-MgO system taking into consideration of high safety margins to not affect the durability at later ages. As shown in Table 4-3, the temperatures mentioned above had the lowest expansion readings (less than $0.100 \%$ ) in specimens made of Class-F fly ash with both particle sizes. Therefore, the use of $750^{\circ} \mathrm{C}_{-} 1,2$ and $900^{\circ} \mathrm{C} \_1$ was omitted from the best calcination system in this research. Finally, all $750^{\circ} \mathrm{C} \_4,900^{\circ} \mathrm{C} \_2$ and $1050^{\circ} \mathrm{C} \_1$ were considered to be the best choice for the best calcination system. However, based on economic reasons, only $900^{\circ} \mathrm{C} \_2 \_45 \mu \mathrm{m}$ was selected as best calcination system and used in this research as mentioned before. 
Table 4-3: MgO powder behavior before* and after* utilizing them into ECC-MgO self-healing system

\begin{tabular}{|c|c|c|c|c|c|c|c|c|c|}
\hline $45 \mu \mathrm{m}$ & \multicolumn{2}{|c|}{ Class-F fly ash } & \multicolumn{2}{|c|}{ Class-CI fly ash } & \multicolumn{5}{|c|}{ MgO Powder } \\
\hline 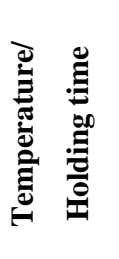 & 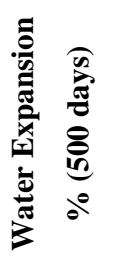 & 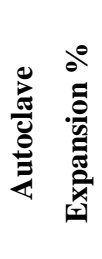 & 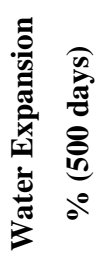 & 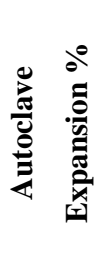 & 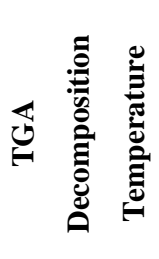 & 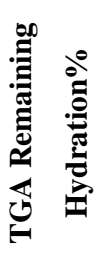 & 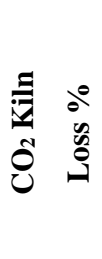 & 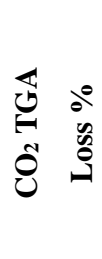 & 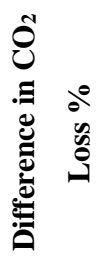 \\
\hline $750-1$ & 0.063 & 0.105 & 0.076 & 0.156 & $630^{\circ} \mathrm{C}$ & 96.92 & 24.28 & 33.17 & -8.90 \\
\hline $750-2$ & 0.078 & 0.149 & 0.128 & 0.221 & $625^{\circ} \mathrm{C}$ & 96.15 & 32.11 & 29.86 & 2.30 \\
\hline 750-4 & $\underline{0.125}$ & $\underline{0.160}$ & $\underline{0.271}$ & $\underline{0.338}$ & $600^{\circ} \mathrm{C}$ & 92.31 & $\underline{42.90}$ & 11.89 & $\underline{31.00}$ \\
\hline $900-1$ & 0.092 & 0.145 & 0.173 & 0.196 & $635^{\circ} \mathrm{C}$ & 97.69 & 32.09 & 28.19 & 3.90 \\
\hline 900-2 & $\underline{0.158}$ & $\underline{0.166}$ & $\underline{0.170}$ & $\underline{0.237}$ & $\underline{615^{\circ} \mathrm{C}}$ & 94.62 & 44.06 & $\mathbf{1 7 . 7 7}$ & 26.30 \\
\hline $900-4$ & 0.217 & 0.218 & 0.191 & 0.286 & $355^{\circ} \mathrm{C}$ & 54.62 & 50.22 & 5.28 & 44.94 \\
\hline$\underline{1050-1}$ & $\underline{0.169}$ & $\underline{0.188}$ & $\underline{0.180}$ & $\underline{0.254}$ & $\underline{590^{\circ} \mathrm{C}}$ & $\underline{90.77}$ & $\underline{44.15}$ & $\underline{19.00}$ & $\underline{25.15}$ \\
\hline $1050-2$ & 0.201 & 0.254 & 0.195 & 0.297 & $390^{\circ} \mathrm{C}$ & 60.00 & 49.30 & 13.14 & 36.16 \\
\hline $1050-4$ & 0.234 & 0.326 & 0.440 & 0.486 & $385^{\circ} \mathrm{C}$ & 59.23 & 49.76 & 4.96 & 44.81 \\
\hline $1200-1$ & 0.216 & 0.222 & 0.233 & 0.264 & $400^{\circ} \mathrm{C}$ & 61.54 & 50.08 & 16.10 & 33.98 \\
\hline $1200-2$ & 0.225 & 0.277 & 0.233 & 0.312 & $395^{\circ} \mathrm{C}$ & 60.77 & 50.32 & 11.62 & 38.70 \\
\hline $1200-4$ & 0.153 & 0.295 & 0.129 & 0.381 & $375^{\circ} \mathrm{C}$ & 57.69 & 50.44 & 3.61 & 46.83 \\
\hline $100 \mu \mathrm{m}$ & \multicolumn{2}{|c|}{ Class-F fly ash } & \multicolumn{2}{|c|}{ Class-CI fly ash } & \multicolumn{5}{|c|}{ MgO Powder } \\
\hline $750-1$ & 0.056 & 0.102 & 0.046 & 0.078 & $640^{\circ} \mathrm{C}$ & 98.46 & 23.56 & 33.70 & -10.15 \\
\hline $750-2$ & 0.083 & 0.121 & 0.087 & 0.134 & $630^{\circ} \mathrm{C}$ & 96.92 & 33.03 & 24.52 & 8.51 \\
\hline $750-4$ & 0.136 & 0.151 & 0.136 & 0.278 & $600^{\circ} \mathrm{C}$ & 92.31 & 43.70 & 10.56 & 33.14 \\
\hline $900-1$ & 0.094 & 0.111 & 0.107 & 0.179 & $625^{\circ} \mathrm{C}$ & 96.15 & 34.52 & 26.04 & 8.47 \\
\hline $900-2$ & 0.144 & 0.146 & 0.168 & 0.225 & $635^{\circ} \mathrm{C}$ & 97.69 & 43.58 & 14.09 & 29.50 \\
\hline $900-4$ & 0.195 & 0.218 & 0.222 & 0.276 & $380^{\circ} \mathrm{C}$ & 58.46 & 49.33 & 4.78 & 44.55 \\
\hline $1050-1$ & 0.152 & 0.170 & 0.131 & 0.210 & $610^{\circ} \mathrm{C}$ & 93.85 & 41.21 & 17.10 & 24.11 \\
\hline $1050-2$ & 0.231 & 0.243 & 0.235 & 0.278 & $400^{\circ} \mathrm{C}$ & 61.54 & 48.87 & 11.76 & 37.11 \\
\hline $1050-4$ & 0.206 & 0.350 & 0.408 & 0.494 & $395^{\circ} \mathrm{C}$ & 60.77 & 49.80 & 4.46 & 45.34 \\
\hline $1200-1$ & 0.198 & 0.231 & 0.199 & 0.213 & $395^{\circ} \mathrm{C}$ & 60.77 & 48.06 & 15.66 & 32.40 \\
\hline $1200-2$ & 0.206 & 0.277 & 0.200 & 0.281 & $395^{\circ} \mathrm{C}$ & 60.77 & 49.16 & 8.97 & 40.19 \\
\hline $1200-4$ & 0.120 & 0.294 & 0.098 & 0.334 & $370^{\circ} \mathrm{C}$ & 56.92 & 49.64 & 2.70 & 46.94 \\
\hline
\end{tabular}

*Before = MgO powder state; *After = MgO powder used in ECC-MgO self-healing system 
From Table 4-3, it can be found that the ultimate expansion reading of water cured ECC-MgO bar specimens produced by $900^{\circ} \mathrm{C} \_2 \_45 \mu \mathrm{m}$ was $0.158 \%$ while the accelerated autoclaved expansion reading for the same specimen was $0.166 \%$. This means that the water cured specimen of $900^{\circ} \mathrm{C} \_2 \_45 \mu \mathrm{m}$ had reached the ultimate point of $\mathrm{MgO}$ hydration and there will be no more expansion at later ages as confirmed by autoclave expansion test. Mo et al. (2010) confirmed the same behavior for normal cement pastes by accelerating the expansion of cement paste bar specimens by curing them in water at $40^{\circ} \mathrm{C}$ to compare them with the ones under same conditions but cured in water at $20^{\circ} \mathrm{C}$. It was shown that the ultimate expansion of MEA prepared at $900^{\circ} \mathrm{C}$ for 1 hour of holding time was around $0.225 \%$ which remained the same even when hydration accelerated when cured in water at $40^{\circ} \mathrm{C}$. It was also shown that the ultimate expansion of MEA prepared at $1200^{\circ} \mathrm{C}$ cured in water at $20^{\circ} \mathrm{C}$ was around $0.075 \%$ and jumped up to $1.7 \%$ when cured in water at $40^{\circ} \mathrm{C}$. When HVFA (55\% of cement replacement) used in this research, the ultimate expansion reading of water cured ECC-MgO bar specimen produced by $1200^{\circ} \mathrm{C} \_1 \_45 \mu \mathrm{m}$ was $0.216 \%$ while under accelerated autoclave expansion test was $0.222 \%$. The presence of HVFA in ECC-MgO specimens produced by using low $\mathrm{CaO}$ content fly ash suppressed or might stop the expansion even under autoclave curing conditions. 

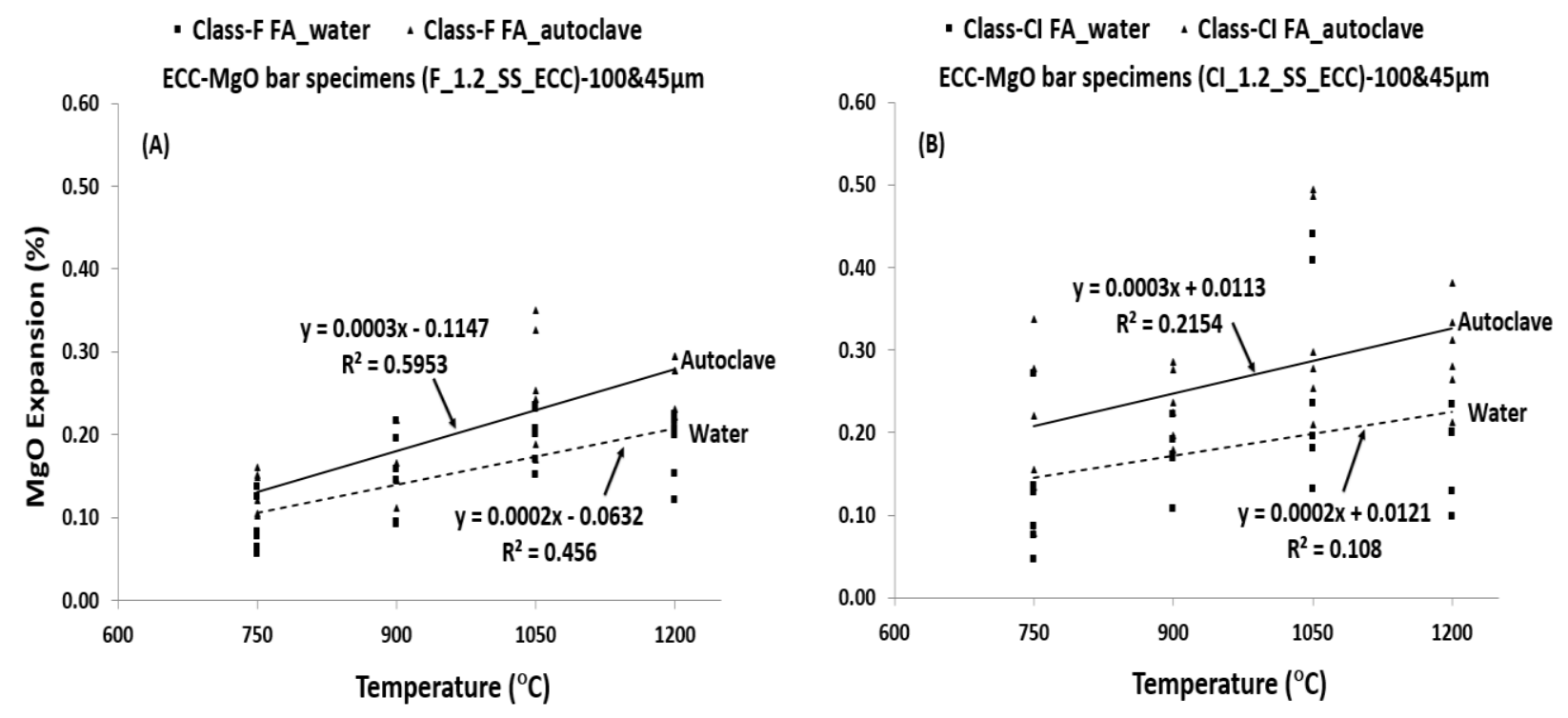

Figure 4-14: Relationship between all $\mathrm{MgO}$ expansion readings for both autoclave and water $\mathrm{ECC}-\mathrm{MgO}$ bar specimens as a function of temperature (A: Class-F fly ash \& B: Class-CI fly ash)

Figure 4-14 shows the relationship between $\mathrm{MgO}$ expansion and calcination temperature for both types of fly ash and both $\mathrm{MgO}$ particle sizes. The more calcination temperature, the more the expansion for both autoclave and water cured ECC-MgO bar specimens. 


\subsubsection{Microstructure of ECC-MgO self-healing system}

For the microstructural investigation, disk specimens were prepared from cracked ECC-MgO prismatic specimens. The prismatic specimens were cured in sealed plastic bags for 14 days (the day of cracking) and then multiple cracks were induced by applying flexural loading under four point loading. The crack induced disk specimens were further cured in water for 21 and 56 days right after cracking before scanning electron microscope (SEM) analysis.

Figure 4-15 shows crack healing after 21 days and 56 days of water curing and chemical composition of healing compound formed due to self-healing of ECC-MgO system. Figure 4-15 (A) shows SEM photographs of the surface of a specimen right after cracking with a maximum crack width of around $100 \mu \mathrm{m}$. The SEM observation clearly revealed crack filling compounds to be consisting of two materials. The first one was $\mathrm{MgO}$ crystals as shown in the zone I with $12.6 \%$ of magnesium while the second one was $\mathrm{CaO}$ crystals as shown in zone II with $43.9 \%$ of calcium. Figure 4-15 confirms the formation of cementitious $\mathrm{MgO}$ crystals within the crack walls which might supplement the $\mathrm{CaCO}_{3}$ crystallization and both together can improve mechanical and durability properties of ECC-MgO self-healing system. 


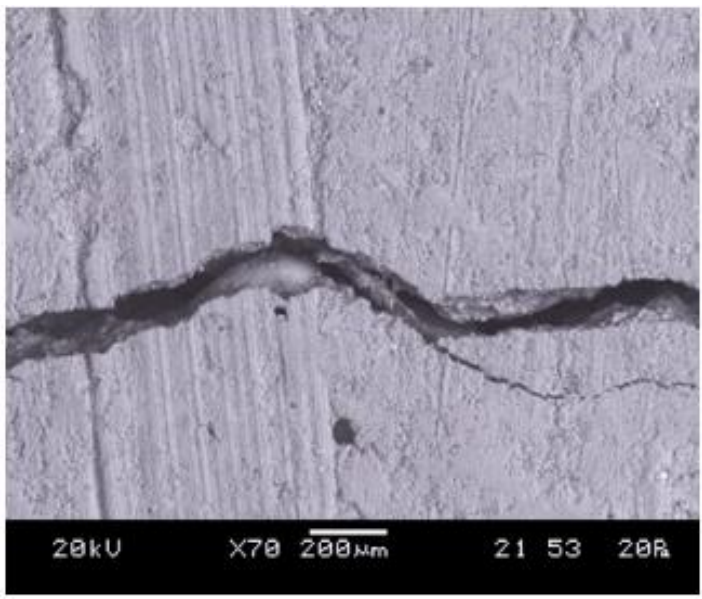

a) 14 days, the day of cracking

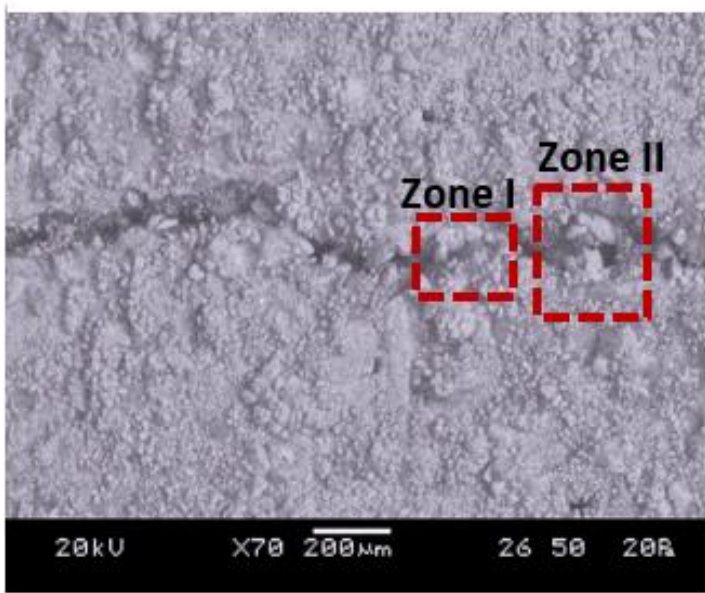

b) 21 days after cracking

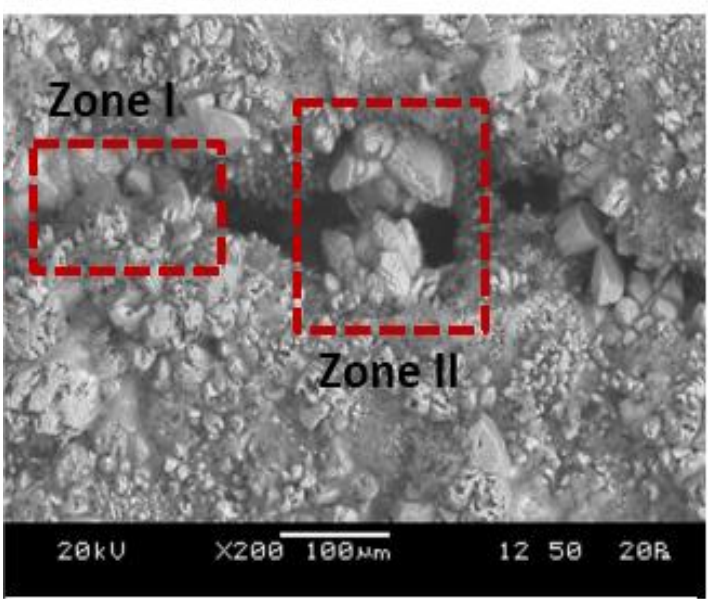

c) 21 days after cracking

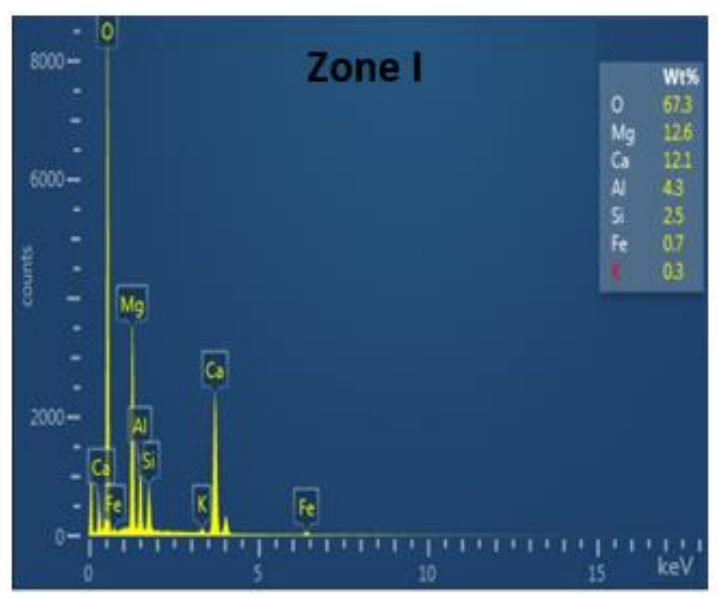

d) Chemical composition, Zone I: $\mathrm{Mg}(\mathrm{CO})_{3}$

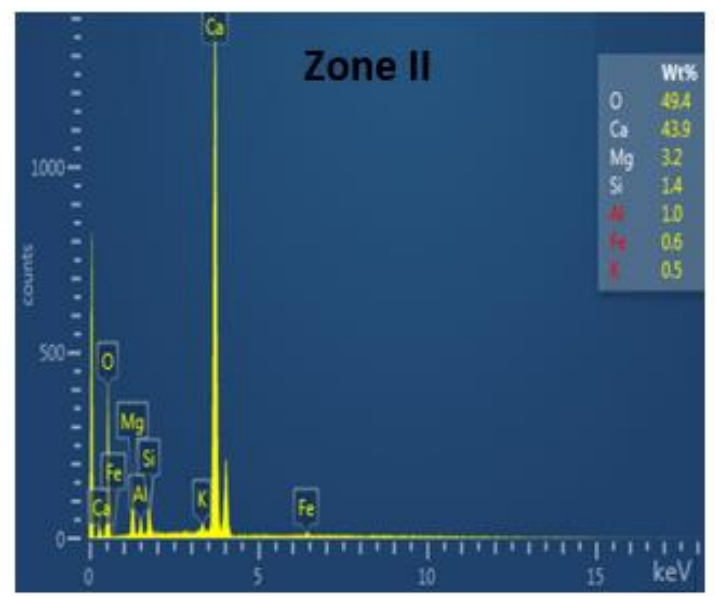

e) Chemical composition, Zone II: $\mathrm{Ca}(\mathrm{CO})_{3}$

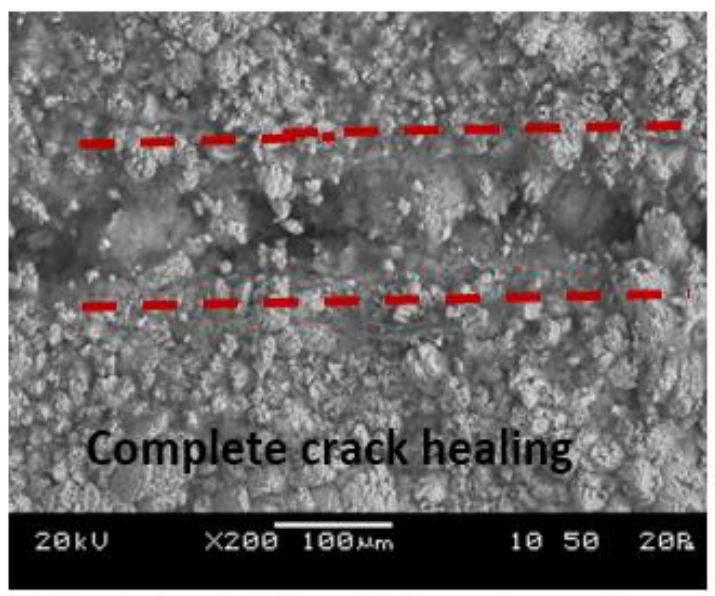

f) Complete crack filling at $\mathbf{5 6}$ days after cracking

Figure 4-15: Self-healing of ECC-MgO concrete at different ages. 


\subsection{Summary}

Engineered Cementitious Composites (ECCs) are found to be a better replacement for traditional concrete due to their ability to induce smaller crack width, higher damage tolerance under maximum loadings and potential crack healing capabilities. MgO-type expansive agent (MEA) (made of lightly burnt $\mathrm{MgO}$ powder) has been widely used as a cementitious additive to compensate shrinkage of mass concrete. This chapter presented an effective ECC-MgO combined self-healing system and the viability of using MEA in the production of ECC-MgO concrete. New measures were implemented in examining the performance of ECC-MgO self-healing system based on accelerated autoclave test to simulate the experimental results relevant to the field conditions. 


\section{CHAPTER FIVE}

\section{SELF-HEALING AND EXPANSION CHARACTERISTICS OF ENGINEERED CEMENTITIOUS COMPOSITES WITH HIGH VOLUME FLY ASH AND MGO-TYPE EXPANSIVE AGENT}

\subsection{Introduction}

The current chapter investigates the effect of both high volume of fly ash (HVFA) and MgO contents involved in the production of ECC-MgO self-healing system and evaluates their effectiveness based on the expansion effect of MEA and microstructural matrix densification through accelerated autoclave/water linear expansion tests and Scanning Electron Microscope (SEM) analysis, respectively.

Also, self-healing characteristics of ECC-MgO system was evaluated through compressive strength recovery of pre-cracked specimens based on accelerated autoclave tests. Supplementary cementing material (SCM) and $\mathrm{MgO}$ contents for the best performance of ECC-MgO self-healing system are quantified. 


\subsection{Experimental Investigations}

Based on chapter 4 , the calcination system of " $900^{\circ} \mathrm{C} \_2$ hours of holding time_45 $\mu \mathrm{m}$ particle size" was used in the production of ECC-MgO bar specimens. According to this system, $\mathrm{MgO}$ was prepared from "lightly burned" pure Magnesite (Magnesium Carbonate, $\mathrm{MgCO}_{3}$ ) exposed to $900^{\circ} \mathrm{C}$ for 2 hours inside the kiln and using $45 \mu \mathrm{m}$ particle size of $\mathrm{MgCO}_{3}$. In this chapter, ECC$\mathrm{MgO}$ bar specimens $(25 \times 25 \times 285 \mathrm{~mm})$ were tested to study the effect of different types of fly ash (Class-F and Class-CI) having two different cement replacements (30\% and 55\%) and different dosages of MEA content of up to 30\%. The effectiveness of ECC-MgO system was assessed based on lower expansion effect of MEA through both accelerated autoclave/water linear expansion tests and SEM analysis of microstructure. The effect of high volumes of MEA (up to $30 \%$ ) combined with HVFA on the microstructure of ECC-MgO system was studied, in particular. The effectiveness of ECC-MgO self-healing system using the best combination of SCM and MEA dosages was assessed based on the compressive strength development/recovery accelerated autoclave cured cube specimens.

\subsubsection{ECC-MgO Mixture Proportions}

To develop and assess the effectiveness of MEA in ECC-MgO self-healing system, three ECC mixtures designed and selected. The first ECC mix was produced by Class-F fly ash and silica sand with cement replacement of $\mathrm{FA} / \mathrm{PC}=1.2(\mathrm{FA} / \mathrm{Binder}=55 \%)$. The second ECC mix was same as the first one except that it was produced by Class-CI fly ash. The third ECC mix was produced as the first mix as well except that the cement replacement of Class-F fly ash was reduced to $\mathrm{FA} / \mathrm{PC}=0.45(\mathrm{FA} / \mathrm{Binder}=30 \%)$ for comparison purposes. The amount of $\mathrm{MgO}$ powder used in the production of standard ECC bar specimens was ranged between $0 \%$ and $30 \%$ measured with respect to binder weight $(\mathrm{PC}+\mathrm{FA})$. The mixture proportions and designations for both ECC mixtures are given in Table 5-1. The water/binder (w/b) ratio was kept in the range of 0.27 for all three ECC mixes and the amount of aggregate was held constant.

In Table 5-1, all ECC mixtures are labeled in a way that the ingredients are identifiable from their Mix IDs. The first number in the mixture designation indicates the percentage of MEA while the 
second letter indicates SCM type (F = Class-F fly ash, CI = Class-CI fly ash). It should be noted that fly ash content was reduced when $\mathrm{MgO}$ content was added to the $\mathrm{ECC}-\mathrm{MgO}$ mixes.

Table 5-1: ECC mixture proportions produced from different combinations of fly ash and $\mathrm{MgO}$ contents

\begin{tabular}{|c|c|c|c|c|c|c|c|c|c|}
\hline \multirow{2}{*}{$\begin{array}{c}\text { Set } \\
\#\end{array}$} & \multirow[b]{2}{*}{ Mixture ID } & \multicolumn{8}{|c|}{ Ingredients per 1 part of binder measured by mass } \\
\hline & & W/B & $\begin{array}{l}\text { Binder } \\
\text { (PC+FA) } \\
\end{array}$ & $\begin{array}{c}\mathrm{MgO} \\
\%\end{array}$ & $\begin{array}{c}\text { FA/Binder after } \\
\text { adding } \mathrm{MgO}\end{array}$ & $\begin{array}{l}\text { Silica } \\
\text { Sand }\end{array}$ & $\begin{array}{l}\mathrm{PVA} \\
\mathrm{kg} / \mathrm{m}^{3}\end{array}$ & $\begin{array}{l}\text { HRWRA } \\
\mathrm{kg} / \mathrm{m}^{3}\end{array}$ & $\mathrm{FA} / \mathrm{PC}$ \\
\hline \multirow{7}{*}{$1^{*}$} & 0\%MEA_F & \multirow{7}{*}{0.27} & \multirow{7}{*}{1} & 0 & 0.55 & \multirow{7}{*}{0.36} & \multirow{7}{*}{26} & \multirow{7}{*}{5.4} & 1.2 \\
\hline & 2\%MEA_F & & & 2 & 0.53 & & & & 1.16 \\
\hline & 4\%MEA_F & & & 4 & 0.51 & & & & 1.11 \\
\hline & 5\%MEA_F & & & 5 & 0.50 & & & & 1.09 \\
\hline & 6\%MEA_F & & & 6 & 0.49 & & & & 1.07 \\
\hline & 8\%MEA_F & & & 8 & 0.47 & & & & 1.02 \\
\hline & 10\%MEA_F & & & 10 & 0.45 & & & & 0.98 \\
\hline \multirow{7}{*}{$2 * *$} & 0\%MEA_Cl & \multirow{7}{*}{0.27} & \multirow{7}{*}{1} & 0 & 0.55 & \multirow{7}{*}{0.36} & \multirow{7}{*}{26} & \multirow{7}{*}{5.4} & 1.2 \\
\hline & 2\%MEA_Cl & & & 2 & 0.53 & & & & 1.16 \\
\hline & 4\%MEA_Cl & & & 4 & 0.51 & & & & 1.11 \\
\hline & 5\%MEA_Cl & & & 5 & 0.50 & & & & 1.09 \\
\hline & 6\%MEA_Cl & & & 6 & 0.49 & & & & 1.07 \\
\hline & 8\%MEA_Cl & & & 8 & 0.47 & & & & 1.02 \\
\hline & 10\%MEA_Cl & & & 10 & 0.45 & & & & 0.98 \\
\hline \multirow{7}{*}{$3+$} & 0\%MEA_F & \multirow{7}{*}{0.27} & \multirow{7}{*}{1} & 0 & 0.30 & \multirow{7}{*}{0.36} & \multirow{7}{*}{26} & \multirow{7}{*}{5.4} & 0.45 \\
\hline & 2\%MEA_F & & & 2 & 0.28 & & & & 0.43 \\
\hline & 4\%MEA_F & & & 4 & 0.26 & & & & 0.40 \\
\hline & 5\%MEA_F & & & 5 & 0.25 & & & & 0.38 \\
\hline & 6\%MEA_F & & & 6 & 0.24 & & & & 0.37 \\
\hline & 8\%MEA_F & & & 8 & 0.22 & & & & 0.34 \\
\hline & 10\%MEA_F & & & 10 & 0.20 & & & & 0.31 \\
\hline \multirow{4}{*}{$4^{*}$} & 15\%MEA_F & \multirow{4}{*}{0.27} & \multirow{4}{*}{1} & 15 & 0.40 & \multirow{4}{*}{0.36} & \multirow{4}{*}{26} & \multirow{4}{*}{5.4} & 0.87 \\
\hline & 20\%MEA_F & & & 20 & 0.35 & & & & 0.76 \\
\hline & 25\%MEA_F & & & 25 & 030 & & & & 0.65 \\
\hline & 30\%MEA_F & & & 30 & 0.25 & & & & 0.54 \\
\hline \multirow{4}{*}{$5+$} & 15\%MEA_F & \multirow{4}{*}{0.27} & & 15 & 0.15 & & & & 0.24 \\
\hline & 20\%MEA_F & & & 20 & 0.10 & & & & 0.16 \\
\hline & 25\%MEA_F & & 1 & 25 & 0.05 & 0.36 & 26 & 5.4 & 0.09 \\
\hline & 30\%MEA_F & & & 30 & 0.00 & & & & 0.00 \\
\hline
\end{tabular}

*First ECC mix; **Second ECC mix; +Third ECC Mix

\subsubsection{Preparation of ECC-MgO bar specimens}

As shown in Table 5-1, 5 sets of ECC-MgO bar specimens were produced from three basic ECC mixes by varying MEA content from 0 to $30 \%$. The first set (seven sub-mixes) was produced by 
using Class-F fly ash with cement replacement of 55\% while $\mathrm{MgO}$ content (replacing fly ash) ranging between $0 \%$ and $10 \%$. The second set (seven sub-mixes) was the same as the first set but made of Class-CI fly ash based second basic ECC mix. The third set (seven sub-mixes) was made of third basic ECC mix with cement replacement of Class-F FA of $30 \%$ with MgO content (ranging between $0 \%$ and $10 \%$. Both fourth and fifth sets (total eight sub-mixes) used high volumes of $\mathrm{MgO}$ content ranging between $15 \%$ and $30 \%$ produced with Class-F fly ash with two cement replacement levels of $55 \%$ and $30 \%$, respectively. The bar/prism specimens of ECC-MgO mixtures were produced in accordance with (ASTM C1038-b, 2014). All specimens of the first three sets were used for water and autoclave linear expansion tests. Only the bar specimens of fourth and fifth sets were used for water cured linear expansion test. At least 3 bar specimens $(25 \times 25 \times 285 \mathrm{~mm}$ in dimensions) were used for each of the $29 \mathrm{ECC}-\mathrm{MgO}$ sub-mixes to monitor water cured linear expansion readings - total 87 bar specimens were prepared for water expansion test. However, two bar specimens were used for each sub-mix in autoclave expansion testing - 42 bar specimens were prepared. After casting, bar specimens were cured in plastic bags at $95 \pm 5 \%$ relative humidity $(\mathrm{RH}), 23 \pm 2{ }^{\circ} \mathrm{C}$. All bar specimens were demolded after 24 hours and initial length measure was taken right after the demolding. After obtaining the initial length comparator readings, bar specimens for the water expansion test were cured in water at $20^{\circ} \mathrm{C}$ until the next length measure while autoclave cured bar specimens were placed in autoclave machine for testing.

At least 3 cubic specimens $(50 \times 50 \times 50 \mathrm{~mm})$ were also cast for each ECC-MgO mixture and cured under water and autoclave conditions. The compression test was carried out on cubic specimens by using a compression testing machine with a capacity of 400,000 lbs. The age of testing was 7 and 28 days for water cured specimens and 28 days for autoclave specimens.

\subsection{Test procedures}

\subsubsection{Water and autoclave expansion tests}

As shown in Table 5-1, five sets of ECC-MgO bar specimens (25x25x285 mm) were produced and used in both water and autoclave linear expansion tests. The length change readings acquired in both tests were analyzed based on lower expansion effect of $\mathrm{MgO}$ agent. For water expansion test, all bar specimens were stored in water until the following length measure in accordance with 
(ASTM C1038-b, 2014). The readings of water cured ECC-MgO bar specimens were taken up to 150 days by using a length comparator in accordance with (ASTM C490, 2011). Autoclave test was used to expedite/disclose the delayed $\mathrm{MgO}$ hydration when involved in the production of $\mathrm{ECC}-\mathrm{MgO}$ bar specimens. Autoclave test setup and procedure were conducted in accordance with (ASTM C151, 2015) and mentioned in Chapter 4 - (section 4.3.2). At the end of autoclave test, the length comparator readings were obtained again as per (ASTM C490, 2011).

\subsubsection{Compressive strength development and recovery tests}

The performance (in terms of feasibility and practicality) of ECC-MgO self-healing system was compared to ECC-control (without MEA) through the compressive strength development of cubic specimens under accelerated autoclaved curing expediting the expansion behavior of MEA and under water curing conditions. All cubic specimens were cured in water at $20^{\circ} \mathrm{C}$ until the day of compressive strength testing. Five arrangements were employed: (i) in the first and the second arrangements, 3 cubic specimens (for each age) were tested for strength after 7 and 28 days of water curing, respectively (ii) in the third arrangement, 3 (28 day water cured) cubic specimens were tested for strength after autoclaved curing and (iii) in the last two arrangements, 28 day water cured pre-cracked (made by applying $50 \%$ and $80 \%$ of maximum strength) cubic specimens were tested for strength right away after autoclaved curing.

It is well known that only bar/prism specimens can be used in autoclave test based on (ASTM C151, 2015). For this reason, special autoclave rack was made for cubic specimens to fit the autoclave vessel (see Figure 3-5). It should be emphasized that during autoclave test, care should be taken to ensure safety due to the emission of sharp fumes which could be toxic due to the presence of PVA fibers and chemical admixtures in ECC- $\mathrm{MgO}$ specimens when exposed to very high pressure and temperature. Consequently, proper ventilation system such as fume hood and complete respiratory protection with ambient air pump must be used. 


\subsection{Results and Discussions}

All expansion data of water and natural cured prism/bar specimens are provided in Appendix C.

\subsubsection{Effect of fly ash on linear expansion}

\subsubsection{Autoclave expansion}

Figure 5-1 shows expansion of autoclaved ECC-MgO bar specimens produced by two types of fly ash (Class-F and Class-CI) with cement replacement up to 55\%. The expansion of ECC-MgO bar specimens increased with the increase of $\mathrm{MgO}$ content. Zheng et al. (1991) observed that the expansion of cement pastes prisms increased with the increase of both $\mathrm{MgO}$ content and elevated environmental temperatures (from $20^{\circ} \mathrm{C}$ to $50^{\circ} \mathrm{C}$ ) due to accelerated hydration process. According to (ASTM C150, 2016), the maximum $\mathrm{MgO}$ content in clinker and the maximum permitted expansions are limited to $6.0 \%$ measured by cement weight and $\leq 0.80 \%$, respectively. Gonnerman et al. (1953) (reported by (Helmuth and West, 1998)) revealed that suddenly quenched clinkers with low free $\mathrm{CaO}$ content showed a sharp increase in autoclave expansions when $\mathrm{MgO}$ content increased beyond 6\%. Du (2005) has limited the addition of $\mathrm{MgO}$ content as cement additive to $5 \%$ of cement weight only. Moreover, Nikiforov \& Zosoulia (1980) limited the use of MgO content in commercial Portland cement to $6 \%$ and may reach up to $11 \%$ if $\mathrm{MgO}$ is uniformly distributed (Nikiforov and Zosoulia, 1980). Also, Zheng et al. (1991) considered cement as unsound (expansion is greater than $0.80 \%$ ) when $\mathrm{MgO}$ content exceeds $4.73 \%$. Although MEA content increased up to $10 \%$ (by weight of cementitious materials) as shown in Figure 5-1, still ECC-MgO bar specimens made of both fly ashes (class F and CI) showed expansions lower than ASTM limit $(<0.80 \%)$. 


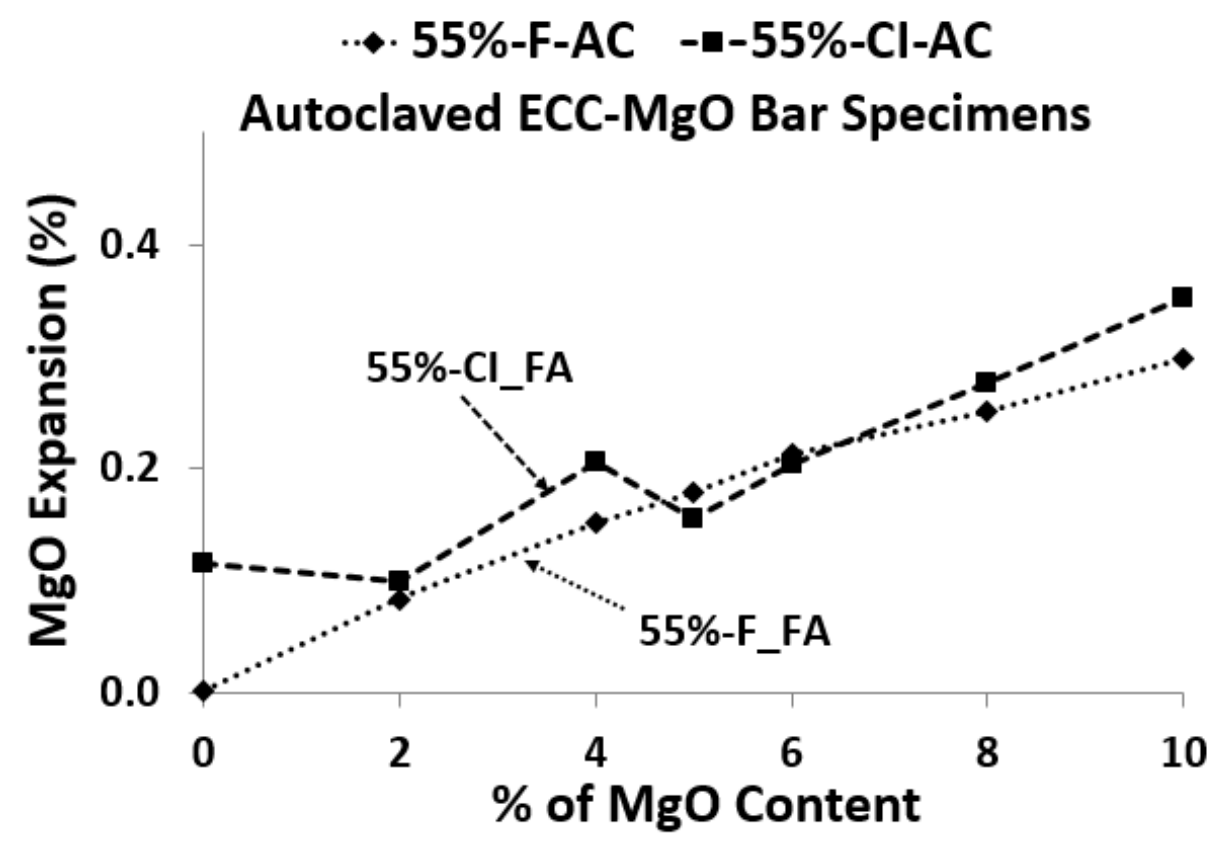

Figure 5-1: Expansion of autoclaved ECC-MgO bar specimens with two types of fly ash

In most cases, the literature have revealed very high expansion for autoclaved bar specimens made of cement pastes without the addition of fly ash. Autoclave test accelerates rapidly the formation of $\mathrm{Mg}(\mathrm{OH})_{2}$ crystals and the hydration of C-S-H as well. Therefore, weaker microstructures might be produced in autoclave curing due to considerable expansive stresses created on the surrounding pores when $\mathrm{Mg}(\mathrm{OH})_{2}$ crystals are formed. Also, the C-S-H hydration will be expedited leading to the loss of cohesive forces within cement paste. After the addition of fly ash to cement pastes, pozzolanic reaction occurs forming strong $\mathrm{C}-\mathrm{S}-\mathrm{H}$ products in the surrounding microstructures and limiting the formation of $\mathrm{Mg}(\mathrm{OH})_{2}$ crystals as well (Ali and Mullick, 1998; Gao et al., 2007; Helmuth and West, 1998). Therefore, Figure 5-1 revealed lower expansion readings for autoclaved $\mathrm{ECC}-\mathrm{MgO}$ bar specimens (less than $0.80 \%$ ) due to the addition of fly ash regardless of the type. The effect of adding fly ash to high magnesium clinkers containing 7.5, 10 and $15 \%$ of $\mathrm{MgO}$ was reported by Reshi (1983). After the autoclave test, the expansion was reduced from $17 \%$ to $0.75 \%$ with the addition of $30 \%$ fly ash even with cement containing $15 \%$ of $\mathrm{MgO}$ content.

It was also observed from Figure 5-1 that bar specimens produced with 55\% FA of Class-F exhibited lower expansions than those made of 55\% fly ash of Class-CI. The maximum expansion 
recorded for 55\%-CI_FA bar specimens was $0.353 \%$ compared to $0.298 \%$ of 55\%-F_FA when $10 \%$ MEA content added. This might be justified due to the presence of higher $\mathrm{CaO}$ content in Class-CI compared to Class-F fly ash leading to the increase of temperature/rate of hydration (Bisaillon et al., 1994; Du, 2005; Langley et al., 1992; Mavani, 2012; Thomas et al., 1995). The rate of $\mathrm{MgO}$ hydration will also be increased leading to more formation of $\mathrm{Mg}(\mathrm{OH})_{2}$ crystals by combining together the available $\mathrm{OH}^{-}$and $\mathrm{Mg}^{2+}$ ions in pore solution. In addition to that, higher $\mathrm{CaO}$, higher $\mathrm{Na}_{2} \mathrm{O}$ and lower $\mathrm{SiO}_{2}$ contents in fly ash (as is the case for Class CI fly ash in Table 3-1) were found to lower the efficiency of fly ash in reducing pore solution alkalinity (Shehata, 2001). Therefore, higher $\mathrm{CaO}$ in fly ash in general will increase the content of $\mathrm{OH}^{-}$ions that might increase the formation of $\mathrm{Mg}(\mathrm{OH})_{2}$ crystals and finally lead to higher expansions as observed for Class-CI fly ash. But after close observation, Figure 5-1 showed slightly lower expansions related to 55\%-CI_FA than 55\%-F_FA when $\mathrm{MgO}$ content was 5\% and 6\%. As revealed in Shehata (2001), the presence of high $\mathrm{CaO}$ content in fly ash increases pore solution alkalinity and then also increases the content of $\mathrm{Ca}(\mathrm{OH})_{2}$ (Shehata, 2001). Rich calcium hydroxide ions in pore solution might also stimulate the pozzolanic reaction to consume more $\mathrm{Ca}(\mathrm{OH})_{2}$ crystals and increase the existence of C-S-H products in cement matrix. The formation of C-S-H will counteract the expansive stresses of $\mathrm{MgO}$ and eventually reduces the expansion as shown in Figure 5-1 (Mo et al., 2014). However, the tendency of having higher MgO expansions in 55\%-CI_FA was observed compared to 55\%-F_FA in general. The fluctuated trend (Figure 5-1) in the expansion curve of 55\%-CI_FA produced by competing $\mathrm{MgO}$ expansive stresses with the formation of C-S-H products might lead to unbalanced expansions at later ages. Therefore, due to the uniform/balanced trend of 55\%-F_FA expansion curve and lower expansion (than 55\%-CI_FA), Class-F fly ash with cement replacement of 55\% was chosen/suggested in ECC-MgO self-healing system as a better choice.

In order to study the effect of low fly ash content on ECC-MgO self-healing system (characterized by low $\mathrm{CaO}$ content), Class-F fly ash content was reduced from 55\% to $30 \%$ as shown in Figure 5-2. It is common in Chinese research and dam construction engineering to limit the cement replacement of fly ash to $30 \%$ only considering the shrinkage compensation of mass concrete (Du, 2005). Most studies conducted on $\mathrm{MgO}$ concrete to achieve shrinkage compensation in dam 
construction used fly ash with low calcium content (Ali and Mullick, 1998; Du, 2005; Gao et al., 2008, 2007; Mo et al., 2014, 2012; Xian Li et al., 2010). The intention of replacing cement with fly ash is to reduce the heat of concrete hydration and limit the formation of $\operatorname{Mg}(\mathrm{OH})_{2}$ to suppress the expansion of $\mathrm{MgO}$ concrete indirectly ( $\mathrm{Du}, 2005)$. However, Figure 5-2 revealed higher expansions of ECC-MgO bar specimens produced by 30\%-F_FA than those of 55\%-F_FA. Obviously, higher $\mathrm{CaO}$ content acquired in 30\%-F_FA microstructure (due to increased Portland cement content) compared to 55\%-F_FA lead to the increase of $\mathrm{OH}^{-}$ions and higher expansions when more $\mathrm{Mg}(\mathrm{OH})_{2}$ crystals formed. Similar behavior was observed in 55\%-CI_FA as mentioned before shown in Figure 5-1. Due to the lowest expansion in bar specimens made of 55\%-F_FA compared to both 30\%-F_FA and 55\%-CI_FA, the former was considered to be the better choice for ECC-MgO self-healing system.

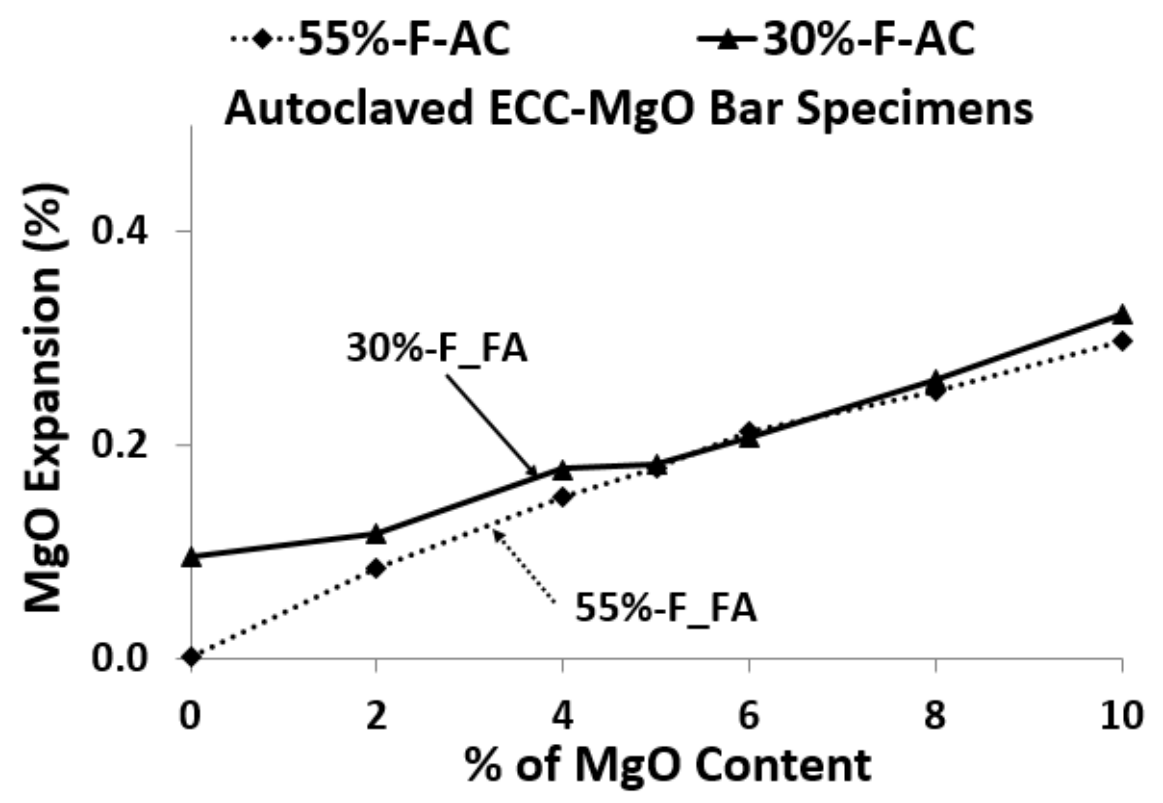

Figure 5-2: Expansions for autoclaved ECC-MgO bar specimens with cement replacement of fly ash in terms of $\mathrm{MgO}$ content 
It should be noted that both 30\%-F_FA and 55\%-CI_FA had similar expansions but higher than 55\%-F_FA when $\mathrm{MgO}$ content was 0\% (Figure 5-1 \& Figure 5-2). This might be attributed to the presence of high $\mathrm{CaO}$ content in former systems causing increased rate of concrete hydration especially for bar specimens cured at elevated temperatures of up to $216 \pm 2^{\circ} \mathrm{C}$ in autoclave curing.

\subsubsection{Water expansion test}

Figure 5-3 shows the expansion effect of different MEA added in different amounts to ECC-MgO bar specimens cured in water at $20^{\circ} \mathrm{C}$. The expansion was monitored for around 160 days until the curves reached a plateau and no more expansion recorded. (ASTM C1038-b, 2014) was used to produce $\mathrm{ECC}-\mathrm{MgO}$ bar specimens by using two kinds of fly ashes (Class-F and Class-CI) with $55 \%$ cement replacement. As expected, the expansion of ECC-MgO bar specimens increased with the increase of $\mathrm{MgO}$ content (from 0 to $10 \%$ of FA) and time. The expansion increase was in the order of $0 \%$ to $10 \%$ and took place significantly up to the age of 28 days. After 28 days, the expansion developed slowly up to 160 days in all ECC-MgO bar specimens. 55\%-CI_FA specimens clearly had higher expansions than those produced by 55\%-F_FA. The maximum expansion of 55\%-CI_FA (with 10\% of MEA) was $0.258 \%$ while it was $0.215 \%$ for ECC-MgO bar specimens made of 55\%-F_FA. Most researchers revealed similar expansion behavior of MEA when used in cement paste prisms - the more $\mathrm{MgO}$ added the more expansion recorded (Xian $\mathrm{Li}$ et al., 2010; Zheng et al., 1991). 

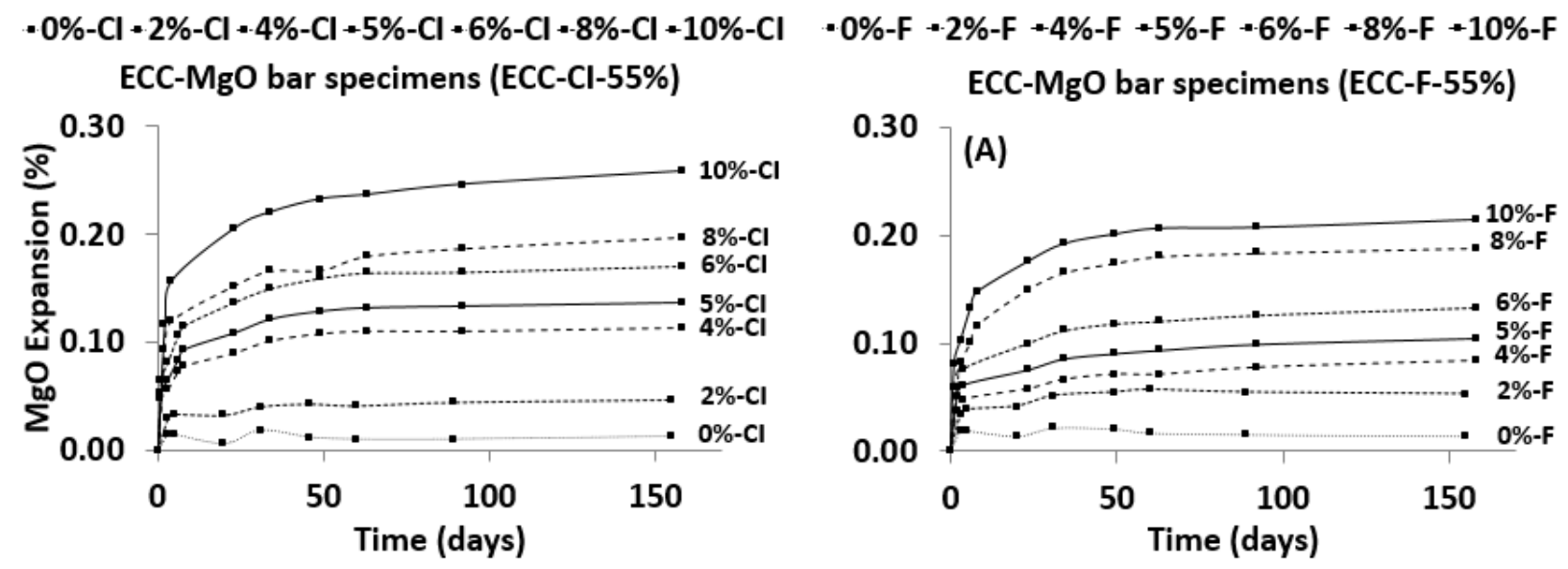

Figure 5-3: Expansion of water cured ECC-MgO specimens made of different fly ash and $\mathrm{MgO}$ content with time

Again similar to autoclave expansion test and, 55\%-F_FA with cement replacement of 55\% was found to be the better choice compared to 55\%-CI_FA considering lower expansion.

Figure 5-4 shows expansion characteristics of $20^{\circ} \mathrm{C}$ water cured ECC-MgO bar specimens with low content of fly ash (characterized by low $\mathrm{CaO}$ content). ECC-MgO bar specimens made of 55\%-F_FA showed higher expansion than those made of 30\%-F_FA during the monitoring period of 160 days (Figure 5-4 part A and B). This finding was totally opposite to autoclave expansion test which revealed that 30\%-F_FA had higher expansions than 55\%-F_FA (Figure 5-2). However, expansion produced by 30\%-F_FA (Figure 5-4 part D) exhibited a sharp rise in most curves showing very strong tendency to develop higher expansions than those produced by 55\%-F_FA (Figure 5-4 part C) especially within the period of first 28 days. Because of the presence of higher $\mathrm{CaO}$ content in 30\%-F_FA microstructure (associated with the higher content of Portland cement), the sudden rise in the expansion curves (as shown in Figure 5-4-part D) represented a higher rate of $\mathrm{Mg}(\mathrm{OH})_{2}$ hydration. Obviously, the higher content of $\mathrm{OH}^{-}$ions due to higher $\mathrm{CaO}$ content will lead for more formation of $\mathrm{Mg}(\mathrm{OH})_{2}$ crystallization and eventually leading to higher expansions. However, after the age of 28 days, the sharp rise observed in the curves not only curbed strongly but also decreased significantly until the curves approached the usual expansion evolution. For instance, considering the curve of 10\%-F (MEA) produced by 30\%-F_FA (Figure 5-4 - part D), the expansion was $0.117 \%$ at the age of 19 days then increased sharply to $0.207 \%$ at the age of 25 
days but decreased strongly to $0.129 \%$ at the age of 27 days and then increased slowly to $0.146 \%$ at the age of 32 days. By removing the expansion value at the age of 25 days from the curve and keeping other values at the ages of 19, 27 and 32 days, the curve will show smooth rise in expansion in the order of $0.117 \%, 0.129 \%$ and $0.146 \%$, respectively.
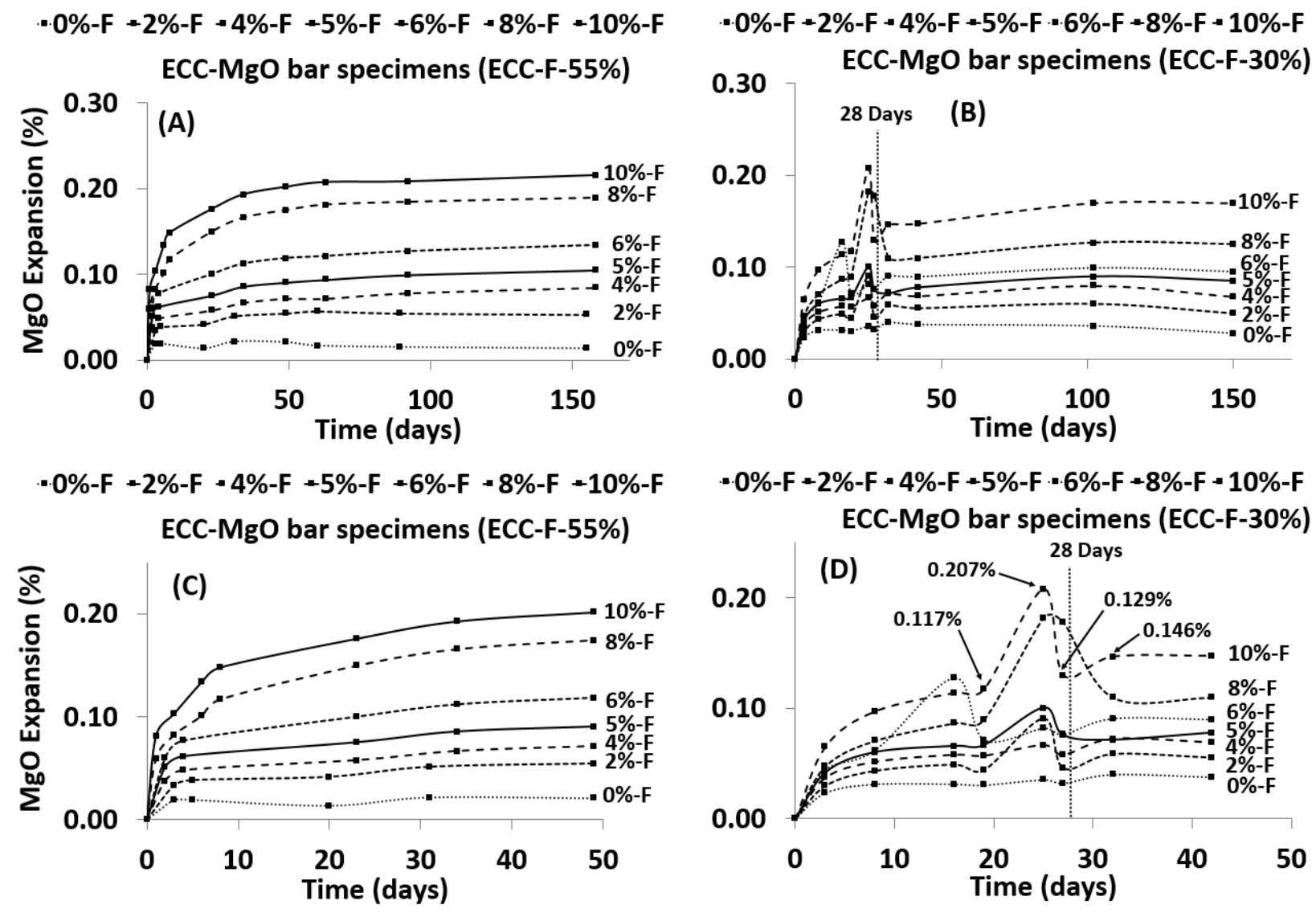

Figure 5-4: Expansion of ECC-MgO specimens made of different FA and $\mathrm{MgO}$ contents as a function of time (part A \& B: 150 days, and part C \& D: 50 days)

The strong counteracting forces that curbed and sharply reduced the expansion as shown in Figure 5-4 (part D) might be attributed to different reasons. Firstly, the presence of PVA fibers enhanced significantly the volume stability of ECC microstructure leading to lower expansions ( $\mathrm{Li}, 2003 ; \mathrm{Li}$ et al., 1995). Secondly, the losses in pre-compressive stresses generated by MEA might be curbing and relaxing the expansive stresses ( $\mathrm{Du}, 2005)$. The linear portion of ECC stress-strain curve is considered to be elastic as long as it does not reach the first cracking threshold to form any cracks (Carpinteri et al., 2013; Kanda and Li, 1998). No cracks were observed in all ECC-MgO bar 
specimens which means that the material will return to its original shape if loading was removed. Hence once the losses in pre-compressive stresses of MEA are in effect, the elastic behavior of ECC will trigger which lead the expanded bar specimen to return to its original state and eventually decreasing the rate of expansion significantly. Thirdly, the sharp rise in expansion curves generated sudden impact forces applied to ECC matrix. In general, enhanced damage tolerance and energy absorbing capacity significantly improve ECC's resistance against multiple impact loadings (Zhang et al., 2005). Therefore, ECC counteracted strongly the sudden rise in expansion curves leading to curbing and reducing the expansions.

At first glance, Figure 5-4 revealed the lowest expansions for 30\%-F_FA specimens which is the favored situation in ECC-MgO self-healing system. However, when considering the sudden rise of expansion curves, it can be seen that 55\%-F_FA had comparable expansions to those produced by 30\%-F_FA. The hydration process of ECC-MgO produced by 30\%-F_FA was not stable and developed unbalanced expansions especially within the period of first 28 days. Therefore, 55\%F_FA was considered conservatively as a better choice for ECC-MgO self-healing system due to balanced and smooth evolution of expansion curves.

\subsubsection{Comparison of water cured and autoclave ECC-MgO bar specimens}

Figure 5-5 analyzed the readings of water cured bar specimens with and without considering the sharp rise in expansion curves produced by 30\%-F_FA. In addition, both autoclave and water expansion results were drawn in one graph for comparison purposes. Figure 5-5 (part A) analyzed the last reading of expansion values for water cured specimens at the age of 160 days from Figure 5-4 (parts A and B). Again, it can be seen from Figure 5-5 (part A) that the counteracting forces formed in ECC-MgO microstructure produced by 30\%-F_FA-Water reduced significantly the expansion values and gave an impression that 30\%-F_FA-Water has the lowest expansions than those produced by 55\%-F_FA-Water. This finding was not similar to the same ECC-MgO bar specimens cured under autoclaved conditions (Figure 5-5 part A). On the other hand, Figure 5-5 (part B) analyzed the highest reading of sudden rise expansion values for water cured specimens within the period of 50 days taken from Figure 5-4 (parts $C$ and D). It can be seen from Figure 5-5 
(part B) that water cured ECC-MgO bar specimens produced by 30\%-F_FA and 55\%-F_FA had shown almost similar behavior to those cured under autoclaved conditions.

As concluded before, 55\%-F_FA was considered as a better choice for ECC-MgO self-healing system due to its expansion behavior under cured under both water and autoclaved conditions as well as due to its balanced and smooth evolution of expansion curves compared to 30\%-F_FA.

By referring to Figure 5-5, it is obvious that the expansions produced by accelerated autoclave test were higher than those obtained from water curing for Class-F fly ash with two different cement replacements (30\% and $55 \%)$.
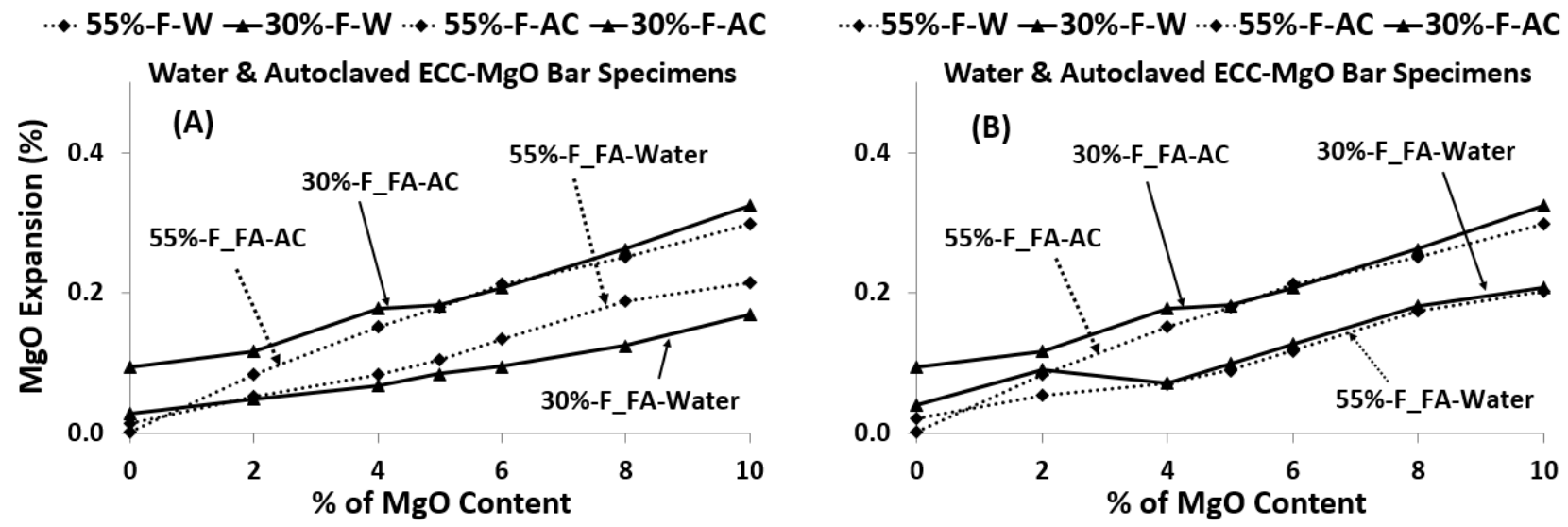

Figure 5-5: Comparison of water and autoclave ECC-MgO expansions as a function of $\mathrm{MgO}$ contents, (part A: last readings at 150 days, part B: highest readings within first 50 days)

\subsubsection{Effect of MgO content on ECC-MgO self-healing system}

The effectiveness of MEA in ECC-MgO self-healing system was assessed based on lower MgO expansion by both water and autoclave linear expansion tests. So far, research results have led to choose "Class-F fly ash with cement replacement of 55\%" as best option for ECC-MgO selfhealing system within SCMs studied. In the following paragraph, the $\mathrm{MgO}$ content which can meet the needs of ECC-MgO self-healing system is quantified and described.

Figure 5-6 shows expansion curves obtained from both autoclave and water linear expansion tests. It should be noted that both water expansion curves presented in Figure 5-6 were the same curves used in Figure 5-5 (part B) - these curves were repeated herein again for the aim of finding the 
content of MEA that can fit the requirements of ECC-MgO self-healing system. From Figure 5-6, it can be seen that both autoclave expansion curves produced by both 30\%-F_FA and 55\%-F_FA matched each other for $5 \%$ and $6 \%$ of $\mathrm{MgO}$ content. In addition, water expansion curves produced by both cement replacements (30\% and 55\% FA) almost matched each other with very small differences for $4 \%, 5 \%, 6 \%, 8 \%$ and $10 \%$ of $\mathrm{MgO}$ contents. The presence of $\mathrm{CaO}$ and $\mathrm{SiO}_{2}$ contents in ECC-MgO microstructure might be the reason for this behavior especially when linear expansion readings were collected at a fixed $\mathrm{MgO}$ content. According to Shehata (2001), when cement replacement of fly ash decreased from $55 \%$ to $30 \%$, higher $\mathrm{CaO}$ and lower $\mathrm{SiO}_{2}$ contents in ECC microstructure might lead to the formation of less counteracting forces that can suppress expansion stresses due to lower formation of C-S-H products. On the other hand, opposite situation existed when cement replacement increased up to 55\% - characterized by higher formation of C$\mathrm{S}-\mathrm{H}$ products and suppressing more expansion stresses. Eventually when both expansion curves of 30\%-F_FA and 55\%-F_FA matched each other in different $\mathrm{MgO}$ contents, equal forces might be presented when C-S-H products formed and then suppressed the expansion stresses formed by $\mathrm{Mg}(\mathrm{OH})_{2}$ crystals leading to balanced counteracting forces. Ali \& Mullick (1998) confirmed similar behavior when additional fly ash was added to cement paste containing fixed $6 \%$ of $\mathrm{MgO}$ content. When fly ash increased from $15 \%$ to $20 \%$ at $6 \% \mathrm{MgO}$ content, the difference in expansion was really small in autoclave test (only $0.030 \%$ ). Similar behavior was observed for water expansion which was $0.011 \%$ at same fly ash and $\mathrm{MgO}$ contents. In this research, the difference was relatively small in autoclave expansion which was $0.006 \%$ at $6 \%$ of $\mathrm{MgO}$ content when fly ash increased from $30 \%$ to $55 \%$; while it was $0.009 \%$ in water expansion at same fly ash and $\mathrm{MgO}$ contents.

According to (ASTM C150, 2016), the maximum content of $\mathrm{MgO}$ in clinker was limited to only $6.0 \%$ by cement weight. Also, Helmuth \& West (1998) emphasized that Portland cement containing less than $6 \%$ of $\mathrm{MgO}$ content with low free $\mathrm{CaO}$ content will not show any disruptive expansions even when mortar or cement paste samples cured in water for 10 to 15 years. Moreover, Zheng et al. (1991) concluded that the percentage of $\mathrm{MgO}$ content in cement is not a fixed value because it depends on many different factors, such as calcination temperature, fineness, dispersibility, cement type and application, and so on. However, according to Chinese State 
Standards on the Autoclave Test for Cement Soundness (GB 750-65), Zheng et al. (1991) found that cement is sound when $\mathrm{MgO}$ content in cement is less than 4.73\%. Referring to Figure 5-6, both water and autoclave expansion curves produced by both 30\%-F_FA and 55\%-F_FA matched and stabilized each other at $5 \%$ and $6 \%$ of $\mathrm{MgO}$ content (Zheng et al., 1991). Therefore, there was no point of adding more than these percentages to ECC-MgO self-healing system, especially when planned to use a low amount of $\mathrm{MgO}$ contents. The goal of this research was to design ECC-MgO mixture with very low expansions just to heal the micro cracks in ECC-MgO self-healing system. Therefore, authors preferred to use only $5 \%$ of $\mathrm{MgO}$ content to have less expansions (as much as possible) accompanied with high safety margins without affecting the durability of ECCs.

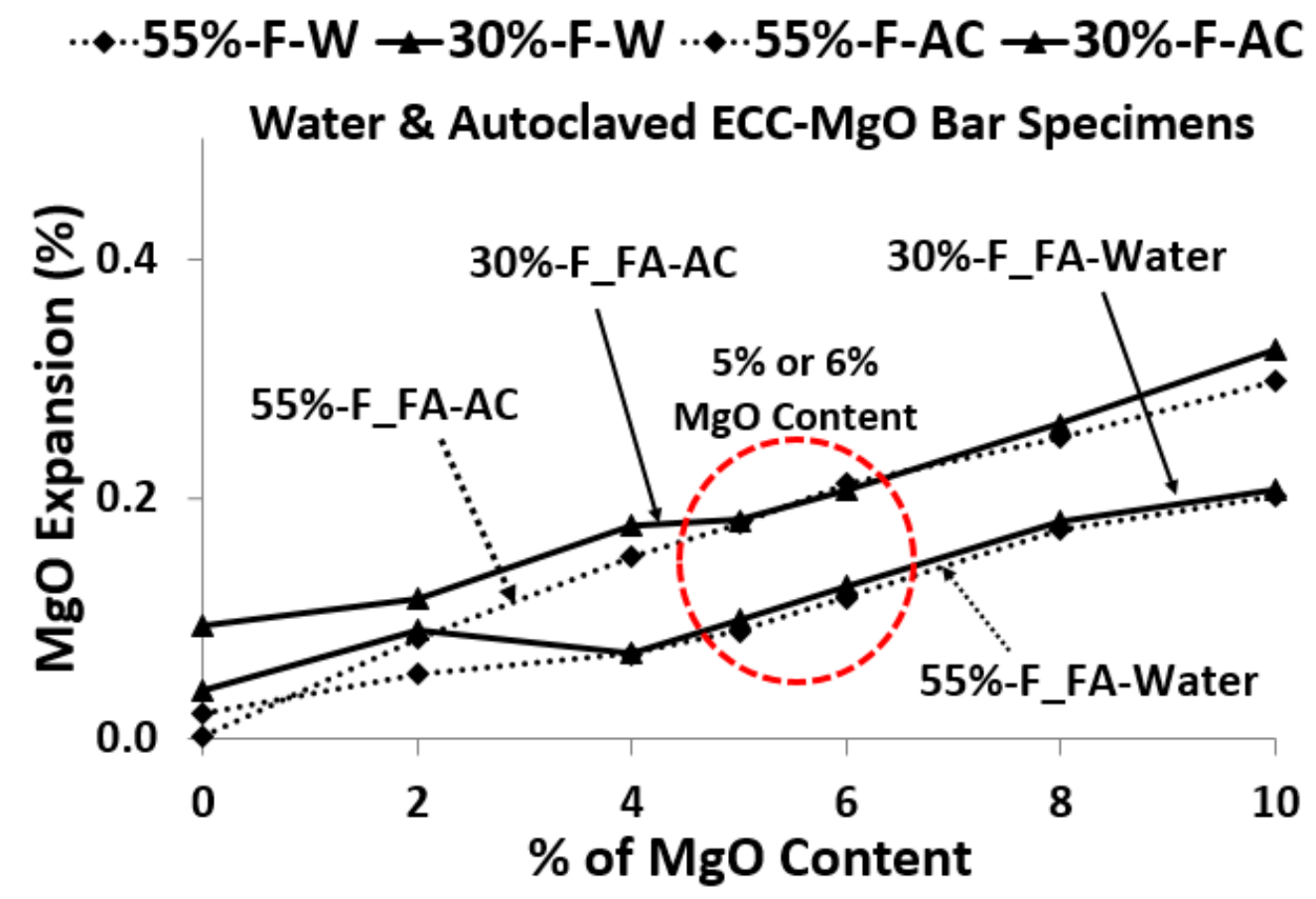

Figure 5-6: Best $\mathrm{MgO}$ content utilized for ECC-MgO self-healing system based on water and Autoclave ECC-MgO bar specimens

To study the effect of high content of MEA on ECC-MgO microstructure, Figure 5-7 presents the expansions of ECC-MgO bar specimens produced by using $\mathrm{MgO}$ contents up to $30 \%$. All bar specimens were cured in water at $20^{\circ} \mathrm{C}$ up to 150 days. Water cured bar specimens made of ClassF fly ash with 55\% cement replacement revealed lower expansion readings than those made of Class-F fly ash with $30 \%$ cement replacement. However, even when a high $\mathrm{MgO}$ content (up to 
$30 \%$ ) was utilized in the production of ECC-MgO bar specimens, still both types of cement replacements (30\% and 55\%) showed expansions less than $0.80 \%$ which is the limitation recommended by (ASTM C150, 2016) standard. This might be related to the presence of both HVFA characterized by low calcium content and the inherent use PVA fibers within ECCs. The use of HVFA (which limited the formation of $\mathrm{Mg}(\mathrm{OH})_{2}$ crystals in ECC microstructure) and the presence of PVA fibers (which enhanced significantly the volume stability) were believed to stimulate the capability of ECCs to produce significant reduction in expansive stresses even with high volumes of $\mathrm{MgO}$ content (Figure 5-7).

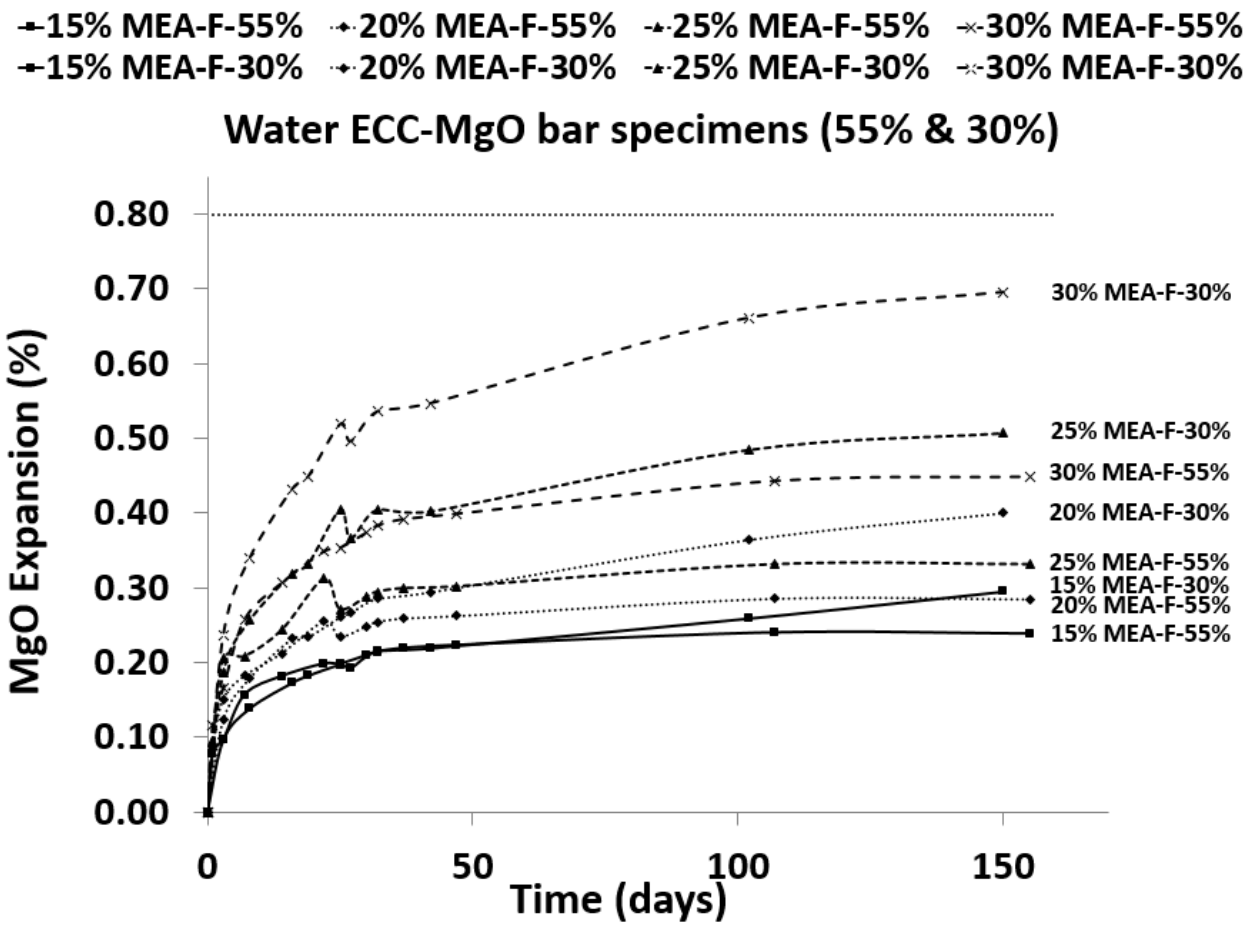

Figure 5-7: Effect of high volumes of $\mathrm{MgO}$ content on water cured $\mathrm{ECC}-\mathrm{MgO}$ bar specimens with different cement replacements as a function of time

\subsubsection{SEM analysis of ECC-MgO self-healing system}

Before studying the mechanism of the compressive strength development/recovery of ECC-MgO self-healing system, SEM analysis was essential to monitor the effect of $\mathrm{MgO}$ hydration on microstructures. SEM analysis was conducted on both ECC-control (without $\mathrm{MgO}$ ) and ECC$\mathrm{MgO}$ mixtures at the ages of 7, 14 and 28 days. The mixture of ECC-MgO was produced by using 
Class-F fly ash with 55\% cement replacement and 5\% of MEA content. The ECC-control mixture was produced with Class-F fly ash with $55 \%$ cement replacement without the addition of MEA. It should be noted that SEM samples were prepared from bar specimens cured in water at $20^{\circ} \mathrm{C}$. The samples were used directly without polishing in order not to affect the crack presence and to observe the disappearance of the surface roughness and cracks within ECC microstructure.

Figure 5-8 shows the densification effect in ECC-control matrix observed clearly at different age sequences. The sample was premature at the age of 7 days and still PVA fibers and micro cracks were shown clearly at the surface (Figure 5-8 A). At the age of 14 days (as shown in 12b), more hydration products started to accumulate leading to increasing in the maturity and enhanced the permeability of ECC matrix. Also, the presence of PVA fibers and surface cracks started to disappear gradually. Complete elimination for PVA fibers was observed at the age of 28 days with improved matrix densification as shown in Figure 5-8 C. However, surface cracks remained on the surface even at the age of 28 days as shown in the microstructure of Zone I (Figure 5-8 D). The chemical composition of Zone I was shown in Figure 5-8 E which confirmed that presence of only calcium and silicon ions with very low existence of magnesium ions in the ECC-control matrix.

Figure 5-9 shows the densification effect in ECC-MgO matrix at different age sequences. At the age of 7 days as shown in (Figure 5-9 A), PVA fibers and micro cracks were noticed. Later, at the age of 14 days (Figure 5-9 B), more condensed matrix was observed compared with ECC-control shown in (Figure 5-8 B). Although both PVA fibers and micro cracks can be observed clearly in ECC-MgO matrix at the age of 14 days, still both of them started to disappear faster than the matrix of ECC-control at a similar age. Contrary to ECC-control matrix, complete elimination for both PVA fibers and surface cracks with significant permeability enhancement were observed in the matrix of ECC-MgO at the age of 28 days (Figure 5-9 C). (Figure 5-9 D) confirmed the elimination of both PVA fibers and micro cracks at the surface of ECC-MgO microstructure. The crack elimination in ECC-MgO mixture was attributed to the expansion effect of MEA in combination with the existence of PVA fibers which both might enhance the volume stability of ECCs and reduced cracking significantly at later ages. Zone II of Figure 5-9 E shows a very condensed and cohesive cementitious material presence within the ECC-MgO matrix. The surface texture of this material is similar to a cement glue-like material which might help to combine ECC-MgO matrix 
together and reduce the surface cracking significantly. The chemical composition of Zone II as shown in Figure 5-9 F confirmed the existence of magnesium ions in ECC-MgO matrix even in higher proportion than the existence of both calcium and silicon ions.

In general, most research studies have confirmed that cracks can be healed partially when concrete samples cured under water, wet/dry and carbon dioxide curing conditions. However, Qian et al. (2010) mentioned that concrete cracks did not heal under air curing conditions and could not form new cracks in other locations due to weak bonding strength of already healed micro-cracks once loading reapplied. Also, even if concrete cracks healed completely, the main component of selfhealing products is Calcium Carbonate $\left(\mathrm{CaCO}_{3}\right)$ which is weaker than $\mathrm{C}-\mathrm{S}-\mathrm{H}$ products. Further, still, the strength of new C-S-H products (formed within the crack openings/ inside crack cavities due to hydration of un-hydrated cementitious) does not have same bonding strength as those presented within the microstructure (Qian et al., 2010). As shown in Figure 5-9 E, the ability of MEA to produce cohesive cementitious products at later ages might supplement the formation of $\mathrm{C}-\mathrm{S}-\mathrm{H}$ products at early ages and contribute to more densification of the microstructure. Also, low water demand of MEA to form cementitious products compared to other expansive additives might help ECCs to heal micro cracks even under air curing conditions. Moreover, not only $\mathrm{CaCO}_{3}$ or $\mathrm{C}-\mathrm{S}-\mathrm{H}$ products will form within the crack openings, magnesium products which have cementitious characteristics might also be formed as well and supplement the strength of already healed cracks and eventually form new cracks in other locations under subsequent loading. All these factors should provide inspiration/justification to employ MEA as a self-healing agent in the production of ECC-MgO self-healing system. 


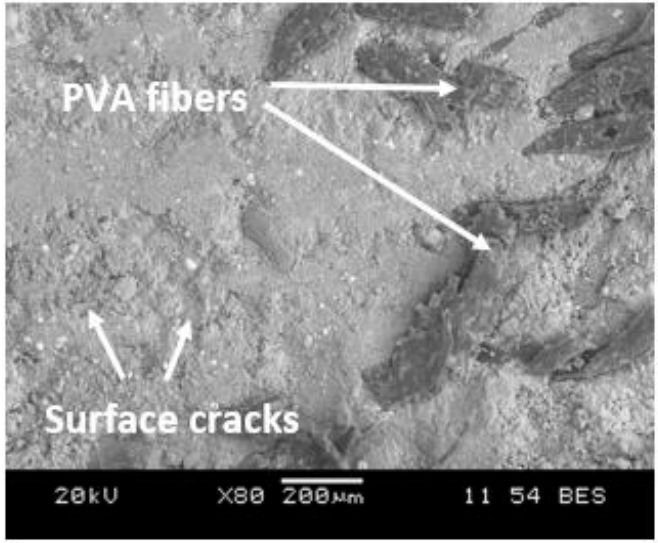

A) 7 days, ECC-control without MEA

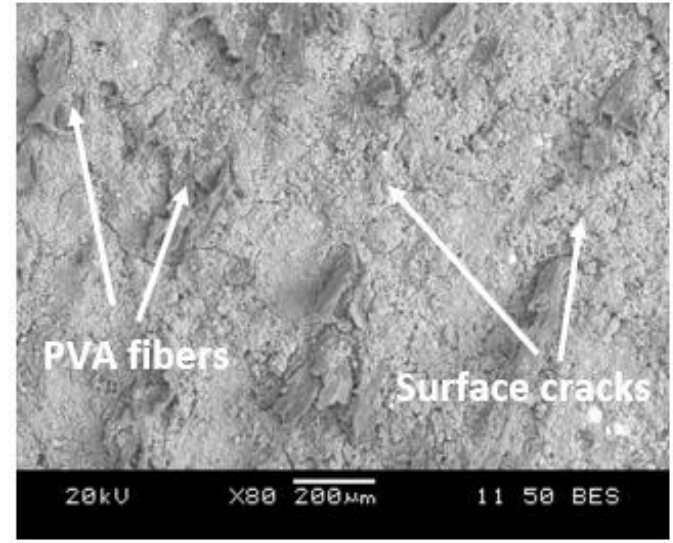

B) 14 days, ECC-control without MEA

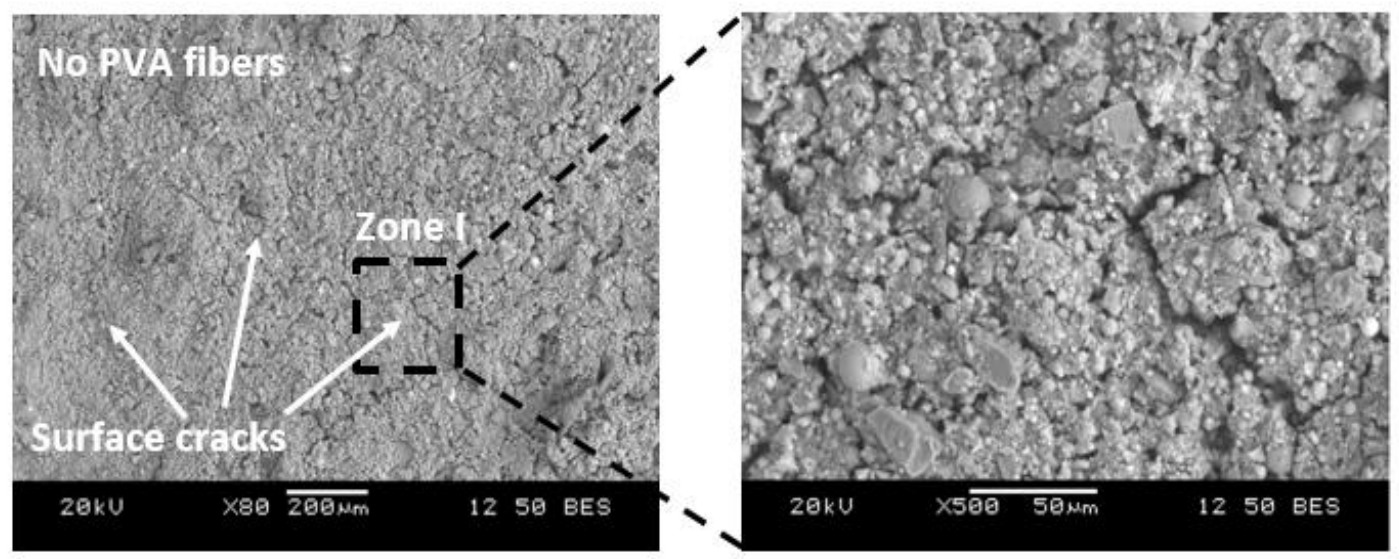

C) 28 days, ECC-control without MEA

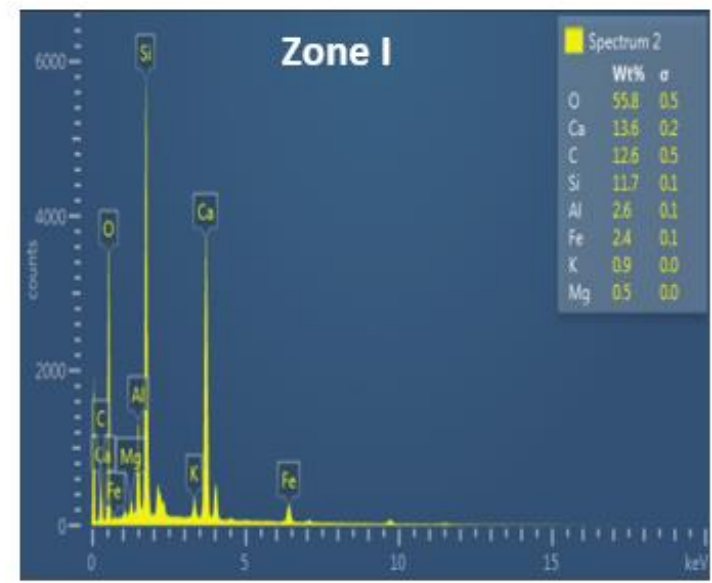

E) Chemical composition, Zone I

Figure 5-8: Microstructure of ECC-control without the addition of MEA at different ages (7, 14 and 28 days), cured in water at $20^{\circ} \mathrm{C}$ 


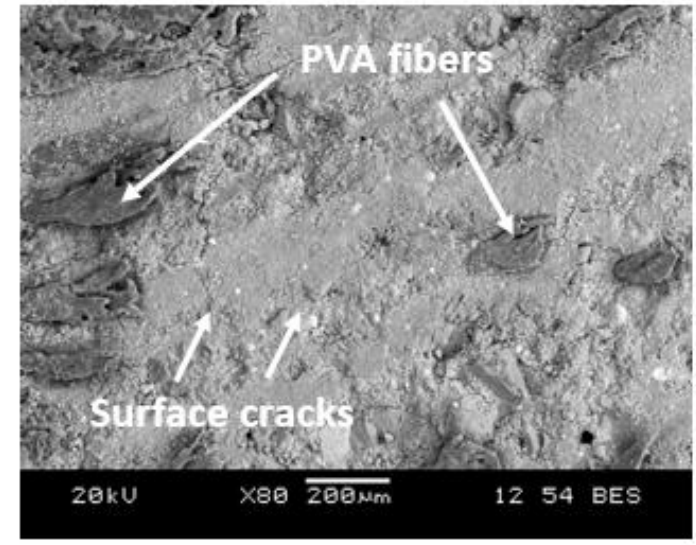

A) 7 days, ECC-MgO with MEA

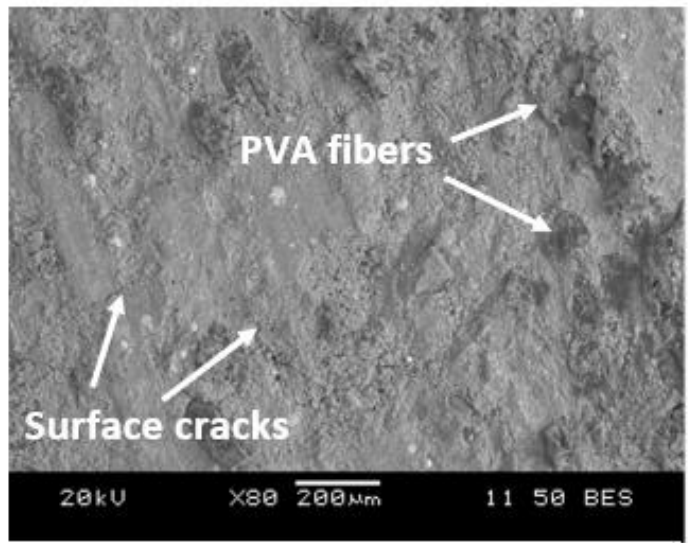

B) 14 days, ECC-MgO with MEA

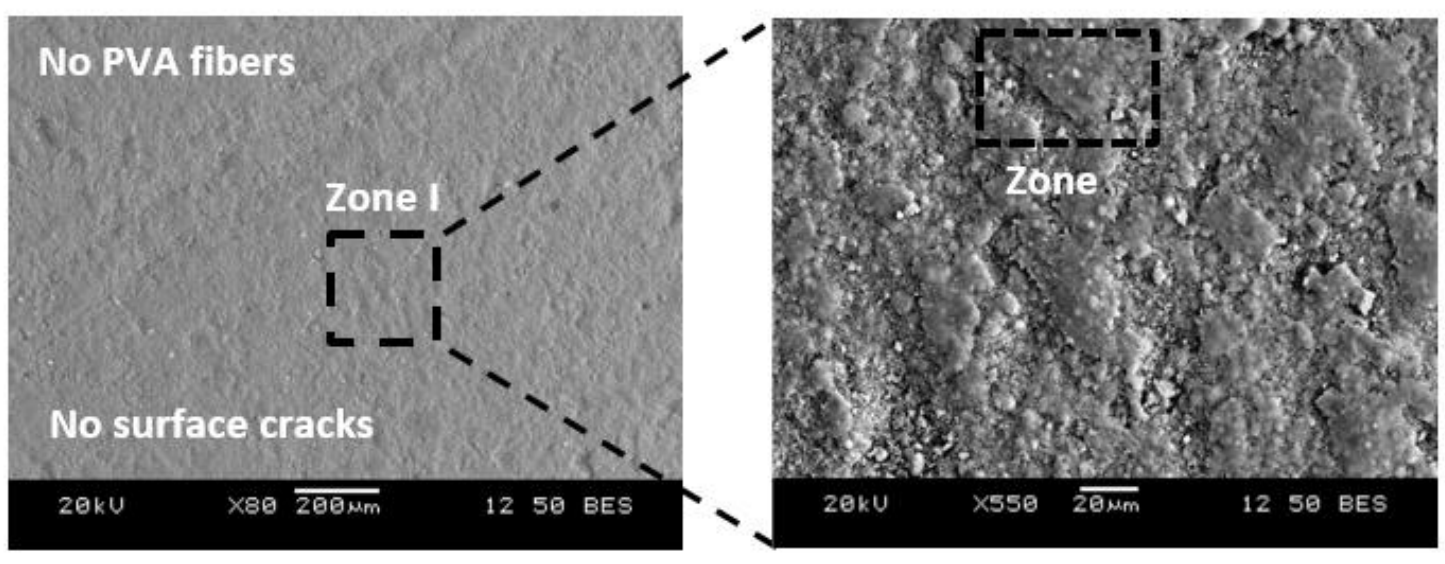

C) 28 days, ECC-MgO with MEA

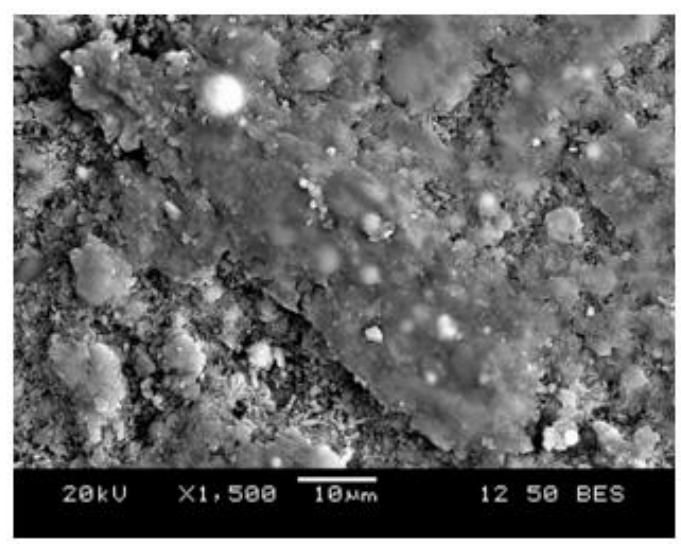

E) Microstructure, Zone II
D) Microstructure, Zone I

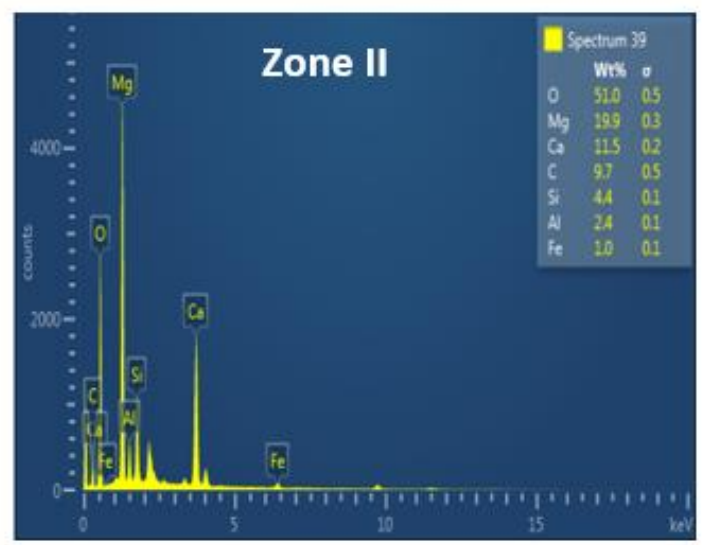

F) Chemical composition, Zone II

Figure 5-9: Microstructure of ECC-MgO matrix with the addition of MEA at different ages (7, 14 and 28 days), cured in water at $20^{\circ} \mathrm{C}$ 


\subsubsection{Compressive strength development/recovery of ECC-MgO self-healing system}

To study the self-healing capability of proposed ECC-MgO system (produced by 55\% cement replacement of Class-F fly ash with 5\% of MEA), the compressive strength development of ECC$\mathrm{MgO}$ mixtures was assessed by accelerating the hydration of $\mathrm{MgO}$ at the age of 28 days through autoclave curing.

Table 5-2: Compressive strength of pre-cracked ECCs with and without MEA after autoclave test at 28 days

\begin{tabular}{|c|c|c|c|c|c|c|}
\hline \multirow{3}{*}{$\begin{array}{c}\text { Mix } \\
\text { Designation }\end{array}$} & \multicolumn{6}{|c|}{ Compressive Strength (MPa) } \\
\hline & \multicolumn{2}{|c|}{$\begin{array}{c}\text { Water cured at } \\
20^{\circ} \mathrm{C} \text { w/o cracking } \\
1^{\text {st }} \text { and } 2^{\text {nd }} \\
\text { arrangement }\end{array}$} & \multirow{2}{*}{$\begin{array}{c}\text { AC cured - } \\
\text { w/o cracking* } \\
3^{\text {rd }} \\
\text { arrangement } \\
\text { AC at } 28 \mathrm{~d}\end{array}$} & \multirow{2}{*}{$\begin{array}{c}\% \text { increase of } \\
\text { Controls at } \\
\text { 28d strength } \\
\text { before \& after } \\
\text { AC }\end{array}$} & \multirow{2}{*}{$\begin{array}{c}50 \% \text { pre } \\
\text { cracked-AC } \\
\text { cured }^{*} \\
4^{\text {th }} \\
\text { arrangement } \\
\text { AC at } 28 \mathrm{~d}\end{array}$} & \multirow{2}{*}{$\begin{array}{c}80 \% \text { pre } \\
\text { cracked-AC } \\
\text { cured }^{*} \\
5^{\text {th }} \\
\text { arrangement } \\
\text { AC at } 28 \mathrm{~d}\end{array}$} \\
\hline & $7 d$ & $28 \mathrm{~d}$ & & & & \\
\hline ECC-control & 44 & 56 & 58 & $3 \%$ & 55 & 48 \\
\hline $\mathrm{ECC}-\mathrm{MgO}$ & 45 & 55 & 64 & $16 \%$ & 64 & 60 \\
\hline
\end{tabular}

* Results after conducting autoclave test, AC: autoclave test

Table 5-2 summarizes the compressive strength of ECC-control and ECC-MgO mixtures tested under five different arrangements as described previously. The compressive strength of water cured ECC-control and ECC-MGO mixtures were almost same even after adding MEA at the ages of 7 and 28 days (Table 5-2 and Figure 5-10). The compressive strengths of ECC-control were 44 $\mathrm{MPa}$ and $56 \mathrm{MPa}$ while $45 \mathrm{MPa}$ and $55 \mathrm{MPa}$ for ECC-MgO at the ages of 7 and 28 days, respectively. This might be related to low water to cement ratio accompanied with low permeability of ECCs (Lepech and Li, 2009a). Also, the age of 28 days might be relatively low due to slow reactivity of $\mathrm{MgO}$ at early ages to assess compressive strength development although $\mathrm{MgO}$ addition could influence the long-term durability of ECC matrix (Gao et al., 2008). However, after autoclaved curing at the age of 28 days, the compressive strength of ECC-MgO mixture was $6 \mathrm{MPa}$ higher than that of ECC-control. The compressive strength ECC-MgO mixture after autoclaved curing was increased by $16 \%$ compared to the same mixture when cured under water only while only $3 \%$ increase in compressive strength was observed for ECC-control after 
autoclaved curing compared to water curing (Table 5-2). This might be attributed to higher microstructure densification due to the use of MEA in the production of ECC-MgO mixture as confirmed by the SEM analyses (Figure 5-8 and Figure 5-9). Similar behavior was observed by Moradpour et al. (2013) who revealed that the compressive strength increased and permeability enhanced when nano-MgO particles were added to cement pastes.

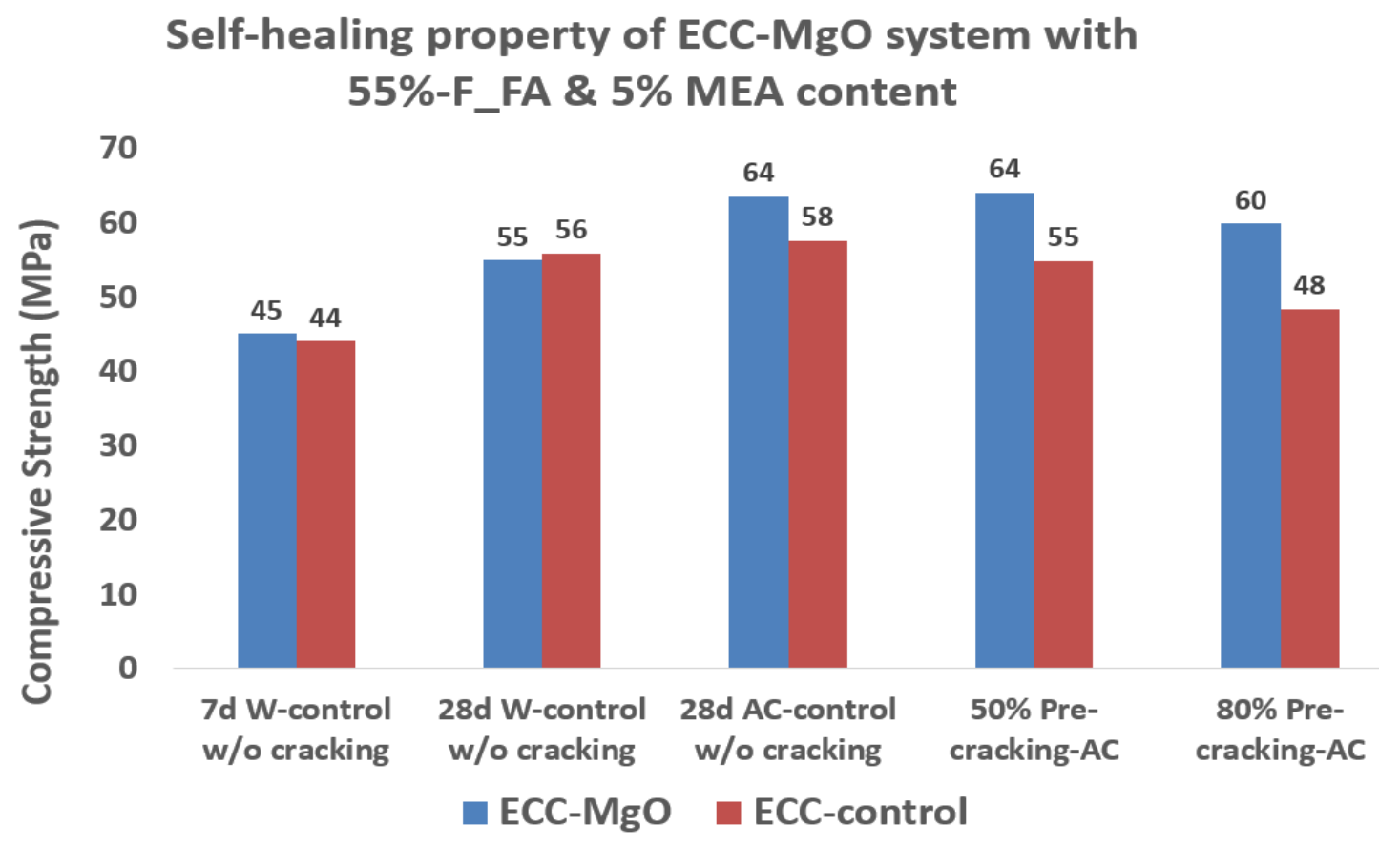

Figure 5-10: Compressive strength development and recovery ECC-MgO specimens before and after autoclave test at the age of 28 days

The compressive strengths of autoclaved cured 50\% and $80 \%$ pre-cracked ECC-control cubic specimens were decreased by $2 \%$ and $14 \%$, respectively compared to virgin specimens (without pre-cracking) cured under water. On the contrary, the compressive strengths autoclaved cured $50 \%$ and $80 \%$ pre-cracked ECC-MgO cubic specimens were increased by $16 \%$ and $9 \%$, respectively compared to virgin specimens cured under water. The development of higher compressive strength of both $50 \%$ and $80 \%$ pre-cracked specimens compared to their virgin counterparts revealed strength recovering capability of ECC-MgO system through healing micro cracks. It should be noted that there was no disintegration observed in all ECC specimens made of MEA even after conducting the autoclave testing conducted at elevated temperatures reached up to $216 \pm 2^{\circ} \mathrm{C}$. This 
confirmed that the use of 5\% MEA content in the production of ECC specimens was totally safe. This agrees with Mehta who concluded that the expansion limit of ASTM standards should be increased up to $10 \%$ and might be perfectly safe when MEA is used in concrete specimens instead of $0.80 \%$ when used in cement pastes (Mehta, 1978). This might be attributed to the difference in the test mechanisms of concrete and bar specimens. 


\subsection{Summary}

Discovering new cement based materials characterized by higher durability and longer service life is essential for sustainable infrastructure. Engineered Cementitious Composite (ECC) with high potential for micro-crack healing can enhance ductility and durability of concrete structures. $\mathrm{MgO}$ type expansive agent (MEA) having low water demand and with the ability of densification of concrete microstructure was utilized in this research to develop ECC-MgO self-healing system. The effect of dosages of MEA and fly ash of different types as cement replacements were investigated based on lower expansion characteristics of ECC-MgO bar specimens through both water and autoclave linear expansion tests. The performance of ECC-MgO self-healing system was examined based on compressive strength recovery of pre-cracked cubic specimens and matrix micro-structural densification through Scanning Electron Microscope (SEM). 


\section{CHAPTER SIX}

\section{MECHANICAL PROPERTIES OF SELF-HEALED CEMENTITIOUS COMPOSITES WITH MGO-TYPE EXPANSIVE AGENT}

\subsection{Introduction}

The recovery of durability through improved permeability and water/air tightness is very important to assess the quality of self-healing technique. However, many researchers have shown interest in the recovery of mechanical properties of self-healed concrete as well and considered it as an important tool in self-healing studies. According to Tittelboom and De Belie (2013) review, the mechanical properties of self-healed cracks were mostly inferior compared to the virgins (Tittelboom and De Belie, 2013). Even after filling the cracks with $\mathrm{CaCO}_{3}$ precipitation and/or newly formed C-S-H gel compared to primary hydration products, these fillers did not have a proper bond with the crack faces and cracks might present again at the same previous locations of damaged regions. This might be attributed to the fact revealed by Edvardsen (1999) who concluded that over $80 \%$ of $\mathrm{CaCO}_{3}$ precipitation formed through the cracks was done within the first 3 to 5 days only while the rest of $\mathrm{CaCO}_{3}$ crystal was formed over longer periods. Therefore, the need for later formation of a cementitious material which can provide a proper bond between the crack faces and supplement the early formation of $\mathrm{CaCO}_{3}$ crystals is important.

The current chapter investigates the effectiveness of proposed ECC-MgO self-healing system based on the recovery of mechanical properties (compressive strength and load resistance capacity/characteristic) and Ultrasonic Pulse Velocity (UPV) measurements. Both mechanical properties and UPVs were assessed based on the comparison between control ECC and ECC-MgO specimens through different methods: comparing the developments in control specimens, the 
development/recovery of re-loading events of pre-cracked/multiple cracked/failed specimens and the development/recovery of multiple events of damages (repeatability test). Additionally, the load resistance property was evaluated based on accelerated autoclave test and long-term development/recovery study including surface crack observation. All ECC and ECC-MgO specimens were cured under water and natural curing conditions for around 500 days. The aim of this chapter was to understand the performance characteristics of proposed ECC-MgO self-healing system when used in practical field construction applications. 


\subsection{Experimental Investigations}

Magnesium Oxide (MgO) was prepared locally by exposing Magnesite (Magnesium Carbonate, $\mathrm{MgCO}_{3}$ ) to $900^{\circ} \mathrm{C}$ for 2 hours inside the kiln " $900^{\circ} \mathrm{C} \_2$ hours of holding time_45 $\mu \mathrm{m}$ particle size of $\mathrm{MgCO}_{3}$ " which was found to be the best calcination system. In addition to the choice of ClassF fly ash as best supplementary cementing material (SCM) for aforementioned calcination system - the combination of SCM and MEA was chosen based on chapter 4. In this chapter, the performance of ECC-MgO self-healing system was tested based on the mechanical properties using cubic (50x50x50 mm) and prismatic (50x76x355 mm) specimens when MEA was employed as self-healing agent to heal micro cracks. The effectiveness of ECC-MgO self-healing technique was assessed based on studying the compressive strength and load/strength development/recovery of the control specimens, reloaded pre-cracked specimens, reloaded multiple damaged same specimens (repeatability of loading) over long period of time and eventually, autoclave cured

specimens in order to expedite the effect of $\mathrm{MgO}$ expansion behavior. Also, surface crack observation, deflection capacity development, ultrasonic pulse velocity and energy absorption were studied. The different evaluation methods were conducted on water and natural cured ECC$\mathrm{MgO}$ specimens and compared with counterpart ECC specimens cured under similar conditions.

\subsubsection{ECC-MgO Mixture Proportions}

To assess the effectiveness of MEA in ECC-MgO self-healing system, two ECC mixtures were designed and selected. The first mix was produced from Class-F fly ash and silica sand with cement replacement of FA/PC $=1.2(\mathrm{FA} / \mathrm{Binder}=55 \%)$ considered as ECC-controls. The second mix was same as the first one except that MEA was added to it.

The amount of $\mathrm{MgO}$ powder used in the production of ECC-MgO specimens was $5 \%$ measured with respect to binder weight $(\mathrm{PC}+\mathrm{FA})$. The mixture proportions and designations for both $\mathrm{ECC}$ mixtures are given Table 6-1. The water/binder (w/b) ratio was kept in the range of 0.27 for two ECC mixes and the amount of aggregate was kept constant. It should be noted that fly ash content was reduced when $\mathrm{MgO}$ content was added to the $\mathrm{ECC}-\mathrm{MgO}$ mixes. 
Table 6-1: Typical ECC mixture proportions with and without the addition of $\mathrm{MgO}$

\begin{tabular}{|c|c|c|c|c|c|c|c|c|}
\hline \multirow{2}{*}{ Mixture ID } & \multicolumn{7}{|c|}{ Ingredients per 1 part of binder measured by mass } \\
\cline { 2 - 9 } & $\begin{array}{c}\text { Water/ } \\
\text { Binder }\end{array}$ & $\begin{array}{c}\text { Binder } \\
\text { (PC+FA) }\end{array}$ & $\mathrm{MgO}$ & $\begin{array}{c}\text { FA/Binder after } \\
\text { adding MgO }\end{array}$ & $\begin{array}{c}\text { Silica } \\
\text { Sand }\end{array}$ & $\begin{array}{c}\text { PVA } \\
\mathrm{kg} / \mathrm{m}^{3}\end{array}$ & $\begin{array}{c}\text { HRWRA } \\
\mathrm{kg} / \mathrm{m}^{3}\end{array}$ & FA/PC \\
\hline ECC & 0.27 & 1 & - & $55 \%$ & 0.36 & 26 & 5.4 & 1.20 \\
\hline ECC-MgO & 0.27 & 1 & $5 \%$ & $50 \%$ & 0.36 & 26 & 5.4 & 1.09 \\
\hline
\end{tabular}

\subsubsection{Preparation of ECC-MgO specimens}

\subsubsection{Specimens for compressive strength}

As shown in Table 6-2, 3 sets consist of ECC and ECC-MgO specimens were produced from the two ECC mixtures shown in Table 6-1. The first set consists of ECC and ECC-MgO controls (specimens without cracking). At least 3 cubic specimens $(50 \times 50 \times 50 \mathrm{~mm})$ were produced for each age cured under water and natural curing conditions - total 54 cubes were prepared to explore the compressive strength performance of ECC and ECC-MgO mixtures (see Table 6-2 - set 1*).

The second set (Table 6-2 - set 2**) consisted of pre-cracked ECC and ECC-MgO specimens. All specimens were pre-loaded at the age of 28 days $(28 \mathrm{~d}+0)$. At least 3 cubic specimens were produced for each age $(28 d+1,28 d+3,28 d+5,28 d+7$ and $28 d+9)$ cured under water and natural curing conditions - total 126 cubes were prepared to explore the compressive strength development/recovery of ECC and ECC-MgO mixtures. The third set (Table 6-2 - set 3+) was the repeatability set which studied the compressive strength development/recovery on the same ECC and ECC-MgO specimens. The specimens were pre-cracked at the age of 28 days and then the reloading was repeated to the same pre-cracking level on the same specimens. At least 3 cubic specimens were prepared for each curing type and pre-cracking level and then reloaded every two months at the ages $(28 d+1,28 d+3,28 d+5,28 d+7$ and $28 d+9)$. At the age $28 d+11$ (360 days), specimens were loaded to failure and compared with the controls. The total number of specimens prepared for ECC and ECC-MgO mixtures especially made for repeatability test was 24 cubes. All compression tests were carried out on cubic specimens by using a compression testing machine with a capacity of 400,000 lbs. 
Table 6-2: Preparation of the compressive strength cubic specimens

\begin{tabular}{|c|c|c|c|c|c|c|c|c|c|}
\hline \multirow{3}{*}{ Set \# } & \multirow{3}{*}{$\begin{array}{c}\text { Mix } \\
\text { designation }\end{array}$} & \multirow{3}{*}{$\begin{array}{c}\text { Type of } \\
\text { specimens }\end{array}$} & \multicolumn{7}{|c|}{ Time of curing } \\
\hline & & & \multicolumn{7}{|c|}{$\begin{array}{c}\text { (controls failed at } 7 \mathrm{~d}, 14 \mathrm{~d} \& \mathrm{28d} \text { days of lab curing) also } \\
\text { (another set of controls failed at } 120 \mathrm{~d}, 240 \mathrm{~d} \& 360 \mathrm{~d} \text { of water/natural curing) }\end{array}$} \\
\hline & & & $7 \mathrm{~d}$ & $14 \mathrm{~d}$ & $28 \mathrm{~d}$ & $120 \mathrm{~d}$ & $240 \mathrm{~d}$ & - & $360 \mathrm{~d}$ \\
\hline \multirow{2}{*}{$1 *$} & $\mathrm{ECC}$ & \multirow{2}{*}{ Controls } & \multirow{2}{*}{\multicolumn{7}{|c|}{$\begin{array}{c}3 \text { cubes/each time and for each curing type: } \\
{[(9 \text { cubes-lab })+(18 \text { cubes-water } \& \text { natural })] \times 2 \text { mixes }=54 \text { cubes }}\end{array}$}} \\
\hline & ECC-MgO & & & & & & & & \\
\hline \multirow{3}{*}{ Set \# } & \multirow{3}{*}{$\begin{array}{c}\text { Mix } \\
\text { designation }\end{array}$} & \multirow{3}{*}{$\begin{array}{c}\text { Type of } \\
\text { specimens }\end{array}$} & $\begin{array}{l}\text { All cubes } \\
\text { preloaded at } 28 \\
\text { days }\end{array}$ & \multicolumn{6}{|c|}{$\begin{array}{l}28 \text { days of lab curing }+300 d \text { of water/natural curing } \\
\text { All cubes pre-cracked at } 28+0 \text {, then failed at } 28 d+1,28 d+3 \text {, } \\
\qquad 28 d+5,28 d+7 \text { and } 28 d+9\end{array}$} \\
\hline & & & $28+0$ & $28+1 \sim$ & $28+3 \sim$ & $28+5 \sim$ & $28+7 \sim$ & $28+9 \sim$ & - \\
\hline & & & $28 \mathrm{~d}$ & $56 \mathrm{~d}$ & $120 \mathrm{~d}$ & $180 \mathrm{~d}$ & $240 \mathrm{~d}$ & $300 \mathrm{~d}$ & - \\
\hline \multirow{4}{*}{$2 * *$} & $\mathrm{ECC}$ & \multirow{2}{*}{$\begin{array}{l}50 \% \text { Pre- } \\
\text { cracked }\end{array}$} & \multirow{4}{*}{\multicolumn{7}{|c|}{$\begin{array}{c}3 \text { cubes/each time, curing type \& pre-cracked level: } \\
{[(3 \text { cubes-lab })+(30 \text { cubes-water } \& \text { natural }) \times 2 \text { types of pre-cracked levels }} \\
(50 \% \& 80)] \times 2 \text { mixes }=126 \text { cubes }\end{array}$}} \\
\hline & ECC-MgO & & & & & & & & \\
\hline & $\mathrm{ECC}$ & \multirow{2}{*}{$\begin{array}{l}80 \% \text { Pre- } \\
\text { cracked }\end{array}$} & & & & & & & \\
\hline & ECC-MgO & & & & & & & & \\
\hline \multirow[t]{3}{*}{ Set \# } & $\begin{array}{l}\text { Mix } \\
\text { desionation }\end{array}$ & \multirow[t]{3}{*}{$\begin{array}{c}\text { Type of } \\
\text { specimens }\end{array}$} & $\begin{array}{l}\text { All cubes } \\
\text { preloaded at } \\
28 \text { days }\end{array}$ & \multicolumn{6}{|c|}{$\begin{array}{c}28 \text { days of lab curing }+360 d \text { of water/natural curing } \\
\text { (cubes pre-cracked at } 28 d+0 \text { then same cubes reloaded to } \\
\text { same level of loading at } 28 d+1 \text {, again at } 28 d+3,28 d+5,28 d+7 \text {, } \\
28 d+9 \text { and finally failed at } 28 d+11\end{array}$} \\
\hline & & & $28+0$ & $28+1 \sim$ & $28+3 \sim$ & $28+5 \sim$ & $28+7 \sim$ & $28+9 \sim$ & $28+11 \sim$ \\
\hline & & & $28 \mathrm{~d}$ & $56 \mathrm{~d}$ & $120 \mathrm{~d}$ & $180 \mathrm{~d}$ & $240 \mathrm{~d}$ & $300 \mathrm{~d}$ & $360 \mathrm{~d}$ \\
\hline \multirow{4}{*}{$3+$} & $\mathrm{ECC}$ & \multirow{2}{*}{$\begin{array}{c}50 \% \\
\text { Repeated }\end{array}$} & \multirow{4}{*}{\multicolumn{7}{|c|}{$\begin{array}{c}3 \text { cubes/each curing type } \& \text { pre-cracked level: } \\
3 \text { cubes x } 2 \text { types of curing } \times 2 \text { types of repeated/pre-cracked levels }(50 \% \\
\& 80) \times 2 \text { mixes }=24 \text { cubes }\end{array}$}} \\
\hline & ECC-MgO & & & & & & & & \\
\hline & $\mathrm{ECC}$ & \multirow{2}{*}{$\begin{array}{c}80 \% \\
\text { Repeated }\end{array}$} & & & & & & & \\
\hline & ECC-MgO & & & & & & & & \\
\hline
\end{tabular}

*First set; **Second set; +Third set; all sets consisted of ECC and ECC-MgO mixes, ECC-MgO: ECC mix produced by

adding $5 \%$ of $\mathrm{MgO}$ with respect to binder; $\sim$ number of months $(28+1$ month, 28+3 months ...etc.)

\subsubsection{Specimens for the study of flexural strength characteristics}

Table 6-3 shows 5 sets consisting of ECC and ECC-MgO specimens produced based on ECC mixtures shown in Table 6-1. The first set consisted of the controls (without cracking) of ECC and ECC-MgO prisms. At least 3 prismatic controls $(50 \times 76 \times 355 \mathrm{~mm})$ were produced for each mixture type and tested at the age of (7 days). Additionally, another 3 prismatic controls were produced for each water and natural cured ECC and ECC-MgO mixtures and tested at the ages $(28 \mathrm{~d}, 120 \mathrm{~d}, 270 \mathrm{~d}$ and 450 days) - total 54 prisms were prepared to explore the strength (load-displacement evolution) performance of ECC and ECC-MgO mixtures when cured for long periods. The second set (Table 
6-3 - set \# 2**) presented a comprehensive study for the self-healing property of ECC-MgO system through strength development/recovery when cured under water and natural curing conditions. All specimens were preloaded at the age of 7 days $(7 d+0)$ based on the maximum multiple cracked deflections of the controls. Then, same preloaded specimens were multiple cracked at different ages and loaded to failure after one month and compared with controls (specimens multiple cracked at $7 d+1,7 d+3,7 d+5,7 d+7$ and $7 d+9$ then same specimens failed after one month at $7 d+2$, $7 \mathrm{~d}+4,7 \mathrm{~d}+6,7 \mathrm{~d}+8$ and $7 \mathrm{~d}+10$ ) as shown in Table $6-3$ (set \# $2^{* *}$ ). The total number of prisms used in the second set was 60 prisms for both ECC and ECC-MgO specimens cured under both water and natural conditions. In third set (long term curing set), all specimens were pre-loaded to the same deflection level of the second set at the age of 7 days; then failure loading case was conducted on same specimens after 450 days $(7 d+15)$ - total 12 prisms were prepared to study the very longterm strength development/recovery of self-healed ECC and ECC-MgO specimens cured under water and natural curing conditions (Table 6-3 - set \# 3+). Application of multiple damages on prismatic specimens was done in the fourth set. Specimens were pre-cracked at the age of 7 days $(7 d+0)$ then reloading events were repeated every 3 months at the ages $(7 d+1,7 d+4,7 d+7$ and $7 \mathrm{~d}+10$ ) on same samples under same curing conditions as shown in Table 6-3 (set \# 4-). 
Table 6-3: Preparation of the load strength prismatic specimens

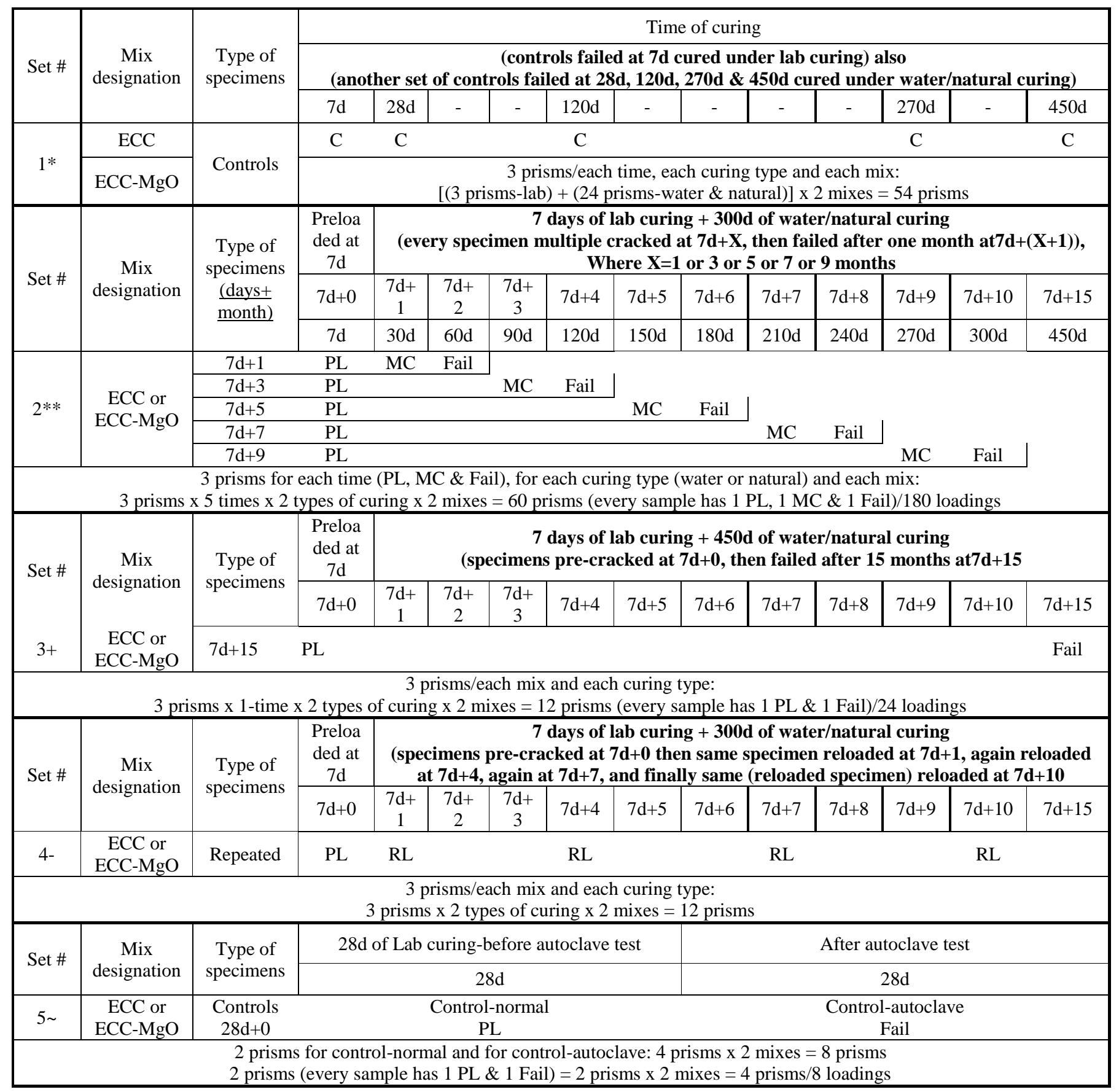

*First set; **Second set; +Third set; - Fourth set; Fifth set; all sets consist of ECC and ECC-MgO mixes, ECC-MgO: ECC

mix produced by adding $5 \%$ of $\mathrm{MgO}$ with respect to binder; $P L$ : pre-loading; $\mathrm{RL}$ : reloading; C: control; Fail: fail case loading;

MC: multiple cracking 
The total number of specimens used for this purpose was 12 prisms to study the strength development/recovery of self-healed ECC and ECC-MgO specimens when exposed to multiple damage events. Finally, in the fifth set, autoclave testing was used to expedite the expansive behavior of $\mathrm{MgO}$; and hence self-healing capability of proposed ECC-MgO system can be evaluated. Autoclave cured controls were tested and related to normally cured controls (inside plastic bags - without applying autoclave test) while pre-cracked specimens were tested before and after autoclave test at the age of 28 days. In total, 8 prisms $(50 \times 76 \times 285 \mathrm{~mm})$ were used for the controls and 4 prisms of pre-cracked specimens as shown in Table 6-3 (set \# 5 ).

\subsection{Test procedures}

\subsubsection{Compressive strength cube specimens preparation}

As shown in Table 6-2, the feasibility of proposed self-healing ECC-MgO system was evaluated through compressive strength development/recovery over a long period when cured under water and natural curing conditions. Then ECC-MgO specimens were compared with the counterpart ECC controls (without MEA). To do so, three arrangements were employed: (i) in the first arrangement, 3 cubic specimens for each age were tested for strength at the ages of 7, 14, 28, 120, 240 and 360 days. Under laboratory curing condition, all cubic specimens were cured inside plastic bags until the day of testing (7, 14 and 28 days only). After 28 days, two curing conditions were applied on specimens for a long period to study the long-term compressive strength performance of ECC and ECC-MgO specimens (up to 360 days). Accordingly, half of the specimens were kept continuously in water (stored in water continuously inside the lab) to conduct water curing condition while the other half of the specimens were taken out to the field site to be cured naturally (see Figure 6-1). The water and natural cured ECC-MgO specimens were tested at the ages of 120, 240 and 360 days and compared with ECC-controls. 


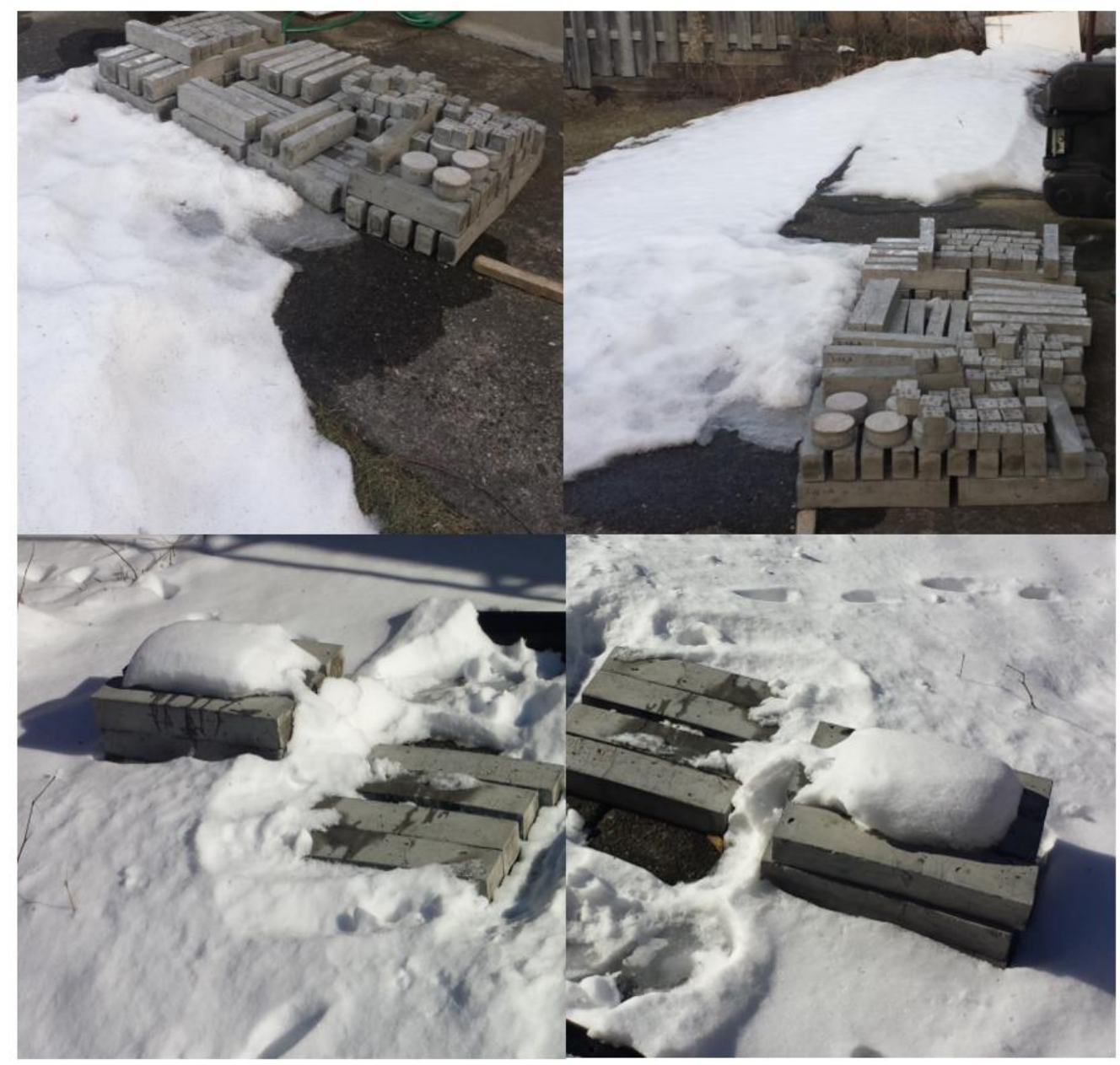

Figure 6-1: Natural curing condition

(ii) in the second arrangement, all specimens were pre-loaded to the level of $50 \%$ and $80 \%$ of the maximum compressive strength of the virgins at the age of 28 days $(28 d+0)$. The pre-loaded specimens were separated into five groups and prepared for reloading again to failure every 2 months over 9 months of reloading events. This means that every group of pre-cracked specimens will reload to failure at different ages $(28 d+1,28 d+3,28 d+5,28 d+7$ and $28 d+9)$; which allows to reveal clearly the self-healing performance of pre-cracked ECC-MgO specimens through compressive strength development/recovery and compared with ECC specimens. The arrangement of the second set also studied the self-healing capability of proposed ECC-MgO system under long water and natural curing conditions. (iii) in the third arrangement, the specimens were pre-cracked to $50 \%$ and $80 \%$ at the age of 28 days and then the re-loading events were repeated to the same 
pre-cracking levels (50\% and 80\%) every two months applied on the same specimens. The reloading was repeated five times up to 300 days and the specimens were loaded to failure at the age of 360 days. At 360 days, the compressive strength recovery of repeated re-loadings of ECC and ECC-MgO specimens (multiple damaged) were compared with ECC and ECC-MgO controls (without cracking) at the same age (360 days) cured under same curing condition (water or natural curing).

\subsubsection{Flexural strength (simulating load-displacement) evolution/recovery and UPV}

The feasibility of proposed self-healing ECC-MgO system was conducted through studying the flexural strength development/recovery of simply supported prism specimens under four-point static monotonic loading (as shown in Figure 6-2) over long period compared with their counterpart ECC controls (without MEA) when cured under water and natural curing conditions (Table 6-3). Accordingly, five arrangements were employed as follows.

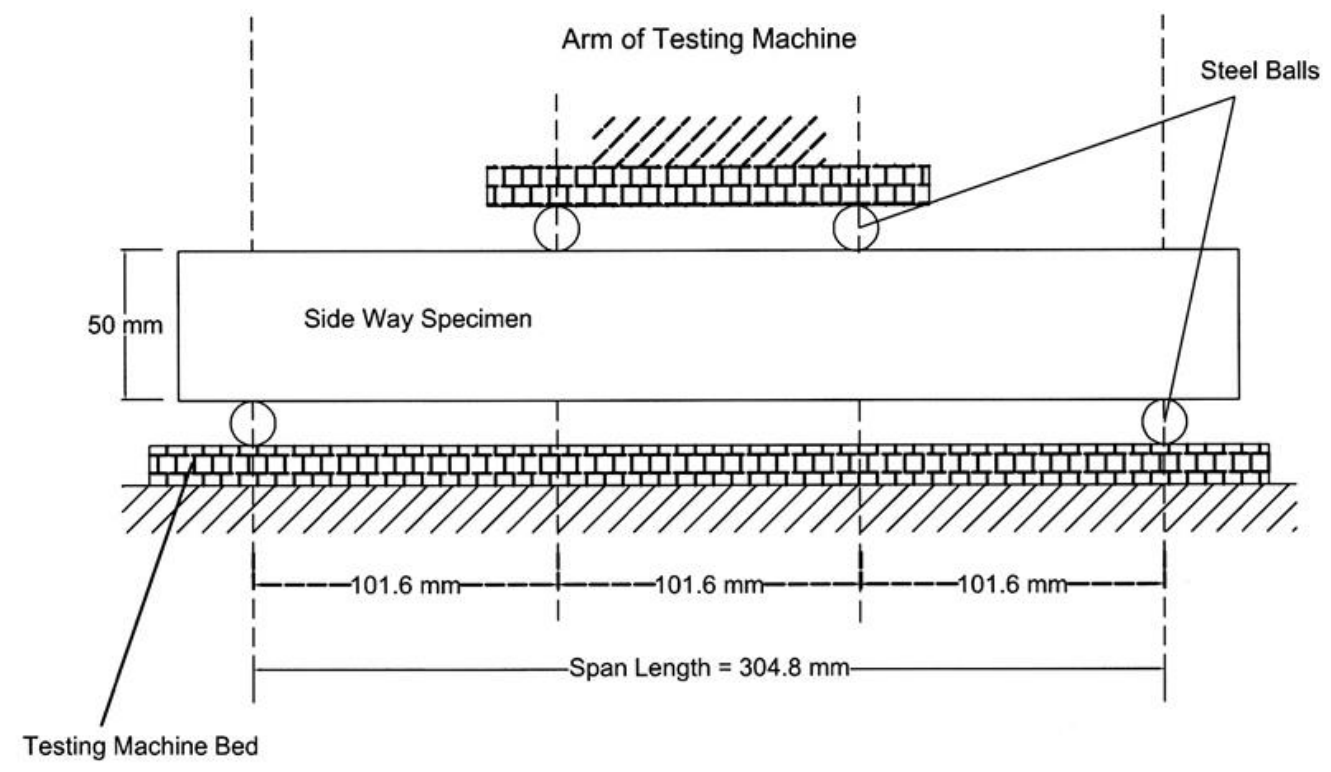

Figure 6-2: Four-point bending test setup for sideway specimens (Sherir, 2012)

In the first arrangement, 3 prismatic specimens for each age (considered as controls) were tested for strength at the ages of 7, 28, 120, 270 and 450 days. All prismatic specimens cured inside 
plastic bags under laboratory condition until the day of testing (at 7 days). At 7 days, only 3 prisms were tested to failure considered as controls cured under lab condition. The rest of prisms were moved out of the plastic bags to new curing conditions. As in the case of compressive strength, some specimens were cured continuously under water curing condition (stored in water inside the lab) while others were taken outside to the field site to be cured naturally. Both water and natural cured ECC and ECC-MgO specimens were cured for extended period of time to study the longterm development of strength of ECC and ECC-MgO specimens (cured up to 450 days) (see Figure 6-1). Eventually, water and natural cured ECC-MgO specimens were tested at the ages of 28,120 , 270 and 450 days and compared with counterpart ECC-controls (see Table 6-3 - set $1^{*}$ ). In addition to flexural strength development, UPV was measured at all different ages to assess the quality of water and natural cured ECC and ECC-MgO controls based on the presence of voids and internal cracks.

In the second arrangement shown in Table 6-3 - set $2^{* *}$, three stages of pre-loading, multiple cracking and failing were applied on every sample. Initially, all prismatic specimens were preloaded (pre-cracked) to the limit of $60 \%$ of the maximum multiple cracked deflections of the virgins acquired at the age of 7 days. The 7 days' pre-loading was utilized to maximizing the damages on specimens by generating micro cracks as much as possible. Then, every set of prismatic specimens were reloaded two times after the initial pre-loading stage. The first reloading was conducted to apply multiple cracks (MC) which manually stopped once the descending loaddeflection curve passed the first cracking (considered as MC stage) to ensure a maximum use of multiple cracking. The second reloading was repeated on the same multiple cracked specimens to fail them after one month of MC stage (considered as failure - Fail stage). Five groups of specimens were used in this method. The first group was multiple cracked after one month of preloading $(7 \mathrm{~d}+1$ - means 7 days followed by one month) then reloaded again to failure stage after another one month $(7 \mathrm{~d}+2)$. Thereafter, the rest of the specimens' groups were multiple cracked at $(7 d+3),(7 d+5),(7 d+7)$ and $(7 d+9)$ then failed at $(7 d+4),(7 d+6),(7 d+8)$ and $(7 d+10)$, respectively. It should be noted that preloaded specimens were kept cured in water or naturally (at the field site) until the day of multiple cracking testing, then multiple cracked specimens were returned back to their allocated curing condition until the day of loading to failure event. The aim of this technique 
was to exhaust the prismatic specimens as much as possible to measure the load strength development/recovery of ECC-MgO specimens and compared them with ECC specimens. Therefore, a complete understanding of self-healing capability/performance of ECC-MgO system (when cured under water and natural curing conditions) can be realized. As in the case of the first arrangement, UPVs of water and natural cured MC/Fail ECC and ECC-MgO specimens were measured and compared with the controls.

The third arrangement studied the long-term behavior of ECC-MgO system over 15 months of curing. As in the case of the second arrangement, all specimens were preloaded to $60 \%$ of multiple cracked deflection of the virgins at 7 days $(7 d+0)$; then cured under water and natural curing conditions and finally reloaded up to failure after 15 months of curing ( $7 d+15$ months). In this arrangement, a complete study was applied on this set by measuring the load/strength development/recovery, deflection development rate, UPVs development and energy absorption which was measured by the area under the load-deflection curve up to the maximum load point only. In addition, a study of crack characteristics through studying surface crack observation, crack widths and number of cracks before and after failure loading case was conducted as well on all ECC and ECC-MgO specimens. Forming new crack locations after applying failure loading case when specimens cured for long periods was considered as an evidence of the feasibility of selfhealing ECC-MgO system (see Table 6-3 - set 3+).

In the fourth arrangement, multiple damages were applied on same prismatic specimens precracked at the age of 7 days $(7 d+0)$ to the loading level of $60 \%$ of multiple cracked deflections of the virgins. The re-loading events to the same re-loading level (60\%) started at 28 days $(7 d+1$ month) and then repeated every 3 months up to 10 months $(7 d+1,7 d+4,7 d+7$ and $7 d+10)$. Although the goal was to reach similar $60 \%$ re-loading level each repeated loading, most specimens started to show load and deflection degradations after couple of re-loadings. In this case, the re-loading was stopped once the descending part of the load-deflection curve passed the first cracking. It should be noted that all events of multiple damages were repeated on the same ECC or ECC-MgO specimens whether cured under water or natural curing condition. 
In the fifth arrangement, the autoclave test was conducted to evaluate the load/strength development of autoclave cured ECC and ECC-MgO prismatic controls and compared with counterpart controls cured under lab curing at the age of 28 days. It should be noted that lab curing means storing the prisms inside sealed plastic bags until the day of testing (28-days). In addition, the development/recovery of pre-loaded prismatic specimens was tested before and after autoclave test as well (see Table 6-3 - set 5 ).

It is well known that only bar/prism specimens can be used in autoclave test based on (ASTM C151, 2015). For this reason, special autoclave rack was made for prismatic specimens to fit the autoclave vessel (see Figure 3-5). It should be emphasized that during autoclave test, care should be taken to ensure safety due to the emission of sharp fumes which could be toxic due to the presence of PVA fibers and chemical admixtures in ECC-MgO specimens when exposed to very high pressure and temperature. Consequently, proper ventilation system such as fume hood and complete respiratory protection with ambient air pump must be used.

\subsection{Results and Discussions}

\subsection{Compressive strength development and recovery}

Table 6-4 \& Figure 6-3 present the compressive strength results of standard ECC mixtures (ECCcontrol) and ECCs using MEA (ECC-MgO-control). The performance of ECC and ECC-MgO controls (virgin specimens without cracking/damage) as a function of time was explored under water and natural curing conditions (based on Table 6-2 - set $1 *$ ).

From Table 6-4 \& Figure 6-3, the compressive strength of both ECC and ECC-MgO controls were almost similar at 7 and 14 days without showing any effect of the addition of MEA at such ages. The compressive strength of ECC-control was $44 \mathrm{MPa}$ and $54 \mathrm{MPa}$ while it was $45 \mathrm{MPa}$ and $53 \mathrm{MPa}$ for ECC-MgO-control at the ages of 7 and 14 days, respectively. Due to the slow activity of $\mathrm{MgO}$ expansive agent, the assessment of the compressive strength development for both controls at early ages (such as 7 and 14 days) was relatively difficult for cubic specimens. At the age of 28 days, ECC-controls began to produce slightly higher compressive strength than ECC$\mathrm{MgO}-$ controls despite both mixtures cured under laboratory condition. The compressive strength 
of ECC-controls was $60.40 \mathrm{MPa}$ while it was 55.33 $\mathrm{MPa}$ for ECC-MgO-controls showing an increase of $9.20 \%$ at 28 days. After 28 days and when the compressive strength evaluated under water and natural curing conditions, ECC-controls developed higher compressive strength than ECC-MgO-controls in a steady increase rate whether cured under water or natural conditions. The steady increase rate was ranging between $4 \%$ and $7 \%$ up to the age of 240 days. The slower strength development of ECC-MgO-controls between 28 days and 240 days might be attributed to the reduction of the amount of cementitious materials when replaced by $\mathrm{MgO}$ expansive agent featured with a slow rate of hydration at such ages.

Table 6-4: Compressive strength of ECC and ECC-MgO controls

\begin{tabular}{|c|c|c|c|c|c|c|}
\hline \multirow{2}{*}{$\begin{array}{l}\text { Time } \\
\text { (days) }\end{array}$} & \multicolumn{2}{|c|}{ ECC-control (MPa) } & \multirow{2}{*}{\multicolumn{2}{|c|}{$\begin{array}{l}\text { ECC-MgO-control (MPa) } \\
\text { ry Curing }\end{array}$}} & \multirow{2}{*}{\multicolumn{2}{|c|}{$\begin{array}{c}\% \text { of ECCs strength } \\
\text { development* }\end{array}$}} \\
\hline & \multicolumn{2}{|c|}{ Laboratory Curing } & & & & \\
\hline 7 & \multicolumn{2}{|c|}{44.00} & \multicolumn{2}{|c|}{45.00} & \multicolumn{2}{|c|}{$-2.22 \%$} \\
\hline 14 & \multicolumn{2}{|c|}{54.00} & \multicolumn{2}{|c|}{53.00} & \multicolumn{2}{|c|}{$+1.9 \%$} \\
\hline 28 & \multicolumn{2}{|c|}{60.40} & \multicolumn{2}{|c|}{55.33} & \multicolumn{2}{|c|}{$+9.20 \%$} \\
\hline \multirow{2}{*}{$\begin{array}{l}\text { Time } \\
\text { (days) }\end{array}$} & \multicolumn{4}{|c|}{ Curing Type } & \multirow{2}{*}{$* *$} & \multirow{2}{*}{$* * *$} \\
\hline & Water & Natural & Water & Natural & & \\
\hline 120 & 71.00 & 68.50 & 66.90 & 64.00 & $+6.13 \%$ & $+7.03 \%$ \\
\hline 240 & 80.00 & 74.50 & 74.70 & 71.50 & $+7.10 \%$ & $+4.20 \%$ \\
\hline 360 & 77.00 & 78.50 & 77.50 & 79.50 & $-0.65 \%$ & $-1.27 \%$ \\
\hline
\end{tabular}

*\% of ECC-control to ECC-MgO-control when cured under laboratory condition; ** under water curing condition; *** under natural curing condition

It was noted by Gao et al. (2008) that only $57 \%$ of $\mathrm{MgO}$ formed chemically stable $\mathrm{Mg}(\mathrm{OH})_{2}(\mathrm{main}$ part in $\mathrm{MgO}$ concrete) within 180 days which indicated that $\mathrm{MgO}$ hydration was very slow at early ages. However, the hydration rate of ECC-MgO-controls whether cured under water or natural environmental conditions, started to increase more than ECC-controls after the age of 300 days. 


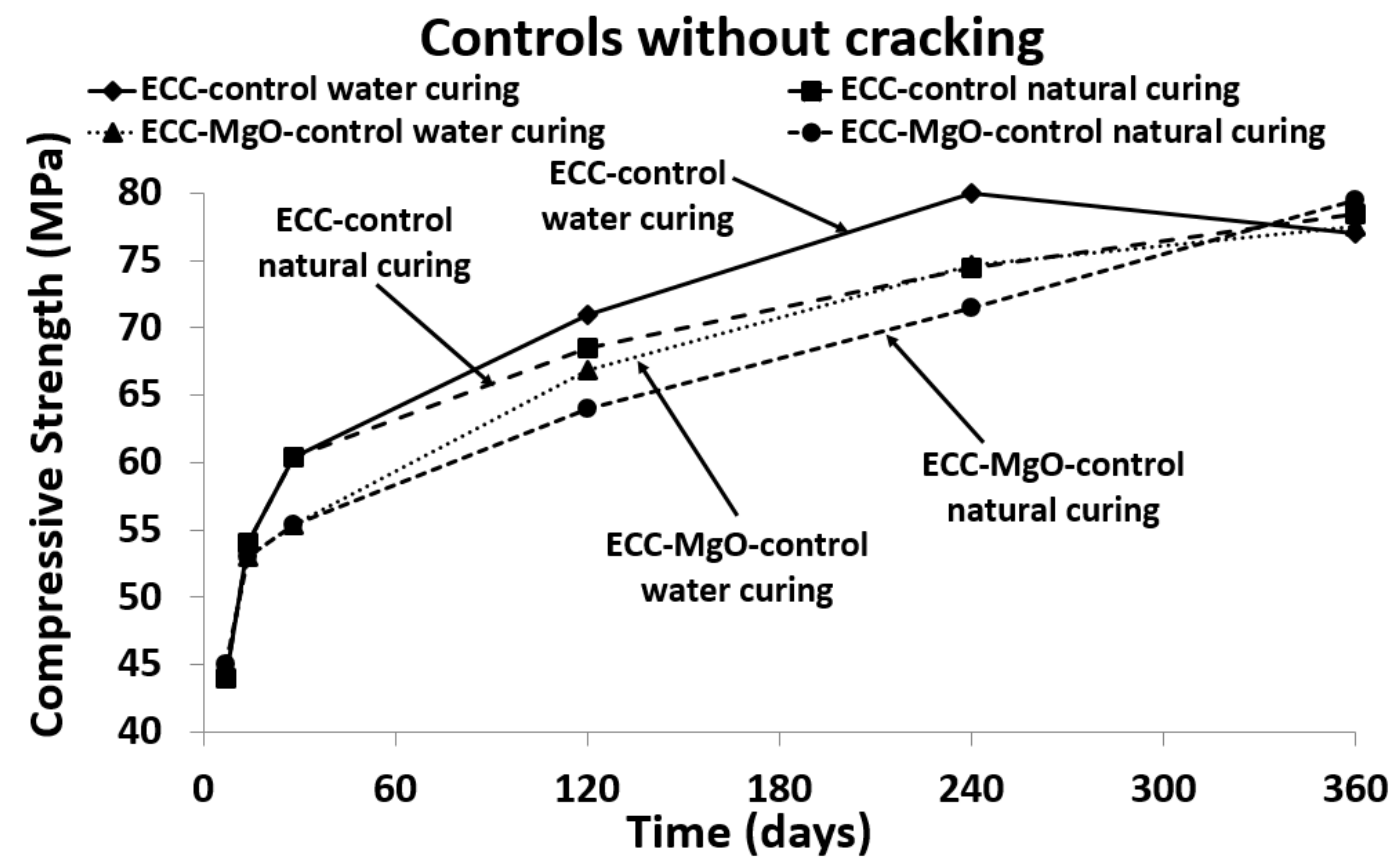

Figure 6-3: Compressive strength of ECC and ECC-MgO controls as a function of time and curing condition.

Furthermore, the compressive strength of ECC and ECC-MgO controls (non-cracked specimens) cured under water condition was always higher than those cured under natural environmental condition (Figure 6-3). The continuous presence of cubic specimens in water stimulated the curing process which may increase the rate of hydration and developed more strength. But after 300 days, it was observed that the specimens cured under the field (natural) conditions started to develop more compressive strength than those cured under water conditions. As long as there was enough moisture along with enough space for un-hydrated cementitious particles to resuming concrete hydration (Mindess et al., 2003), the field (natural) curing condition provided prolonged curing process and was able to develop higher compressive strength than water curing condition 


\subsubsection{Compressive strength recovery of self-healed ECC \& ECC-MgO specimens}

The self-healing property of ECC-MgO system was assessed through studying the long-term development of compressive strength characteristic of pre-loaded (cracked/damaged) ECC and ECC-MgO cubic specimens. All specimens herein were arranged based on Table 6-2 - set 2**.

For ECC mixtures under water curing condition (Table 6-5 and Figure 6-4), reloaded 50\% and $80 \%$ pre-cracked ECC cubic specimens did not recover the strength of ECC-controls. After 240 days, both $50 \%$ and $80 \%$ pre-cracked ECCs started to recover and catch up the compressive strength of the ECC-controls. 50\% and 80\% pre-cracked field (natural) cured ECC cubic specimens recovered the compressive strength of natural cured ones right after 60 days. Referring

to Table 6-5, a comparison between ECC and ECC-MgO based on curing and loading level conditions was done at 300 days. Under the same curing condition, the recovery of water and natural cured $80 \%$ pre-cracked ECC cubic specimens was $6.6 \%(88.5 \mathrm{MPa} / 83.0 \mathrm{MPa})$ and $4.9 \%$ (85.0MPa/81.0MPa) more compressive strength than similar $50 \%$ pre-cracked ECC cubic specimens at the age of 300 days. Under same loading level at 300 days, the recovery of 50\% and $80 \%$ water cured pre-cracked ECC cubic specimens developed $2.4 \%$ and $4.0 \%$ more compressive strength of similar natural cured specimens.

According to Table 6-5 and Figure 6-4, the 50\% and $80 \%$ pre-cracked ECC-MgO specimens whether cured under water or natural environmental curing conditions exhibited almost similar compressive strength development behavior compared to their ECC counterparts. More specifically, the similarity between them was observed when $50 \%$ and $80 \%$ water cured precracked ECC-MgO cubic specimens recovered the strength of controls after 240 days while field (natural) cured pre-cracked ECC-MgO specimens at both levels started to recover the strength right after 60 days. Also, under same curing condition, the recovery of water and natural cured $80 \%$ pre-cracked ECC-MgO cubic specimens developed $3.7 \%$ and $8.5 \%$ more compressive than similar 50\% pre-cracked specimens at the age of 300 days. Different to pre-cracked ECC specimens, under same loading level - at 300 days, the recovery of 50\% natural cured pre-cracked ECC-MgO cubic specimens was same to the compressive strength of similar water cured 
specimens while the recovery of $80 \%$ natural cured pre-cracked ECC-MgO cubic specimens developed $4.7 \%$ more compressive strength of similar water cured specimens.

Table 6-5: Compressive strength of pre-cracked ECC mixtures

\begin{tabular}{|c|c|c|c|c|c|}
\hline \multirow[b]{2}{*}{ Mix } & \multirow{2}{*}{$\begin{array}{c}\text { Time days }+ \text { months } \\
\text { (days) }\end{array}$} & \multicolumn{2}{|c|}{ Water Curing $(\mathrm{MPa})$} & \multicolumn{2}{|c|}{ Natural Curing (MPa) } \\
\hline & & $\begin{array}{c}\text { Reloading } \\
(50 \%)\end{array}$ & $\begin{array}{c}\text { Reloading } \\
(80 \%)\end{array}$ & $\begin{array}{c}\text { Reloading } \\
(50 \%)\end{array}$ & $\begin{array}{c}\text { Reloading } \\
(80 \%)\end{array}$ \\
\hline \multirow{9}{*}{$\begin{array}{l}\text { Standard } \\
\text { ECC } \\
\text { mixtures }\end{array}$} & $28+0 *$ (28 days $)$ & $\underline{30.20}$ & 48.30 & 30.20 & 48.30 \\
\hline & $28+1 * *$ (56 days) & 53.00 & 57.40 & 71.00 & 68.00 \\
\hline & $28+3 * *(120$ days $)$ & 66.00 & 60.00 & 72.00 & 68.00 \\
\hline & $28+5 * *$ (180 days) & 62.00 & 62.00 & 69.00 & 79.00 \\
\hline & $28+7 * *$ (240 days) & 74.00 & 67.00 & 76.00 & 84.00 \\
\hline & $28+9 * *$ (300 days) & 83.00 & 88.50 & 81.00 & 85.00 \\
\hline & Recovery of & \multicolumn{2}{|c|}{ Same loading level } & \multicolumn{2}{|c|}{ Same curing condition } \\
\hline & $\begin{array}{c}\text { compressive } \\
\text { strength at } 28+9 * *\end{array}$ & $\begin{array}{l}(\underline{50 \%}-\mathrm{W}) / \\
(\underline{50 \%}-\mathrm{N})\end{array}$ & $\begin{array}{l}(\underline{80 \%}-\mathrm{W}) / \\
(\underline{80 \%}-\mathrm{N})\end{array}$ & $\begin{array}{c}(80 \%-\underline{\mathbf{W}}) / \\
(50 \%-\underline{\mathbf{W}})\end{array}$ & $\begin{array}{c}(80 \%-\mathbf{N}) / \\
(50 \%-\underline{\mathbf{N}})\end{array}$ \\
\hline & (300 days) & $+2.4 \%$ & $+4.0 \%$ & $+6.6 \%$ & $+4.9 \%$ \\
\hline \multirow{9}{*}{$\begin{array}{l}\text { ECC-MgO } \\
\text { mixtures }\end{array}$} & $28+0 *$ (28 days) & 27.70 & 44.30 & 27.70 & 44.30 \\
\hline & $28+1 * *$ (56 days) & 56.60 & 61.10 & 71.86 & 69.00 \\
\hline & $28+3 * *(120$ days $)$ & 53.50 & 63.30 & 74.40 & 66.00 \\
\hline & $28+5 * *(180$ days $)$ & 63.53 & 67.80 & 74.00 & 69.90 \\
\hline & $28+7 * *(240$ days $)$ & 72.80 & 72.00 & 74.52 & 80.22 \\
\hline & $28+9 * *$ (300 days) & 82.00 & 85.00 & 82.00 & 89.00 \\
\hline & Recovery of & \multicolumn{2}{|c|}{ Same loading level } & \multicolumn{2}{|c|}{ Same curing condition } \\
\hline & $\begin{array}{c}\text { compressive } \\
\text { strength at } 28+9 * *\end{array}$ & $\begin{array}{l}(50 \%-\mathrm{N}) / \\
(\underline{50 \%}-\mathrm{W})\end{array}$ & $\begin{array}{l}(\underline{80 \%}-\mathrm{N}) / \\
(\underline{80 \%}-\mathrm{W})\end{array}$ & $\begin{array}{l}(80 \%-\underline{\mathbf{W}}) / \\
(50 \%-\underline{\mathbf{W}})\end{array}$ & $\begin{array}{l}(80 \%-\mathbf{N}) / \\
(50 \%-\underline{\mathbf{N}})\end{array}$ \\
\hline & (300 days) & $0.0 \%$ & $+4.7 \%$ & $+3.7 \%$ & $+8.5 \%$ \\
\hline
\end{tabular}

$* 50 \%$ \& $80 \%$ of the maximum load of the virgins at 28 days; **Reloading values of pre-cracked specimens at different ages

It could be concluded that long period of curing (up to 300 days), natural cured $80 \%$ pre-cracked ECC-MgO specimens developed (8.5\%) the highest compressive strength recovery amongst all loading levels and curing conditions when related to similar pre-cracked ECC and ECC-MgO specimens. 

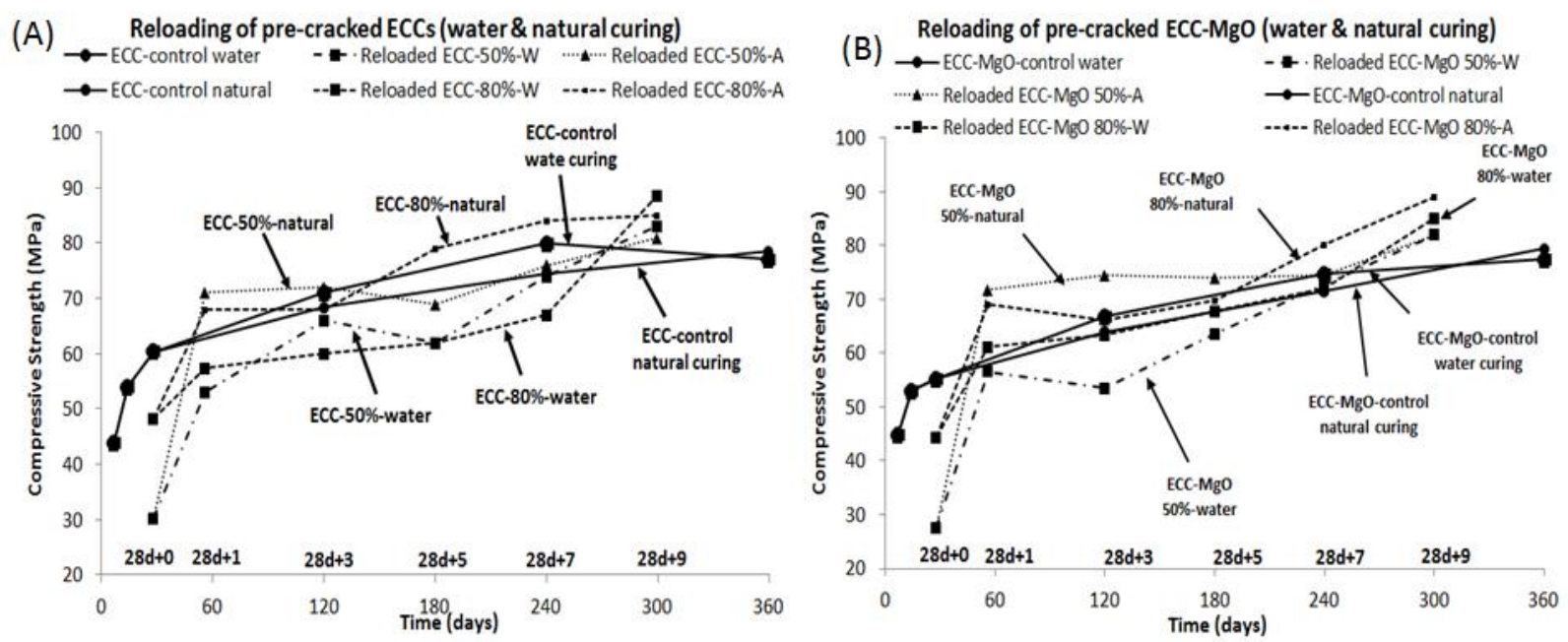

Figure 6-4: (A); The evaluation of self-healing property of ECC mixtures and (B); ECC-MgO mixtures based on compressive strength as a function of time
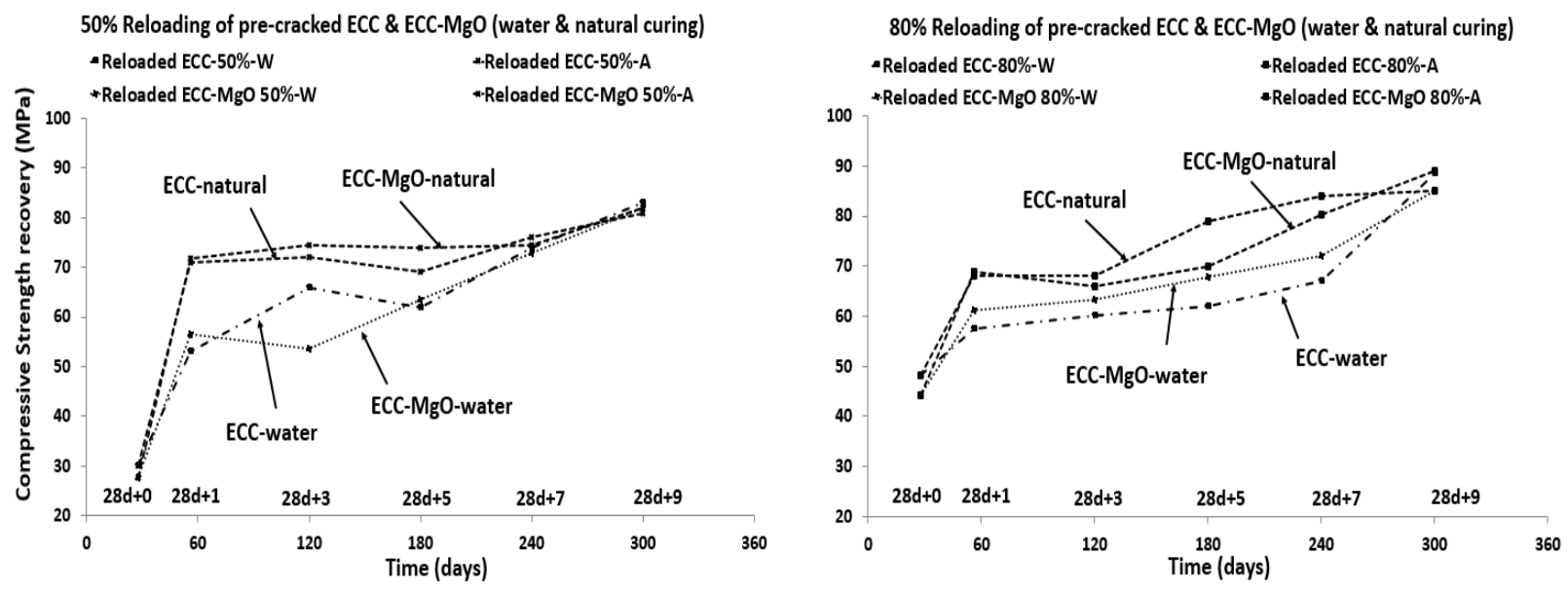

Figure 6-5: Reloading of pre-cracked water and natural cured ECC \& ECC-MgO specimens, 50\% (left) and $80 \%$ (right)

For in-depth study of the performance of reloaded ECC and ECC-MgO specimens, the compressive strength recovery of all water and natural cured $50 \%$ and $80 \%$ pre-cracked ECC and ECC-MgO specimens were combined together in one graph as shown in Figure 6-5. For 50\% precracked group, both natural cured pre-cracked ECC and ECC-MgO specimens recovered more compressive strength than those cured under water condition. Generally, all $50 \%$ pre-cracked 
specimens cured under both conditions recovered almost the same strength (ranged between 81 $\mathrm{MPa}$ and $83 \mathrm{MPa}$ ) (see Table 6-5).

Similar to $50 \%$, the $80 \%$ pre-cracked ECC and ECC-MgO specimens cured under natural condition developed the highest strength recovery at 300 days than those cured under water condition. Again, field/natural cured ECC-MgO specimens started to develop the highest strength recovery in the $80 \%$ pre-cracked group after the age of 240 days. Eventually, all $80 \%$ pre-cracked specimens developed more strength recovery $(85 \mathrm{MPa} \sim 89 \mathrm{MPa})$ than $50 \%$ pre-cracked specimens. The highest strength recovery was observed for $80 \%$ pre-cracked ECC-MgO mixture when cured under the field/natural condition ( $89 \mathrm{MPa})$ with an increase of $4.7 \%$ when related to counterpart ECC one $(89 \mathrm{MPa} / 85 \mathrm{MPa}=104.7 \%$ - see Table 6-5). It should be noted that the compressive strength values of $50 \%$ and $80 \%$ water and natural cured ECC-MgO controls acquired at the age of $28+0$ were $27.7 \mathrm{MPa}$ and $44.3 \mathrm{MPa}$; which were lower by $2.5 \mathrm{MPa}$ and $4.0 \mathrm{MPa}$ than ECC controls at the same age. Although this was the case, the pre-cracked ECC-MgO specimens developed/recovered more compressive strength than counterpart ECC specimens after a long period of curing. This can confirm the remarkable tendency of self-healing property in ECC-MgO system especially when cured under natural curing condition (see Table 6-5).

\subsubsection{Effect of multiple damage (repeatability of reloading) on compressive strength recovery of self-healed ECC \& ECC-MgO mixtures}

To assess the self-healing property of ECC and ECC-MgO mixtures, multiple reloading events were applied on pre-cracked ECC and ECC-MgO specimens (see third specimens' arrangement shown in Table 6-2 - set 3+).

As per Figure 6-6, water cured 50\% pre-cracked multiple reloaded ECC specimens did not recover the compressive strength of ECC-control while the $80 \%$ pre-cracked multiple reloaded ones almost recovered the strength of control. The compressive strength percentages of multiple reloaded $50 \%$ and $80 \%$ pre-cracked ECC specimens when related to controls were $94.8 \%$ and $101.3 \%$, respectively compared to water cured ECC-controls. Similar to water curing, natural cured $50 \%$ and $80 \%$ pre-cracked multiple reloaded ECC specimens did not recover the compressive strength of ECC-controls. The percentages of compressive strength of multiple reloaded $50 \%$ and $80 \%$ pre- 
cracked natural cured ECC specimens was $98.1 \%$ compared to natural cured ECC-controls. Different than ECC, the $50 \%$ pre-cracked multiple re-loaded water or natural cured ECC-MgO specimens almost recovered the strength of ECC-MgO-control. The compressive strength of water and natural cured $50 \%$ pre-cracked multiple reloaded ECC-MgO specimens was $99.4 \%$ compared to $\mathrm{ECC}-\mathrm{MgO}$-controls cured under water and natural conditions, respectively. Additionally, the water and natural cured $80 \%$ pre-cracked multiple reloaded ECC-MgO specimens developed significantly higher strength recovery than ECC-MgO-controls cured under both conditions. The percentages of compressive strength of water and natural cured $80 \%$ pre-cracked multiple reloaded ECC-MgO specimens were $105.8 \%$ and $103.1 \%$ respectively, compared to ECC-MgO-controls.

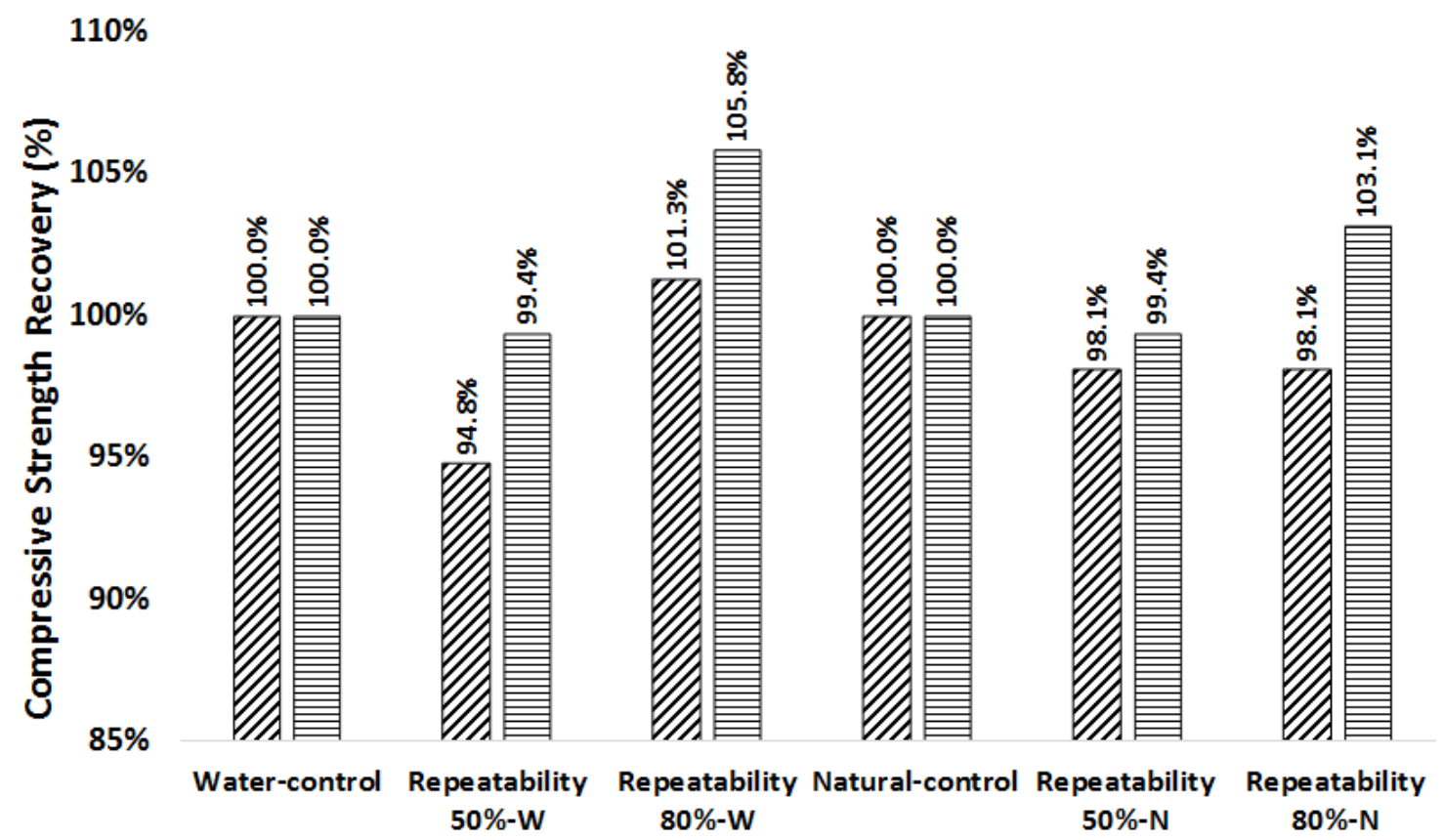

Figure 6-6: Self-healing assessment of multiple re-loading of ECC \& ECC-MgO specimens through compressive strength recovery under water and natural curing

The enhanced compressive strength recovery of water and natural cured multiple damaged $80 \%$ pre-cracked ECC-MgO cubic specimens indicated the strong self-healing capability of ECC-MgO system through the healing of micro cracks which signified by enhancing the mechanical properties. 


\subsection{Flexural load/strength development/recovery and UPV measurements}

Table 6-6 \& Figure 6-7 show the maximum load/deflection values and load-displacement responses, respectively of standard ECC and ECC-MgO control specimens at the ages of 7, 28, 120, 270 and 450 days based on the arrangement shown in Table 6-3 - set $1 *$. The maximum loads/strengths of ECC and ECC-MgO control specimens varied from $7.40 \mathrm{kN}$ to $9.80 \mathrm{kN}$ and $5.95 \mathrm{kN}$ to $8.33 \mathrm{kN}$, respectively. Similar to load/strength, the ultimate span deflection (deflection capacity) of specimens made of ECC and ECC-MgO controls varied respectively from $2.91 \mathrm{~mm}$ to $10.80 \mathrm{~mm}$ and $3.12 \mathrm{~mm}$ to $9.00 \mathrm{~mm}$ depending on curing type and age. The 7-day specimens (cured under laboratory condition inside sealed plastic bags until the age of testing -7 days) showed lowest ultimate load/strength and highest ultimate displacement capacities (Figure 6-7).

Table 6-6: Maximum load/strength and ultimate deflection of ECC and ECC-MgO controls at different ages and curing conditions

\begin{tabular}{|c|c|c|c|c|c|}
\hline \multirow{2}{*}{$\begin{array}{c}\text { Mix } \\
\text { Designation }\end{array}$} & \multirow{2}{*}{$\begin{array}{l}\text { Time } \\
\text { (days) }\end{array}$} & \multicolumn{4}{|c|}{ Laboratory Curing } \\
\hline & & \multicolumn{2}{|c|}{ Max. Strength (kN) } & \multicolumn{2}{|c|}{ Max. Deflection (mm) } \\
\hline \multirow{7}{*}{$\begin{array}{c}\text { ECC } \\
\text { controls }\end{array}$} & 7 & \multicolumn{2}{|c|}{7.50} & \multicolumn{2}{|c|}{10.80} \\
\hline & \multirow{2}{*}{$\begin{array}{l}\text { Time } \\
\text { (days) }\end{array}$} & \multicolumn{2}{|c|}{ Water Curing } & \multicolumn{2}{|c|}{ Natural Curing } \\
\hline & & $\begin{array}{c}\text { Strength } \\
(\mathrm{kN})\end{array}$ & $\begin{array}{l}\text { Deflection } \\
(\mathrm{mm})\end{array}$ & $\begin{array}{l}\text { Strength } \\
(\mathrm{kN})\end{array}$ & $\begin{array}{l}\text { Deflection } \\
\text { (mm) }\end{array}$ \\
\hline & 28 & 8.80 & 7.42 & 7.40 & 3.26 \\
\hline & 120 & 9.80 & 7.38 & 7.40 & 4.07 \\
\hline & 270 & 9.40 & 2.91 & 8.80 & 7.10 \\
\hline & 450 & 9.20 & 6.17 & 8.60 & 6.99 \\
\hline \multirow{2}{*}{$\begin{array}{c}\text { Mix } \\
\text { Designation } \\
\end{array}$} & \multirow{2}{*}{$\begin{array}{l}\text { Time } \\
\text { (days) }\end{array}$} & \multicolumn{4}{|c|}{ Laboratory Curing } \\
\hline & & \multicolumn{2}{|c|}{ Max. Strength $(\mathrm{kN})$} & \multicolumn{2}{|c|}{ Max. Deflection (mm) } \\
\hline \multirow{7}{*}{$\begin{array}{l}\mathrm{ECC}-\mathrm{MgO} \\
\text { controls }\end{array}$} & 7 & \multicolumn{2}{|c|}{6.45} & \multicolumn{2}{|c|}{9.00} \\
\hline & \multirow{2}{*}{$\begin{array}{l}\text { Time } \\
\text { (days) }\end{array}$} & \multicolumn{2}{|c|}{ Water Curing } & \multicolumn{2}{|c|}{ Natural Curing } \\
\hline & & $\begin{array}{l}\text { Strength } \\
(\mathrm{kN})\end{array}$ & $\begin{array}{c}\text { Deflection } \\
(\mathrm{mm})\end{array}$ & $\begin{array}{l}\text { Strength } \\
(\mathrm{kN})\end{array}$ & $\begin{array}{l}\text { Deflection } \\
(\mathrm{mm})\end{array}$ \\
\hline & 28 & 7.03 & 5.57 & 8.20 & 4.97 \\
\hline & 120 & 7.20 & 3.12 & 5.95 & 4.67 \\
\hline & 270 & 8.33 & 7.13 & 7.84 & 6.22 \\
\hline & 450 & 8.03 & 4.75 & 6.60 & 5.50 \\
\hline
\end{tabular}


ECC-Controls (natural curing)

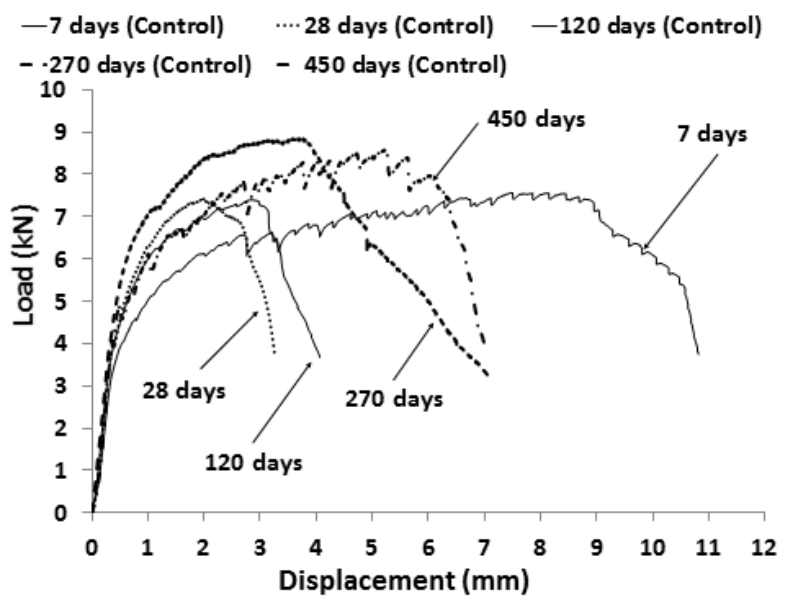

ECC-MgO Controls (natural curing)

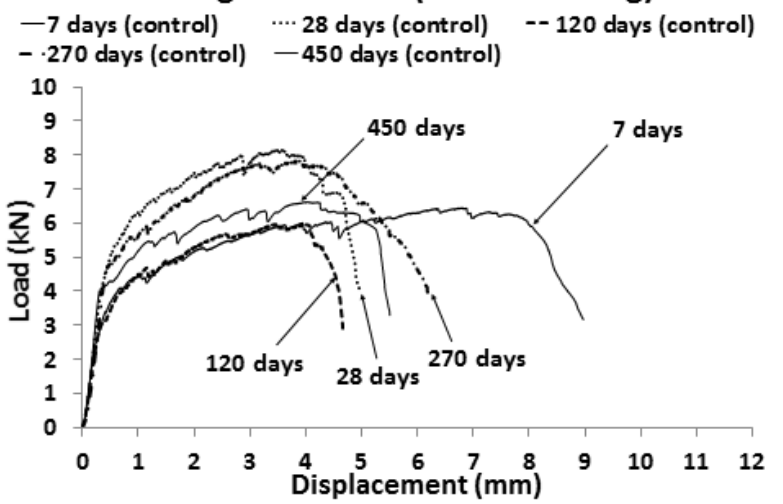

ECC-Controls (water curing)

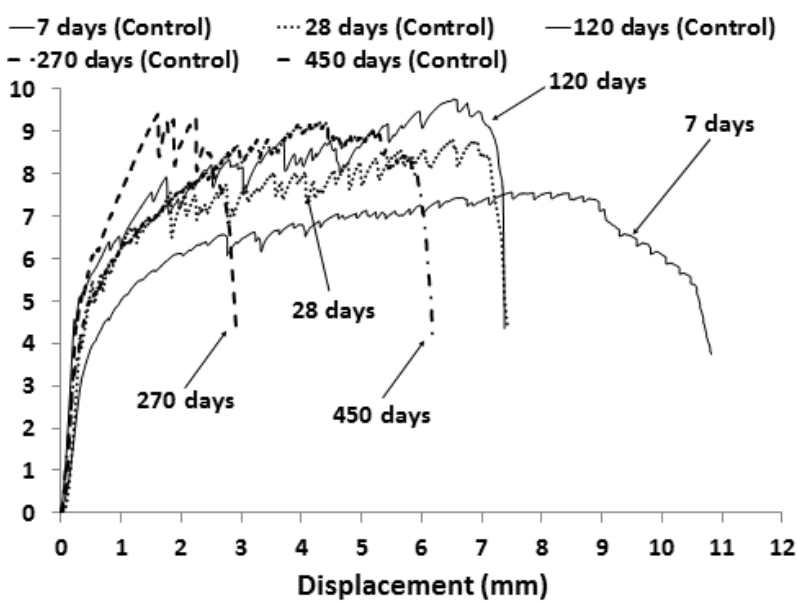

ECC-MgO Controls (water curing)

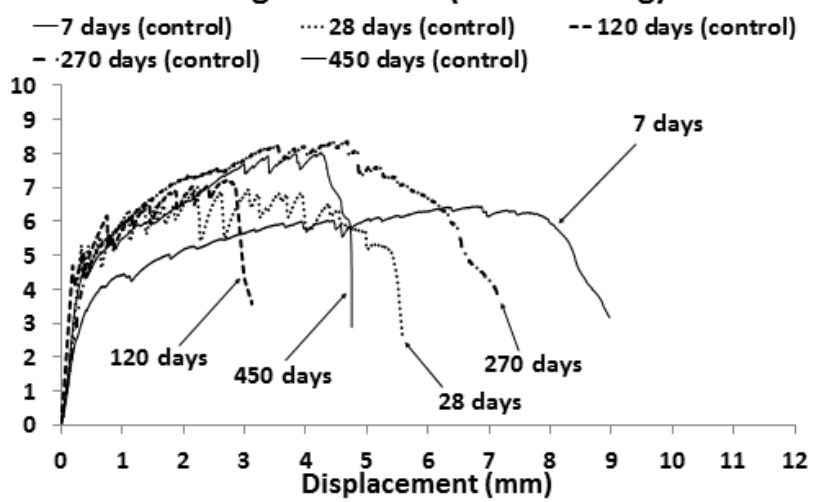

Figure 6-7: Maximum flexural strength and ultimate deflection of ECC and ECC-MgO controls as a function of time cured under water and natural conditions.

To differentiate between the flexural load-deflection curves shown in Figure 6-7, Figure 6-8 presents the trend of development of maximum load/strength of specimens made of ECC and ECC$\mathrm{MgO}$ controls as a function of time. As in the case of compressive strength (see Figure 6-3), Figure 6-8 shows ECC controls developed the highest load/strength than ECC-MgO controls cured under water and natural curing conditions. The highest trend of load/strength development was for water cured ECC controls while the lowest one was for the natural cured ECC-MgO controls (see Figure 6-8). It was also observed that the water cured controls, whether ECC or ECC-MgO, developed higher load/strength than the ones cured under natural condition. Contrary to compressive strength, the flexural load/strength of ECC-MgO controls did not show any improvement after a long-term 
of curing under both water and natural conditions and remained below the strength development of ECC controls (after 450 days). The lowest load/strength development of ECC-MgO-controls at later ages might be related to two reasons. First, replacement of cementitious materials with very slow reactive $\mathrm{MgO}$ expansive agent and second, the size effect of bigger prismatic specimens might not reveal clearly the effect of $\mathrm{MgO}$ expansion as in the case of cubic specimens. Hence, 450 days of testing may not be enough to reveal the expansion effect of $\mathrm{MgO}$ on the load/strength performance. Accordingly, accelerated tests (such as autoclave test) might be needed to expedite the rate of $\mathrm{MgO}$ hydration.

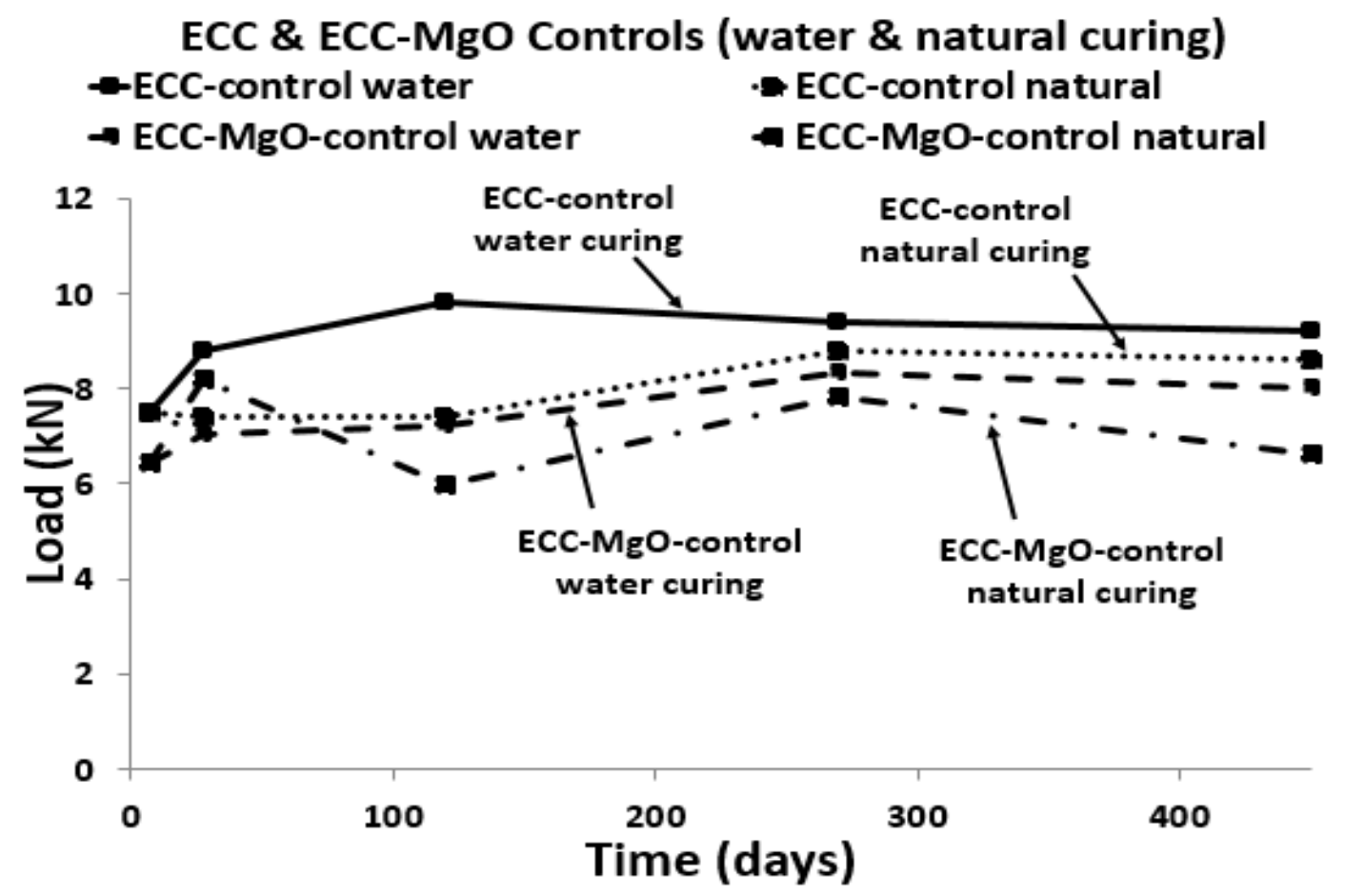

Figure 6-8: Trend development of maximum load of ECC and ECC-MgO controls as a function of time

Figure 6-9 (A) monitors the trend of development of maximum ultrasonic pulse velocity (UPV) of the ECC control specimens as a function of time. In general, UPV increased with the increase of time irrespective of types of ECC and curing conditions. 

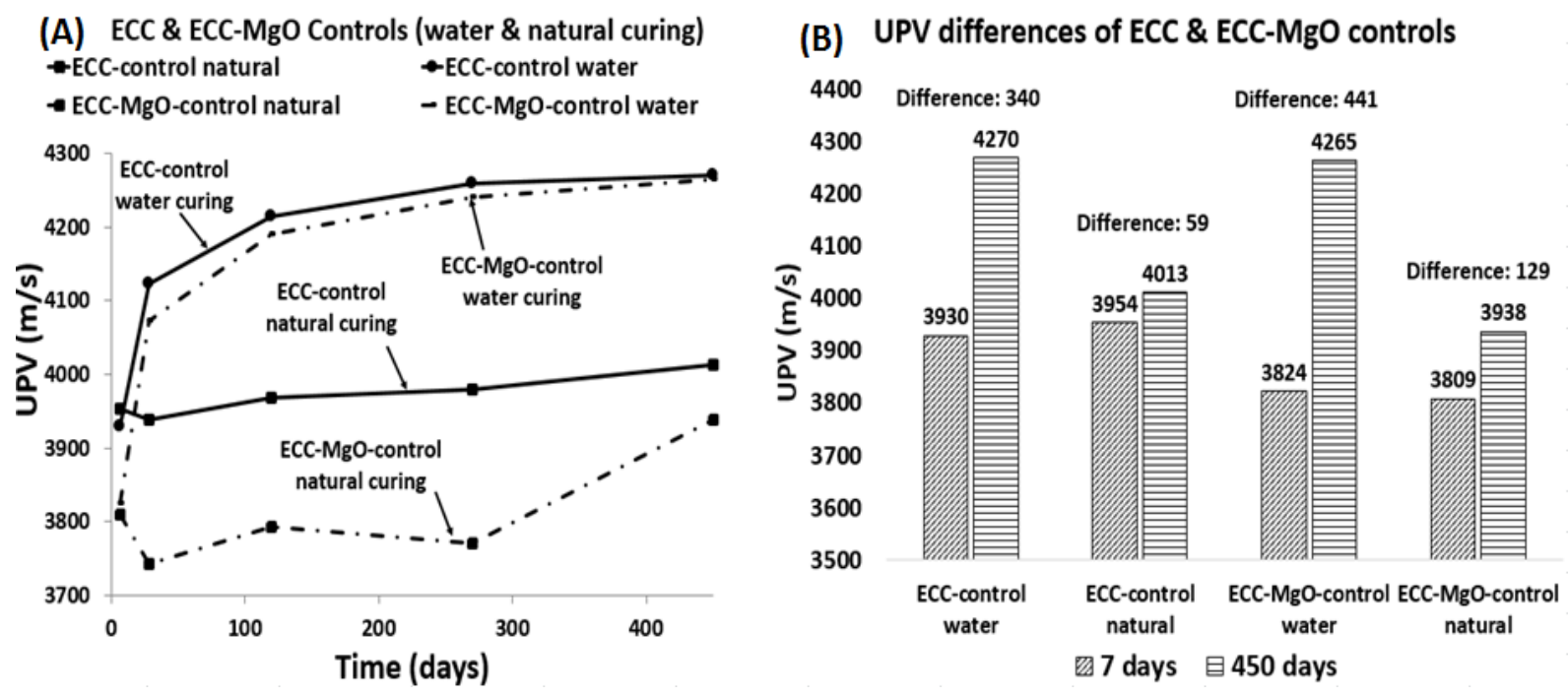

Figure 6-9: (A) Trend of development of UPV of ECC \& ECC-MgO controls as a function of time and (B) differences in UPVs between first and last day of curing

As expected, the water cured specimens of both ECC and ECC-MgO controls developed higher UPVs than those cured under natural condition. This was attributed to filing effect of more hydrated products when specimens were stored continuously in water. Particularly and similar to load strength shown in Figure 6-8, the trends of UPV increase were highest for ECC-controls whether cured under water or natural conditions compared to ECC-MgO-controls cured under same conditions. However, ECC-MgO-controls cured under both conditions showed a stronger rate of UPV increase than ECC-controls beyond 300 days of curing. The difference between UPVs at 7 days and 450 days of water cured ECC and ECC-MgO controls was $340 \mathrm{~m} / \mathrm{s}$ and $441 \mathrm{~m} / \mathrm{s}$, respectively; while it was $59 \mathrm{~m} / \mathrm{s}$ and $129 \mathrm{~m} / \mathrm{s}$ for the same controls cured under natural condition (Figure 6-9 - B). Furthermore, the UPV values of natural cured ECC-MgO control specimens did not reach those of water cured ECC controls even after long period of curing (450 days). Only water cured ECC-MgO-controls reached almost similar UPVs of ECC-controls cured under similar condition. 


\subsubsection{Flexural load/strength recovery of self-healed ECC \& ECC-MgO specimens}

To appraise the self-healing behavior of ECC-MgO system over time, a comprehensive technique was adopted through studying the strength property on water and natural cured prismatic specimens based on Table 6-3 - set $2^{* *}$. In this technique, the pre-loading flexural deflection of four-point loaded ECC specimens was fixed to $5.5 \mathrm{~mm}$ while it was fixed to $4.5 \mathrm{~mm}$ for ECC$\mathrm{MgO}$ specimens based on the virgins (see Table 6-7, Figure 6-10 \& Figure 6-11). To evaluate the self-healing property of proposed ECC-MgO mixtures, it should be emphasized that the focus in this method was on the load/strength recovery not the deflection rate development. In this method, all specimens were pre-cracked at the age of $7 d+0$ for crack initiation to the limit of $60 \%$ of the maximum multiple cracked deflections of the controls. Then every specimen was reloaded again for two times, first is multiple cracking (MC) events at $7 d+1,7 d+3,7 d+5,7 d+7$ and $7 d+9$ and second re-loading to failure was after one month of multiple cracking (at $7 d+2,7 d+4,7 d+6,7 d+8$ and $7 d+10)$. The load/strength of multiple cracked/failed specimens was then compared with the controls at similar ages.

Under pre-loading strength event $(7 \mathrm{~d}+0)$, ECC specimens produced consistently higher preloading strengths than ECC-MgO specimens cured under water and natural conditions (see Table 6-7). For instance, under the time frame $(7 \mathrm{~d}+1)$, the pre-loading strength of water cured ECC specimens at $(7 \mathrm{~d}+0)$ was $7.5 \mathrm{MPa}$ greater than $6.45 \mathrm{MPa}$ of water cured ECC-MgO specimens. Moreover, ECC specimens produced higher multiple cracks (MC) and failure loads/strengths than ECC-MgO specimens at early ages as well (time frames: $7 d+1,7 d+3$ and $7 d+5$ ). However, the

ECC-MgO specimens cured under both conditions started to develop higher MC and failure strengths than their ECC counterparts at later ages $(7 d+7$ and $7 d+9)$. For convenience, all similar or higher multiple crack and failure strength in addition to ultimate deflections of ECC-MgO specimens (compared to ECC specimens) were highlighted in bold as shown in Table 6-7.

From Figure 6-10 \& Figure 6-11, it was observed that the load-deflection curves of water cured multiple cracked/failed ECC specimens developed better load/strength development than ECC$\mathrm{MgO}$ specimens up to $7 \mathrm{~d}+7$. It is well known that the presence of water in most studies of self- 
healing is very important to initiate the autogenous healing property (Qian et al., 2010). However, the situation of fully submerging concrete structures continuously in water is impossible during the lifetime of any concrete element. Therefore, the focus should be on the natural cured multiple cracked ECC or ECC-MgO specimens. It was shown in Figure 6-10 \& Figure 6-11 that the enhancement in load/strength development of multiple cracked/failed ECC specimens were reduced earlier less than counterpart ECC-MgO ones at the age of $7 \mathrm{~d}+3$. The significant enhancement in multiple cracked ECC-MgO specimens could be related to the enhancement of tensile strain capacity of $\mathrm{MgO}$ expansive agent which is always prescribed to reduce concrete microcracking (Du, 2005).

Table 6-7: Maximum load/strength and ultimate deflection of self-healed ECC and ECC-MgO specimens over 10 months of water and natural curing conditions

\begin{tabular}{|c|c|c|c|c|c|c|c|c|c|c|}
\hline \multirow[b]{3}{*}{$\begin{array}{l}\text { Time } \\
\text { Frame }\end{array}$} & \multirow[b]{3}{*}{$\begin{array}{c}\text { Time } \\
\text { Days + Months } \\
\text { (Days + Days) }\end{array}$} & \multirow[b]{3}{*}{$\begin{array}{l}\text { Loading } \\
\text { Stage }\end{array}$} & \multicolumn{4}{|c|}{ ECC Specimens } & \multicolumn{4}{|c|}{ ECC-MgO Specimens } \\
\hline & & & \multicolumn{2}{|c|}{ Water } & \multicolumn{2}{|c|}{ Natural } & \multicolumn{2}{|c|}{ Water } & \multicolumn{2}{|c|}{ Natural } \\
\hline & & & 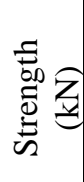 & 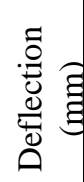 & 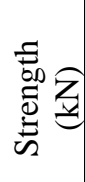 & 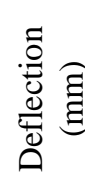 & 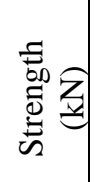 & 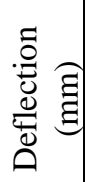 & 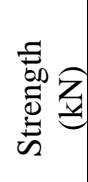 & 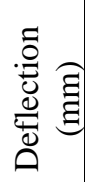 \\
\hline $7 \mathrm{~d}+1$ & $7 d+1(7 d+30)$ & Control & 8.8 & 7.4 & 7.4 & 3.3 & 7.03 & 5.6 & 8.2 & 5.0 \\
\hline \multirow{3}{*}{$7 d+1$} & $7 d+0(7 d+0)$ & Pre-Loading & 7.5 & 5.5 & 7.5 & 5.5 & 6.45 & 4.5 & 6.45 & 4.5 \\
\hline & $7 d+1(7 d+30)$ & $\mathrm{MC}$ & 8.6 & 4.4 & 7.5 & 6.2 & 6.6 & 4.4 & 6.6 & 4.0 \\
\hline & $7 d+2(7 d+60)$ & Fail & 6.4 & 2.6 & 5.5 & 5.5 & 6.4 & 3.3 & 4.8 & 2.7 \\
\hline $7 d+4$ & $7 d+4(7 d+120)$ & Control & 9.8 & 7.4 & 7.4 & 4.1 & 7.2 & 3.1 & 5.95 & 4.7 \\
\hline \multirow{3}{*}{$7 d+3$} & $7 d+0(7 d+0)$ & Pre-Loading & 9.3 & 5.5 & 8.4 & 5.5 & 6.5 & 4.5 & 6.5 & $\overline{4.5}$ \\
\hline & $7 d+3(7 d+90)$ & $\mathrm{MC}$ & 9.8 & 5.4 & 8.0 & 4.2 & 8.0 & 5.4 & 6.7 & 3.6 \\
\hline & $7 d+4(7 d+120)$ & Fail & 6.3 & 3.9 & 6.6 & 3.2 & 6.6 & 3.5 & 5.2 & 3.1 \\
\hline $7 d+9$ & $7 d+9(7 d+270)$ & Control & 9.4 & 2.9 & 8.8 & 7.1 & 8.33 & $\underline{7.1}$ & 7.84 & 6.2 \\
\hline \multirow{3}{*}{$7 d+5$} & $7 d+0(7 d+0)$ & Pre-Loading & 7.4 & 5.5 & 7.7 & 5.5 & 6.5 & 4.5 & 7.8 & 4.5 \\
\hline & $7 d+5(7 d+150)$ & $\mathrm{MC}$ & 8.2 & 3.7 & 8.2 & 4.9 & 7.4 & 2.7 & $\overline{8.4}$ & 3.1 \\
\hline & $7 d+6(7 d+180)$ & Fail & 6.4 & 2.6 & 7.0 & 4.7 & 5.4 & 1.5 & 5.3 & 2.6 \\
\hline $7 d+9$ & $7 d+9(7 d+270)$ & Control & 9.4 & 2.9 & 8.8 & 7.1 & 8.3 & 7.1 & 7.84 & 6.2 \\
\hline \multirow{3}{*}{$7 d+7$} & $7 d+0(7 d+0)$ & Pre-Loading & 8.2 & 5.5 & 7.3 & 5.5 & 7.7 & 4.5 & 7.2 & 4.5 \\
\hline & $7 d+7(7 d+210)$ & $\mathrm{MC}$ & 8.8 & 4.3 & 7.8 & 5.0 & 8.33 & 3.0 & 8.7 & 5.4 \\
\hline & $7 d+8(7 d+240)$ & Fail & 6.7 & 2.3 & 5.6 & 5.2 & 6.8 & 2.5 & $\overline{6.0}$ & $\overline{3.9}$ \\
\hline $7 d+9$ & $7 d+9(7 d+270)$ & Control & 9.4 & 2.9 & 8.8 & 7.1 & 8.33 & $\underline{7.2}$ & 7.84 & 6.2 \\
\hline \multirow{3}{*}{$7 d+9$} & $7 d+0(7 d+0)$ & Pre-Loading & 8.2 & 5.5 & 9.7 & 5.5 & 6.8 & $\overline{4.5}$ & 8.2 & 4.5 \\
\hline & $7 d+9(7 d+270)$ & $\mathrm{MC}$ & 8.4 & 3.1 & 9.0 & 3.9 & 8.5 & 2.2 & $\underline{9.5}$ & $\underline{4.2}$ \\
\hline & $7 d+10(7 d+300)$ & Fail & 7.9 & 3.3 & 7.5 & 3.2 & 6.3 & 1.8 & 8.9 & $\overline{4.0}$ \\
\hline
\end{tabular}

*7d+0: 7 days; $7 d+1: 7$ days +30 days; MC: multiple cracking stage; Fail: failure stage of multiple cracked specimens 

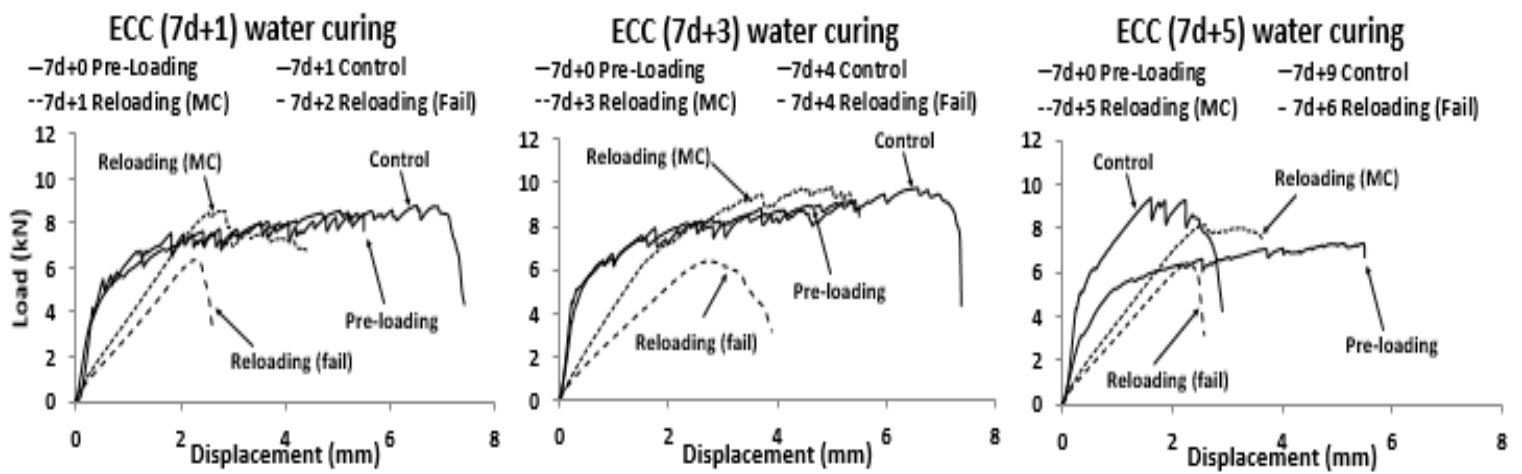

$\mathrm{ECC}(7 \mathrm{~d}+7)$ water curing

$-7 \mathrm{~d}+0$ Pre-Loading

$-7 d+9$ control

$\mathrm{ECC}(7 \mathrm{~d}+9)$ water curing

$--7 \mathrm{~d}+7$ Reloading (MC) $\quad-7 \mathrm{~d}+8$ Reloading (Fail)

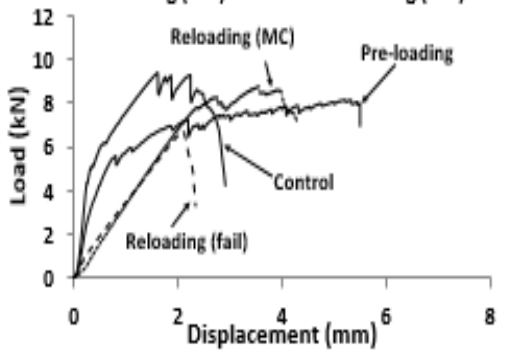

$-7 \mathrm{~d}+0$ Pre-Loading $\quad-7 \mathrm{~d}+9$ control

$-7 \mathrm{~d}+9$ Reloading (MC) $\quad-7 \mathrm{~d}+10$ Reloading (Fail)

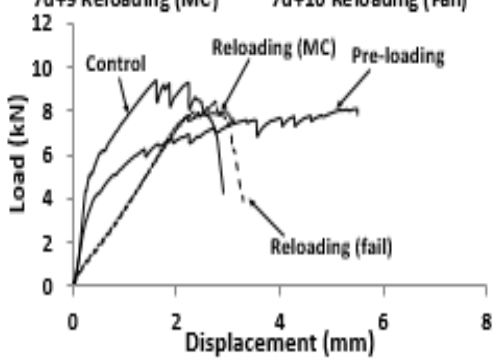

$\mathrm{ECC}(7 \mathrm{~d}+1)$ natural curing

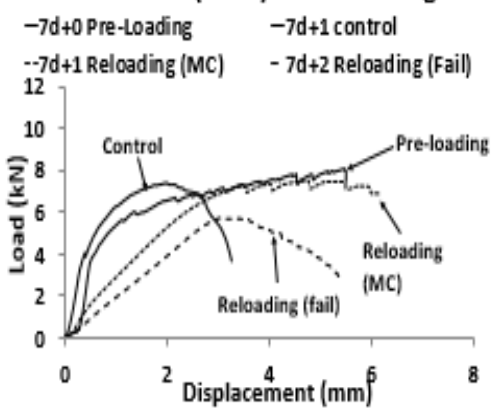

$\operatorname{ECC}(7 d+3)$ natural curing

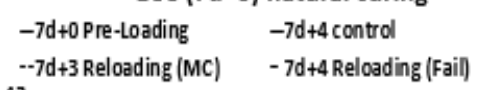

$-7 \mathrm{~d}+4$ Reloading (Fail)

ECC $(7 d+5)$ natural curing

$--7 \mathrm{~d}+3$ Reloading (MC)

$-7 \mathrm{~d}+0$ Pre-Loading

$-7 \mathrm{~d}+5$ Reloading (MC)

$-7 \mathrm{~d}+9$ control
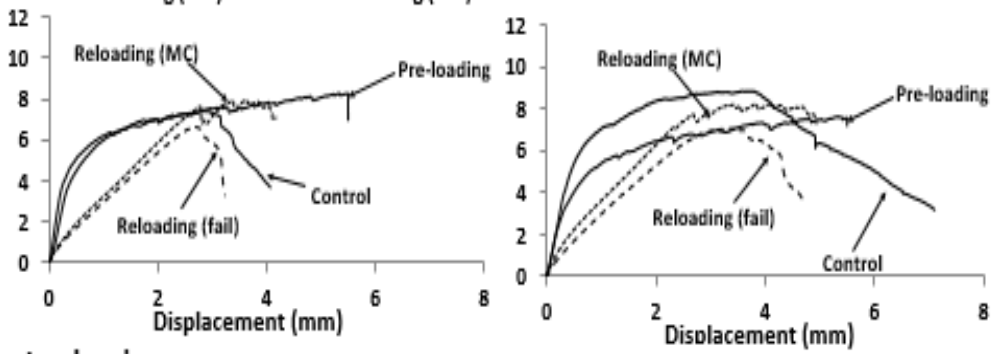

$\operatorname{ECC}(7 d+7)$ natural curing

$-7 d+0$ Pre-Loading $\quad-7 d+9$ control

$-7 \mathrm{~d}+9$ control
$-7 \mathrm{~d}+8$ Reloading (Fai)

ECC $(7 d+9)$ natural curing
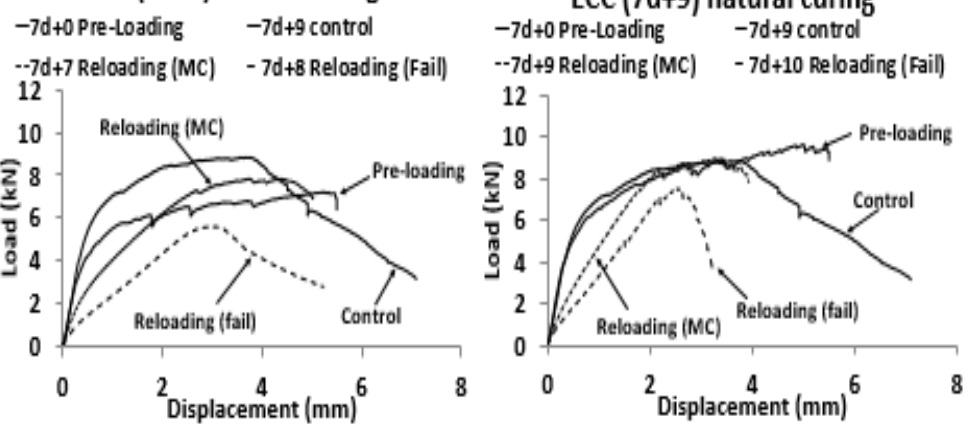

Figure 6-10: Load vs deflection curves of water and natural cured control and self-healed ECC specimens over different periods 

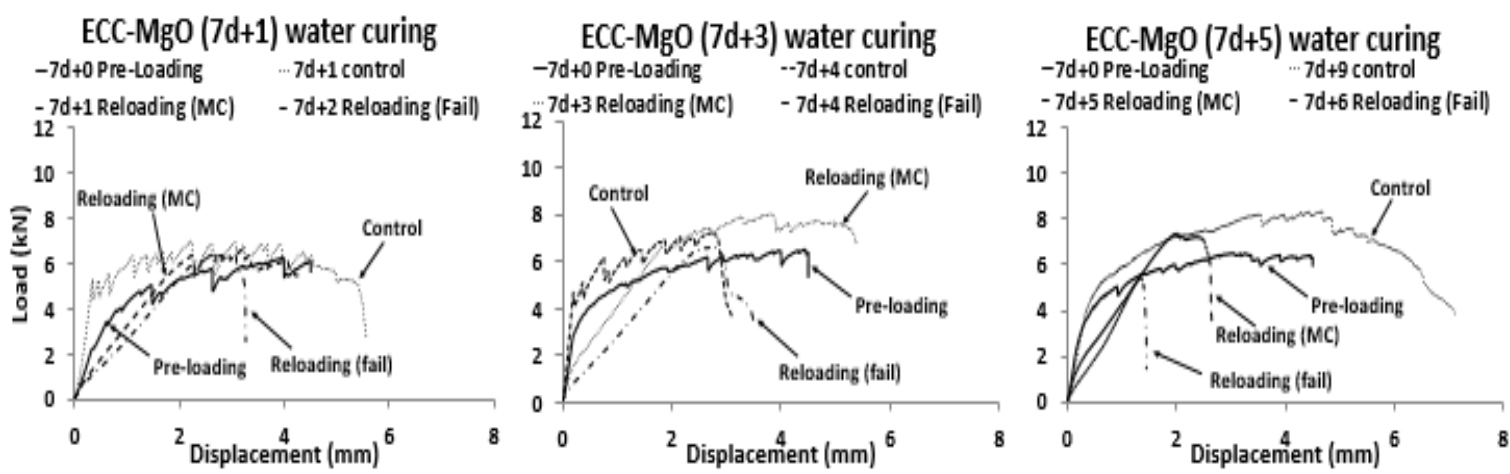

$\mathrm{ECC}-\mathrm{Mg} 0(7 \mathrm{~d}+7)$ water curing

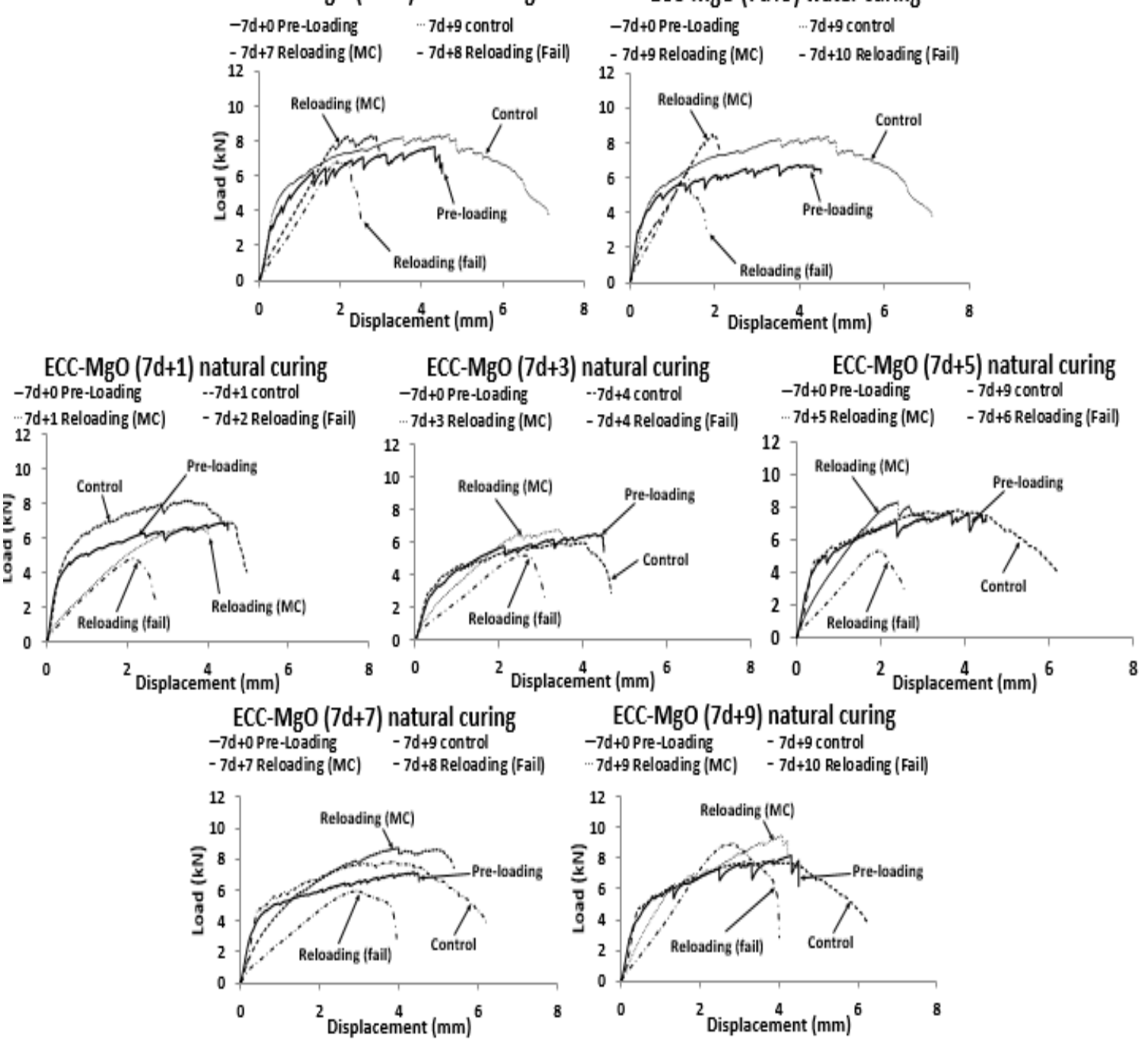

$\mathrm{ECC}-\mathrm{MgO}(7 \mathrm{~d}+9)$ water curing

Figure 6-11: Load vs deflection curves of water and natural cured control and self-healed ECC-MgO specimens over different periods 

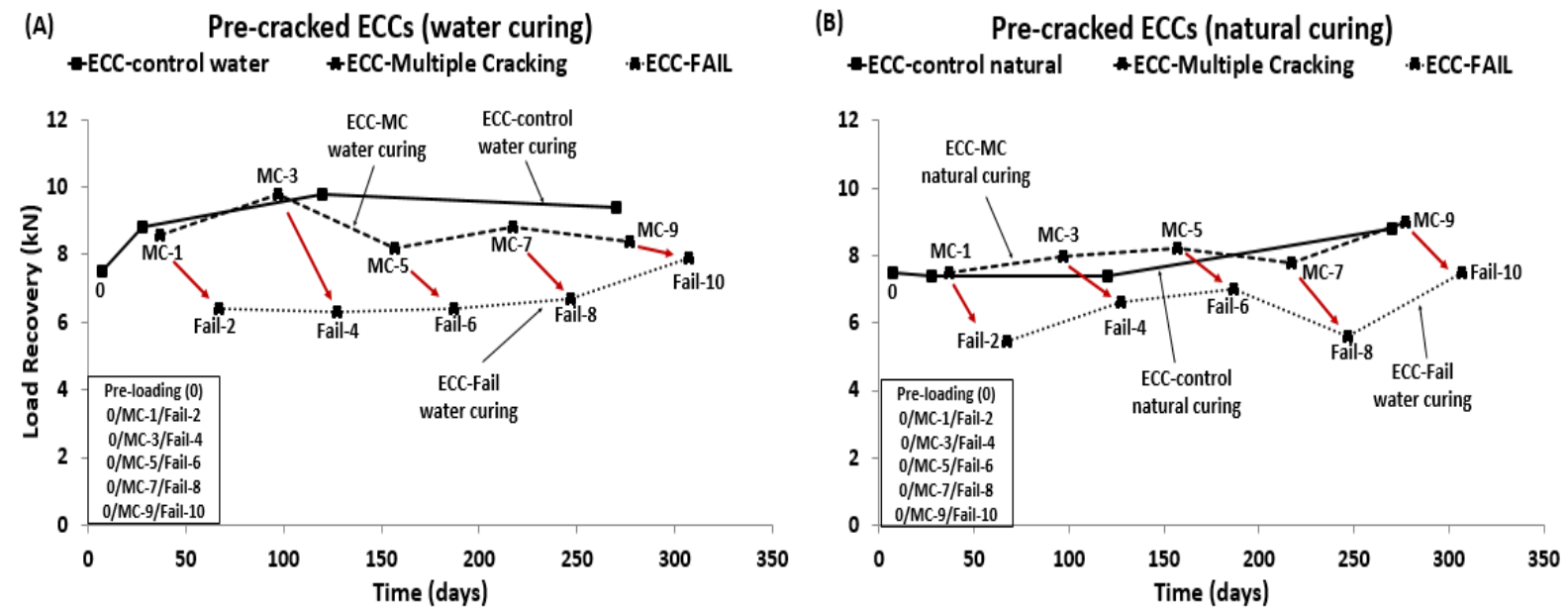

(C) Pre-cracked ECC-MgOs (water curing)

(D) Pre-cracked ECC-MgOs (natural curing)

-ECC-MgO-control water *ECC-MgO Multiple Cracking ECCC-MgO falL

-ECC-MgO-control natural \#ECC-MgO Multiple Cracking *ECC-MgO FAIL
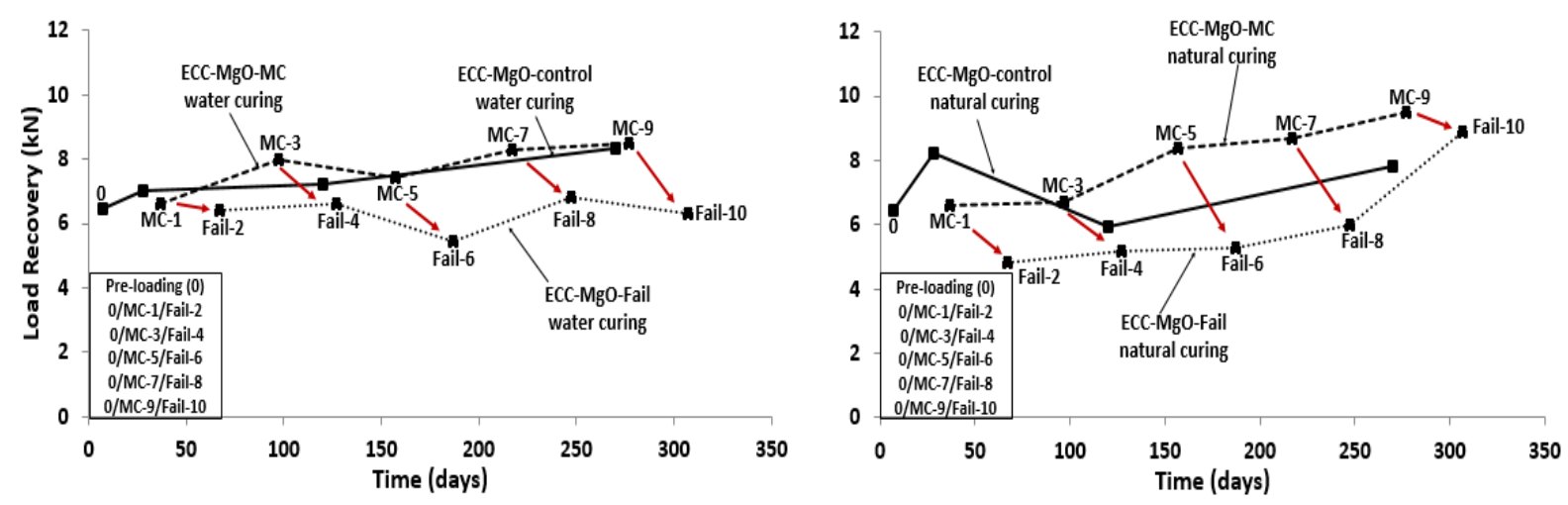

Figure 6-12: Recovery loads of water and natural cured ECC and ECC-MgO specimens loaded at different stage loadings as a function of time

To figure out the performance (based on load vs. deflection curves shown in Figure 6-10 \& Figure 6-11), all pre-loading, multiple cracking and failure strengths were compared in terms of controls (up to 270 days) under both curing conditions in Figure 6-12. The marked points shown in Figure 6-12 signify the strength recovery of every loading stage at different times. For instance, if the numbers (0/MC-1/Fail-2) for water cured ECC specimens shown in Figure 6-12 part (A) are considered - (0) means the preloading strength of ECC specimens at the age of ( $7 d+0$ days) based on $60 \%$ maximum multiple cracked deflection of the virgins; (MC-1) means the strength recovery of multiple cracked ECC sample (after preloading stage) at the age of ( $7 d+1$ month or $7 d+30$ days); and (Fail-2) means the strength recovery of failed ECC sample (after one month of multiple cracking stage $)$ at the age of $(7 d+2$ months or $7 d+60$ days $)$. It should be emphasized that all stages 
of loadings mentioned above (the case 0/MC-1/Fail-2: multiple cracked at the age of 1 month and then cured for another month before loaded to failure) were repeated on the same specimens and related to the controls' curves shown in Figure 6-8 drawn up to $7 d+9$ months or $7 d+270$ days only. Under multiple cracking loading case, it was observed from Figure 6-12 part (A) that only multiple cracked ECC sample (ECC-MC-3) at the age of ' $7 \mathrm{~d}+3$ ' almost recovered the load/strength of ECCcontrols. It should be noted that the water cured ECC-MC-3 specimen showed the highest strength $(9.8 \mathrm{kN})$ recovery compared to multiple cracked/failed ECC and ECC-MgO specimens cured under water and natural conditions. Different to water curing condition as shown in Figure 6-12 part (B), all natural cured ECC-MC specimens recovered the load/strength of ECC-controls except the natural cured ECC-MC-7 specimen. Figure 6-12 part (C \& D) reveals that all water cured ECC$\mathrm{MgO}-\mathrm{MC}$ specimens recovered the load/strength of ECC-MgO-controls except for ECC-MgOMC-1 specimens under both curing conditions. It should be mentioned that the natural cured ECC$\mathrm{MgO}-\mathrm{MC}$ specimens had shown enhanced strength, way better than the ECC-MgO-controls. Under failure loading, specimens (ECC-Fail and ECC-MgO-Fail) in all loading stages did not recover the strength of either control or multiple cracked specimens under both water and natural conditions. The only specimen that produced higher strength $(8.9 \mathrm{kN})$ than all other controls was natural cured ECC-MgO-Fail-10. Further, the natural cured ECC-MgO-Fail-2 specimen developed the lowest strength recovery $(4.8 \mathrm{kN})$ compared to all multiple cracked/failed ECC and ECC-MgO specimens cured under water and natural conditions. In general, strength increased with the increase of age for control, multiple cracked and failed ECC or ECC-MgO specimens whether water or natural cured (Figure 6-12).

Figure 6-13 studies MC/Failed ECC and ECC-MgO specimens in terms of high strength recovery under both water and natural curing conditions. After long period of curing (300 days), it was concluded (from Figure 6-12 and Figure 6-13) that natural cured ECC-MgO specimens developed the highest strength recovery in MC/Failed loading cases $(\mathrm{MC}-9=9.5 \mathrm{kN}$ and Fail-10 $=8.9 \mathrm{kN})$ while in contrast, same failed specimens developed the lowest strength recovery under water curing (Fail-10 =6.3 kN). The natural curing at later ages stimulated the self-healing phenomena (as exhibited in MC/Failed ECC-MgO specimens) better than water curing by showing as enhanced strength recovery. This can be attributed to the flow of carbon dioxide $\left(\mathrm{CO}_{2}\right)$ present in 
air (which usually loaded in moisture, $\mathrm{H}_{2} \mathrm{O}$ ) through concrete cracks stimulating $\mathrm{Ca}^{2+}$ ions (exist at the surface of crack walls) to form $\mathrm{CaCO}_{3}$ crystals - a phenomenon classified as surfacecontrolled crystal growth by Edvardsen (1999) who concluded that this phase was responsible to form the major $\mathrm{CaCO}_{3}$ crystals at the first 3 to 5 days. Due to slow kinetic of moisture flow through cracks, Edvardsen also concluded that $\mathrm{CO}_{2}$ will keep diffusing more $\mathrm{Ca}^{2+}$ ions from deeper locations of hardened cement paste and forming around $20 \%$ of $\mathrm{CaCO}_{3}$ crystals over long period of curing - this phase was called diffusion-controlled crystal growth which is slower than the surface-controlled phase. The enhancement of strength recovery in this research could be due to the precipitation of $\mathrm{CaCO}_{3}$ crystals through cracks as observed by Edvardsen (1999). Because of delayed effect of slow MgO hydration (Du, 2005; Mo et al., 2014), similar kinetic of diffusioncontrolled crystal growth could be occurred also to magnesium ions $\left(\mathrm{Mg}^{2+}\right)$ after long period of curing. The presence of $\mathrm{CO}_{2}$ in moisture can combine with $\mathrm{Mg}^{2+}$ ions located in both crack surfaces and deep locations of hardened cement pastes to forming $\mathrm{MgCO}_{3}$ crystals. The late formation of $\mathrm{MgCO}_{3}$ crystals can supplement the early formation of $\mathrm{CaCO}_{3}$ for sealing the cracks of natural cured MC/Failed ECC-MgO specimens and hence, developed more strength recovery than water cured specimens.

At later ages, the weakness of strength recovery of water cured failed ECC-MgO specimens was due to the continuous curing in water. Excessive water curing might aggravate the rate of $\mathrm{MgO}$ hydration to have an extreme filling effect due to expansive nature of $\mathrm{MgO}$ leading to brittleness in ECC-MgO microstructure. Mo et al. (2012) reported that low water demand is required in $\mathrm{MgO}$ concrete to form chemically stable Magnesium Hydroxide $\mathrm{Mg}(\mathrm{OH})_{2}$ - a dominant product producing autogenous expansion to compensate concrete autogenous shrinkage. 

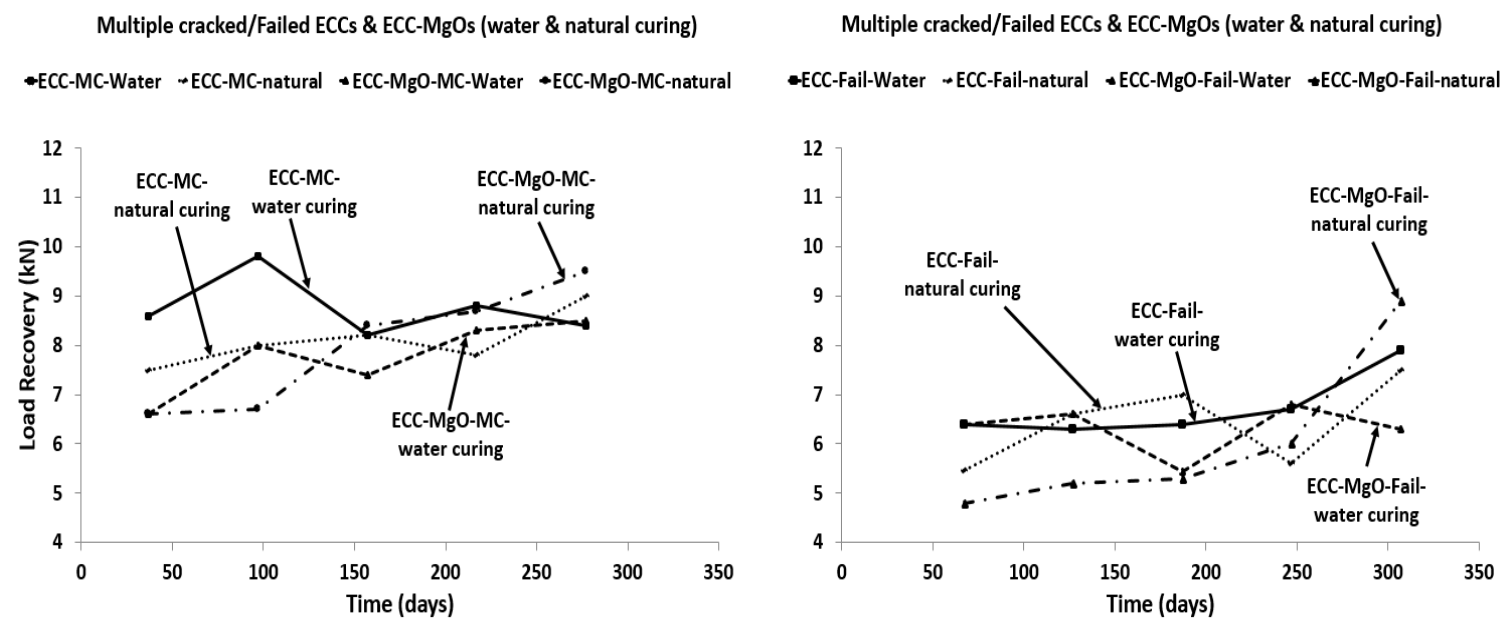

Figure 6-13: Comparison between load recovery of multiple cracked (left) and failed (right) ECC and

ECC-MgO specimens cured under water and natural conditions as a function of time

The variation of ultrasonic pulse velocity with time for MC/Failed ECC, ECC-MgO and ECCcontrol specimens cured under water and natural conditions presented in Figure 6-14. Generally, cracking UPV increased with the increase of age irrespective of ECC types, specimen characteristics (cracked or control/virgin) and curing types (water or natural). All water cured MC/Failed ECC and ECC-MgO specimens developed higher UPVs than their natural cured counterparts. From Figure 6-14 (A), it can be noted that none of MC/Fail ECC specimens recovered the UPVs of ECC-control cured under both conditions. 

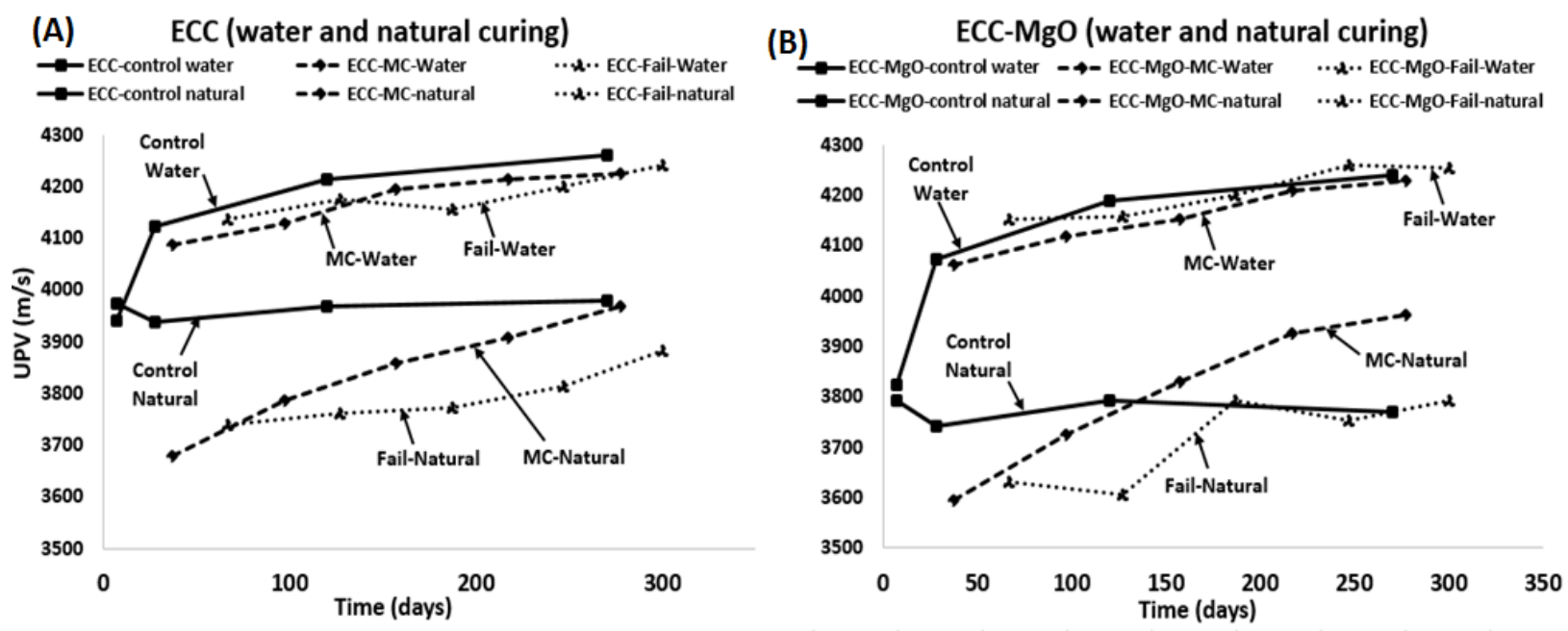

Figure 6-14: (A) Trend of UPV development with time for water and natural cured MC/Failed ECC and

(B) $\mathrm{MC} /$ Failed ECC-MgO specimens compared to ECC-controls

On the other hand, Figure 6-14 (B) shows that only natural cured-MC and water cured-Failed ECC$\mathrm{MgO}$ specimens recovered the UPV's of controls at a later age. It should be noted that water curedFailed ECC-MgO specimens produced the highest UPV readings $(4260 \mathrm{~m} / \mathrm{s}$ ) amongst all specimens. This might be related to the presence of lots of micro cracks in $\mathrm{MgO}$ specimens. After applying multiple crack (MC) loading case, the number of cracks in multiple cracked ECC-MgO specimens in addition to the crack width increased. Storing the specimens in water continuously for one month until the next reloading to failure allowed more water to flow through cracks and hence - aggravates the hydration of both calcium and magnesium crystals. This lead to more crack filling/sealing produced by $\mathrm{MgO}$ agent (due to the expansive effect of $\mathrm{MgO}$ ) and consequently producing higher UPVs for ECC-MgO specimens than ECC ones. This might lead to the fact that most of the cracks present in water cured-Failed ECC-MgO specimens were sealed faster than their ECC counterparts. However, higher UPVs do not necessarily mean higher strength recovery of specimens. The UPV test is generally used to evaluate the quality of concrete based on the presence of voids and internal cracks but excellent UPVs may lead to a stiff and brittle concrete (Panzera et al., 2011). Referring to Figure 6-13 (A), water cured-Failed ECC-MgO specimens showed the lowest strength recovery $(6.3 \mathrm{kN})$ after 300 days. 
Table 6-8: Differences in UPVs between first and last day of water and natural cured MC/Failed ECC and ECC-MgO specimens

\begin{tabular}{|c|c|c|c|c|}
\hline Mix Designation & Curing Type & $\begin{array}{c}7+30 \text { days } \\
(\mathrm{m} / \mathrm{s})\end{array}$ & $\begin{array}{c}7+270 \text { days } \\
(\mathrm{m} / \mathrm{s})\end{array}$ & $\begin{array}{l}\text { UPV difference } \\
(\mathrm{m} / \mathrm{s})\end{array}$ \\
\hline ECC-MC & \multirow{2}{*}{ Water } & 4086 & 4225 & 139 \\
\hline ECC-MgO-MC & & 4063 & 4230 & $\underline{167}$ \\
\hline ECC-MC & \multirow{2}{*}{ natural } & 3678 & 3969 & $\overline{291}$ \\
\hline ECC-MgO-MC & & 3595 & 3964 & 369 \\
\hline Mix Designation & Curing Type & $\begin{array}{c}7+60 \text { days } \\
(\mathrm{m} / \mathrm{s})\end{array}$ & $\begin{array}{c}7+300 \text { days } \\
(\mathrm{m} / \mathrm{s})\end{array}$ & $\begin{array}{l}\text { UPV difference } \\
(\mathrm{m} / \mathrm{s})\end{array}$ \\
\hline ECC-Fail & \multirow{2}{*}{ Water } & 4137 & 4240 & 103 \\
\hline ECC-MgO-Fail & & 4152 & 4260 & $\underline{108}$ \\
\hline ECC-Fail & \multirow{2}{*}{ natural } & 3738 & 3883 & 145 \\
\hline ECC-MgO-Fail & & 3632 & 3793 & $\underline{161}$ \\
\hline
\end{tabular}

As shown in Table 6-8, all MC/failed ECC-MgO specimens showed a tendency to develop higher UPVs than ECC specimens over a long period of curing $(7 d+270 \& 7 d+300$ days). This was indicated by the difference between UPVs at 7d+30 and 7d+270 days which were $139 \mathrm{~m} / \mathrm{s}$ and 167 $\mathrm{m} / \mathrm{s}$, respectively of water cured ECC and ECC-MgO MC specimens compared to $291 \mathrm{~m} / \mathrm{s}$ and $369 \mathrm{~m} / \mathrm{s}$, respectively of natural cured ECC-MC and ECC-MC-MgO specimens. Additionally, the UPVs at 7d+60 and 7d+300 days of water cured ECC and ECC-MgO Failed specimens were $103 \mathrm{~m} / \mathrm{s}$ and $108 \mathrm{~m} / \mathrm{s}$, respectively; while they were $145 \mathrm{~m} / \mathrm{s}$ and $161 \mathrm{~m} / \mathrm{s}$ for the same Failed specimens cured under natural condition (see Table 6-8).

\subsubsection{Long-term study of strength recovery of self-healed ECC \& ECC-MgO specimens}

The self-healing behavior of ECC-MgO system over long period of curing time is studied based on strength recovery, deflection development, energy absorption, UPV development and crack characteristics of a set of prismatic specimens cured under water and natural conditions over 15 months (7d+450 days) of curing (Table 6-9 and Figure 6-15). All specimens were arranged based on Table 6-3 - set 3+. Similar to the specimens (as described earlier) cured up to $7 d+9$ (7 days and 9 months), water and natural cured ECC-MgO specimens cured up to $7 \mathrm{~d}+15$ developed almost 
same or even better failure strengths, ultimate deflections and UPV values than ECC specimens (highlighted in bold in Table 6-9).

Table 6-9: Maximum load/strength and ultimate deflection of water and natural cured self-healed ECC and $\mathrm{ECC}-\mathrm{MgO}$ specimens over long term

\begin{tabular}{|c|c|c|c|c|c|c|c|c|c|c|}
\hline \multirow[b]{2}{*}{$\begin{array}{c}\text { Mix } \\
\text { Designation }\end{array}$} & \multirow[b]{2}{*}{$\begin{array}{c}\text { Time (days + } \\
\text { Months) }\end{array}$} & \multirow[b]{2}{*}{$\begin{array}{l}\text { Loading } \\
\text { Stage }\end{array}$} & \multicolumn{2}{|c|}{ Water } & \multicolumn{2}{|c|}{ Natural } & \multicolumn{2}{|c|}{ Water } & \multicolumn{2}{|c|}{ Natural } \\
\hline & & & 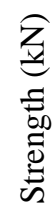 & 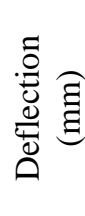 & 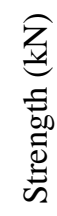 & 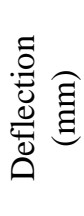 & 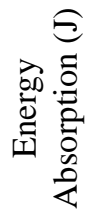 & 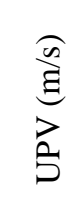 & 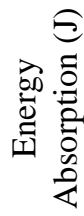 & 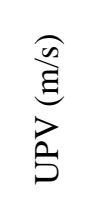 \\
\hline$\overline{E C C}$ & $7 d+15$ & Control & 9.2 & 6.2 & 8.6 & 7.0 & 44.9 & 4270 & 45.1 & 4013 \\
\hline Specimens & $7 d+15$ & Fail & 7.4 & 3.2 & 9.4 & 4.2 & 11.0 & 4295 & 24.7 & 3969 \\
\hline ECC-MgO & $7 d+15$ & Control & 8.0 & 4.8 & 6.6 & 4.5 & 29 & 4265 & 29.9 & 3938 \\
\hline Specimens & $7 d+15$ & Fail & 7.6 & 3.1 & 9.3 & $\mathbf{5 . 0}$ & 15.9 & 4270 & $\underline{29.4}$ & 4027 \\
\hline
\end{tabular}

*Control: Specimens without cracking failed at 7d+15months; Fail: Cracked specimens at $7 d$ reloaded to failure at $7 d+15$ months

Long term cured specimens reloaded to failure exhibited a reduction in stiffness and deflection capacities compared to control specimens showing higher (usually associated with air curing) or similar strength development (Figure 6-15). 

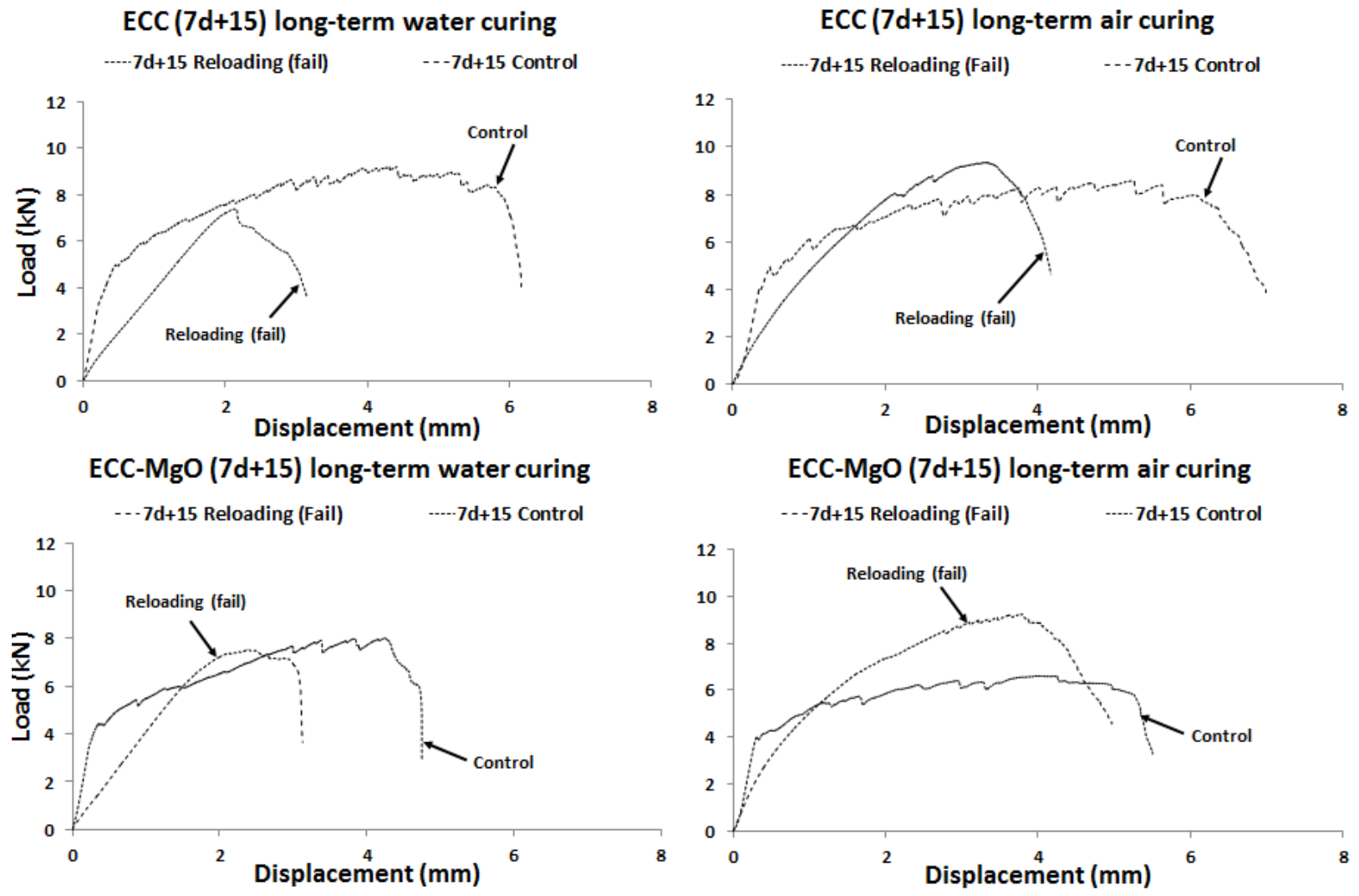

Figure 6-15: Maximum load vs ultimate deflection curves of self-healed ECC and ECC-MgO specimens over 15 months of curing under water and natural conditions

Figure 6-16 summarized the strength recovery, deflection development and energy absorption capacity recovery as derived from load vs. deflection curves shown in Figure 6-15 by relating failure (at $7 d+450$ ) loading case to the control (at $7 d+450$ ) cured under both curing conditions in addition to UPV development. As shown in Figure 6-16 (A), under long-term water curing condition, none of ECC or ECC-MgO specimens recovered the strength of the controls but specimens cured under long-term natural conditions recovered the strength of controls. Although the strength of ECC control specimens was higher than those of ECC-MgO cured under both conditions, the ability of strength recovery for Failed-ECC-MgO specimens to recover the strength of controls was higher than ECC specimens. This was signified by the high strength percentage between Failed and control specimens of ECC-MgO (95.0\%) than ECC specimens (80.4\%) under water curing condition. The strength percentage between Failed and control strengths of similar 
specimens cured under natural condition were 109.3\% (ECC) and 140.9\% (ECC-MgO), respectively (see Figure 6-16 - A). Additionally, under same kind of specimens (either ECC or ECC-MgO), the water cured controls developed greater strengths $(9.2 \mathrm{kN}>8.6 \mathrm{kN}$ in ECC and $8 \mathrm{kN}$ $>6.6 \mathrm{kN}$ in ECC-MgO controls) than the ones cured under natural conditions. However, the natural cured Failed specimens of both mixes recovered more strength than same specimens cured under water condition. It should be noted that natural cured Failed ECC-MgO specimens developed $(140.9 \%)$ the highest strength recovery under all loading cases.

Similar to strength recovery, long term deflection development of ECC and ECC-MgO specimens is shown in Figure 6-16 - B. Of all, only natural cured Failed-ECC-MgO specimens recovered the deflection of controls with a percentage of $111.0 \%$ between failed loading cases. Although the load/strength of ECC controls was higher than ECC-MgO controls (Figure 6-16 - A), the deflection development in ECC controls was higher than ECC-MgO controls after a long term of water and natural curing. Higher strength development in ECC controls (with no crack) did not indicate better performance compared to ECC-MgO controls as in field condition, it would be rare to have crack-free concrete structures because of the presence of very small micro cracking formed once formwork removed. Even with low load/strength of ECC-MgO controls, the deflection recovery of Failed ECC-MgO specimens was almost similar $(3.1 \mathrm{~mm})$ to Failed ECC specimens $(3.2 \mathrm{~mm})$ under long-term water curing condition while it was better under long-term natural curing condition $(5.0 \mathrm{~mm}>4.2 \mathrm{~mm})$. Therefore, the high deflection recovery in pre-cracked (damaged) ECC-MgO specimens provided a vital means of assessing the self-healing capability of ECC mixtures in this study. Again, under same kind of specimens (either ECC or ECC-MgO), the natural cured control or failed specimens developed greater deflection than the ones cured under water curing conditions. The highest deflection recovery was observed for natural cured Failed ECC (4.2 mm) and ECC-MgO specimens (5.0 mm) (Figure 6-16 - B). 


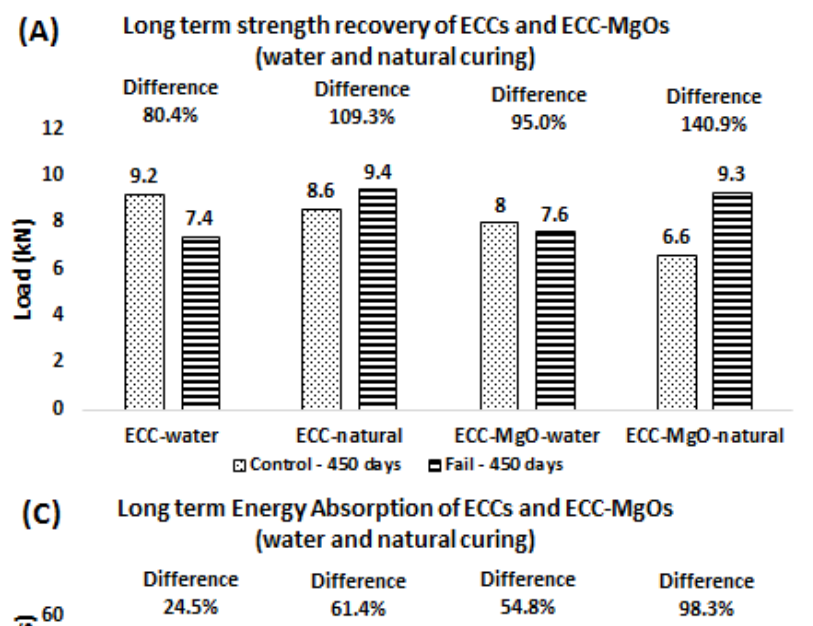

(B) Long term deflection development of ECCs and ECC-MgOs
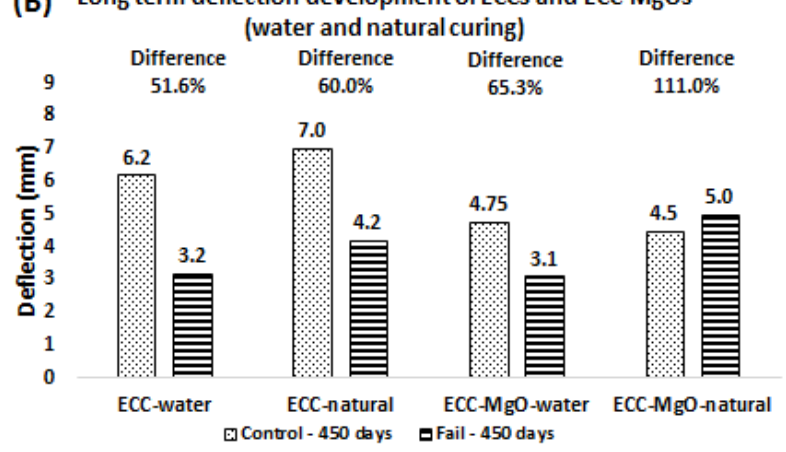

(D) Long term UPV development of ECCs and ECC-MgOs

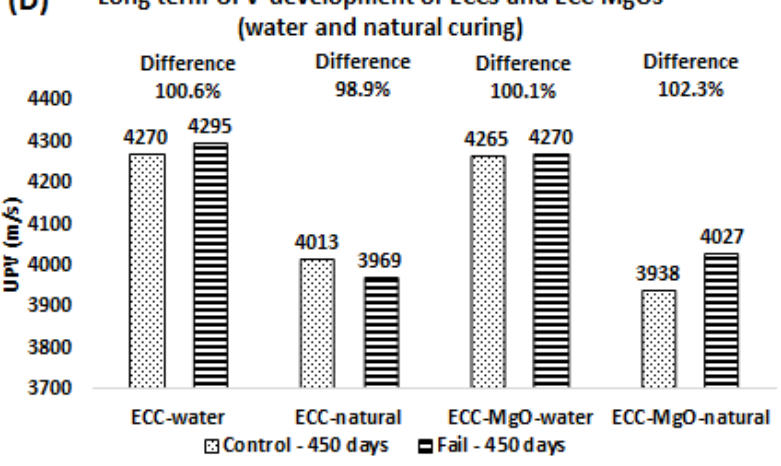

Figure 6-16: Strength recovery (part-A), deflection development (part-B), Energy absorption recovery (part-C) and UPV development (part-D) of water \& natural cured ECC and ECC-MgO specimens over 15 months of curing

Figure 6-16 - C shows that the energy absorption of control ECC specimens was higher than the ECC-MgO ones cured under both conditions. The Failed-ECC-MgO specimens cured under water and natural curing conditions recovered the greatest energy absorption capacity amongst similar ECC specimens cured under same conditions. However, none of Failed ECC or ECC-MgO specimens recovered the energy absorption capacity of the controls. The capacity of energy recovery of Failed ECC specimens to recover the energy of controls was lower than ECC-MgO specimens under both curing conditions. The percentage of energy absorption between the control and Failed ECC specimens was $24.5 \%$ and $61.4 \%$ when cured under water and natural conditions, respectively. On the other hand, the percentage of similar ECC-MgO specimens cured under similar conditions was 54.8\% and 98.3\%, respectively (see Figure 6-16 - C). Under same specimen type (either ECC or ECC-MgO), it was noted that the natural curing condition developed higher recovery in energy absorption capacity of controls/Failed specimens than water curing condition. 
This might be related to higher deflection recovery of natural cured control/Failed ECC and ECC$\mathrm{MgO}$ specimens than the ones cured under water (see Figure 6-16 - B). For instance, the recovery of natural cured Failed ECC and ECC-MgO specimens developed highest energy absorption $61.4 \%$ and $98.3 \%$ and highest deflection recovery (60.0\% and $111.0 \%$ ), respectively (as shown in Figure 6-16 - B and C).

Figure 6-16 - D compares the long term UPV development of water and natural cured ECC and ECC-MgO specimens. Similar to UPV development shown in Figure 6-9, the water cured control/Failed ECC and ECC-MgO specimens developed higher UPV values than natural cured ones. According to Figure 6-14, only 10-month water cured Failed-ECC-MgO specimens recovered the UPV of controls in Failed specimens. However, based on Figure 6-16 - D, all 15month water or natural (field) cured Failed specimens recovered the UPV of controls except the natural cured Failed ECC specimens (98.9\%). Similar to 10 months water cured Failed-ECC-MgO specimens (mentioned in Figure 6-14 - B), 15 months water cured Failed ECC specimen developed $(4295 \mathrm{~m} / \mathrm{s})$ the highest UPV amongst all other specimens - this explains the weakness in strength recovery of the same specimen $(7.4 \mathrm{kN}$ ) (see Figure 6-16 - A). It can be concluded that extreme UPVs can produce brittleness in concrete. However, from Figure 6-16-D, although the natural cured Failed-ECC-MgO specimens developed/recovered (102.3\%) the highest UPVs amongst all other specimens, it developed/recovered (140.9\%) the highest load/strength along with (111.0\%) the highest deflection capacity and (98.3\%) the highest energy absorption of the control specimens amongst all other specimens (Figure 6-16). This confirms the strongest tendency to self-heal the micro cracks than ECC specimens which indicated through enhanced mechanical performance, energy absorption capacity and UPVs development; which might be related to the presence of MEA in ECC-MgO mixtures which characterized by enhanced tensile strain capacity.

\subsubsection{Crack characteristics}

Table 6-10 compares the crack pattern of 450-day water and natural cured ECC and ECC-MgO specimens formed before and after failure loading. The old cracks formed after pre-loading case (at 7 days) were marked in green by a green colored marker. Then the failed specimens were 
marked in blue by a blue colored marker to reveal the newly formed cracks at new locations considered as an evidence of self-healing process (Figure 6-17).

Table 6-10: Crack characteristics of ECC and ECC-MgO specimens before and after failure cured under water and natural conditions

\begin{tabular}{|c|c|c|c|c|c|c|}
\hline \multirow{2}{*}{ Designation } & \multirow{2}{*}{$\begin{array}{c}\text { Curing } \\
\text { Type }\end{array}$} & \multicolumn{2}{|c|}{$\begin{array}{c}\text { Old cracks formed } \\
\text { after pre-loading } \\
\text { (at 7 days)* }\end{array}$} & \multicolumn{2}{|c|}{$\begin{array}{c}\text { New cracks formed } \\
\text { after loading to failure } \\
\text { (at 450 days) }\end{array}$} & \multirow{2}{*}{$\begin{array}{c}\text { of new cracks formed } \\
\text { at new locations }\end{array}$} \\
\cline { 3 - 6 } & Number & $\begin{array}{c}\text { Width } \\
(\mu \mathrm{m})\end{array}$ & Number & $\begin{array}{c}\text { Width } \\
(\mu \mathrm{m})\end{array}$ & \\
\hline \multirow{2}{*}{$\begin{array}{c}\text { ECC } \\
\text { specimens }\end{array}$} & Water & 30 & $<110$ & 15 & same & $50 \%$ \\
\cline { 2 - 6 } $\begin{array}{c}\text { ECC-MgO } \\
\text { specimens }\end{array}$ & Watural & 50 & $<200$ & 13 & same & $26 \%$ \\
\cline { 2 - 6 } & Natural & 44 & $<150$ & 24 & same & $47 \%$ \\
\hline
\end{tabular}

*These cracks were observed just before the failure loading at the age of 450 days

From Table 6-10, it was observed that the number of cracks and the crack width just before reloading to the failure of water and natural cured ECC and ECC-MgO specimens were almost similar. After reloading to failure after 450 days, the percentage of new cracks formed compared to old ones for water cured ECC and ECC-MgO specimens was almost similar. Under natural curing condition, the percentage (55\%) of formed new cracks for ECC-MgO specimens was almost double that (26\%) of ECC specimens (Table 6-10). Figure 6-17 - (C) revealed that natural cured ECC-MgO specimens developed the highest number of new cracks ( 24 cracks) after reloading to failure. This might indicate the superior self-healing characteristics of natural cured $\mathrm{ECC}-\mathrm{MgO}$ specimens - exhibited by the capability of developing more micro-cracking associated with high strain hardening and ductility behavior. It can asspciate with the slow rate of reactivity of $\mathrm{MgO}$ expansive agent over the long extended period of curing producing crack healing compounds. According to Figure 6-16 (B-C), the natural cured Failed-ECC-MgO specimens developed the highest deflection recovery and energy absorption capacity compared to all other specimens cured for 450 days. 


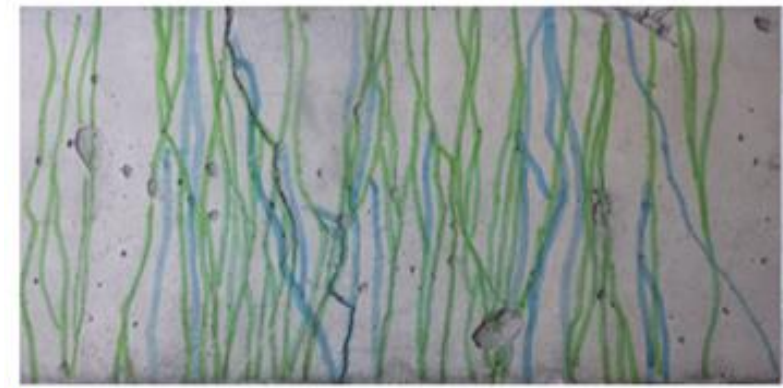

(A) ECC-natural curing

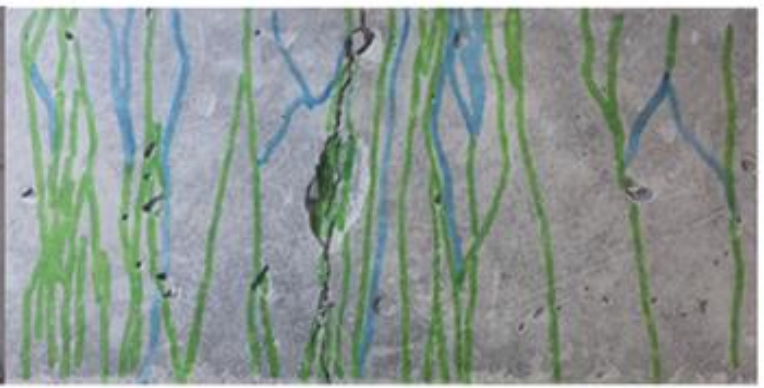

(B) ECC-water curing

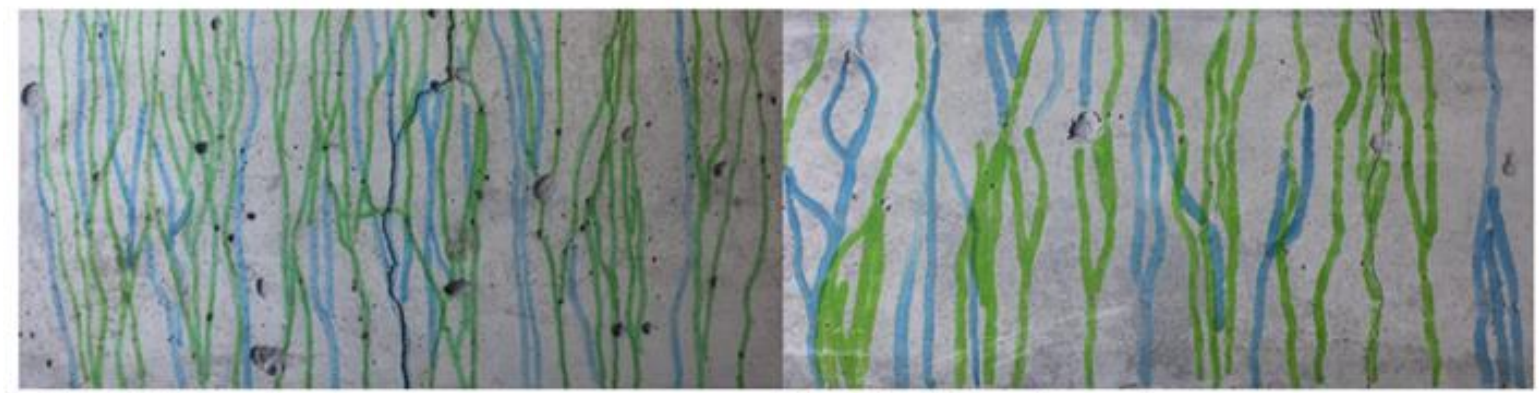

(c) ECC-MgO-natural curing

(D) ECC-MgO-water curing

Figure 6-17: Formation of new cracks after reloading to failure of self-healed natural or water cured ECC and ECC-MgO specimens: old cracks in green formed at 7 days (after pre-loading case), new cracks in blue formed after failure loading applied after 450 days of self-healing

Figure 6-18 shows surface observation of self-healed cracks of 450 days water and natural cured ECC and ECC-MgO specimens. It should be noted that the cracks were observed before loading to failure at 450 days. All cracks less than $30 \mu \mathrm{m}$ wide in natural cured specimens were sealed

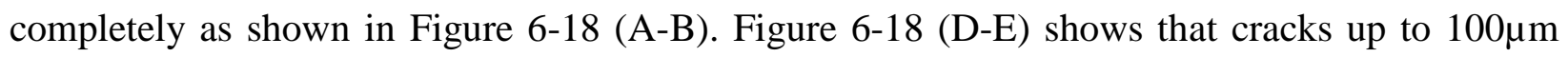
width in water cured specimens had higher tendency to seal/close completely - which was not the case for natural cured specimens. Generally, self-healed water cured cracks were sealed and filled completely by precipitation of white healing crystals (which can be seen/observed by naked eye which was not the case for natural cured cracks (Figure 6-18 C and F). 


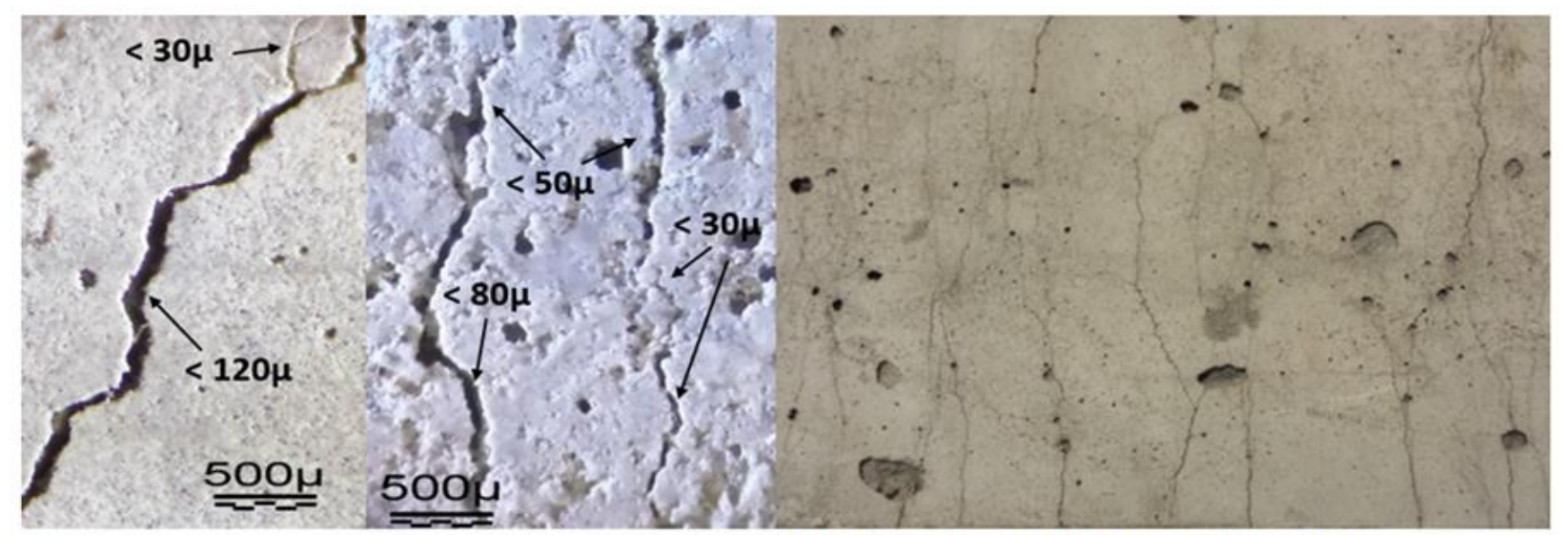
A) ECC-natural
B) ECC-MgO-natural
C) Crack layout of 450 days natural curing

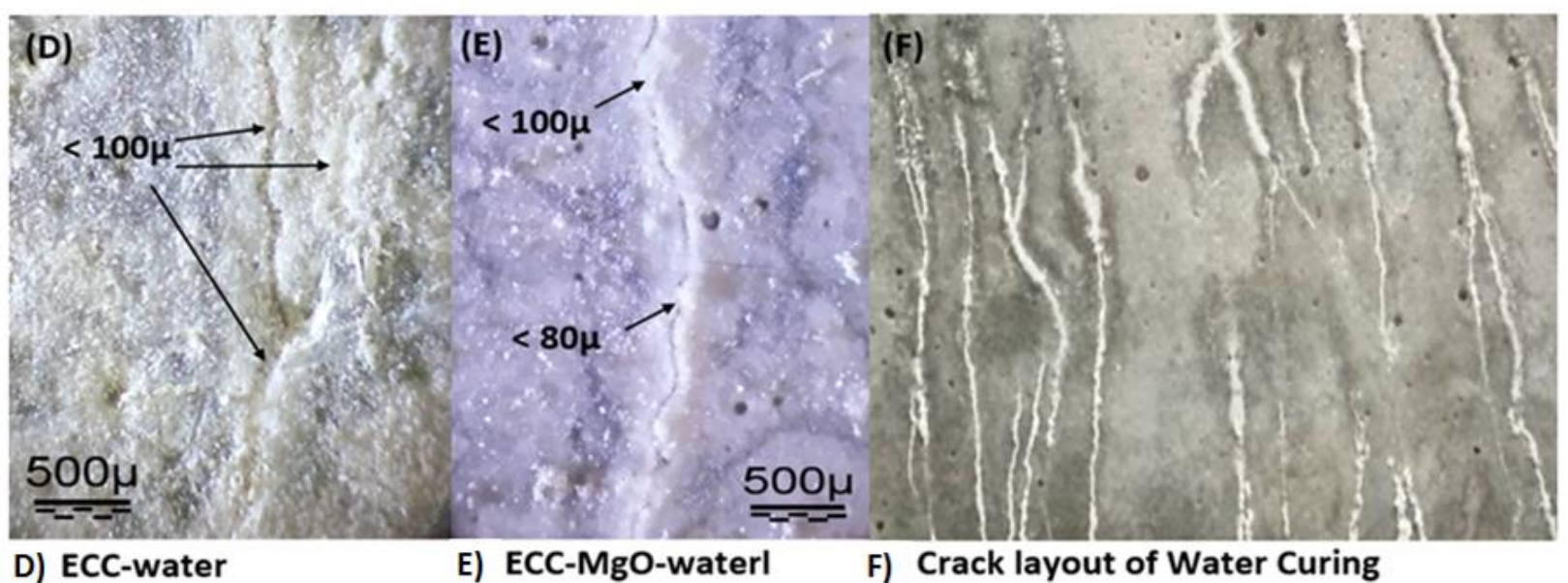

Figure 6-18: Surface observation of self-healed ECC and ECC-MgO specimens under water and natural curing conditions over 450 days - cracks were observed before loading to failure at 450 days

The harsh Canadian environment accompanied with heavy snowfall might be the reason of washing out of the deposited healing products inside cracks in natural cured specimens. However, it is believed that the washing out of healing products occurred only at the surface of crack and it did not appear to happen deep inside the cracks. Also, most studies in the literature have revealed that the healing products are formed of C-S-H and $\mathrm{CaCO}_{3}$ which are very difficult to be washed out easily even by heavy snowfalls. This fact was emphasized by Herbert \& Li (2013) who believed that the washed out healing products were calcium hydroxide (formed during cement hydration) before being consumed by the slow pozzolanic reaction. The satisfactory mechanical 
performance of natural cured specimens compared to water cured ones (as observed in this research) has confirmed that the natural curing is much better/effective than water curing - even with the lack of healing products at the surface of the natural cured cracks.

\subsubsection{Effect of multiple damages under repeated reloading on load/strength recovery of self-healed ECC \& ECC-MgO specimens}

Multiple reloading events were repeated on same water and natural cured pre-cracked ECC and ECC-MgO prismatic specimens - the strength and deflection characteristics are summarized in Table 6-11. The effect of repeated re-loading was assessed based on load carrying capacity as per arrangement in Table 6-3 - set 4-. It should be noted that repeatability in this research was defined by reloading events applied 4 times every 3 months up to 10 months (at $7 d+1,7 d+4,7 d+7$ and $7 \mathrm{~d}+10)$ on the same specimens cured under the same curing condition to the same pre-loading level $(60 \%)$ at 7 days. Further, all re-loading events of multiple damages were repeated on the same ECC or ECC-MgO specimens whether cured under water or natural curing condition. All results are drawn in one graph to show the load/strength recovery and deflection development (Figure 6-19).

Table 6-11: Maximum load strength and ultimate deflection of multiple damaged ECC and ECC-MgO specimens over long term of water and natural curing conditions

\begin{tabular}{|c|c|c|c|c|c|c|c|c|c|}
\hline \multirow[b]{3}{*}{$\begin{array}{c}\text { Time } \\
\text { days + months } \\
\text { (days + days) }\end{array}$} & \multirow[b]{3}{*}{$\begin{array}{l}\text { Loading } \\
\text { Stage }\end{array}$} & \multicolumn{4}{|c|}{ ECC Specimens } & \multicolumn{4}{|c|}{ ECC-MgO Specimens } \\
\hline & & \multicolumn{2}{|c|}{ Water } & \multicolumn{2}{|c|}{ Natural } & \multicolumn{2}{|c|}{ Water } & \multicolumn{2}{|c|}{ Natural } \\
\hline & & 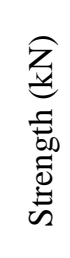 & 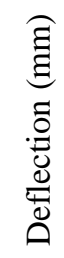 & 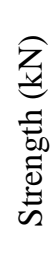 & 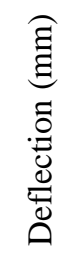 & 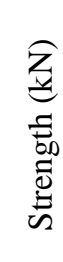 & 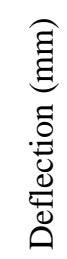 & 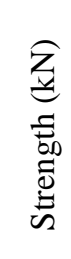 & 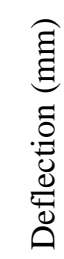 \\
\hline $7 \mathrm{~d}+0$ & Pre-Loading & $\underline{8.7}$ & $\underline{5.5}$ & $\underline{8.5}$ & $\underline{5.5}$ & 7.2 & 4.5 & 6.2 & 4.5 \\
\hline $7 d+1(7 d+30)$ & Re-Loading & $\overline{8.8}$ & $\overline{5.5}$ & $\overline{8.6}$ & $\overline{5.5}$ & 7.7 & 4.5 & 6.6 & 4.5 \\
\hline $7 d+4(7 d+120)$ & Re-Loading & 8.1 & $\underline{3.3}$ & 7.3 & 3.7 & 7.9 & 3.0 & 7.0 & 4.5 \\
\hline $7 d+7(7 d+210)$ & Re-Loading & $\overline{5.8}$ & $\overline{2.5}$ & $\overline{5.7}$ & 2.9 & 5.9 & 2.5 & 6.4 & $\overline{3.4}$ \\
\hline $7 d+10(7 d+300)$ & Re-Loading & 4.4 & 2.2 & 4.6 & 2.5 & 5.0 & $\overline{1.9}$ & 5.0 & 3.1 \\
\hline
\end{tabular}


As shown in Table 6-11 \& Figure 6-19, the maximum load/strength of re-loaded ECC and ECC$\mathrm{MgO}$ specimens varied from $8.80 \mathrm{kN}$ to $4.40 \mathrm{kN}$ and $7.90 \mathrm{kN}$ to $5.00 \mathrm{kN}$, respectively. Similar to load/strength, the ultimate deflection (deflection capacity) of re-loaded ECC and ECC-MgO specimens varied from $5.5 \mathrm{~mm}$ to $2.20 \mathrm{~mm}$ and $4.50 \mathrm{~mm}$ to $1.90 \mathrm{~mm}$, respectively, depending on curing type and the age of specimens. Table 6-11 shows that ECC specimens at early ages $(7 \mathrm{~d}+0$, $7 \mathrm{~d}+1$ and $7 \mathrm{~d}+4$ ) produced higher load/strength than their ECC-MgO counterparts cured under both water and natural conditions. However, ECC-MgO specimens started to develop consistently reloading strength greater than ECC ones weather cured under water or natural curing condition at later ages $(7 d+7$ and $7 d+10)$. For convenience, all similar or enhanced re-loading strengths of ECC-MgO specimens were highlighted in Table 6-11.
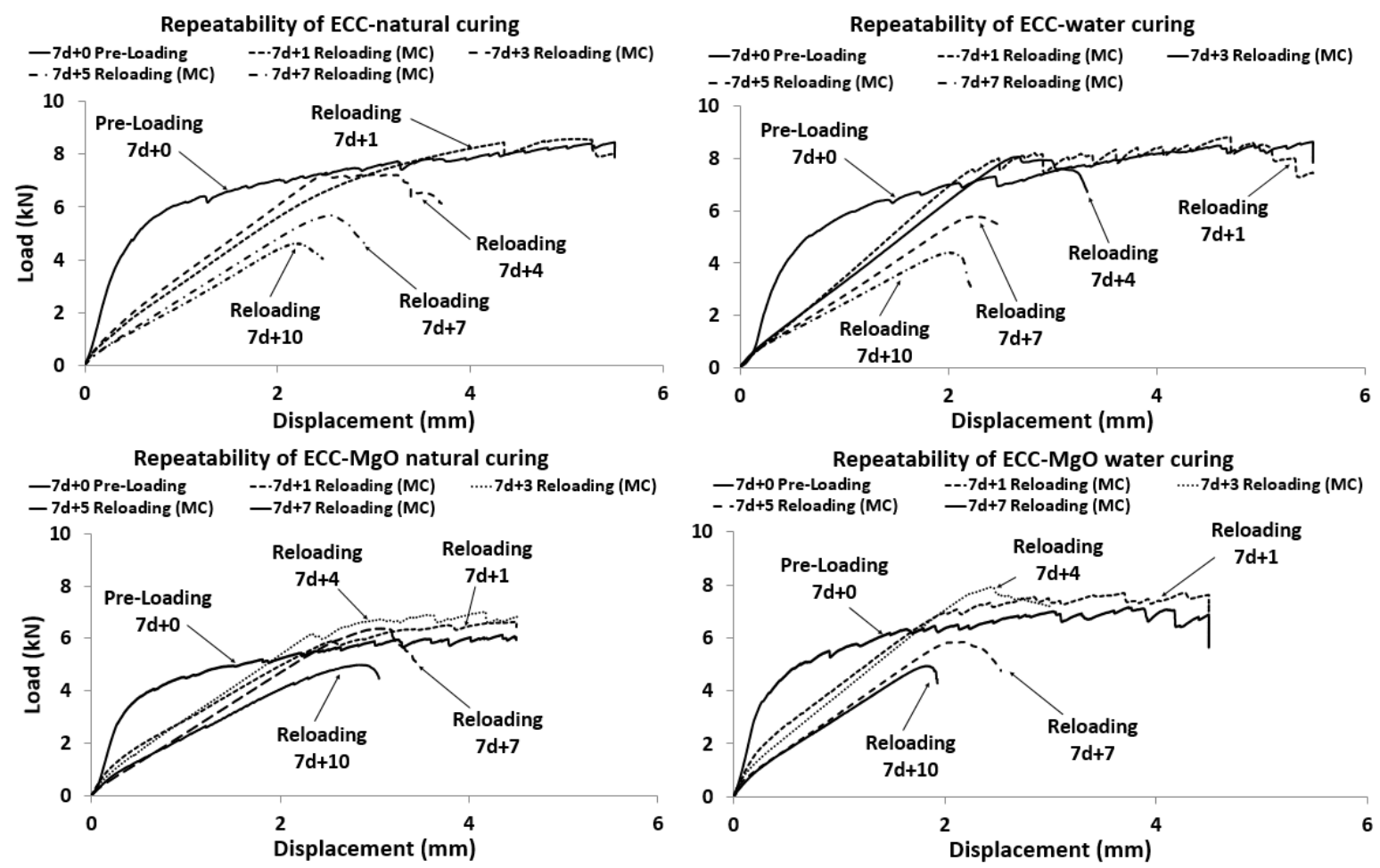

Figure 6-19: Load vs ultimate deflection curves of multiple damaged long term water and natural cured $\mathrm{ECC}$ and $\mathrm{ECC}-\mathrm{MgO}$ specimens

Figure 6-20 shows the percentages of strength and UPV development when related to the controls (at 7-days) as a function of time. Every point in this figure signifies the percentage of strength recovery and UPV development at different times and curing conditions - the first point at $7 \mathrm{~d}+0$ 
considered as an origin point (control point). From Figure 6-20 (A-B), the water cured ECC-MgO specimens recovered the pre-loaded strength at both $7 d+1$ and $7 d+4$ re-loading while natural cured ECC-MgO specimens recovered the pre-loaded strength even after 7 months of re-loading $(7 \mathrm{~d}+7)$. Eventually, the slope of strength reduction of water and natural cured ECC-MgO specimens were flat and recovered $69.4 \%$ to $80.6 \%$ of pre-loaded strength at the end of re-loading events $(7 \mathrm{~d}+10)$ (see Figure 6-20 -B). On the other hand, only the strength of $7 d+1$ reloading of ECC specimens (whether natural cured or water cured) almost recovered the strength of the pre-loaded event. Afterward, the slope of strength reduction of water and natural cured ECC specimens started to decrease steeply until reached around $50.9 \%$ to $54.4 \%$ of pre-loaded strength. In general, the natural cured specimens started to develop more strengths than water cured ones in ECC specimens after $7 d+7$ re-loading event and only after 7d+1 in ECC-MgO specimens (see Figure 6-20 A-B). As was expected, Figure 6-20 (C-D) show that the water cured re-loaded ECC and ECC-MgO specimens developed greater UPVs than similar specimens cured under natural condition. All reloaded specimens at the age of $7 d+10$ recovered the pre-loaded UPVs (at $7 d+0$ ) except the natural cured ECC specimens $(98.5 \%$ at $7 \mathrm{~d}+10)$ than the control (Figure 6-20 - C). Furthermore, the water cured re-loaded ECC-MgO specimens developed the highest UPVs development than all other specimens (110.3\%) which was $107.4 \%$ in water cured re-loaded ECC specimens at $7 \mathrm{~d}+10$ (Figure 6-20 - D). This indicates that the water cured ECC-MgO specimens has a stronger tendency of recovering the UPVs of pre-loaded specimens (controls at origins) than the counterpart ECC specimens. Under natural curing condition, the re-loaded event of $7 \mathrm{~d}+10$ for ECC-MgO specimens recovered the UPVs of pre-loaded event with only $0.1 \%$ extra while ECC specimens did not recover the pre-loaded UPV and the percentage of UPVs was $-1.5 \%$ between the first and last day of re-loading events $(98.5-100 \%=-1.5 \%)$ (Figure 6-20 C-D).

Figure 6-21 shows the percentages of deflection development and energy absorption capacity (derived from load vs. deflection curves shown in Figure 6-19) as a function of time. From Figure 6-21 (A-B), at the first re-loading event $(7 \mathrm{~d}+1)$, all water and natural cured ECC and ECC-MgO specimens recovered the $60 \%$ deflection of $7 d+0$ (pre-loaded deflection capacity). The recovery of pre-loaded deflection capacity was extended to $7 \mathrm{~d}+4$ only in the case of natural cured ECC$\mathrm{MgO}$ specimens $(100 \%=4.5 \mathrm{~mm}$ at $7 \mathrm{~d}+1$ and $7 \mathrm{~d}+4)($ Figure 6-21 B). After the age of $7 \mathrm{~d}+4$, water 
and natural cured ECC-MgO specimens developed deflection capacity more than ECC specimens cured under similar conditions. It should be noted that natural curing condition in both ECC and ECC-MgO specimens developed greater deflection capacity than water curing condition (Figure 6-21 - A \& B).
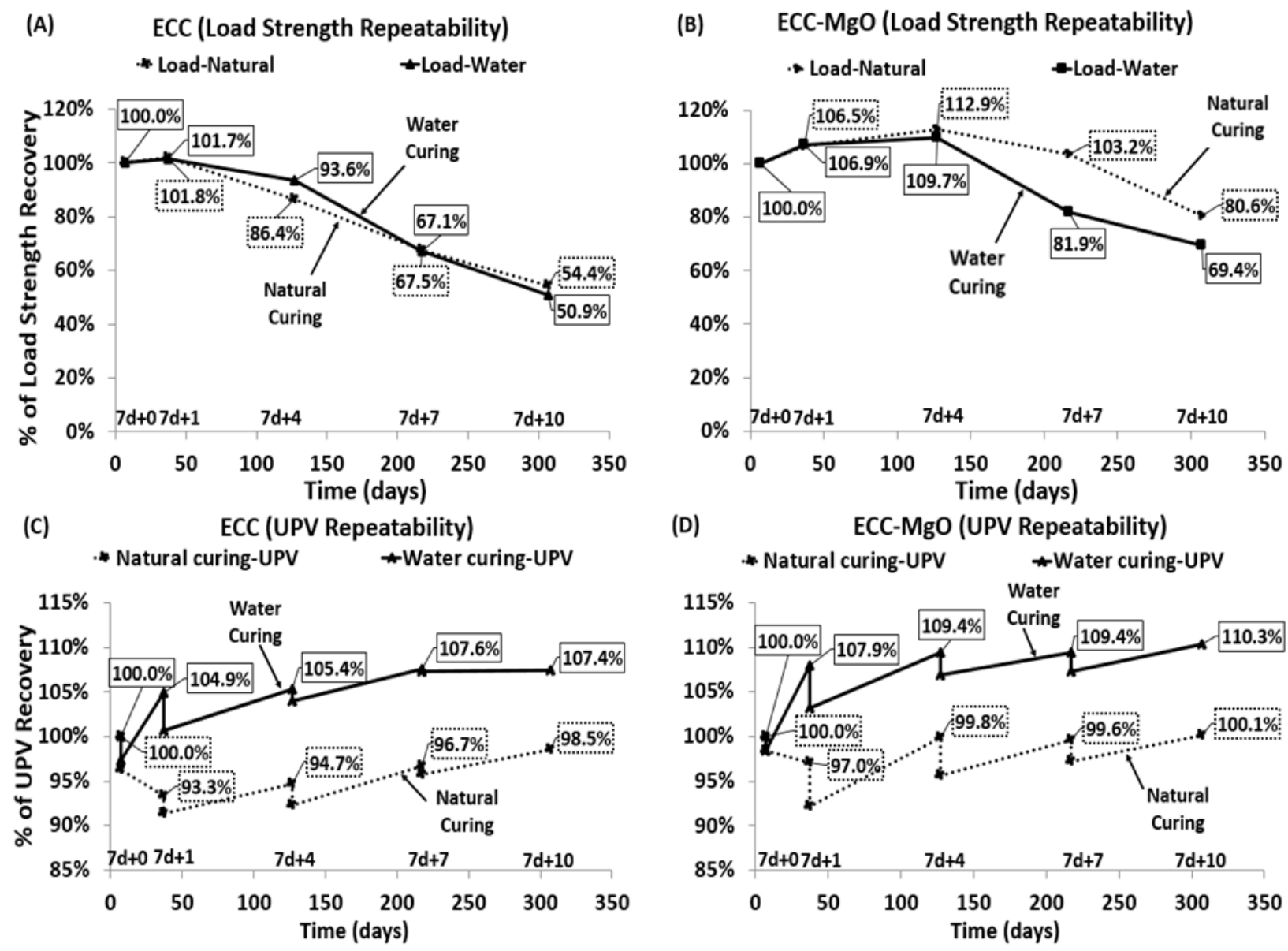

Figure 6-20: Strength recovery (part-A \& B) and UPV development (part-C \& D) of multiple damaged $\mathrm{ECC}$ and ECC-MgO specimens over long term of water and natural curing conditions

Due to greater percentages of load/strength and deflection recoveries of water and natural cured ECC-MgO specimens than ECC specimens (see Figure 6-20 - B \& Figure 6-21 - B), ECC-MgO specimens developed the greatest energy absorption capacity than their ECC counterparts (Figure 6-21 C-D). Consistently, ECC-MgO specimens whether cured under water or natural conditions developed the highest energy absorption than ECC specimens cured under similar conditions. Again, the natural curing condition produced higher energy absorption capacity of ECC-MgO 
specimens than water curing condition (Figure 6-21 - C \& D) and started to increase slightly after $7 \mathrm{~d}+7$ in counterpart ECC specimens. It could be concluded that the natural cured ECC-MgO specimens developed the highest strength recovery (80.6\%), almost the lowest UPVs (100.1\%), the highest deflection capacity (67.8\%) and the highest energy absorption (37.2\%) of the control specimens amongst of all other specimens after applying 4 multiple damage events (through 10 months of repeated loading) on the specimens. The enhanced mechanical properties of natural cured ECC-MgO specimens indicated the strongest capability to self-heal the micro cracks than ECC specimens.
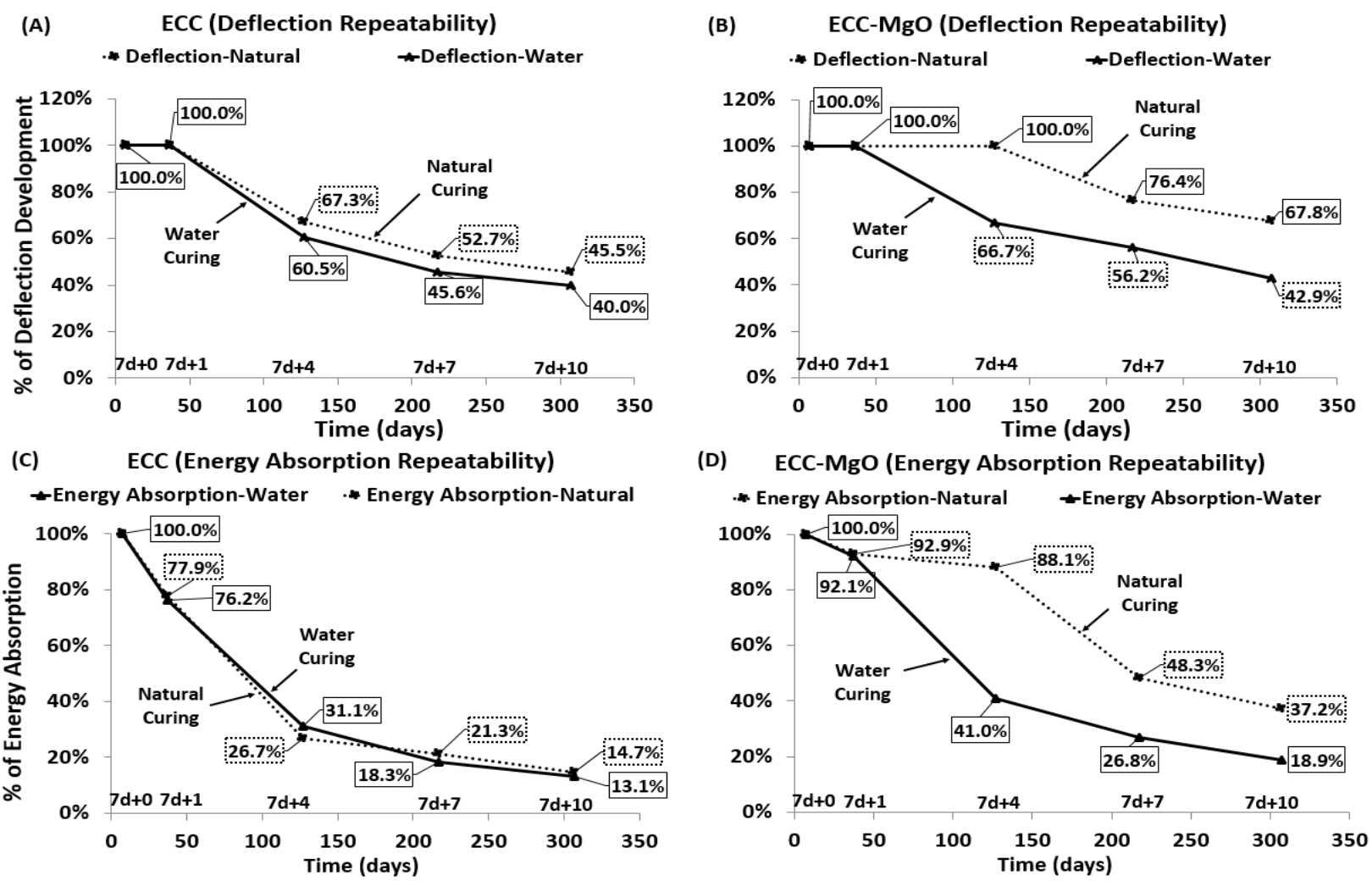

Figure 6-21: Deflection development (A \& B) and energy absorption (C \& D) of multiple damaged ECC and $\mathrm{ECC}-\mathrm{MgO}$ specimens over long-term water and natural curing 


\subsubsection{Strength recovery of ECC-MgO self-healing system under autoclave curing}

The self-healing capability of proposed ECC-MgO system was assessed through the load strength development of ECC-MgO mixtures by accelerating the hydration of $\mathrm{MgO}$ at the age of 28 days through autoclave curing. The arrangement of the specimens was done based on Table 6-3 - set 5 . In this method, the goal was to evaluate the strength/development of the filling effect of MEA in ECC-MgO controls after expediting the $\mathrm{MgO}$ hydration; in addition to assessing the strength/recovery of pre-cracked specimens under autoclave curing. Enhancement in mechanical performance is an evidence of the feasibility of self-healing ECC-MgO system

As seen from Figure 6-22 - A, both ECC and ECC-MgO controls before applying autoclave test showed almost similar load/strength at the age of 28 days $(\mathrm{ECC}=10.5 \mathrm{kN}$ and $\mathrm{ECC}-\mathrm{MgO}=10.8$ $\mathrm{kN})$. However, after applying autoclave test, the strength development of ECC-MgO control was significantly improved $(15.2 \mathrm{kN})$ compared to ECC control $(11.5 \mathrm{kN})$. Due to the presence of $\mathrm{MgO}$ in $\mathrm{ECC}-\mathrm{MgO}$ specimens, autoclave test accelerated quickly the hydration of $\mathrm{MgO}$ leading to a quick growth of $\mathrm{Mg}(\mathrm{OH})_{2}$ crystals by combining together the available $\mathrm{OH}^{-}$with $\mathrm{Mg}^{2+}$ ions. Moradpour et al. (2013) confirmed that larger volumes will be formed due to the expansive nature of $\mathrm{MgO}$ hydration which might cause better filling effect in microstructure and hence, leading to a significant improve in strength development as shown in Figure 6-22 - A. Figure 6-22 - B shows the self-healing capability of pre-cracked ECC and ECC-MgO specimens after conducting autoclave test. The ECC and ECC-MgO specimens were pre-loaded before autoclave test based on the limit of $50 \%$ of multiple cracked deflections of the virgins at 28 days. The residual strength of ECC-MgO specimens was almost doubled $(1.8 \mathrm{kN})$ compared to ECC specimens $(0.8 \mathrm{kN})$. The enhanced strength recovery of autoclave cured pre-cracked ECC-MgO specimens than their ECC counterparts indicated that ECC-MgO had more ability to seal the micro cracks due to filling effect of $\mathrm{MgO}$ expansive agent (see Figure 6-22 - B). 

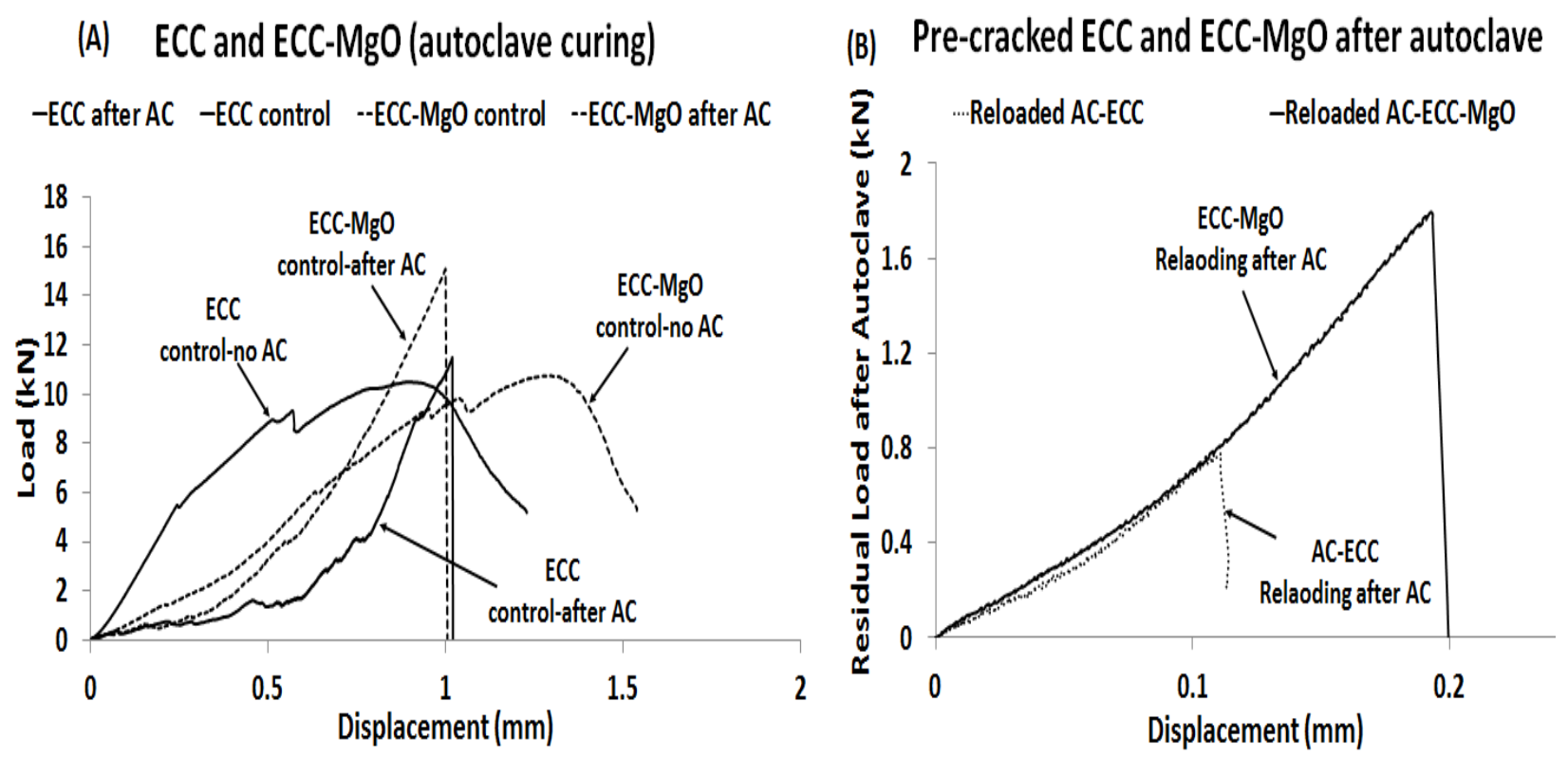

Figure 6-22: (A) Load vs. ultimate deflection curves of ECC and ECC-MgO controls and (B) pre-cracked ECC and ECC-MgO specimens before and after autoclave curing

Figure 6-23 - A shows UPVs of ECC and ECC-MgO controls before and after autoclave test shown in Figure 6-15 - A. It can be seen that the tendency of developing more UPVs of autoclave cured ECC-MgO controls was higher than ECC controls due to filling effect of MEA. The percentage between ECC-MgO controls before and after autoclave test was $98.8 \%$ while it was 96.2\% in ECC controls. Figure 6-23 - B shows UPVs for pre-cracked ECC and ECC-MgO specimens before and after autoclave test. It can be seen that autoclave cured pre-loaded ECC$\mathrm{MgO}$ specimens recovered (100.4\%) of their origin of UPVs before autoclave test while ECC counterpart did not recover their origin UPVs (96.0\%). This might be related to the filling effect of the expansive nature of MEA hydration. It should be noted that all UPVs in Figure 6-23 (A-B) were measured on same ECC or ECC-MgO specimens before and after autoclave test. 
(A)

UPV development of ECCS and ECC-Mg0 controls

(autoclave curing)

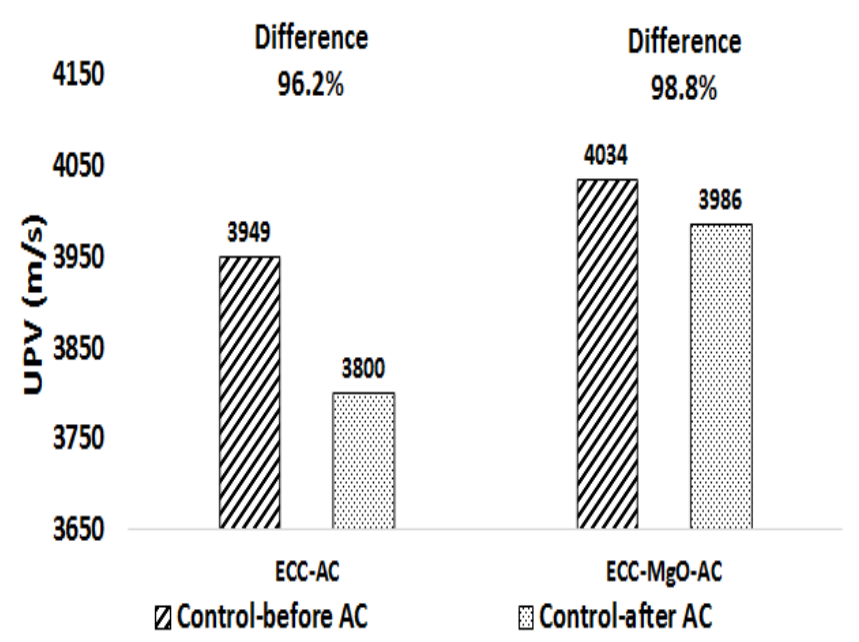

(B)

UPV development of pre-cracked ECCs and ECC-Mg0

(autoclave curing)

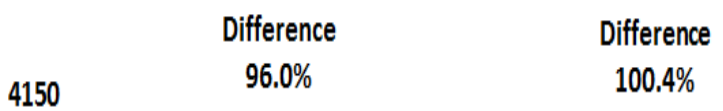

4050

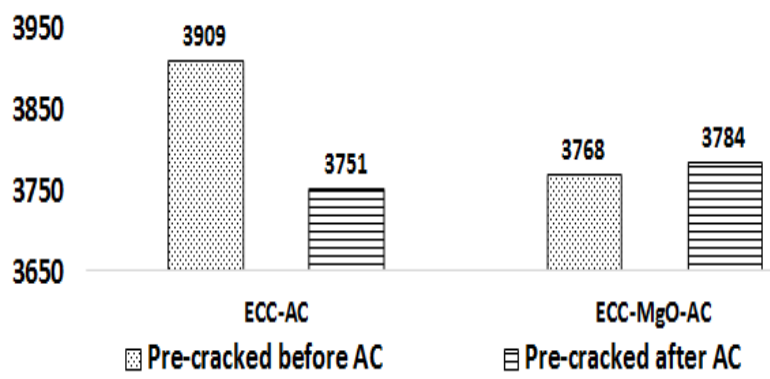

Figure 6-23: (A) UPV development of ECC and ECC-MgO controls and (B) pre-cracked ECC and ECC$\mathrm{MgO}$ specimens before and after autoclave curing (AC)

It can be concluded that autoclave curing accelerated the slow activity of $\mathrm{MgO}$ in ECC-MgO specimens which stimulated the load/strength development and UPVs of control/pre-loaded ECC$\mathrm{MgO}$ specimens than their ECC counterparts. 


\subsection{Summary}

This chapter presented the performance of engineered cementitious composites (ECCs) when produced with $\mathrm{MgO}$ type expansive agent (MEA) and utilized as a self-healing agent to heal micro cracks. The influence of the addition of 5\% MEA as fly ash replacement and water/natural curing in addition to autoclave curing condition was investigated through studying the recovery of compressive strength and load/strength performance and UPV values. All mechanical properties recovery/development characteristics of ECC- $\mathrm{MgO}$ self-healing system were compared and related to counterpart ECC controls. Several methods were used to quantify the long-term selfhealing capability of proposed $\mathrm{ECC}-\mathrm{MgO}$ system by studying the strength development of control and the development/recovery of pre-loaded/multiple cracked/failed/repeated multiple damaged (repeatability test) specimens. Crack characterizations, surface crack observation, length/width/number of cracks were also studied under the long term of curing. 


\section{CHAPTER SEVEN}

\section{MECHANICAL AND DURABILITY PROPERTIES OF SELF-HEALED CEMENTITIOUS COMPOSITES WITH MGO-TYPE EXPANSIVE AGENT}

\subsection{Introduction}

The current chapter presents the performance of ECC and ECC-MgO mixtures based on the heat of hydration and expansion/drying shrinkage of bar specimens. Additionally, the effectiveness of proposed ECC-MgO self-healing system was evaluated based on the recovery of mechanical and durability properties (rapid chloride ion penetration 'RCP', sorptivity, extended freeze/thaw resistance and recovered load/strength and maximum deflection) in addition to Thermogravimetric Analysis (TGA), Differential Scanning Calorimetry (DSC) and Scanning Electron Microscope (SEM) analyses. All properties studied in this chapter were assessed when controls (virgin without cracking) and pre-loaded (cracked or damaged) ECC and ECC-MgO specimens were cured under laboratory, water, natural or accelerated autoclave curing conditions for different ages. The subject matter of this Chapter is significant as it provides useful information on performance characteristics of proposed ECC-MgO self-healing system under various curing conditions including those exists in the field - hence engineers who will involve in constructing structures using proposed ECC-MgO self-healing system in practical field applications will be benefited. 


\subsection{Experimental Investigations}

Magnesium Oxide (MgO) was prepared based on chapter 4. In order to do so, the best calcination system was considered when Magnesite (Magnesium Carbonate, $\mathrm{MgCO}_{3}$ ) was exposed to $900^{\circ} \mathrm{C}$ for 2 hours by using $45 \mu \mathrm{m}$ particle size of $\mathrm{MgCO}_{3}$. Class-F fly ash was found to be the best supplementary cementing material (SCM) to produce ECC-MgO system. In this chapter, the performance of ECC and ECC-MgO mixtures was assessed based on fresh state and mechanical properties while durability properties were evaluated to reveal the expansive behavior of MEA when used as a self-healing agent to heal ECC micro cracks. The efficiency of ECC-MgO selfhealing system was assessed based on examining the permeation properties (RCP and sorptivity), frost resistance (freeze-thaw resistance) and the residual load strength of controls/preloaded prism specimens after conducting freeze/thaw over a long period of cycles. In addition, autoclave cured disk specimens were tested for RCP and sorptivity in order to expedite the effect of $\mathrm{MgO}$ expansion. TGA/DSC and SEM analyses were conducted, as well. The properties and performance characteristics of laboratory, water, natural and autoclave cured $\mathrm{ECC}-\mathrm{MgO}$ specimens were compared with their ECC counterparts.

\subsubsection{ECC-MgO Mixture Proportions}

To assess the effectiveness of MEA in ECC-MgO self-healing system, two ECC mixtures were designed and selected. The first mix was produced by Class-F fly ash and silica sand with cement replacement of $\mathrm{FA} / \mathrm{PC}=1.2(\mathrm{FA} / \mathrm{Binder}=55 \%)$ considered as ECC-control. The second mix (ECC-MgO) was same as the control but MEA was added to it. The mixture proportions and designations for both ECC mixtures are given in Chapter 6 - Table 6-1.

\subsubsection{Preparation of ECC-MgO specimens}

Fresh, mechanical and durability properties of ECC and ECC-MgO mixtures namely heat of hydration, expansion/drying shrinkage, rapid chloride permeability, sorptivity, freeze/thaw cycles and residual load strength of prismatic specimens exposed to freezing and thawing cycling were evaluated. 


\subsubsection{Heat of hydration}

Cylindrical samples of $\varnothing 150 \times 300 \mathrm{~mm}$ were used in the heat of hydration test of both EC mixtures in accordance with (ASTM C1679, 2014). Calorimeter apparatus (isothermal calorimetry) was used to measure the total heat of hydration released during the hydration process as a function of chemical composition and the amount of cementitious materials in ECC and ECC-MgO mixes. Before putting into the calorimeter, the temperature of all mixed materials (even water) should be identical to the room temperature, $23 \pm 2{ }^{\circ} \mathrm{C}$. At the end of mixing process, ECC or ECC-MgO cylindrical samples were placed into calorimeter within $5 \mathrm{~min}$ and the data acquisition for the heat of hydration started immediately and continued recording for 72 hours. Figure 7-2 (A) shows the setup for the heat of hydration test.

\subsubsection{Bar specimens}

Four sets, consisting of $3 \mathrm{bars} /$ prisms $(25 \times 25 \times 285 \mathrm{~mm}$ in dimensions $)$ for each set, were produced for both ECC and ECC-MgO mixtures cured under water and laboratory conditions. Therefore, a total number of 12 bars were prepared for both mixtures cured under both curing conditions. After casting, all bar specimens were cured in plastic bags at $95 \pm 5 \%$ relative humidity $(\mathrm{RH}), 23 \pm 2$ ${ }^{\circ} \mathrm{C}$. All bars were demolded after 24 hours and initial length measure was taken right after the demolding in accordance with (ASTM C490, 2011). After obtaining the initial length, two sets of bars out of four were cured under water condition while the two sets were cured naturally to measure the effect of expansion/drying shrinkage of bar specimens produced with or without $\mathrm{MgO}$ agent, respectively. The length measurement readings of all bar specimens were collected up to 300 days of curing. Bar specimens for water expansion test were cured in water at $20^{\circ} \mathrm{C}$ until the next length measure in accordance with (ASTM C1038-b, 2014); while drying shrinkage of bar specimens was measured when specimens cured naturally in accordance with (ASTM C157, 2008).

\subsubsection{RCP test}

Four sets, consist of 3 disc specimens for each set, were produced for both ECC and ECC-MgO mixtures cured under water and natural conditions. Therefore, a total number of 12 disc specimens 
were prepared for both mixtures cured under both curing conditions. For RCP test, disc specimens were cut from the mid portion of $100 \times 200 \mathrm{~mm}$ cylinders with a size of $100 \mathrm{~mm}$ in diameter and $50 \mathrm{~mm}$ in thickness. Until the day of testing, specimens were cured in plastic bags at $95 \pm 5 \%$ relative humidity $(\mathrm{RH}), 23 \pm 2{ }^{\circ} \mathrm{C}$ and then prepared for RCP test in accordance with (ASTM C1202, 2012). At the age of 21 days, RCP test was conducted and permeation properties were collected for all disc specimens (12 discs). After 21 days - RCP test, two out of three discs in each set were preloaded to $90 \%$ of a maximum load of previously tested failed controls under axial compression; while the third disc was left without cracking as a control for comparison purposes.

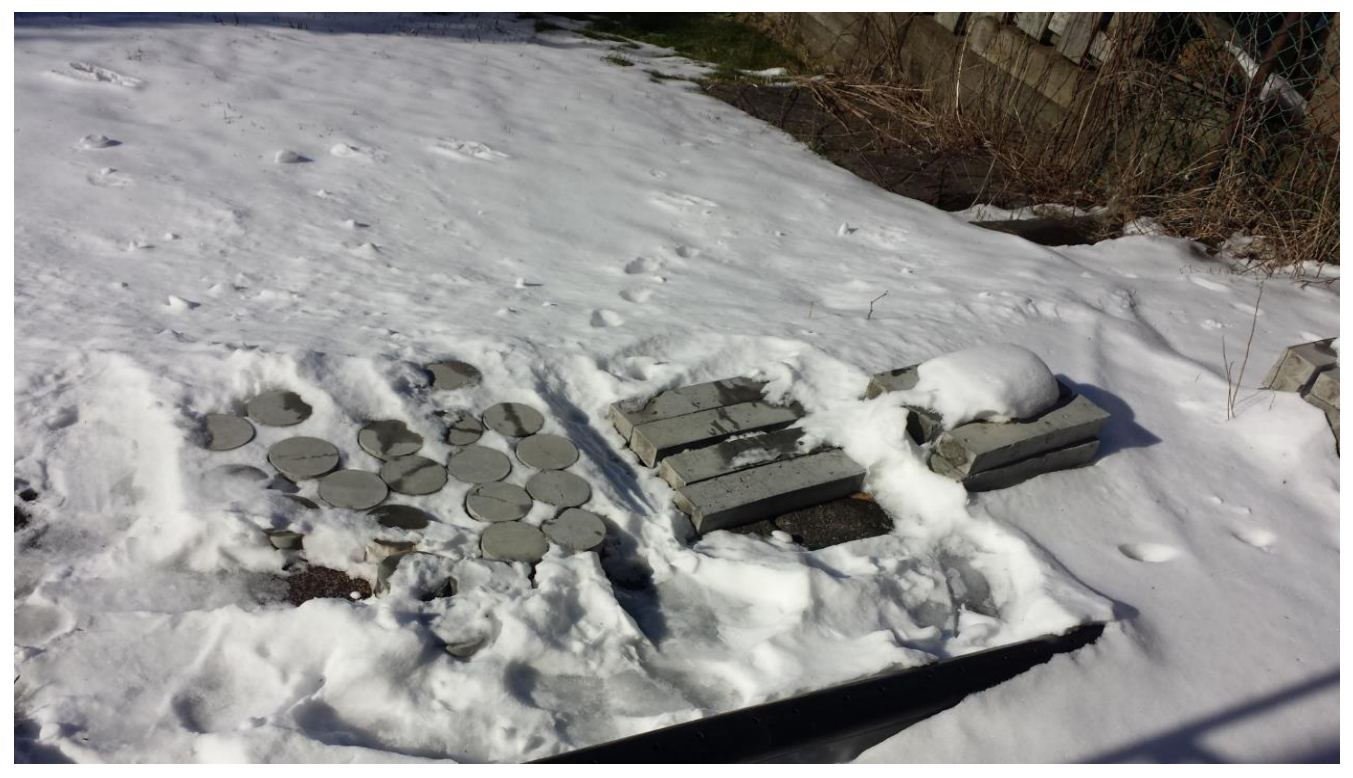

Figure 7-1: Natural curing condition

However, if no cracking occurred after applying the $90 \%$ pre-loading level, application of loading continued after $90 \%$ until a considerable damage can be seen on the specimen's surface. After the pre-loading was released, all pre-loaded cracked specimens were tested immediately so no crack healing was recorded and this test was considered as $21+0$ days $-\mathrm{RCP}$ test. After the age of $21+0$ days, two sets produced with ECC and ECC-MgO mixtures, respectively stored in water until the following testing; while other two sets cured under natural condition (Figure 7-1). RCP test was then repeated on control and preloaded specimens at the ages of 21+45, 21+90 and 21+135 days (total of 156 days). During testing, control and preloaded specimens were subjected to a potential difference of $60 \mathrm{~V}$ for 6 hours when one flat side of the specimen was in contact with sodium 
chloride solution and the other with sodium hydroxide solution. The total charge (measured in Coulombs) passed through the ECC and ECC-MgO disc specimens was determined to assess chloride penetration resistance. Therefore, the quality of ECC-MgO self-healing system was assessed based on charge passed - low charge (coulomb) meant better chloride resistance of ECC mixtures. Figure 7-2 (B) shows the setup of rapid chloride permeability test.

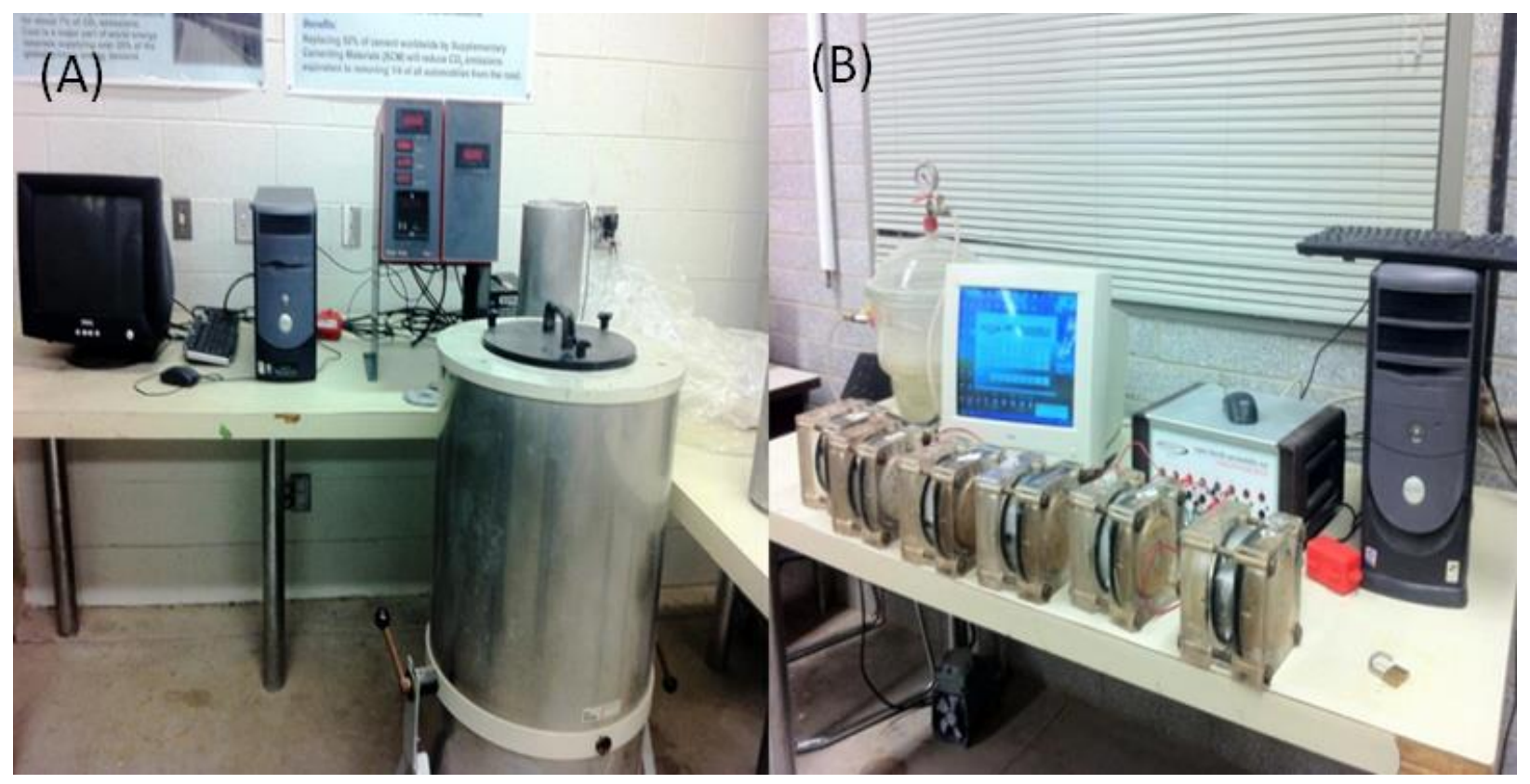

Figure 7-2: Test setup for heat of hydration (A) and rapid chloride permeability test (B)

\subsubsection{Sorptivity test}

Sorptivity test was conducted on four sets consisting of five disc specimens for each of ECC and ECC-MgO mixtures cured under water and natural curing conditions. A total of 20 disc specimens were prepared for both mixtures. The size of sorptivity specimens was $\varnothing 100$ x $50 \mathrm{~mm}$ cut from external portions of $\varnothing 100 \times 200 \mathrm{~mm}$ cylinders. Similar to RCP test, sorptivity specimens were cured in plastic bags at $95 \pm 5 \%$ relative humidity $(\mathrm{RH}), 23 \pm 2{ }^{\circ} \mathrm{C}$ up to 21 days and then prepared for sorptivity test in accordance with (ASTM C1585, 2013). The preparation of specimens included placing the test specimens inside an oven at a temperature of $50 \pm 2{ }^{\circ} \mathrm{C}$ for 3 days and then storing them inside a sealable container for at least 15 days before starting the absorption 
procedure. At the age of 21 days including the 18 days of specimen's preparation, the sorptivity test was conducted and transport properties were collected for all disc specimens (20 discs) at the age of 21+18 days. After the first sorptivity test (conducted at 21+18 days), three out of five discs in each set were preloaded to $90 \%$ of the maximum load of a previously loaded failed controls; while the last two discs were left without cracking as a control for comparison purposes. The preloading proceeded if no cracking occurred until a considerable damage in the specimen's surface was observed. After the pre-loading was released and damages were induced on specimen's surface, the rate of absorption was measured immediately for all control and pre-loaded sorptivity specimens so that no crack healing was permitted and this test was considered as the second sorptivity test conducted at 21+36 days. Finally, after 21+180 days of water and natural curing (see Figure 7-1), the 18 days of specimen's preparation was repeated and the rate of absorption was measured again for ECC and ECC-MgO control and preloaded specimens to assess the effect of ECC-MgO self-healing system. Generally, sorptivity test measures the rate of water penetration through unsaturated concrete by capillary suction.

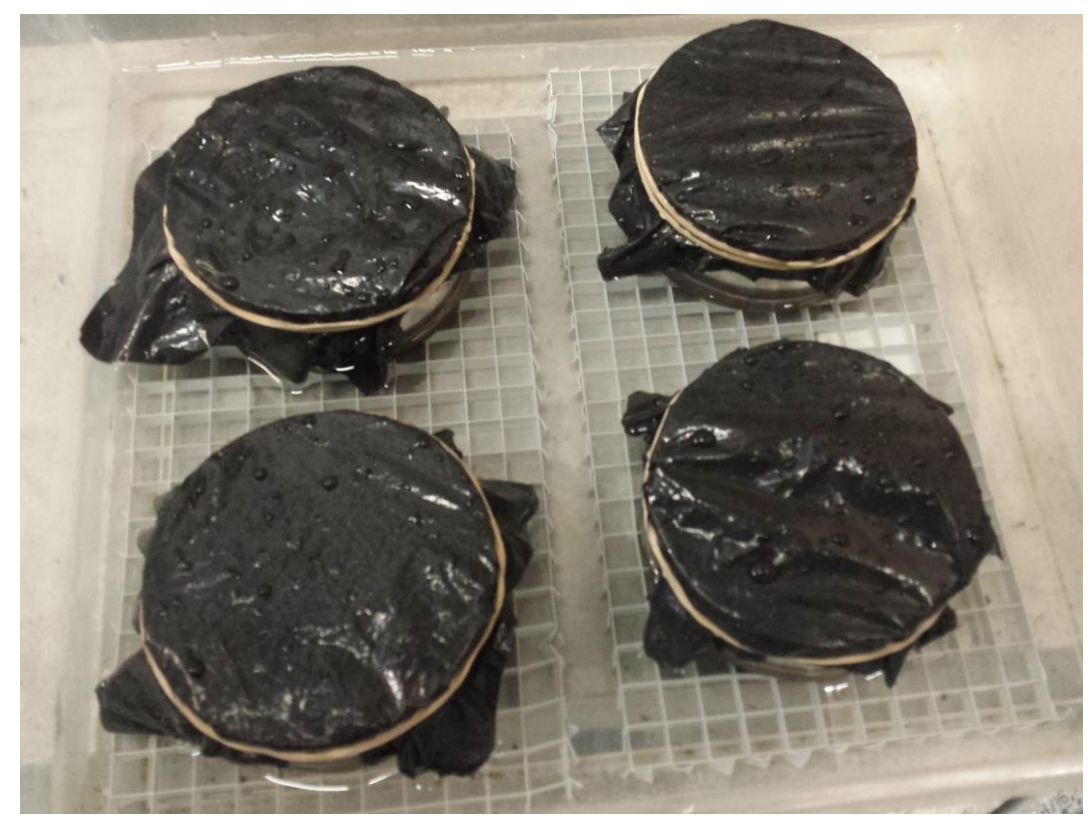

Figure 7-3: Test set-up for sorptivity test 
During the test, mass increase of disc specimens was recorded at recommended time intervals $(0$, $1,5,10,20,30,60,120,180,240,300$ and $360 \mathrm{~min}$ ) due to water absorption. Based on ASTM standard, specimens should be submerged in water to a depth of $2 \pm 1 \mathrm{~mm}$ only from the bottom surface after sealing the side surface by proper sealing material (such as aluminum tape). The other top surface of specimens is sealed with a plastic sheet to control the evaporation of water as shown in Figure 7-3.

\subsubsection{Autoclave cured sorptivity properties of ECC and ECC-MgO specimens}

Autoclave cured sorptivity test was conducted on four sets consisting of four disc specimens made from each of ECC and ECC-MgO mixtures. The total number of disc specimens was 16 produced for both mixtures cured under both curing conditions. Similar to water and natural cured sorptivity test, sorptivity test was conducted at the age of $21+18$ days. After that, specimens were preloaded to $90 \%$ of virgins or pre-loading was proceeded until a considerable damage occurred in specimen's surface. Again and at the age of 21+36 days, sorptivity test was conducted immediately after the loading events were released so no crack healing was permitted. After the second sorptivity test (at $21+36$ ), specimens were cured under laboratory condition for the long term of curing (180 days) and then autoclave test was conducted on all disc specimens made of ECC and ECC-MgO. Eventually, at the age of $21+180$ days, the 18 days of specimen's preparation was repeated on autoclave cured specimens and the rate of absorption was measured again for ECC and ECC-MgO control and preloaded specimens to assess the effect of ECC-MgO self-healing system under autoclave curing.

It is well known that only bar/prism specimens can be used in autoclave test based on (ASTM C151, 2015). For this reason, special autoclave rack was made for disc specimens to fit the autoclave vessel (see Figure 3-5). It should be emphasized that during autoclave test, care should be taken to ensure safety due to the emission of sharp fumes which could be toxic due to the presence of PVA fibers and chemical admixtures in ECC-MgO specimens when exposed to very high pressure and temperature. Consequently, proper ventilation system such as fume hood and complete respiratory protection with ambient air pump must be used. 


\subsubsection{Freezing and thawing cycles}

Under frost resistance test, four sets of prisms were produced from $\mathrm{ECC}$ and $\mathrm{ECC}-\mathrm{MgO}$ mixtures specially to conduct freeze-thaw cycle test. Each set consisted of three prismatic samples with a total number of twelve prisms having dimensions of 50x76x355 mm. The prisms were cured in plastic bags at $95 \pm 5 \%$ relative humidity $(\mathrm{RH}), 23 \pm 2{ }^{\circ} \mathrm{C}$ up to 14 days and then placed into freeze/thaw chamber. During the test, prisms were subjected to alternate freeze/thaw cycles up to 300 cycles in accordance with (ASTM C666, 2015) "Procedure A". After all prisms being subjected to the first 300 freeze/thaw cycles, one out of three prisms in each set was loaded to failure completely (under simply supported four-point loading) and considered as the virgin while the other two prisms in the same set were pre-loaded to $60 \%$ of the maximum deflection of the failed virgin. After pre-loadings were released, prisms were transferred immediately into freeze/thaw chamber for another 300 freeze/thaw cycles - to observe the effect of additional freeze-thaw on cracked samples and self-healing during the freeze-thaw duration. At the end of the second 300 cycles (after 600 cycles), the residual strength and maximum deflection were measured for preloaded $\mathrm{ECC}$ and $\mathrm{ECC}-\mathrm{MgO}$ prisms which subjected to long-term frost damage. Due to the lack of resonance frequency measurement which was recommended by (ASTM C666, 2015), UPV measurement was used in this study similar to many other researchers to measure the damage induced to prisms by freeze/thaw cycles (Kan and Ramazan, 2009; Özbay et al., 2013b). It should be noted that mass loss and Relative Dynamic Modulus of Elasticity (RDME) based on changes in Ultrasonic Pulse Velocity (UPV) were calculated at the end of every 30 cycles and up to 600 cycles. The setup of freezing and thawing cycles is shown in Figure 7-4. 


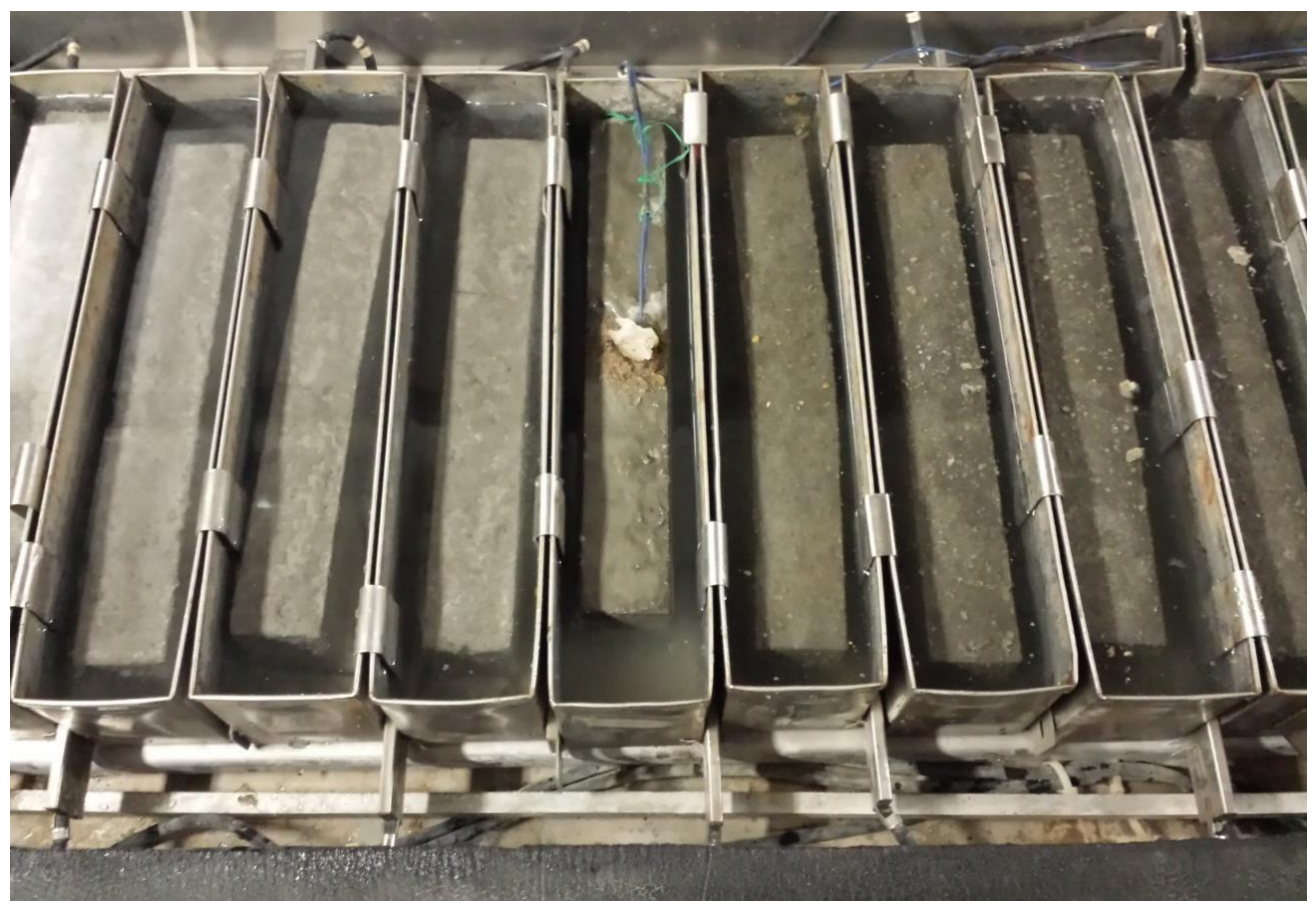

Figure 7-4: Test setup for freezing and thawing cycles test

\subsection{Results and Discussions}

\subsubsection{Heat of Hydration of ECC and ECC-MgO}

The rate of the heat of hydration with time can be measured by the isothermal calorimetry test. More heat evolution is an evidence of more reactivity of the cementitious materials. This indicated by drawing isothermal hydration curves which can provide more knowledge of the properties such as setting time, early strength development, curing methods and mixing times of cementing materials (Mavani, 2012; Mindess et al., 2003).

The evolution of heat of hydration with time for both ECC and ECC-MgO mixes conducted in accordance with (ASTM C1679, 2014) is shown in Figure 7-5. It can be noted from Figure 7-5 that the addition of $\mathrm{MgO}$ expansive agent to $\mathrm{ECC}-\mathrm{MgO}$ mixture had a significant effect to delay the peak of the accelerated period than ECC mixture, i.e. prolonged the induction period. The ECC mix reached the maximum rate of the heat of hydration within 11.25 hours compared to 11.45 hours of ECC-MgO. Similar behavior was observed in Chengmu's investigation (reported by 
Zheng et al. (1992)) who revealed that the time to reach the maximum peak of heat evolution increased when $\mathrm{MgO}$ content increased.

In general, when cement grains are mixed with water, the first hydration products $(\mathrm{C}-\mathrm{S}-\mathrm{H})$ will form and start to coat the cement grains as semi-permeable protective hydrate layers. Therefore, after further hydration, more time is required for water to contact cement grains due to the formation of more protective layers and then dormant period initiated. Simultaneously, $\mathrm{C}_{3} \mathrm{~S}$ starts to release rapidly both calcium and hydroxide ions when water contacts cement grains as well. This stage of hydration slows down quickly (usually within 15 minutes) but continues slowly during the dormant period. During the dormant period, the more protective layers of C-S-H formed around cement grains, the longer diffusion paths produced and hence, more time is needed for calcium and hydroxide ions to diffuse and react with un-hydrated $\mathrm{C}_{3} \mathrm{~S}$ grains. The dormant period might take several hours waiting for calcium and hydroxide ions to reach the maximum of a certain concentration to form rapidly $\mathrm{Ca}(\mathrm{OH})_{2}$ crystals in pore solution. Hence, ending the dormant period and reaching the maximum rate of hydration heat at the end of the accelerated period (Mindess et al., 2003; Zheng et al., 1992).

Referring to Figure 7-5, this case of hydration is compatible with ECC mixtures. However, by adding MEA which has cementitious properties to ECC-MgO mixture, the accelerated period was delayed by about 20 minutes than ECC mixture. Due to smaller solubility product constant of $\mathrm{Mg}(\mathrm{OH})_{2}$ than $\mathrm{Ca}(\mathrm{OH})_{2}$, Zheng et al. (1992) revealed that $\mathrm{Mg}(\mathrm{OH})_{2}$ precipitated in the liquid phase faster than $\mathrm{Ca}(\mathrm{OH})_{2}$ did. Therefore, in addition to forming protective $\mathrm{C}-\mathrm{S}-\mathrm{H}$ layers around the cement grains, Ding Min's studies (reported by (Zheng et al., 1992)) revealed that MgO hydrates can also form protective layers on the surface of cement grains that can retard further cement hydration. Thus, more delay occurs at the peak of the accelerated period as observed in Figure 7-5 which is indicated by the shifting of the heat of hydration curve to the right.

Furthermore, a higher rate of the heat of hydration was observed at the end of the accelerated period for ECC-MgO mixture compared to ECC mixture as shown in Figure 7-5. The ECC-MgO mixture developed $67.37 \mathrm{~mW} / \mathrm{g}$ of the heat of hydration compared to $63.36 \mathrm{~mW} / \mathrm{g}$ of ECC mixture. The amount of fly ash in ECC-MgO mixture was 50\% compared to 55\% (as cement replacement) 
of ECC mix as 5\% of $\mathrm{MgO}$ added to ECC-MgO replaced 5\% fly ash. The presence of 5\% more fly ash in ECC as cement replacement is equivalent to reducing the content of highly reactive $\mathrm{C}_{3} \mathrm{~S}$ in cement by silicon ions. Due to prolonged pozzolanic reaction, a delay might occur to reach the critical concentration of calcium and hydroxide ions to form hydration products leading to decrease the rate of the heat of hydration at the end of the accelerated period of ECC mixtures (Mindess et al., 2003).

Simply, the addition of $\mathrm{MgO}$ to ECC-MgO mixtures delayed the cement hydration which was indicated by increasing the time of accelerated period. Furthermore, the presence of more fly ash in ECC mixtures decreased the maximum rate of the heat of hydration at the end of the accelerated period.

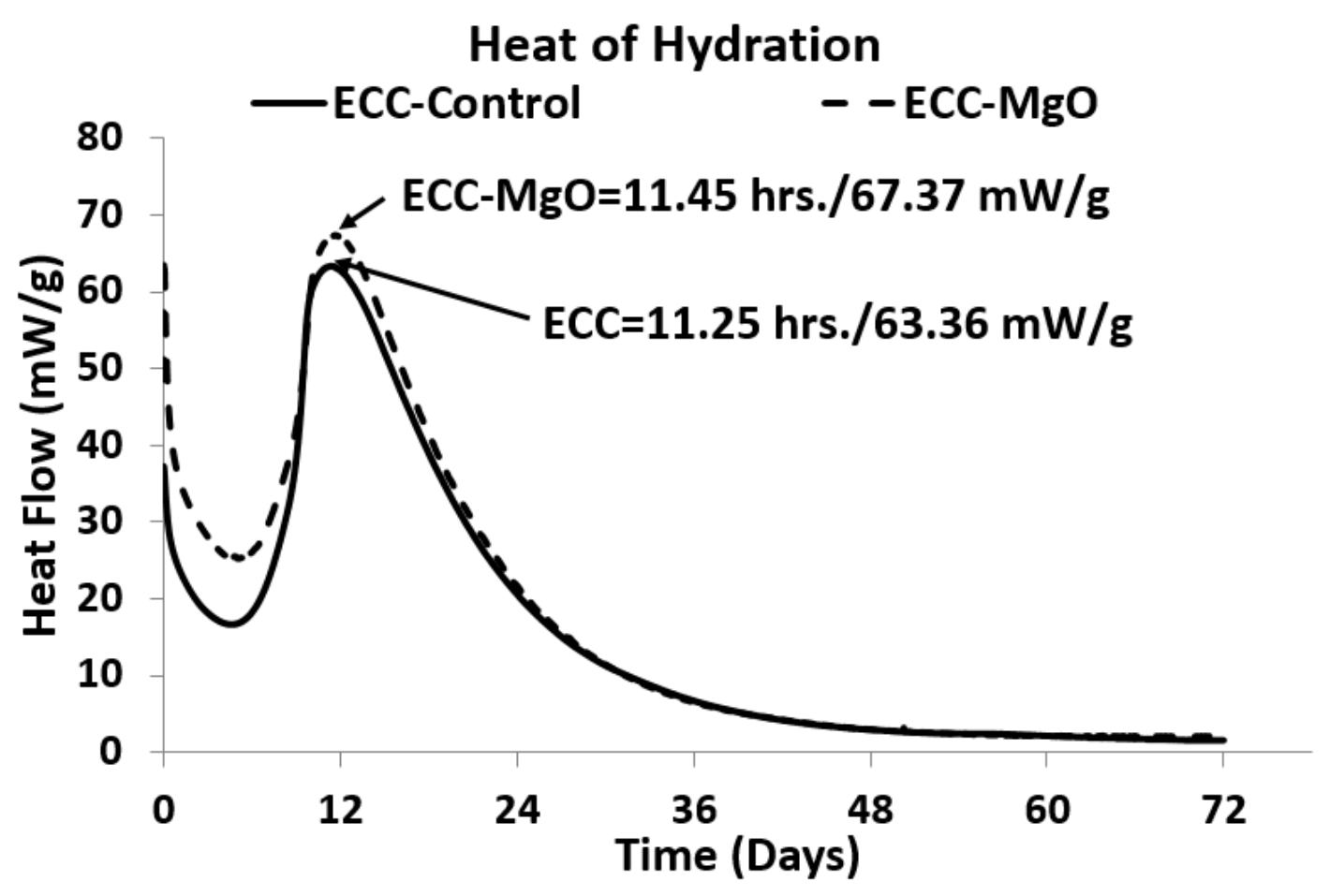

Figure 7-5: Heat of hydration of ECC and ECC-MgO mixes 


\subsubsection{Drying shrinkage and expansion of ECC and ECC-MgO bar specimens}

The drying shrinkage of ECC and ECC-MgO bar specimens cured in water and laboratory (air) condition for 300 days were evaluated. The variation drying shrinkage and expansion with time (each curve shown represents the average measurements of three specimens) for both mixes is presented in Figure 7-6. In general, both expansion and drying shrinkage increased with the increase of age (time) showing a higher rate of increase up to 7 days.

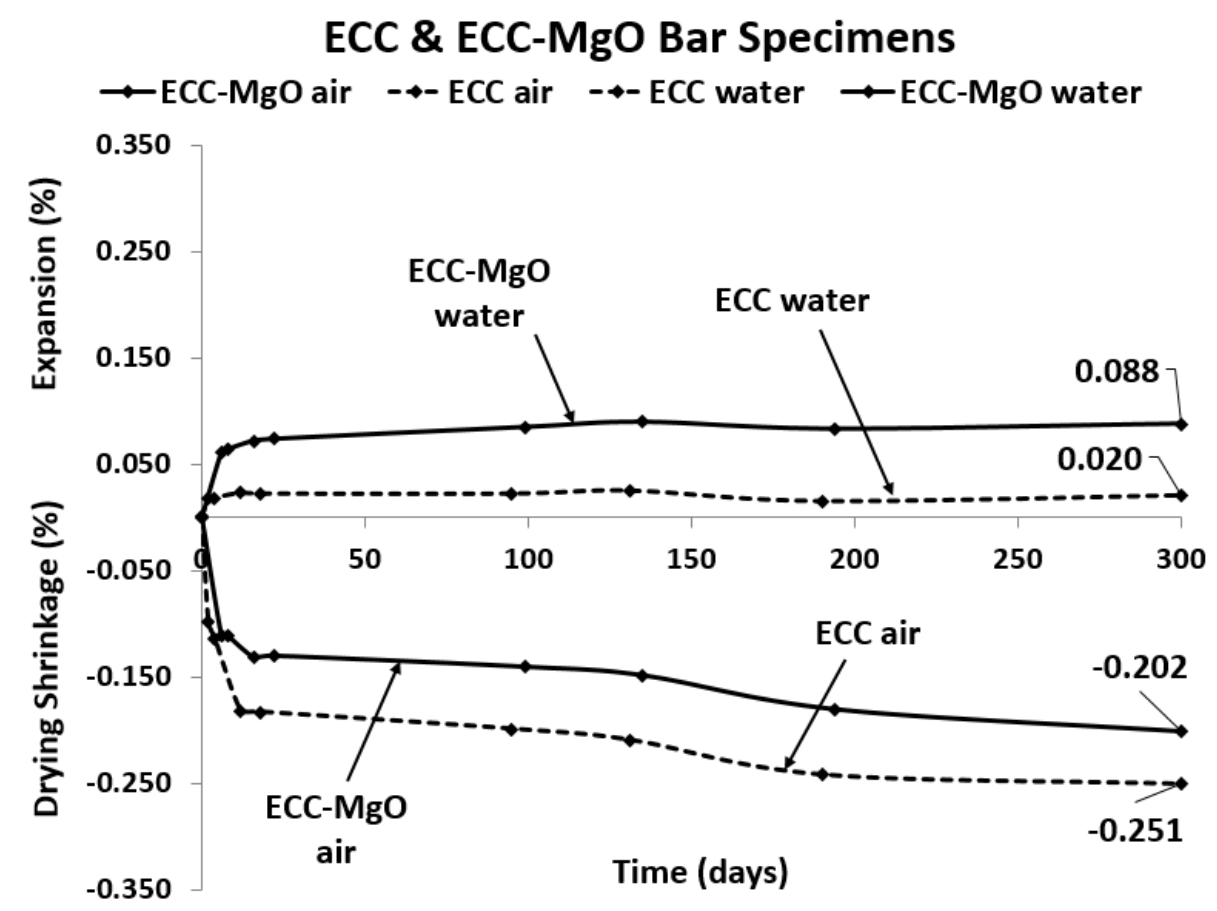

Figure 7-6: Expansion/drying shrinkage of water and laboratory (air) cured ECC and ECC-MgO specimens

As shown in Figure 7-6, the addition of MEA to water cured ECC-MgO bar specimens produced higher expansions than water cured ECC specimens. At 300 days, the expansion was $0.088 \%$ for water cured ECC-MgO specimens while it was $0.020 \%$ for ECC specimens. This might be related to the formation of lots of small interior pores within $\mathrm{MgO}$ microstructures when $\mathrm{MgCO}_{3}$ was calcined at a lower temperature and shorter holding times (Mo et al., 2010). Therefore, this might facilitate $\mathrm{OH}^{-}$ions in pore solution to diffuse into the interior pores saturated with $\mathrm{Mg}^{2+}$ ions to 
form $\mathrm{Mg}(\mathrm{OH})_{2}$. The formation of $\mathrm{Mg}(\mathrm{OH})_{2}$ crystals increased the expansion pressure on the walls of interior pores leading to higher expansions.

Similar to water curing condition, Figure 7-6 also shows that air cured ECC-MgO bar specimens produced lower drying shrinkage than ECC ones cured under similar condition. The drying shrinkage of air cured ECC-MgO specimens was $-0.202 \%$ at 300 days compared to $-0.251 \%$ of their ECC counterparts. Although air cured bar specimens were tested under very dry condition, ECC-MgO bar specimens showed less drying shrinkage than those made with ECC. The water demand in $\mathrm{MgO}$ concrete to form chemically stable $\mathrm{Mg}(\mathrm{OH})_{2}$ crystals is relatively low compared to other expansive agents which used to compensate concrete autogenous shrinkage (Mo et al., 2012). Therefore, the growth of air cured $\mathrm{Mg}(\mathrm{OH})_{2}$ crystals inside plenty of small pores within $\mathrm{MgO}$ microstructure forms counteracted pressure that might reduce the contraction mechanism of ECC-MgO specimens more than their ECC counterparts. Sahmaran et al. (2009) reported that ECC mixtures produced with a FA/PC ratio of 1.2 and micro silica sand with a size of $0.2 \mathrm{~mm}$ (similar to this study) exhibited the highest drying shrinkage at the end of 90 days. It was revealed that the addition of high volume fly ash (FA/PC ratio up to 2.2) densified the ECC matrix and reduced the drying shrinkage by 20\%. The drying shrinkage results of Sahmaran et al. (2009) was expressed as micro-strain instead of percentages - so making a comparison with the results of this study is difficult. However, ECC specimens exhibited more drying shrinkage than ECC-MgO specimens as shown in Figure 7-6.

It should be noted that the drying shrinkage of air cured ECC-MgO specimens at 300 days was 2.3 $(=0.202 / 0.088)$ times higher than the expansion of similar specimens cured under water. Further, the drying shrinkage of ECC specimens at 300 days was $12.55(=0.251 / 0.020)$ times higher than the expansion. This might give an indication that ECC-MgO mixtures can resist more damages than their ECC counterparts when exposed to wetting/drying harsh environments without undergoing sudden changes in material's microstructure. It could be one of the reasons for the wide use of $\mathrm{MgO}$ to compensate the thermal shrinkage effectively in mass concrete at a later age. $\mathrm{MgO}$ in concrete as an expansive agent is effectively used in China as a novel concrete technology and to date; over 30 dams have been constructed. It should be emphasized that conclusions derived 
from this study should be valid for the ECC mixtures examined herein and more research should be conducted.

\subsubsection{Rapid Chloride Permeability (RCP) of ECC and ECC-MgO mixtures}

Table 7-1 summarizes the permeations properties (in terms of RCP) of water and natural cured ECC and ECC-MgO specimens at 21, 21+0, 21+45, 21+90 and 21+135 days (total of 156 days).

Figure 7-7 shows the variation of RCP values in Coulombs for water and natural cured specimens - in general, RCP values decreased with age as expected.

Table 7-1: RCP property of water and natural cured ECC and ECC-MgO specimens

\begin{tabular}{|c|c|c|c|c|c|c|c|c|}
\hline \multirow{4}{*}{$\begin{array}{c}\text { Time } \\
\text { (days) }\end{array}$} & \multicolumn{8}{|c|}{ ECC Mixtures (Coulombs) } \\
\hline & \multicolumn{4}{|c|}{ Water Curing } & \multicolumn{4}{|c|}{ Natural Curing } \\
\hline & \multirow{2}{*}{ Control } & \multirow{2}{*}{$\begin{array}{c}\text { Pre- } \\
\text { loaded }\end{array}$} & \multicolumn{2}{|c|}{ Difference } & \multirow{2}{*}{ Control } & \multirow{2}{*}{$\begin{array}{c}\text { Pre- } \\
\text { loaded }\end{array}$} & \multicolumn{2}{|c|}{ Difference } \\
\hline & & & Coulombs & $\%$ & & & Coulombs & $\%$ \\
\hline 21 & $1345^{x+}$ & 1254 & & & $1175^{x}$ & 1280 & & \\
\hline $21+0$ & - & 1730 & & & - & 2019 & & \\
\hline $21+45$ & 550 & 628 & $1102^{*}$ & & 485 & 491 & $1528^{*}$ & \\
\hline $21+90$ & 428 & 405 & & & 204 & 296 & & \\
\hline $21+135$ & 311 & $281^{\mathrm{xx}}$ & $347^{* *}$ & $80 \%+$ & 134 & 167 & $324^{* *}$ & $86 \%+$ \\
\hline \multirow{4}{*}{ Time } & \multicolumn{8}{|c|}{ ECC-MgO Mixtures (Coulombs) } \\
\hline & \multicolumn{4}{|c|}{ Water Curing } & \multicolumn{4}{|c|}{ Natural Curing } \\
\hline & \multirow{2}{*}{ Control } & Pre- & \multicolumn{2}{|c|}{ Difference } & \multirow{2}{*}{ Control } & \multirow{2}{*}{$\begin{array}{c}\text { Pre- } \\
\text { loaded }\end{array}$} & \multicolumn{2}{|c|}{ Difference } \\
\hline & & loaded & Coulombs & $\%$ & & & Coulombs & $\%$ \\
\hline 21 & $1824^{\mathrm{x}}$ & 1758 & & & $1816^{\mathrm{x}}$ & 1774 & & \\
\hline $21+0$ & - & 2296 & & & - & 2159 & & \\
\hline $21+45$ & 635 & 675 & $1621^{*}$ & & 476 & 511 & $1648^{*}$ & \\
\hline $21+90$ & 368 & 343 & & & 239 & 209 & & \\
\hline $21+135$ & 259 & $303^{x x}$ & $372^{* *}$ & $84 \%+$ & 124 & $100^{\mathrm{xx}}$ & $411^{* *}$ & $95 \%+$ \\
\hline
\end{tabular}

*The difference in coulombs of pre-loaded: at the age 21+0 - at the age 21+45; **The difference in coulombs of pre-loaded: at the age of $21+45-$ at the age of $21+135 ;+$ The $\%$ between the controls $\left({ }^{x+}\right)$ at the age 21 and pre-loaded at the age $21+135$ $\left({ }^{x}\right)$

At an early age (21 days), the un-cracked (water or natural cured ECC-MgO specimens (controls) developed higher RCP values than their ECC counterparts (Table 3 and Figure 9). The 21 days RCP values for water and natural cured ECC controls were 1345 and 1175 coulombs, respectively compared to 1824 and 1816 coulombs of their ECC-MgO counterparts. The relatively high 
difference in RCP values at an early age might be related to the slow reactivity of $5 \% \mathrm{MgO}$ expansion agent and reduction of fly ash content by $\mathrm{MgO}$ replacement (Table 3-2). It was reported by Gao et al. (2008) that $\mathrm{MgO}$ hydration is very slow and only $57 \%$ of $\mathrm{MgO}$ formed $\mathrm{Mg}(\mathrm{OH})_{2}$ crystals within the first 180 days of hydration - this explains the reduced chloride ion penetration resistance of ECC-MgO specimens compared to their ECC counterparts due to slow $\mathrm{MgO}$ hydration especially at early age (Figure 7-7). Moreover, it is recommended by (ASTM C1202, 2012) that the addition of fly ash positively influenced the permeation property of concrete samples which is attributed to producing denser microstructure through improving pore sizes in transition zones (Amrutha et al., 2011; Mehta and Monteiro, 2006; Sahmaran and Li, 2009; Sherir et al., 2016). Therefore, the increased RCP values (permeation property) of ECC-MgO controls containing 5\% lower fly ash (as replaced by $\mathrm{MgO}$ ) than ECC controls at an early age is justified. However, ECC-MgO controls at the age of 21+135 days developed showed better chloride ion penetration resistance (lower RCP values) than ECC controls. The RCP values water and natural cured ECC-MgO controls at 21+135 were 259 and 124 coulombs, respectively which were lower than 311 and 134 coulombs, respectively of their ECC counterparts. Similar behavior was observed by Choi et al. (2014) who revealed that the addition of $5 \% \mathrm{MgO}$ to concrete specimens could improve the long-term chloride resistance when tested at the age of 360 days rather than 28 days (Choi et al., 2014). Although ECC controls at an early age developed better (lower RCP values) resistance to chloride ion penetration than ECC-MgO controls, both controls fall within the low range (1000 2000 coulombs) as per (ASTM C1202, 2012) at the age of 21 days.

Table 7-1 and Figure 7-7 show the effect of self-healing on pre-loaded (cracked) ECC and ECC$\mathrm{MgO}$ specimens. The specimens were pre-loaded (cracked) at 21 days and then cured under water and natural conditions up to $21+135$ (156 days) to evaluate the ECC-MgO self-healing system. Similar to the controls (un-cracked), the pre-loaded ECC-MgO specimens developed more damage and less resistance to chloride ion penetration than similar ECC specimens (right after releasing the pre-loadings at $21+0$ days) and hence developed higher coulombs to be classified as 'moderate' (between 2000 4000 coulombs) as per (ASTM C1202, 2012). The charge passed for water and natural cured pre-loaded ECC-MgO specimens at 21+0 days were 2296 and 2159 coulombs, respectively which were higher than 1730 and 2019 coulombs of their pre-loaded ECC 
counterparts. Up to 66 days $(21+45$ days $)$ of both water and natural curing, the effect of selfhealing was revealed clearly in Table 7-1 and Figure 7-7 by a steep reduction in the permeation properties (reduction in RCP values) of pre-loaded ECC and ECC-MgO specimens. Beyond 66 days and up to 166 days of curing, a gradual reduction in the trend of self-healed permeation properties was observed. The steep differences in coulombs of water and natural cured pre-loaded ECC specimens between the ages $21+0$ and $21+45$ were 1102 and 1528 coulombs compared to 1621 and 1648 coulombs of counterpart ECC-MgO specimens cured and tested under similar conditions. Also, the differences were 347 and 324 coulombs for ECC specimens compared to 372 and 411 coulombs of ECC-MgO specimens between the ages 21+45 and 21+135 (Table 7-1 and Figure 7-7).
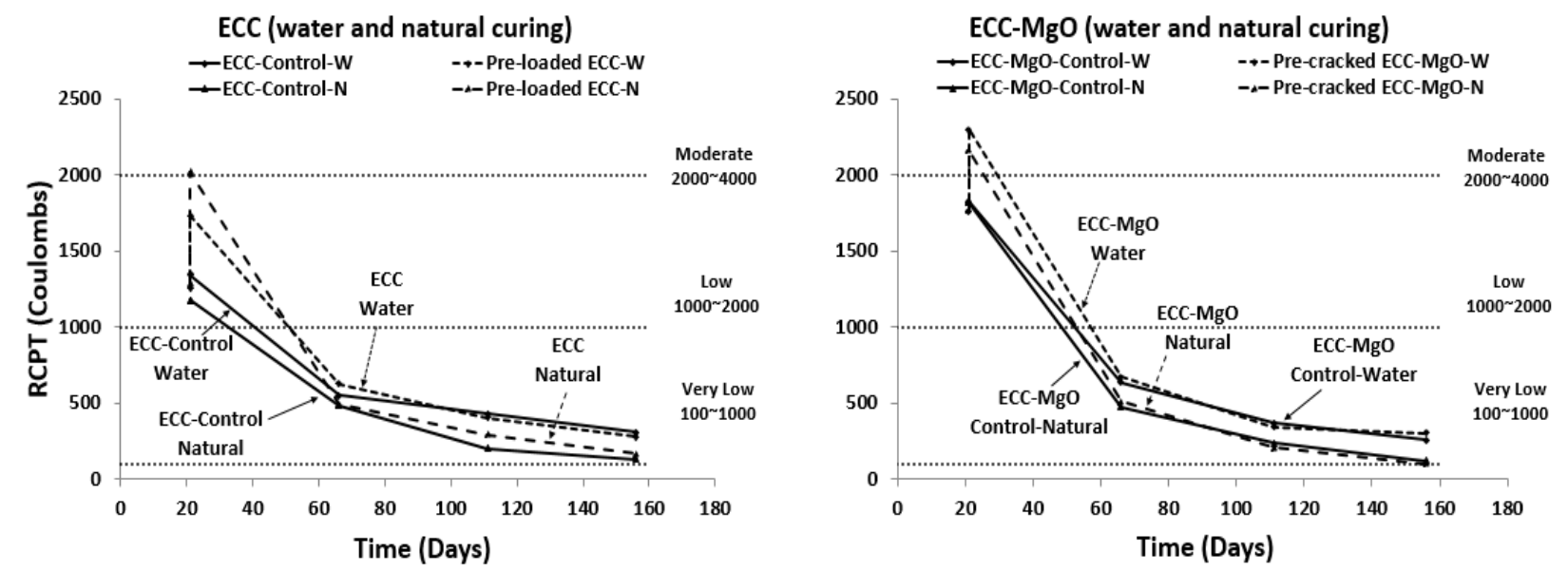

Figure 7-7: RCP property of water and natural cured ECC and ECC-MgO specimens as a function of time

Eventually and at the age of 21+135 days (Figure 7-7), the permeation properties of both preloaded (cracked) and control (un-cracked) specimens of water or natural cured ECC and ECC$\mathrm{MgO}$ specimens were matched to each other (classified as 'very low'). This signifies that precracked specimens self-healed the cracks and develop the same RCP values (permeability) compared to un-cracked specimens. To compare the self-healing capability of ECC and ECC-MgO specimens, the percentage difference of RCP values of pre-loaded (cracked) specimens at the age of 21+135 days and corresponding controls (un-cracked) at 21 days was calculated (Table 7-1). The $\%$ reduction for water cured ECC specimen was $80 \%$ compared to $84 \%$ of ECC-MgO counterpart while the \% reduction for natural cured ECC specimen was $86 \%$ compared to $95 \%$ of 
ECC-MgO counterpart. The higher reduction in permeation properties (RCP values) of damaged ECC-MgO specimens in 156 days compared to their ECC counterparts indicated that $5 \% \mathrm{MgO}$ addition produced better self-healing. This might be related to the expansive nature of $\mathrm{MgO}$ hydration that could cause better filling effect in ECC-MgO microstructure and hence, leading to a significant improvement in permeation properties as shown in Table 7-1 and Figure 7-7 (Moradpour et al., 2013).

It should be noted that the natural cured ECC and ECC-MgO specimens either pre-loaded (cracked) or control developed better chloride ion penetration resistance (lower RCP values) from the first day of long term curing than water curing condition. Referring to UPV measurements conducted in chapter 6, it was revealed that both water cured ECC and ECC-MgO specimens developed higher UPVs than counterpart natural cured specimens; this was attributed to the enhanced filling effect on microstructure (leading to micro-structure densification) when specimens stored continuously in water. Therefore, better (lower) permeability properties of natural cured disk specimens than water cured ones were not expected. However, the main conclusion from RCP test could be referred to the high tendency of ECC-MgO mixtures to undergo better self-healing properties than counterpart ECC ones as described.

\subsubsection{Sorptivity properties}

Table 7-2 summarizes the sorptivity index (So) of ECC and ECC-MgO specimens (control or preloaded/cracked) at $21+18$ days ( $1^{\text {st }}$ time $), 21+36$ days $\left(2^{\text {nd }}\right.$ time $)$ and $21+180$ days $\left(3^{\text {rd }}\right.$ time $)$. For ECC specimens (control or cracked), in general, sorptivity index (as a measure of the rate of water absorption) increased with time in both water and natural curing conditions while for ECC$\mathrm{MgO}$ specimens, sorptivity reduced (Table 7-2). 
Table 7-2: Sorptivity index, $\mathrm{S}_{\mathrm{o}}$ of ECC and ECC-MgO specimens cured under water and natural conditions

\begin{tabular}{|c|c|c|c|c|c|c|c|c|c|}
\hline & & \multicolumn{6}{|c|}{ Sorptivity index, $\mathrm{S}_{\mathrm{o}}\left(\mathrm{mm} / \mathrm{min}^{1 / 2}\right)$} \\
\hline \multirow{2}{*}{$\begin{array}{c}\text { Time } \\
\text { (days) }\end{array}$} & \multirow{2}{*}{$\begin{array}{c}\text { Sorptivity } \\
\text { test order }\end{array}$} & \multicolumn{3}{|c|}{ ECC Mixtures } & \multicolumn{3}{c|}{ ECC-MgO Mixtures } \\
\cline { 3 - 10 } & & Water Curing & Natural Curing & Water Curing & Natural Curing \\
\hline $21+18$ & $1^{\text {st }}$ time & 0.078 & 0.077 & 0.082 & 0.077 & 0.082 & 0.079 & 0.085 & 0.087 \\
\hline $21+36$ & $2^{\text {nd }}$ time & 0.063 & 0.136 & 0.066 & 0.135 & 0.073 & 0.149 & 0.074 & 0.131 \\
\hline $21+180$ & $3^{\text {rd }}$ time & 0.089 & 0.188 & 0.185 & 0.239 & 0.048 & 0.052 & 0.055 & 0.078 \\
\hline
\end{tabular}

Preloaded means cracked

The first time sorptivity test was conducted for ECC and ECC-MgO controls (without cracking) cured under laboratory condition at the age of $21+18$ days (considered $=100 \%$ ). In order to quantify the rate of absorption, the second and third sorptivity test results (sorptivity indexes) are expressed as $\%$ of the $1^{\text {st }}$ test as shown in Figure $7-8$. The values above $100 \%$ meant that the cumulative volume of water absorbed per unit surface area $\left(\mathrm{mm}^{3} / \mathrm{min}^{2}\right)$ in the specimen increased as a function of square root of time from $1^{\text {st }}$ test. This situation is not preferred due to the increase in transport properties which implies a lack of hydration. Therefore, a value of less than $100 \%$ of the control is favored as it signifies lower rate of absorption as time increases. It can be noted from Figure 7-8 that when the sorptivity test was repeated for the second time at the age of $21+36$ days, un-cracked control ECC specimens cured under laboratory condition developed less rate of absorption than their ECC-MgO counterparts. The absorption rates of water and natural cured ECC controls at $21+36$ days were $82 \%$ and $80 \%$ of the $1^{\text {st }}$ test, respectively compared to $89 \%$ and $87 \%$ of the $1^{\text {st }}$ test for ECC-MgO controls tested under similar conditions. Similar to RCP, the addition of 5\% MEA to ECC-MgO controls featured with slow reactivity at early ages might be the reason to develop a higher rate of absorption than ECC controls. It was reported that the lightly burnt $\mathrm{MgO}$ had very slow expansive activity during very long periods after the concrete hardens (between 7 to 1000 days) (Du, 2005). After a long term of water and natural curing of 21+180 days, a considerable reduction in the rate of absorption/sorptivity of ECC-MgO controls was observed while it was increased remarkably for ECC controls. The rate of absorption at $21+180$ days were $58 \%$ and $64 \%$ of water and natural cured ECC-MgO controls, respectively which were better than $116 \%$ and $225 \%$ of their ECC control counterparts. 

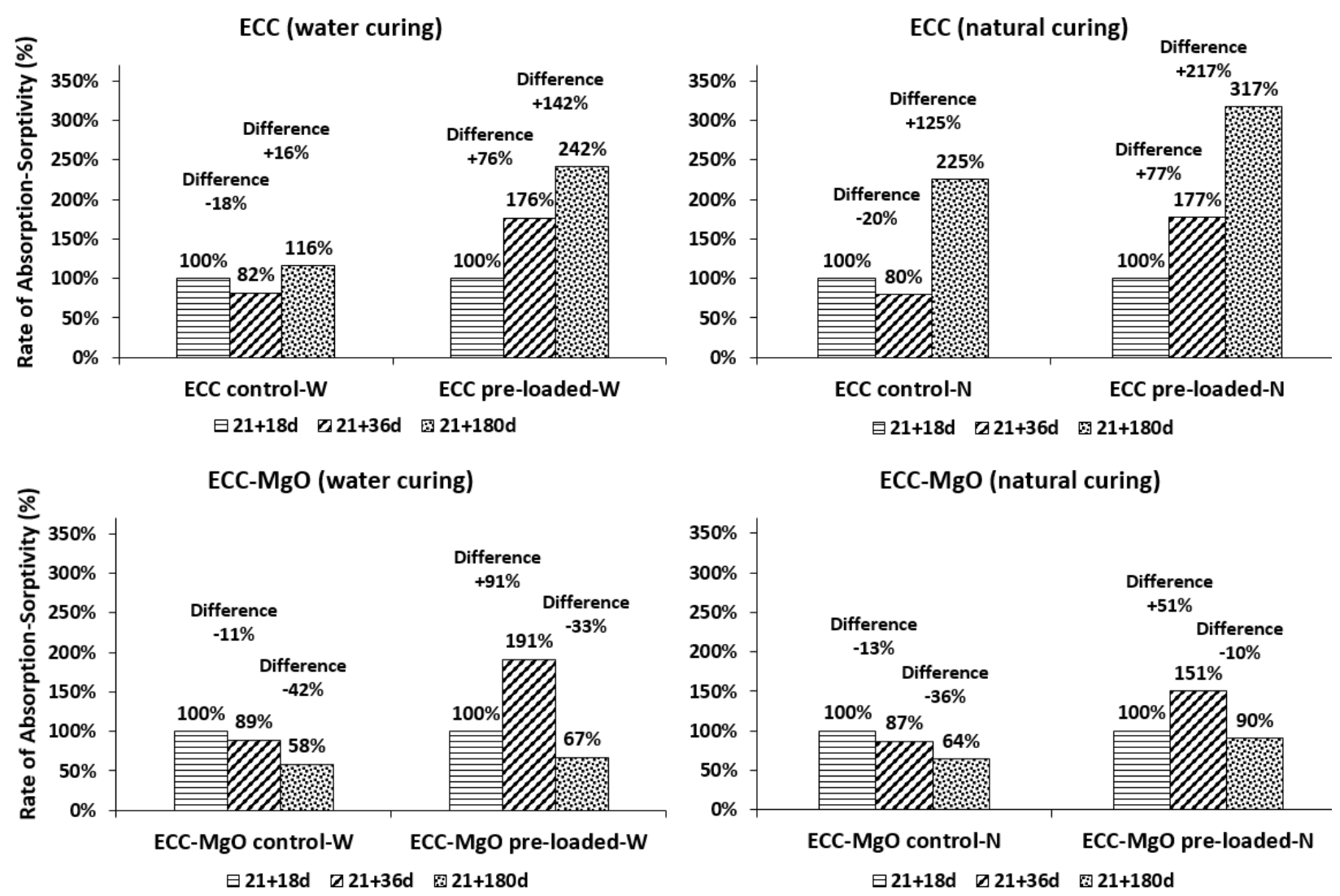

Figure 7-8: Sorptivity index $\left(\mathrm{S}_{\mathrm{o}}\right)$ as percentages for water and natural cured ECC and ECC-MgO controls and pre-loaded specimens as a function of time

This significant improvement in transport property of ECC-MgO compared to ECC as shown in Figure 7-8 might be related to the delayed filling effect at a later age due to the expansive nature of MEA as confirmed by Moradpour et al. (2013). The inferior (higher) sorptivity of ECC mixtures after $21+180$ days of curing might be related to the nature of the test method itself. As mentioned before, placing the specimens inside the oven for 3 days before conducting every sorptivity test might increase the self-desiccation phenomenon of ECC controls due to low w/c ratio (less than $30 \%$ ) and to the addition of Pozzolans, as well (Mindess et al., 2003). In this study, the w/c ratio used in both ECC and ECC-MgO mixtures was 0.27 while 50 55\% high volume fly ash was used as cement replacement. The addition of 5\% of MEA was responsible for improving (reducing sorptivity values) significantly the transport properties of control ECC-MgO mixtures compared to their ECC counterparts at later ages of 21+180 days as mix ingredients/proportions and curing conditions were kept similar. 
In order to quantify the rate of absorption/sorptivity characteristics in the damaged/cracked state, both pre-loaded (cracked) ECC and ECC-MgO specimens were cured under water and natural conditions for additional 180 days. Again before conducting the last sorptivity test, all specimens were placed inside the oven for 3 days followed by 15 days inside a sealable container. From Figure 7-8, both types of ECC mixtures developed more than 100\% sorptivity/absorption rate with respect to that at the age of $21+36$ days (second sorptivity test) based on the damage applied to the specimens. It should be noted that the value of the second sorptivity conducted at the age of $21+36$ days was not the target of observation. This was because, in some cases, the specimens did not crack after applying $90 \%$ of the maximum load of the virgins and thereby the loading was continued until considerable cracking was observed. Mainly, the damage on specimen's surface was quantified/approved as long there was a significant increase in the rate of absorption after conducting the second sorptivity test. Therefore, the main focus was on the last sorptivity test conducted at the age of $21+180$ which gave a good indication of self-healing under long-term water and natural curing conditions assessed by sorptivity index. From Figure 7-8, it was observed that none of pre-loaded (cracked) ECC specimens recovered the sorptivity of controls whether water or natural cured, while their ECC-MgO counterparts recovered those of controls. The percentage rate of absorption/sorptivity of water and natural cured pre-loaded ECC specimens at 21+180 days were $242 \%$ and $317 \%$, respectively compared to $67 \%$ and $90 \%$ for ECC-MgO controls tested under similar conditions. Like controls, the presence of 5\% of MEA to pre-loaded ECC-MgO specimens had improved positively (lowering absorption rate/sorptivity due to self-healing causing crack filling) their transport properties at later ages $(21+180$ days $)$ than pre-loaded ECC specimens.

Opposite to RCP and as was expected, the water cured pre-loaded ECC/ECC-MgO specimens in addition to the controls developed better self-healing producing lower absorption rate/sorptivity index than natural cured ones. 


\subsubsection{RCP \& Sorptivity Properties of ECC \& ECC-MgO specimens under Autoclave Curing Condition}

To confirm the self-healing capability of proposed ECC-MgO system, the permeation properties (RCP and Sorptivity) of ECC-MgO mixture was assessed by accelerating the hydration of $\mathrm{MgO}$ at the age of $21+180$ days through autoclave curing.
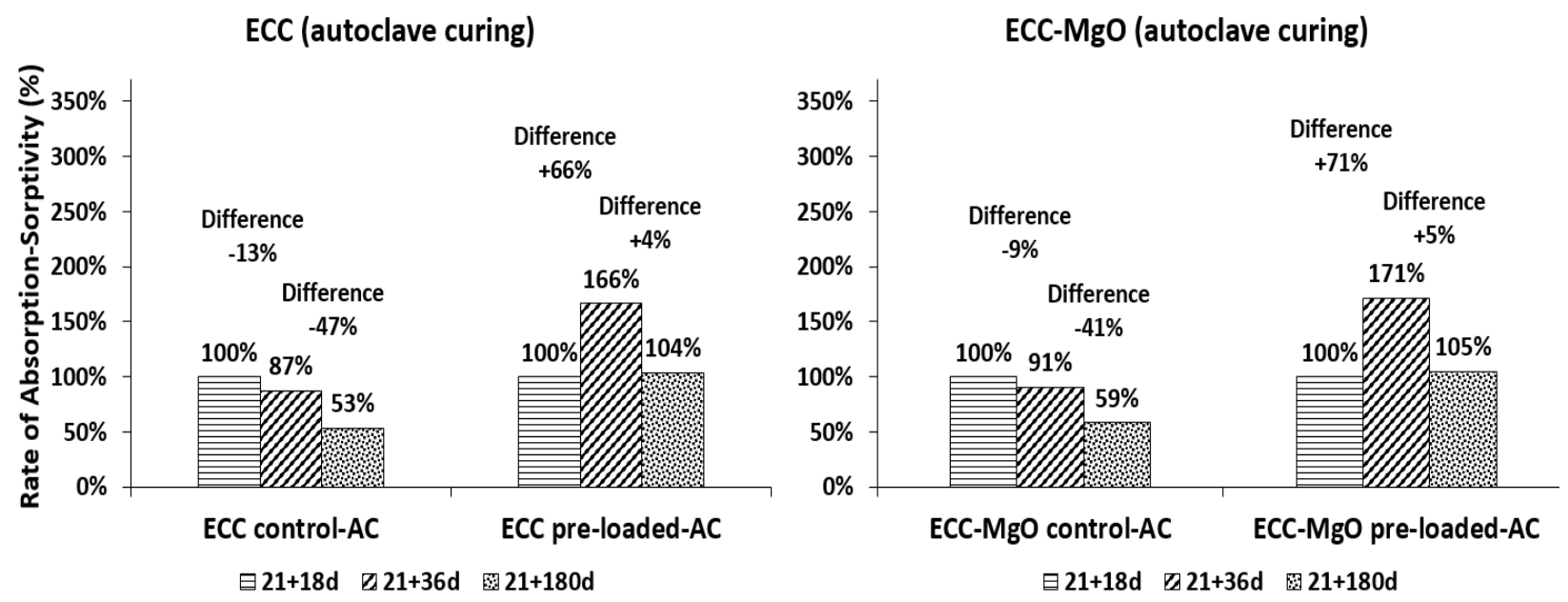

Figure 7-9: Sorptivity index $\left(\mathrm{S}_{\mathrm{o}}\right)$ as percentages for autoclave cured ECC and ECC-MgO controls and pre-loaded specimens

Similar to water and natural curing conditions, sorptivity/absorption rate values lower than $100 \%$ was favored under autoclave curing condition. Similar to Figure 7-8, Figure 7-9 shows that laboratory cured ECC controls at the age of $21+36$ days developed less rate of absorption than ECC-MgO controls. The difference in percentage sorptivity for ECC controls (un-cracked) at the age of $21+36$ days was $-13 \%$ which was less than $-9 \%$ of ECC-MgO controls cured under similar condition. After the age of $21+36$ days, the controls were cured under the laboratory condition for another 180 days then autoclave curing was conducted on ECC and ECC-MgO specimens to assess the ECC-MgO self-healing system through sorptivity index. From Figure 7-9, it was observed that autoclave cured ECC controls developed less rate of absorption than counterpart ECC-MgO controls cured under similar condition. The differences in autoclave cured sorptivity at the age of 
21+180 days were $-47 \%$ and $-41 \%$ for ECC and ECC-MgO controls, respectively. Even though the difference in the rate of absorption between them (ECC and ECC-MgO controls) was very small, the result was not expected because ECC-MgO mixtures should experience the expansive effect of $\mathrm{MgO}$ hydration and exhibit better filling in $\mathrm{MgO}$-concrete microstructure causing an increase in resistance to absorption property. Generally, accelerating $\mathrm{MgO}$ hydration through autoclave curing can cause a quick formation of $\mathrm{Mg}(\mathrm{OH})_{2}$ crystals and then develop considerable expansive stresses on the surrounding pores. In addition to $\mathrm{MgO}$ hydration, autoclave curing will accelerate the C-S-H hydration leading to destroying the cohesive forces in cement paste microstructure. Consequently, bigger pores and weaker autoclaved microstructures will form due to the quick growth of $\mathrm{Mg}(\mathrm{OH})_{2}$ crystals in addition to the destroyed cohesive forces in concrete microstructures (Ali and Mullick, 1998; Du, 2005; Helmuth and West, 1998). Referring to Figure 7-9, only the cohesive forces in ECC microstructure can be negatively affected after conducting the autoclave curing. On the other hand, due to the presence of $\mathrm{MgO}$ within $\mathrm{ECC}-\mathrm{MgO}$ microstructure, the quick growth of $\mathrm{Mg}(\mathrm{OH})_{2}$ crystals in addition to the destroyed cohesive forces in $\mathrm{ECC}-\mathrm{MgO}$ controls after autoclave curing can form weaker microstructures with bigger pores due to extreme filling effect than ECC controls. Further, self-desiccation may occur in both types of autoclave cured specimens before conducting the sorptivity test due to placing the specimens inside the oven for 3 days. Therefore, these reasons might help to form less or equal absorption resistance in autoclave cured ECC-MgO controls than ECC controls. Similar to the controls, autoclave cured pre-loaded (cracked) ECC and ECC-MgO specimens produced almost same sorptivity values to each other at the age of 21+180 days. The difference in sorptivity for ECC controls after conducting autoclave test was $+4 \%$ which was almost similar to $+5 \%$ for ECC-MgO controls cured under similar condition.

Due to the lack of information in autoclaved sorptivity test which did not differentiate clearly the absorption performance between ECC and ECC-MgO mixtures shown in Figure 7-9, it was preferred to conduct RCP test on same sorptivity specimens to confirm the self-healing capability of proposed ECC-MgO system (Figure 7-10). From Figure 7-10, an improvement was observed in permeation properties (lower RCP values) of the control/pre-loaded ECC-MgO specimens than similar ECCs. The reason for the improvement in RCP property of autoclave cured ECC-MgO 
mixtures was unknown especially after developing inferior performance showing higher sorptivity property. However, the nature of the RCP test itself might help somehow to improve the permeation properties of ECC-MgO specimens - associated with the filling of the specimen's microstructure with water before starting the test as the RCP test depends mainly on maintaining the relative humidity of specimens $\geq 95 \%$.

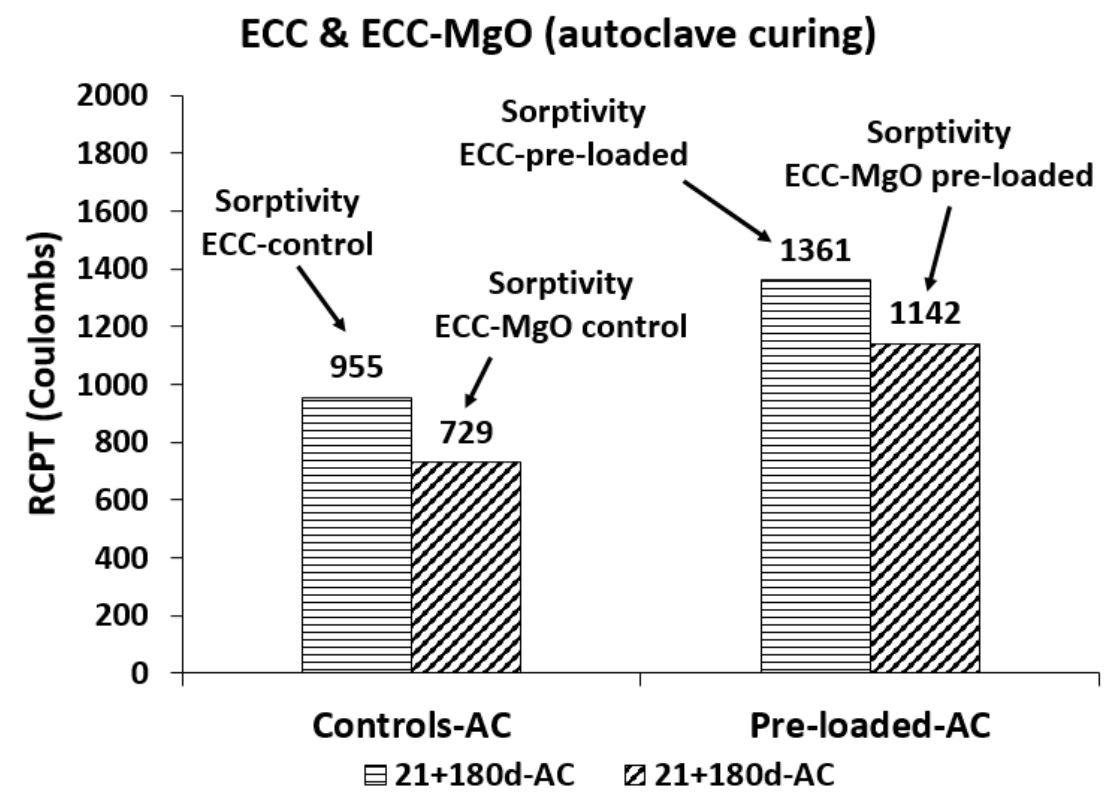

Figure 7-10: RCP property of autoclave cured ECC and ECC-MgO sorptivity specimens at $21+180$ days

\subsubsection{Freeze and Thaw resistance of ECC and ECC-MgO prisms and self- healing against frost damage}

Figure 7-11 shows the frost resistance in terms of the relative dynamic modulus of elasticity (RDME) of ECC and ECC-MgO prisms when subjected to alternate freeze/thaw cycles in accordance with (ASTM C666, 2015) "Procedure A". The test was used to assess the performance of prismatic samples through two aspects. Firstly, the measurement of the internal damage of prismatic controls (without cracking) caused by 300 freeze/thaw cycles through measuring mass loss and RDME based on changes in UPVs. Secondly, the use of frost resistance to evaluate the self-healing capability of preloaded ECC and ECC-MgO prisms by measuring flexural 
performance in terms of residual strength and maximum deflection, dynamic modulus and mass loss at the end of the second 300 freeze/thaw cycles followed right after the first 300 cycles.

As noted from Figure 7-11, only for ECC-MgO specimens, there was a slight improvement (increase) in RDME 30 freeze/thaw cycles ranging between $0.50 \%$ and $1.50 \%$. This might be related to the continuous hydration during the 14 days curing process conducted before placing the prisms inside the freeze/thaw chamber. However, a gradual decrease in RDME of ECC-MgO mixtures was started after 60 freeze/thaw cycles. Generally, both ECC and ECC-MgO mixtures developed lower RDME (RDME generally decreased with increased number of cycles) when exposed to alternate 300 cycles of freezing and thawing cycling. At the end of 300 cycles, the RDME of ECC prisms ranged between $94.9 \%$ and $95.3 \%$ compared to the original fixed as $100 \%$ at 0 freeze/thaw cycles. Exactly same behavior was observed for ECC-MgO prisms which developed almost similar RDME compared to ECC prisms between 60 and 150 freeze/thaw cycles. A significant reduction occurred in RDME of ECC-MgO prisms starting after 150 cycles. At 150 cycles, the RDME of ECC-MgO prisms ranged between $96.7 \%$ and $97.4 \%$ while the sudden reduction ranged between 85.7 and $86.2 \%$ at 270 cycles of the original (100\% at 0 cycles). At the end of 300 cycles, the RDME of ECC-MgO prisms increased slightly to $87.4 \%$ 87.8\% which was still much lower than the ones of ECC prisms at similar freeze/thaw cycles. In general, the accumulated damage in concrete microstructures after applying successive freeze/thaw cycles might depend on expansion and contraction mechanisms of frozen water in capillaries and pores of cement paste. When water begins to freeze in wet concrete, it expands $9 \%$ of its original volume and thereby producing more hydraulic pressures in capillaries and pores. Therefore, if the formed pressure is greater than the tensile strength of cement paste, voids in microstructures will rupture and develop cracking (Nawy, 2008). Although ECC prisms underwent successive 300 freeze/thaw cycles, the presence of PVA fibers in its microstructure was believed to play a significant role to enhance the volume stability significantly. This was indicated by the development of only $5 \%$ less RDME in ECC prisms at the end of first 300 freeze/thaw cycles. In addition to PVA effect, the addition of MEA to ECC-MgO microstructure might be the reason of developing $15 \%$ less RDME at the end of first 300 cycles. 


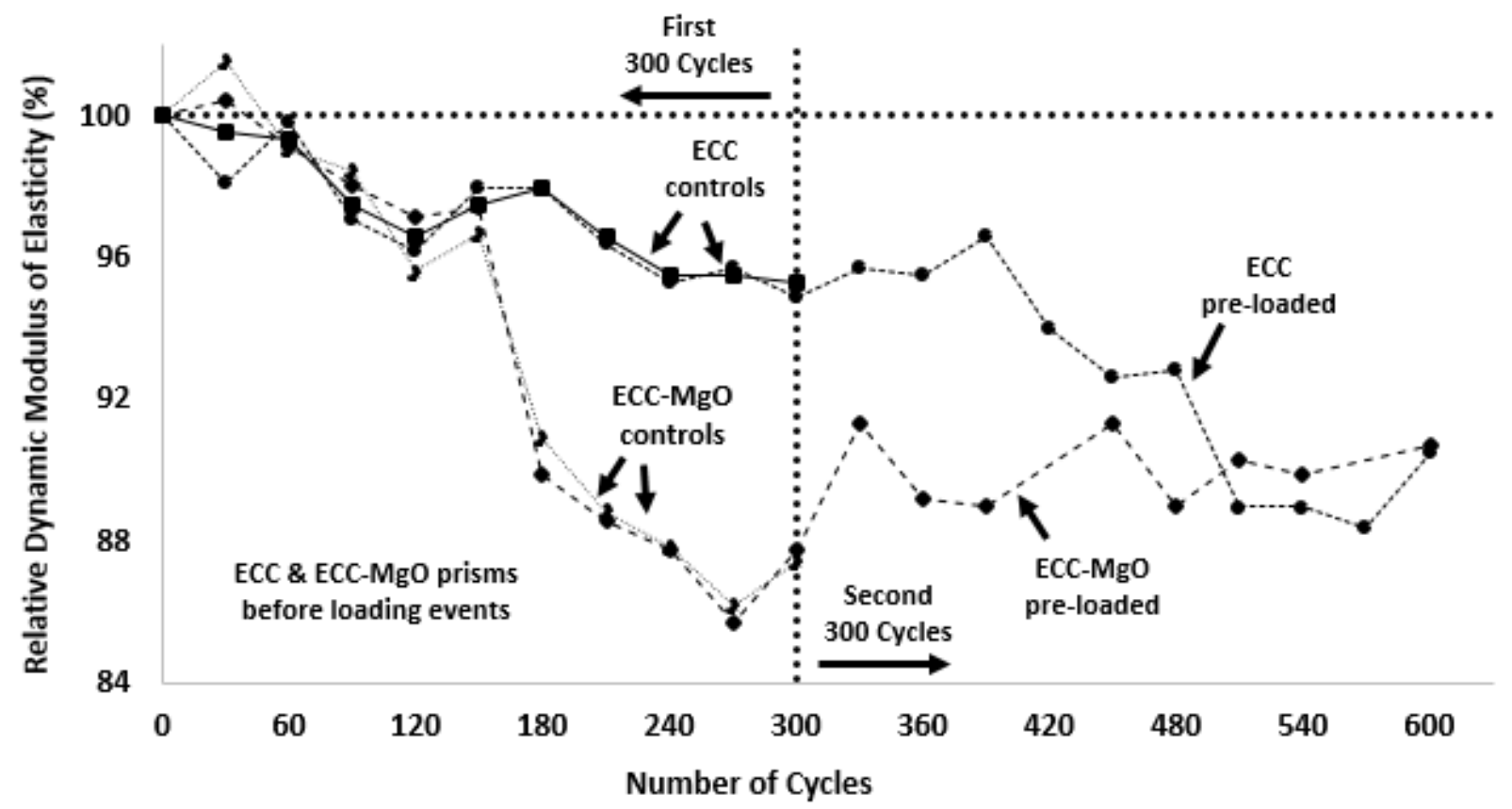

Figure 7-11: Relative dynamic modulus of ECC and ECC-MgO prisms based on UPV change after 300 and 600 freeze/thaw cycles

Based on Du (2005), the addition of MEA can uniformly distribute the pre-compressive stresses applied by $\mathrm{MgO}$ expansion to compensate the tensile stresses at early ages partially while the losses in pre-compressive deformations can compensate the tensile stresses at a later age. Therefore, a significant enhancement in tensile strain capacity in ECC-MgO microstructure will occur which is always prescribed to reduce concrete micro cracking. The flexibility in tensile strain capacity in the voids of ECC-MgO microstructures could provide more rooms to accommodate the volume changes effectively without developing severe concrete micro cracking. However, due to fast alteration between freezing and thawing cycles, the filling effect due to the expansive nature of MEA might be accelerated and thereby extreme formation/packing of new hydration products might be formed in capillaries and pores. Consequently, higher UPVs will be developed which might lead to a brittleness in concrete microstructure (Panzera et al., 2011). Therefore, weakening in ECC-MgO mixtures occurred which indicated by the sudden significant reduction in RDME values starting after 150 cycles to 300 freeze/thaw cycles (Figure 7-11). 
At the end of the first 300 freeze/thaw cycles, one of ECC and ECC-MgO prisms (designated as control) were loaded to failure under four-point loading while the other prisms were pre-loaded to $60 \%$ of the maximum deflection of the failed controls in order to assess the self-healing capability of ECC-MgO system by subjecting to further 300 cycles. Subsequently, UPVs were collected for all pre-loaded prisms placed back inside the freezing-thawing chamber right after the pre-loading event during additional 300 cycles. As can be seen from Figure 7-11, the RDME of pre-loaded ECC specimens started to deteriorate significantly until reached to $88.4 \%$ at 570 cycles then slightly increased to $90.4 \%$ at the end of total 600 cycles of freezing and thawing $(300+300$ cycles). In summary, the RDME of preloaded ECC specimens was $95.0 \%$ at 300 cycles and ended up with $90.4 \%$ at 600 cycles with a deterioration of 4.6\%. Moreover, the RDME of preloaded ECC-MgO specimens showed a gradually increasing trend during the second 300 cycles. The maximum RDME of preloaded ECC-MgO specimens in the second 300 cycles was $91.3 \%$ at 330 and 450 cycles. In addition, the RDME of preloaded ECC-MgO specimens at 300 cycles was $87.7 \%$ and ended up with $90.7 \%$ at $600 \%$ cycles showing an enhancement of $3 \%$. Accumulating the capillaries and pores of ECC-MgO paste with lots of hydration products at the first 300 cycles signified by the sudden decrease in the trend of RDME might be the reason of developing a slight increase in RDME of ECC-MgO specimens during the second 300 cycles (almost 3\%). Application of relatively low pre-loading levels had definitely formed micro cracks at specimen's surface but might not affect negatively on old formed hydration products in capillaries and pores within the entire microstructures. Therefore, accumulation of new hydration products might be valid only for the new room formed within the crack cavities. As long there was enough room to accommodate newly formed hydration products within the crack cavities without applying extra pressures on crack walls, a slight increase in RDME of ECC-MgO mixtures during the second 300 cycles (as observed) was justified (Figure 7-11). This might give an indication that the flexibility in tensile strain capacity of ECC-MgO mixtures due to the presence of MEA still existed even after applying long-term frost damage. In addition, enhanced RDME of ECC-MgO prisms might give an indication of the effectiveness of ECC-MgO self-healing system even after extreme frost damage. On the other hand, the pre-cracked ECC specimens showed continued trend of deterioration of RDME even after applying surface cracking which might provide enough rooms to accommodate more newly formed hydration products. Again, due to lower flexibility in ECC 
microstructure compared to ECC-MgO one, it was believed that the long term frost resistance of ECC specimens kept deteriorating due to accumulation of lots of newly formed hydration products within micro cracked Interfacial Transition Zones (ITZ) in addition to the newly formed crack cavities leading to rupturing of more capillaries and pores within the entire ECC microstructure.

It could be concluded that ECC controls developed better frost damage resistance than ECC-MgO at the first 300 freeze/thaw cycles while preloaded ECC-MgO specimens developed better resistance than their ECC counterparts at the second 300 cycles. However, both preloaded ECC and ECC-MgO prisms reached the same level of RDME even after applying prolonged 600 freeze/thaw cycles as shown in Figure 7-11 (around 90.5\%). Unfortunately, the freezing and thawing cycling was stopped at 600 cycles due to the research's time limitation. Therefore, it is recommended that pre-loading events should be applied at the beginning of frost damage procedure in order to assess clearly the proposed ECC-MgO self-healing system.

In order to confirm the performance based on RDME of both ECC and ECC-MgO specimens, Figure 7-12 (A) shows that in general both types of specimens exhibited minor mass gain (an almost $2 \%$ ). As mentioned before, the mass gain might be related to the formation of new hydration products within capillaries and pores of ECC microstructures. Therefore, more mass gain will lead to lower RDME. As shown in Figure 7-12(A), ECC-MgO control developed the highest mass gain (around $2 \%$ ) after 150 cycles while it was $1.4 \%$ at the end of first 300 cycles for ECC control. By referring to Figure 7-11, the $15 \%$ sudden reduction in the trend of RDME of ECC-MgO controls started after 150 cycles up to 300 cycles; while the reduction was $5 \%$ for ECC controls at the end of first 300 cycles. In addition, during the second 300 freeze/thaw cycles, pre-loaded ECC specimens were kept developing a higher mass gaining $(2.2 \%)$ than the counterpart ECC-MgO specimens $(1.7 \%)$; which might be related to the gradual deterioration in the trend of RDME of ECC controls (Figure 7-11). The minor increase in the trend of mass gain for preloaded ECC-MgO specimens (almost flat) might explain the small increase in RDME's trend for preloaded ECC$\mathrm{MgO}$ specimens (Figure 7-11).

Moreover, the common frost damage in concrete elements exposed to freezing and thawing cycling is associated with cracking and spalling. As per Figure 7-12 (B), slight frost damage was only 
applied on the top surface layers of ECC and ECC-MgO specimens which had no significant effect on the physical properties of ECC's mass. Therefore, instead of mass loss, both ECC specimens developed a gradual minor mass gain with increased number of cycles as shown in Figure 7-12 (A).
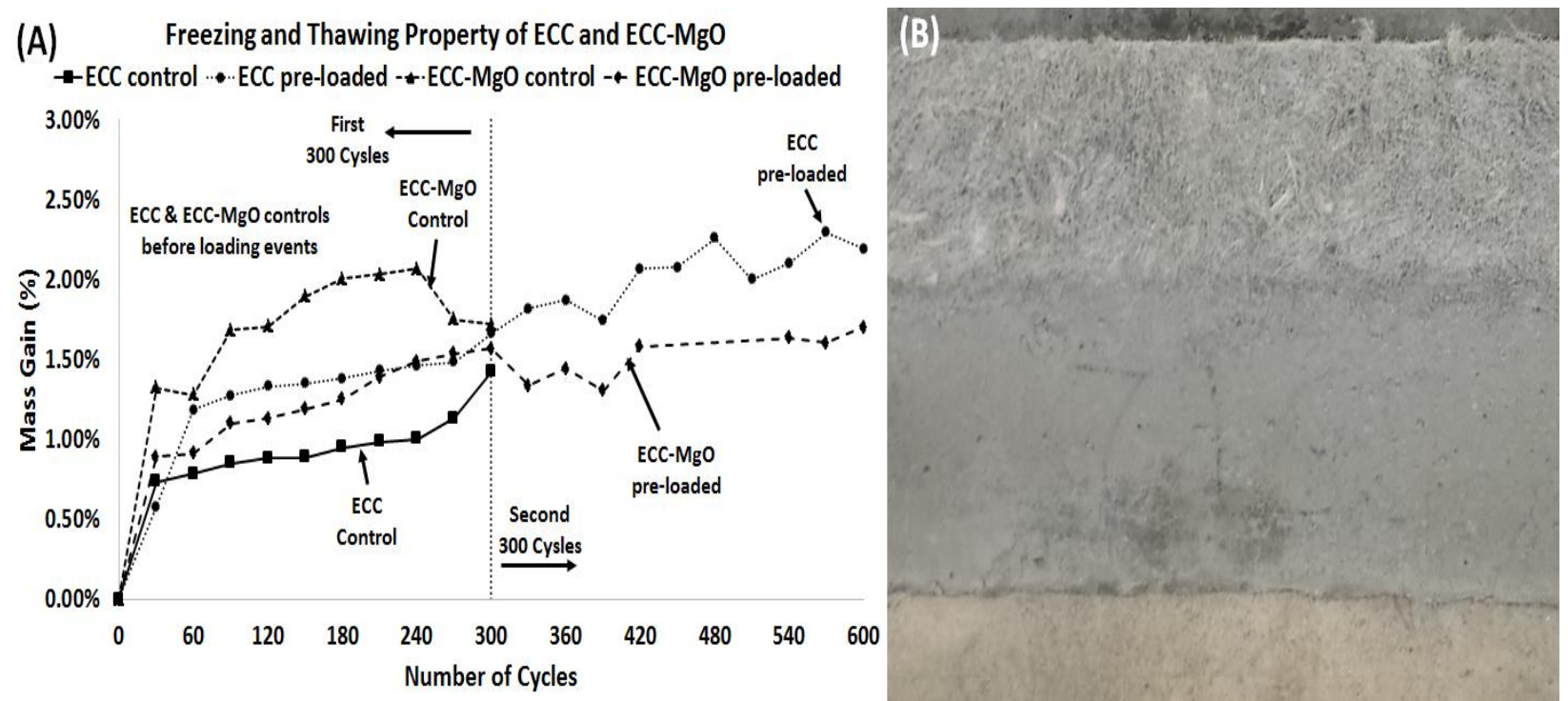

Figure 7-12: Mass gain changes of ECC and ECC-MgO prisms with freezing and thawing cycles (left); surface cracking (right)

In addition to the measurement of mass loss and RDME, the effectiveness of self-healing ECC$\mathrm{MgO}$ system was evaluated based on flexural performance in terms of residual strength and maximum deflection of ECC and $\mathrm{ECC}-\mathrm{MgO}$ prisms after being subjected to frost damage. Figure 7-13 presents the residual strength and mid-span deflection evolution of failed ECC and ECC$\mathrm{MgO}$ controls after conducting 300 freeze/thaw cycles. From this figure, it was observed that ECC controls after subjected to 300 cycles developed higher residual strength and maximum deflection than ECC-MgO controls subjected to similar freeze/thaw cycles. Referring to Figure 7-11, this result was expected due to the higher reduction in RDME developed by ECC-MgO controls at the first 300 cycles than ECC controls. As mentioned before, the sudden decrease in the trend of RDME of ECC-MgO controls after conducting 150 cycles was affected by packing more cementitious hydration products within the capillaries and pores of ECC-MgO paste featured with enhanced strain capacity than ECC control. 


\section{Residual Strength/Freeze-Thaw of ECC and ECC-MgO Controls}

-ECC-MgO control-300 cycles -ECC control-300 cycles

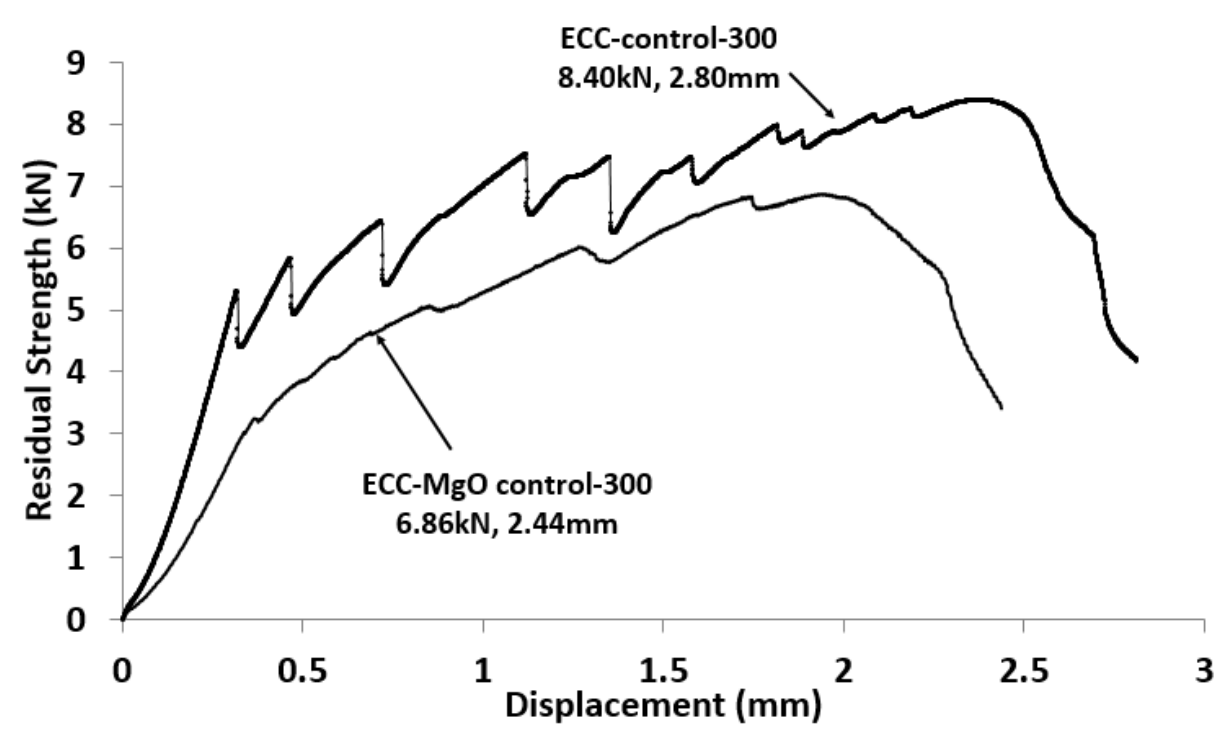

Figure 7-13: Residual strength and maximum deflection of ECC and ECC-MgO controls at the end of 300 freeze/thaw cycles

Therefore, and as a confirmation of RDME results, ECC-MgO controls performed slightly less than ECC controls in terms of residual strength and maximum deflection. In addition, Figure 7-12 (A) shows that more mass was gained by ECC-MgO controls developed less flexural performance in terms of residual strength and maximum deflection than ECC controls. The reductions were $19 \%$ and $13 \%$ in the residual strength and maximum deflection of ECC-MgO controls, respectively than ECC controls at the first 300 cycles.

Figure 7-14 presents the residual strength and mid-span deflection evolution of pre-loaded ECC and $\mathrm{ECC}-\mathrm{MgO}$ specimens after being subjected to total 600 freeze/thaw cycles. The specimens were pre-loaded to $60 \%$ of maximum deflection of the controls at the conclusion of the first 300 cycles then loaded to failure at the end of the second 300 cycles (600 cycles in total). Opposite to the flexural performance of ECC and ECC-MgO controls presented in Figure 7-13, the pre-loaded ECC specimens developed less residual strength and maximum deflection than counterpart ECC$\mathrm{MgO}$ specimens. Simply, this result was expected due to the continuous reduction in the trend of RDME of pre-loaded ECC specimens during the second 300 cycles (Figure 7-11). Again, 
accumulation of extra hydration products inside ruptured capillaries and pores within ITZ and less flexible ECC microstructures than ECC-MgO might be the reasons of lower flexural performance (lower strength and deformation/ductility) at the end of the second 300 cycles. The reduction was $12 \%$ and $11 \%$ in the residual strength and maximum deflection of pre-loaded ECC specimens at the end of second 300 cycles, respectively compared to pre-loaded ECC-MgO specimens.

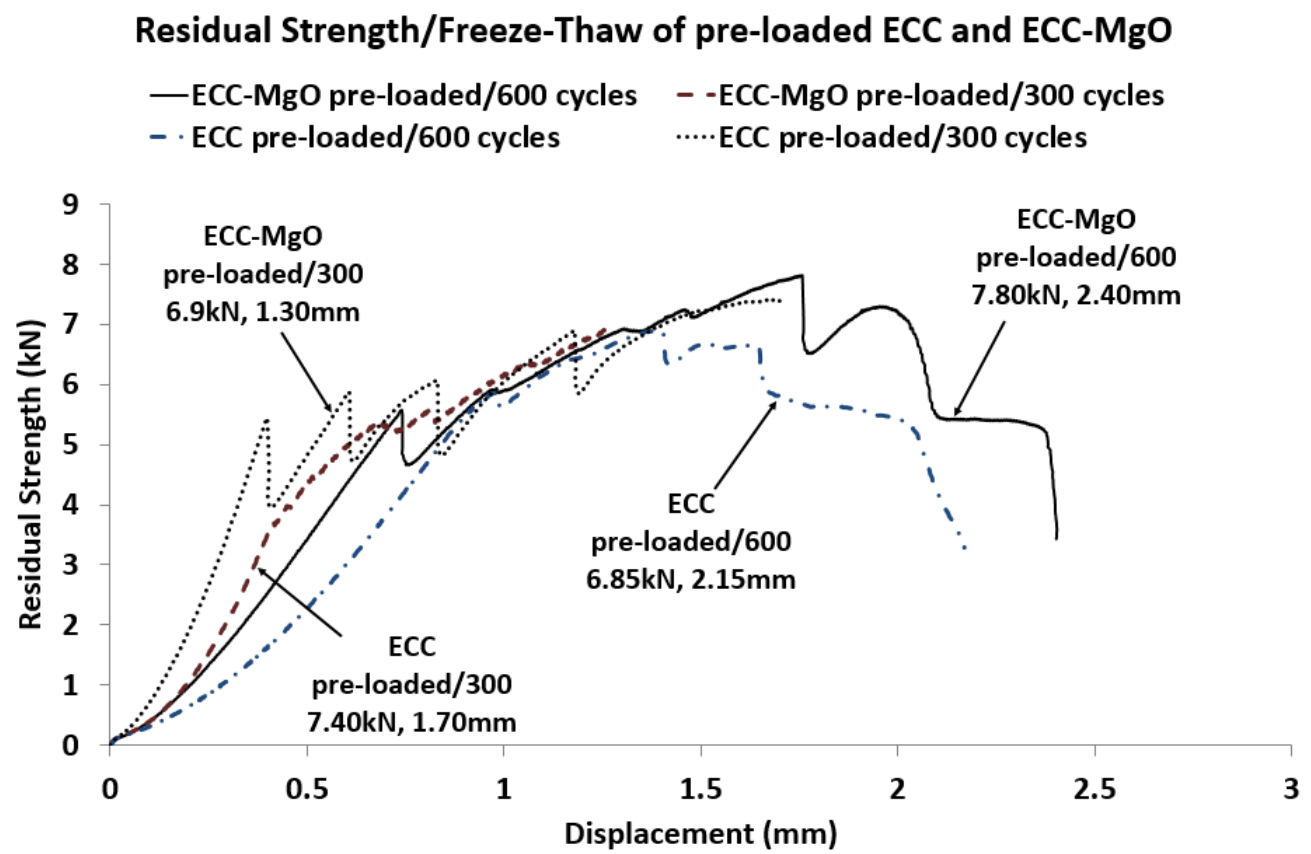

Figure 7-14: Residual strength and maximum deflection evolution of pre-loaded ECC and ECC-MgO prisms at 300 and 600 freeze/thaw cycles

It was believed that the small amounts of hydration products accumulated within the crack walls of pre-loaded ECC-MgO specimens (responsible for crack healing) during the second 300 cycles (Figure 7-11) (which was indicated by the trend mass gain shown in Figure 7-12) was adequate to develop more residual strength and maximum deflection than their pre-loaded ECC counterparts. This gave an indication that self-healing phenomenon of ECC-MgO system could proceed at all times even under extreme frost damage. As long there is enough room that can accommodate developing new hydration products within ECC-MgO microstructures featured with high tensile strain capacity, the delayed formation of magnesium hydration products can supplement the early 
formation of C-S-H products in order to fill the cracks- thereby self-healing ECC-MgO system can be useful in regions with the very harsh weather.

\subsubsection{TGA/DSC and SEM analysis of ECC-MgO self-healing system}

To validate the performance of ECC-MgO self-healing system, it is essential to discover the degradation peaks of healed cementitious materials produced from self-healing mechanism through conducting TGA/DSC analyses. The cementitious materials were scratched and collected from the crack wall surfaces and compared with the ones located within the cementitious pastes.
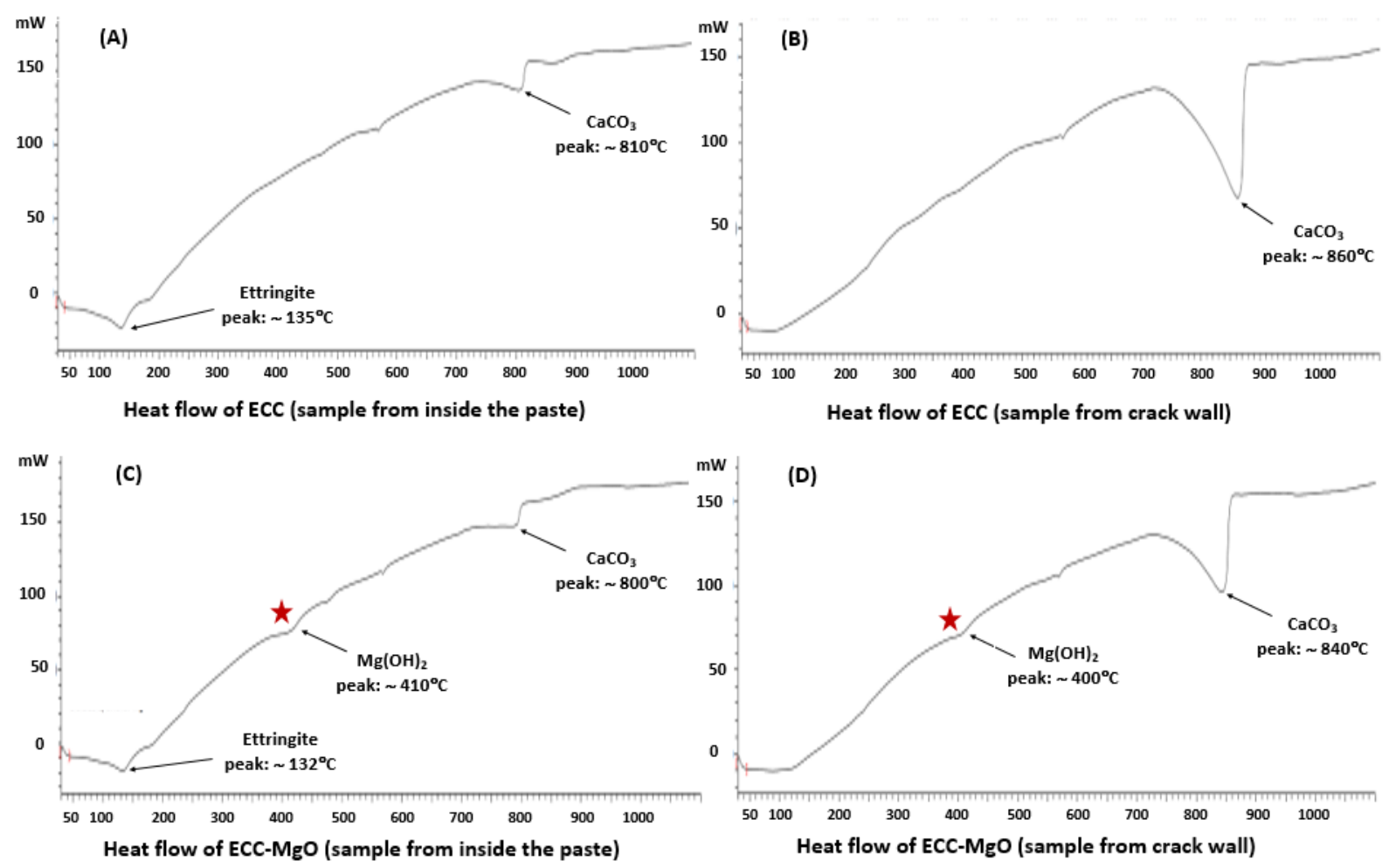

Figure 7-15: TGA/DSC analyses of ECC/ECC-MgO materials collected from inside the paste (A/C) and from crack wall (B/D) at the age of 450 days 
Figure 7-15 (part A and B) show the degradation peak of ECC materials collected from inside the paste and crack walls, respectively. Similarly, the degradation peak of ECC-MgO materials are also shown in Figure 7-15 (part C and D). From Figure 7-15, it is observed clearly that the amount of crystals within the crack walls is higher than the ones located within the cement pastes. This was indicated by higher degradation peaks as shown in Figure 7-15 (B and D) than Figure 17 (A and $\mathrm{C}$ ) whether ECC or ECC-MgO mixtures.

Additionally, Figure 7-16 shows that the precipitations due to self-healing process in every specimen consisted of $\mathrm{CaCO}_{3}$. The degradation temperature of $\mathrm{CaCO}_{3}$ ranged between $800^{\circ} \mathrm{C}$ and $860^{\circ} \mathrm{C}$ depending on the sample's type and location. It was mentioned that the decomposition of $\mathrm{CaCO}_{3}$ takes place between $635^{\circ} \mathrm{C}$ and $865^{\circ} \mathrm{C}$ (Halikia et al., 2001). Further, Ettringite was observed in the samples inside the cement pastes and did not exist within the crack walls with a degradation temperature ranged between $132^{\circ} \mathrm{C}$ and $135^{\circ} \mathrm{C}$ (Figure 7-15).

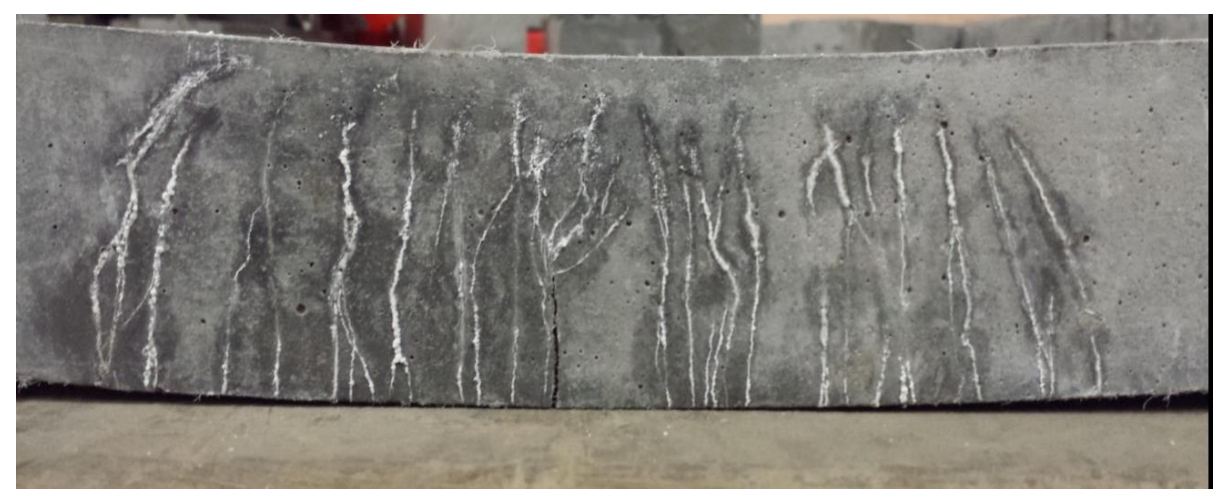

Figure 7-16: white crystals within the crack walls of ECC and ECC-MgO prisms

It was reported that Magnesium hydroxide $\mathrm{Mg}(\mathrm{OH})_{2}$ is the chemically stable product in $\mathrm{MgO}$ concrete which is considered to be the key factor in producing an autogenous expansion that can compensate autogenous shrinkage in mass concrete (Mo et al., 2012). Therefore, for validation of this concept, it was expected that more hydration products of $\mathrm{MgO}$ to be found within the crack walls of proposed self-healing ECC-MgO system. Figure 7-15 (C and D) show that the decomposition temperature of $\mathrm{Mg}(\mathrm{OH})_{2}$ ranged between $410^{\circ} \mathrm{C}$ and $400^{\circ} \mathrm{C}$, depending on the location of samples. Although the massive knowledge of $\mathrm{MgO}$ expansive cement was not accessible because most $\mathrm{MgO}$ research publications were in Chinese and Korean languages, 
according to Choi et al. (2013) (in Korean) the decomposition temperature of $\mathrm{Mg}(\mathrm{OH})_{2}$ was around $405^{\circ} \mathrm{C}$ at the age of 360 days of $\mathrm{MgO}$ hydration and the degradation peak was increased as a function of time (Choi et al., 2013). Although the TGA/DSC analysis was conducted on ECC$\mathrm{MgO}$ samples at later age (450 days), the degradation peaks of $\mathrm{Mg}(\mathrm{OH})_{2}$ shown in Figure 7-15 (C and D) were relatively small either in sample located in crack walls or within $\mathrm{MgO}$ cement paste and thereby these peaks might not be considered (marked by red stars in Figure 7-15). According to $\mathrm{Du}$ (2005), the hydration of lightly burnt $\mathrm{MgO}$ could be extended up to 1000 days due to the slow activity of $\mathrm{MgO}$ and this might be the reason of not showing clear degradation peaks (see Figure 7-15-C and D). Therefore, the use of SEM analysis could be effective to disclose more intensity of MEA within the crack walls of ECC-MgO prisms to evaluate the effectiveness of crack healing process in ECC-MgO self-healing system.

Figure 7-17 shows SEM analysis conducted on ECC-MgO samples only to check the existence of both calcium and magnesium precipitations within the same crack walls. It should be noted that SEM samples were prepared from pre-loaded prism specimens stored in water at $20^{\circ} \mathrm{C}$ for 450 days (see Figure 7-16). As shown in Figure 7-17(A and B), the crack cavity is self-healed and filled/closed completely due to the high maturity of ECC-MgO specimen. As expected, the filled material within the crack cavity should be a mix of calcium and magnesium crystallization. Figure 7-17 C (Zone I) shows that the chemical composition of the self-healed materials consisted of magnesium and calcium hydration products with a percentage of $9.0 \%$ and $22.0 \%$, respectively. Also, the magnesium hydration products in Zone II reduced to $4.90 \%$ while calcium crystals increased up to $35.4 \%$ of total weight (see Figure 7-17 D). This means that the calcium precipitations within same crack cavity could be supplemented by forming new magnesium hydration products. Qian et al. (2010) revealed that the main newly formed healed products within the crack cavity are $\mathrm{CaCO}_{3}$ which does not have similar cementitious bonding like original C-S-H products. Therefore, the formation of delayed $\mathrm{MgO}$ hydration products featured with cementitious characteristics might be the solution to compensate the weakness in cementitious bonding and thereby to increase the strength of already healed micro cracks. Finally, Figure 7-17 E shows that the magnesium hydration products in Zone III located inside ECC-MgO paste were a bit higher (5.3\% of total weight) than similar ones in Zone II located within the crack wall (4.90\% of total 
weight). This result is compatible with TGA/DSC results presented in Figure 7-15 (C \& D) which indicated that the degradation peak of $\mathrm{Mg}(\mathrm{OH})_{2}$ inside the $\mathrm{ECC}-\mathrm{MgO}$ paste was a bit higher than similar one within the crack wall. 


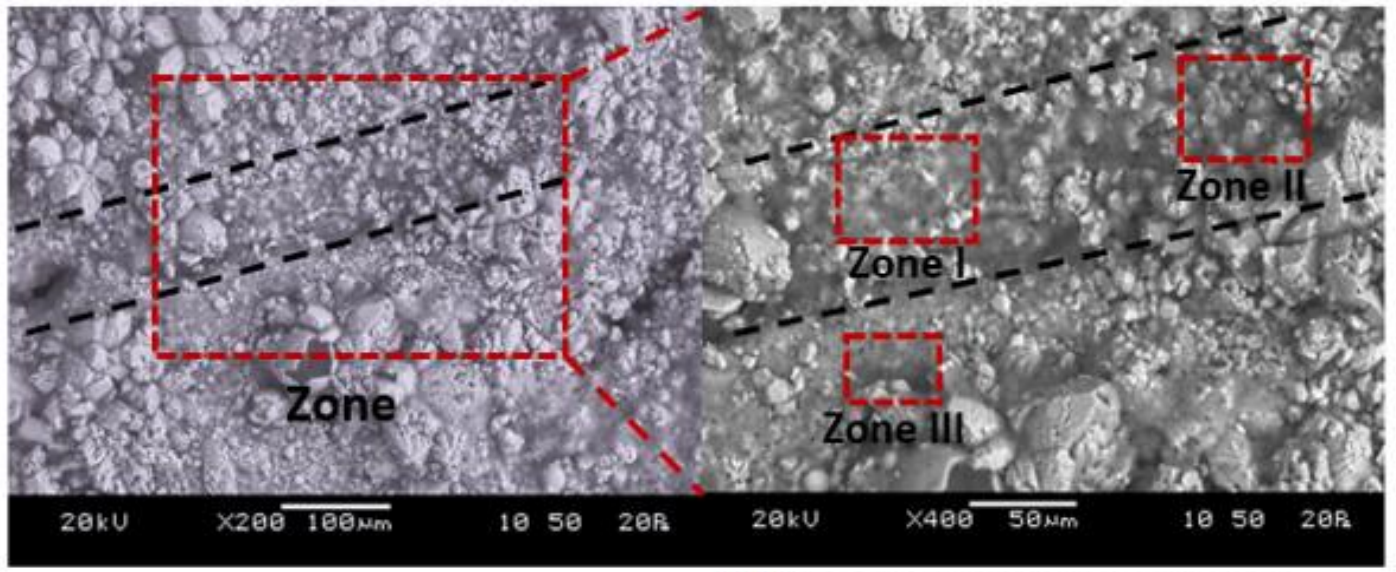

A) 450 days, ECC-MgO microstructure

B) Zone I \& II within crack wall; Zone III in cement paste

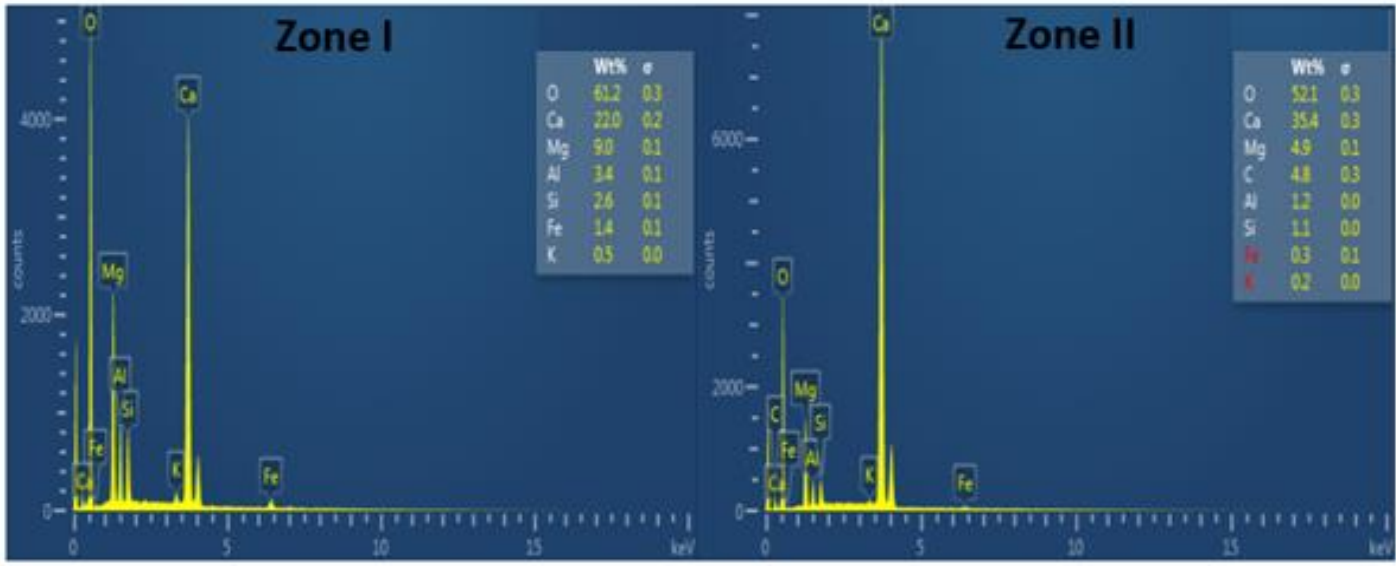

C) Chemical Composition, Zone I

D) Chemical Composition, Zone II

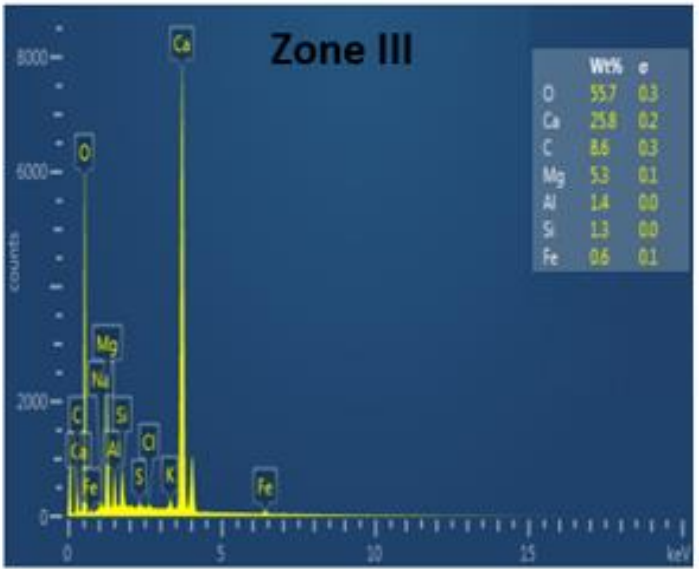

E) Chemical Composition, Zone III

Figure 7-17: Self-healing of ECC-MgO concrete at the age of 450 days - SEM analyses 


\subsection{Summary}

This chapter studied the self-healing behavior of engineered cementitious composites (ECCs) produced from the combination of 5\% MEA and 50\% Class-F fly ash when cured under laboratory, water, natural and autoclave curing conditions. The performance of proposed ECC-MgO selfhealing system was investigated through studying fresh, mechanical and durability properties. The crack healing ability of ECC-MgO self-healing system was assessed through development/recovery of mechanical and durability properties such as RCP, sorptivity, freeze/thaw resistance and recovery of strength/deflection capacity as well as through microstructural characterization by TGA/DSC and SEM analyses. 


\section{STRUCTURAL PERFORMANCE OF WATER AND NATURAL CURED SELF-HEALED LINK SLABS UNDER MONOTONIC LOADING}

\subsection{Introduction}

An experimental program has been conducted for studying the self-healing performance of developed ECC-MgO mixture (using $5 \% \mathrm{MgO}$ as replacement of Class-F fly ash) in link slabs proposed for joint-free bridge deck construction (as introduced in Chapter 2). Six model link slab specimens $\left(1 / 4^{\text {th }}\right.$ scale of the prototype) were constructed by using the ECC-MgO mixture and a traditional ECC mixture (without $\mathrm{MgO}$ ). This chapter describes the details of the experimental investigations illustrating geometric dimensions, casting and curing, testing scheme and instrumentation of model link slab specimens as well as test set-up and testing procedure.

The self-healing capability of pre-cracked/damaged (after 28-days of lab curing) link slab specimens (made of ECC and ECC-MgO mixtures) cured under water or natural/field conditions for four months duration (December $2015 \sim$ April 2016) is described based on the analysis of loaddeflection/moment-rotation response, concrete/steel strain developments, cracking/crack propagation, energy absorption and stiffness properties. The self-healing performance was quantified by strength/stiffness/energy absorbing capacity recovery and crack healing/development of new cracks of healed link slab specimens loaded to failure under static monotonic loading compared to their virgin/un-cracked counterparts. 


\subsection{Experimental program using one-fourth scale ECC link slabs}

The self-healing ability of developed ECC-MgO system in structural elements was evaluated by testing six (two control without damage and four (damaged/pre-cracked) one-fourth scale model link slab specimens as shown in Table 8-1. The design of the link slab model specimens was conducted based on previous research studies (Caner and Zia, 1998; Gahtrehsamani, 2015; Hossain et al., 2015). The typical deformed shape (showing end span rotation, $\theta$ ) and moment distribution due to the applied loading $(\mathrm{P})$ for a two span (span length, $\mathrm{L}_{\mathrm{sp}}$ ) simply supported bridge structure with a link slab is schematically represented in Figure 8-1. Flexural crack formations were expected at the top of the link slab due to the development of tensile stresses. The link slab section was designed between the points of inflection (Figure 8-1 - B). Based on the stiffness of the link slab, the location of the inflection points varies from 0 to $20 \%$ of the adjacent span's length (Caner and Zia, 1998; Kim et al., 2004). 

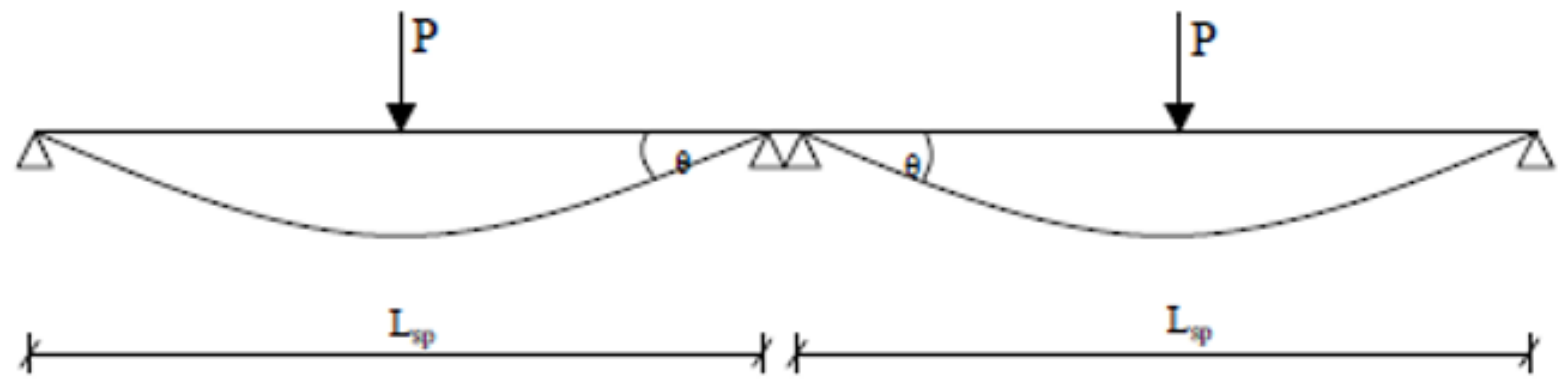

(A) Deformed Shape of the bridge

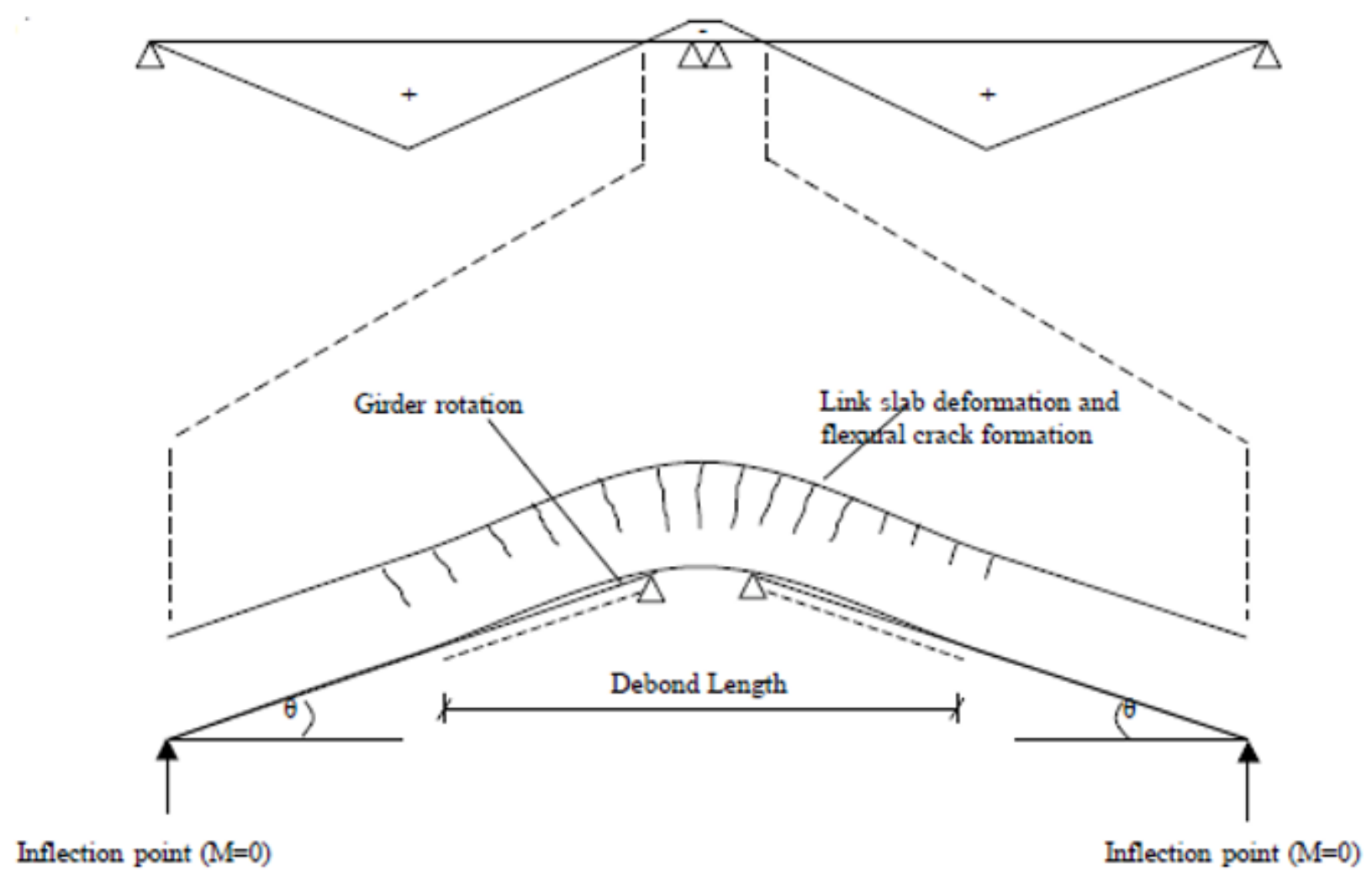

(B) Moment Distribution and flexural crack formation in link slab

Figure 8-1: Two-span bridge deformation with focus on link slab section (Kim et al., 2004)

The dimensions of the $1 / 4^{\text {th }}$ scale link slab specimens were derived based on full-scale prototype $711 \mathrm{~mm}$ wide and $3251 \mathrm{~mm}$ long between the inflection points of the adjacent deck slabs (having W14 x 82 steel I-beams) as shown in Figure 8-2 (Kim et al., 2004). The location of inflection points (length of the link slab) based on the stiffness of the section (ECC and the steel rebar) was determined as $6.5 \%$ of the adjacent span's length. The summary of the link slab specimen's 
configurations with loading and curing conditions are presented in Table 8-1. Overall, the total length of the link slab for the $1 / 4^{\text {th }}$ scale model made of two W100 x 19.3 steel I-sections was 810 $\mathrm{mm}$ as shown Figure 8-3 and Table 8-1. The length of the debonding zone based on the proposed design guidelines by Caner and Zia (1998), was set as 5\% of simple spans - a total length of 330 $\mathrm{mm}$. The length of the transition zone with additional 50\% shear stud connector (total of 16 studs) was selected as $300 \mathrm{~mm}$ following Qian et al. (2009). The cross-sectional dimensions for the $1 / 4^{\text {th }}$ link slab models were $176 \mathrm{~mm}$ in width and $60 \mathrm{~mm}$ in depth. A minimum longitudinal steel reinforcing ratio of $1.1 \%$ (three $6 \mathrm{~mm}$ bars) were provided for maintaining a low structural stiffness of the link slab. Additional transverse reinforcements at $210 \mathrm{~mm} \mathrm{c} / \mathrm{c}$ spacing were provided using $6 \mathrm{~mm}$ bars (Figure 8-3 and Table 8-1).

Table 8-1: Link Slab Specimens configurations, loading and curing conditions

\begin{tabular}{|c|c|c|c|c|c|}
\hline Link Slabs & $\begin{array}{c}\text { Length* } \\
(\mathrm{mm})\end{array}$ & $\begin{array}{c}\text { Debonding } \\
\text { Zone Length } \\
5 \%(\mathrm{~mm})\end{array}$ & $\begin{array}{c}\text { Transition } \\
\text { Zone } \\
\text { Length } \\
2.5 \%(\mathrm{~mm})\end{array}$ & $\begin{array}{c}\text { Concrete in } \\
\text { Transition } \\
\text { Zone }\end{array}$ & $\begin{array}{c}\text { Concrete in } \\
\text { Debonding } \\
\text { Zone }\end{array}$ \\
\hline \multicolumn{7}{|c|}{ Control-link slab specimens - tested to failure under static monotonic } \\
loading after 28-days of lab curing
\end{tabular}

*center to center distance between supports 


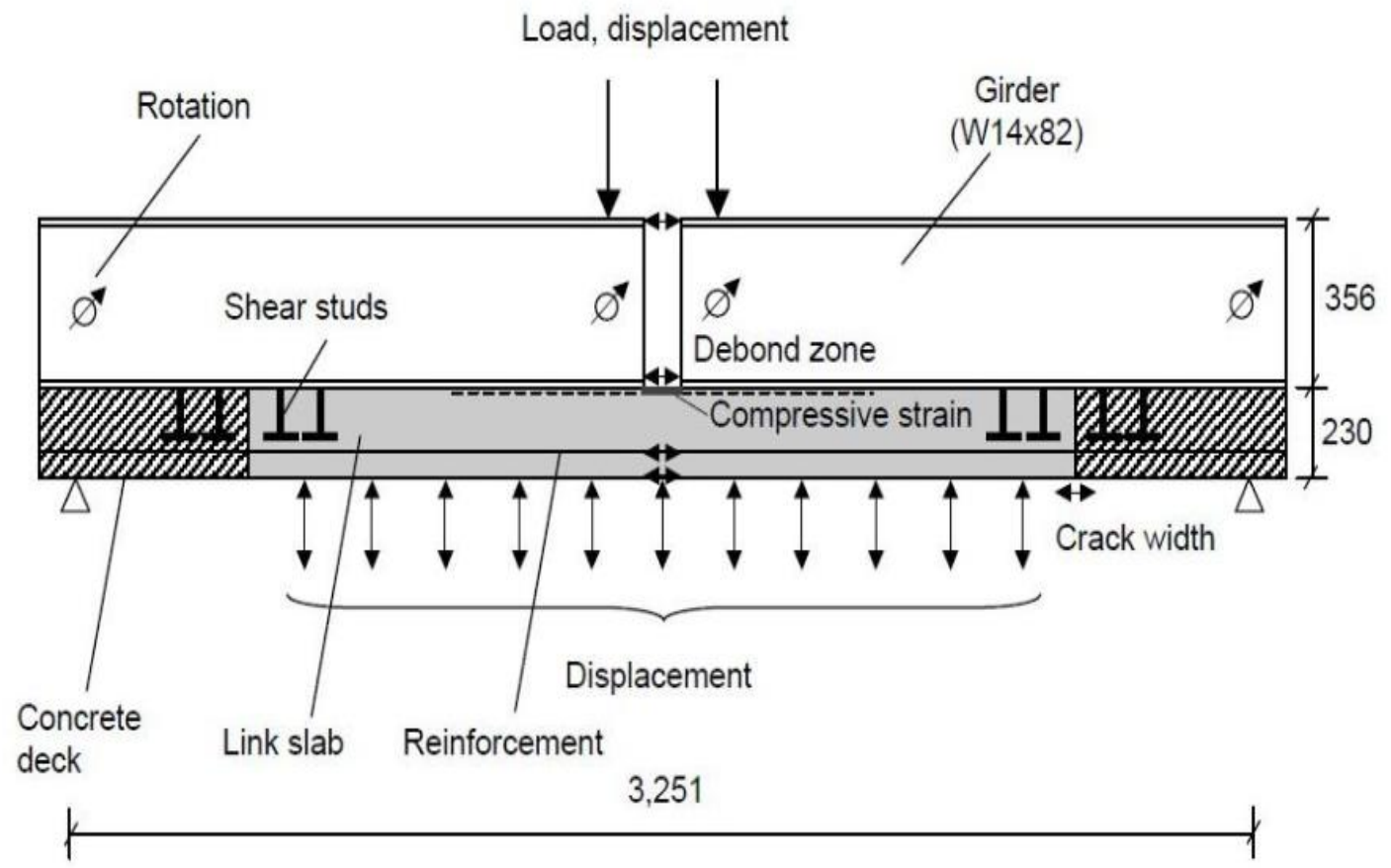

Figure 8-2: Representative full-scale prototype section of link slab, dimensions in mm (Kim et al., 2004) 


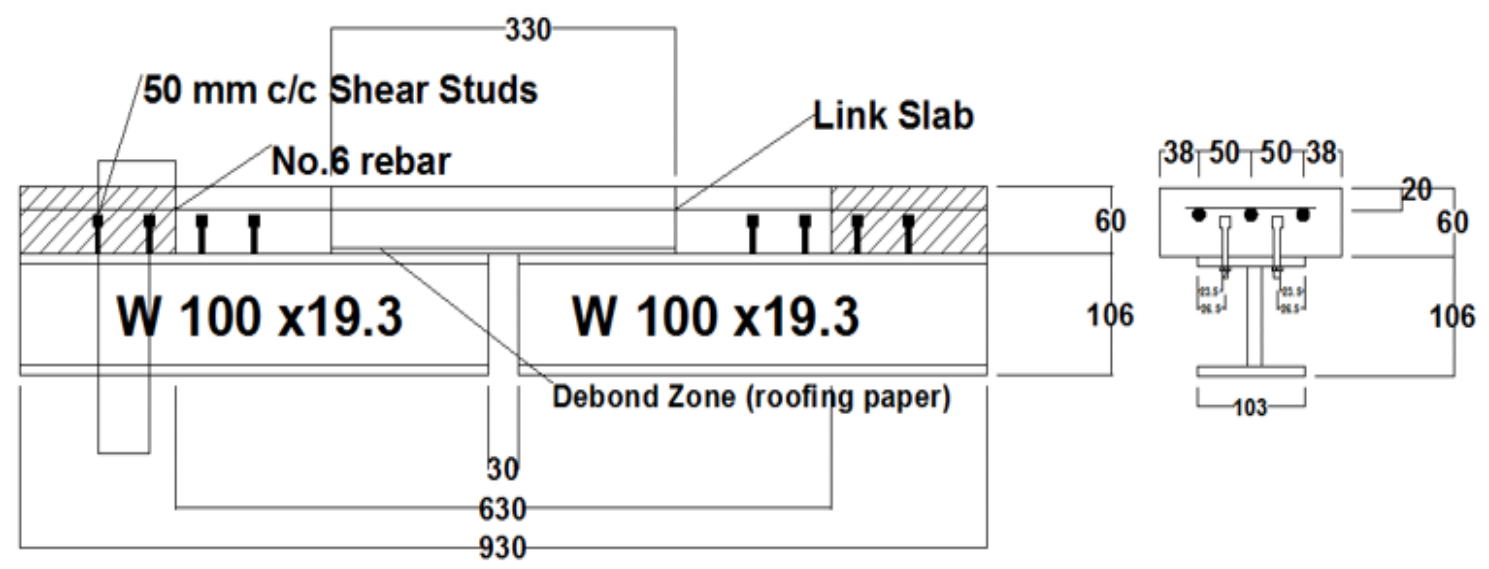

(b) SCC Bridge ECC Link
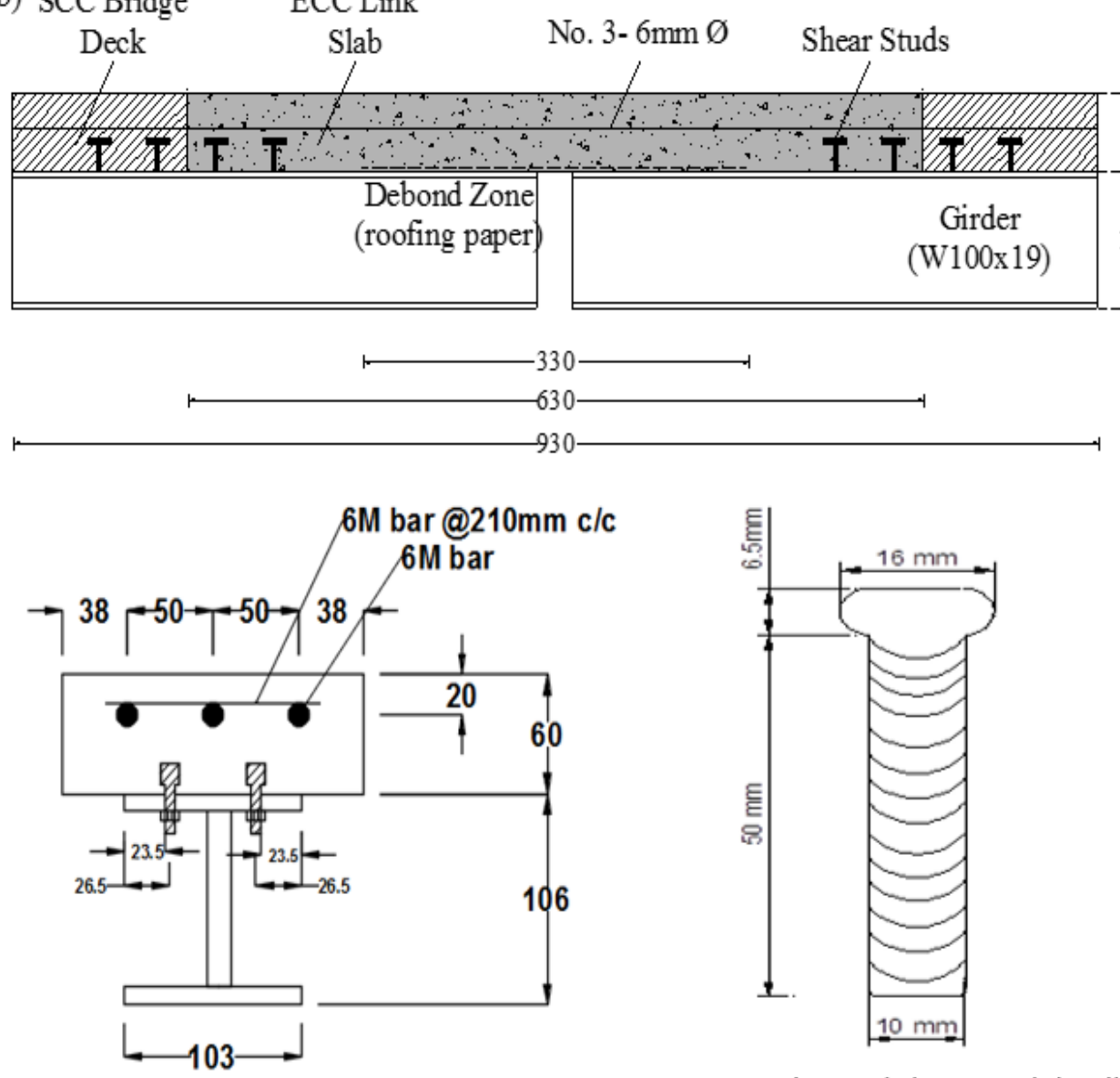

Cross-section and shear-stud details

Figure 8-3: Geometry and reinforcement details of model link slabs (dimensions in $\mathrm{mm}$ ) 
The shear studs (having a shank diameter of $10 \mathrm{~mm}$, shank height of $50 \mathrm{~mm}$, head diameter of 16 $\mathrm{mm}$ and head height of $6.5 \mathrm{~mm}$ shown in Figure 8-3 for the composite action of the steel girder and the concrete bridge decks were selected in accordance with (CISC, 2010).

\subsubsection{Materials and Properties}

Standard ECC, proposed ECC-MgO and a ready mix commercial SCC mixtures were used for the construction of the link slab specimens as indicated in Table 8-1. The ECC was designed by incorporating silica sand and Class-F fly ash (FA) as cement (PC) replacement having FA/PC = $1.2(\mathrm{FA} / \mathrm{Binder}=55 \%)$. ECC-MgO was similar to ECC but had 5\% MEA as fly ash replacement $(\mathrm{FA} /$ Binder $=50 \%)$. The bridge deck portion was cast with SCC and the link slab segment was cast with either ECC or ECC-MgO mixtures as shown in Figure 8-3 and Table 8-1. The mixture proportions of ECC and ECC-MgO mixtures are shown in Table 8-2 while chemical and physical properties of the Portland cement and fly ash are presented in (Chapter three - Table 3-1). Commercial SCC was made of $10 \mathrm{~mm}$ nominal size aggregate, Portland cement, silica fume and air-entraining admixtures. For each $30 \mathrm{~kg}$ pre-packaged bag, a 2.4 liter of water was added to developing SCC mixture. The $6 \mathrm{~mm}$ dia. Reinforcing steel bars had a mean yield and ultimate strength of $407 \mathrm{MPa}$ and $550 \mathrm{MPa}$, respectively. The compressive strength of SCC at 28 days was ranging between 60 and $63 \mathrm{MPa}$, modulus of elasticity was $33.59 \mathrm{GPa}$ and its flexural strength value was $3 \mathrm{MPa}$ at $1 \mathrm{~mm}$ of ultimate mid-span deflection. The compressive and flexural strength properties of ECC and ECC-MgO mixtures under various curing conditions and at different curing/test ages are presented in Table 8-3 \& Table 8-4.

Table 8-2: ECC and ECC-MgO mixture proportions

\begin{tabular}{|c|c|c|c|c|c|c|c|c|}
\hline \multirow{2}{*}{ Mixture ID } & \multicolumn{8}{|c|}{ Ingredients per 1 part of binder measured by mass } \\
\cline { 2 - 10 } & $\begin{array}{c}\text { Water/ } \\
\text { Binder }\end{array}$ & $\begin{array}{c}\text { Binder } \\
(\mathrm{PC}+\mathrm{FA})\end{array}$ & $\mathrm{MgO}$ & $\begin{array}{c}\text { FA/Binder after } \\
\text { adding MgO }\end{array}$ & $\begin{array}{c}\text { Silica } \\
\text { Sand }\end{array}$ & $\begin{array}{c}\text { PVA } \\
\mathrm{kg} / \mathrm{m}^{3}\end{array}$ & $\begin{array}{c}\text { HRWRA } \\
\mathrm{kg} / \mathrm{m}^{3}\end{array}$ & FA/PC \\
\hline ECC & 0.27 & 1 & - & $55 \%$ & 0.36 & 26 & 5.4 & 1.20 \\
\hline ECC-MgO & 0.27 & 1 & $5 \%$ & $50 \%$ & 0.36 & 26 & 5.4 & 1.09 \\
\hline
\end{tabular}


Table 8-3: Compressive strength of ECC mixtures

\begin{tabular}{|c|c|c|c|c|}
\hline \multirow{2}{*}{$\begin{array}{c}\text { Time } \\
\text { (days) }\end{array}$} & \multicolumn{3}{|c|}{ ECC-control (MPa) } & \multicolumn{2}{c|}{ ECC-MgO-control (MPa) } \\
\cline { 2 - 5 } & \multicolumn{3}{|c|}{ Laboratory Curing* } \\
\hline $28 *$ & \multicolumn{2}{|c|}{60.40} & \multicolumn{2}{c|}{55.33} \\
\hline & Water & Natural & Water** & Natural $^{* *}$ \\
\hline $120^{* *}$ & 71.00 & 68.50 & 66.90 & 64.00 \\
\hline
\end{tabular}

*Lab curing means specimens cured inside plastic bags until 28-days, **water and natural curing mean specimens stored in water continuously and in natural environment until 120-days

Table 8-4: Flexural and deformation properties of ECC mixture

\begin{tabular}{|c|c|c|c|c|c|}
\hline \multirow[b]{2}{*}{$\begin{array}{c}\text { Mix } \\
\text { Designation }\end{array}$} & \multirow[b]{2}{*}{$\begin{array}{l}\text { Time } \\
\text { (days) }\end{array}$} & \multicolumn{2}{|c|}{ Water Curing* } & \multicolumn{2}{|c|}{ Natural Curing* } \\
\hline & & $\begin{array}{c}\text { Flexural } \\
\text { Strength } \\
(\mathrm{MPa})\end{array}$ & $\begin{array}{l}\text { Deflection } \\
\quad(\mathrm{mm})\end{array}$ & $\begin{array}{c}\text { Flexural } \\
\text { Strength } \\
(\mathrm{MPa})\end{array}$ & $\begin{array}{l}\text { Deflection } \\
\quad(\mathrm{mm})\end{array}$ \\
\hline \multirow{2}{*}{$\begin{array}{c}\mathrm{ECC} \\
\text { controls }\end{array}$} & 28 & 14.12 & 7.42 & 11.9 & 3.26 \\
\hline & 120 & 15.69 & 7.38 & 11.9 & 4.07 \\
\hline \multirow{2}{*}{$\begin{array}{c}\text { ECC-MgO } \\
\text { controls }\end{array}$} & 28 & 11.3 & 5.57 & 13.1 & 4.97 \\
\hline & 120 & 11.6 & 3.12 & 9.55 & 4.67 \\
\hline
\end{tabular}

\subsubsection{Casting of link slab specimens}

Long wooden boards were fastened together for preparing the deck slab of the $1 / 4^{\text {th }}$ scale link slabs. For each specimen, the wooden deck mold was clamped and locked into the two separate steel girders at $30 \mathrm{~mm}$ distanced apart. As a barrier for steel and concrete, a 15-lb roofing paper was provided in the debonding zone of the link slab. The casting procedure of the link slab specimens was followed the general field practice. The bridge deck portion was cast initially with flowable ready SCC mix and left for 24 hours for setting. Then the link slab segment was cast with ECC or ECC-MgO after initial 24 hours as shown in Figure 8-4. The specimens were cured for 28 days at the laboratory temperature while covered with the burlap. The relative humidity $(\mathrm{RH})$ and the temperature of the laboratory were $45 \underline{ \pm} \%$ and $24 \underline{ \pm} 2^{\circ} \mathrm{C}$, respectively. The sequential casting process of the $1 / 4^{\text {th }}$ scale link slab specimens is presented in Figure 8-4 (A, B and C). 


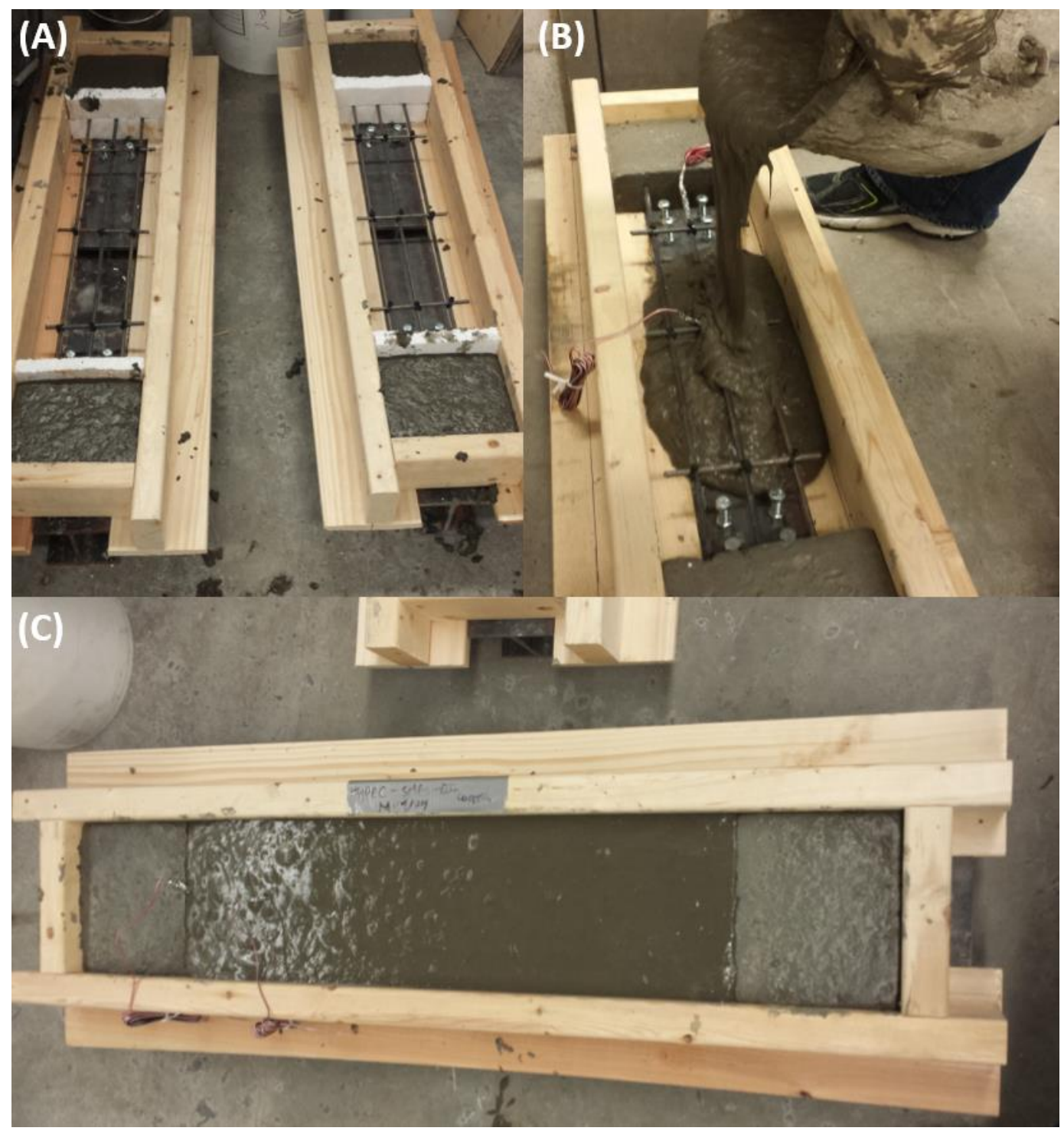

Figure 8-4: Casting Process of $1 / 4^{\text {th }}$ Scale link slab specimens; A) mold preparation and casting SCC bridge deck portion; B) casting ECC or ECC-MgO Link slab segment; C) finished link slab specimen

\subsubsection{Test Set-Ups, Instrumentation and Testing Procedure}

Test set-up for link slab specimens are shown in Figure 8-5. The inflection points spanning 810 $\mathrm{mm}$ were simulated by the pin and roller supports. For practical purpose, the link slab was tested in an inverted orientation (as shown in Figure 8-5) for simulating actual link slab behavior in a 
joint-free bridge deck. During casting two strain gauges were installed on the steel bar (as shown in Figure 8-5 - the first one was at the mid-span location in between the two loading points (called as abutment-strain) while the other one at the $1 / 4^{\text {th }}$ span location (called as mid-strain) for recording the tensile strain development. Also, two strain gauges were also installed on the surface (top and bottom) of the concrete slab at mid-span (as shown in Figure 8-5) to record concrete strain development. Besides an inclinometer was installed at the support location for recording the support-end rotation.

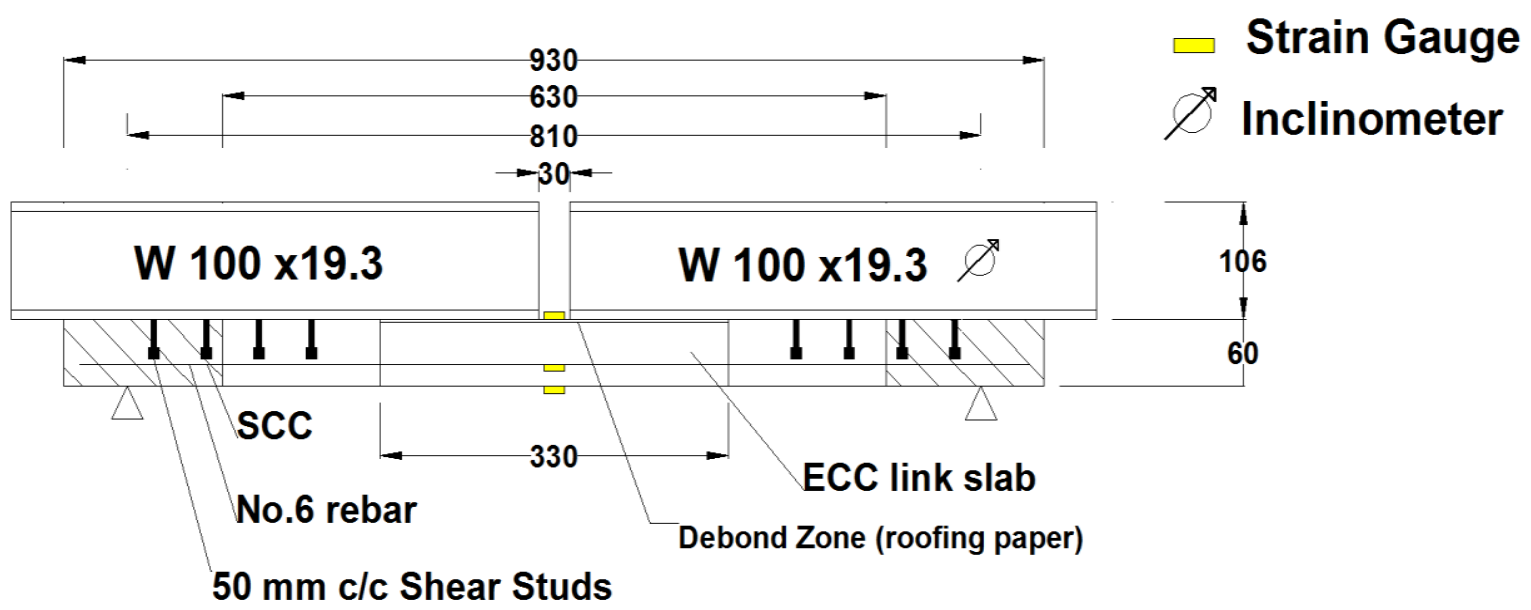

Figure 8-5: Schematic representation of test set-up for $1 / 4^{\text {th }}$ scale link slab specimens showing instrumentation (dimensions in $\mathrm{mm}$ )

The monotonic testing (either loading to failure or pre-cracking to induce damage) was performed using a closed-loop controlled servo-hydraulic system under displacement condition at a loading rate of $0.005 \mathrm{~mm} / \mathrm{s}$ (as shown in Figure 8-6). During loading history, load-mid-span displacement was recorded by the MTS machine while support rotation and steel/concrete strain development were recorded by additional data acquisition system. Also, crack propagations and failure modes were visually observed and crack width/length was measured using the crack scope. 


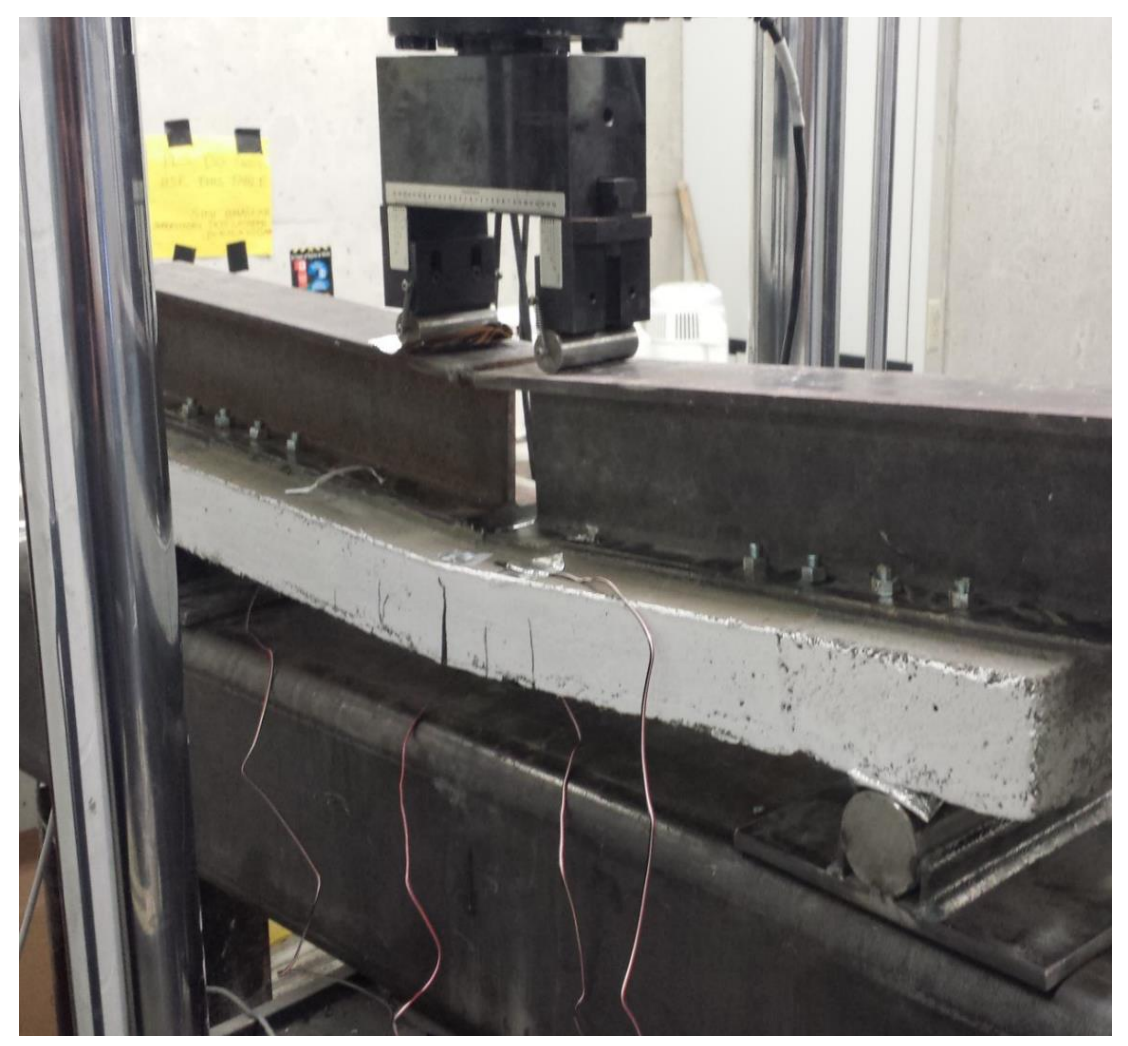

Figure 8-6: Testing and instrumentation of link slab specimen showing cracking and deformation

\subsubsection{Self-healing curing methods, testing scheme and post-healing performance evaluation}

Two link slab specimens (ECC-control and ECC-MgO-control as per Table 8-1) after 28 days of lab curing were loaded to failure under static monotonic loading. After 28-days of lab curing, distributed cracks were formed by pre-loading remaining four ECC and ECC-MgO link slab specimens up to $65 \%$ of the ultimate strength of the control specimens (ECC-control and ECC$\mathrm{MgO}$-control as indicated in Table 8-1). Then the crack-formed damaged specimens were subjected to two exposure conditions (to induce self-healing) - cured in water and cured naturally. Accordingly, two preloaded link slab specimens (ECC-water and ECC-MgO-water as per Table 8-1) were kept continuously in a sealed water container to conduct water curing while the other 
two specimens (ECC-Natural and ECC-MgO-natural as per Table 8-1) were taken out to the field to be cured naturally under harsh Canadian environment (Figure 8-7 natural curing). Figure 8-8 shows water curing process of pre-cracked link slab specimens in sealed water tank. Bricks were placed underneath the link slab specimens to create a gap between the specimen and the water surface. Very hot water was poured into the tank simulating water vapor curing environment to induce self-healing process. All specimens were placed cautiously inside the tank (as shown in Figure 8-8) without damaging attached strain gauges. Hot water was poured at the final stage of preparation before sealing the container by polyethylene sheets. Also, all strain gauges were sealed firmly in small plastic bags to keep safe from hot water vapor. Water vapor curing was continued for 120 days (four months) in the sealed tank. At the end of water curing, a small gap was created through polyethylene sheets and a sensor was inserted proving that the link slab specimens were cured at a relative humidity $(\mathrm{RH})$ and temperature of $52 \pm 2 \%$ and $23 \pm 2^{\circ} \mathrm{C}$, respectively.

After water or natural curing period of over 120 days, four self-healed pre-cracked link slab specimens were loaded to failure under static monotonic loading. The post self-healed loading to failure event was similar to the monotonic flexural loading conducted on ECC-control or ECC$\mathrm{MgO}$ control specimens. During loading history, development of load-deflection/moment-rotation response and concrete/steel strain development were recorded through LVDT, strain gauges and inclinometer readings in addition to monitoring crack development/propagation and failure modes. The performance of control and post-healed cracked/damaged link slab specimens was compared based on load-deflection/moment-rotation response, initial stiffness, concrete/steel strain development, peak load/ strength, steel yielding, energy absorption capacity, new crack formation, and crack width to quantify the effect of self-healing. 


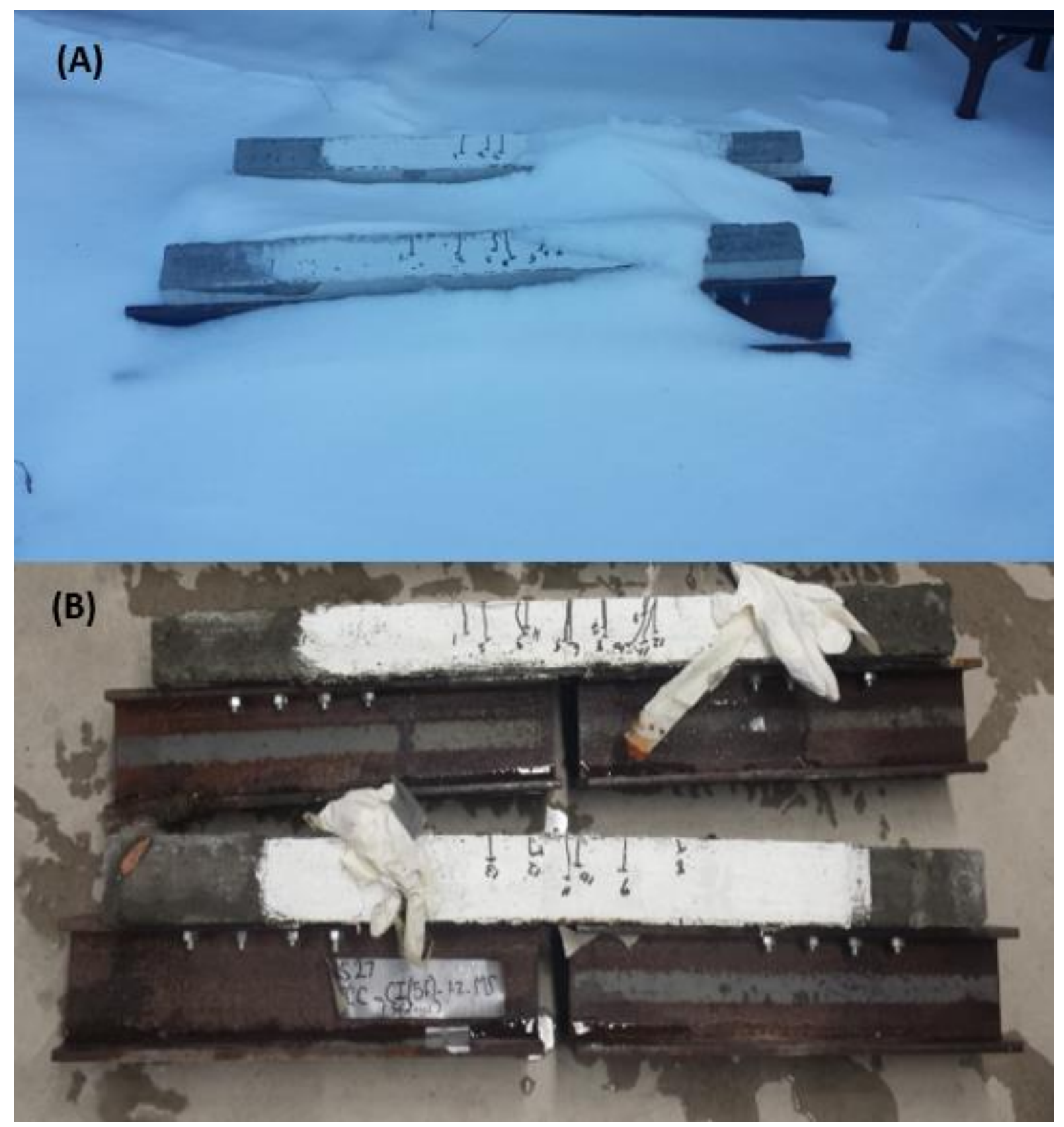

Figure 8-7: Link slab natural curing; a) specimens cured under harsh environment for 120 days; b) natural cured specimens at the day of testing after bringing them to the lab 

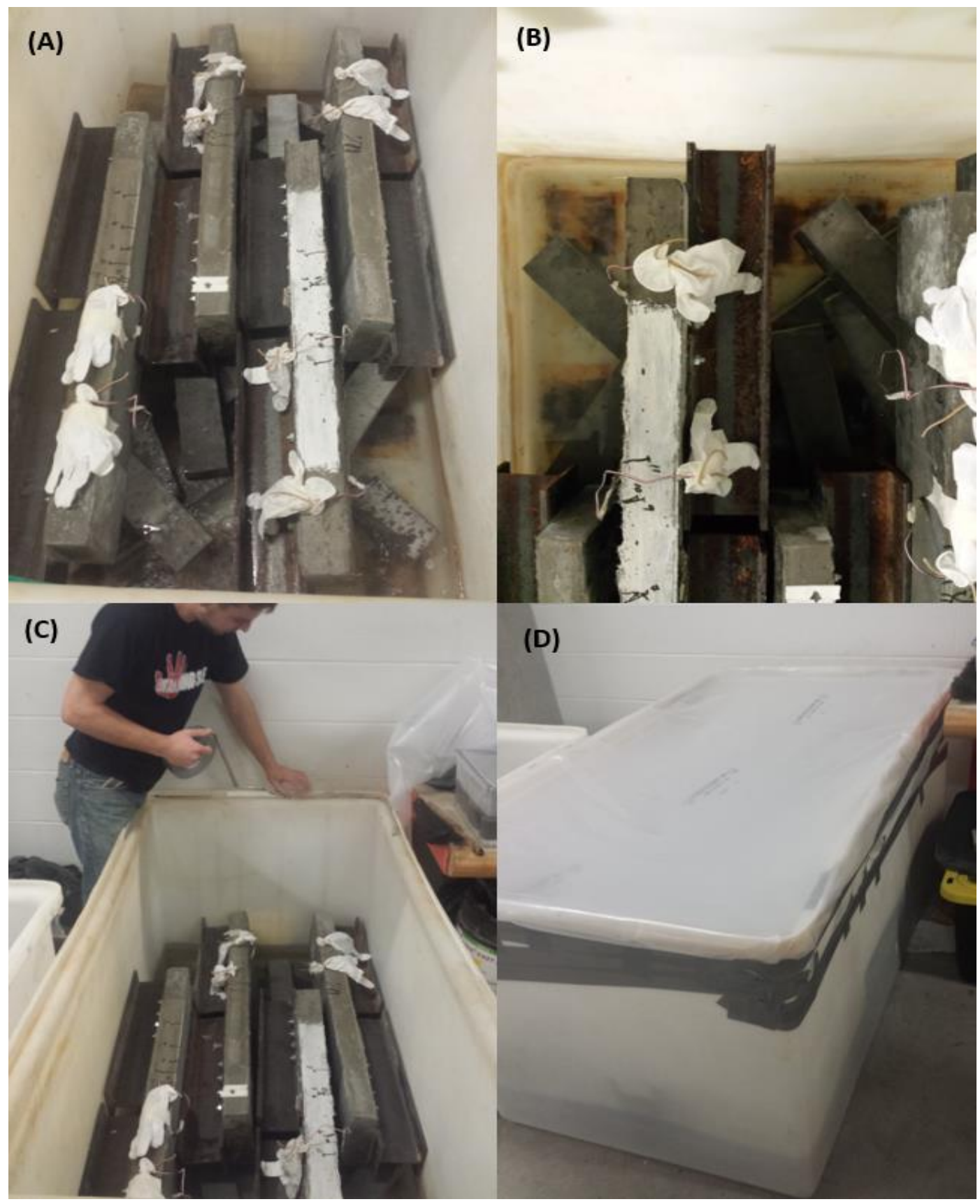

Figure 8-8: Link slab water curing steps; A) placing the link slab specimens above hot water; B) securing the strain gauges firmly; C) sealing the water container; D) curing link slab specimens for 120 


\subsection{Results and Discussions}

\subsubsection{Structural performance of ECC and ECC-MgO link slabs subjected to monotonic loading and evaluation of self-healing potential}

The structural performance of the $1 / 4^{\text {th }}$ scale link slab specimens subjected to monotonic loading is described and compared with special reference to self-healing effect ECC and ECC-MgO mixtures.

\subsubsection{Comparative structural performance and self-healing characterization using load-deflection and moment-rotation responses}

The key results derived from load-deflection responses (shown in Figure 8-9) including ultimate (peak) load/moment, $1^{\text {st }}$ cracking load/deflection, failure deflection/rotation, pre-cracking stiffness and energy absorbing capacity and ultimate moment capacity are summarized in Table 8-5. 


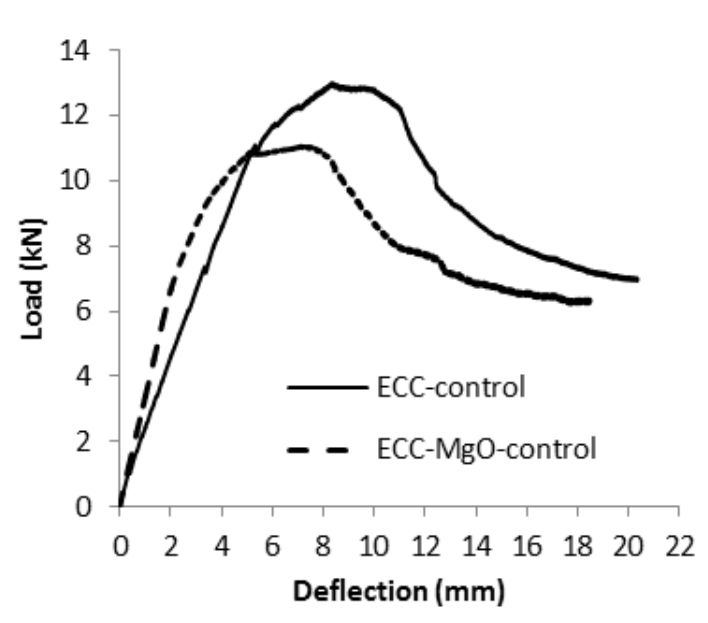

(A)

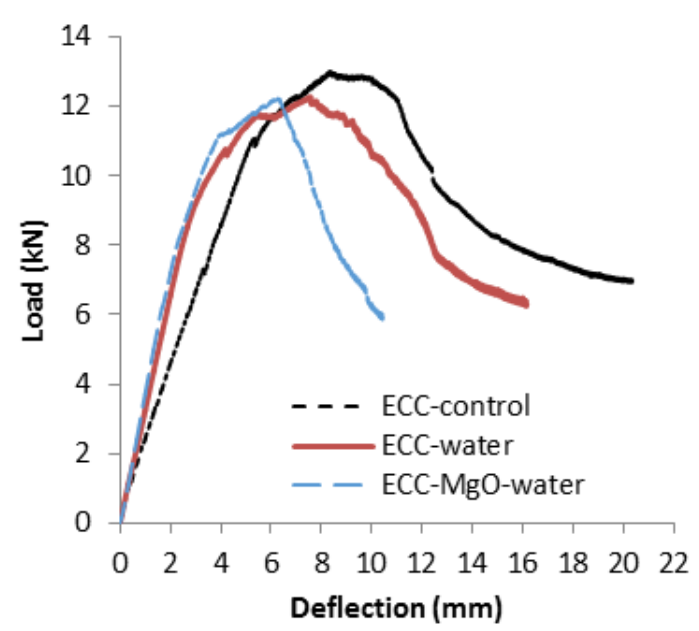

(B)

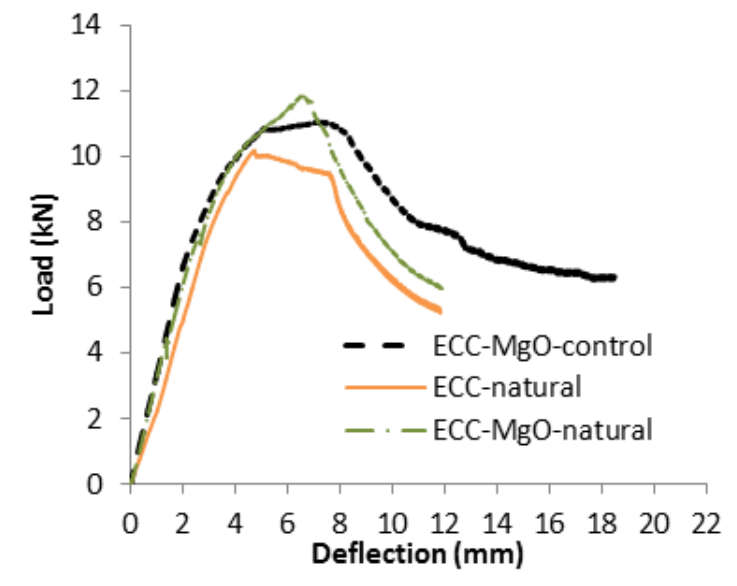

(C)

Figure 8-9: Load-deflection responses of link slabs

Figure 8-9 (B) compares load-deflection responses of post-healed ECC-water and ECC-MgOwater link slab specimens with their ECC-control counterparts. Post-healed ECC-water and ECC$\mathrm{MgO}$-water specimens developed lower ultimate (peak) load/moment and deflection capacities compared to their ECC-control counterpart. Although post-healed ECC-water and ECC-MgOwater specimens achieved similar load/moment capacities but ECC-MgO-water one showed lower deflection capacities. Post-healed ECC-MgO-water/ECC-water specimens showed higher precracking stiffness (slope of initial part of the response) than their ECC-control counterpart with ECC-MgO-water specimen showing higher stiffness. 
Figure 8-9 (C) compares load-deflection responses of post-healed ECC-natural and ECC-MgOnatural link slab specimens with their ECC-MgO-control counterpart. Post-healed ECC-MgOnatural link slab specimen developed higher strength (peak load)/moment capacity while ECCnatural developed lower strength than ECC-MgO-control counterpart. Both post-healed ECCnatural and ECC-MgO-natural ones developed lower deflection capacities than their ECC-MgOcontrol counterpart.
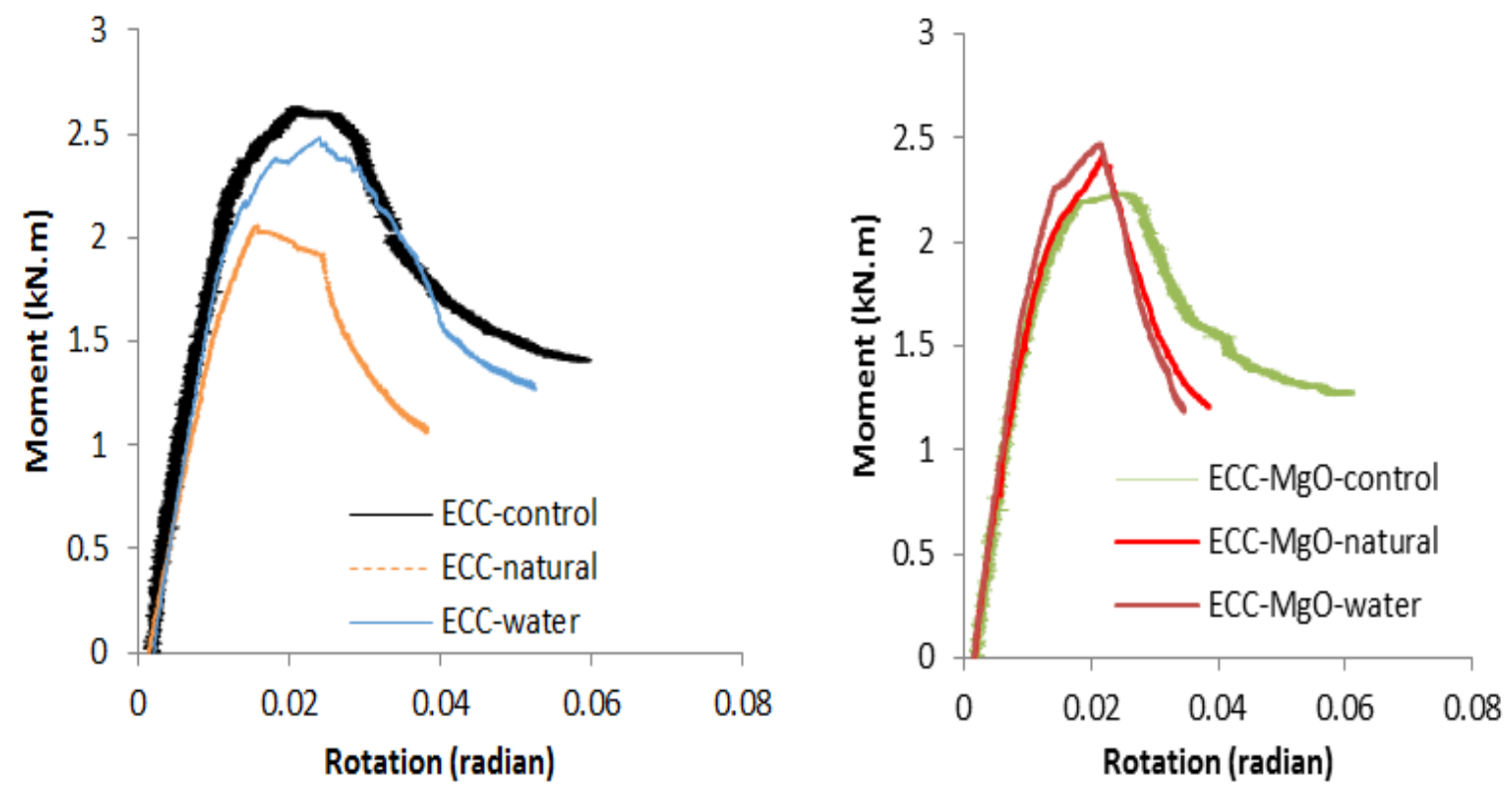

Figure 8-10: Moment-rotation responses of link slabs 
Table 8-5: Summary of load-deflection and moment-rotation responses

\begin{tabular}{|c|c|c|c|c|c|c|c|c|c|}
\hline \multirow{3}{*}{ Link slab } & \multirow{3}{*}{$\begin{array}{l}\text { Loading } \\
\text { to }\end{array}$} & \multicolumn{2}{|c|}{ Load } & \multicolumn{3}{|c|}{ Deflection } & \multicolumn{2}{|c|}{ Rotation } & \multirow{2}{*}{$\begin{array}{c}\text { Moment } \\
\text { Peak }\end{array}$} \\
\hline & & Peak & $\begin{array}{l}1 \text { st } \\
\text { crack }\end{array}$ & Peak & $\begin{array}{l}1 \text { st } \\
\text { crack }\end{array}$ & Failure & Peak & Failure & \\
\hline & & \multicolumn{2}{|c|}{$(\mathrm{kN})$} & \multicolumn{2}{|c|}{$(\mathrm{mm})$} & & \multicolumn{2}{|c|}{ (radian) } & (kN.m) \\
\hline \multicolumn{10}{|c|}{$\begin{array}{l}\text { Link slab specimens - Pre-cracked (damaged) and then water cured for } 4 \text { months before loaded to failure under } \\
\text { static monotonic loading }\end{array}$} \\
\hline $\begin{array}{l}\text { ECC- } \\
\text { control }\end{array}$ & $\begin{array}{l}\text { Virgin- } \\
\text { failure }\end{array}$ & 13.0 & 7.4 & 8.25 & 3.3 & 18.48 & 0.0206 & 0.0604 & 2.63 \\
\hline $\begin{array}{l}\text { ECC-MgO- } \\
\text { control }\end{array}$ & $\begin{array}{l}\text { Virgin- } \\
\text { failure }\end{array}$ & 11.1 & 7.0 & 7.46 & 2.1 & 18.50 & 0.0254 & 0.0620 & 2.23 \\
\hline \multicolumn{10}{|c|}{$\begin{array}{l}\text { Link slab specimens - Pre-cracked (damaged) and then water cured for } 4 \text { months before loaded to failure under } \\
\text { static monotonic loading }\end{array}$} \\
\hline \multirow[b]{2}{*}{ ECC-water } & pre-cracking & 8.40 & - & 4.30 & - & 4.30 & 0.0140 & 0.0140 & 1.69 \\
\hline & $\begin{array}{l}\text { Post-healed } \\
\text { failure }\end{array}$ & 12.3 & 9.0 & 7.54 & 2.8 & 16.14 & 0.0241 & 0.0526 & 2.48 \\
\hline \multirow{2}{*}{$\begin{array}{l}\text { ECC-MgO- } \\
\text { water }\end{array}$} & pre-cracking & 7.32 & - & 3.26 & - & 3.26 & 0.0430 & 0.0430 & 1.45 \\
\hline & $\begin{array}{l}\text { Post-healed } \\
\text { failure }\end{array}$ & 12.2 & 8.2 & 6.30 & 2.3 & 10.45 & 0.0213 & 0.0347 & 2.47 \\
\hline \multicolumn{10}{|c|}{$\begin{array}{l}\text { Link slab specimens - Pre-cracked (damaged) and then natural cured for } 4 \text { months before loaded to failure under } \\
\text { static monotonic loading }\end{array}$} \\
\hline \multirow{2}{*}{$\begin{array}{l}\text { ECC- } \\
\text { natural }\end{array}$} & pre-cracking & 8.40 & - & 4.30 & - & 4.30 & 0.0200 & 0.0200 & 1.70 \\
\hline & $\begin{array}{l}\text { Post-healed } \\
\text { failure }\end{array}$ & 10.2 & 4.8 & 4.73 & 1.9 & 11.83 & 0.0158 & 0.0383 & 2.06 \\
\hline \multirow{2}{*}{$\begin{array}{c}\text { ECC-MgO- } \\
\text { natural }\end{array}$} & pre-cracking & 7.32 & - & 3.26 & - & 3.26 & 0.0120 & 0.0120 & 1.48 \\
\hline & $\begin{array}{l}\text { Post-healed } \\
\text { failure }\end{array}$ & 11.9 & 4.5 & 6.60 & 1.5 & 11.9 & 0.0217 & 0.0386 & 2.41 \\
\hline
\end{tabular}

Moment capacity: Peak load $(\mathrm{kN}) * 405 \mathrm{~mm} / 1000$

For all ECC link slabs, a steady increase in load with the increase of deflection was observed up to the peak load. The post-peak behavior was characterized by a gradual decrease in the load before failure (Figure 8-9). This proves that all ECC link slabs clearly defined strain hardening and ductility behaviour by showing no major drop in load-deflection responses up to the peak load at the onset of multi-crack formation (as normally happens in traditional concrete where a single crack formation is accompanied by sudden drop in load which marks the transfer of load to the steel reinforcement) and also by showing post-peak descending branch before failure. The moment-rotation responses also showed similar patterns as observed in load-deflection responses (Figure 8-10). 
ECC-control link slab specimen developed higher peak (ultimate load) and moment capacity compared to its ECC-MgO counterparts (Figure 8-9 - A \& Table 8-5) - which can be associated with the higher compressive and flexural strength of ECC mixture as indicated in Table 8-3 and Table 8-4.

Table 8-6: Peak load/deflection/energy absorption/stiffness capacity of link slabs w.r.to control

\begin{tabular}{|c|c|c|c|c|c|c|c|c|c|}
\hline \multirow{3}{*}{ Link slab } & \multirow{3}{*}{$\begin{array}{l}\text { Loading } \\
\text { to }\end{array}$} & \multicolumn{2}{|c|}{ Load } & \multicolumn{2}{|c|}{ Deflection } & \multicolumn{2}{|c|}{ Energy } & \multicolumn{2}{|c|}{$\begin{array}{c}\text { Stiffness at first } \\
\text { crack }\end{array}$} \\
\hline & & Peak & $\begin{array}{l}\% \text { of } \\
\text { control }\end{array}$ & Peak & $\begin{array}{l}\% \text { of } \\
\text { control }\end{array}$ & $\begin{array}{l}\text { Peak } \\
\text { load }\end{array}$ & $\begin{array}{c}\% \\
\text { control }\end{array}$ & & $\begin{array}{l}\% \text { of } \\
\text { control }\end{array}$ \\
\hline & & $(\mathrm{kN})$ & & $(\mathrm{mm})$ & & Joules & & $\mathrm{kN} / \mathrm{mm}$ & \\
\hline \multicolumn{10}{|c|}{ Control-link slab specimens - tested to failure under static monotonic loading after 28 -days of lab curing } \\
\hline $\begin{array}{l}\text { ECC- } \\
\text { control }\end{array}$ & $\begin{array}{l}\text { Virgin- } \\
\text { failure }\end{array}$ & 13.0 & - & 8.25 & - & 67.31 & - & 2.16 & - \\
\hline $\begin{array}{l}\text { ECC-MgO- } \\
\text { control }\end{array}$ & $\begin{array}{l}\text { Virgin- } \\
\text { failure }\end{array}$ & 11.1 & - & 7.46 & - & 63.02 & - & 3.16 & - \\
\hline \multicolumn{10}{|c|}{$\begin{array}{l}\text { Link slab specimens - Pre-cracked (damaged) and then water cured for } 4 \text { months before loaded to failure under } \\
\text { static monotonic loading }\end{array}$} \\
\hline \multirow[b]{2}{*}{ ECC-water } & Pre-cracking & 8.40 & 65.0 & 4.30 & 52.1 & 19.54 & - & 1.95 & - \\
\hline & $\begin{array}{l}\text { Post-healed } \\
\text { failure }\end{array}$ & 12.3 & 95.0 & 7.54 & 91.4 & 65.50 & 97.3 & 3.00 & 139 \\
\hline \multirow{2}{*}{$\begin{array}{l}\text { ECC-MgO- } \\
\text { water }\end{array}$} & Pre-cracking & 7.32 & 66.5 & 3.26 & 43.7 & 12.13 & - & 2.24 & - \\
\hline & $\begin{array}{l}\text { Post-healed } \\
\text { failure }\end{array}$ & 12.2 & 110.5 & 6.30 & 84.5 & 53.00 & 84.1 & 3.40 & 108 \\
\hline \multicolumn{10}{|c|}{$\begin{array}{c}\text { Link slab specimens - Pre-cracked (damaged) and then natural cured for } 4 \text { months before loaded to failure under } \\
\text { static monotonic loading }\end{array}$} \\
\hline \multirow{2}{*}{$\begin{array}{l}\text { ECC- } \\
\text { natural }\end{array}$} & Pre-cracking & 8.40 & 65.0 & 4.30 & 52.1 & 19.44 & - & 1.95 & - \\
\hline & $\begin{array}{l}\text { Post-healed } \\
\text { failure }\end{array}$ & 10.2 & 78.5 & 4.73 & 57.3 & 27.00 & 40.1 & 2.45 & 113 \\
\hline \multirow{2}{*}{$\begin{array}{c}\text { ECC-MgO- } \\
\text { natural }\end{array}$} & Pre-cracking & 7.32 & 66.2 & 3.26 & 43.7 & 13.17 & & 2.24 & \\
\hline & $\begin{array}{l}\text { Post-healed } \\
\text { failure }\end{array}$ & 11.9 & 108.0 & 6.60 & 88.5 & 51.10 & 81.0 & 3.00 & 95 \\
\hline
\end{tabular}

Table 8-5 shows that the ultimate (peak) load and moment capacity of ECC-control link slab are $13.0 \mathrm{kN}$ and $2.63 \mathrm{kN} . \mathrm{m}$, respectively compared to $11.1 \mathrm{kN}$ and $2.3 \mathrm{kN} . \mathrm{m}$, respectively of ECC$\mathrm{MgO}$ link slab. The first cracking load of ECC-control and ECC-MgO-control are 56\% and 64\% of peak load - this signified enhanced flexural strength of ECC-MgO mixture which led to the better structural performance of delayed transfer of load to steel reinforcement. The initial/precracking stiffness of ECC-control specimen $(2.16 \mathrm{kN} / \mathrm{mm})$ was lower (0.68 times) than its ECC- 
MgO-control counterpart $(3.16 \mathrm{kN} / \mathrm{m})$ and hence, showed higher pre-peak deflection/rotation/energy absorbing capacity but subsequently, produced higher post-peak deflection/rotation capacity. Both ECC-control (34 cracks) and ECC-MgO-control (18 cracks) showed multi-cracking characteristics before failure.

Self-healing performance is evaluated based on strength, pre-cracking stiffness and deflection recovery of post-healed link slab specimens compared to their control counterparts as summarized in Table 8-6. Post-healed ECC-water specimen recovered 95\% of the ultimate (peak) load of ECCcontrol compared to $78.5 \%$ of ECC-natural one. Similarly, post-healed ECC-MgO-water specimen recovered $110.5 \%$ of the ultimate (peak) load of ECC-MgO-control compared to $108 \%$ of ECC$\mathrm{MgO}$-natural one. Hence, water curing showed better strength recovery than natural curing indicating a beneficial effect of water (vapor) curing in enhancing the underlying process of selfhealing. On the other hand, ECC-MgO-specimens showed better strength recovery (even exceeded the strength of control specimens)/self-healing capability compared to their ECC specimen counterparts.

In terms of deflection, post-healed ECC-water specimen recovered $91.4 \%$ of peak deflection of ECC-control compared to $57.3 \%$ of ECC-natural one (Table 8-6). Similarly, post-healed ECC$\mathrm{MgO}$-water specimen recovered $84.5 \%$ of peak deflection of ECC-MgO-control compared to $88.5 \%$ of ECC-MgO-natural one. Hence, water curing showed better peak deflection recovery than natural curing. On the other hand, ECC-MgO-specimens showed lower peak deflection recovery compared to their ECC counterparts. It should be noted that post-healed ECC or ECC-MgO specimens either water or natural cured did not recover $100 \%$ of deflection capacity of control.

With respect to energy, post-healed ECC-water specimen recovered $97.3 \%$ of energy absorbing capacity (up to peak load) of ECC-control compared to $40.1 \%$ of ECC-natural one (Table 8-6). Similarly, post-healed ECC-MgO-water specimen recovered $84.1 \%$ of energy absorbing capacity of ECC-MgO-control compared to $81 \%$ of ECC-MgO-natural one. Overall, water curing showed better energy recovery than natural curing. In terms of energy recovery, it was not clear whether ECC-MgO or ECC specimens performed better although ECC-water seemed to have shown better 
energy recovery. It should be noted that post-healed ECC or ECC-MgO specimens either water or natural cured did not recover $100 \%$ of energy capacity of control.

With respect to pre-cracking stiffness (Table 8-6), post-healed ECC-water specimen recovered $139 \%$ of the pre-cracking stiffness of ECC-control compared to $113 \%$ of ECC-natural one. Similarly, post-healed ECC-MgO-water specimen recovered $108 \%$ of the pre-cracking stiffness of ECC-MgO-control compared to $95 \%$ of ECC-MgO-natural one. Overall, water curing showed better stiffness recovery than natural curing. In terms of stiffness recovery, it was not clear whether ECC-MgO or ECC specimens performed better although ECC-water ones seemed to have shown better stiffness recovery. It should be noted that post-healed ECC or ECC-MgO specimens either water or natural cured did recover more than $100 \%$ or close to $100 \%$ of the pre-cracking stiffness of control.

\subsubsection{Comparative structural performance and self-healing characterization using strain developments}

Figure 8-11 (A) compares the development of strain in reinforcing steel in ECC-control and ECC$\mathrm{MgO}$ control link slab specimens. It can be noted that steel yielded before the peak (ultimate) load for both control specimens. Reinforcing steel in ECC-MgO-control specimen yielded at $10.3 \mathrm{kN}$ (92.8\% of $11.1 \mathrm{kN}$ peak load) compared to $11.65 \mathrm{kN}$ (89.6\% of $13.0 \mathrm{kN}$ peak load) of ECC-control (Table 8-7). 


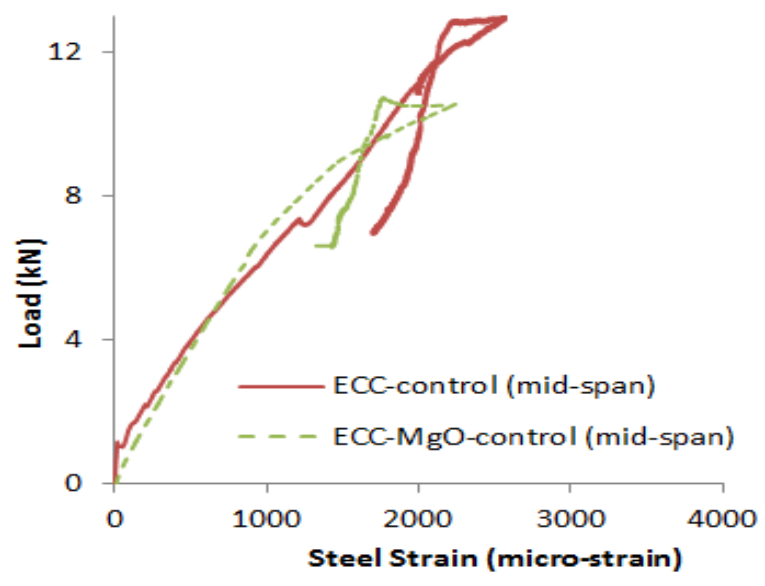

(A)

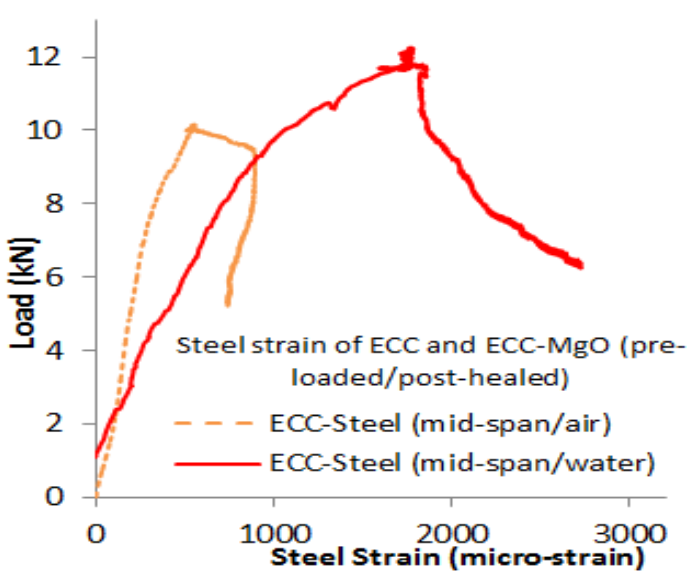

(B)

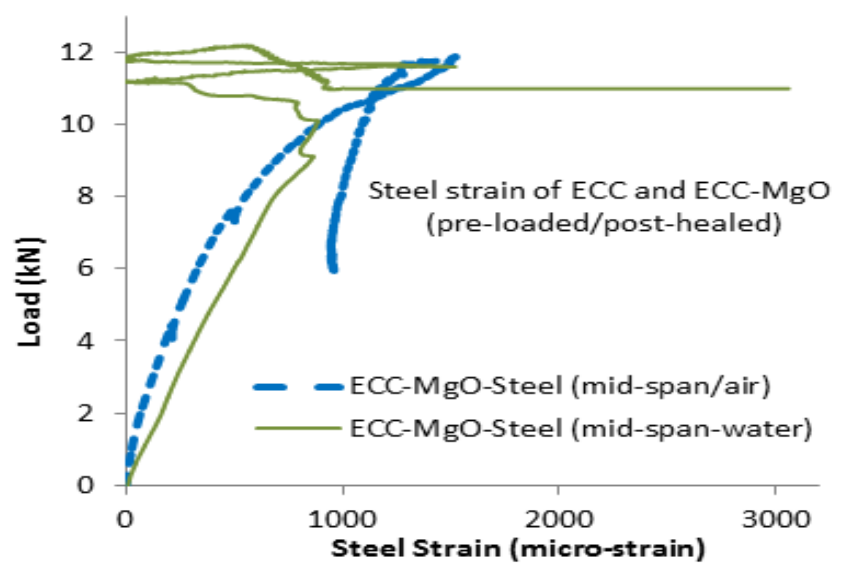

(C)

Figure 8-11: Strain development in reinforcing steel for link slab specimens

The rebar yielding at higher load in ECC-MgO specimen indicated better performance of ECC$\mathrm{MgO}$ in terms of micro-cracking and fiber bridging that allowed ECC matrix to take more contribution to the load resistance during the cracking stage that reduced the subsequent transfer of load to the reinforcing steel and hence, yielding of rebar occurred at higher load. In general, fiber bridging and micro-cracking characteristics of ECC normally allow the load to be gradually transferred to the steel bar. Hence, after the formation of the crack, ECC's tensile strain increased gradually (as can be seen from the development of higher maximum tensile strain of about 4000 micro-strain which is substantially higher than conventional concrete could have developed, Table 8-7). It can also be noted that ECC-MgO developed higher tensile strain than ECC in both control 
and post-healed link slab specimens. The increased tensile strain in ECC is normally caused by the fiber bridging phenomena where PVA fiber is ruptured to its ultimate tensile strength capacity rather than a pull out ( $\mathrm{Li}$ et al., 2001) as observed in the current study. This confirmed the superiority of ECC-MgO matrix (compared to ECC) in contributing more to the load resistance, thereby, reducing the amount reinforcing steel in the link slab. Overall, this study and other research studies (Fischer and Li, 2003; Hossain and Anwar, 2014; Hossain et al., 2015) revealed that the combination of the superior tensile behavior of ECC and steel rebar should result in high ductility, increased ultimate load and high energy absorption capacity for ECC link slabs.

Figure 8-11 (B) and Figure 8-11 (C) compare the reinforcing steel strain development in posthealed water and natural (air) cured ECC and ECC-MgO link slab specimens. The reinforcing steel in both water and natural cured ECC and ECC-MgO link slab specimens during preloading stage did not reach yielding strain of 2100 micro-strain, as expected (Table 8-7). However, ECC-MgO specimens showed lower strain development compared to ECC specimens in pre-cracking loading stage for the reason discussed earlier. Post-healed ECC-water and ECC-MgO-water link slabs exhibited steel yielding (strain > 2100 micro-strain) respectively at $69.9 \%$ and $90.1 \%$ of peak load of corresponding control link slabs (Table 8-7). Again steel yielding at higher load exhibited a better performance of post-healed ECC-MgO-water specimens compared to post-healed ECC one - this signified superior crack healing ability (during four months of curing period after precracking) of ECC-MgO (compared to ECC) that lead to the recovery of matrix strength/rigidity/fiber bridging/multi-cracking properties. Such self-healing capability induced damage repair and hence, increased load carrying capacity of ECC matrix which subsequently increased the yield load of reinforcing steel bar. Similar behavior (based on steel strain) was expected from post-healed ECC-natural (air) and ECC-MgO-natural (air) specimens - but due to strain gauge during natural (air) curing, lower strains were recorded during loading to failure and the reinforcements were not yielded. 
Table 8-7: Maximum strain in reinforcing steel/ECC and load at steel yielding

\begin{tabular}{|c|c|c|c|c|c|c|c|}
\hline \multirow{3}{*}{ Link slab } & \multirow{3}{*}{$\begin{array}{l}\text { Loading } \\
\text { to }\end{array}$} & Load & \multicolumn{2}{|c|}{ Maximum strain } & \multicolumn{2}{|c|}{ Steel yield load } & \multirow{3}{*}{$\begin{array}{c}\text { Steel } \\
\text { yielding }\end{array}$} \\
\hline & & Peak & Concrete & Steel & & $\begin{array}{l}\% \text { of } \\
\text { peak } \\
\text { load }\end{array}$ & \\
\hline & & $\mathrm{kN}$ & \multicolumn{2}{|c|}{ Micro-strain } & $\mathrm{kN}$ & & \\
\hline \multicolumn{8}{|c|}{ Control-link slab specimens - tested to failure under static monotonic loading after 28 -days of lab curing } \\
\hline $\begin{array}{l}\text { ECC- } \\
\text { control }\end{array}$ & Virgin-failure & 13.0 & 3822 & $2571 *$ & 11.65 & 89.6 & Yes \\
\hline $\begin{array}{l}\text { ECC-MgO- } \\
\text { control }\end{array}$ & Virgin-failure & 11.1 & 4000 & $2244 *$ & 10.30 & 92.8 & Yes \\
\hline \multicolumn{8}{|c|}{$\begin{array}{c}\text { Link slab specimens - Pre-cracked (damaged) and then water cured for } 4 \text { months before loaded to failure } \\
\text { under static monotonic loading }\end{array}$} \\
\hline \multirow{2}{*}{ ECC-water } & pre-cracking & 8.4 & - & 1326 & & & No \\
\hline & Post-healed failure & 12.3 & 2114 & $2731 *$ & 8.6 & 69.9 & Yes \\
\hline \multirow{2}{*}{$\begin{array}{l}\text { ECC-MgO- } \\
\text { water }\end{array}$} & pre-cracking & 7.32 & & 1367 & & & No \\
\hline & Post-healed failure & 12.2 & - & $3058 *$ & 11.0 & 90.1 & Yes \\
\hline \multicolumn{8}{|c|}{$\begin{array}{c}\text { Link slab specimens - Pre-cracked (damaged) and then natural cured for } 4 \text { months before loaded to failure } \\
\text { under static monotonic loading }\end{array}$} \\
\hline \multirow{2}{*}{$\begin{array}{c}\text { ECC- } \\
\text { natural }\end{array}$} & pre-cracking & 8.40 & - & 599 & & & No \\
\hline & Post-healed failure & 10.2 & 1806 & $896+$ & + & + & + \\
\hline \multirow{2}{*}{$\begin{array}{c}\text { ECC-MgO- } \\
\text { natural }\end{array}$} & pre-cracking & 7.32 & - & 837 & & & No \\
\hline & Post-healed failure & 11.9 & 2034 & $1531+$ & + & + & + \\
\hline
\end{tabular}

*reinforcing steel yielded + strain gauge damaged during natural curing

\subsubsection{Comparative performance and self-healing characterization using crack development}

Table 8-8 summarizes the number of cracks, crack width and length of crack before and after loading to the failure of self-healed link slab specimens. It should be noted that all the cracks were formed in ECC portion of the link slabs. They were flexure cracks and all link slabs failed in flexure. The width of the cracks remained less than $50 \mu \mathrm{m}$ before and after loading to the failure of a post-healed water or natural cured ECC or ECC-MgO link slab specimens - such crack width is suitable for crack healing (Table 8-8). 
Table 8-8: Crack characterization of ECC and ECC-MgO link slabs

\begin{tabular}{|c|c|c|c|c|c|c|c|c|}
\hline \multirow[b]{2}{*}{ Link slab } & \multirow{2}{*}{$\begin{array}{c}\text { Curing } \\
\text { Type }\end{array}$} & \multicolumn{3}{|c|}{$\begin{array}{l}\text { Old cracks before } \\
\text { loading to failure } \\
\text { (120d healing) }\end{array}$} & \multicolumn{3}{|c|}{$\begin{array}{l}\text { New cracks after } \\
\text { loading to failure } \\
\quad \text { (at } 120 \mathrm{~d})\end{array}$} & \multirow{2}{*}{$\begin{array}{c}\% \text { of new cracks } \\
\text { formed at } \\
\text { new locations }\end{array}$} \\
\hline & & $\begin{array}{l}\dot{\bar{D}} \\
\stackrel{\Xi}{\Xi} \\
\dot{Z}\end{array}$ & 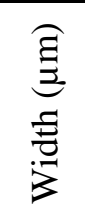 & 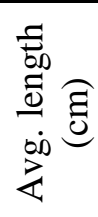 & $\begin{array}{l}\grave{\bar{D}} \\
\stackrel{\Xi}{\Xi} \\
\grave{Z}\end{array}$ & 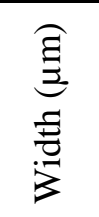 & 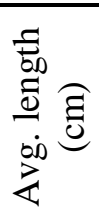 & \\
\hline ECC -water & Water & 20 & $<50$ & 3.75 & 22 & $<50$ & 3.6 & $110 \%$ \\
\hline ECC-natural & Natural & 29 & $<50$ & 3.59 & 33 & $<50$ & 3 & $114 \%$ \\
\hline ECC-MgO-water & Water & 9 & $<50$ & 2.2 & 22 & $<50$ & 4.5 & $244 \%$ \\
\hline ECC-MgO-natural & Natural & 13 & $<50$ & 3.2 & 31 & $<50$ & 3 & $240 \%$ \\
\hline
\end{tabular}

In both ECC and ECC-MgO link slab specimens, old cracks formed during pre-loading were selfhealed (Figure 8-12) and upon reloading to the failure of post-healed, new cracks were formed. The average length of the old cracks ranged between $2.20 \mathrm{~cm}$ and $3.75 \mathrm{~cm}$ compared to $3.00 \mathrm{~cm}$ and $4.50 \mathrm{~cm}$ of new cracks (Table 8-8). It can also be noticed that both water and natural cured ECC-MgO specimens developed less number of cracks during preloading compared to their ECC counterparts - that provided evidence for exhibiting better performance under service load.

Self-healing of cracks can be visualized from the deposition of white calcium and magnesium hydration products in the crack cavity (Figure 8-12 -C) as confirmed by the TGA/DSC and SEM analyses described in Chapter 7. It was also concluded that the calcium precipitations (mainly $\mathrm{CaCO}_{3}$ ) within the same crack cavity could be supplemented by the formation of delayed $\mathrm{MgO}$ hydration products featured with cementitious characteristics in ECC-MgO system - that can compensate the weakness in cementitious bonding by early deposition of $\mathrm{CaCO}_{3}$ and thereby increase the strength of already healed micro cracks. Hence, the formation of delayed $\mathrm{MgO}$ hydration products causing an increase in the strength of already healed micro-cracks is an indicator of the better performance of ECC-MgO system compared to its ECC counterparts. 


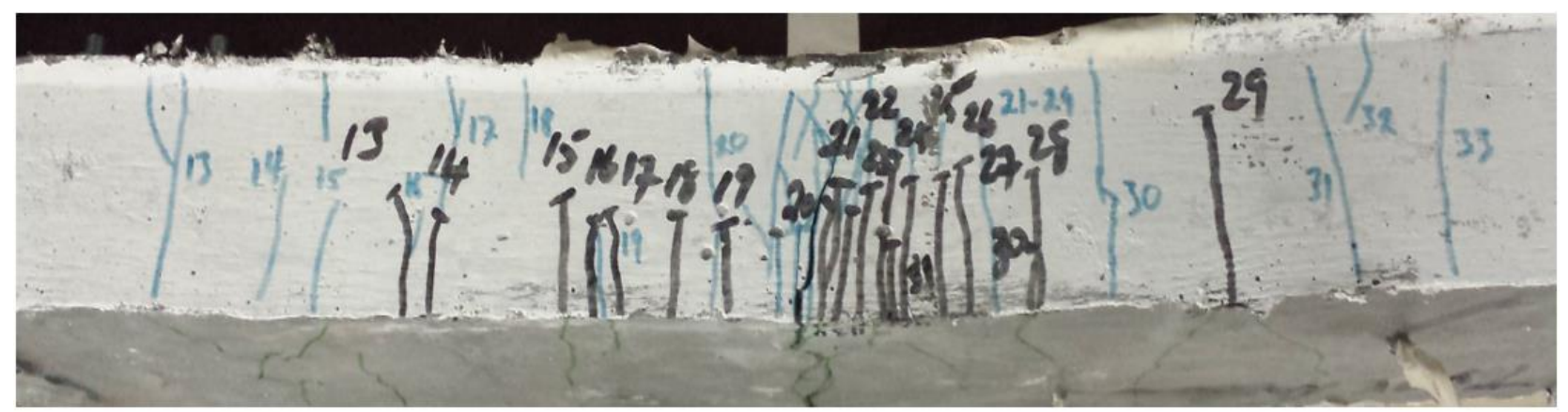

A) Old pre-loaded cracks (in black) and new post-loaded cracks (in blue) at one side of EC link slab

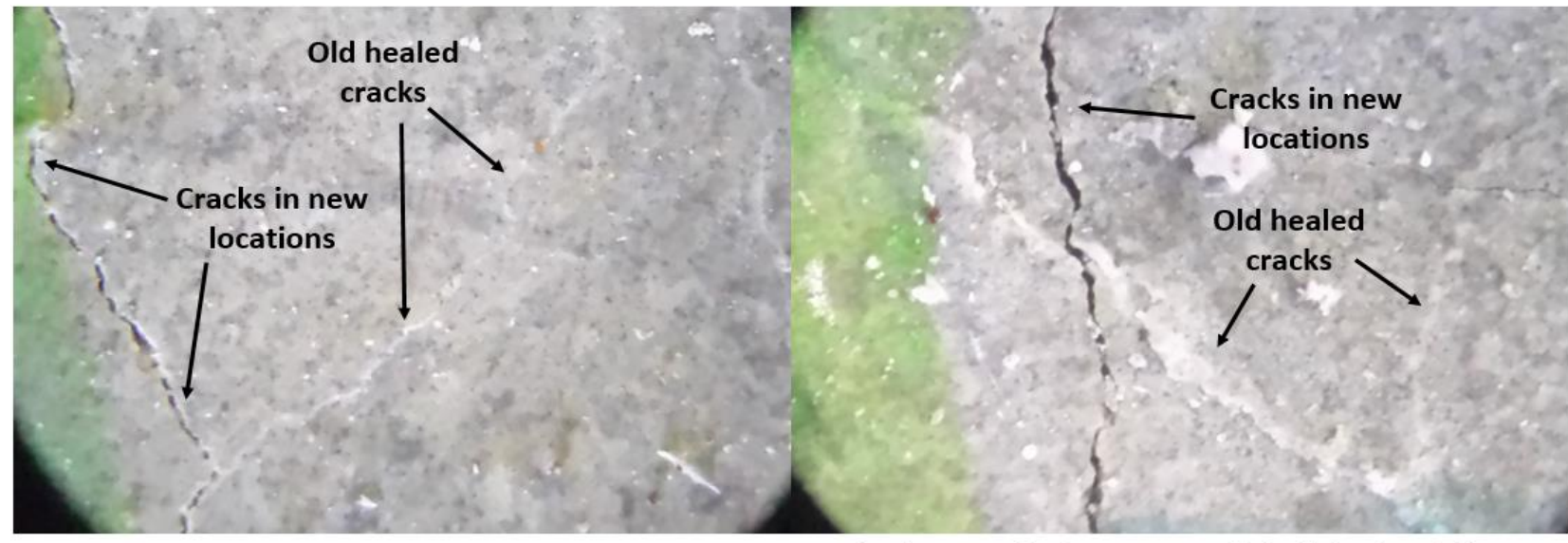

B) Micro cracks in ECC link slab after failure

C) Micro cracks in ECC-MgO link slab after failure

Figure 8-12: A) Crack propagation and failure of pre-loaded link slab specimen; B \& C) Forming cracks in new locations after 28 days +4 months of self-healing

This will allow ECC-MgO system to develop more new cracks and prevent extension of old cracks during the post-healed loading to failure, as observed in the current study (Figure 8-12 - A). ECC$\mathrm{MgO}$ link slab specimens developed $240 \%$ to $244 \%$ higher new cracks (with respect to old cracks) compared to only $110 \%$ to $114 \%$ of their ECC counterparts (Table 8-8). Overall (based on Table 8-8), a total number of cracks (sum of old and new cracks) was higher for ECC link slabs (ranged between 42 and 62) compared to between 31 and 44 of their ECC-MgO counterparts - with natural curing producing higher number cracks compared to water (vapor curing). 


\subsection{Summary}

This chapter described the results of experimental investigations on the self-healing capability of $1 / 4^{\text {th }}$ scale pre-cracked/damaged (after 28-days of lab curing) link slab specimens (made of ECC and ECC-MgO mixtures) cured under water or natural/field condition for four months duration. The self-healing performance was judged based on strength, stiffness and energy absorbing capacity recovery and cracking characteristics of post-healed link slab specimens compared to virgin/un-cracked ones loaded to failure under static monotonic loading. Damaged (pre-cracked) ECC link slabs showed good recovery of strength, stiffness and energy during the curing period through the healing of old cracks and producing cracks in new locations - with ECC-MgO link slabs showing better self-healing performance compared to their ECC counterparts. 


\section{CONCLUSIONS}

This thesis presents the self-healing capability of engineered cementitious composites (ECCs) made of 5\% $\mathrm{MgO}$ type expansive agent (MEA) when utilized as a self-healing agent in addition to $50 \%$ Class-F fly ash under different environmental exposures. The effectiveness of proposed ECC-MgO self-healing system was evaluated through an in-depth experimental investigation considering material exploration, development/recovery of fresh state, mechanical and durability properties in addition to micro/macro crack characterization/observations and compared with its ECC counterpart. Also, the self-healing ability of proposed ECC-MgO system was evaluated based on the recovery of the structural performance of damaged/crack link slab (used in joint-free bridge deck construction) specimens subjected to various curing conditions.

The following conclusions were drawn from this thesis based on each chapter individually:

Chapter 4: This chapter presents an in-depth experimental investigation studying the effectiveness of $\mathrm{MgO}$ as self-healing agent in engineered cementitious composite (ECC) considering the influence of calcination temperature/duration, $\mathrm{MgO}$ particle size and high volume of fly ash of different types (class F and class CI) through various tests (accelerated autoclave/water linear bar expansion test under water/autoclave curing). Hydration activity of $\mathrm{MgO}$-type expansive agent (MEA) was evaluated through kiln burning process and TGA test. The self-healing capability of ECC-MgO system was also evaluated through micro-structural investigation using scanning electron microscopy (SEM). The following conclusions were drawn from this study:

- The increase of calcination temperature and holding time increased the loss of $\mathrm{CO}_{2}$ content as well as reduced the grain fineness (through continued sintering) of $\mathrm{MgO}$ (MEA) powder samples. The increased loss of $\mathrm{CO}_{2}$ content during high temperature-longer holding time calcination process shifted burning level of $\mathrm{MgO}$ samples from "lightly burnt" to "heavily burnt" which might have a harmful effect on concrete structure at later ages. Therefore, lower temperature calcination was suggested for ECC-MgO self-healing system to get a higher 
degree of hydration activity. Within the range of temperatures studied, the best calcination system was considered to be " $900^{\circ} \mathrm{C} \_2$ hours of holding time_45 $\mu \mathrm{m}$ particle size" based on the higher degree of hydration of $\mathrm{MgO}$ powder samples.

- Accelerated autoclaved cured ECC-MgO bar specimens exhibited higher linear expansion compared to their normal water cured counterparts. This was due to the rapid formation of $\mathrm{Mg}(\mathrm{OH})_{2}$ crystals (which produced considerable expansive stresses) and accelerated C-S-H hydration causing the destruction of cohesive forces in ECC-MgO microstructure. Also, ECC$\mathrm{MgO}$ bar specimens produced with high temperature calcinated MEA showed higher expansions than their lower temperature counterparts under accelerated autoclave curing conditions.

- The addition of HVFA in ECC-MgO played a significant role in controlling the expansion effect of MEA. The addition of 55\% of the high volume of fly ash (HVFA) in ECC-MgO bar specimens produced expansions lower than $0.80 \%$ as recommended by (ASTM C150, 2016) in both water and autoclave curing conditions. This was attributed to the replacement of weaker $\mathrm{Ca}(\mathrm{OH})_{2}$ by stronger $\mathrm{C}-\mathrm{S}-\mathrm{H}$ products due to the pozzolanic reaction and to the reduction of expansion stresses on pore walls by limiting $\mathrm{Mg}(\mathrm{OH})_{2}$ formation causing fortification of microstructure leading to an increase in the counteracting forces against $\mathrm{MgO}$ expansions.

- HVFA incorporated ECC-MgO bar specimens made with low temperature calcinated MEA produced lower expansion. This was attributed to the presence of more interior pores with a smaller pore size in low temperature MEA (than high temperature ones) causing a higher production of $\mathrm{C}-\mathrm{S}-\mathrm{H}$ in $\mathrm{MgO}$ pores (due to pozzolanic reaction) leading to a denser microstructure which in turn provides more resistance to expansion pressure producing lower expansion.

- Bar specimens made of Class-F fly ash had lower autoclave expansion readings than their Class-CI counterparts - the presence of low $\mathrm{CaO}$ in Class-F fly ash reduced the temperature of ECC-MgO bar specimens leading to lower expansions. Within the types of fly ashes studied, 
"low calcium Class-F fly ash" was recommended to be the best supplementary cementing material based on the assessment of the effectiveness in lowering the expansion of both water and autoclave cured ECC-MgO bar specimens.

- Comparable behavior was shown by the $\mathrm{MgO}$ in powder state in terms of loss of $\mathrm{CO}_{2}$ content and remaining hydration activity as well as after utilizing it in the ECC-MgO bar specimens in water and autoclave cured linear expansion tests.

- The SEM observation clearly revealed crack filling compounds to be consisting of cementitious $\mathrm{MgO}$ and $\mathrm{CaO}$ crystals. The $\mathrm{MgO}$ crystals might supplement the $\mathrm{CaCO}_{3}$ crystallization and both together can improve mechanical and durability properties of ECC$\mathrm{MgO}$ self-healing system.

- The study confirmed the viability of using ECC-MgO system as self-healing material for durable constructions.

Chapter 5: This chapter presents the self-healing capability of engineered cementitious composites (ECCs) made of fly ash and MgO type expansive agent (MEA). The influences of fly ash content (30\% and 55\% cement replacement), fly ash types (Class-F and Class-CI) and $\mathrm{MgO}$ content up to $30 \%$ were investigated by using twenty-two ECC mixtures through accelerated autoclave/water cured linear expansion tests on bar specimens, Scanning Electron Microscope (SEM) analysis and compressive strength development/recovery of cube specimens. The following conclusions were drawn from this study:

- ECC-MgO self-healing system should be designed for very low expansions without compromising the durability. The use of $5 \%$ lightly burnt $\mathrm{MgO}$ in combination with Class-F fly ash with cement replacement of $55 \%$ by mass (instead of $55 \%$ Class-CI or $30 \%$ Class-F fly ash) is found to be the best choice for the production of ECC-MgO self-healing system. The hydration process of ECC-MgO system produced by using 30\% class F fly ash was not stable 
and developed unbalanced expansions especially within the period of first 28 days. Hence the use $55 \%$ class $\mathrm{F}$ fly ash was considered a better choice due to the balanced and smooth evolution of expansion curves.

- ECC specimens showed the strong capability to produce lower expansion less than $0.80 \%$ recommended by ASTM standards in both water and autoclave linear expansion tests even when a high volume of $\mathrm{MgO}$ content up to $30 \%$ was used in the production of ECC-MgO bar specimens. This might be related to the use of the high volume of FA (HVFA) with 55\% cement replacement which limited the formation of $\mathrm{Mg}(\mathrm{OH})_{2}$ crystals in microstructure and PVA fibers which enhanced the volume stability of ECCs significantly.

- MEA was found to be effective in producing ECC-MgO system with self-healing capability. This was attributed to the MEA's the ability to densification of microstructure through producing $\mathrm{MgO}$ crystals at later ages (characterized by cohesive cementitious properties) supplementing the formation of C-S-H products at early ages as confirmed by the SEM analysis. Also, magnesium products also supplement the formation of $\mathrm{CaCO}_{3}$ and $\mathrm{C}-\mathrm{S}-\mathrm{H}$ products formed within the crack openings enhancing the strength of already healed cracks especially when loadings reapplied.

- Development of maximum 16\% higher compressive strength of pre-cracked cube specimens under accelerated autoclave curing compared to their water cured virgin counterparts revealed strength recovering capability of the proposed ECC-MgO system (with Class-F fly ash with $55 \%$ cement replacement and 5\% of MEA content) through the healing of micro cracks and microstructure densification.

- The 7 and 28 days compressive strengths of water cured virgin cubic specimens (without precracking) made of ECC-control (without MEA) and ECC-MgO were found almost same due to slow reactivity/rate of hydration of $\mathrm{MgO}$ at such early ages - hence to accelerate the hydration of $\mathrm{MgO}$, autoclave curing was employed in this research to assess the self-healing capability. To assess the feasibility of proposed ECC-MgO self-healing system, a long-term 
study assessing mechanical and durability properties should be conducted under both water and normal air curing (field) conditions.

Chapter 6: This chapter presents the performance of engineered cementitious composites (ECCs) when produced with $\mathrm{MgO}$ type expansive agent (MEA) and utilized as a self-healing agent to heal micro cracks. The influence of the addition of 5\% MEA as fly ash replacement and water and natural curing conditions were investigated through studying the recovery of load/strength performance, deflection capacity development, macroscopic crack characteristics, energy absorption and ultrasonic pulse velocity by using cubic (50x50x50 mm) specimens and prismatic (50x76x355 mm) specimens. The following conclusions are drawn from the study:

- The cubic ECC control specimens developed 9.2\% more of compressive strength than ECC$\mathrm{MgO}$ counterparts at the age of 28 days. However, after long-term water and natural curing, ECC-MgO cubic controls developed $0.65 \%$ and $1.27 \%$ more compressive strength, respectively than ECC cubic controls. The slow reactivity of $\mathrm{MgO}$ might be the reason of developing slower compressive strength improvement of ECC-MgO specimens at early ages. During the long term curing, water curing condition enhanced the compressive strength of cubic ECC and ECC-MgO controls than natural curing consistently.

- Under long-term of water and natural curing, both 50\% and 80\% preloaded ECC and ECC$\mathrm{MgO}$ specimens developed almost similar compressive strength development/recovery. However, up to 300 days, natural cured $80 \%$ pre-cracked ECC-MgO specimens developed the highest compressive strength recovery (8.5\%) amongst all loading levels and curing conditions when related to all pre-cracked ECC and ECC-MgO specimens. Even after applying multiple damages events every 2 months up to 10 months on water and natural cured ECC-MgO specimens, the $80 \%$ pre-cracked multiple damaged ECC-MgO cubic specimens developed $105.8 \%$ and $103.0 \%$, respectively (of control virgin specimens) which were the highest compressive strength recovery observed in this study. 
- Similar to compressive strength, ECC prismatic controls at an early age (7-days) developed $7.5 \mathrm{kN}$ load/strength which was higher than $6.45 \mathrm{kN}$ of counterpart ECC-MgO controls; however, after long periods of water and natural curing (up to 450-days), ECC-MgO prismatic controls could not develop higher load/strength than similar ECC ones. This can be related to the replacement of cementitious materials of ECC-MgO mix with very slow active 5\% MEA in addition to the size effect of prismatic specimens which might not reveal clearly the effect of $\mathrm{MgO}$ expansion.

- To assess effectively the self-healing property of ECC-MgO system, the recovery of both precracked ECC and ECC-MgO prismatic specimens was studied by applying different multiple cracking and failure events under both water and natural curing conditions on pre-cracked specimens.

- It was found that the multiple cracked failed ECC specimens developed higher loads/strengths than similar ECC-MgO specimens when stored in water up to 7-months. However, under natural curing condition, the load/strength development of multiple cracked/failed ECC specimens was reduced less than their counterpart ECC-MgO specimens at 3-months.

- Even though the pre-cracked ECC-MgO specimens developed $8.2 \mathrm{kN}$ load/strength which was lower than $9.7 \mathrm{kN}$ of counterpart ECC specimens at 7-days, after 10 months of natural curing (300 days), the multiple cracked and failed ECC-MgO specimens recovered the strength of controls; which was $9.5 \mathrm{kN}$ and $8.9 \mathrm{kN}$ (considered as the highest) compared to $9.0 \mathrm{kN}$ and $7.5 \mathrm{kN}$ of counterpart ECC specimens, respectively.

- After 15 months of curing, the macroscopic observation confirmed that cracks sealed completely under water curing which was not the case for natural curing. However, the formation of 55\% new cracks in new locations after loading the natural cured pre-cracked ECC-MgO specimens to failure revealed the superior self-healing characteristics of natural cured ECC-MgO specimens compared to counterpart ECC ones. The harsh Canadian environment accompanied with heavy snowfall might wash out the white crystals from the 
crack surface only and not from deep locations - this can be attributed to better performance on natural cured specimens.

- Natural cured ECC-MgO specimens developed the highest strength recovery $(80.6 \%$ of control), almost the lowest UPVs (100.1\% of control), the highest deflection capacity (67.8\% of control) and the highest energy absorption (37.2\% of control) amongst all other specimens after applying 4 multiple damage (through repeated loading) over 10 months period.

- It should be noted that even though both natural and water cured ECC controls developed higher early strength than ECC-MgO controls, still the deflection recovery of pre-cracked ECC-MgO specimens (when subjected to different cracking events) was higher than their ECC counterparts especially when cured under long-term natural curing. Higher strength development in ECC controls (with no crack) did not indicate better performance compared to ECC-MgO controls as observed in field/natural curing condition; it would be rare to have crack-free concrete structures because of the presence of very small micro-cracking formed once formwork is removed.

- It general, water cured prismatic healed specimens when subjected to different loading events (simulating repeated damage) over 10 months developed higher UPVs than natural cured ones. However, higher UPVs not necessarily mean higher strength recovery of specimens because excellent UPVs may lead to a stiff and brittle concrete.

- $\quad$ Based on 15 months of curing (long term), it was concluded that natural cured pre-cracked specimens developed higher flexural load/strength performance, energy absorption capacity and UPVs compared to their water cured counterparts. Specifically, the natural cured failed ECC-MgO specimens recovered the highest of load/strength (140.9\%), deflection capacity (111.0\%), energy absorption (98.3\%) and the highest UPVs (102.3\%) of the control specimens amongst of all other specimens cured under both curing conditions. 
The enhanced recovery in strength (both compressive and flexural)/deflection/energy absorption /ultrasonic pulse velocity and better crack healing characteristics, of long-term natural cured precracked/multiple damaged ECC-MgO specimens indicated the strong self-healing capability of proposed ECC-MgO system. The recovery of mechanical properties due to self-healing validates the concept of the proposed ECC-MgO system and justifies its use in practical construction.

Chapter 7: This chapter evaluates the effectiveness of self-healing ECC-MgO system produced with the combination of $\mathrm{MgO}$-type expansive agent considered as self-healing agent and Class-F fly ash under laboratory, water, natural and autoclave curing conditions. The behavior of ECC$\mathrm{MgO}$ system was evaluated through development of fresh and mechanical properties (heat of hydration and bar/prism specimens) while its self-healing behavior was assessed based on development/recovery of mechanical and durability properties (RCP test, sorptivity, extended freeze and thaw cycles and the residual of load/strength-deflection capacities). TGA/DSC and SEM analysis were conducted as well. The following conclusions are drawn from the study:

- The addition of $\mathrm{MgO}$ to ECC-MgO mixtures delayed the cement hydration which delayed the time of hydration (increasing accelerated period by shifting the heat of hydration curve slightly to the right) compared to counterpart ECC. Furthermore, the presence of 55\% fly ash in ECC mixtures decreased the maximum rate of the heat of hydration compared to 50\% fly ash in $\mathrm{ECC}-\mathrm{MgO}$ mix.

- Within the scope of this research study, the drying shrinkage of ECC-MgO bar specimens was 2.3 times more than their expansion property while it was 12.55 times more for ECC bar specimens. This gives an indication that $\mathrm{ECC}-\mathrm{MgO}$ specimens can resist more damages than those made with ECC when exposed to wet/dry successive harsh environments without undergoing sudden changes in material's microstructure.

- At an early age, the permeation properties (sorptivity/water absorption and RCP) of both ECC$\mathrm{MgO}$ controls and preloaded (cracked) specimens whether water or natural cured were higher 
than their ECC counterparts cured under similar conditions. The slow activity of MEA at an early age might be the reasons for developing less chloride penetration resistance (RCP) than ECC ones. However, the delayed hydration of MEA developed better resistance to chloride ion penetration for preloaded ECC-MgO specimens with $84 \%$ and $95 \%$ compared to ECC ones with $80 \%$ and $86 \%$ when cured under water and natural curing conditions, respectively. Based on RCP test results, it was observed that specimens made of ECC-MgO with $5 \% \mathrm{MgO}$ had higher tendency to undergo a significant self-healing process which was signified by the steep reduction in RCP measurements.

- Under long term curing (around 180 days), the sorptivity index of water and natural cured uncracked ECC-MgO controls were 58\% and 64\%, respectively which were much better than $116 \%$ and $225 \%$ of ECC controls cured under similar conditions. By using sorptivity index as a tool to assess long-term cured ECC-MgO self-healing property, it was concluded that water and natural cured pre-loaded (cracked) ECC-MgO specimens recovered the rate of absorption of control (un-cracked) specimens with lower sorptivity indexes (67\% and 90\%, respectively) than their water or natural ECC counterparts (exhibiting sorptivity indexes of $242 \%$ and $317 \%$, respectively). This might be related to the filling effect of MEA at a later age due to its expansive nature.

- Under frost resistance, preloaded ECC-MgO specimens developed/recovered $87.7 \%$ of RDME and ended up with $90.7 \%$ at $600 \%$ cycles with an enhancement of $3 \%$ while ECC counterpart developed/recovered $95.0 \%$ at 300 cycles and ended up with $90.4 \%$ at 600 cycles with a deterioration of $4.6 \%$. The deterioration in ECC-MgO frost resistance at the first 300 cycles was attributed to the filling effect caused by the expansive nature of MEA hydration which might be accelerated by successive freezing and thawing cycles. This was leading to extreme formation/packing of new hydration products in ECC-MgO capillaries/pores and subsequent weakening of micro-structure. Beyond 300 cycles and up to 600 cycles, the preloaded ECC$\mathrm{MgO}$ specimens developed better frost resistance than counterpart ECC specimens. This was attributed to the lower tensile strength capacity of ECC microstructure compared to ECC-MgO - long term frost damage might rupture more pores in ECC microstructures. 
- Although ECC-MgO controls exhibited inferior flexural performance compared to their ECC counterparts at the first 300 freeze-thaw cycles while showing better flexural performance at the second 300 cycles, the expected behavior of both ECC or ECC-MgO (with 5\% MEA as fly ash replacement) specimens subjected to frost damage in terms of strain hardening and multiple cracking characteristics was maintained.

- Based on TGA/DSC and SEM analyses, it can be inferred that the formation of delayed $\mathrm{MgO}$ hydration products within the crack walls (causing crack healing) featured with cementitious characteristics might compensate the weakness in newly formed cementitious bonding (C-S$\mathrm{H}$ products). This might increase the strength of already healed micro-cracks and form crack at new locations when re-loading events applied again to the specimen.

Chapter 8: This chapter studies the structural performance of proposed self-healing ECC-MgO system in join-less bridge deck link slab applications. The influence of adding 5\% $\mathrm{MgO}$ as fly ash replacement and water and natural curing conditions were investigated through studying the recovery of load-deflection/moment-rotation responses, concrete and steel strain developments, energy absorption and stiffness properties in addition to crack macro observations by using 6 model link slab specimens $\left(1 / 4^{\text {th }}\right.$ scale of prototype) and compared with counterpart ECC specimens constructed without $\mathrm{MgO}$. The following conclusions are drawn from the study:

- In terms of peak load/strength, deflection capacity, energy absorption capacity and precracking stiffness recovery, water (vapor) curing showed better recovery (self-healing efficiency) than natural (field) curing.

- Compared to their ECC counterparts, ECC-MgO-specimens showed better strength recovery (even exceeded the strength of controls) and lower peak deflection recovery (ECC or ECC$\mathrm{MgO}$ specimens either water or natural cured did not recover full deflection capacity of control). 
- In terms of energy and initial stiffness recovery, it was not clear whether ECC-MgO or ECC specimens performed better. However, post-healed ECC or ECC-MgO specimens (water or natural) cured did not recover $100 \%$ of energy capacity of control while did recover more than $100 \%$ or close to $100 \%$ of the pre-cracking stiffness of control.

- Steel yielding at higher load exhibited a better performance of post-healed ECC-MgO specimens compared to their ECC counterparts signified superior their crack healing ability during four months of curing period.

- The formation of delayed $\mathrm{MgO}$ hydration products causing an increase in the strength of already healed micro-cracks is an indicator of the better performance of ECC-MgO system compared to its ECC counterparts.

- ECC-MgO link slab specimens showed better self-healing performance (compared to their ECC counterparts) by developing a higher number of new cracks and preventing extension of old cracks during the post-healed loading to failure.

Overall, both ECC and ECC-MgO systems showed good self-healing capability to be used in the in the construction of link slabs for joint-free bridge deck construction with ECC-MgO system showing better performance. More investigations are necessary, especially for the long-term, to explore the self-healing performance of proposed ECC-MgO system in various structural systems. 


\subsection{Recommendations for future studies}

The following recommendations are made for future research studies:

- It was observed consistently that early strength of ECC-MgO control specimens was less than those made with standard ECC; while pre-cracked ECC-MgO specimens developed/recovered full strength of or even higher strength than their ECC counterparts after long term curing. This was due to the slow activity of $\mathrm{MgO}$ at early ages. Therefore, tailoring new mix design with early age strength properties similar to standard ECC mixtures and having long term self-healing properties similar to ECC ECC-MgO mixtures is essential. To do so, it is recommended to study the effect of the addition of different percentages of silica fume (which is amorphous in nature and highly reactive) to ECC$\mathrm{MgO}$ mixtures to increase the strength development at early ages. However, the addition of silica fume will increase the silicon ions in ECC microstructure along with the addition of $55 \%$ of Class-F fly ash as cement replacement. This will increase the consumption of $\mathrm{Ca}(\mathrm{OH})_{2}$ and limit the presence of $\mathrm{OH}$ ions in alkaline solution and thereby affect the formation of $\mathrm{Mg}(\mathrm{OH})_{2}$ and reduce the expansion ability at later ages. Therefore, using more than $5 \%$ of $\mathrm{MgO}$ is recommended in the production of ECC-MgO mixtures incorporating silica fume in combination with a high volume of SCMs. The increase in MgO content should not interfere with ECC durability.

- It was also observed that the number of cracks formed in ECC-MgO link slab specimens was less than their ECC counterparts even under ultimate loads. Also, most of the steel bars in pre-cracked ECC-MgO link slab specimens did not yield after re-loadings to failure. This was related to the high strength capacity of ECC-MgO matrix causing transfer of less loads to steel bars. Therefore, reduction of steel reinforcement to induce steel yielding in link slab bridge deck specimens made with ECC-MgO is recommended. Future research should be addressed in this direction. 
- It is essential to study the durability of ECC-MgO specimens under chloride and sulfate environments. Also, delayed ettringite formation should be investigated as well when ECC$\mathrm{MgO}$ bars/prisms specimen experienced temperatures above $70^{\circ} \mathrm{C}$.

- Evaluation of long-term self-healing performance of pre-cracked/damaged ECC-MgO prisms and link slab specimens under fatigue and creep loading is recommended. 
APPENDICES 


\section{Appendix A}

Relative humidity, temperature, maximum snow on ground and total precipitations 
(Monthly data starts from January 2015 to May 2016)

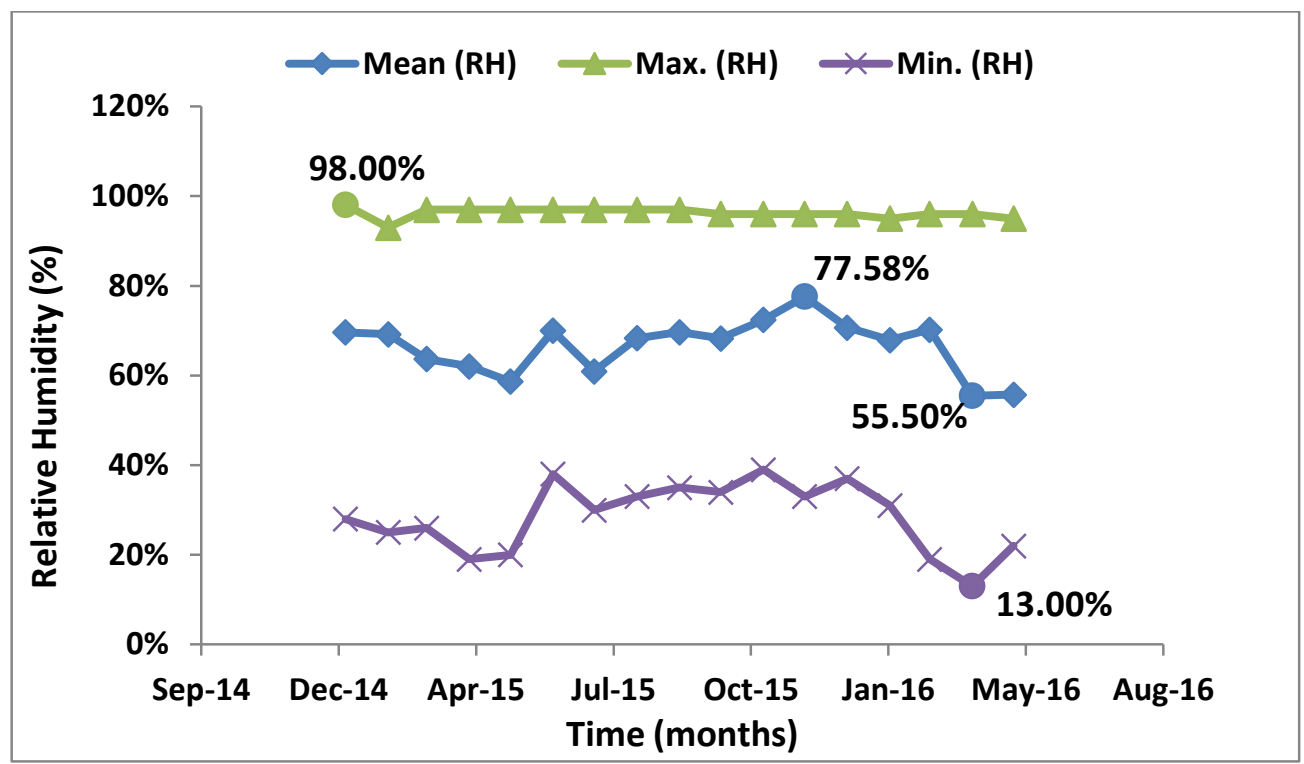

Maximum, minimum and mean relative humidity as a function of time

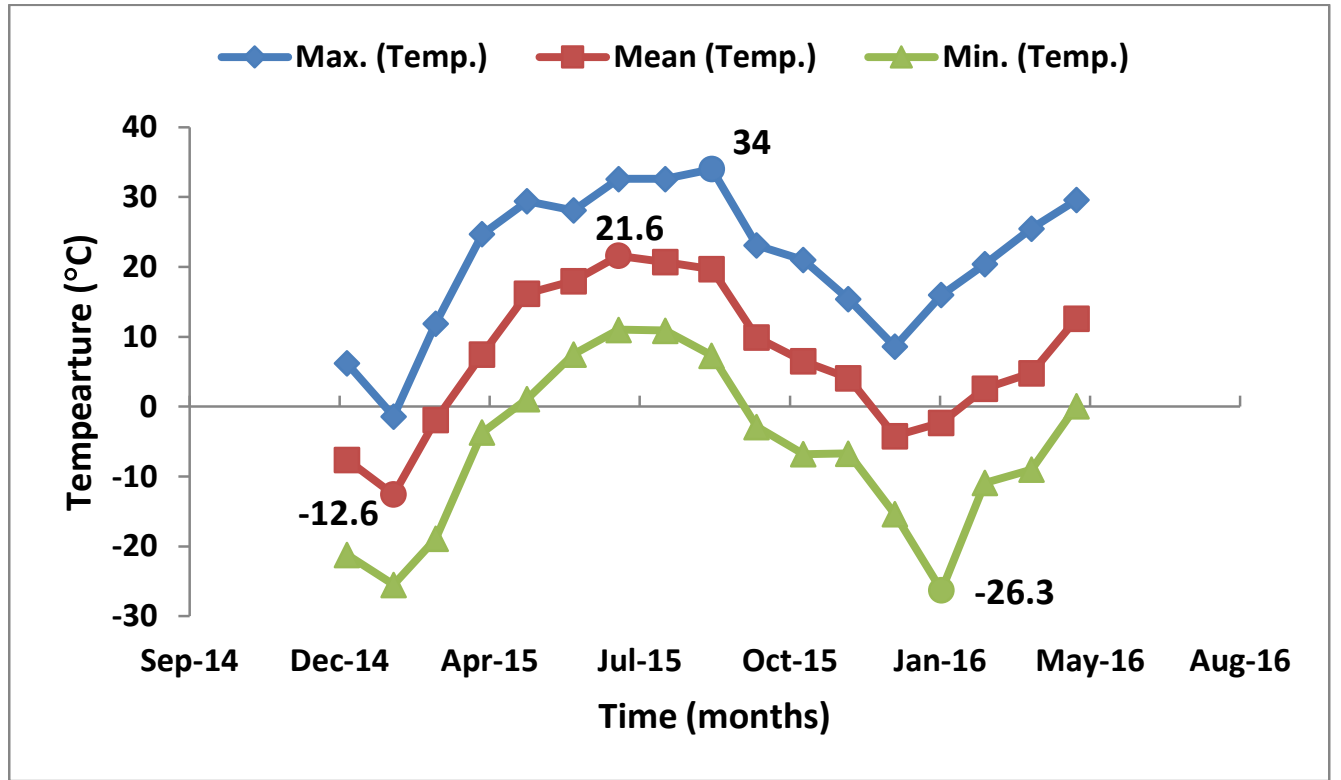

Maximum, minimum and mean temperature as a function of time 


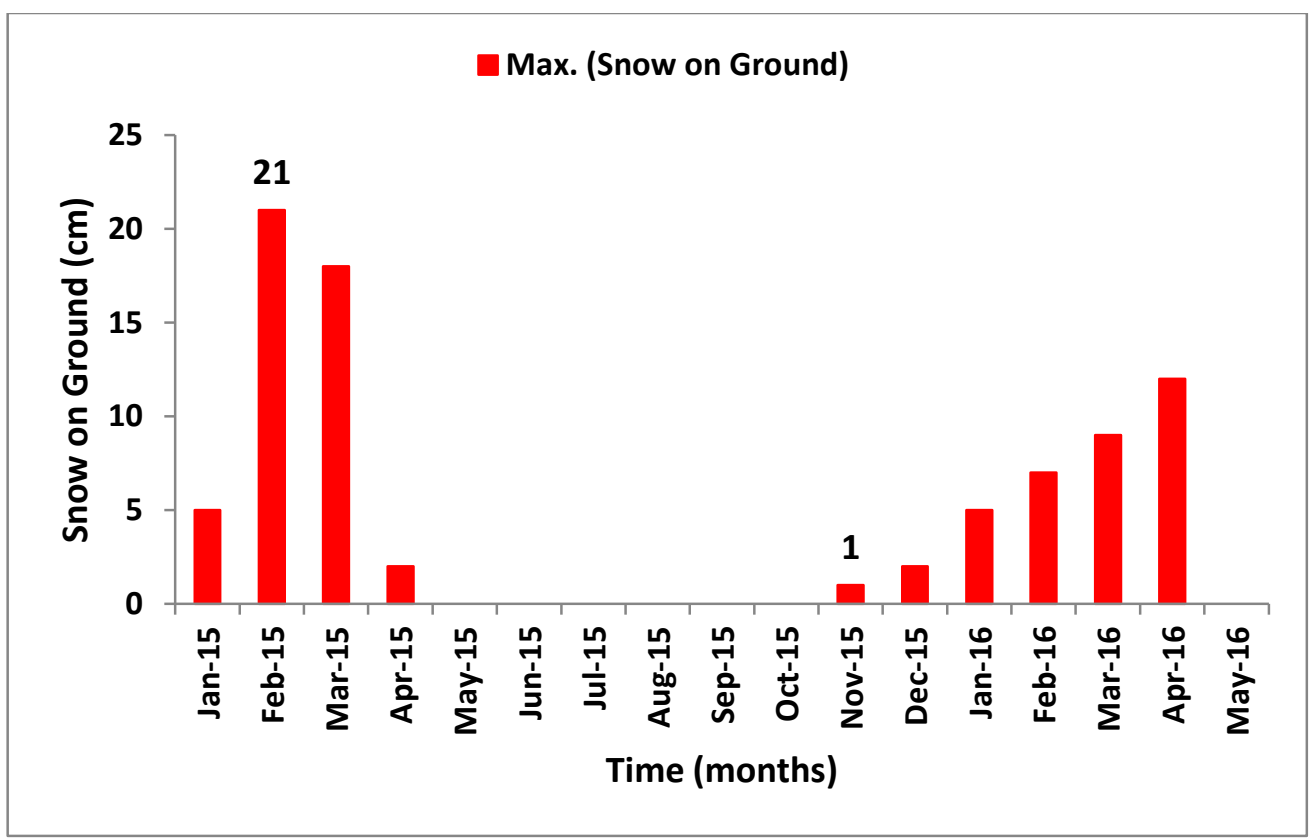

Maximum snow on ground as a function of time

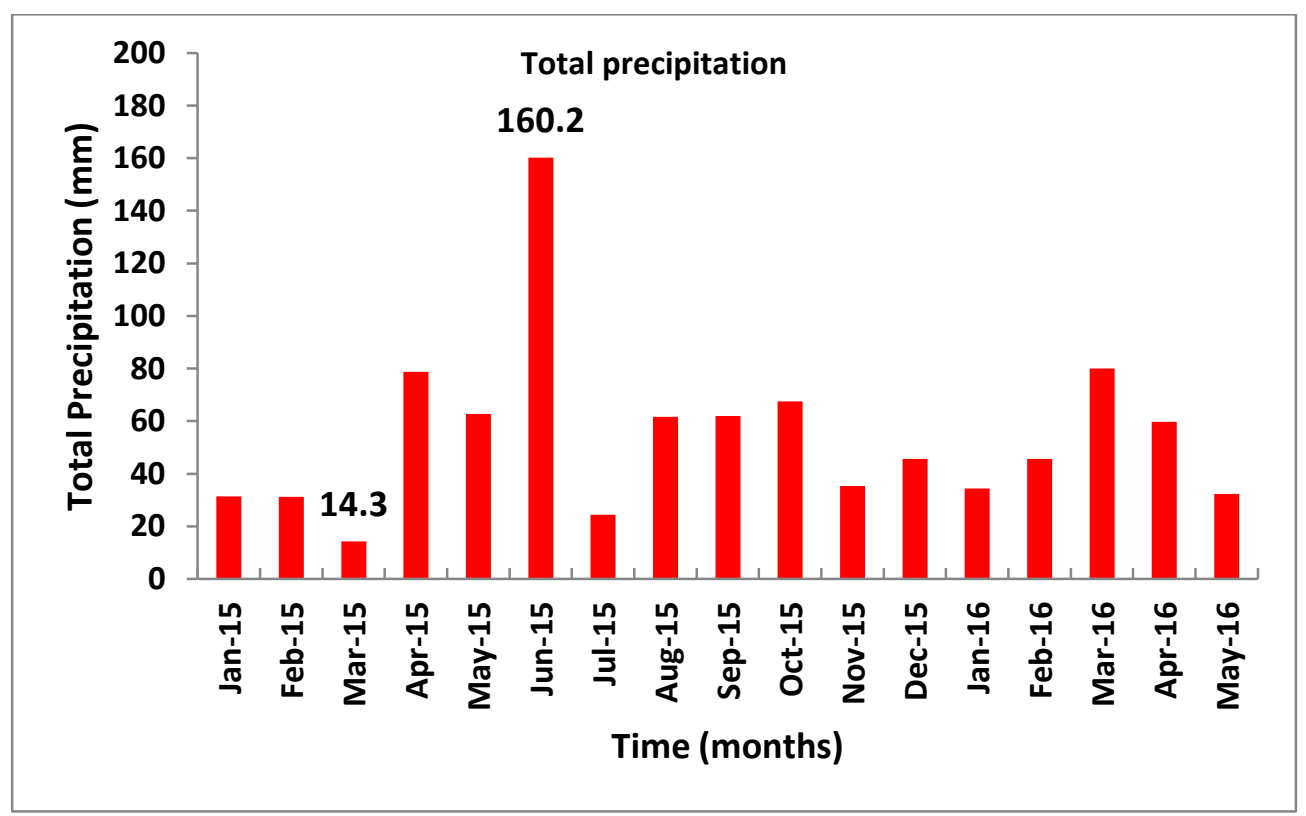

Total precipitation as a function of time (monthly data from Jan-15 to May-16) 


\section{Relative humidity (daily data starts from 21:00pm)}
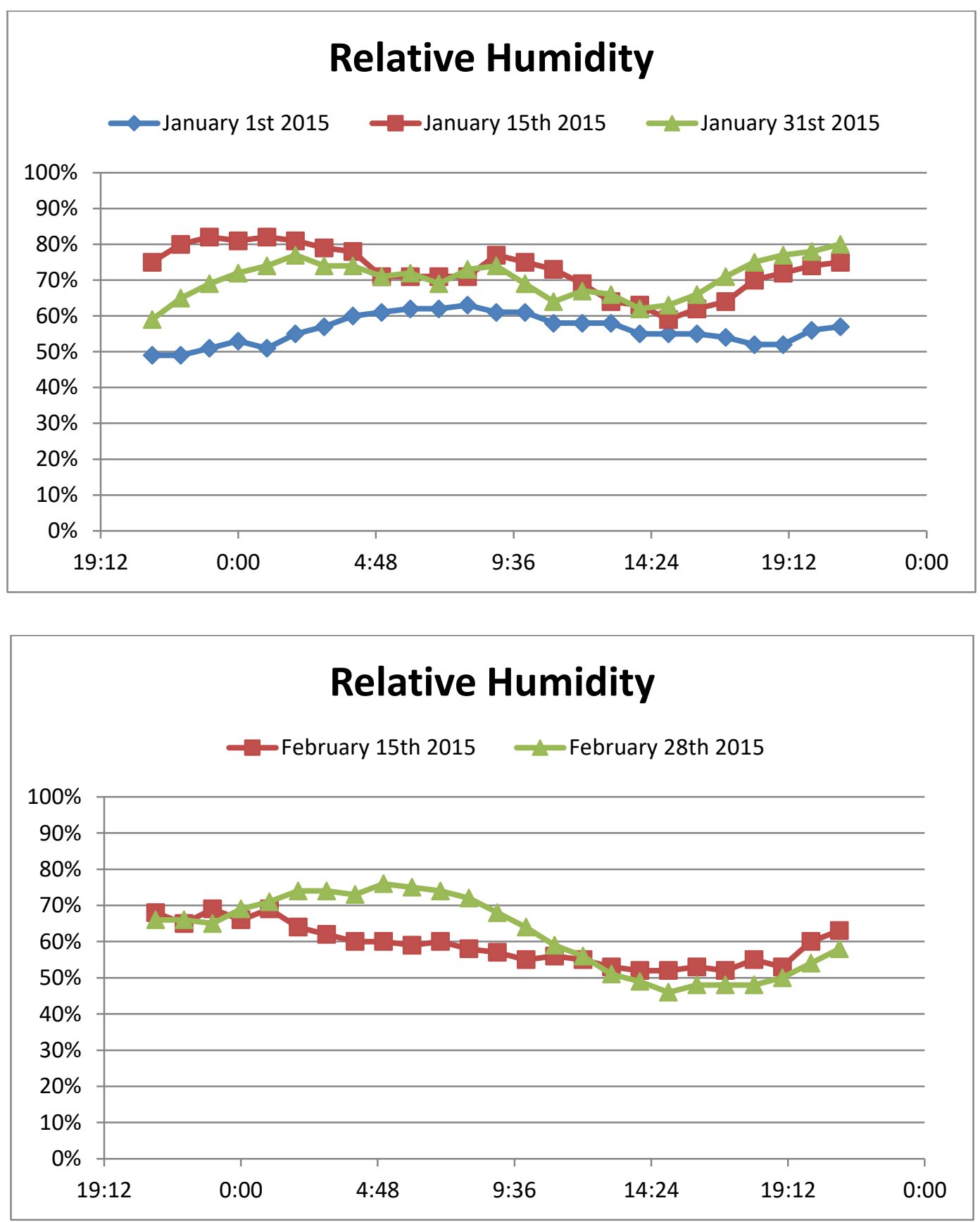

ccxlvi 

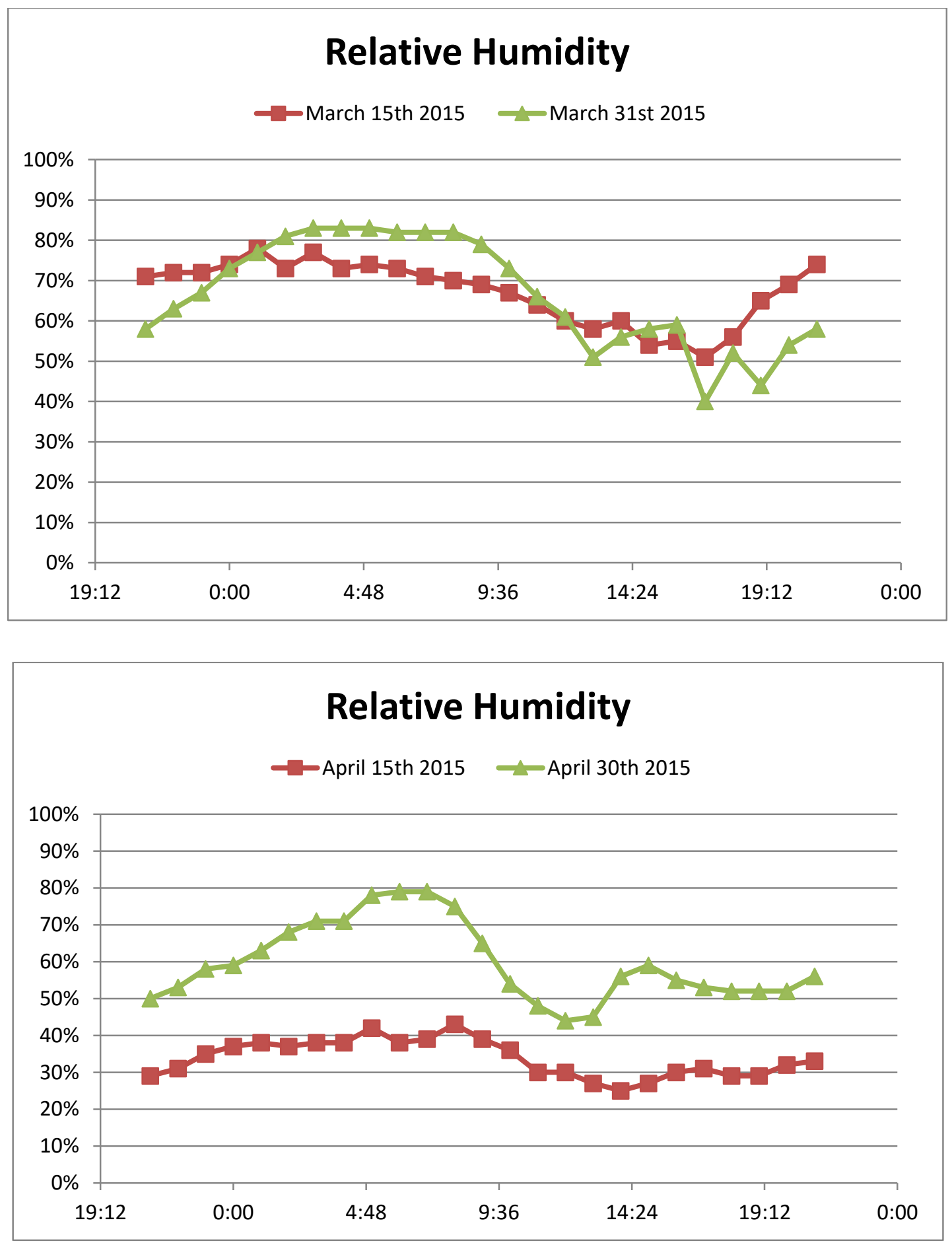

ccxlvii 

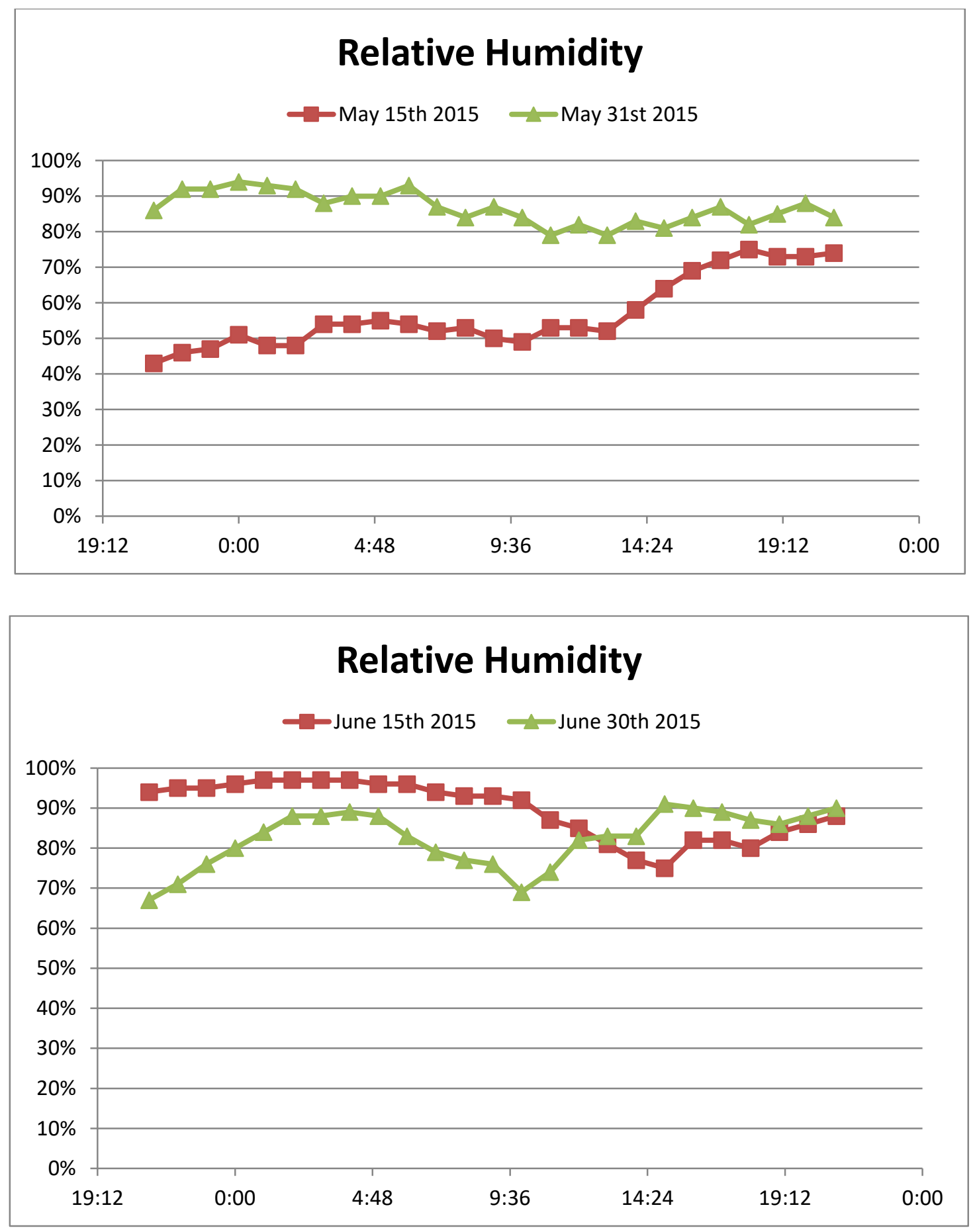

ccxlviii 

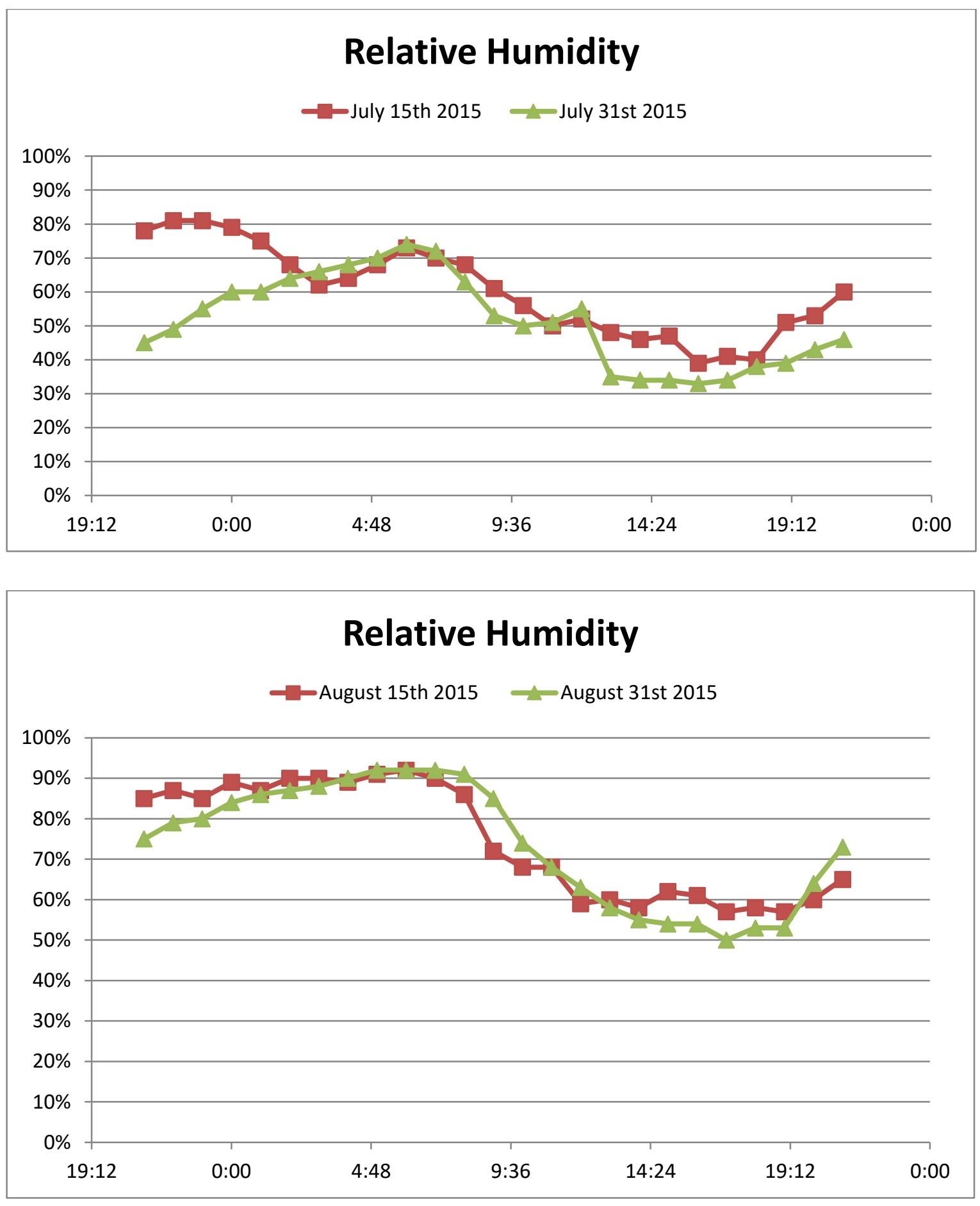

ccxlix 

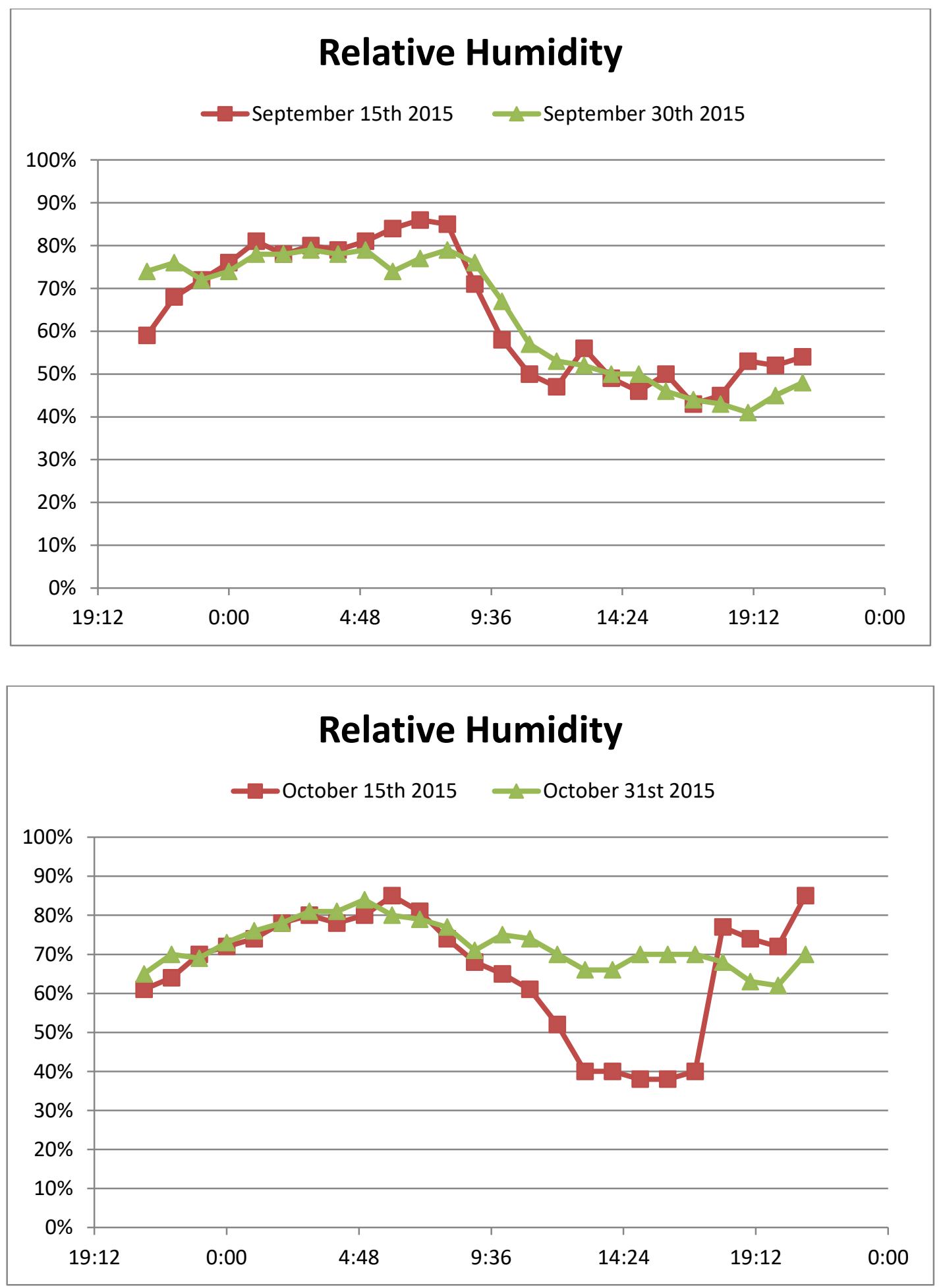

ccl 

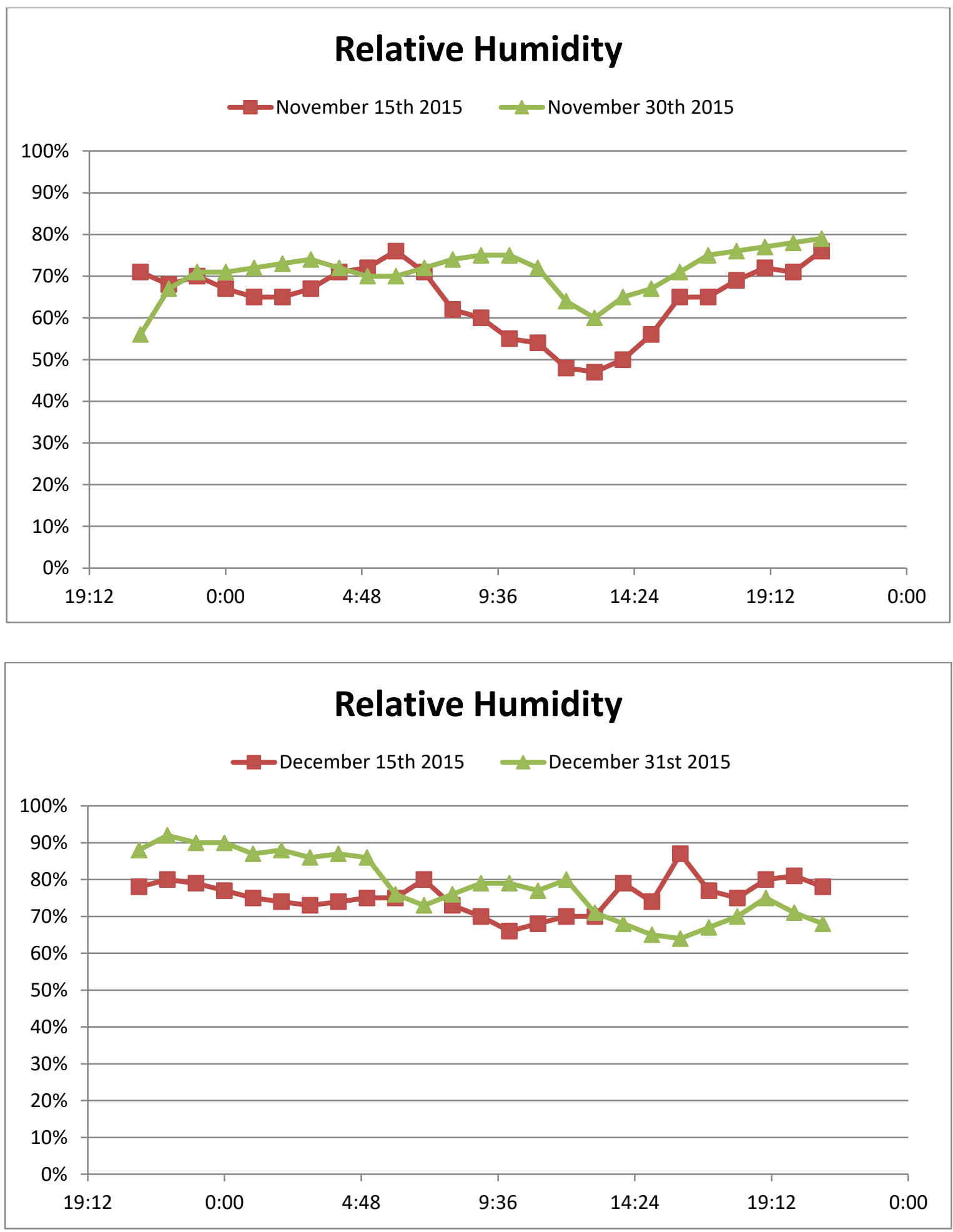

ccli 

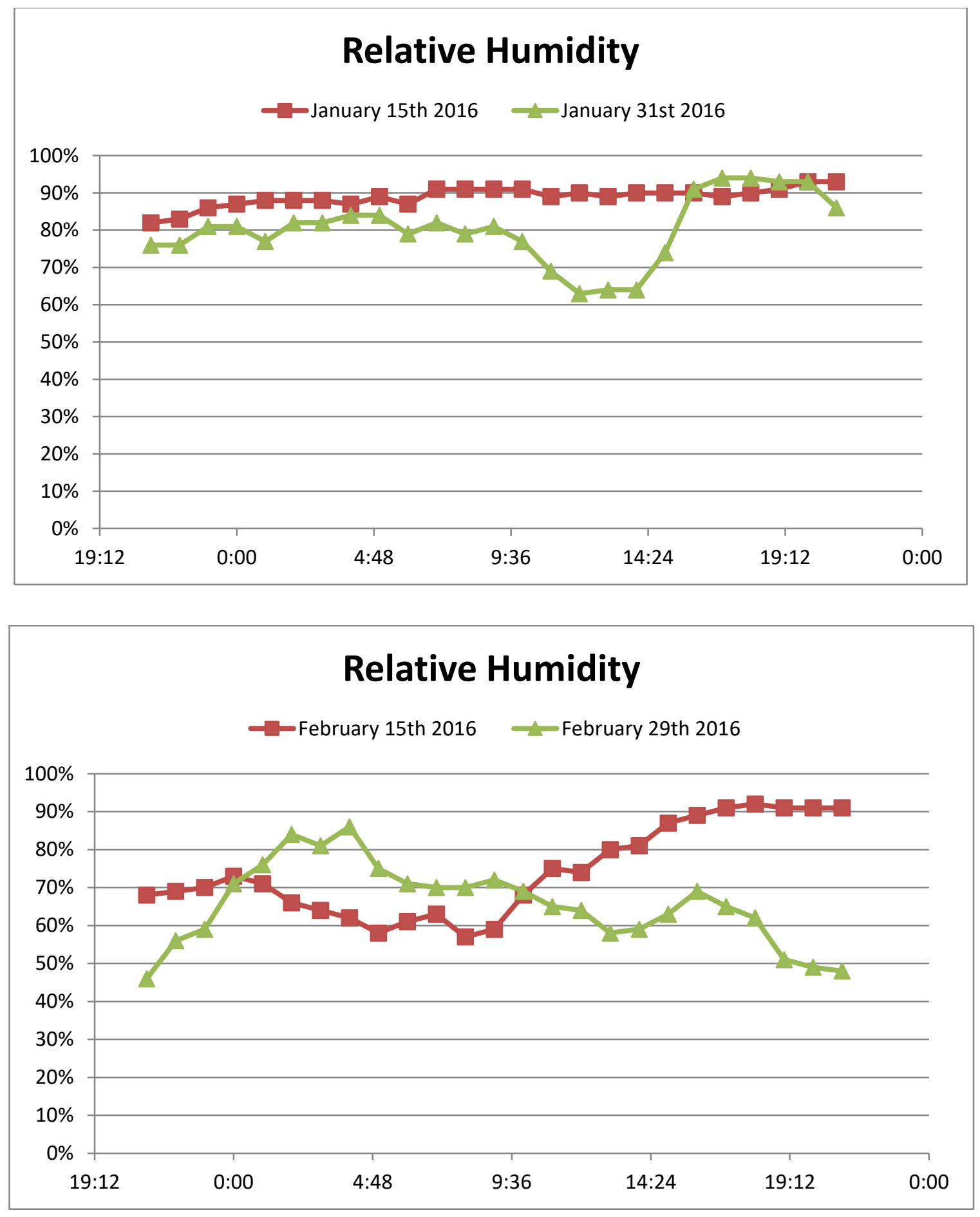

cclii 

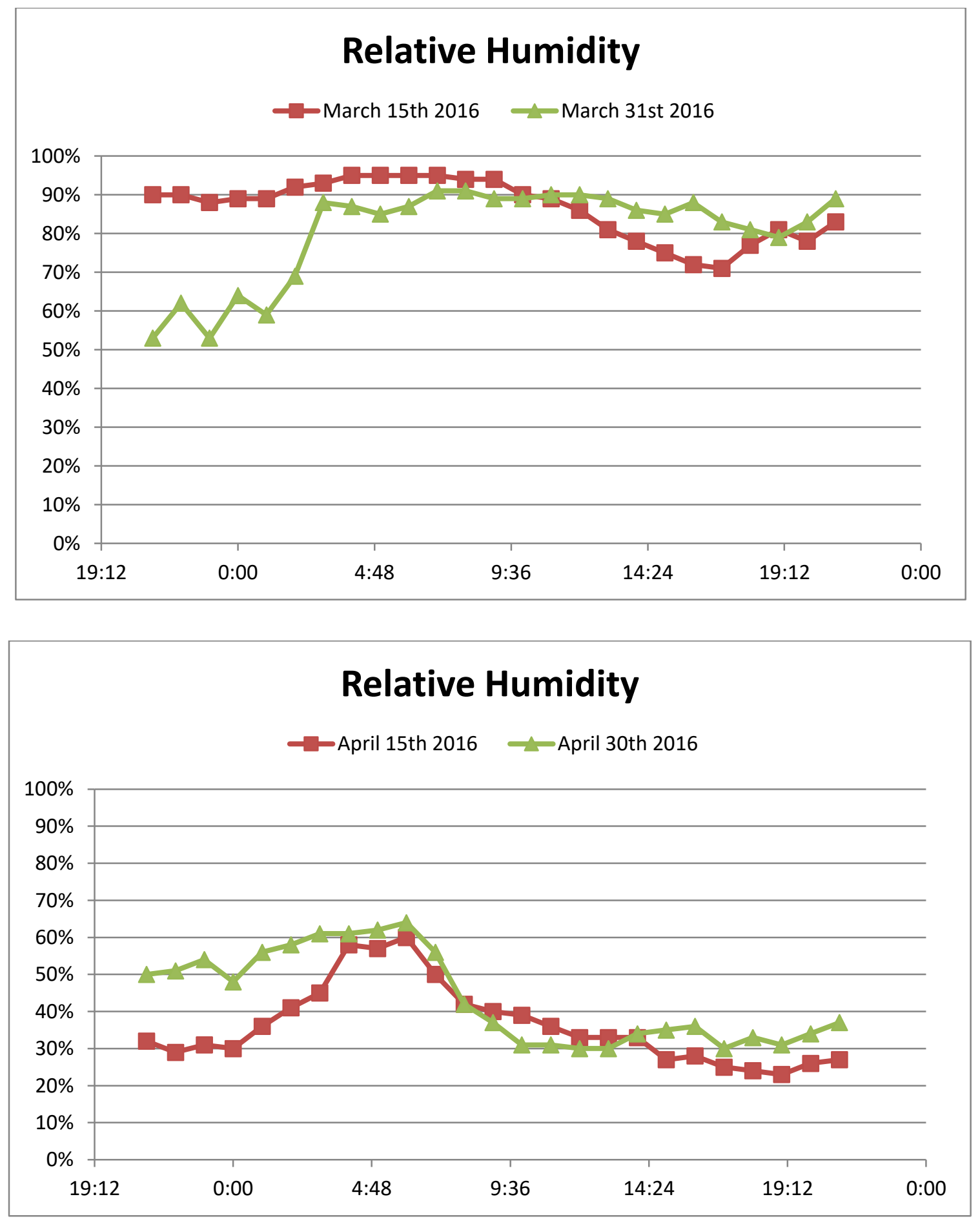

ccliii 


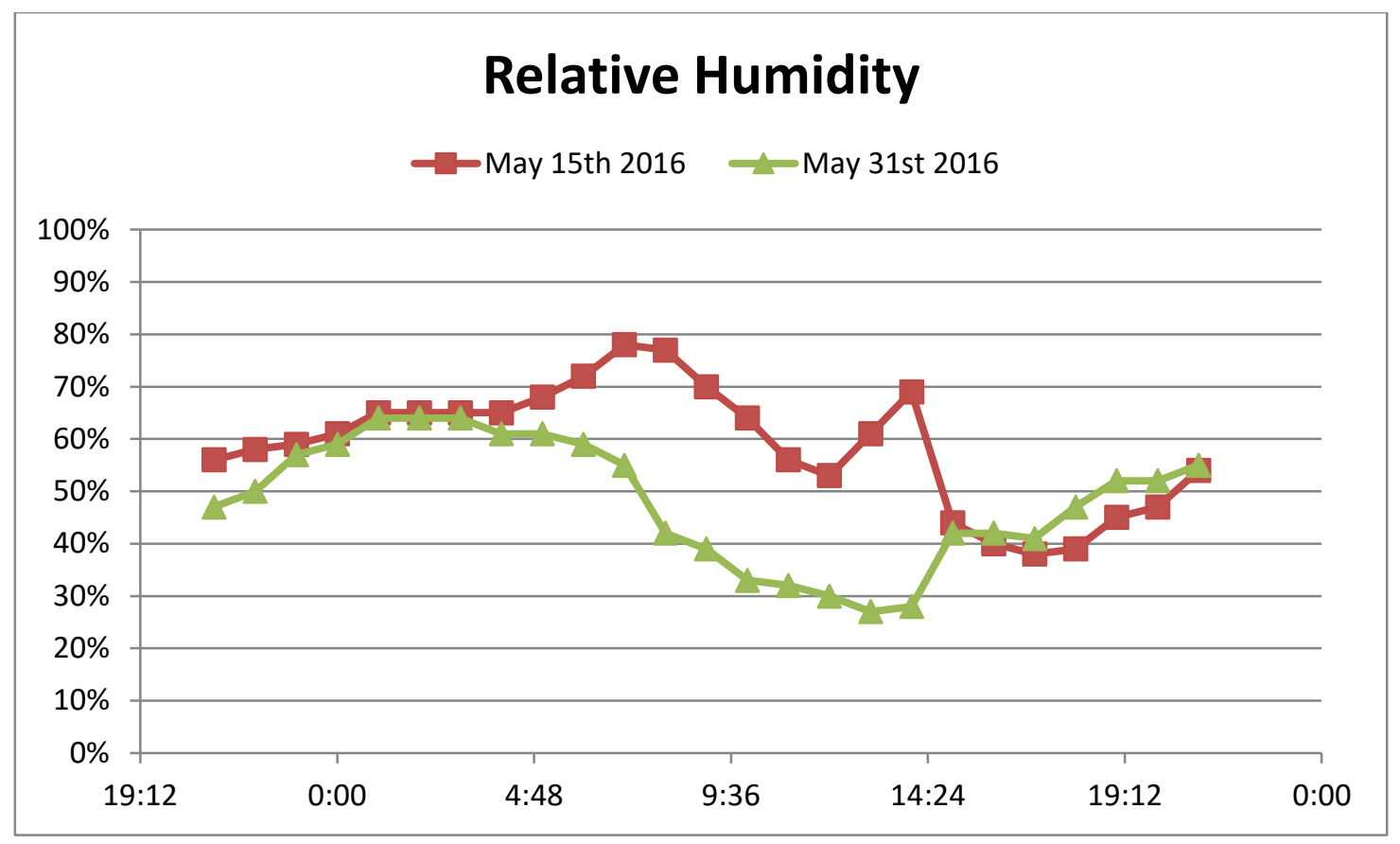

ccliv 


\section{Temperature (daily data starts from 22:00pm)}
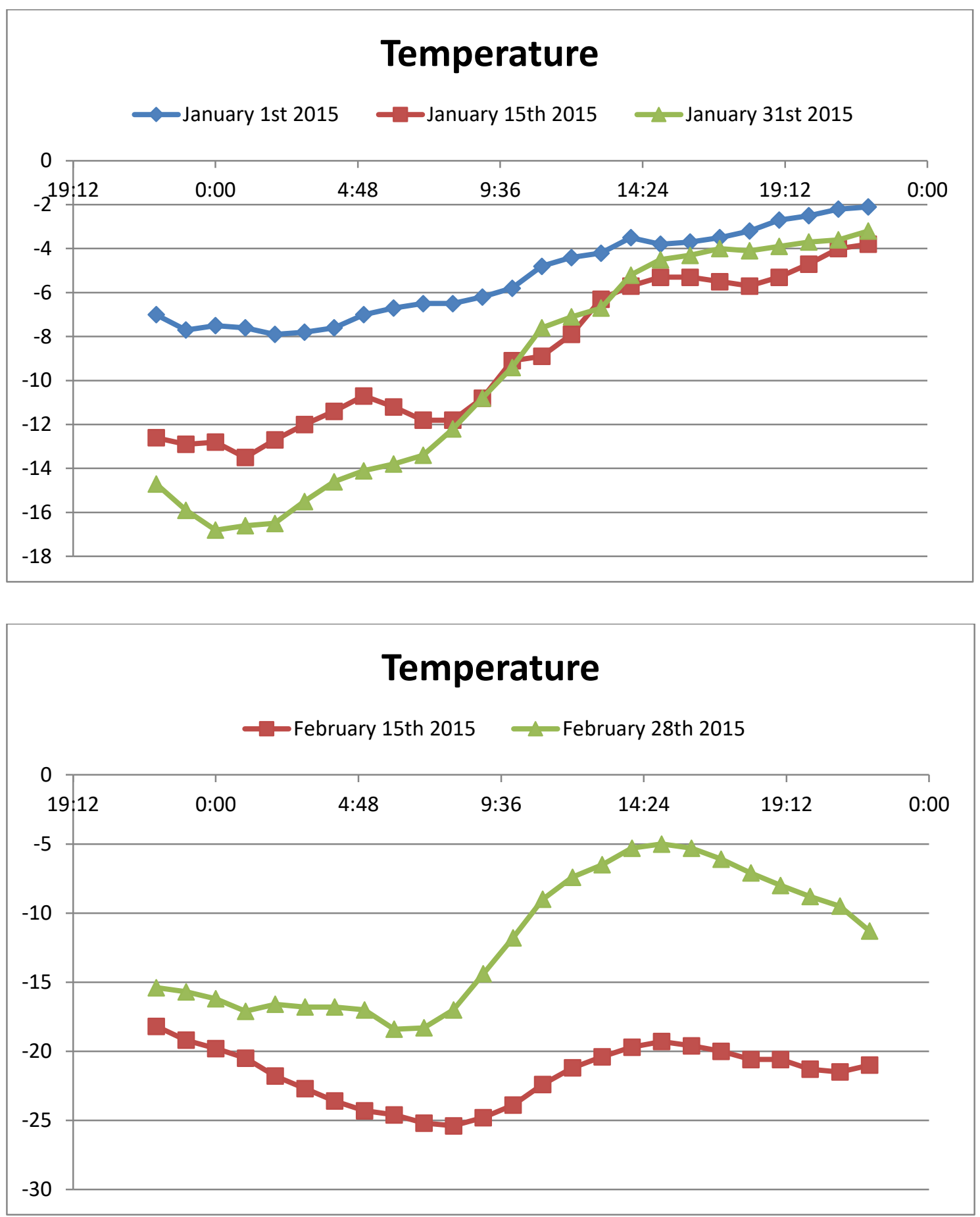

cclv 

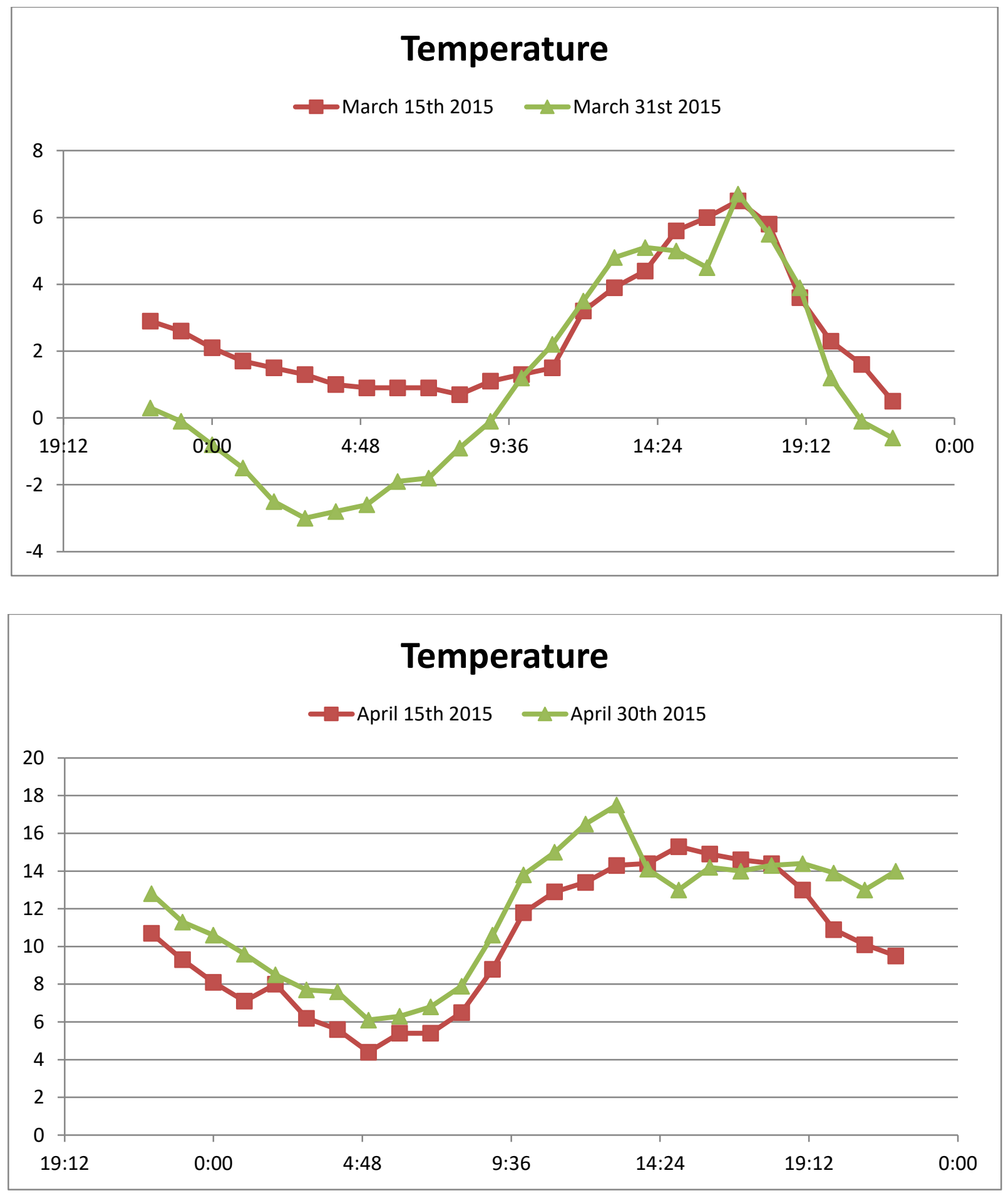

cclvi 

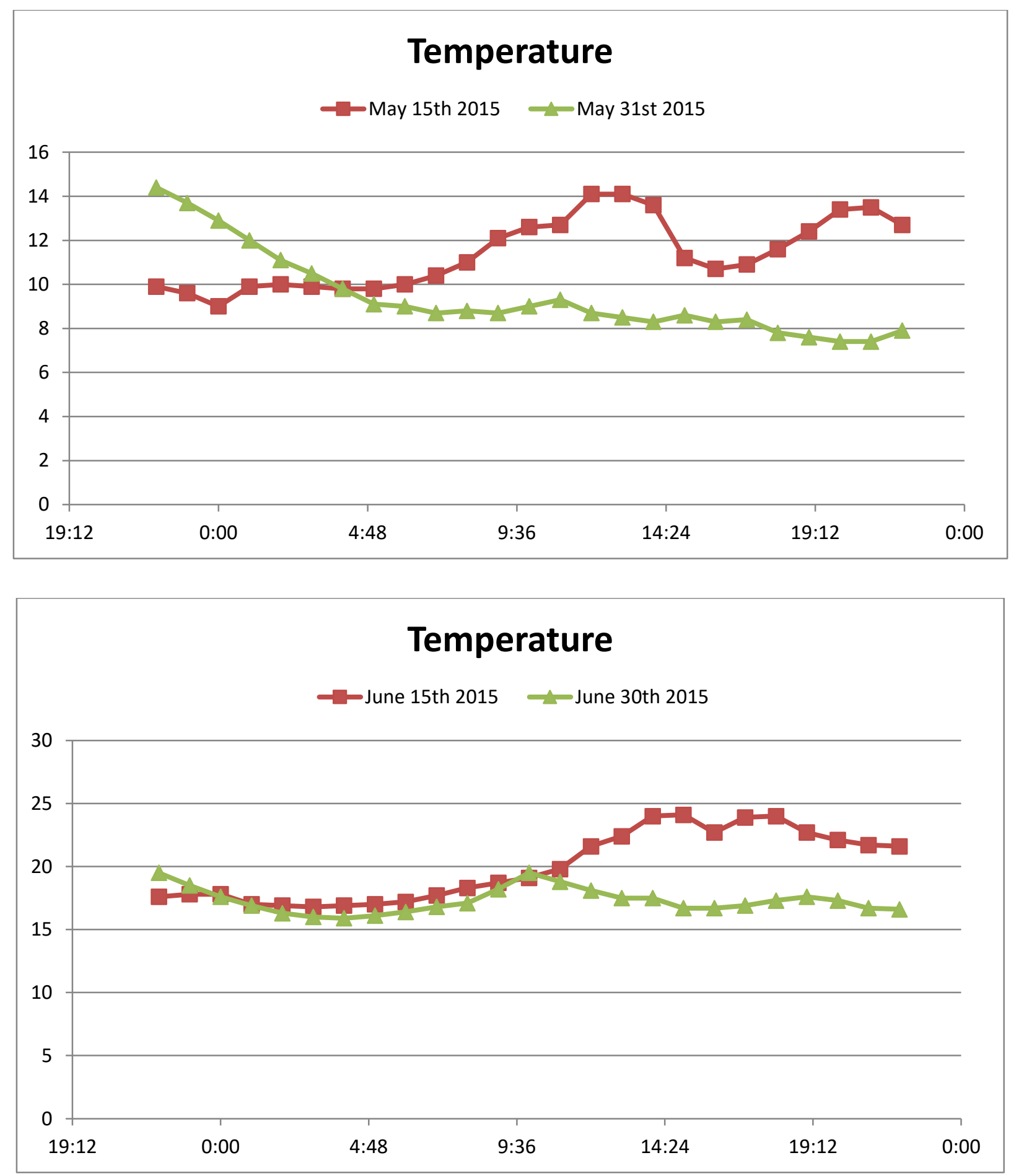

cclvii 

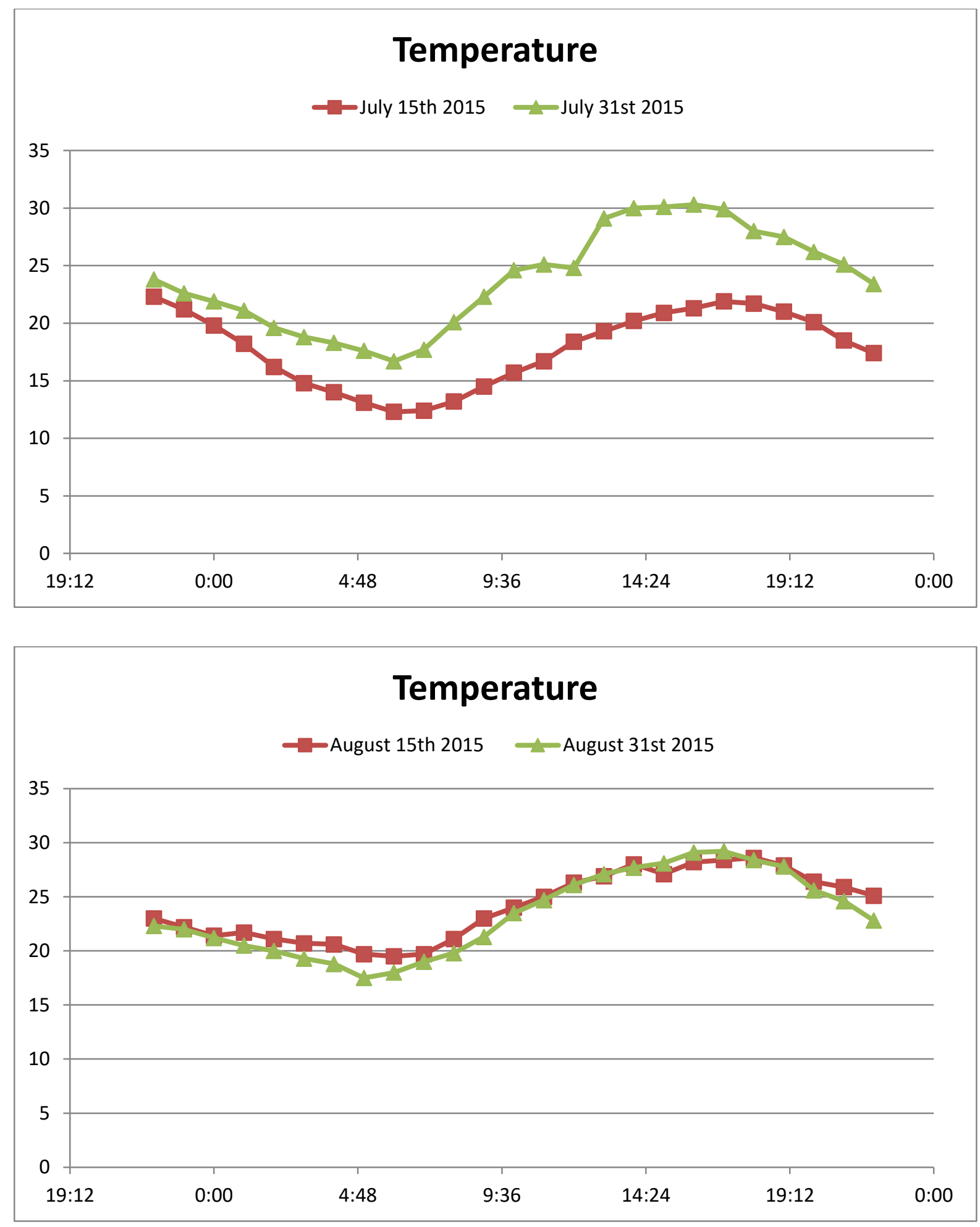

cclviii 

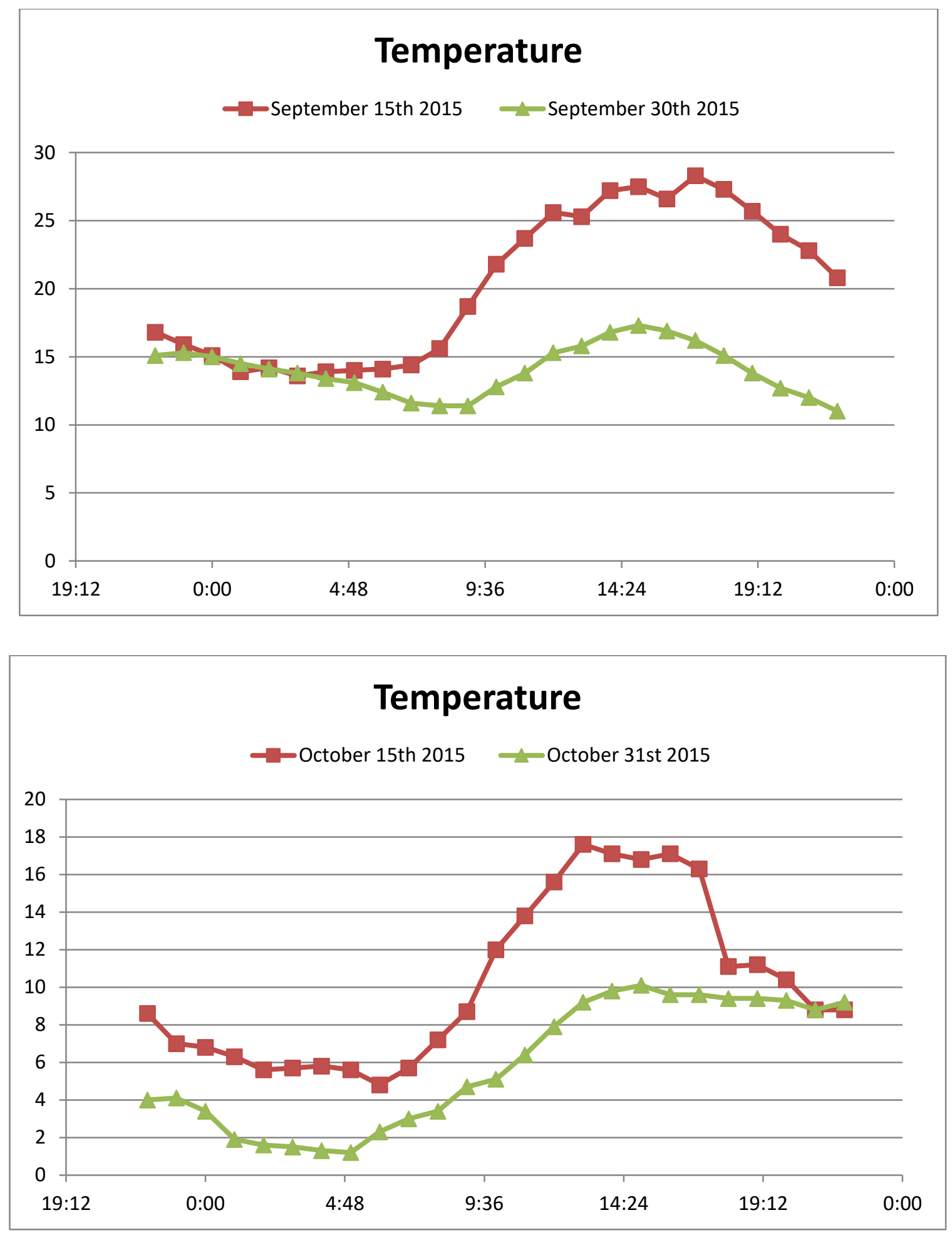

cclix 

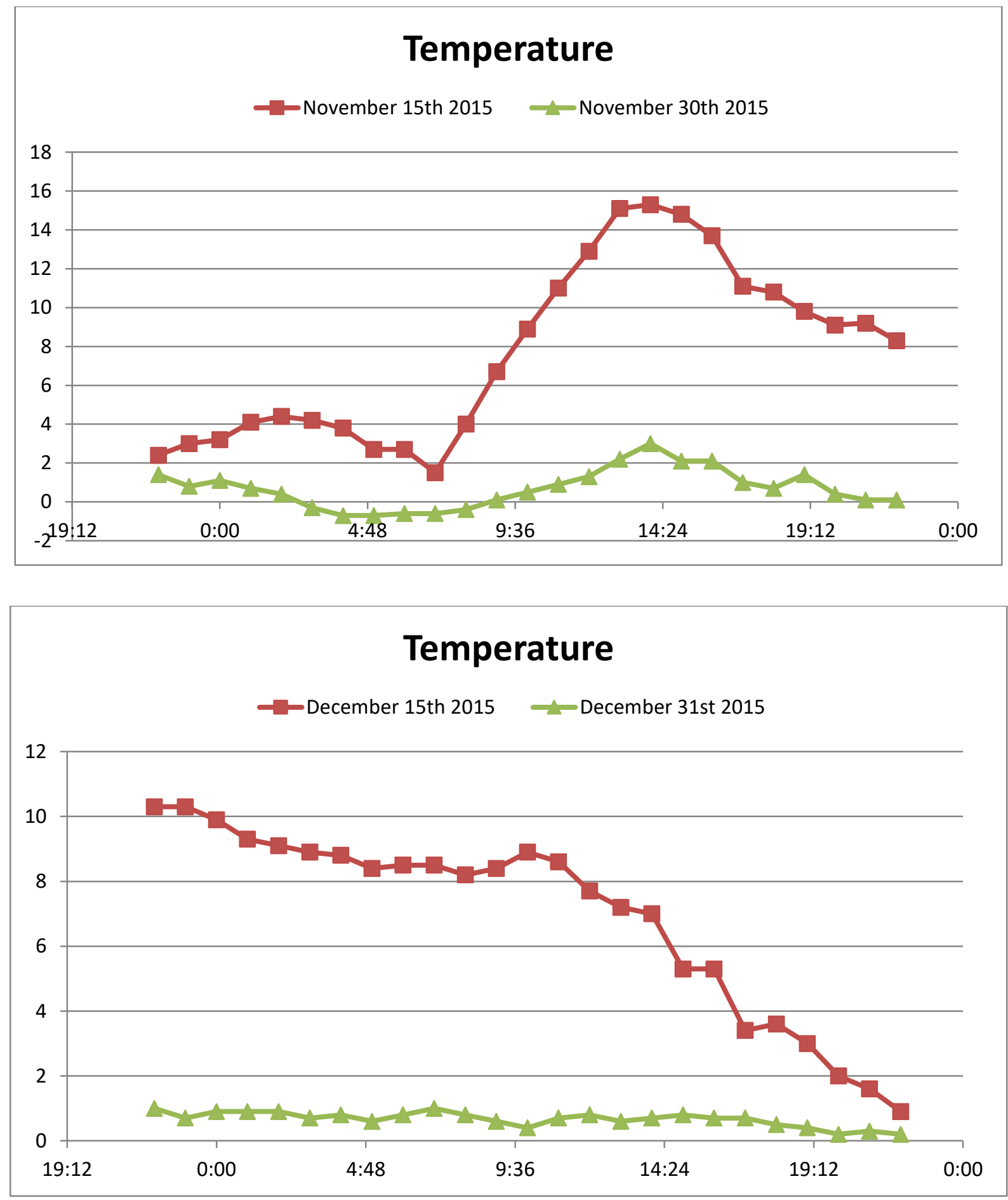

cclx 

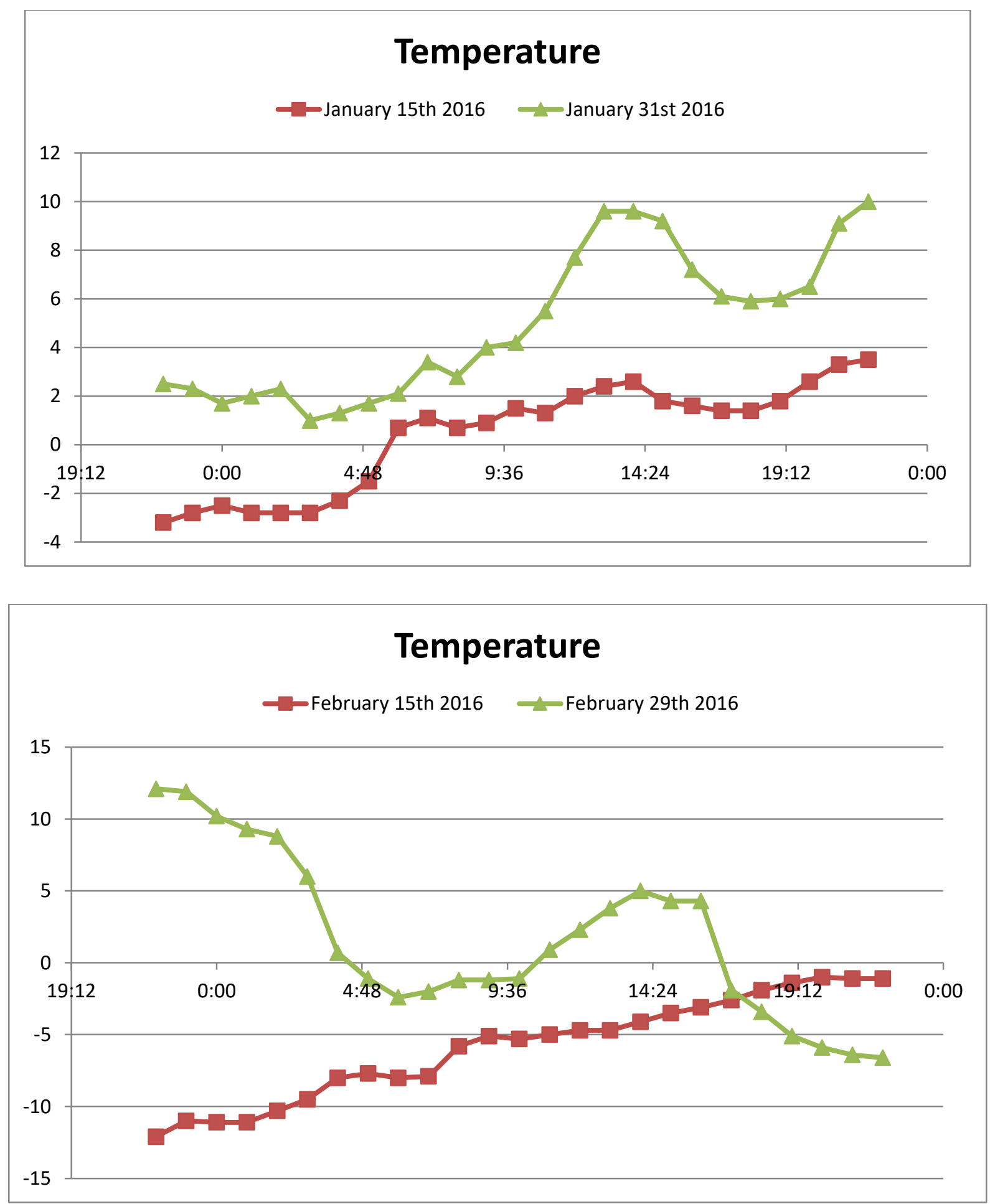

cclxi 

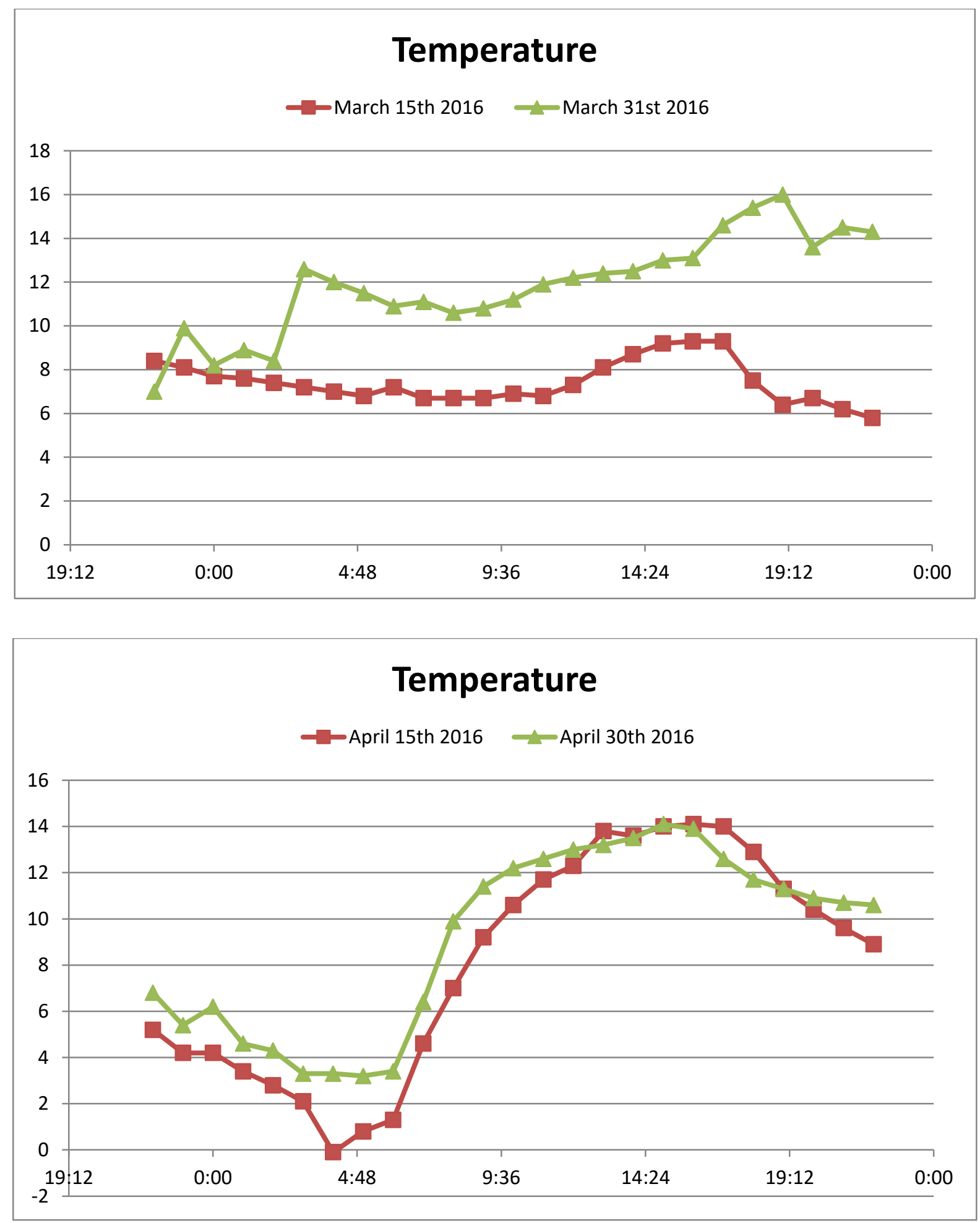

cclxii 


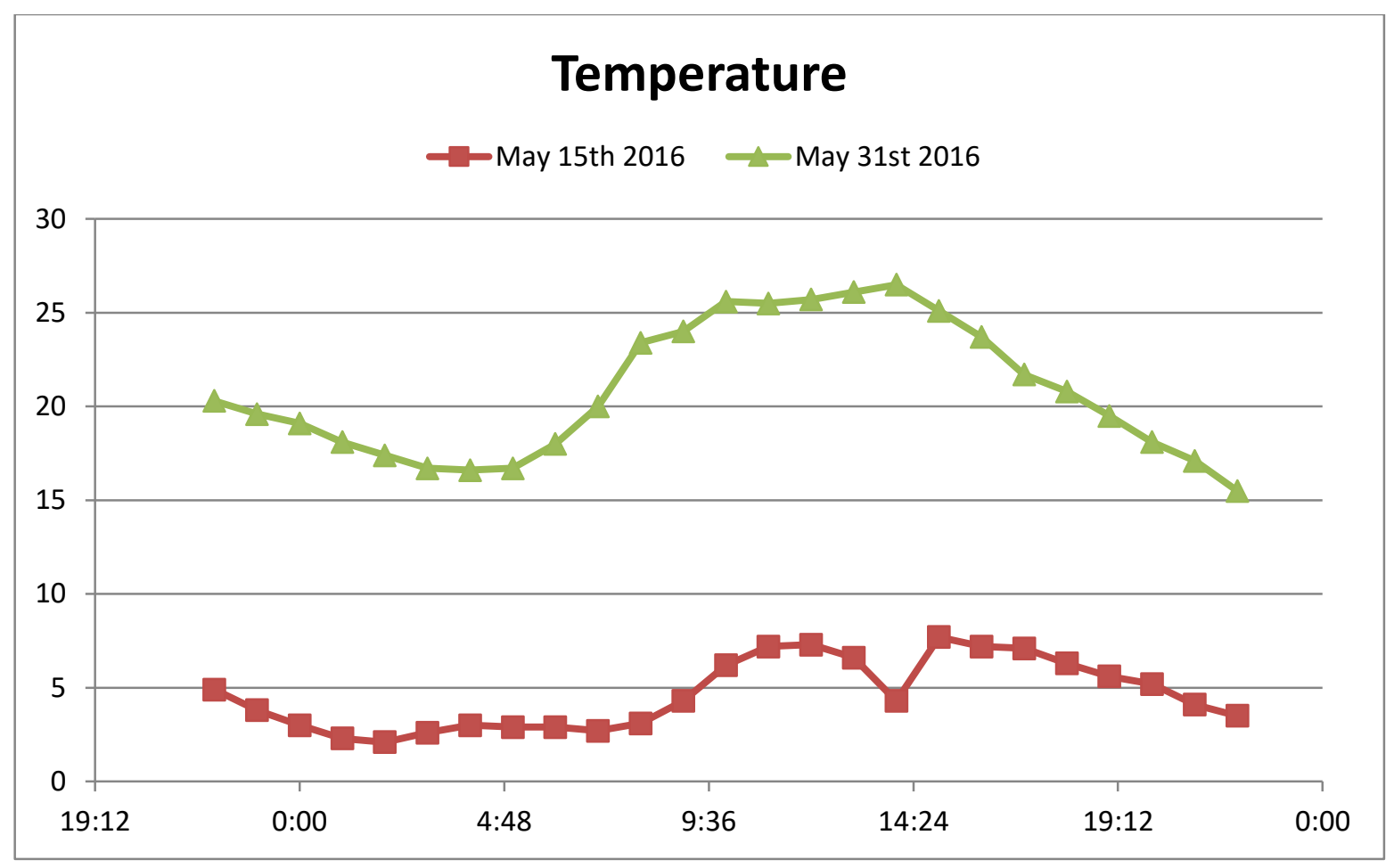

cclxiii 


\section{Snow on Ground (daily data)}
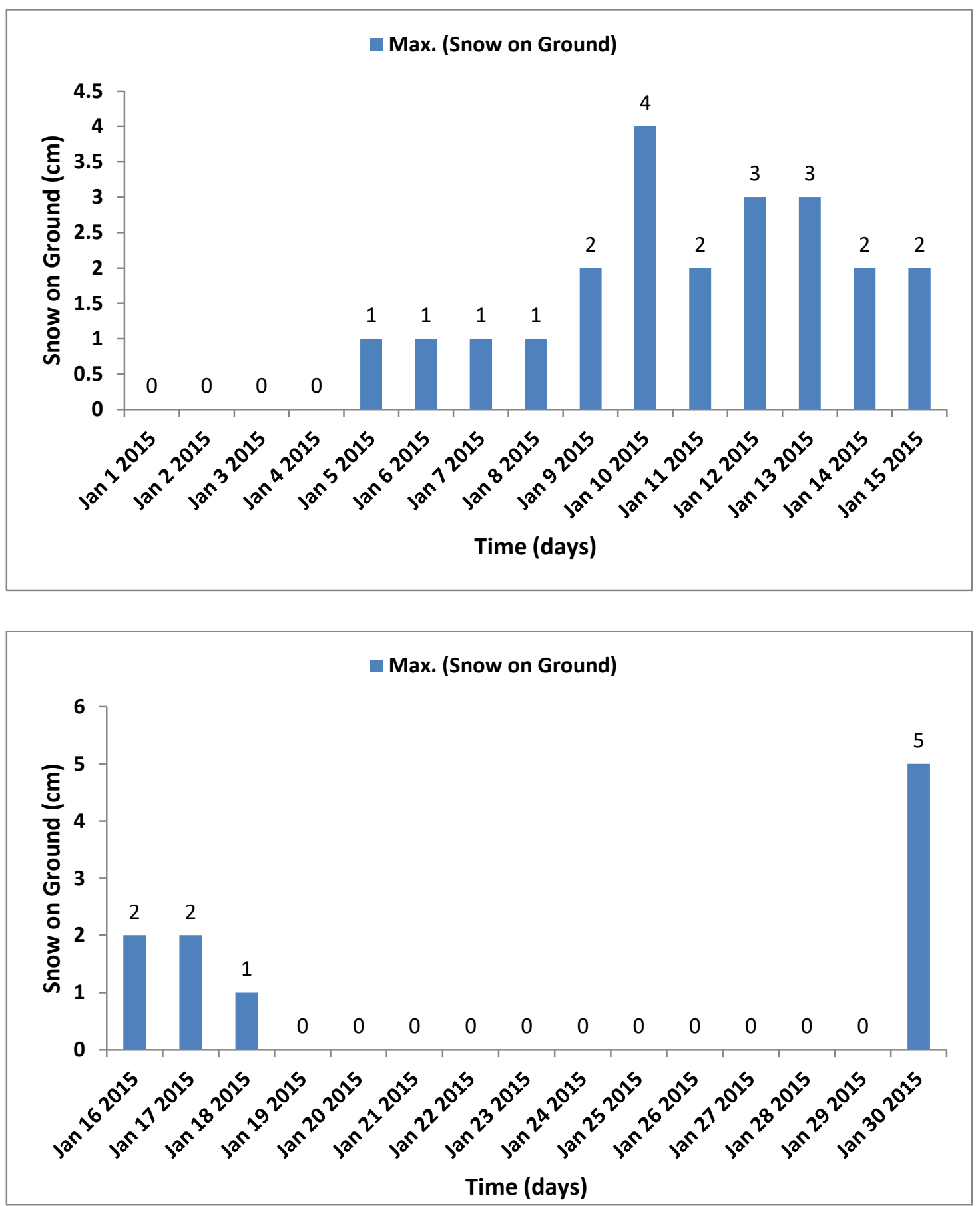

cclxiv 

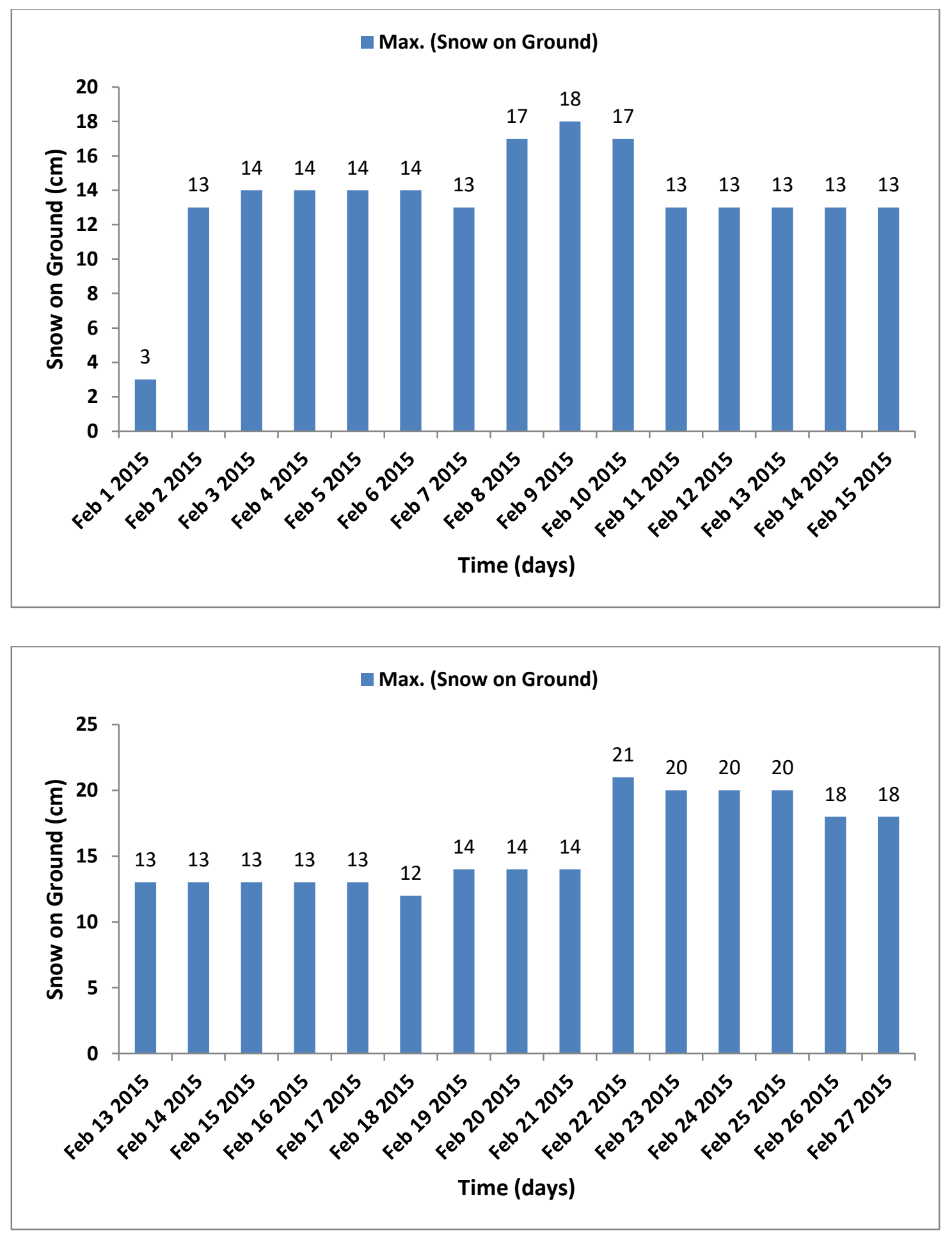

cclxv 

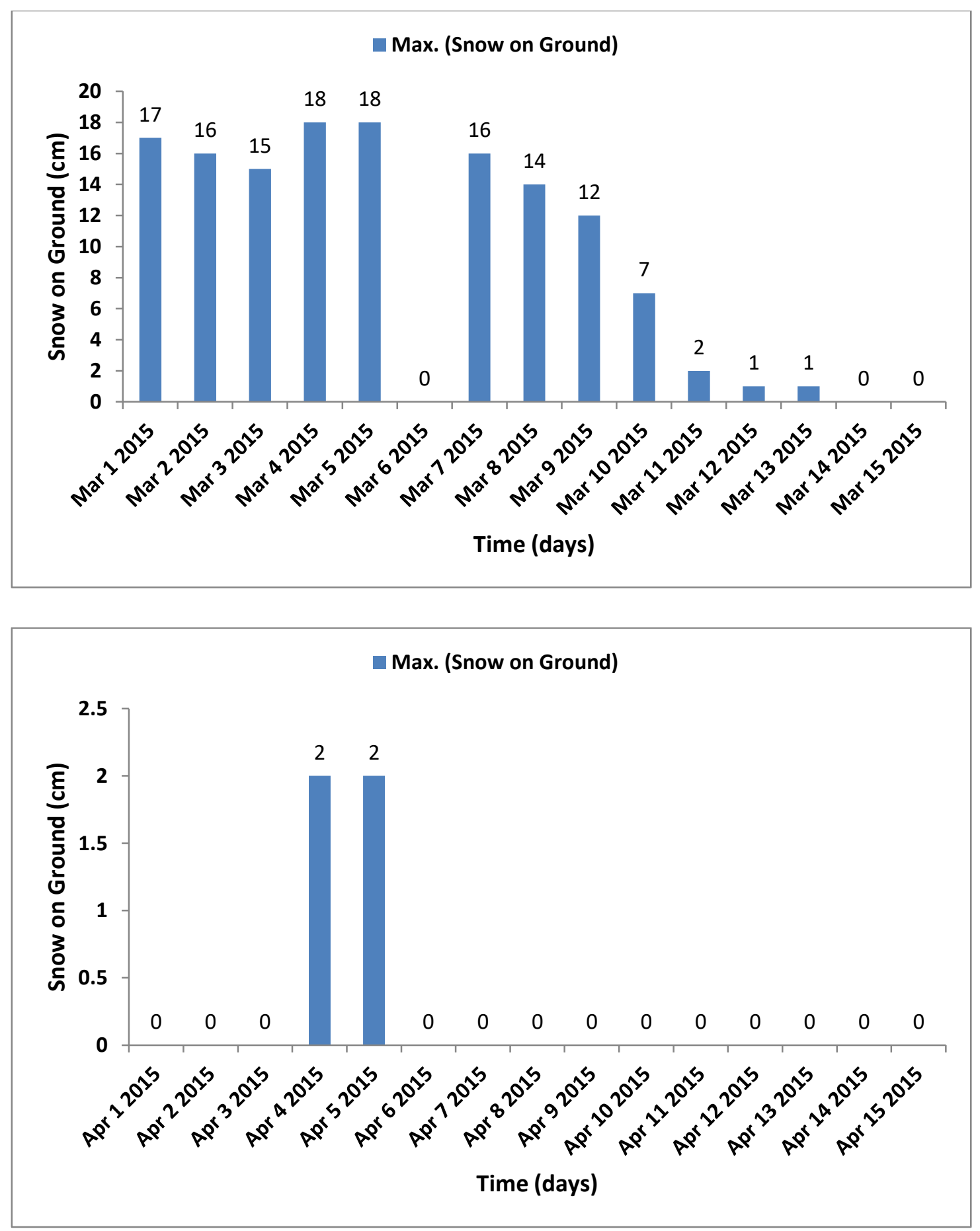

cclxvi 

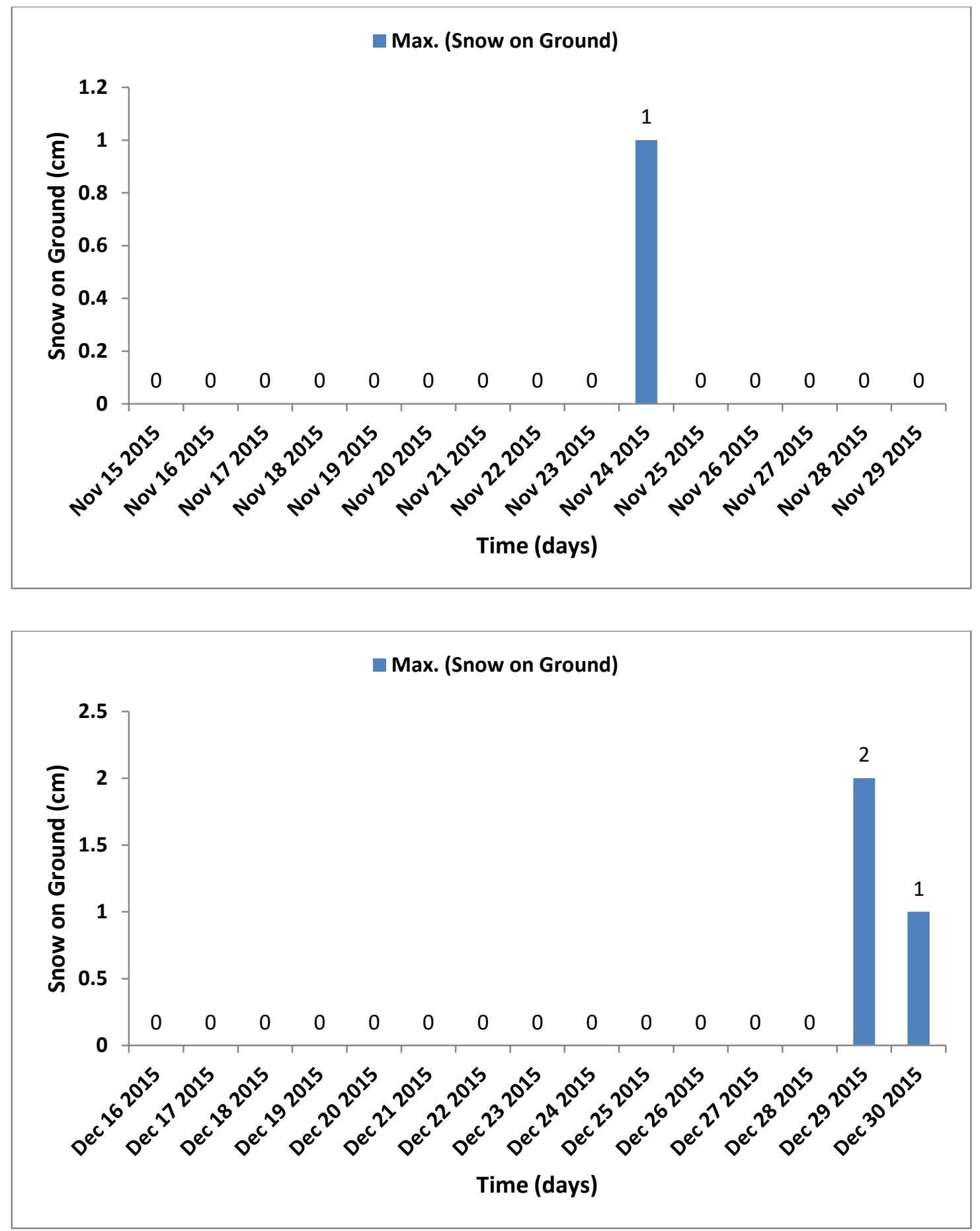

cclxvii 

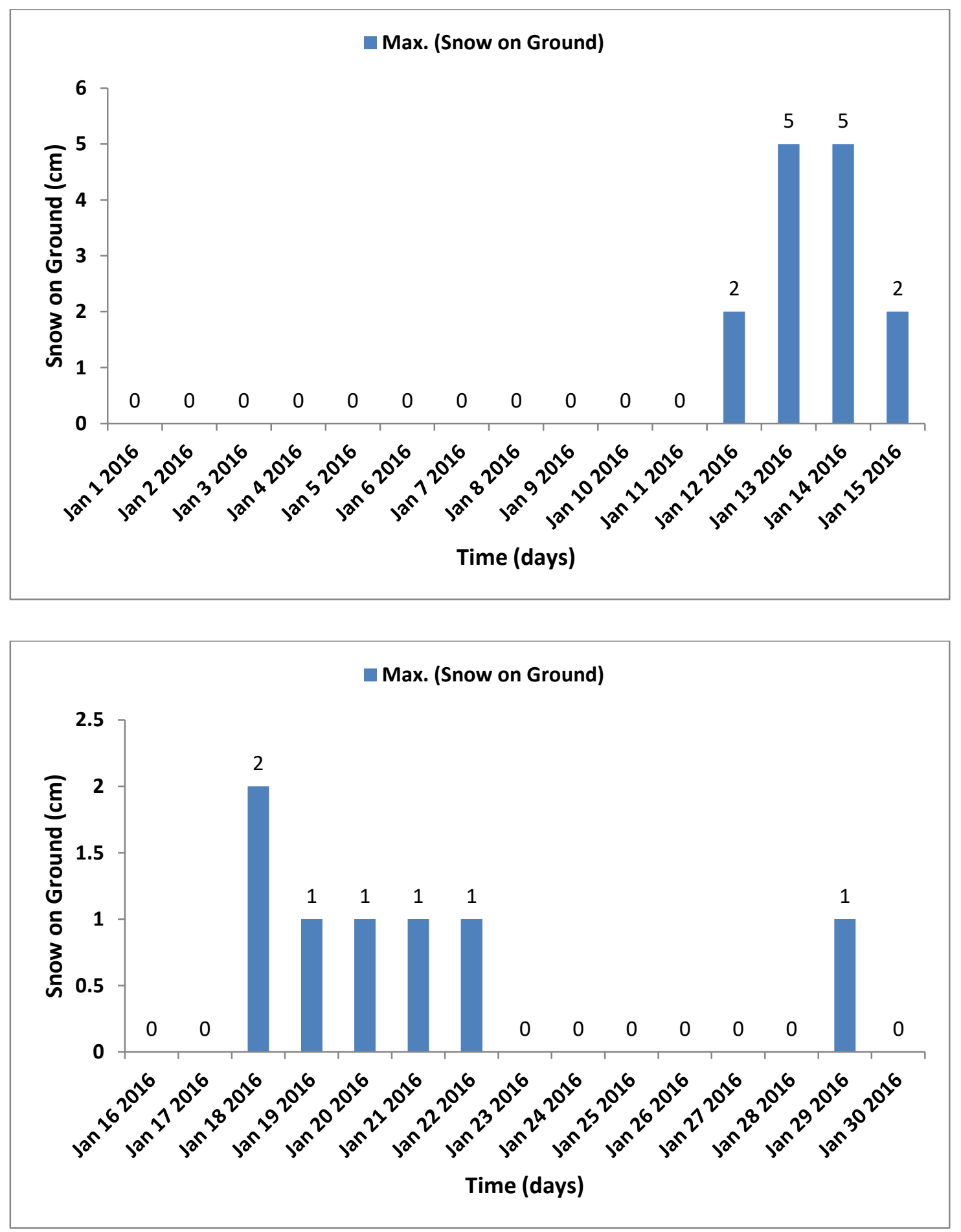

cclxviii 

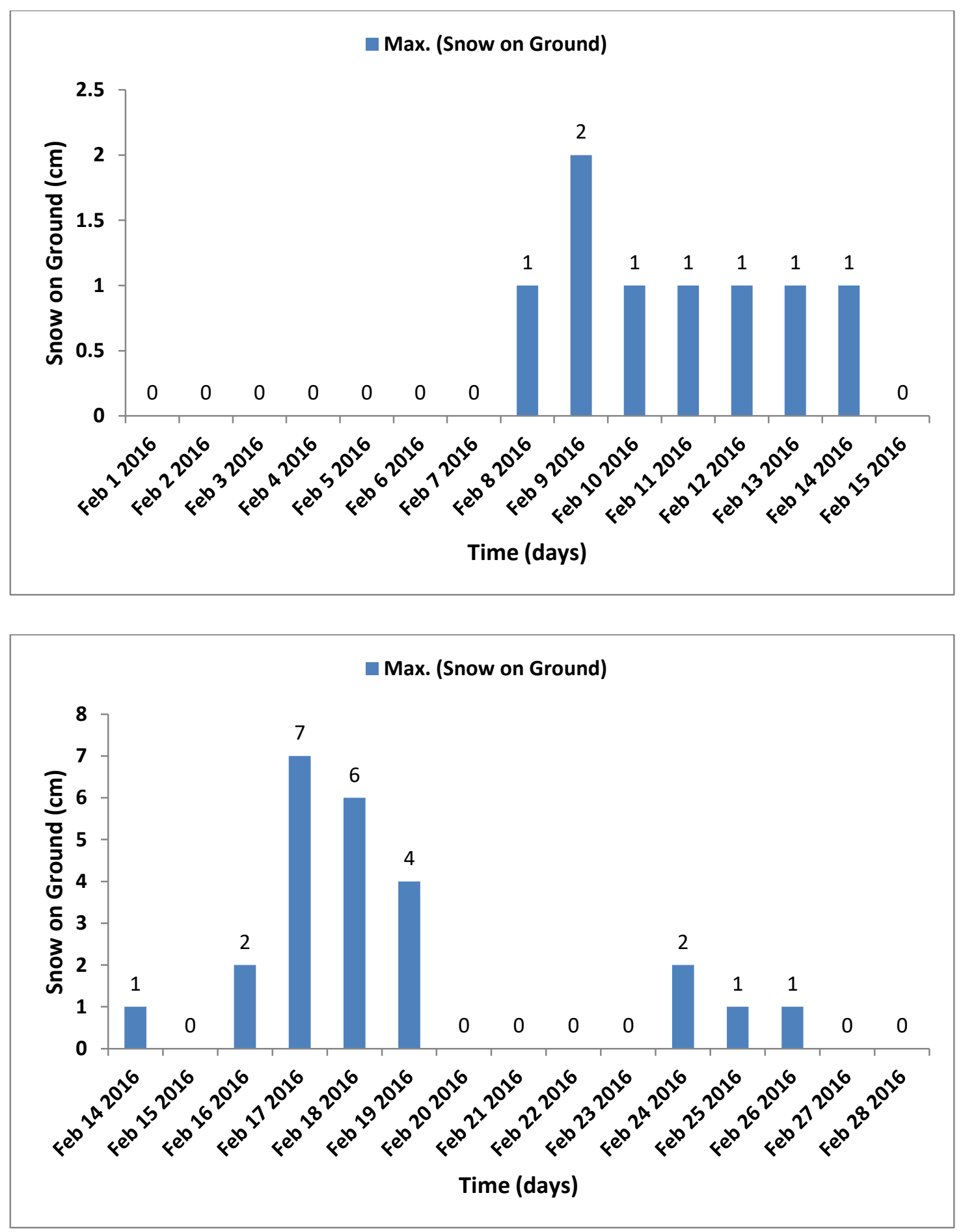

cclxix 

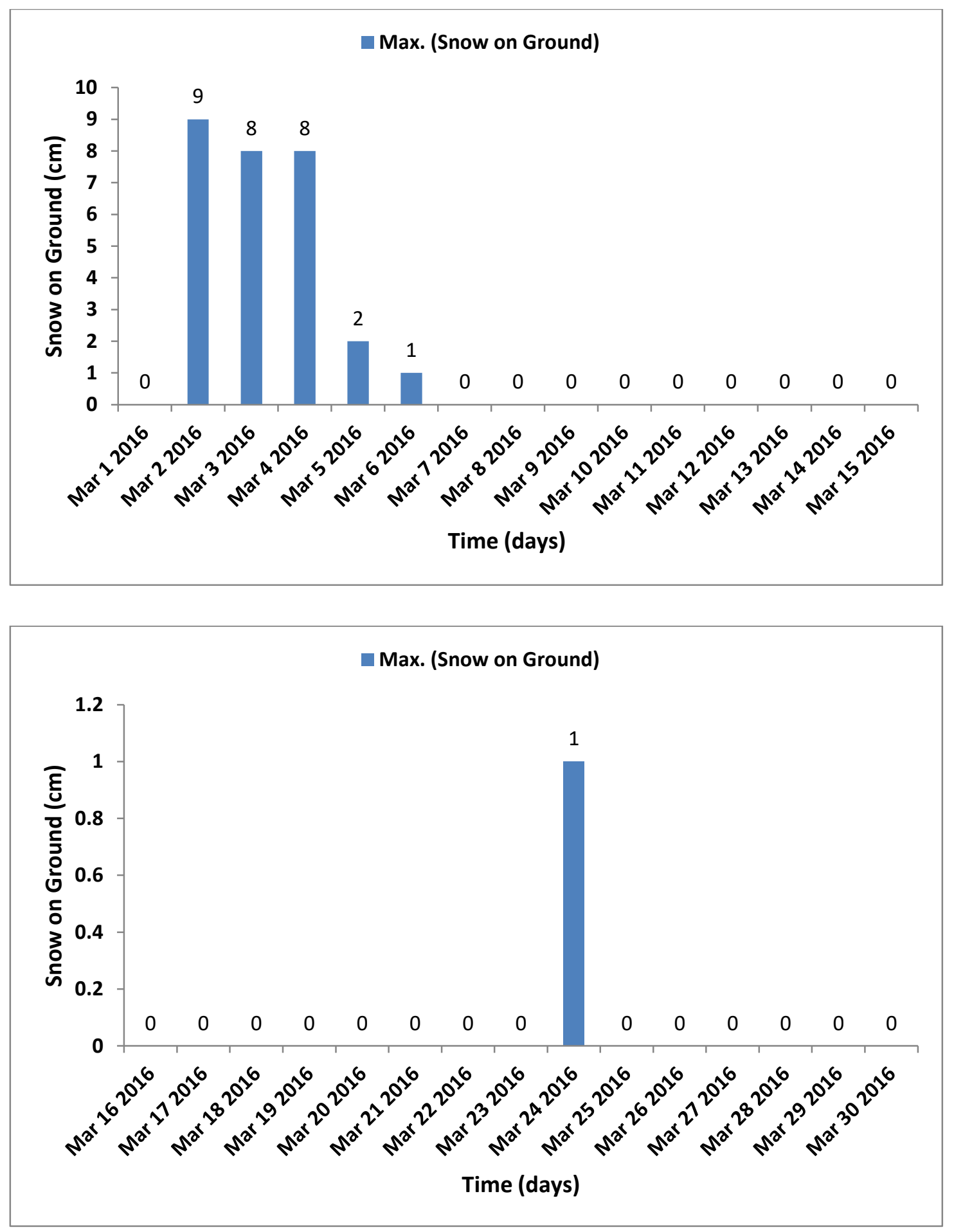

$\operatorname{cclxx}$ 


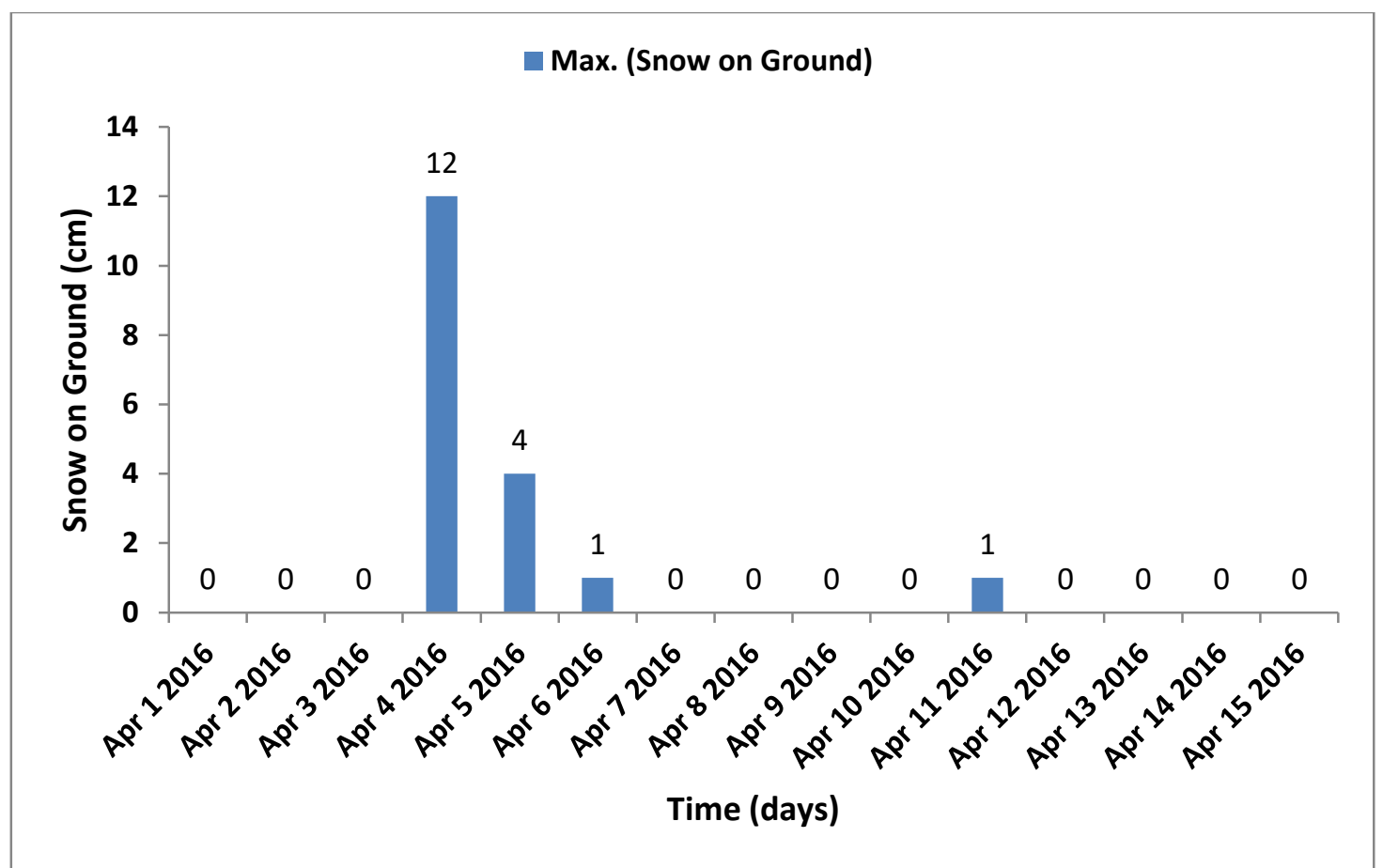

cclxxi 


\section{Total precipitation (daily data)}
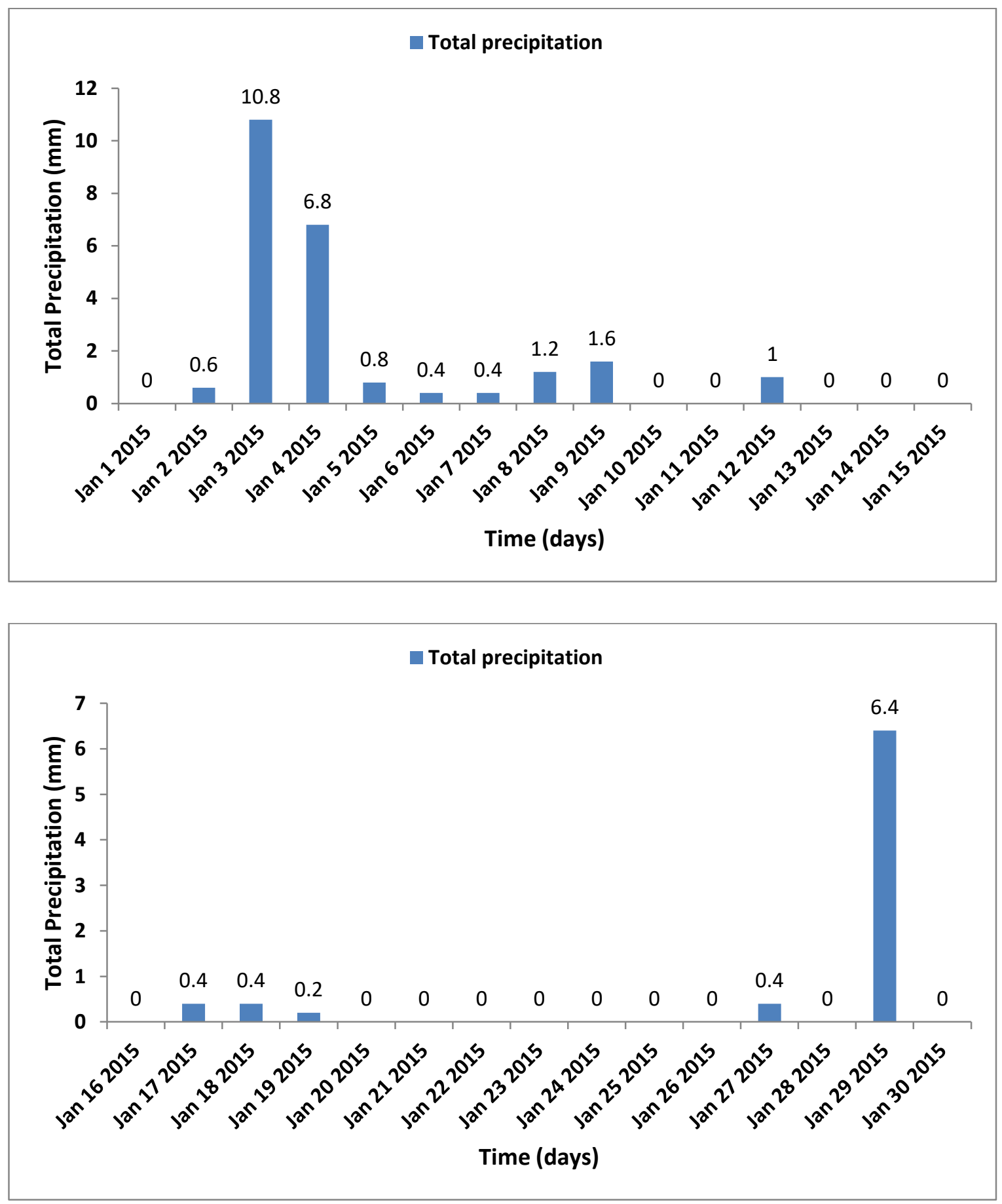

cclxxii 

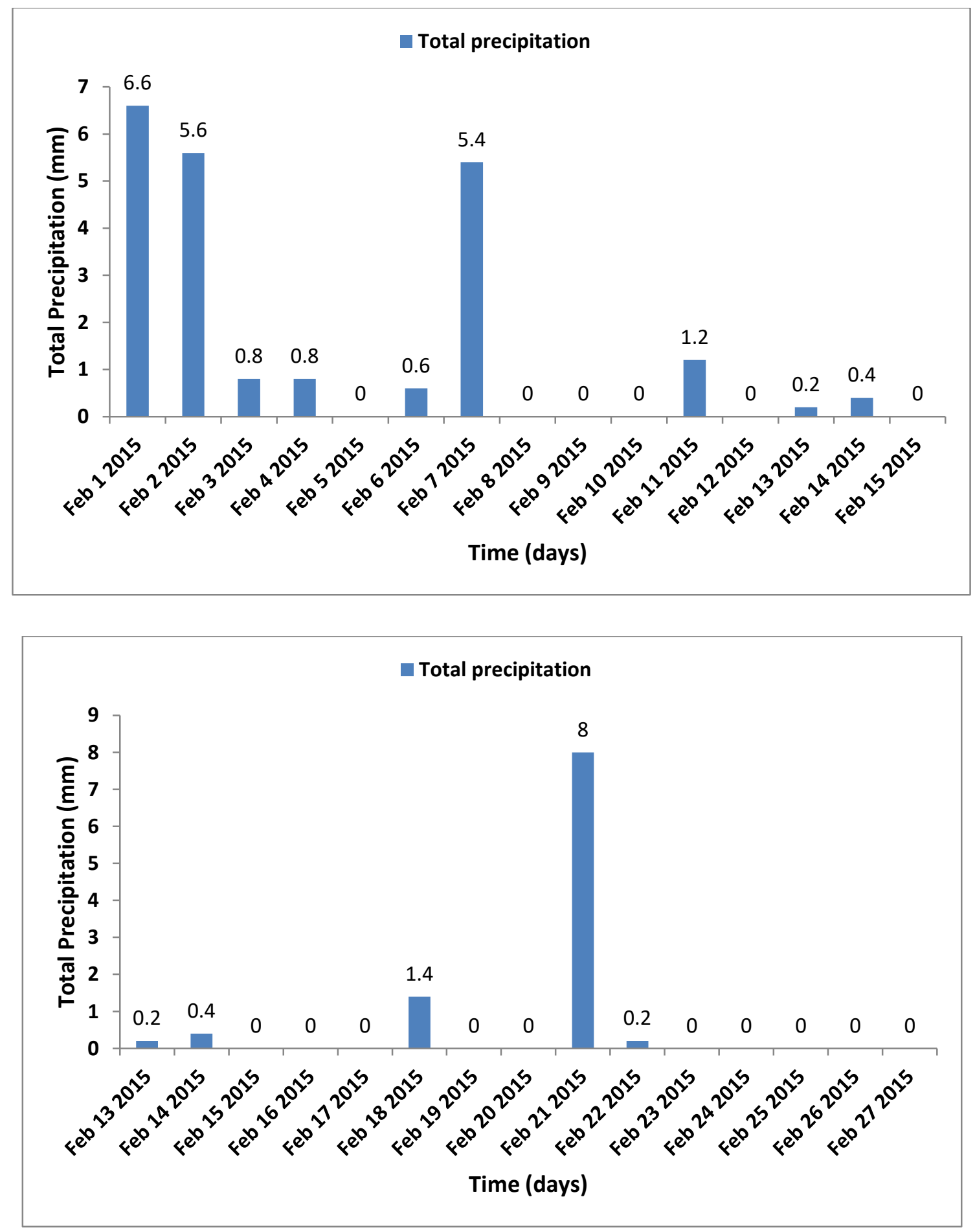

cclxxiii 

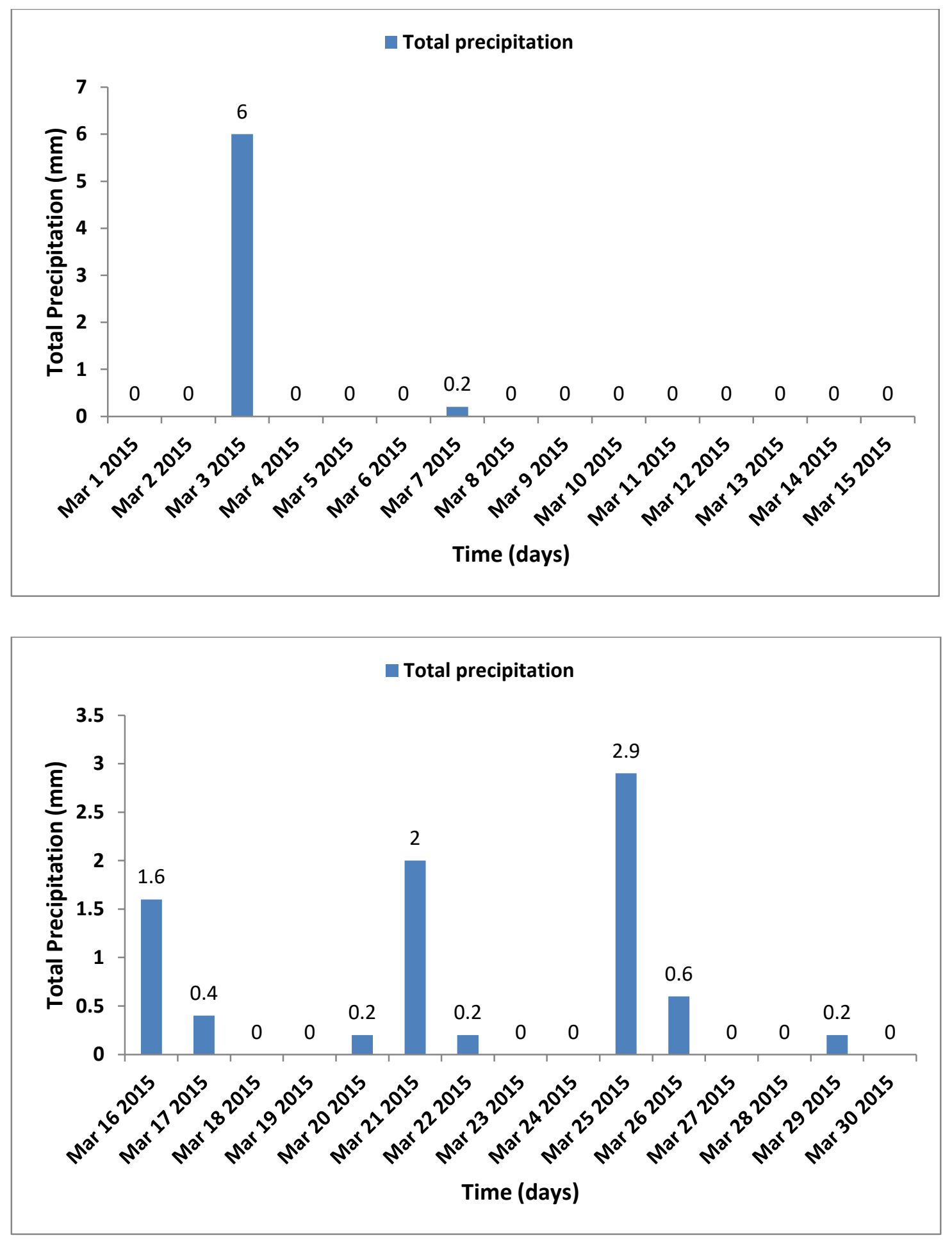

cclxxiv 

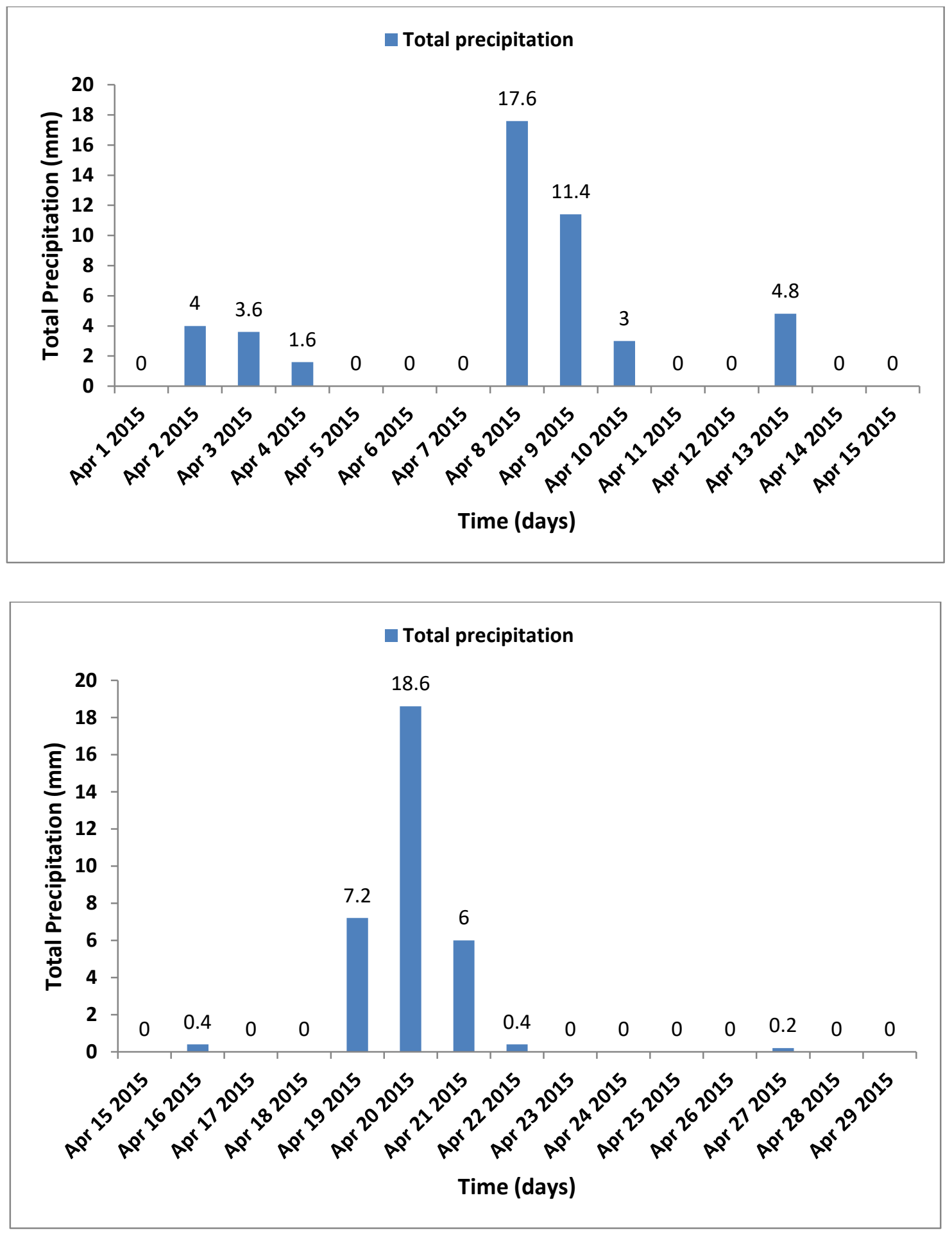

cclxxv 

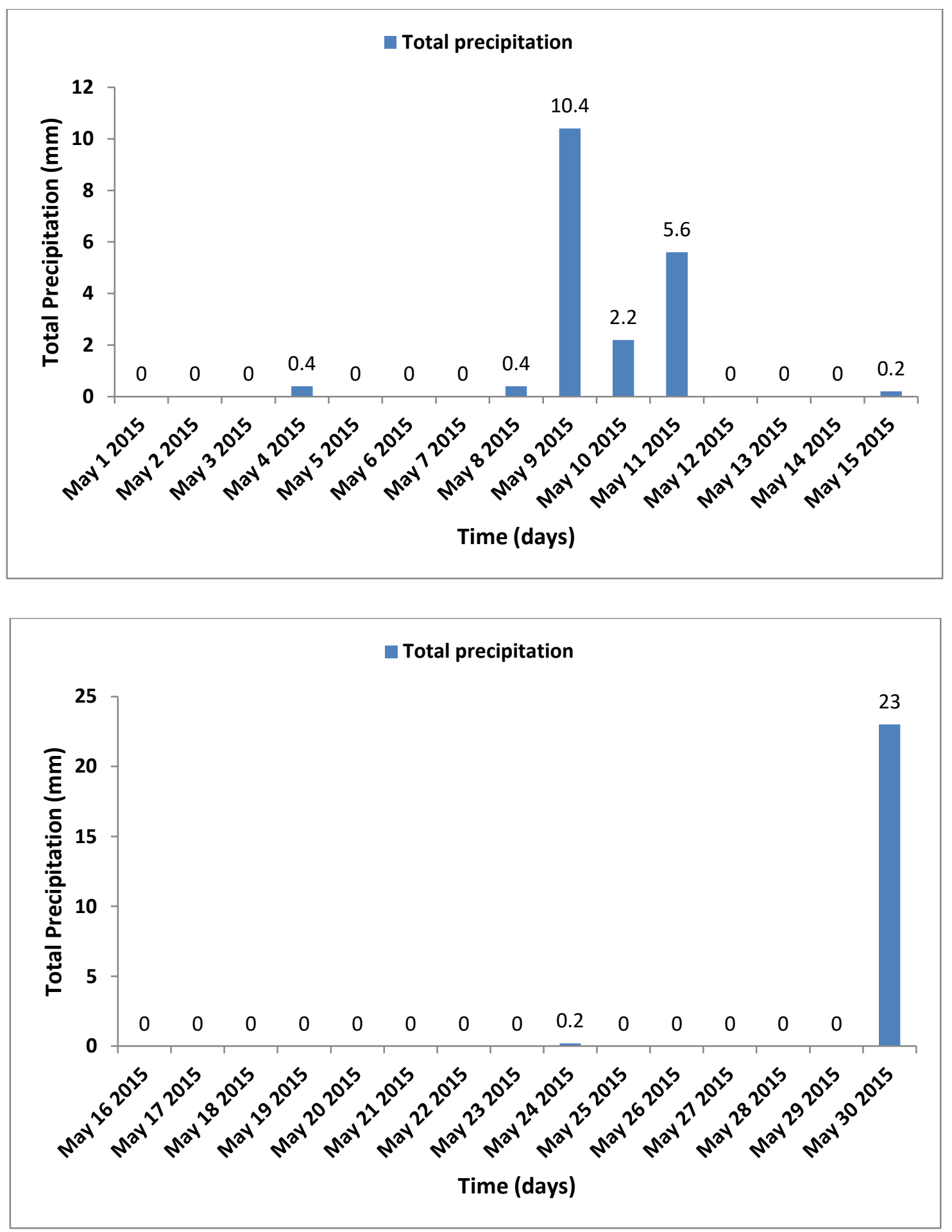

cclxxvi 

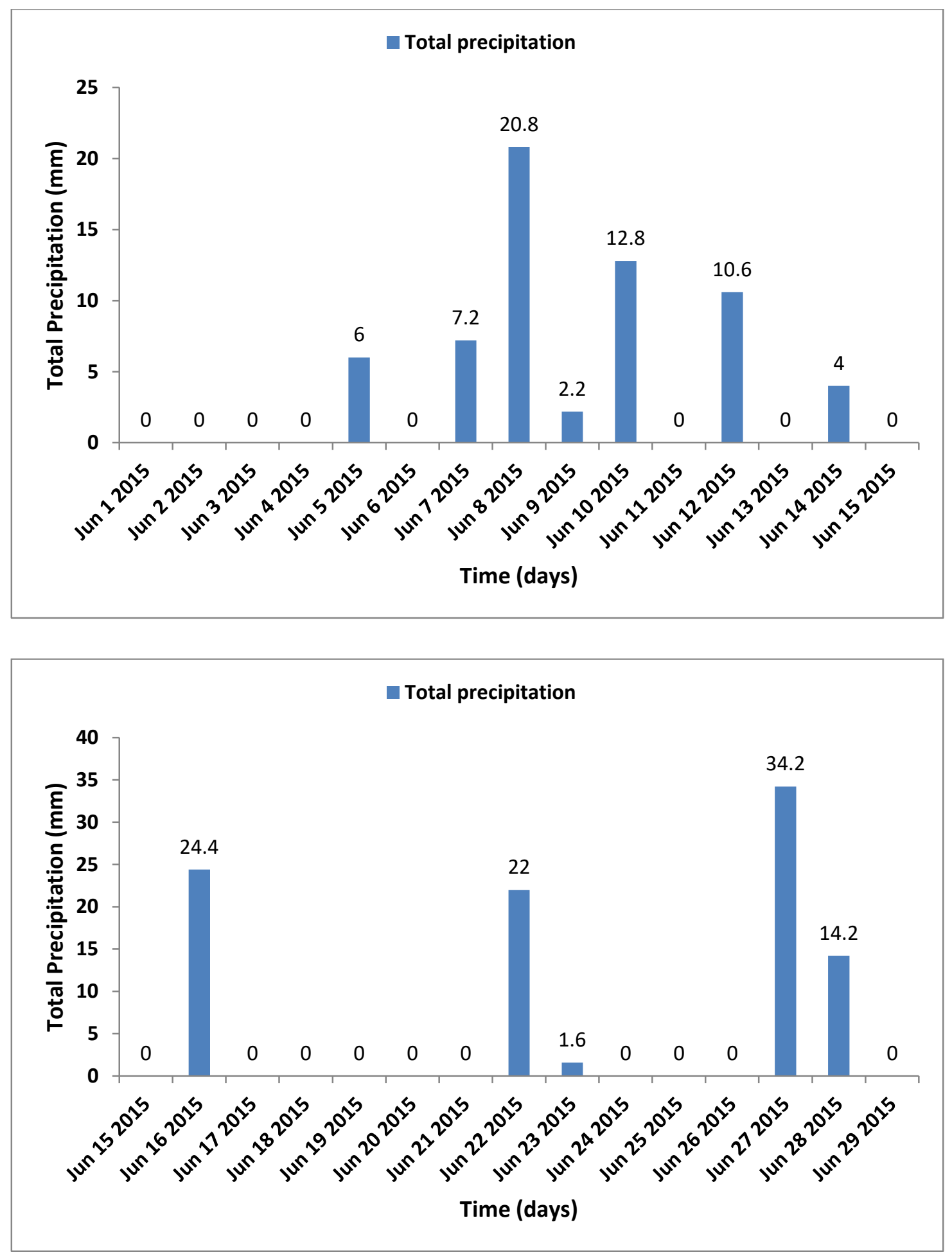

cclxxvii 

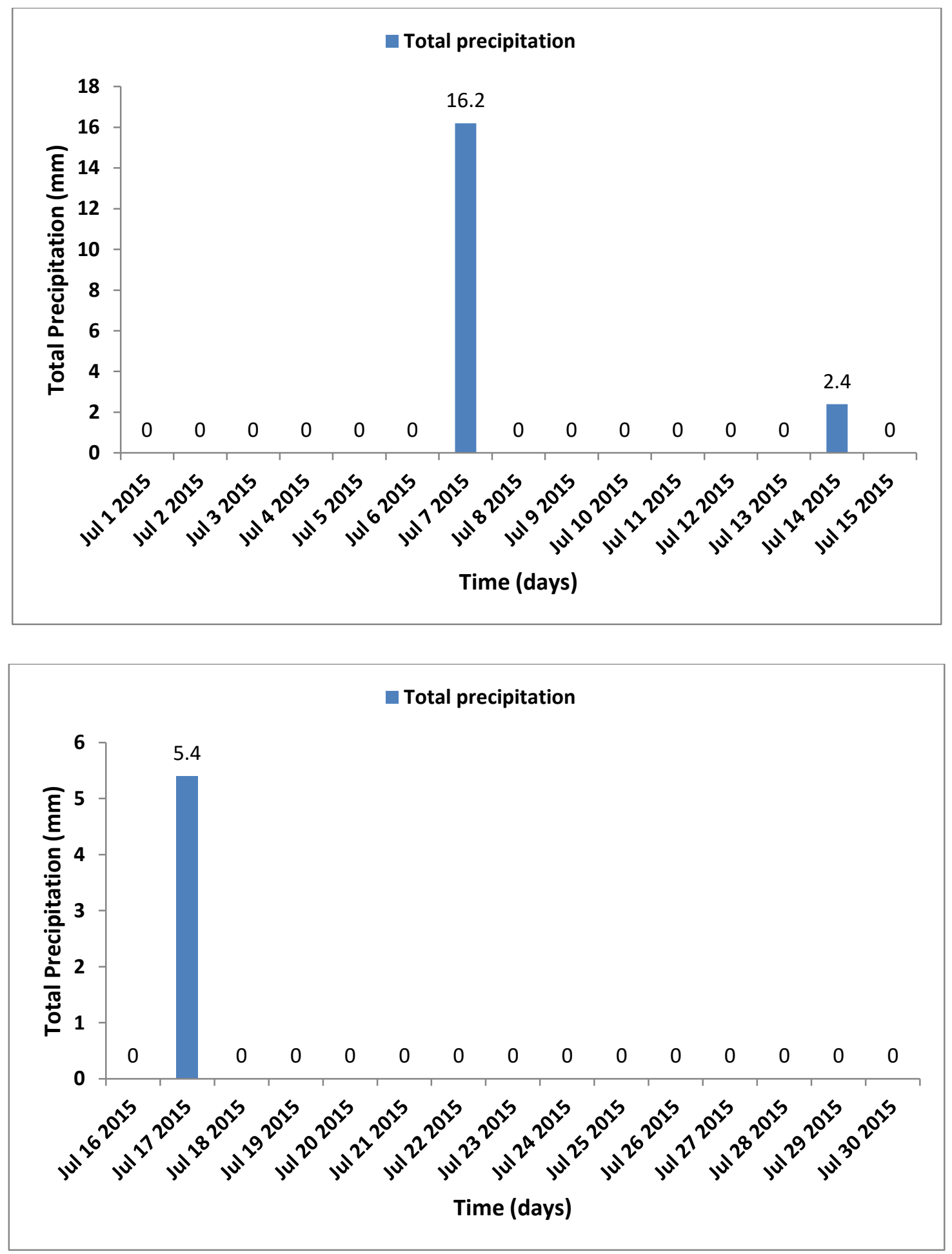

cclxxviii 

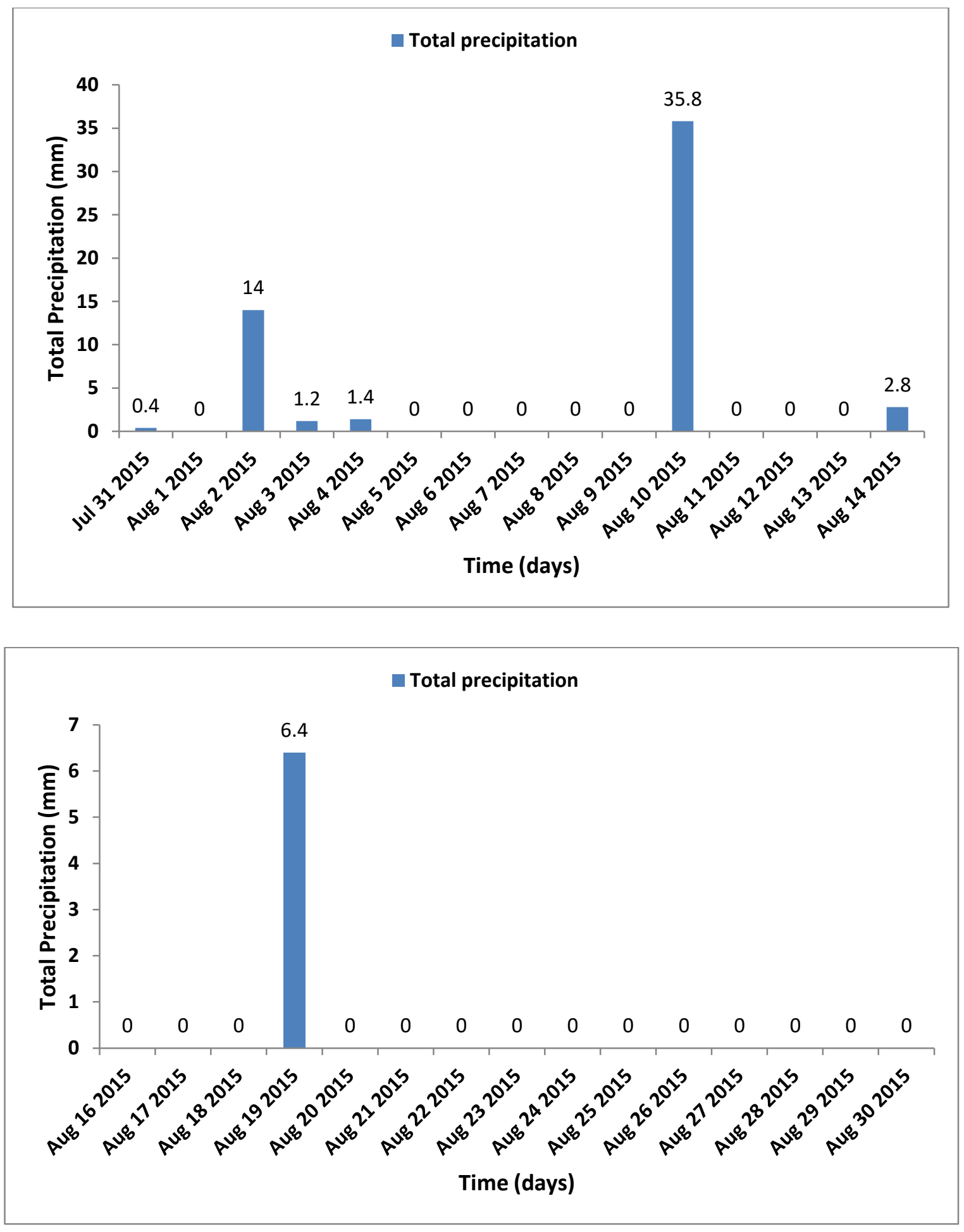

cclxxix 

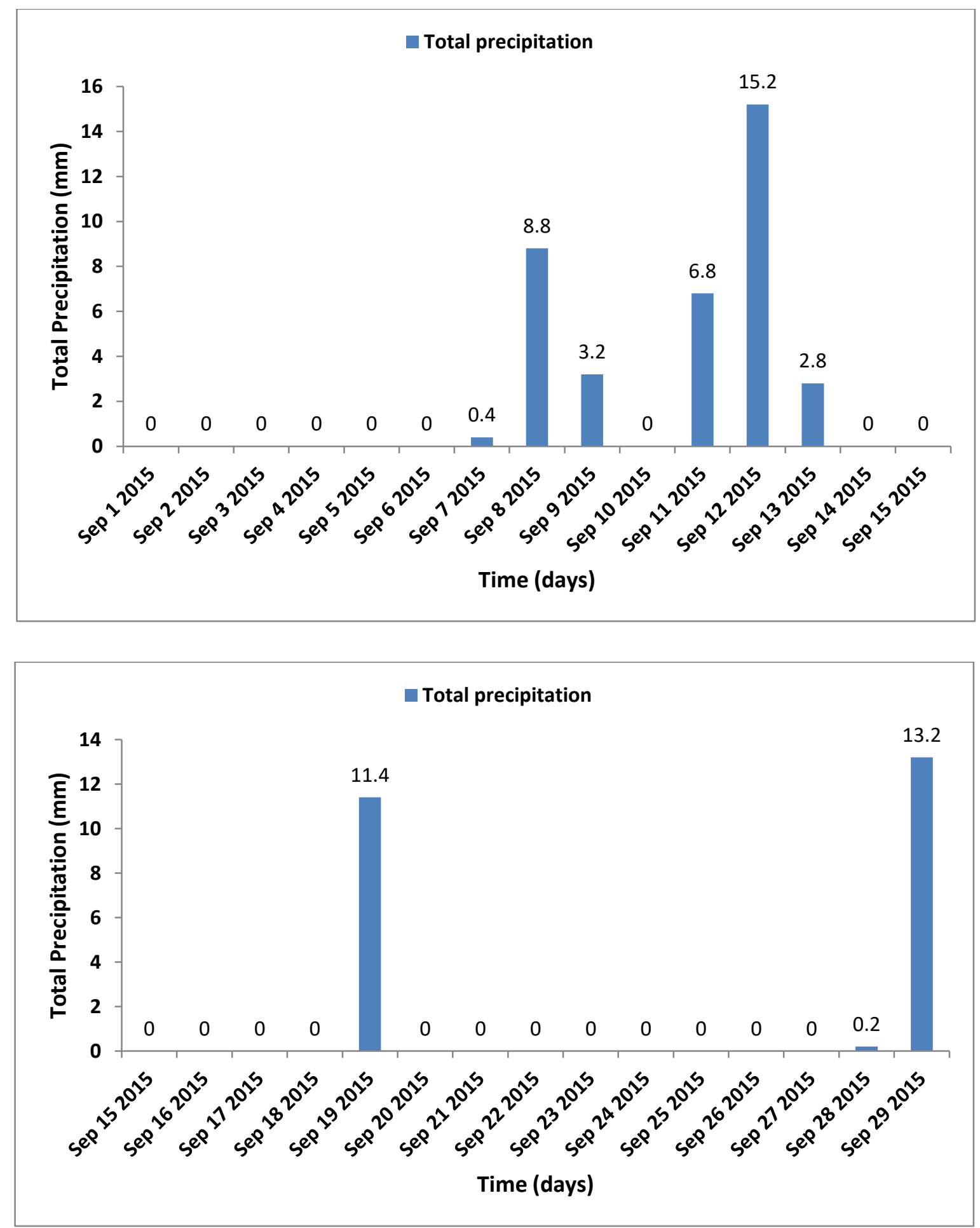

$\operatorname{cclxxx}$ 

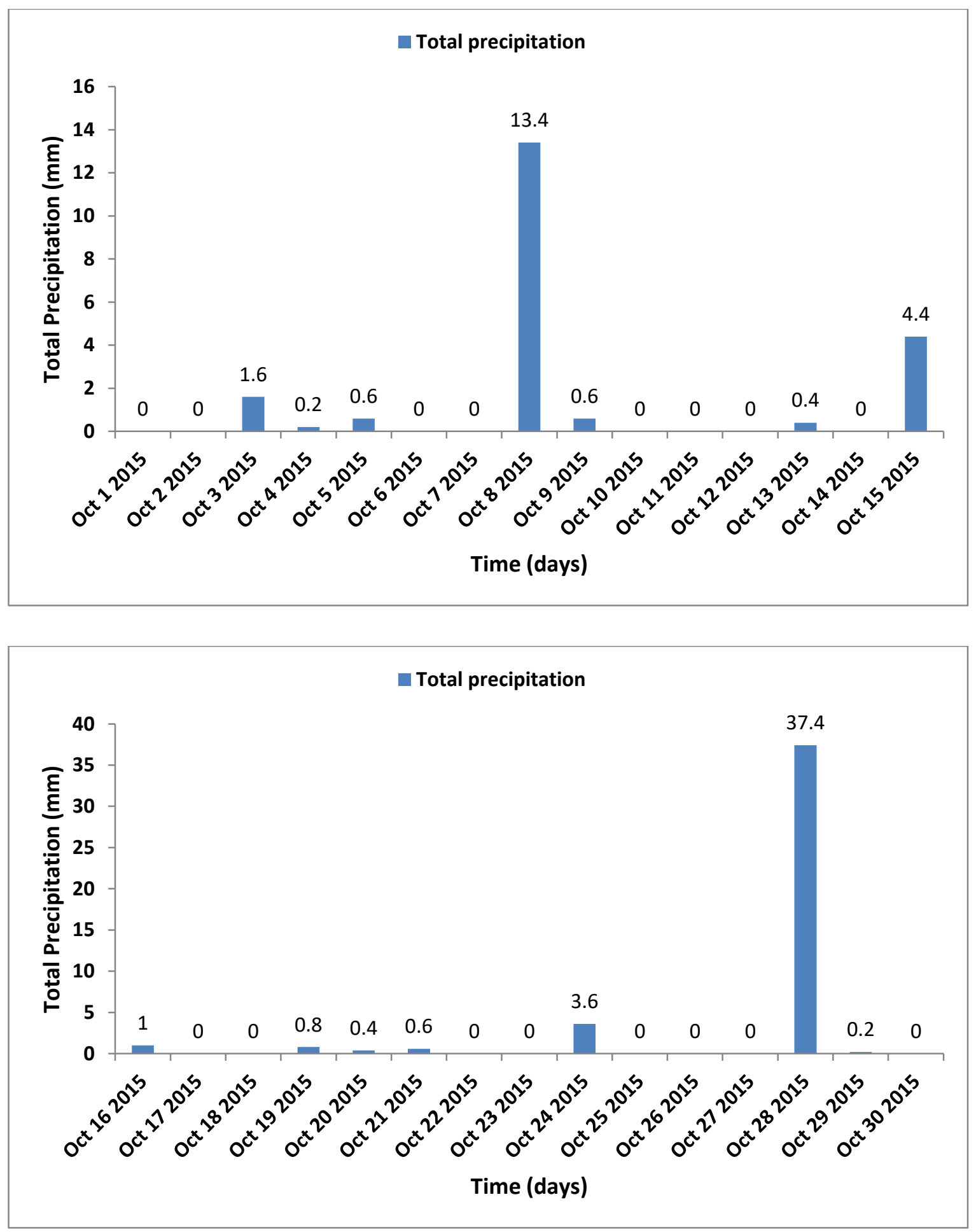

cclxxxi 

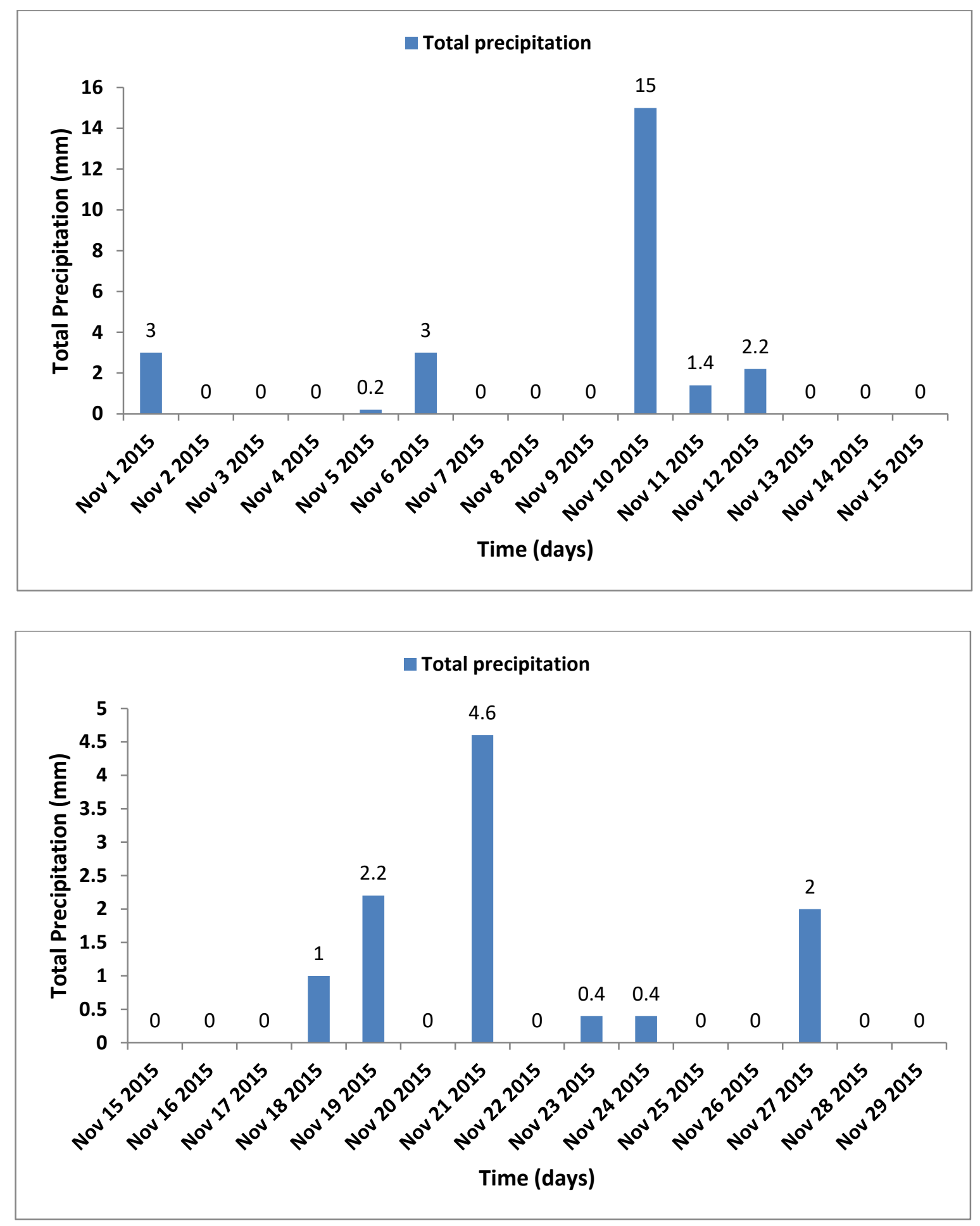

cclxxxii 

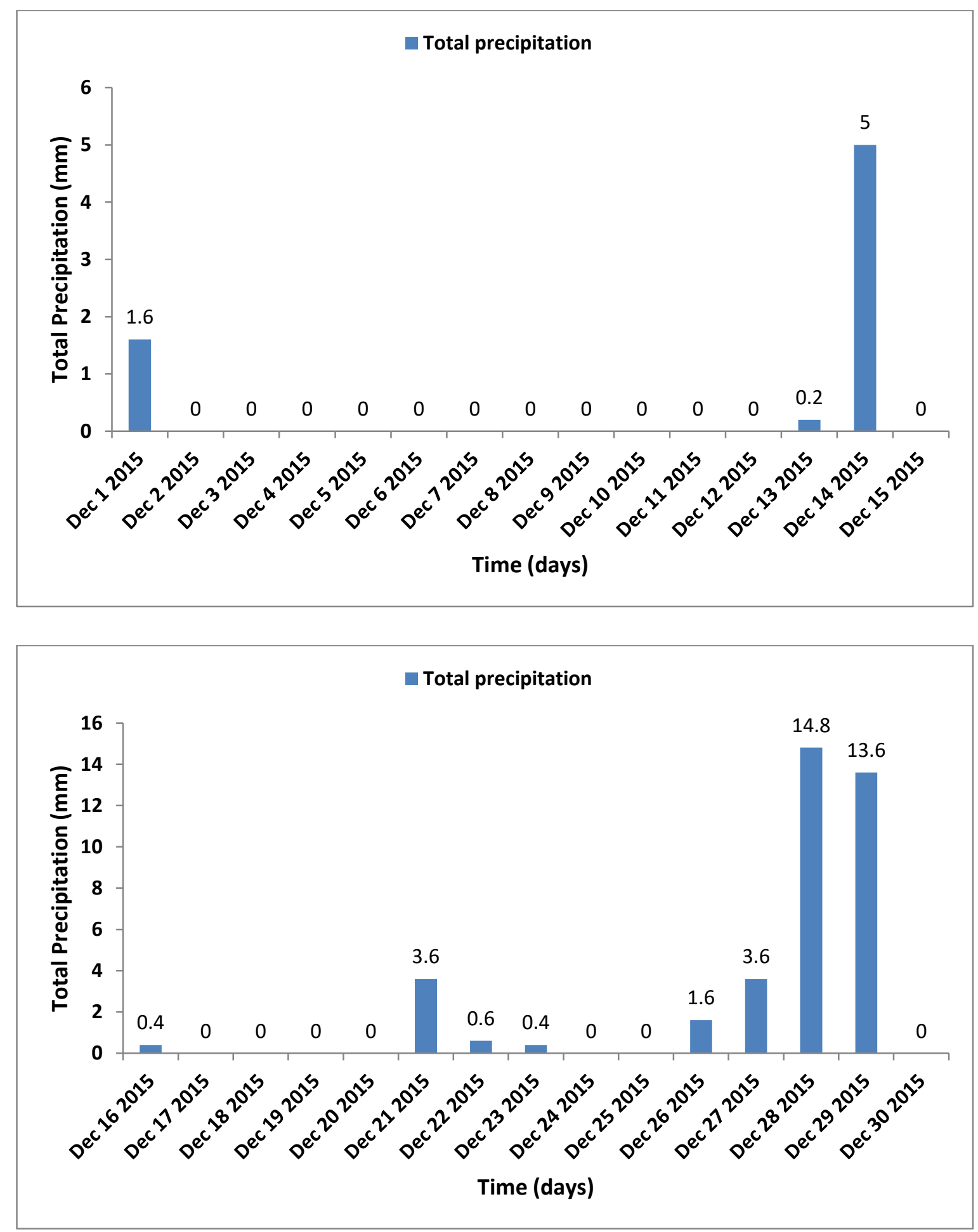

cclxxxiii 

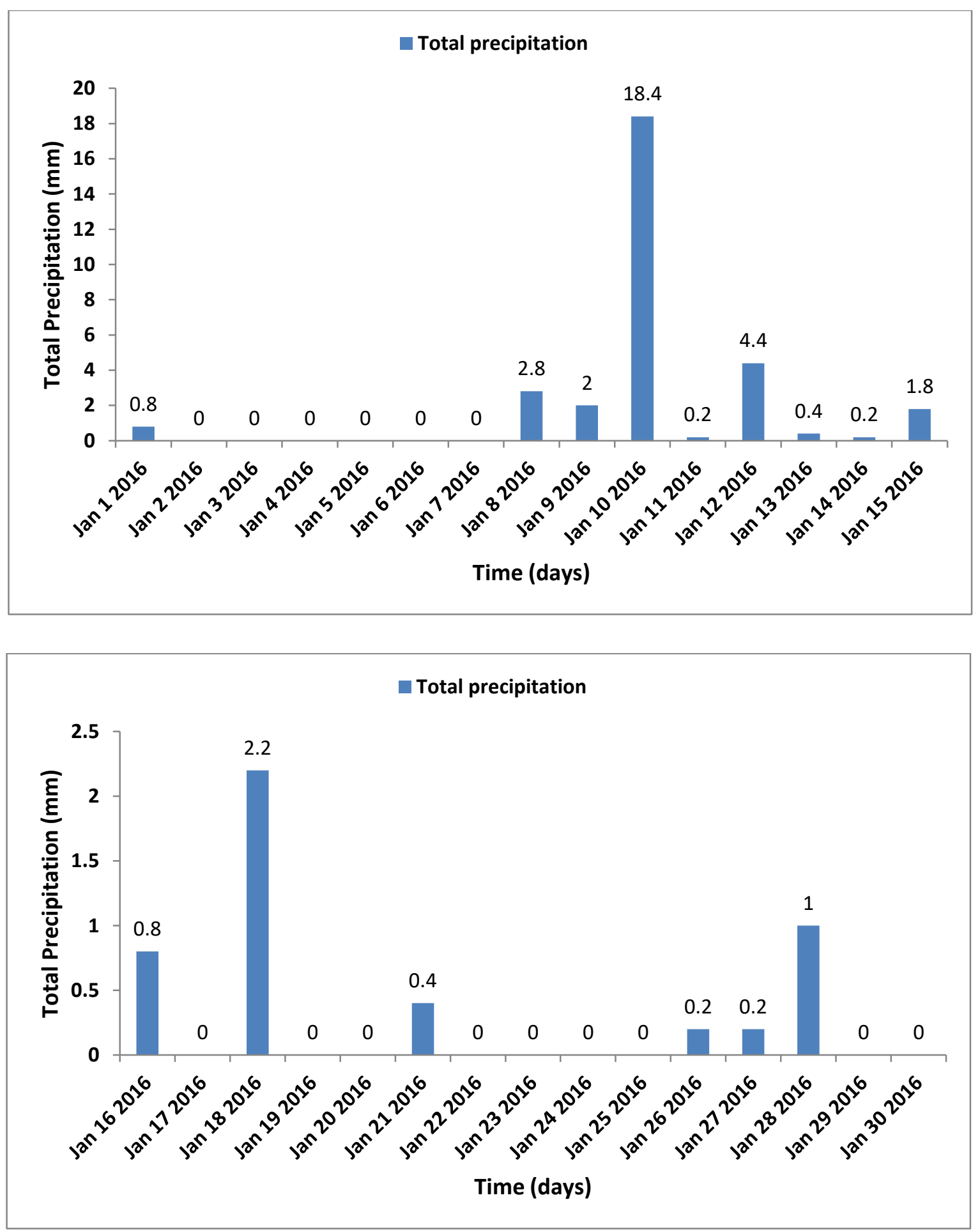

cclxxxiv 

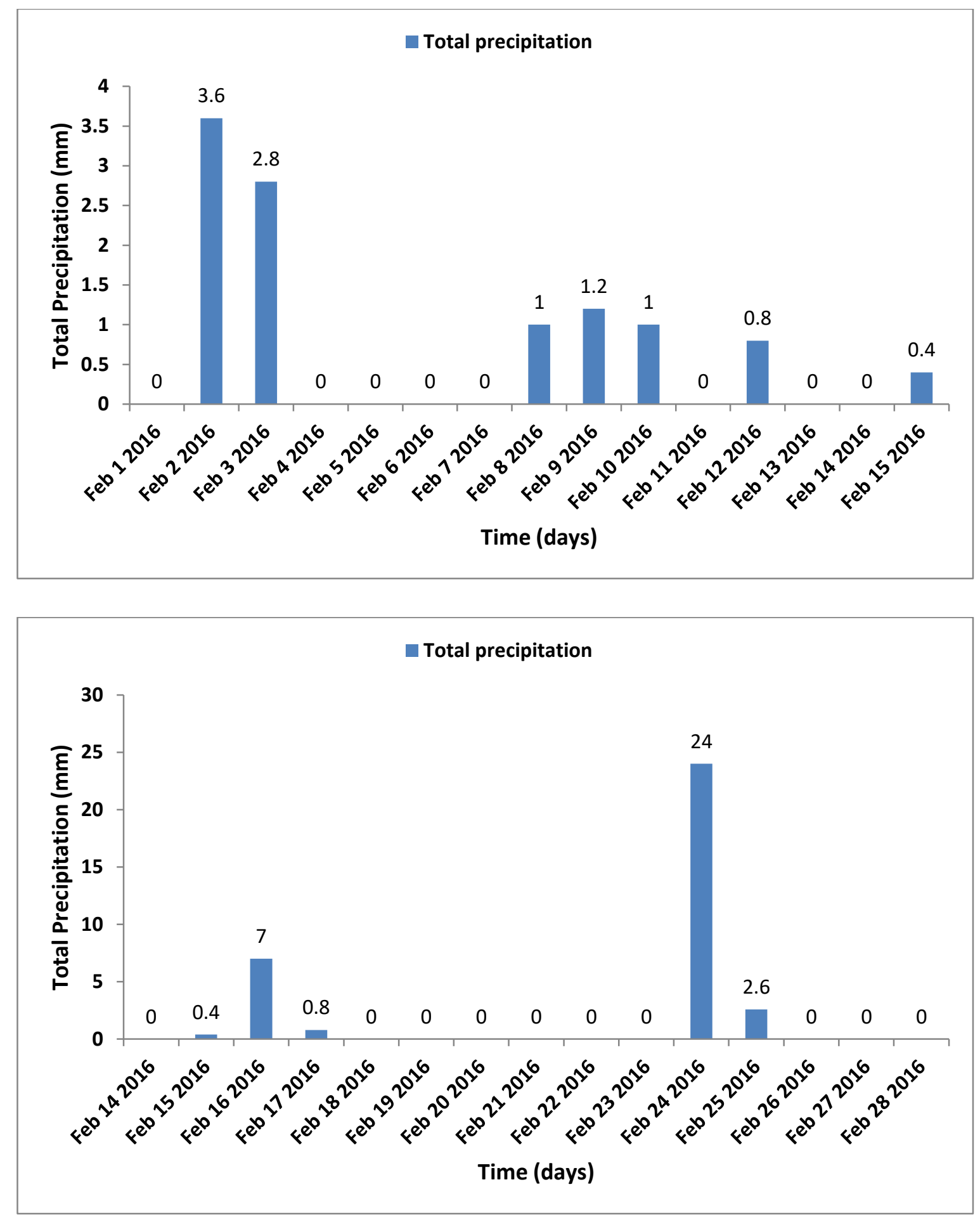

$\operatorname{cclxxxv}$ 

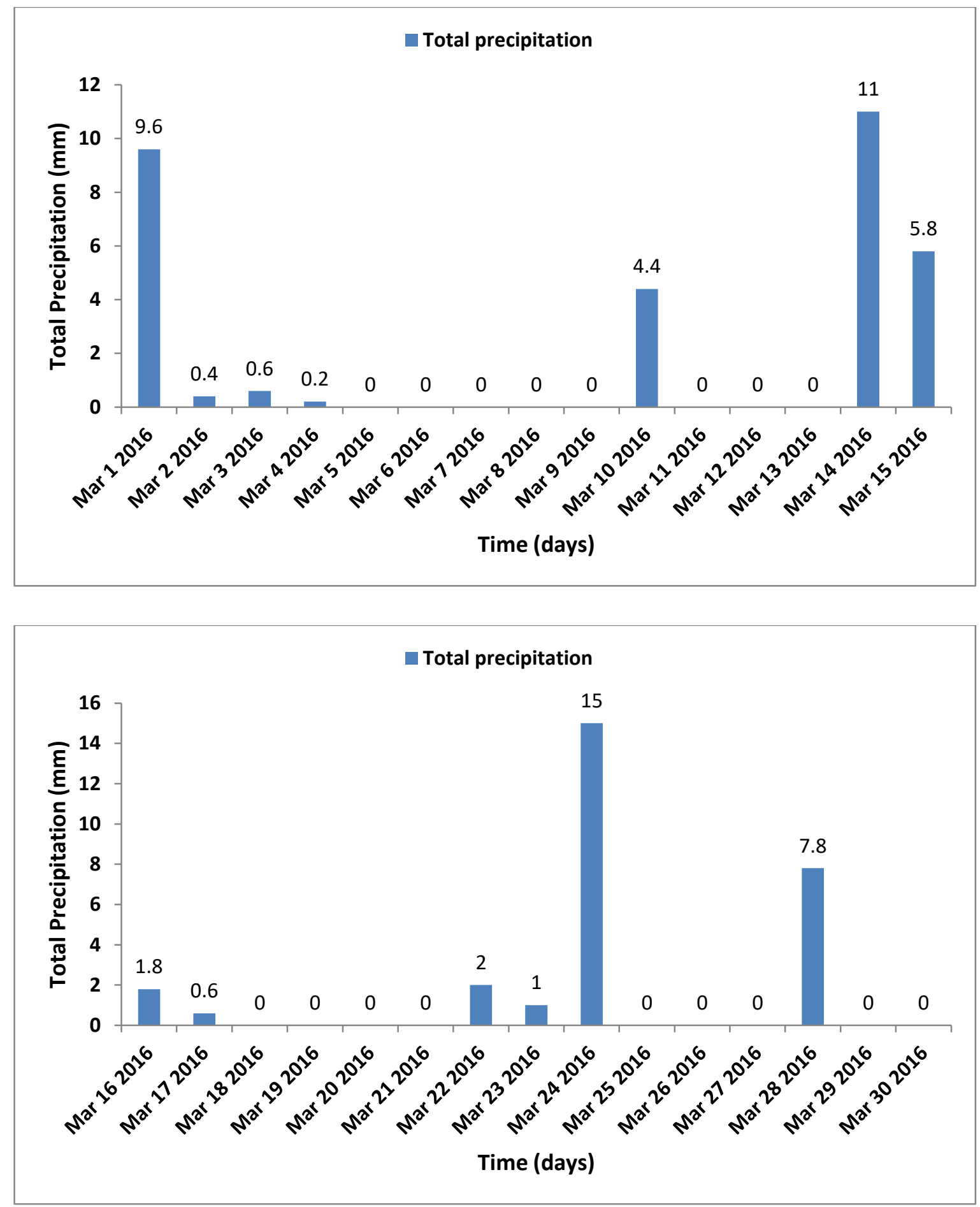

cclxxxvi 

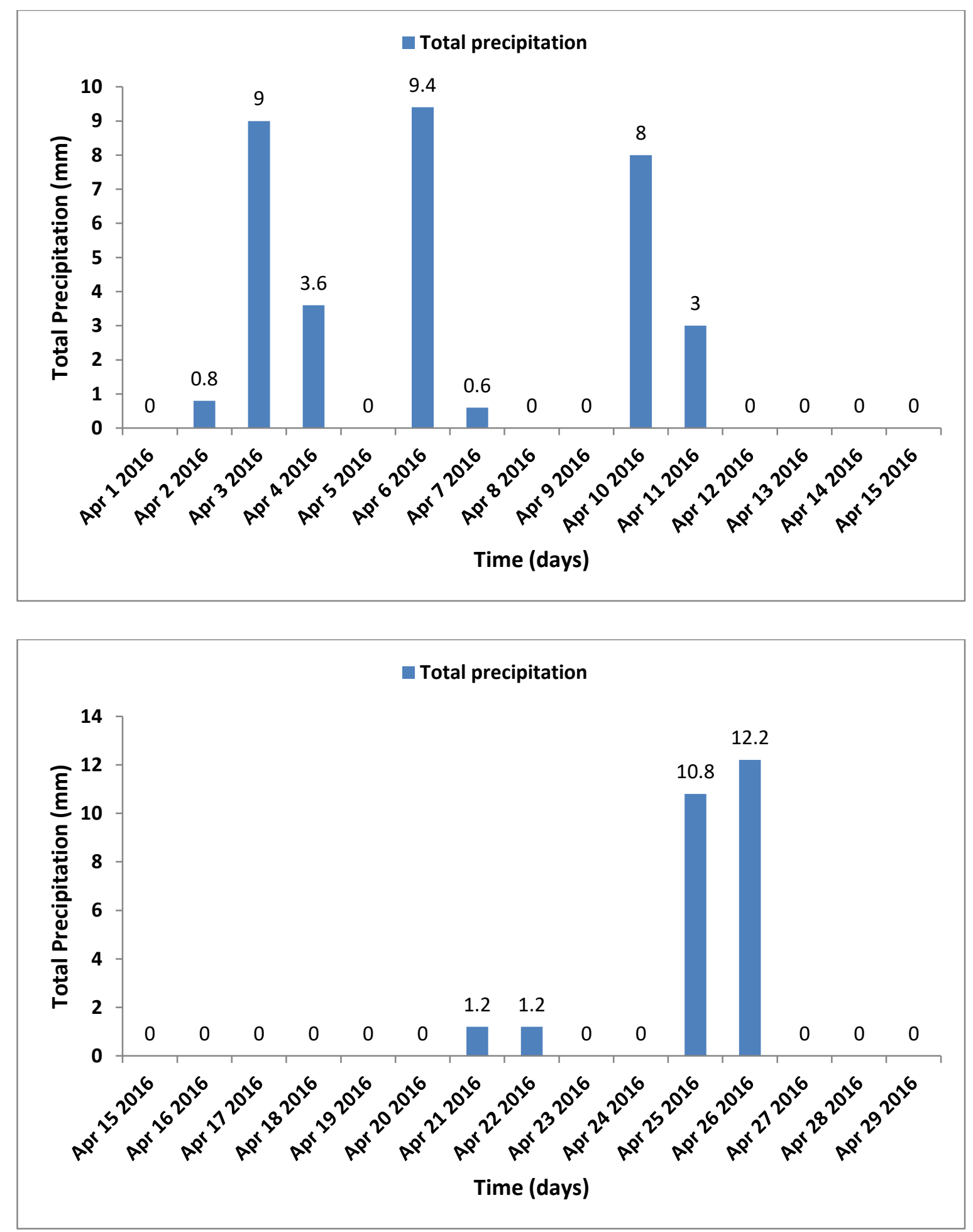

cclxxxvii 

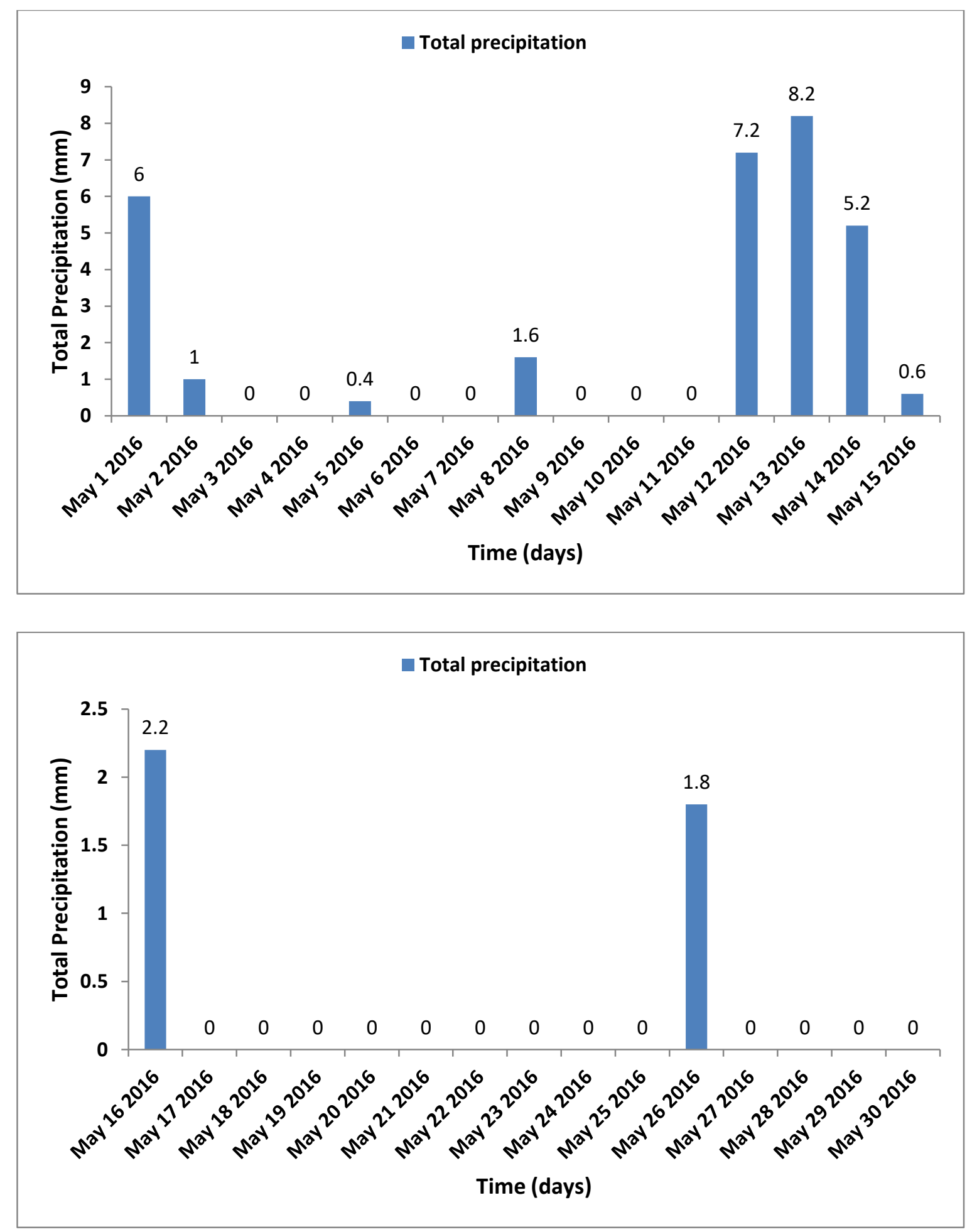

cclxxxviii 
Appendix B

Chapter 4 raw data 
Weight loss of MgCO3 powder samples after kiln burning process

\begin{tabular}{|c|c|c|c|c|c|c|}
\hline $\begin{array}{c}\text { Weight before } \\
\text { burning } \\
\text { (MgCO3) } \\
\text { grams (a) }\end{array}$ & $\begin{array}{c}\text { Kiln } \\
\text { Burning } \\
\text { Temp. }\end{array}$ & $\begin{array}{c}\text { Holding } \\
\text { Time } \\
\text { inside } \\
\text { Kiln }\end{array}$ & $\begin{array}{l}\text { particle size } \\
\text { of sample }\end{array}$ & $\begin{array}{l}\text { Weight after } \\
\text { burning } \\
(\text { MgO) } \\
\text { grams (b) }\end{array}$ & $\begin{array}{c}\% \text { of } \\
\text { remaining } \\
\text { weight of } \\
\mathrm{CO}_{2} \\
\left(\mathrm{c}=\mathrm{b} / \mathbf{a}^{*} * 100\right)\end{array}$ & $\begin{array}{c}\% \text { of weight } \\
\text { loss of } \mathrm{CO}_{2} \\
(100-\mathrm{c})\end{array}$ \\
\hline 450 & $750 \mathrm{C}^{\circ}$ & 1 & 100 & 344 & 76.44 & 23.56 \\
\hline 450 & $750 \mathrm{C}^{\circ}$ & 2 & 100 & 301.35 & 66.97 & 33.03 \\
\hline 450 & $750 \mathrm{C}^{\circ}$ & 4 & 100 & 253.37 & 56.30 & 43.70 \\
\hline 450 & $750 \mathrm{C}^{\circ}$ & 1 & 45 & 340.75 & 75.72 & 24.28 \\
\hline 450 & $750 \mathrm{C}^{\circ}$ & 2 & 45 & 305.5 & 67.89 & 32.11 \\
\hline 450 & $750 \mathrm{C}^{\circ}$ & 4 & 45 & 256.96 & 57.10 & 42.90 \\
\hline 450 & $900 \mathrm{C}^{\circ}$ & 1 & 100 & 294.68 & 65.48 & 34.52 \\
\hline 450 & $900 \mathrm{C}^{\circ}$ & 2 & 100 & 253.9 & 56.42 & 43.58 \\
\hline 450 & $900 \mathrm{C}^{\circ}$ & 4 & 100 & 228.03 & 50.67 & 49.33 \\
\hline 450 & $900 \mathrm{C}^{\circ}$ & 1 & 45 & 305.6 & 67.91 & 32.09 \\
\hline 450 & $900 \mathrm{C}^{\circ}$ & 2 & 45 & 251.72 & 55.94 & 44.06 \\
\hline 450 & $900 \mathrm{C}^{\circ}$ & 4 & 45 & 224.03 & 49.78 & 50.22 \\
\hline 450 & $1050 \mathrm{C}^{\circ}$ & 1 & 100 & 264.55 & $\begin{array}{l}58.79 \\
\end{array}$ & 41.21 \\
\hline 450 & $1050 \mathrm{C}^{\circ}$ & 2 & 100 & 230.08 & 51.13 & 48.87 \\
\hline 450 & $1050 \mathrm{C}^{\circ}$ & 4 & 100 & 225.9 & 50.20 & 49.80 \\
\hline 450 & $1050 \mathrm{C}^{\circ}$ & 1 & 45 & 251.34 & 55.85 & 44.15 \\
\hline 450 & $1050 \mathrm{C}^{\circ}$ & 2 & 45 & 228.13 & 50.70 & 49.30 \\
\hline 450 & $1050 \mathrm{C}^{\circ}$ & 4 & 45 & 226.06 & 50.24 & 49.76 \\
\hline 450 & $1200 \mathrm{C}^{\circ}$ & 1 & 100 & 233.73 & 51.94 & 48.06 \\
\hline 450 & $1200 \mathrm{C}^{\circ}$ & 2 & 100 & 228.77 & 50.84 & 49.16 \\
\hline 450 & $1200 \mathrm{C}^{\circ}$ & 4 & 100 & 226.61 & 50.36 & 49.64 \\
\hline 450 & $1200 \mathrm{C}^{\circ}$ & 1 & 45 & 224.66 & 49.92 & 50.08 \\
\hline 450 & $1200 \mathrm{C}^{\circ}$ & 2 & 45 & 223.57 & 49.68 & 50.32 \\
\hline 450 & $1200 \mathrm{C}^{\circ}$ & 4 & 45 & 223.04 & 49.56 & 50.44 \\
\hline
\end{tabular}




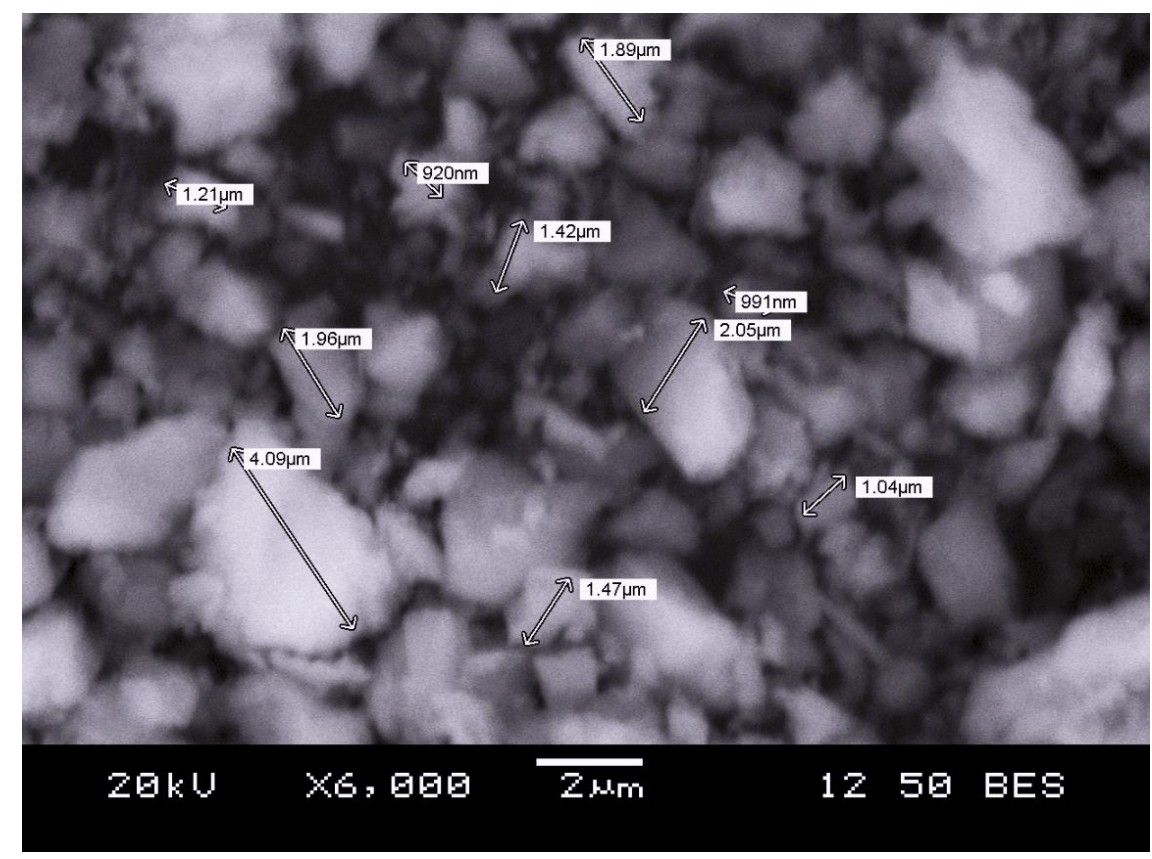

$750^{\circ} \mathrm{C}-45 \mu \mathrm{m}-2$ hours particle size

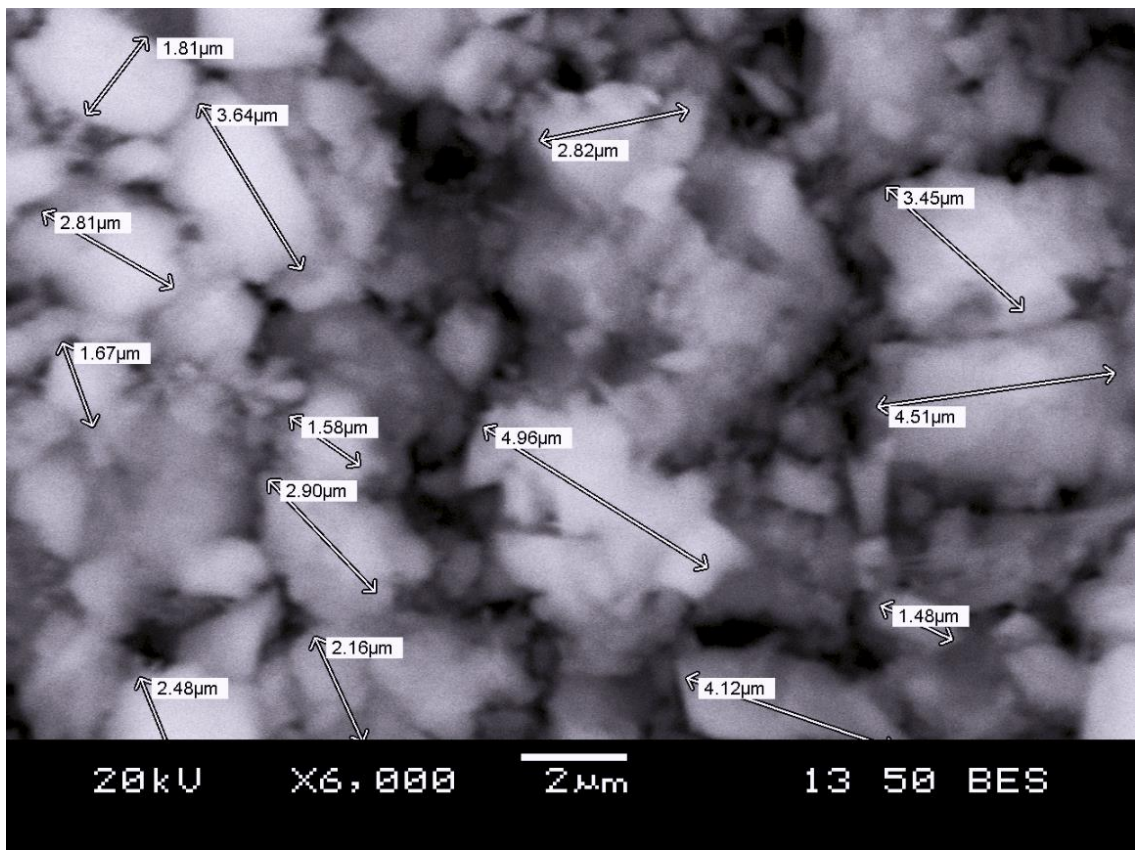

$900^{\circ} \mathrm{C}-45 \mu \mathrm{m}-2$ hours particle size

$\operatorname{ccxci}$ 


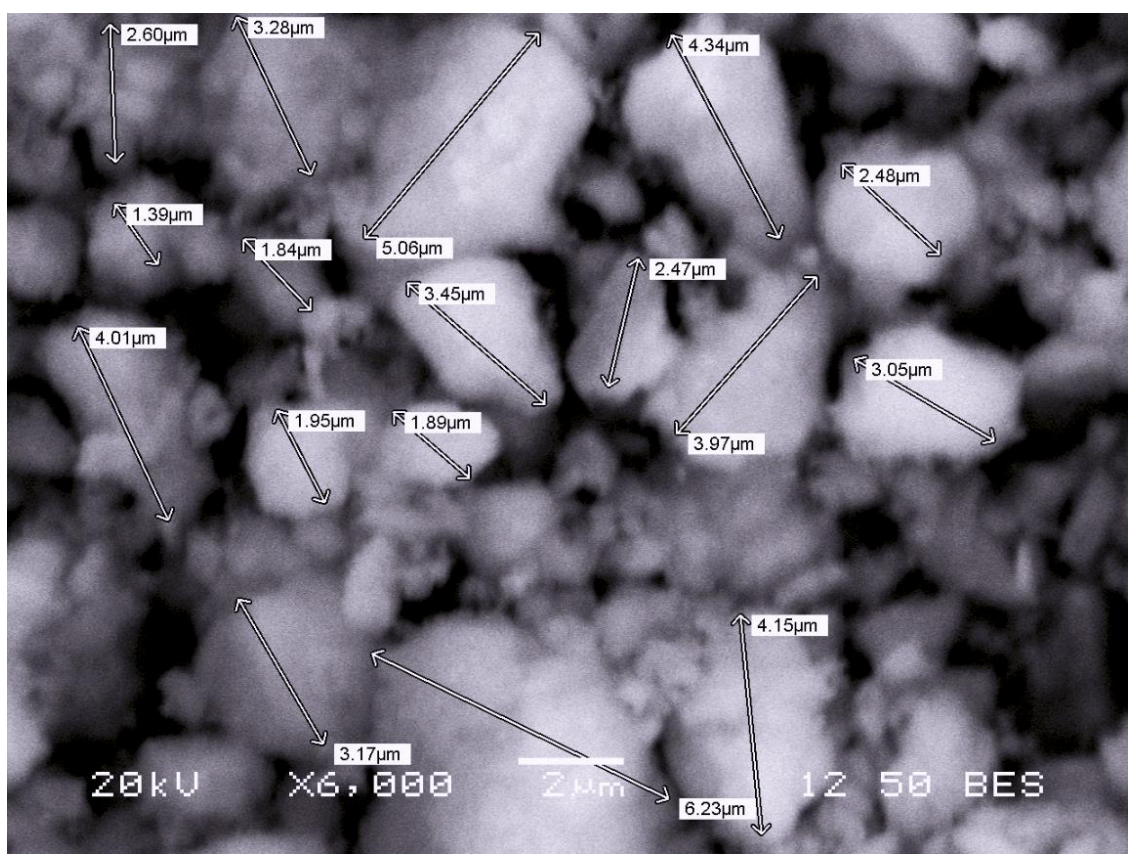

$1050^{\circ} \mathrm{C}-45 \mu \mathrm{m}-2$ hours particle size

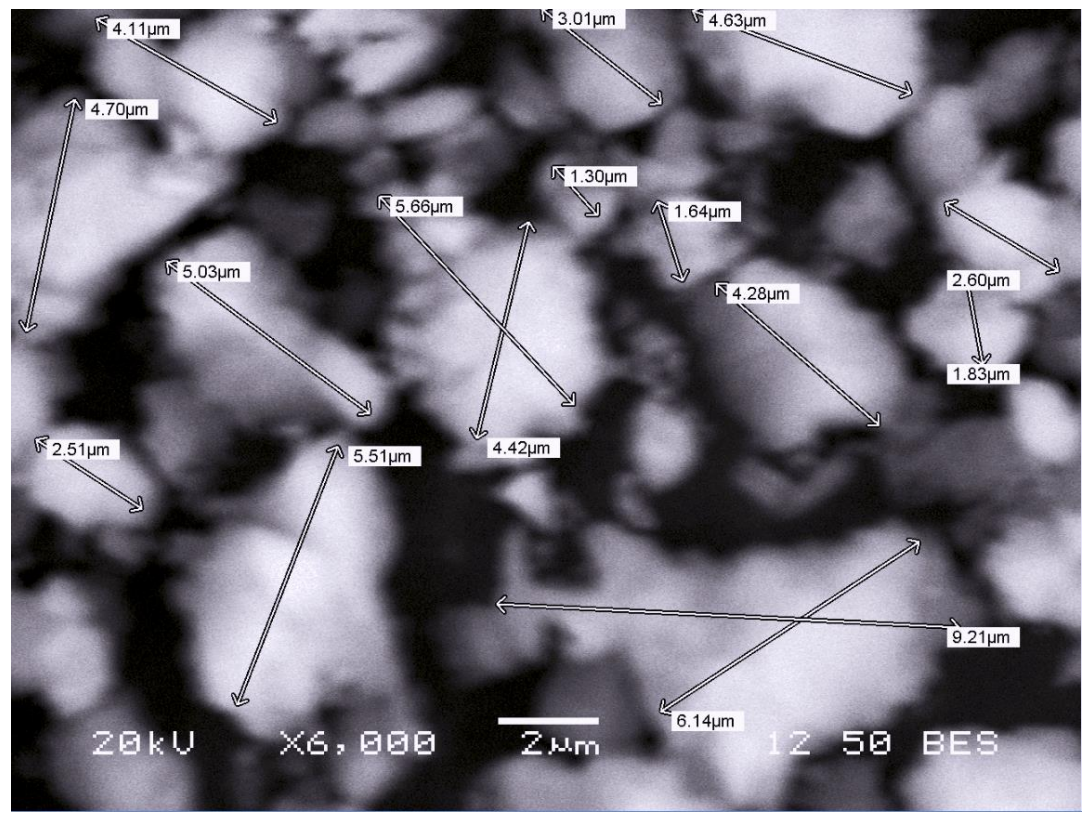

$1200^{\circ} \mathrm{C}-45 \mu \mathrm{m}-2$ hours particle size 


\section{TGA/DSC tests for MgO powder samples after kiln burning process}

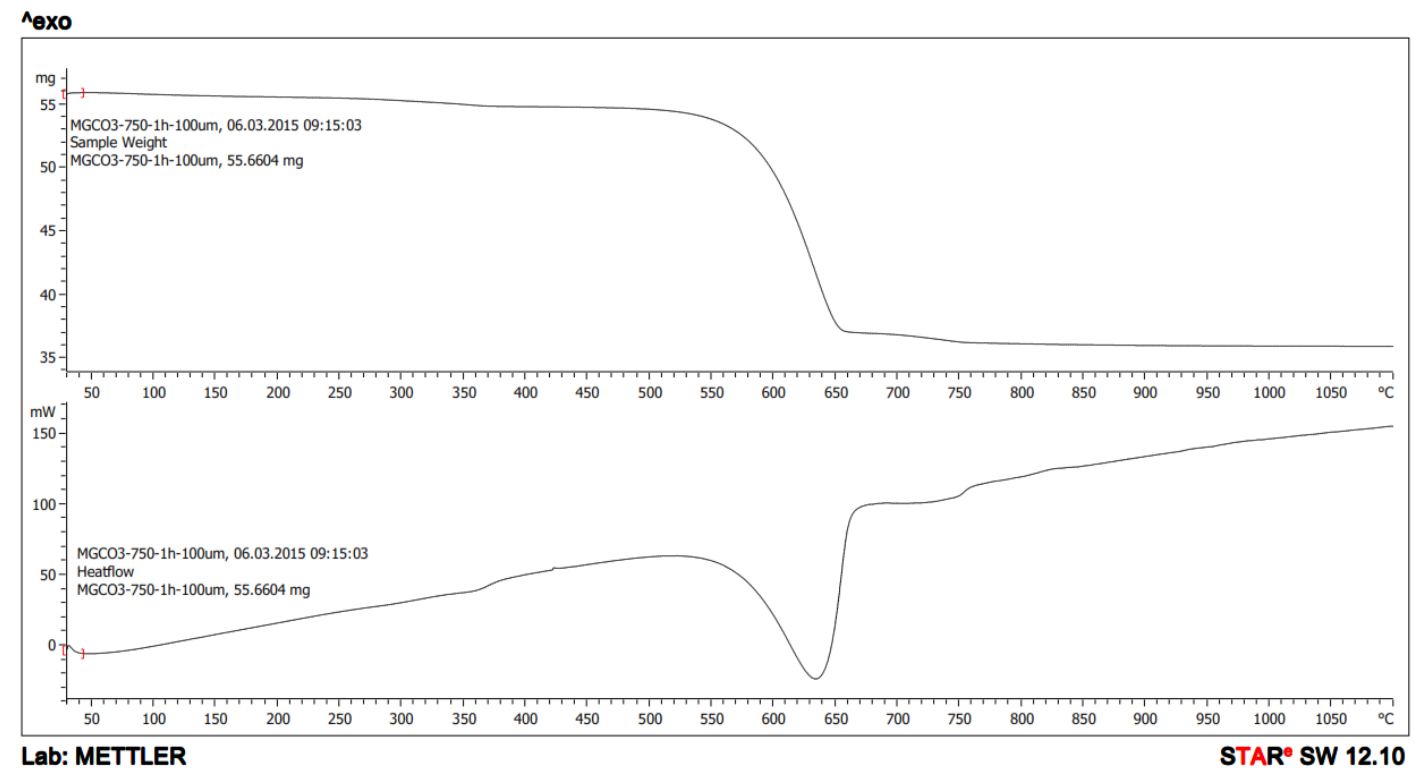

$750^{\circ} \mathrm{C}-100 \mu \mathrm{m}-1$ hour/TGA test

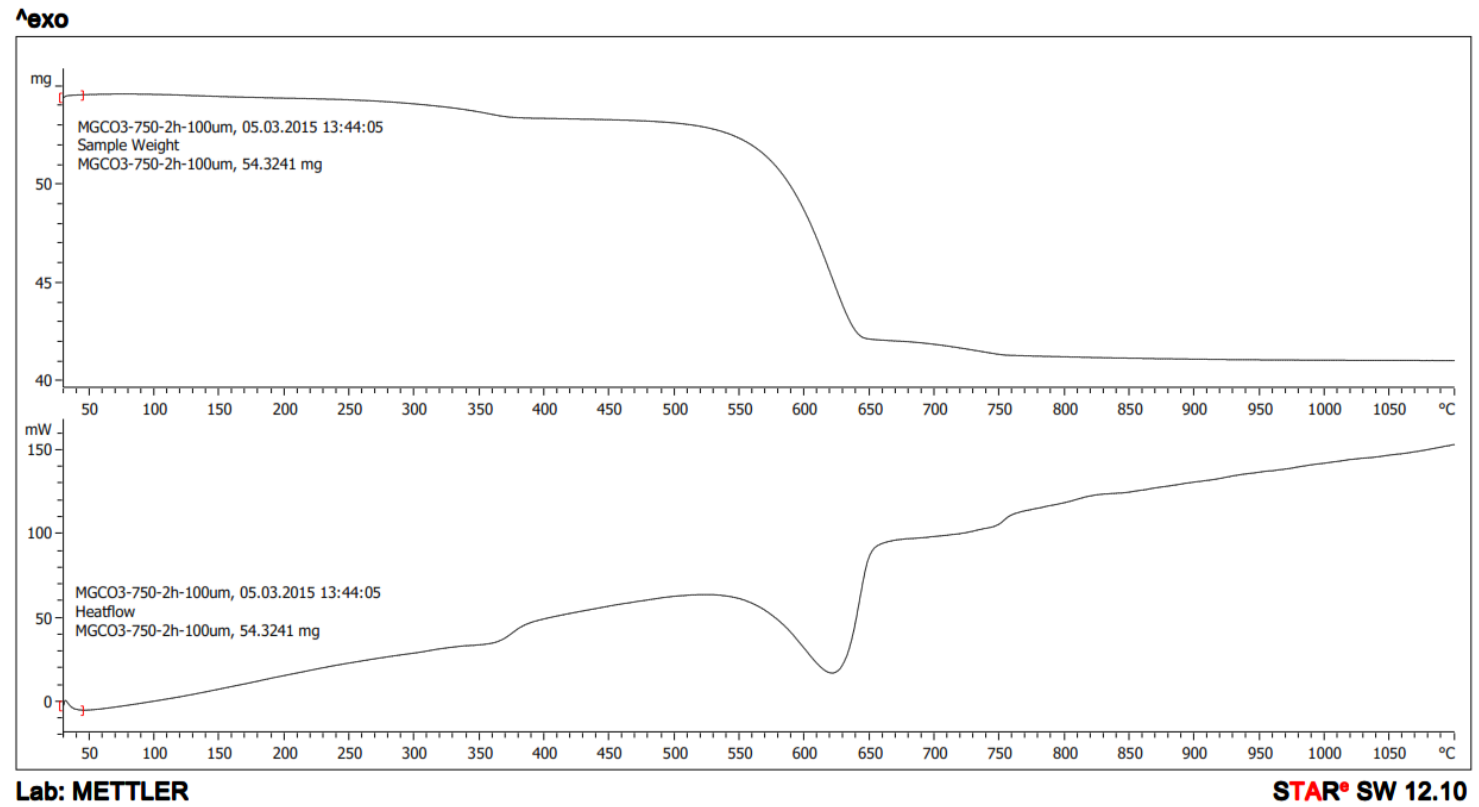

$750^{\circ} \mathrm{C}-100 \mu \mathrm{m}-2 \mathrm{hour} / \mathrm{TGA}$ test

ccxciii 


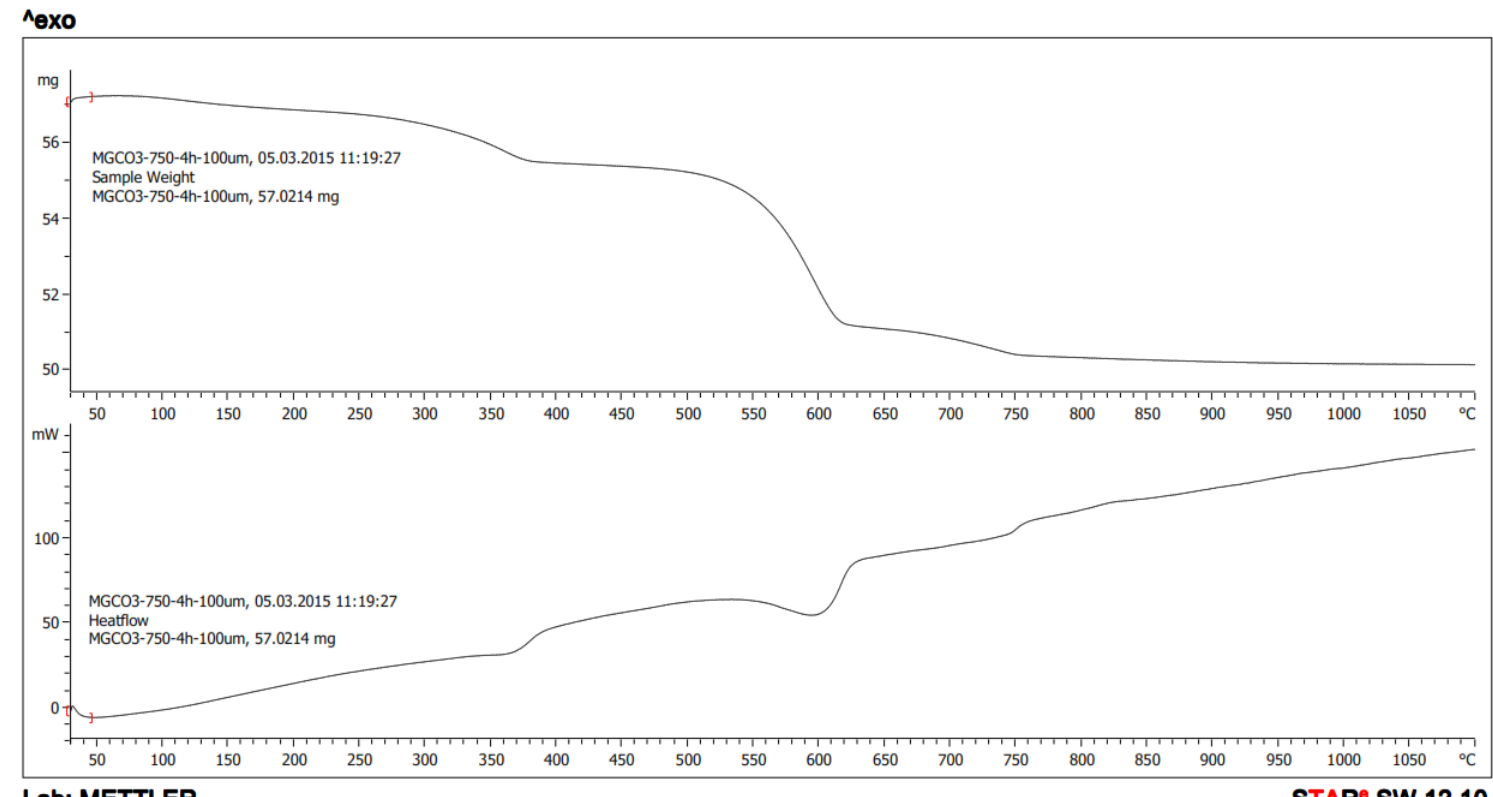

Lab: METTLER

STAR ${ }^{\ominus}$ SW 12.10

\section{$750^{\circ} \mathrm{C}-100 \mu \mathrm{m}-4$ hour/TGA test}

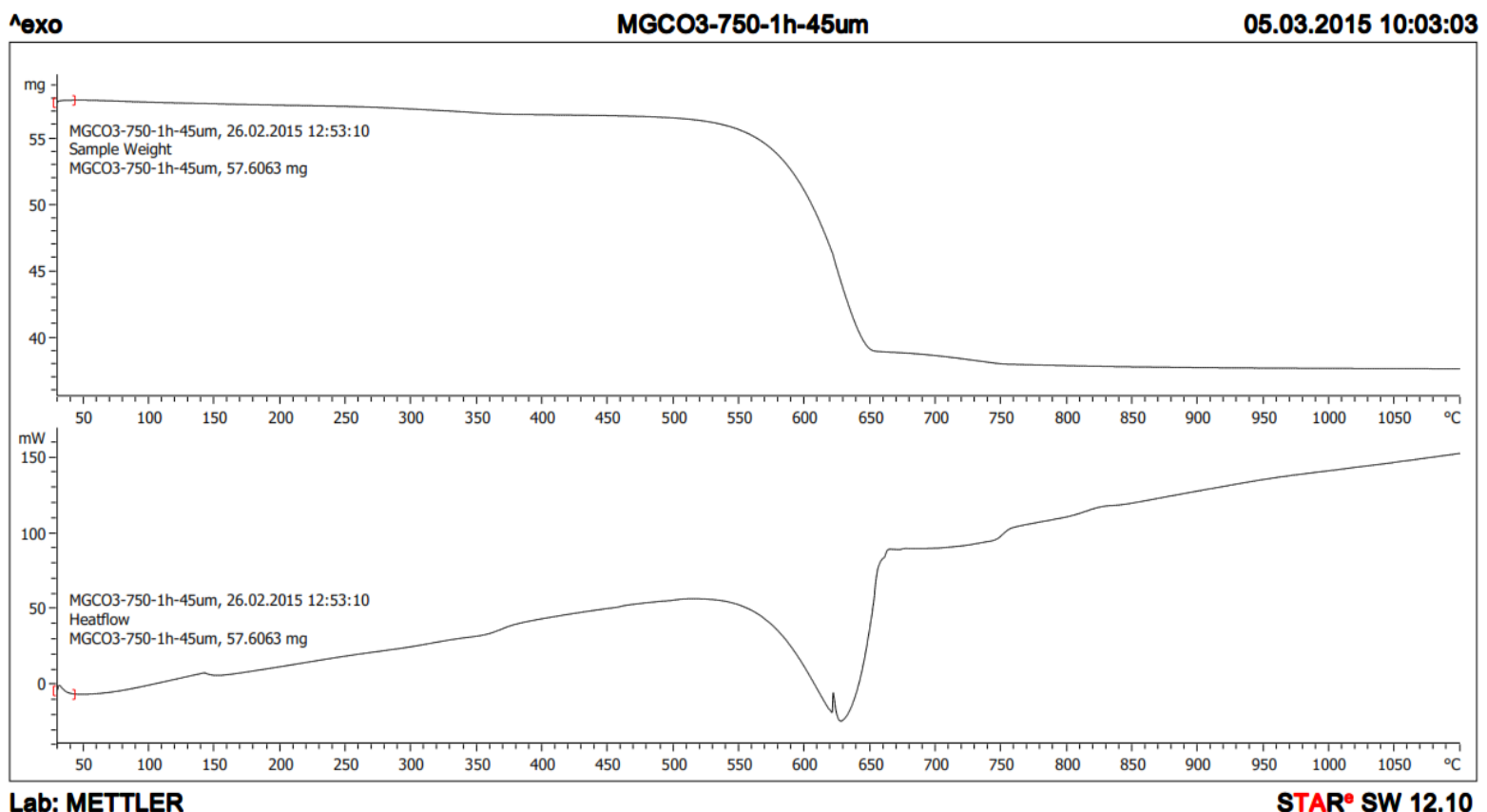

$750^{\circ} \mathrm{C}-45 \mu \mathrm{m}-1$ hour/TGA test

cexciv 


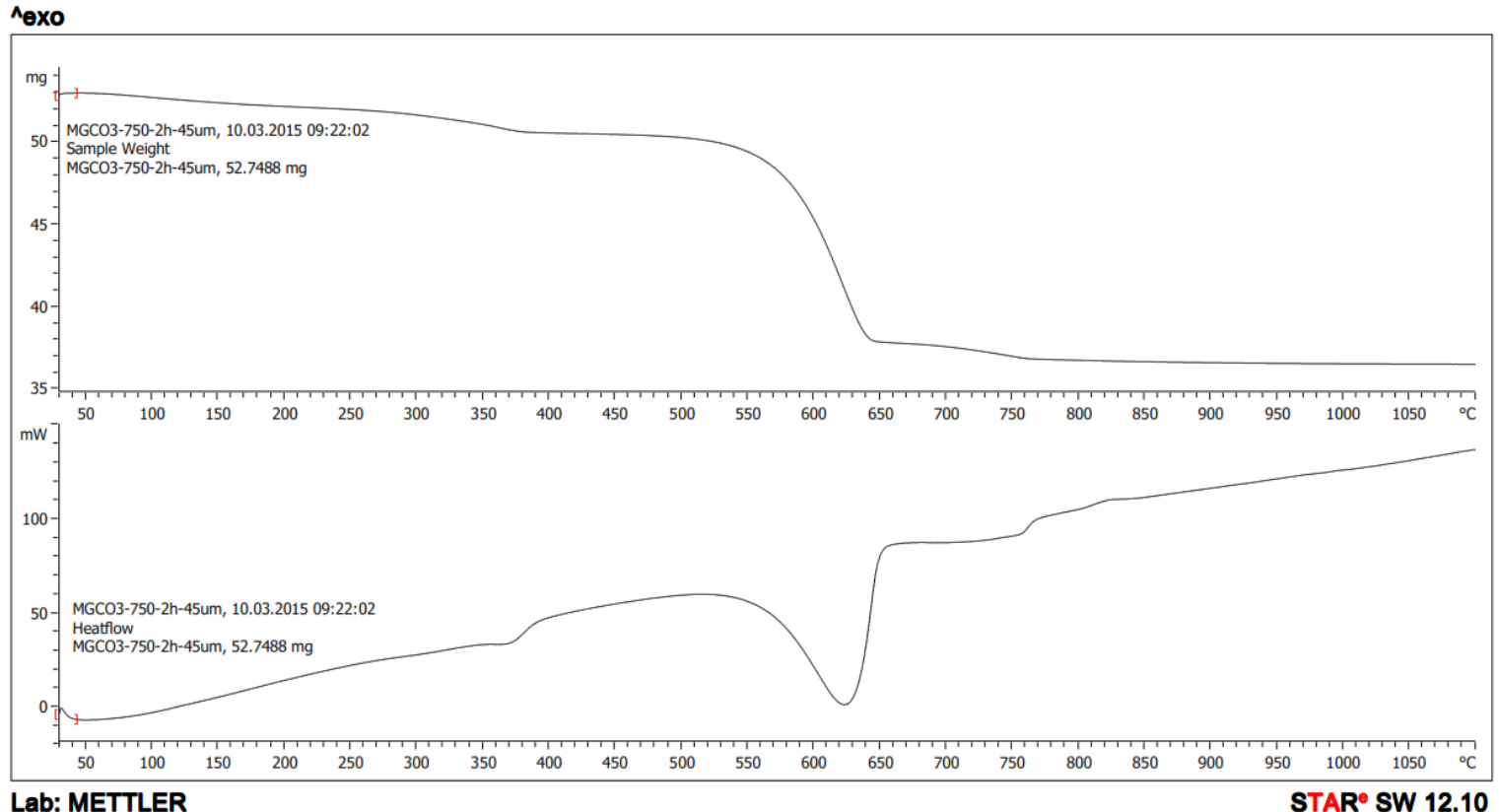

$750^{\circ} \mathrm{C}-45 \mu \mathrm{m}-2 \mathrm{hour} / \mathrm{TGA}$ test

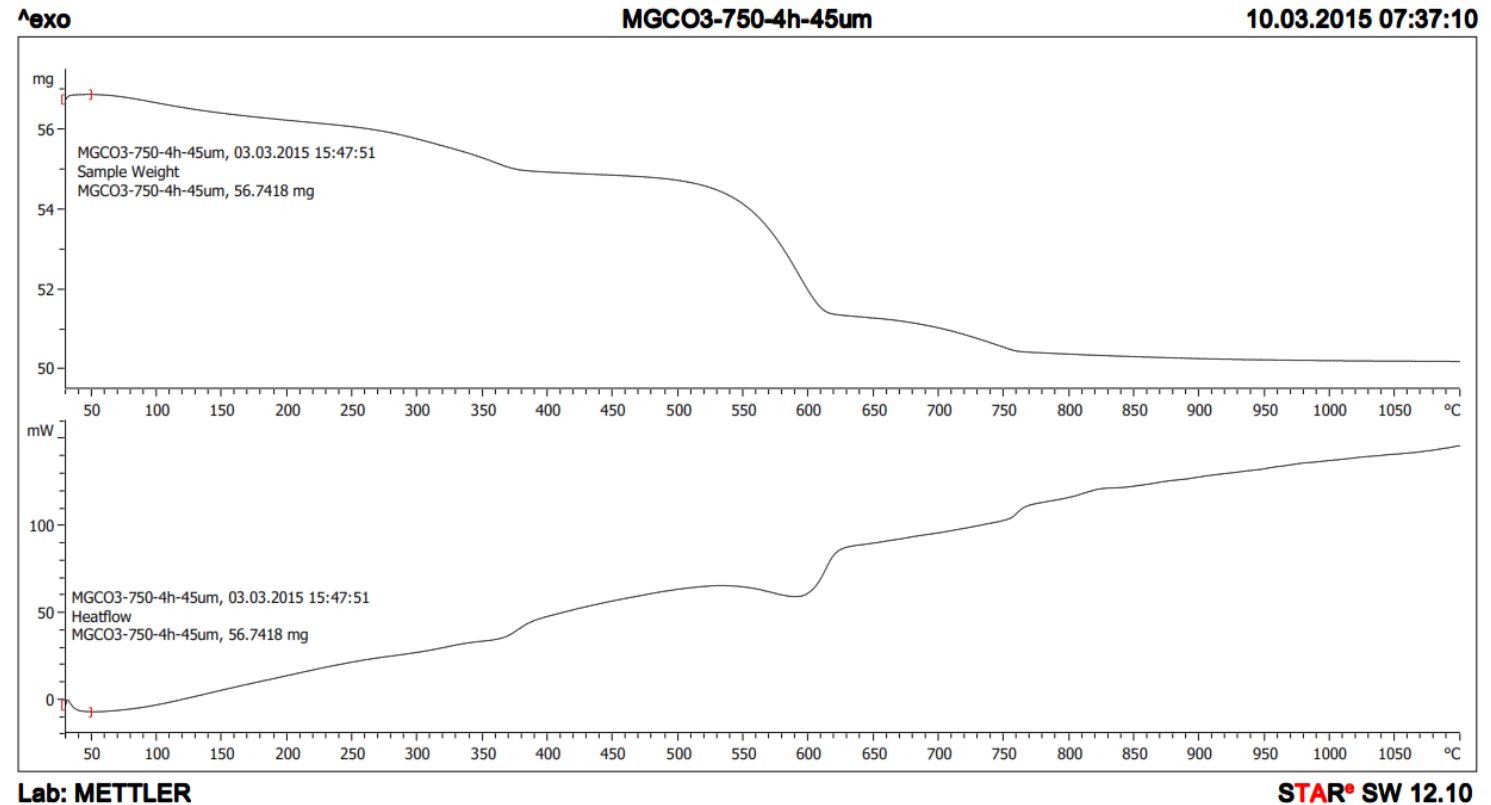

$750^{\circ} \mathrm{C}-45 \mu \mathrm{m}-4$ hour/TGA test 


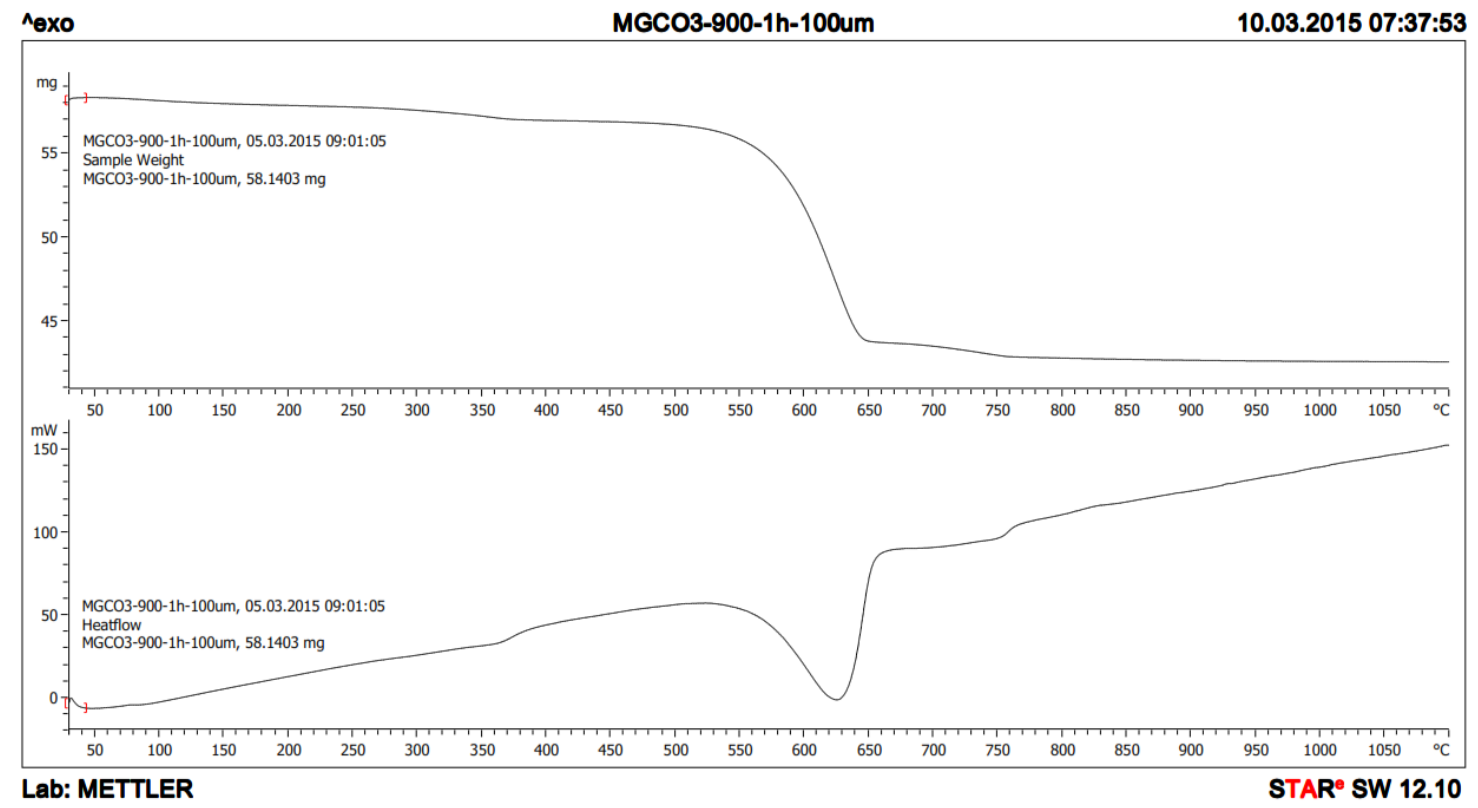

$900^{\circ} \mathrm{C}-100 \mu \mathrm{m}-1$ hour/TGA test

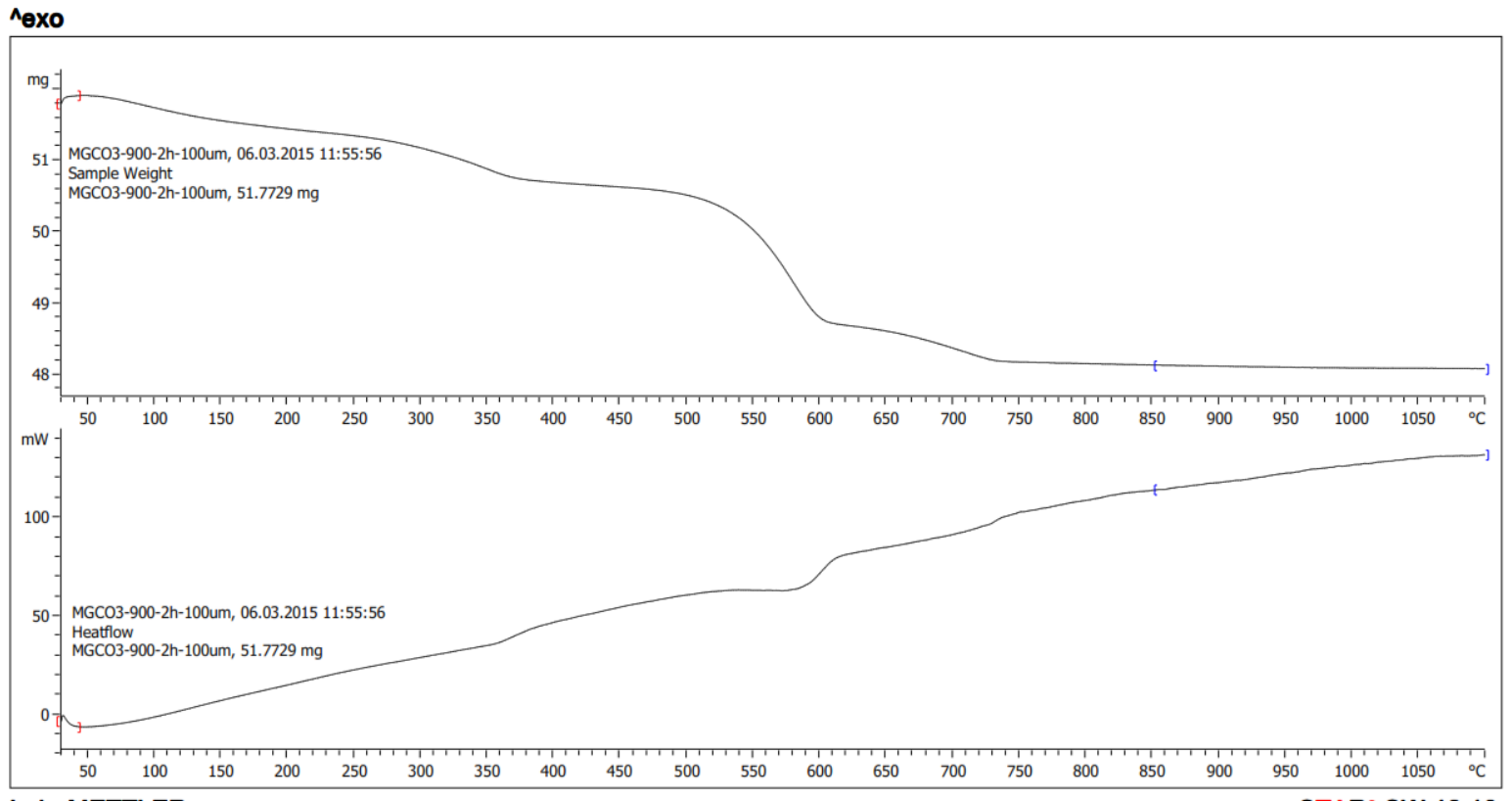

Lab: METTLER

$900^{\circ} \mathrm{C}-100 \mu \mathrm{m}-2$ hour/TGA test 


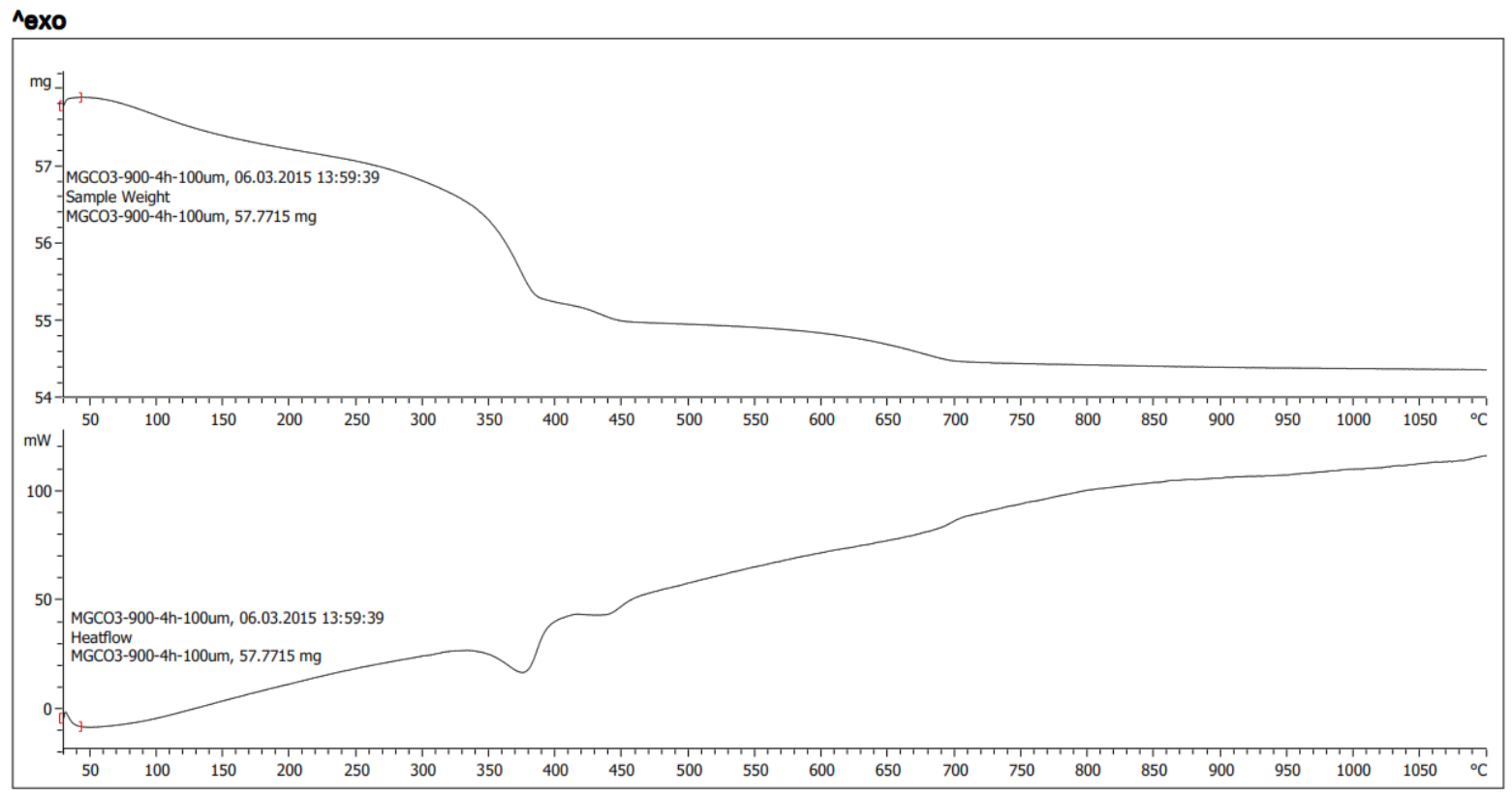

Lab: METTLER

STAR SW 12.10

$900^{\circ} \mathrm{C}-100 \mu \mathrm{m}-4$ hour/TGA test

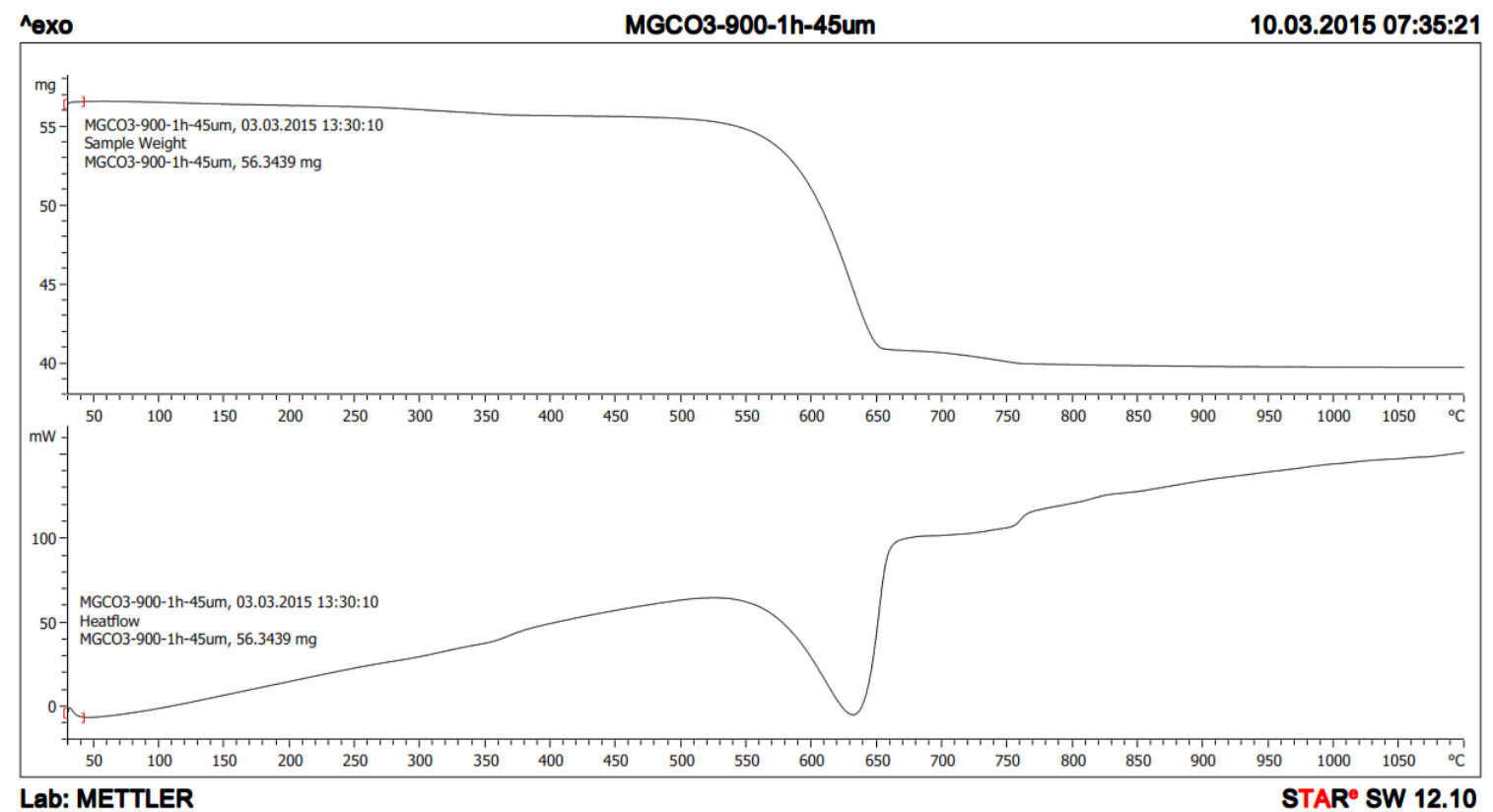

$900^{\circ} \mathrm{C}-45 \mu \mathrm{m}-1$ hour/TGA test

cexcvii 


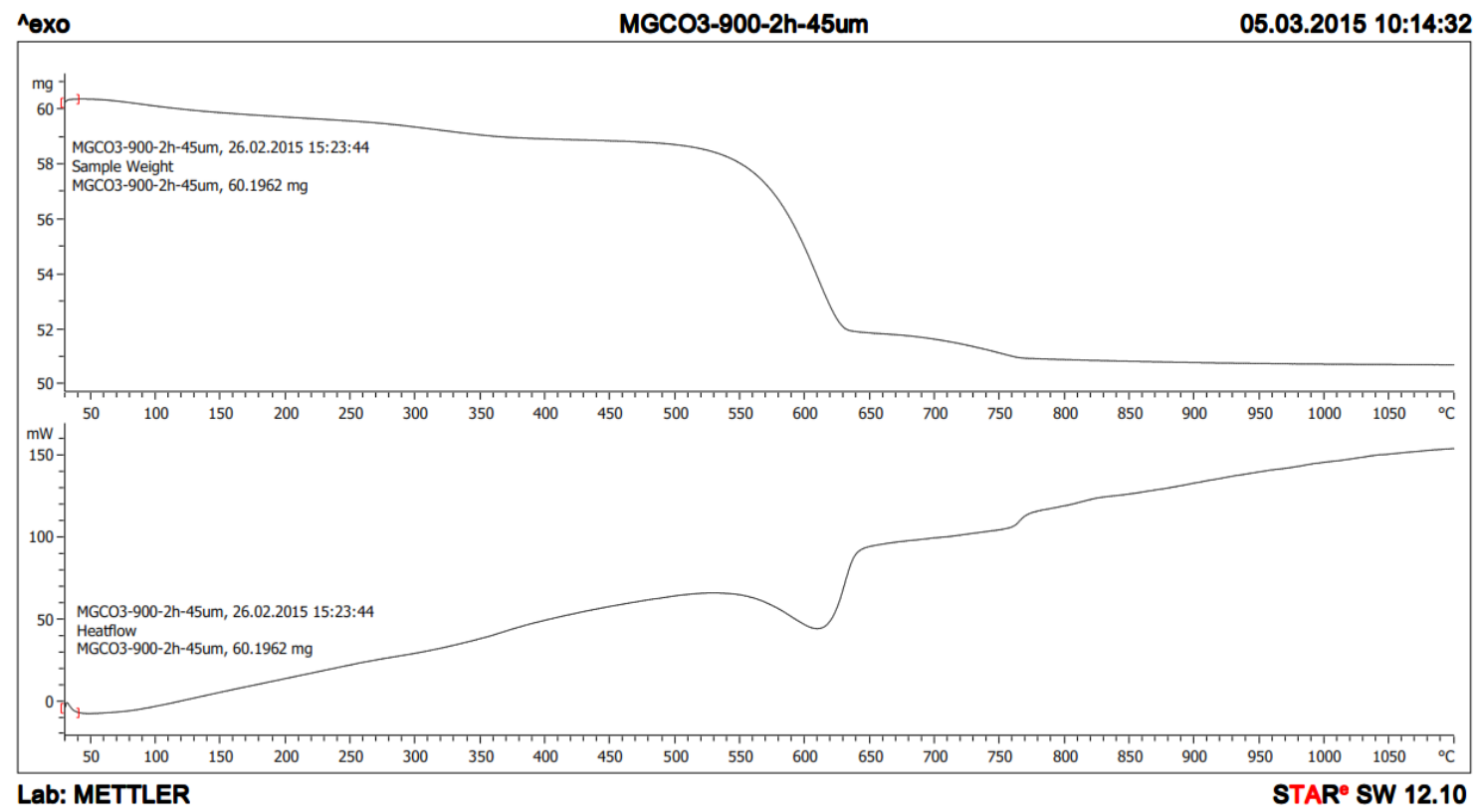

$900^{\circ} \mathrm{C}-45 \mu \mathrm{m}-2 \mathrm{hour} / \mathrm{TGA}$ test

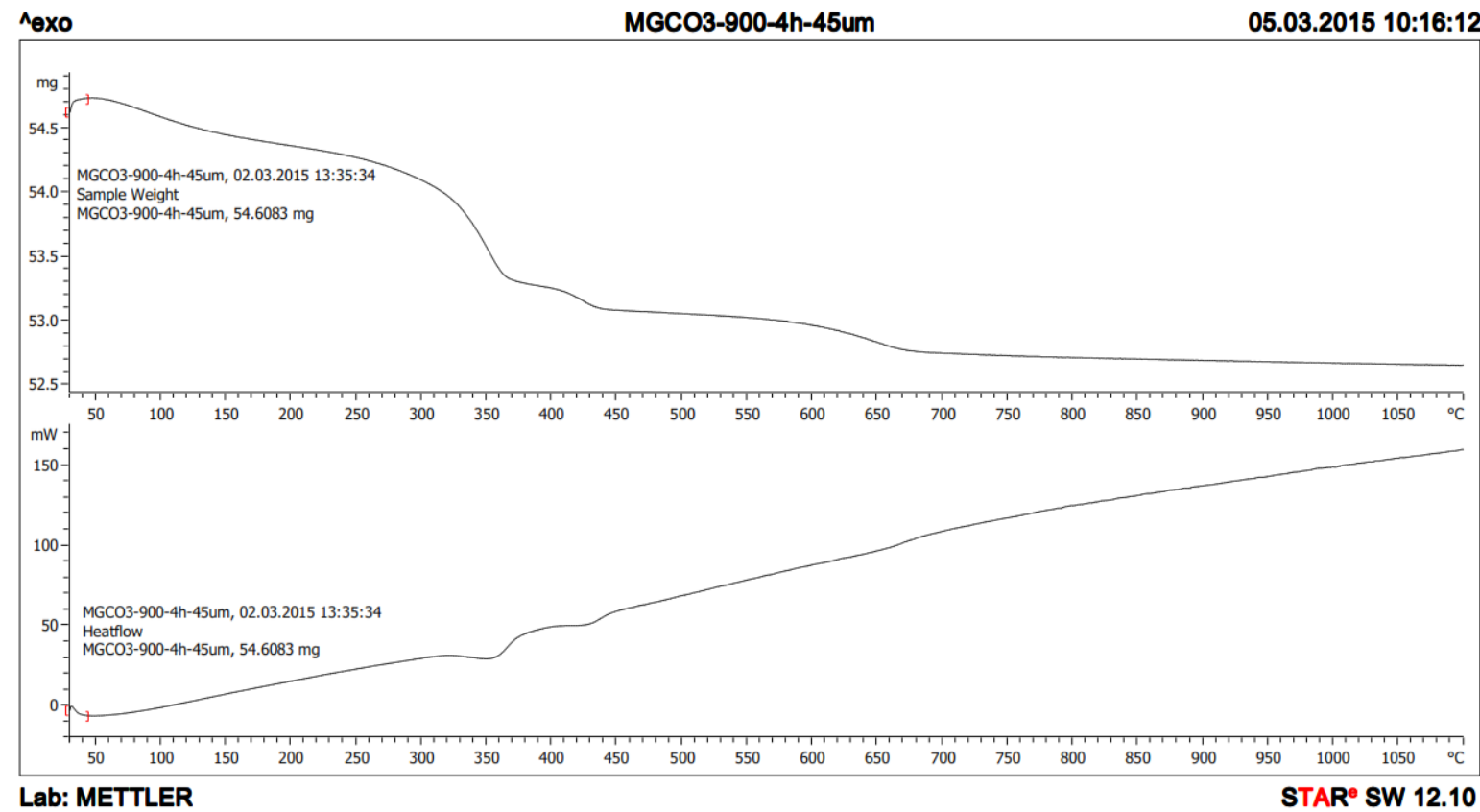

$900^{\circ} \mathrm{C}-45 \mu \mathrm{m}-4$ hour/TGA test

cexcviii 


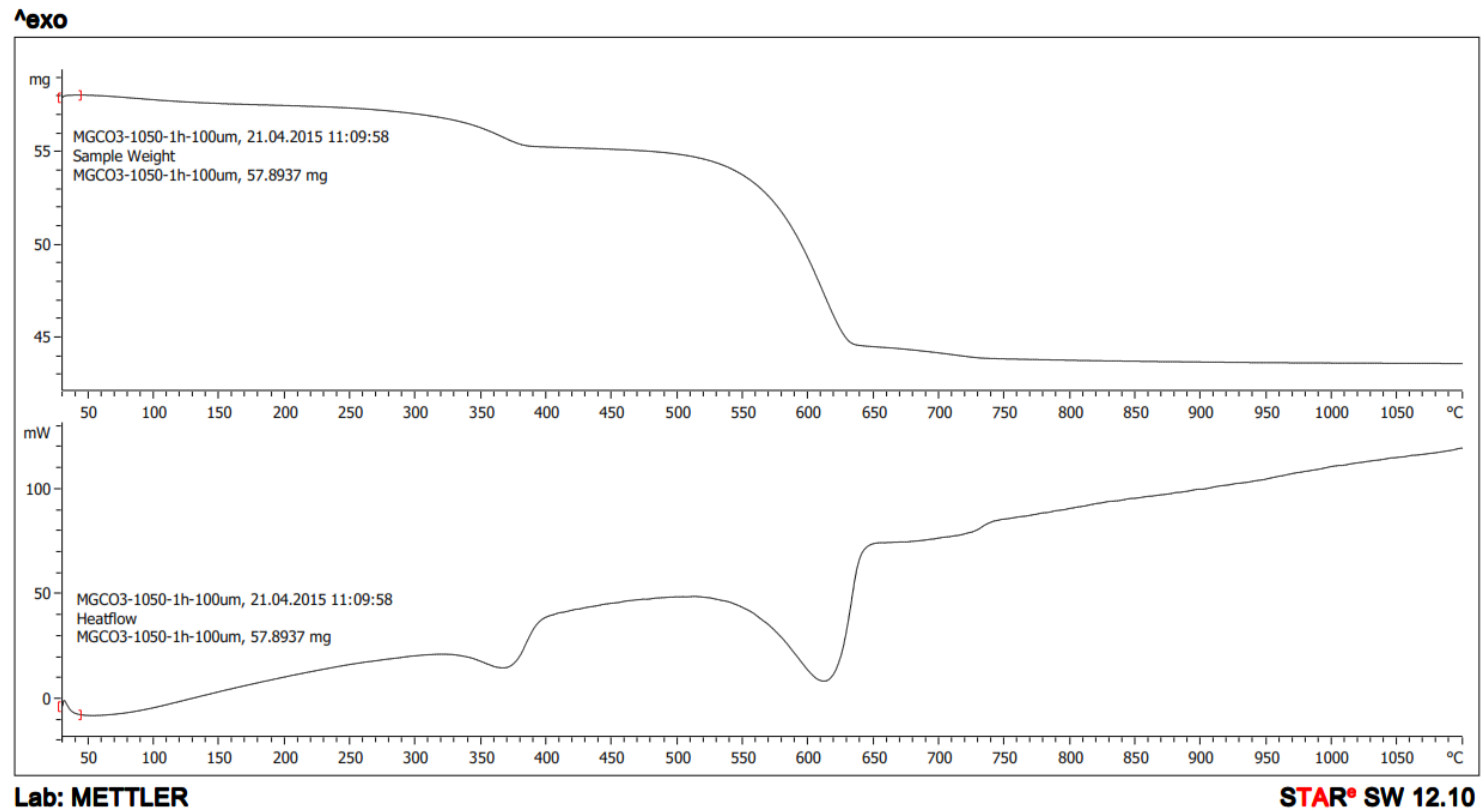

$1050^{\circ} \mathrm{C}-100 \mu \mathrm{m}-1$ hour/TGA test

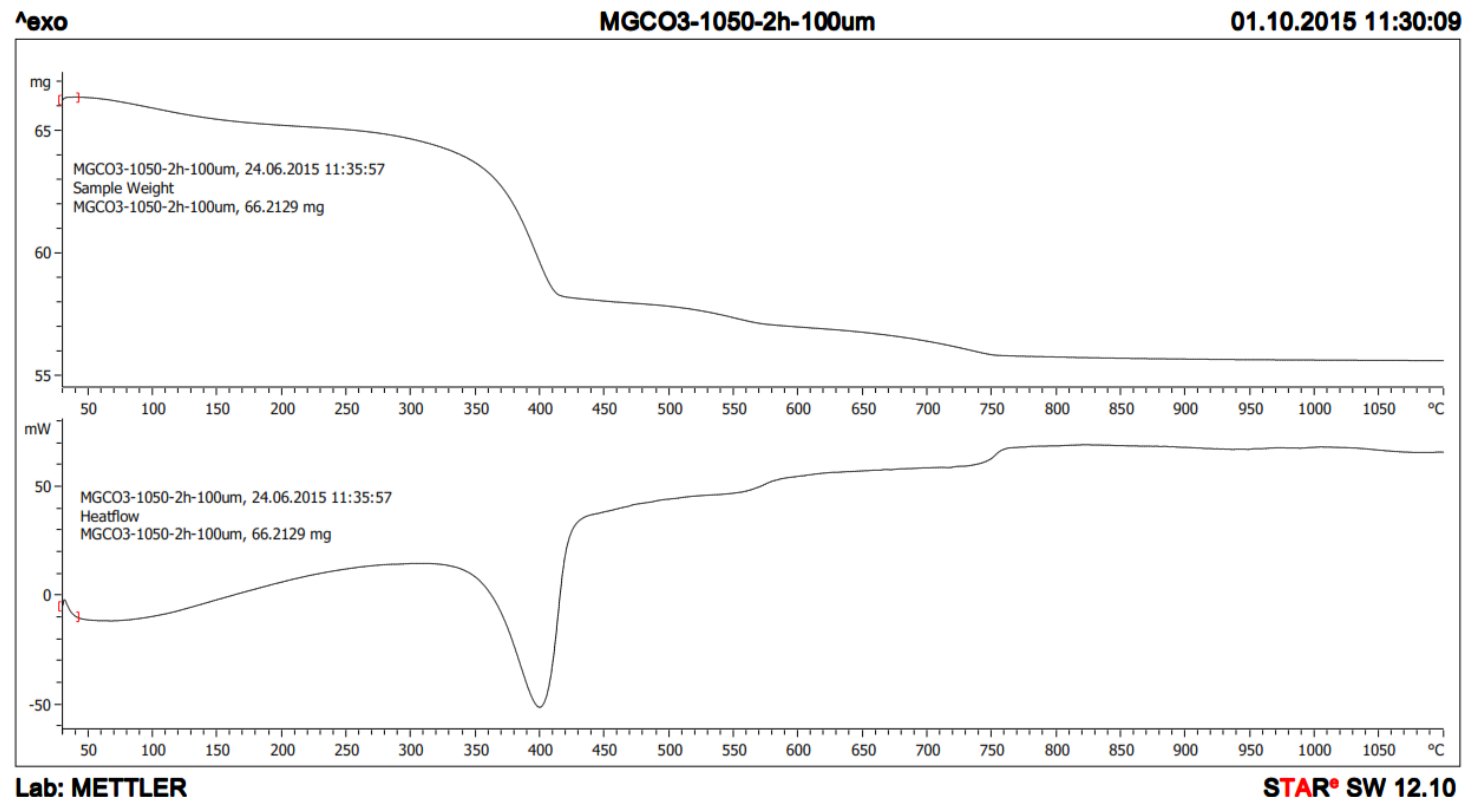

$1050^{\circ} \mathrm{C}-100 \mu \mathrm{m}-2 \mathrm{hour} / \mathrm{TGA}$ test

ccxcix 


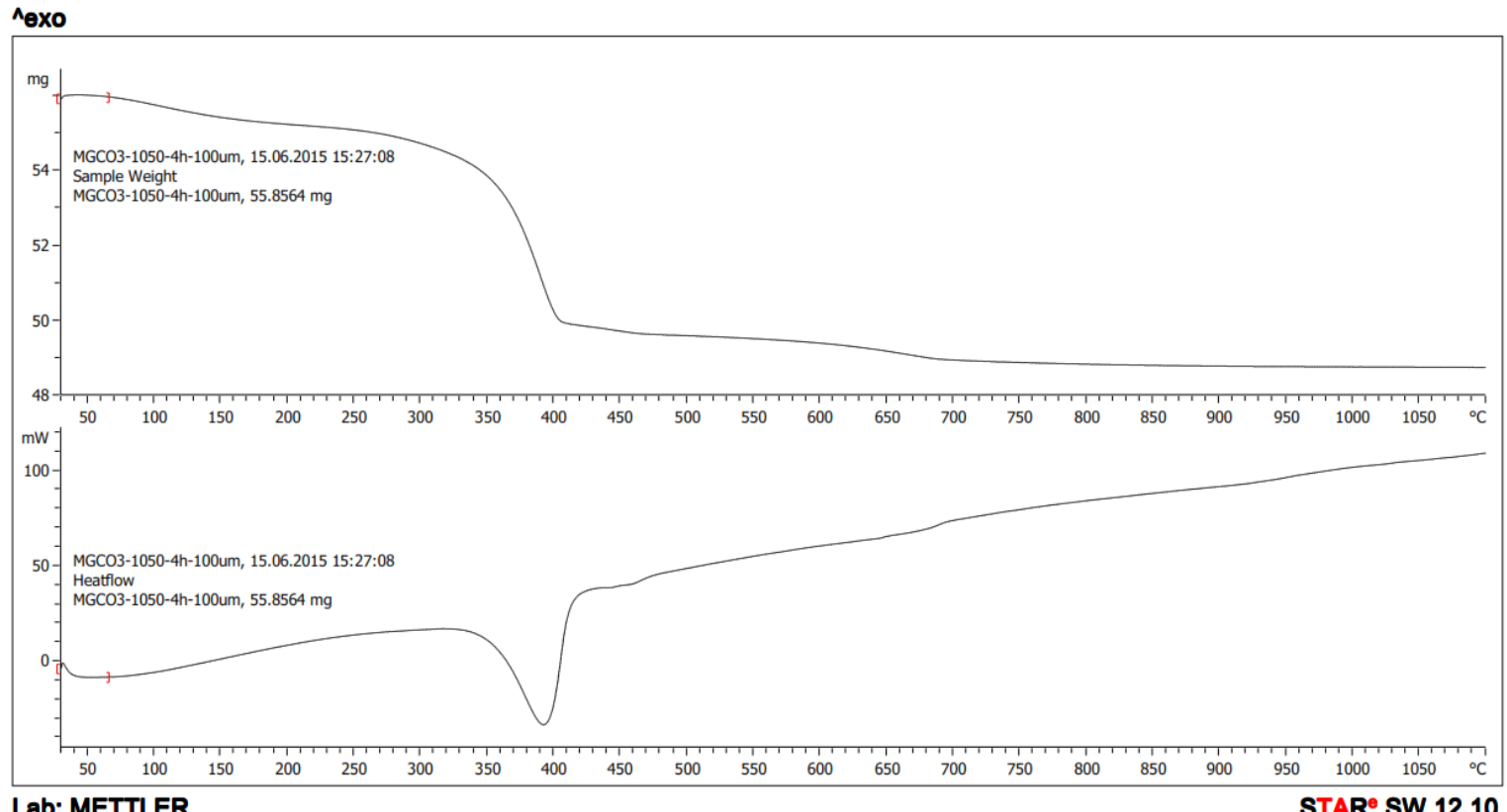

$1050^{\circ} \mathrm{C}-100 \mu \mathrm{m}-4$ hour/TGA test

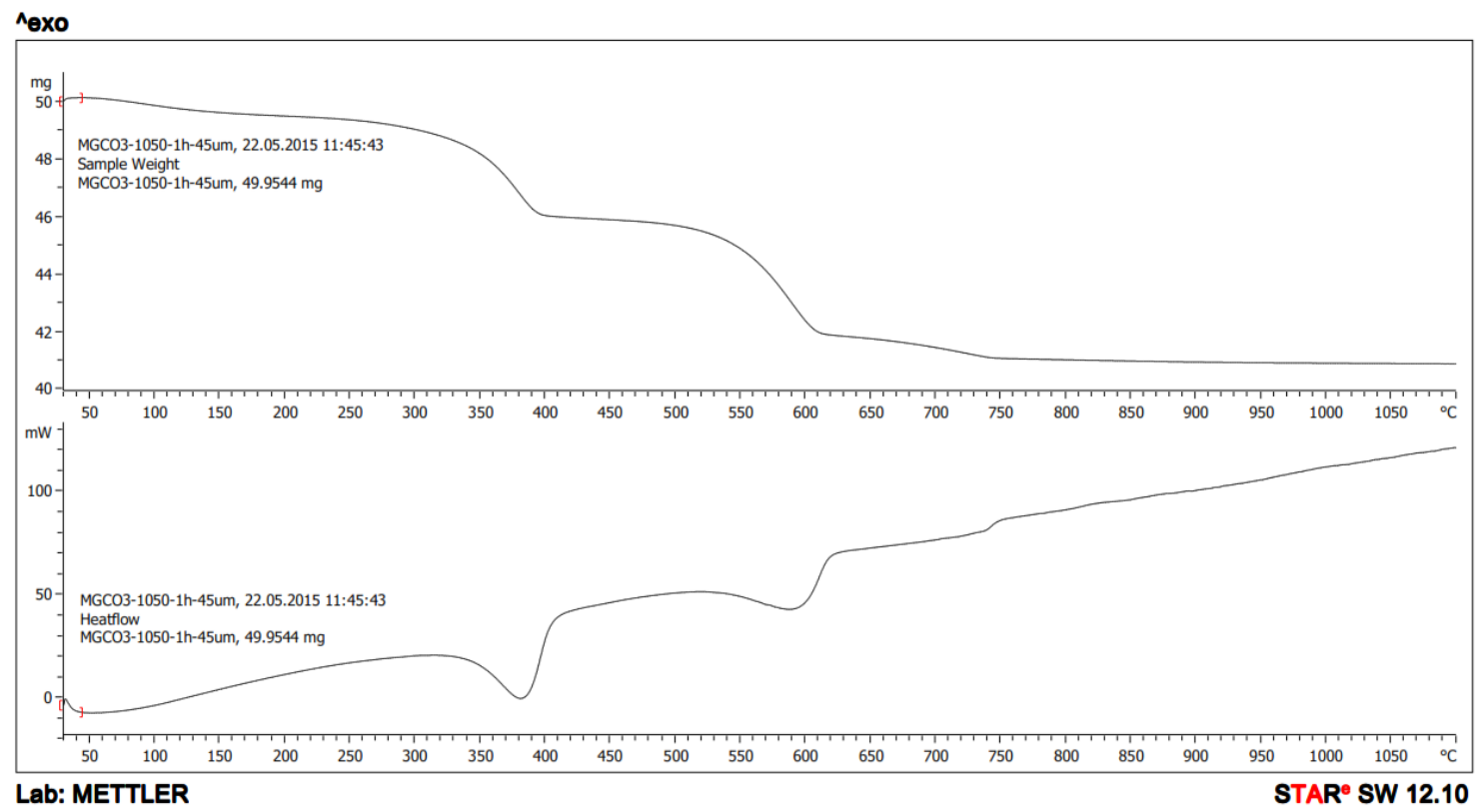

$1050^{\circ} \mathrm{C}-45 \mu \mathrm{m}-1$ hour/TGA test 


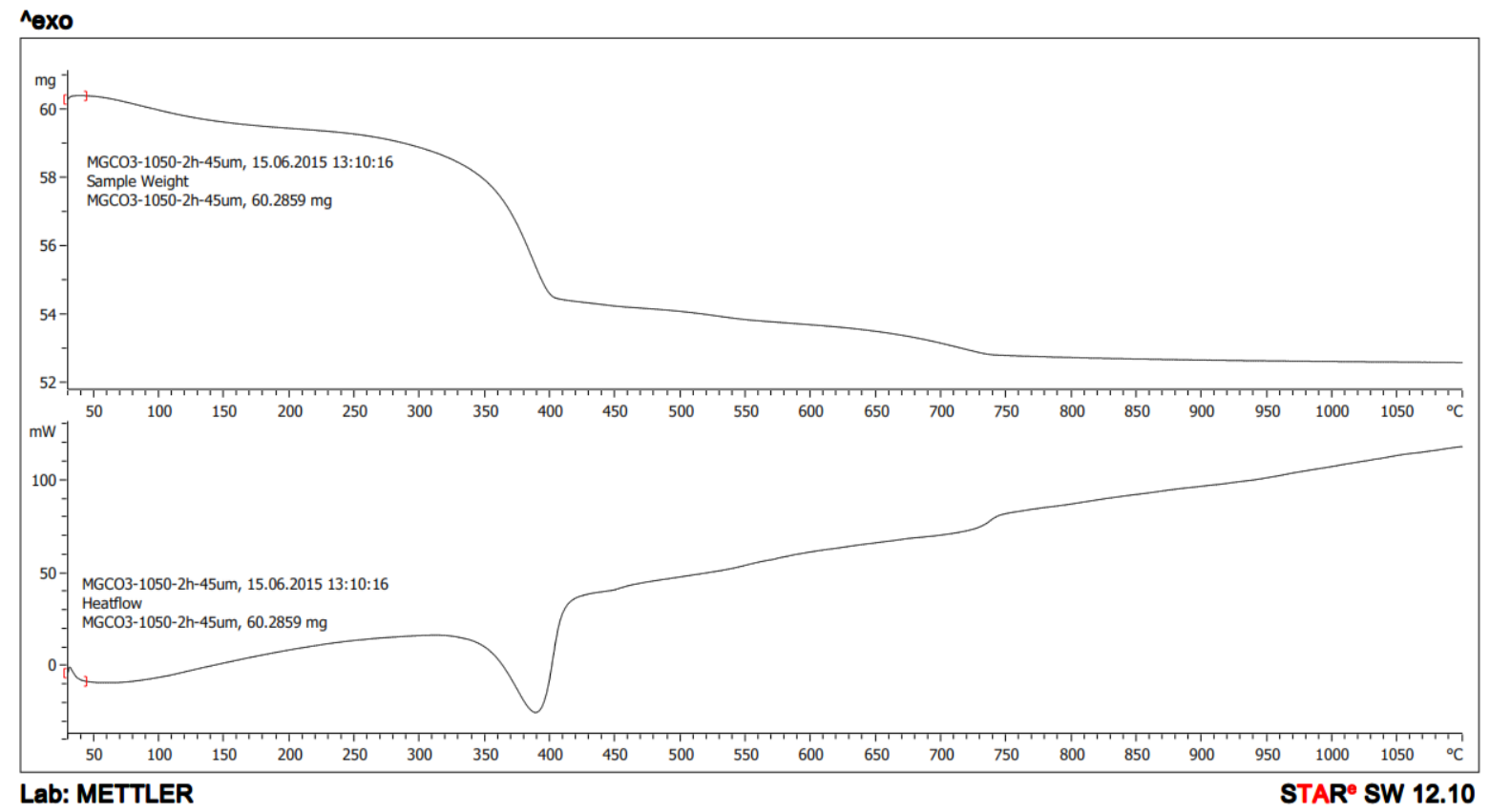

$1050^{\circ} \mathrm{C}-45 \mu \mathrm{m}-2 \mathrm{hour} / \mathrm{TGA}$ test

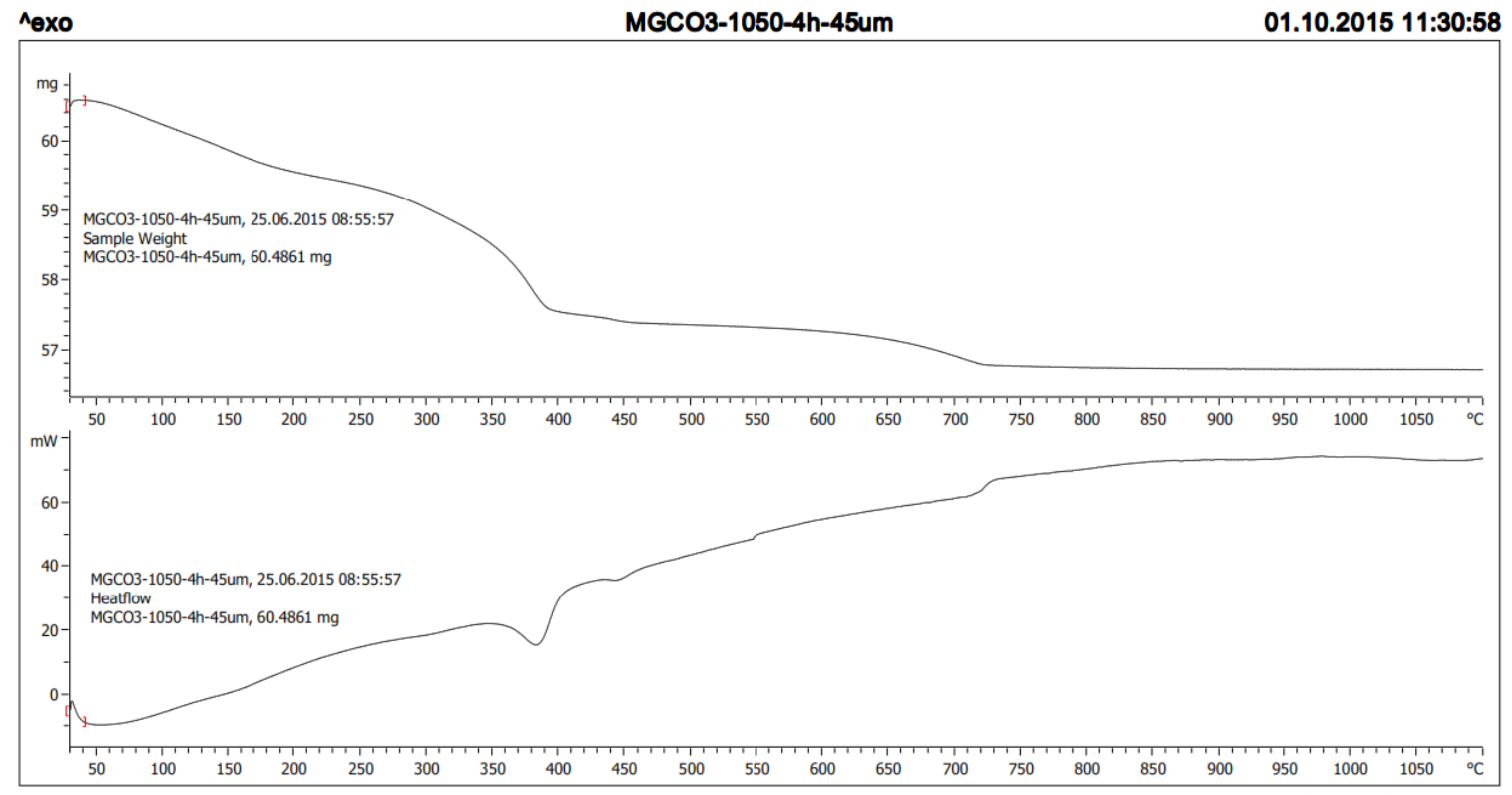

Lab: METTLER

STAR ${ }^{\ominus}$ SW 12.10

$1050^{\circ} \mathrm{C}-45 \mu \mathrm{m}-4$ hour/TGA test

ccci 


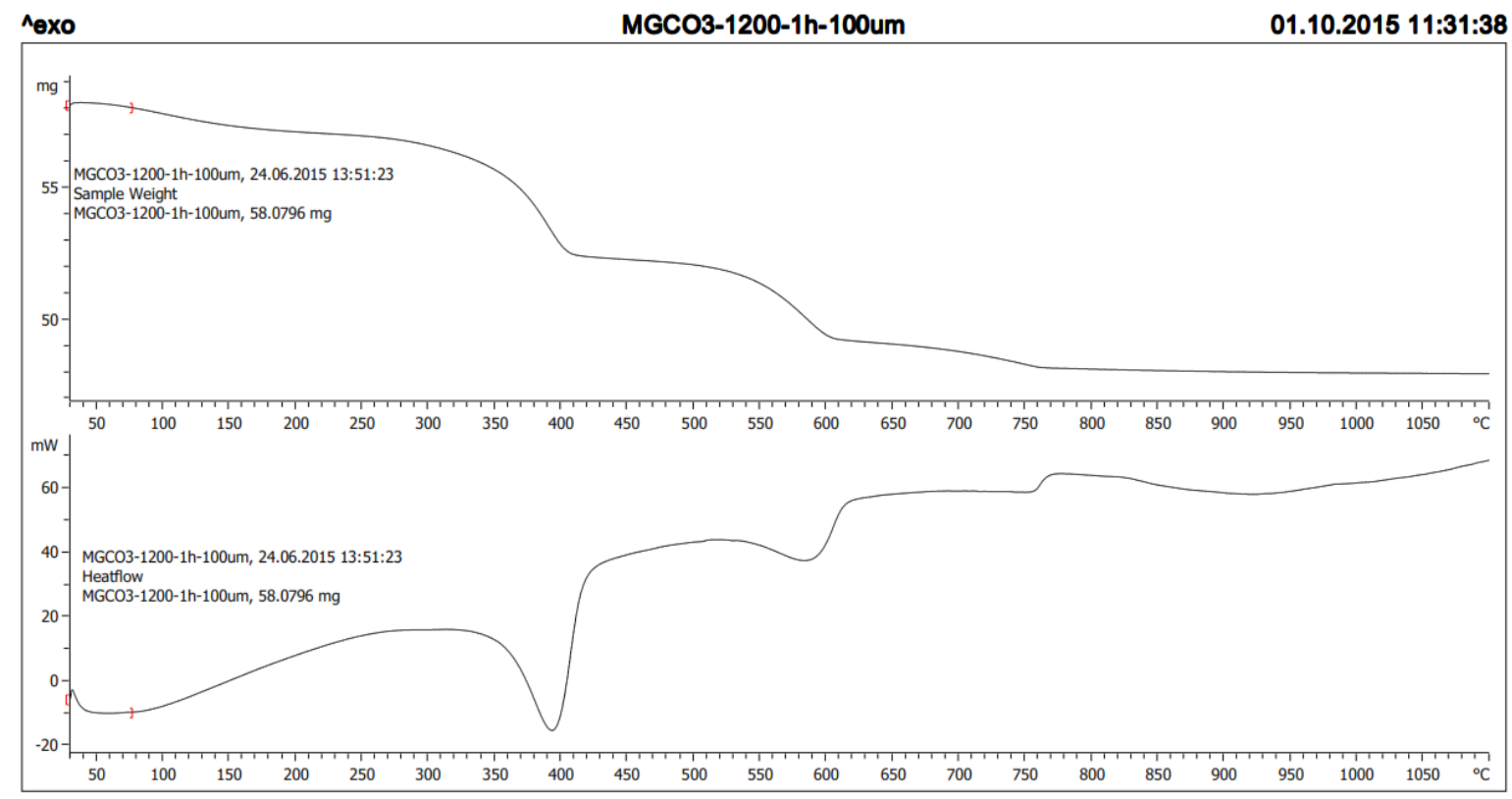

Lab: METTLER

STAR $^{\text {SW }} 12.10$

\section{$1200^{\circ} \mathrm{C}-100 \mu \mathrm{m}-1$ hour/TGA test}

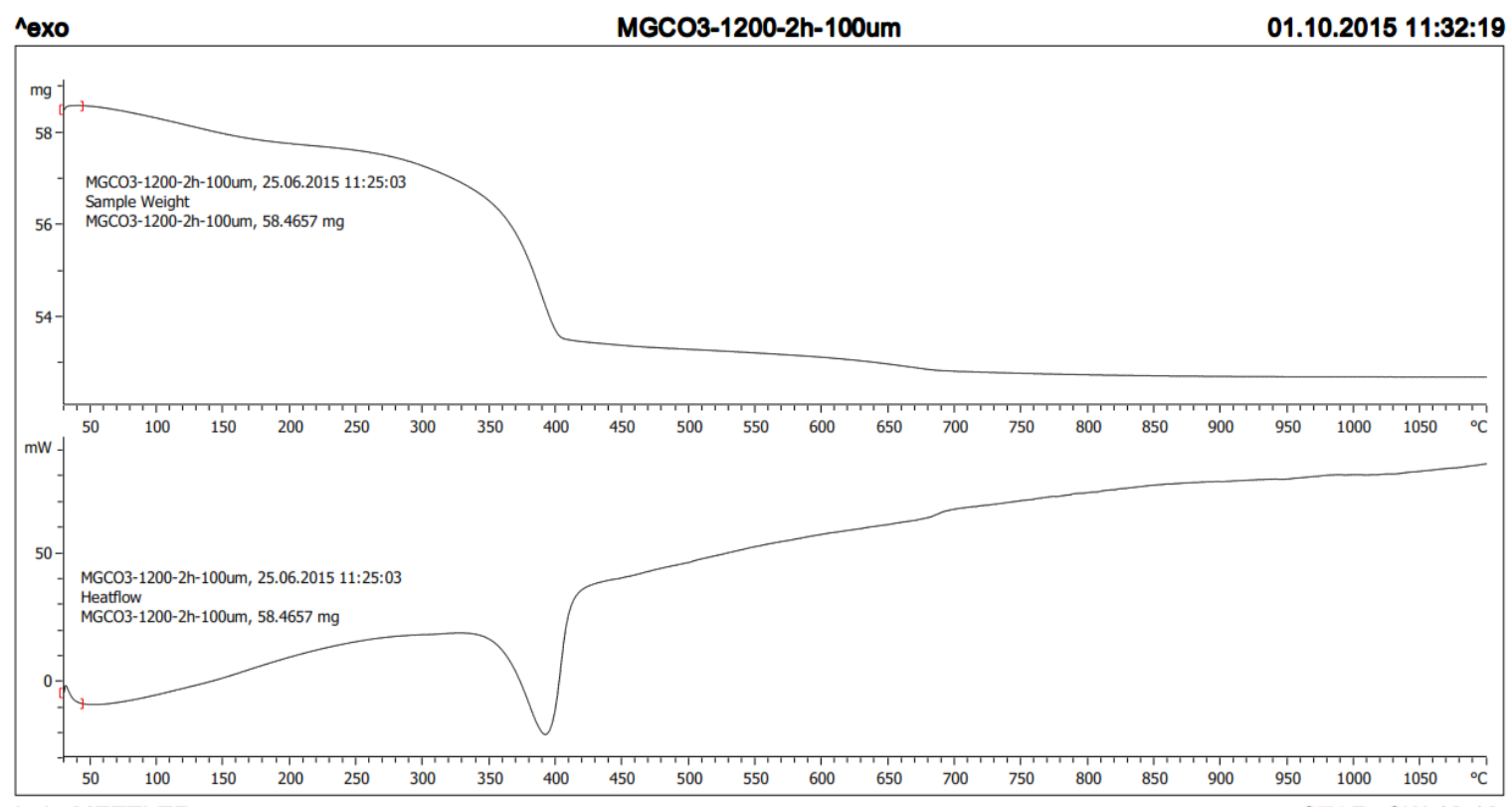

Lab: METTLER

STAR ${ }^{\text {SW }} 12.10$

$1200^{\circ} \mathrm{C}-100 \mu \mathrm{m}-2$ hour/TGA test

cccii 


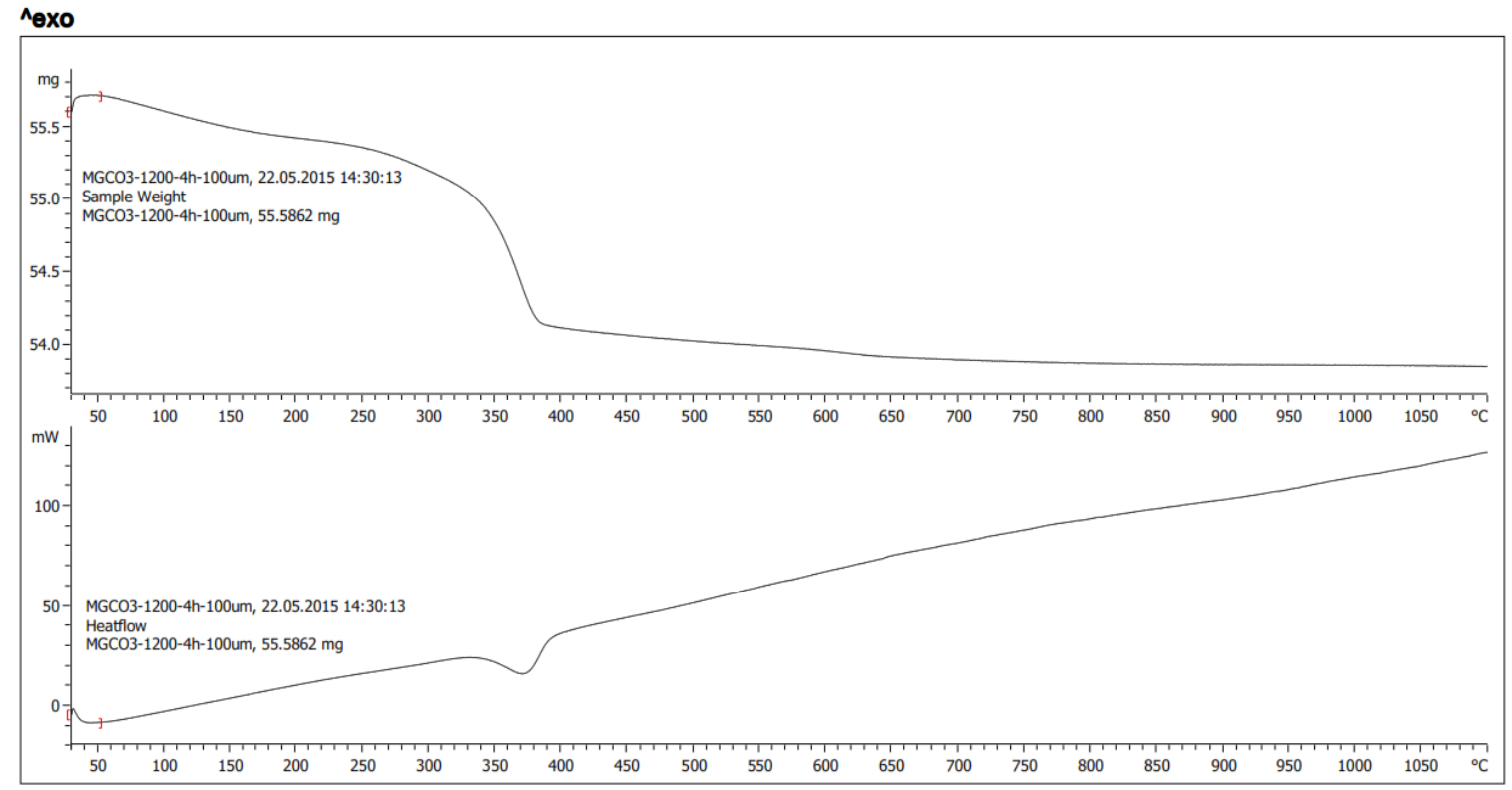

Lab: METTLER

STAR SW 12.10

\section{$1200^{\circ} \mathrm{C}-100 \mu \mathrm{m}-4$ hour/TGA test}

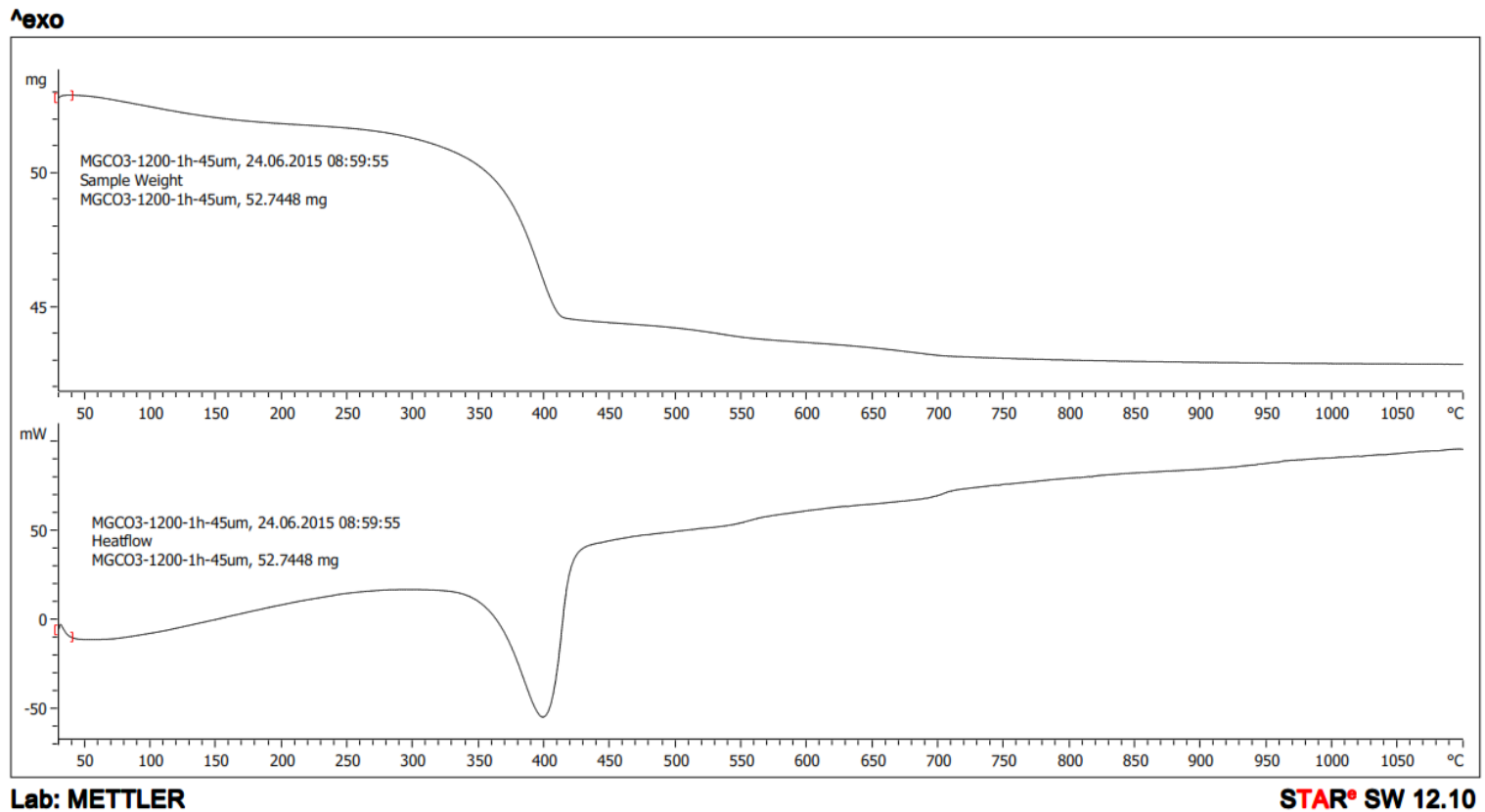

$1200^{\circ} \mathrm{C}-45 \mu \mathrm{m}-1$ hour/TGA test

ccciii 


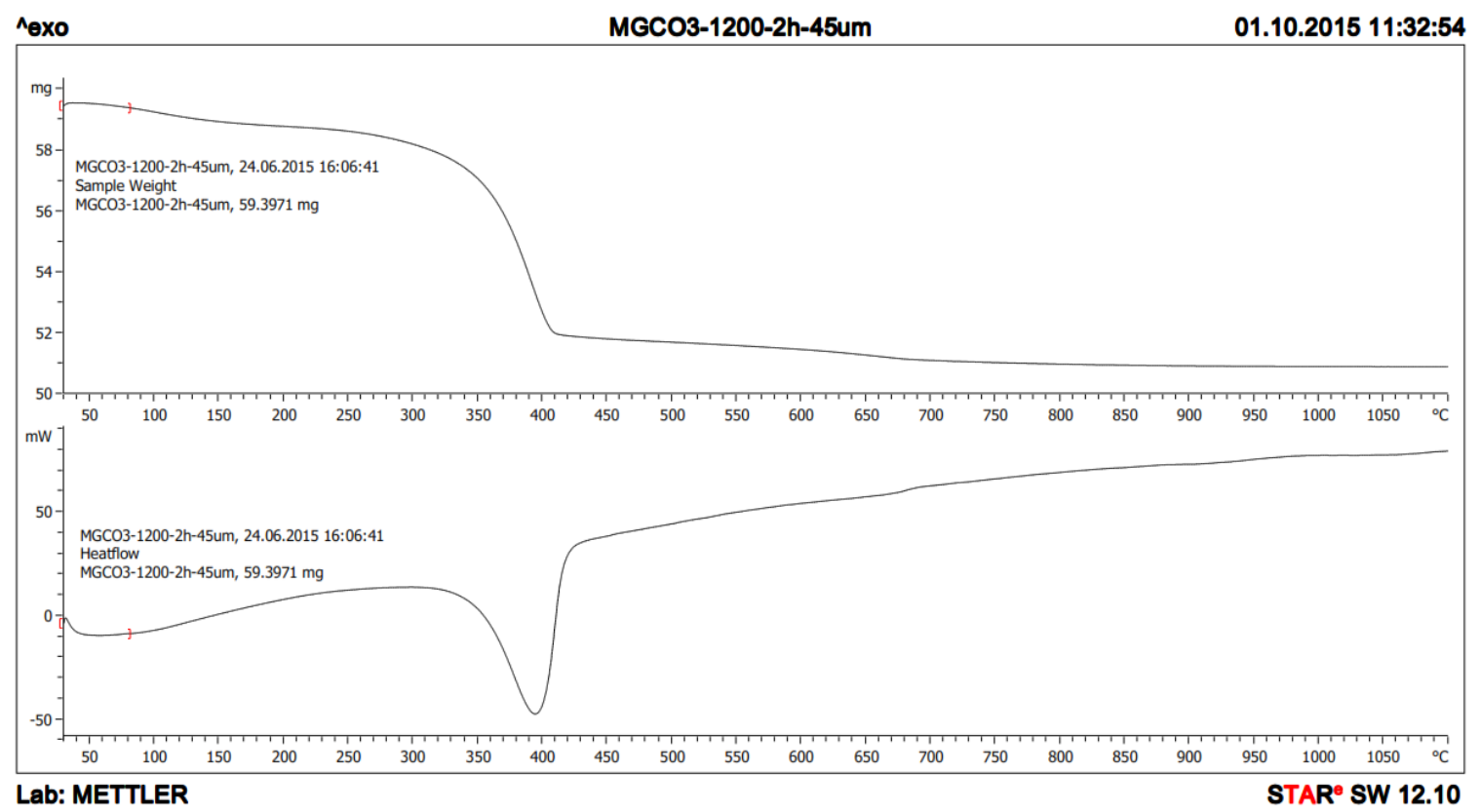

$1200^{\circ} \mathrm{C}-45 \mu \mathrm{m}-2$ hour/TGA test

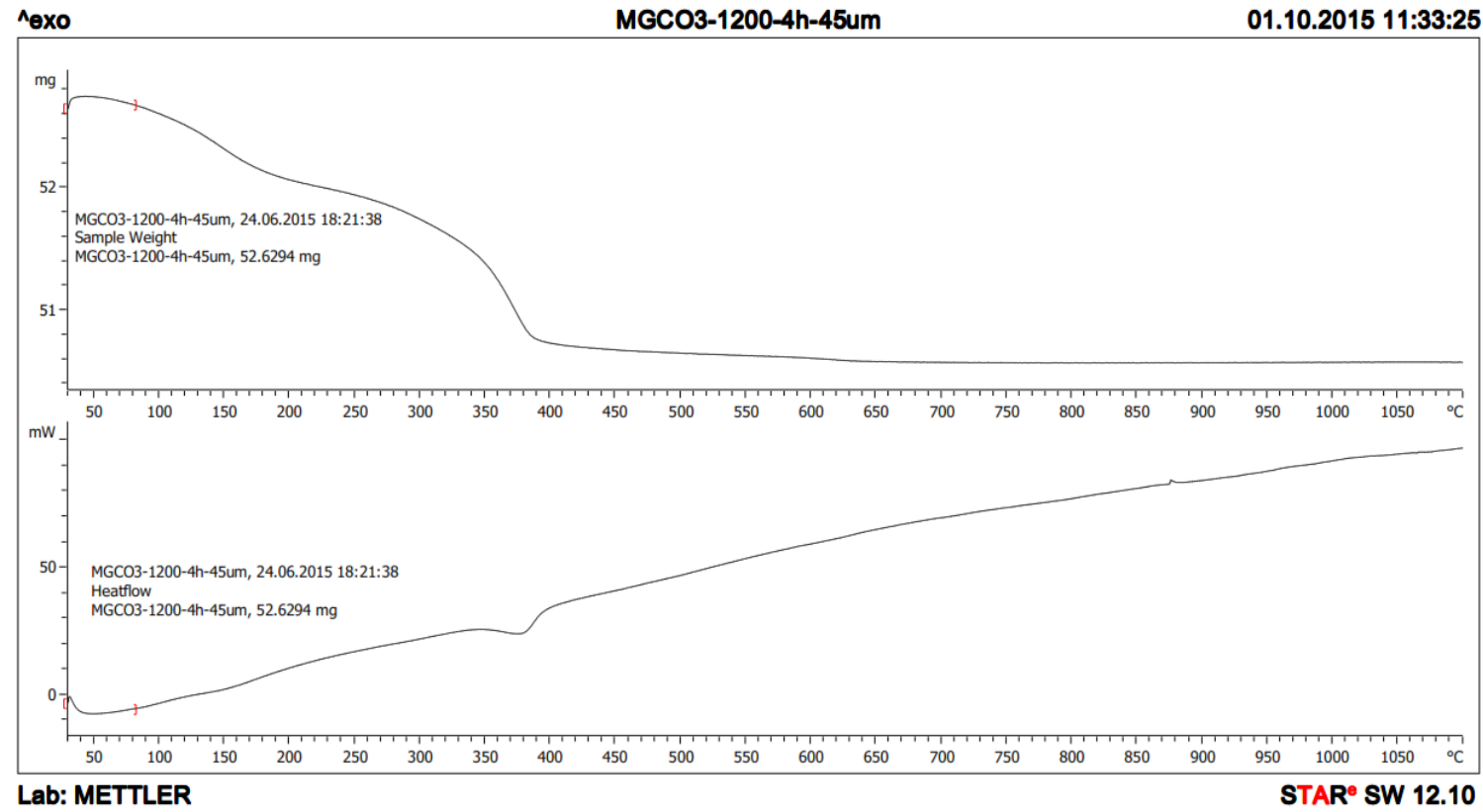

$1200^{\circ} \mathrm{C}-45 \mu \mathrm{m}-4$ hour/TGA test

ccciv 
Difference in weight loss of MgO samples (TGA/Kiln burning processes)

\begin{tabular}{|c|c|c|c|c|c|c|}
\hline & \multicolumn{6}{|c|}{ Holding time - particle size } \\
\hline & $\begin{array}{c}1 \text { Hour - } \\
45 \mu \mathrm{m}\end{array}$ & $\begin{array}{l}2 \text { Hours } \\
-45 \mu \mathrm{m}\end{array}$ & $\begin{array}{l}4 \text { Hours } \\
-45 \mu \mathrm{m}\end{array}$ & $\begin{array}{l}1 \text { Hour } \\
-100 \mu \mathrm{m}\end{array}$ & $\begin{array}{l}2 \text { Hours } \\
-100 \mu \mathrm{m}\end{array}$ & $\begin{array}{l}4 \text { Hours - } \\
100 \mu \mathrm{m}\end{array}$ \\
\hline $\begin{array}{l}\text { Original wt. of MgO } \\
\text { sample before TGA } \\
\text { (grams) }=\text { (a) }\end{array}$ & 57.61 & 52.75 & 56.75 & 55.66 & 54.32 & 57.02 \\
\hline $\begin{array}{l}\text { Residual wt. of MgO } \\
\text { sample (grams) = (b) }\end{array}$ & 38.5 & 37 & 50 & 36.9 & 41 & 51 \\
\hline $\begin{array}{c}\text { (\%) between a \& b } \\
(\mathrm{C}=\mathrm{b} / \mathbf{a})\end{array}$ & 66.83 & 70.14 & 88.11 & 66.30 & 75.48 & 89.44 \\
\hline $\begin{array}{l}\text { TGA wt. Loss }(\%) \\
=100-\mathrm{C}\end{array}$ & 33.17 & 29.86 & 11.89 & 33.70 & 24.52 & 10.56 \\
\hline Kiln wt. Loss (\%) & 24.28 & 32.11 & 42.90 & 23.56 & 33.03 & 43.70 \\
\hline $\begin{array}{l}\text { Original wt. of MgO } \\
\text { sample before TGA } \\
\quad(\text { grams })=(\text { a) }\end{array}$ & 56.4 & 60.2 & 57.8 & 58.14 & 51.8 & 54.61 \\
\hline $\begin{array}{l}\text { Residual wt. of MgO } \\
\text { sample (grams) = (b) }\end{array}$ & 40.5 & 49.5 & 54.75 & 43 & 44.5 & 52 \\
\hline $\begin{array}{c}\text { (\%) between a \& b } \\
(\mathrm{C}=\mathbf{b} / \mathbf{a})\end{array}$ & 71.81 & 82.23 & 94.72 & 73.96 & 85.91 & 95.22 \\
\hline $\begin{array}{l}\text { TGA wt. Loss }(\%) \\
=100-\mathrm{C}\end{array}$ & 28.19 & 17.77 & 5.28 & 26.04 & 14.09 & 4.78 \\
\hline Kiln wt. Loss (\%) & 32.09 & 44.06 & 50.22 & 34.52 & 43.58 & 49.33 \\
\hline $\begin{array}{l}\text { Original wt. of MgO } \\
\text { sample before TGA } \\
\text { (grams) }=(\text { a) }\end{array}$ & 50 & 66.2 & 60.5 & 57.9 & 66.3 & 56 \\
\hline $\begin{array}{l}\text { Residual wt. of MgO } \\
\text { sample (grams) = (b) }\end{array}$ & 40.5 & 57.5 & 57.5 & 48 & 58.5 & 53.5 \\
\hline $\begin{array}{c}\text { (\%) between a \& b } \\
(C=b / a)\end{array}$ & 81.00 & 86.86 & 95.04 & 82.90 & 88.24 & 95.54 \\
\hline $\begin{array}{l}\text { TGA wt. Loss }(\%) \\
=100-\mathrm{C}\end{array}$ & 19.00 & 13.14 & 4.96 & 17.10 & 11.76 & 4.46 \\
\hline Kiln wt. Loss (\%) & 44.15 & 49.30 & 49.76 & 41.21 & 48.87 & 49.80 \\
\hline $\begin{array}{l}\text { Original wt. of MgO } \\
\text { sample before TGA } \\
(\text { grams })=(\text { a) }\end{array}$ & 52.8 & 59.4 & 52.7 & 58.1 & 58.5 & 55.6 \\
\hline $\begin{array}{l}\text { Residual wt. of MgO } \\
\text { sample (grams) = (b) }\end{array}$ & 44.3 & 52.5 & 50.8 & 49 & 53.25 & 54.1 \\
\hline $\begin{array}{c}\text { (\%) between a \& b } \\
(\mathrm{C}=\mathbf{b} / \mathbf{a})\end{array}$ & 83.90 & 88.38 & 96.39 & 84.34 & 91.03 & 97.30 \\
\hline $\begin{array}{l}\text { TGA wt. Loss }(\%) \\
=100-\mathrm{C}\end{array}$ & 16.10 & 11.62 & 3.61 & 15.66 & 8.97 & 2.70 \\
\hline
\end{tabular}




\section{\begin{tabular}{r|c|c|c|c|c|} 
Kiln wt. Loss (\%) & $\mathbf{5 0 . 0 8}$ & $\mathbf{5 0 . 3 2}$ & $\mathbf{5 0 . 4 4}$ & $\mathbf{4 8 . 0 6}$ & $\mathbf{4 9 . 1 6}$ \\
Approach II: Linear expansion effect of MgO expansive additive
\end{tabular}}

Autoclave expansion test - Class-F fly ash

\begin{tabular}{|c|c|c|c|c|}
\hline Sample \# & Designation & $\begin{array}{c}\text { Before } \\
\text { Autoclave }\end{array}$ & $\begin{array}{c}\text { After } \\
\text { Autoclave }\end{array}$ & $\begin{array}{c}\text { Expansion } \\
\%\end{array}$ \\
\hline $1 / 1$ & 750_1_100 & -0.548 & -0.292 & 0.102 \\
\hline $1 / 2$ & 750_1_100 & -0.528 & -0.28 & 0.099 \\
\hline $4 / 1$ & 750_1_45 & -0.508 & -0.256 & 0.101 \\
\hline $4 / 2$ & 750_1_45 & -0.526 & -0.264 & 0.105 \\
\hline $2 / 1$ & 750_2_100 & -0.482 & -0.18 & 0.121 \\
\hline $2 / 2$ & 750_2_100 & -0.524 & -0.226 & 0.119 \\
\hline $5 / 1$ & 750_2_45 & -0.564 & -0.276 & 0.115 \\
\hline $5 / 2$ & 750_2_45 & -0.716 & -0.344 & 0.149 \\
\hline $3 / 1$ & 750_4_100 & -0.414 & -0.12 & 0.118 \\
\hline $3 / 2$ & 750_4_100 & -0.402 & 0.06 & 0.185 \\
\hline $6 / 1$ & $750 \_4 \_45$ & -0.698 & -0.408 & 0.116 \\
\hline $6 / 2$ & 750_4_45 & -0.404 & -0.004 & 0.160 \\
\hline $7 / 1$ & 900_1_100 & -0.554 & -0.276 & 0.111 \\
\hline $7 / 2$ & 900_1_100 & -0.556 & -0.302 & 0.102 \\
\hline $10 / 1$ & 900_1_45 & -0.608 & -0.246 & 0.145 \\
\hline $10 / 2$ & 900_1_45 & -0.552 & -0.198 & 0.142 \\
\hline $8 / 1$ & 900_2_100 & -0.528 & -0.260 & 0.107 \\
\hline $8 / 2$ & 900_2_100 & -0.488 & -0.124 & 0.146 \\
\hline $11 / 1$ & $900 \_2 \_45$ & -0.628 & -0.226 & 0.161 \\
\hline $11 / 2$ & $900 \_2 \_45$ & -0.6 & -0.186 & 0.166 \\
\hline $9 / 1$ & 900_4_100 & -0.602 & -0.058 & 0.218 \\
\hline $9 / 2$ & $900 \_4 \_100$ & -0.58 & -0.300 & 0.112 \\
\hline $12 / 1$ & 900_4_45 & -0.662 & -0.118 & 0.218 \\
\hline
\end{tabular}

cccvi 


\begin{tabular}{|c|c|c|c|c|}
\hline $12 / 2$ & 900_4_45 & -0.656 & -0.124 & 0.213 \\
\hline $13 / 1$ & 1050_1_100 & -0.422 & 0.002 & 0.170 \\
\hline $13 / 2$ & 1050_1_100 & -0.402 & 0.016 & 0.167 \\
\hline $16 / 1$ & 1050_1_45 & -0.784 & -0.314 & 0.188 \\
\hline $16 / 2$ & 1050_1_45 & -0.74 & -0.28 & 0.184 \\
\hline $14 / 1$ & 1050_2_100 & -0.704 & -0.1 & 0.242 \\
\hline $14 / 2$ & 1050_2_100 & -0.169 & 0.438 & 0.243 \\
\hline $17 / 1$ & 1050_2_45 & -1.046 & -0.76 & 0.114 \\
\hline $17 / 2$ & 1050_2_45 & -0.17 & 0.464 & 0.254 \\
\hline $15 / 1$ & 1050_4_100 & -0.632 & 0.086 & 0.287 \\
\hline $15 / 2$ & 1050_4_100 & -0.634 & 0.242 & 0.350 \\
\hline $18 / 1$ & 1050_4_45 & -0.614 & 0.174 & 0.315 \\
\hline $18 / 2$ & 1050_4_45 & -0.624 & 0.192 & 0.326 \\
\hline $19 / 1$ & 1200_1_100 & -0.6 & -0.022 & 0.231 \\
\hline $19 / 2$ & 1200_1_100 & -0.632 & -0.062 & 0.228 \\
\hline $22 / 1$ & 1200_1_45 & -0.618 & -0.068 & 0.220 \\
\hline $22 / 2$ & 1200_1_45 & -0.602 & -0.048 & 0.222 \\
\hline $20 / 1$ & 1200_2_100 & -1.04 & -0.658 & 0.153 \\
\hline $20 / 2$ & 1200_2_100 & -0.564 & 0.128 & 0.277 \\
\hline $23 / 1$ & 1200_2_45 & -0.596 & 0.096 & 0.277 \\
\hline $23 / 2$ & 1200_2_45 & -0.622 & 0.054 & 0.270 \\
\hline $21 / 1$ & 1200_4_100 & -0.468 & 0.26 & 0.291 \\
\hline $21 / 2$ & 1200_4_100 & -0.488 & 0.246 & 0.294 \\
\hline $24 / 1$ & 1200_4_45 & -0.706 & 0.032 & 0.295 \\
\hline $24 / 2$ & 1200_4_45 & -0.678 & 0.054 & 0.293 \\
\hline
\end{tabular}

*The maximum value was used 
Autoclave expansion test - Class-CI fly ash

\begin{tabular}{|c|c|c|c|c|}
\hline Sample \# & Designation & $\begin{array}{c}\text { Before } \\
\text { Autoclave }\end{array}$ & $\begin{array}{c}\text { After } \\
\text { Autoclave }\end{array}$ & $\begin{array}{c}\text { Expansion } \\
\%\end{array}$ \\
\hline $1 / 1$ & 750_1_100 & -0.69 & -0.494 & 0.078 \\
\hline $1 / 2$ & 750_1_100 & -0.698 & -0.504 & 0.078 \\
\hline $4 / 1$ & 750_1_45 & -1.048 & -0.82 & 0.091 \\
\hline $4 / 2$ & 750_1_45 & -0.864 & -0.312 & 0.221 \\
\hline $2 / 1$ & 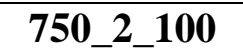 & -0.704 & -0.368 & 0.134 \\
\hline $2 / 2$ & 750_2_100 & -0.686 & -0.352 & 0.134 \\
\hline $5 / 1$ & 750_2_45 & -0.592 & -0.04 & 0.221 \\
\hline $5 / 2$ & 750_2_45 & -0.628 & -0.28 & 0.139 \\
\hline $3 / 1$ & 750_4_100 & -0.59 & 0.034 & 0.250 \\
\hline $3 / 2$ & 750_4_100 & -0.592 & 0.104 & 0.278 \\
\hline $6 / 1$ & 750_4_45 & -0.694 & -0.049 & 0.258 \\
\hline $6 / 2$ & 750_4_45 & -1.054 & -0.208 & 0.338 \\
\hline $7 / 1$ & 900_1_100 & -0.472 & -0.024 & 0.179 \\
\hline $7 / 2$ & 900_1_100 & -1.054 & * & * \\
\hline $10 / 1$ & 900_1_45 & -0.826 & -0.336 & 0.196 \\
\hline $10 / 2$ & 900_1_45 & -0.642 & -0.346 & 0.118 \\
\hline $8 / 1$ & 900_2_100 & -0.422 & 0.14 & 0.225 \\
\hline $8 / 2$ & 900_2_100 & -0.324 & * & * \\
\hline $11 / 1$ & 900_2_45 & -1.054 & $*$ & $*$ \\
\hline $11 / 2$ & 900_2_45 & -0.46 & 0.132 & 0.237 \\
\hline $9 / 1$ & 900_4_100 & -0.336 & $*$ & * \\
\hline $9 / 2$ & 900_4_100 & -0.418 & 0.272 & 0.276 \\
\hline $12 / 1$ & 900_4_45 & $\begin{array}{l}-0.84 \\
\end{array}$ & -0.126 & 0.286 \\
\hline $12 / 2$ & 900_4_45 & -1.054 & * & * \\
\hline $13 / 1$ & 1050_1_100 & -0.724 & -0.254 & 0.188 \\
\hline
\end{tabular}

cccviii 


\begin{tabular}{|c|c|c|c|c|}
\hline $13 / 2$ & 1050_1_100 & -0.708 & -0.184 & 0.210 \\
\hline $16 / 1$ & 1050_1_45 & -0.628 & 0.006 & 0.254 \\
\hline $16 / 2$ & 1050_1_45 & -0.628 & $\mathbf{0}$ & 0.251 \\
\hline $14 / 1$ & 1050_2_100 & -0.64 & 0.03 & 0.268 \\
\hline $14 / 2$ & 1050_2_100 & -0.646 & 0.05 & 0.278 \\
\hline $17 / 1$ & 1050_2_45 & -0.478 & 0.254 & 0.293 \\
\hline $17 / 2$ & 1050_2_45 & -0.514 & 0.228 & 0.297 \\
\hline $15 / 1$ & 1050_4_100 & -1.054 & 0.18 & 0.494 \\
\hline $15 / 2$ & 1050_4_100 & 0.686 & 1.91 & 0.490 \\
\hline $18 / 1$ & 1050_4_45 & -0.668 & 0.546 & 0.486 \\
\hline $18 / 2$ & 1050_4_45 & -0.698 & 0.118 & 0.326 \\
\hline $19 / 1$ & 1200_1_100 & -0.694 & -0.17 & 0.210 \\
\hline $19 / 2$ & 1200_1_100 & -0.668 & -0.136 & 0.213 \\
\hline $22 / 1$ & 1200_1_45 & -0.51 & 0.124 & 0.254 \\
\hline $22 / 2$ & 1200_1_45 & -0.536 & 0.124 & 0.264 \\
\hline $20 / 1$ & 1200_2_100 & -0.376 & 0.326 & 0.281 \\
\hline $20 / 2$ & 1200_2_100 & -0.52 & 0.18 & 0.280 \\
\hline $23 / 1$ & 1200_2_45 & -0.396 & 0.38 & 0.310 \\
\hline $23 / 2$ & 1200_2_45 & -0.28 & 0.5 & 0.312 \\
\hline $21 / 1$ & 1200_4_100 & 0.14 & 0.976 & 0.334 \\
\hline $21 / 2$ & 1200_4_100 & $*$ & $*$ & $*$ \\
\hline $24 / 1$ & 1200_4_45 & -1.054 & -0.168 & 0.354 \\
\hline $24 / 2$ & 1200_4_45 & -0.572 & 0.38 & 0.381 \\
\hline
\end{tabular}

*broken sample; *the maximum value was used

$\operatorname{cccix}$ 
Water expansion - Class-F fly ash/100 $\mu \mathrm{m}$

\begin{tabular}{|c|c|c|c|c|c|c|c|c|c|c|c|c|c|c|c|c|c|c|c|}
\hline \multirow{3}{*}{ 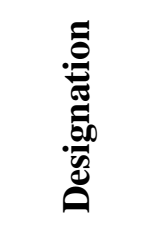 } & \multicolumn{19}{|c|}{ Day } \\
\hline & $\mathbf{0}$ & 1 & 3 & 7 & 14 & 21 & 28 & 35 & 42 & 50 & 65 & 76 & 91 & 105 & 134 & 210 & 250 & 336 & 477 \\
\hline & \multicolumn{19}{|c|}{ Expansion } \\
\hline 750_1 & 0.000 & 0.024 & 0.027 & 0.037 & 0.043 & 0.041 & 0.045 & 0.042 & 0.046 & 0.047 & 0.042 & 0.047 & 0.049 & 0.047 & 0.050 & 0.049 & 0.050 & 0.052 & 0.053 \\
\hline 750_1 & 0.000 & 0.027 & 0.033 & 0.042 & 0.048 & 0.045 & 0.052 & 0.046 & 0.053 & 0.050 & 0.046 & 0.053 & 0.057 & 0.052 & 0.056 & 0.053 & 0.056 & 0.057 & 0.059 \\
\hline 750_1 & 0.000 & 0.025 & 0.030 & 0.038 & 0.044 & 0.045 & 0.046 & 0.044 & 0.049 & 0.049 & 0.042 & 0.049 & 0.051 & 0.049 & 0.051 & 0.049 & 0.050 & 0.052 & 0.057 \\
\hline \multirow[t]{4}{*}{ Average } & 0.000 & 0.025 & 0.030 & 0.039 & 0.045 & 0.043 & 0.047 & 0.044 & 0.049 & 0.049 & 0.044 & 0.050 & 0.052 & 0.049 & 0.052 & 0.050 & 0.052 & 0.054 & 0.056 \\
\hline & \multicolumn{19}{|c|}{ Day } \\
\hline & $\mathbf{0}$ & 1 & 6 & 13 & 20 & 26 & 33 & 41 & 49 & 64 & 75 & 90 & 104 & 133 & 209 & 249 & 335 & 476 & \\
\hline & \multicolumn{19}{|c|}{ Expansion } \\
\hline 900_1 & 0.000 & 0.038 & 0.055 & 0.069 & 0.070 & 0.074 & 0.074 & 0.079 & 0.078 & 0.074 & 0.082 & 0.086 & 0.082 & 0.085 & 0.086 & 0.088 & 0.090 & 0.092 & \\
\hline 900_1 & 0.000 & 0.042 & 0.058 & 0.073 & 0.074 & 0.078 & 0.077 & 0.084 & 0.084 & 0.079 & 0.086 & 0.092 & 0.088 & 0.090 & 0.090 & 0.093 & 0.095 & 0.096 & \\
\hline 900_1 & 0.000 & 0.038 & 0.055 & 0.068 & 0.071 & 0.075 & 0.074 & 0.079 & 0.078 & 0.075 & 0.082 & 0.086 & 0.082 & 0.086 & 0.087 & 0.087 & 0.090 & 0.095 & \\
\hline \multirow[t]{4}{*}{ Average } & 0.000 & 0.039 & 0.056 & 0.070 & 0.072 & 0.076 & 0.075 & 0.081 & 0.080 & 0.076 & 0.083 & 0.088 & 0.084 & 0.087 & 0.088 & 0.089 & 0.092 & 0.094 & \\
\hline & \multicolumn{19}{|c|}{ Day } \\
\hline & $\mathbf{0}$ & 1 & 7 & 14 & 21 & 28 & 35 & 43 & 58 & 69 & 84 & 98 & 127 & 203 & 243 & 329 & 470 & & \\
\hline & \multicolumn{19}{|c|}{ Expansion } \\
\hline 1050_1 & 0.000 & 0.055 & 0.094 & 0.108 & 0.117 & 0.119 & 0.128 & 0.130 & 0.127 & 0.130 & 0.138 & 0.138 & 0.138 & 0.142 & 0.143 & 0.156 & 0.152 & & \\
\hline
\end{tabular}




\begin{tabular}{|c|c|c|c|c|c|c|c|c|c|c|c|c|c|c|c|c|c|c|c|}
\hline 1050_1 & 0.000 & 0.054 & 0.095 & 0.107 & 0.115 & 0.118 & 0.124 & 0.126 & 0.126 & 0.129 & 0.132 & 0.133 & 0.139 & 0.140 & 0.143 & 0.145 & 0.151 & & \\
\hline Average & 0.000 & 0.055 & 0.095 & 0.108 & 0.116 & 0.118 & 0.126 & 0.128 & 0.126 & 0.130 & 0.135 & 0.135 & 0.139 & 0.141 & 0.143 & 0.150 & 0.152 & & \\
\hline & \multicolumn{19}{|c|}{ Day } \\
\hline & $\mathbf{0}$ & 1 & 7 & 14 & 21 & 28 & 35 & 43 & 58 & 69 & 84 & 98 & 127 & 203 & 243 & 329 & 470 & & \\
\hline & \multicolumn{19}{|c|}{ Expansion } \\
\hline 1200_1 & 0.000 & 0.057 & 0.117 & 0.138 & 0.150 & 0.154 & 0.162 & 0.158 & 0.170 & 0.172 & 0.178 & 0.178 & 0.184 & 0.189 & 0.193 & 0.198 & 0.199 & & \\
\hline 1200_1 & 0.000 & 0.057 & 0.116 & 0.138 & 0.150 & 0.154 & 0.162 & 0.172 & 0.168 & 0.172 & 0.179 & 0.179 & 0.183 & 0.190 & 0.194 & 0.198 & 0.198 & & \\
\hline \multirow[t]{4}{*}{ Average } & 0.000 & 0.057 & 0.116 & 0.138 & 0.150 & 0.154 & 0.162 & 0.165 & 0.169 & 0.172 & 0.178 & 0.179 & 0.184 & 0.189 & 0.193 & 0.198 & 0.198 & & \\
\hline & \multicolumn{19}{|c|}{ Day } \\
\hline & $\mathbf{0}$ & 1 & 3 & 7 & 14 & 21 & 28 & 35 & 42 & 50 & 65 & 76 & 91 & 105 & 134 & 210 & 250 & 336 & 477 \\
\hline & \multicolumn{19}{|c|}{ Expansion } \\
\hline 750_2 & 0.000 & 0.037 & 0.044 & 0.056 & 0.066 & 0.063 & 0.068 & 0.067 & 0.073 & 0.073 & 0.068 & 0.074 & 0.078 & 0.074 & 0.077 & 0.078 & 0.078 & 0.082 & 0.084 \\
\hline 750_2 & 0.000 & 0.034 & 0.040 & 0.053 & 0.062 & 0.061 & 0.067 & 0.064 & 0.072 & 0.074 & 0.066 & 0.072 & 0.075 & 0.072 & 0.075 & 0.074 & 0.076 & 0.078 & 0.081 \\
\hline 750_2 & 0.000 & 0.037 & 0.044 & 0.055 & 0.064 & 0.066 & 0.068 & 0.066 & 0.071 & 0.073 & 0.067 & 0.074 & 0.075 & 0.074 & 0.078 & 0.074 & 0.077 & 0.079 & 0.084 \\
\hline \multirow[t]{4}{*}{ Average } & 0.000 & 0.036 & 0.043 & 0.055 & 0.064 & 0.063 & 0.068 & 0.066 & 0.072 & 0.073 & 0.067 & 0.073 & 0.076 & 0.074 & 0.077 & 0.075 & 0.077 & 0.080 & 0.083 \\
\hline & \multicolumn{19}{|c|}{ Day } \\
\hline & $\mathbf{0}$ & 1 & 6 & 13 & 20 & 26 & 33 & 41 & 49 & 64 & 75 & 90 & 104 & 133 & 209 & 249 & 335 & 476 & \\
\hline & \multicolumn{19}{|c|}{ Expansion } \\
\hline 900_2 & 0.000 & 0.051 & 0.077 & 0.098 & 0.101 & 0.108 & 0.108 & 0.116 & 0.114 & 0.112 & 0.119 & 0.123 & 0.124 & 0.125 & 0.131 & 0.135 & 0.138 & 0.142 & \\
\hline 900_2 & 0.000 & 0.052 & 0.078 & 0.100 & 0.104 & 0.113 & 0.112 & 0.119 & 0.120 & 0.117 & 0.124 & 0.129 & 0.127 & 0.131 & 0.136 & 0.136 & 0.142 & 0.145 & \\
\hline
\end{tabular}




\begin{tabular}{|c|c|c|c|c|c|c|c|c|c|c|c|c|c|c|c|c|c|c|c|}
\hline $900 \_2$ & 0.000 & 0.051 & 0.081 & 0.102 & 0.107 & 0.113 & 0.113 & 0.121 & 0.119 & 0.118 & 0.126 & 0.130 & 0.127 & 0.131 & 0.134 & 0.138 & 0.142 & 0.145 & \\
\hline Average & 0.000 & 0.051 & 0.079 & 0.100 & 0.104 & 0.111 & 0.111 & 0.119 & 0.118 & 0.116 & 0.123 & 0.127 & 0.126 & 0.129 & 0.134 & 0.136 & 0.141 & 0.144 & \\
\hline & \multicolumn{19}{|c|}{ Day } \\
\hline & 0 & 1 & 7 & 14 & 21 & 28 & 35 & 43 & 58 & 69 & 84 & 98 & 127 & 203 & 243 & 329 & 470 & & \\
\hline & \multicolumn{19}{|c|}{ Expansion } \\
\hline 1050_2 & 0.000 & 0.074 & 0.140 & 0.162 & 0.176 & 0.181 & 0.190 & 0.193 & 0.197 & 0.202 & 0.210 & 0.213 & 0.218 & 0.224 & 0.226 & 0.232 & 0.230 & & \\
\hline 1050_2 & 0.000 & 0.070 & 0.134 & 0.158 & 0.172 & 0.177 & 0.187 & 0.188 & 0.191 & 0.195 & 0.204 & 0.205 & 0.210 & 0.217 & 0.221 & 0.226 & 0.233 & & \\
\hline \multirow[t]{4}{*}{ Average } & 0.000 & 0.072 & 0.137 & 0.160 & 0.174 & 0.179 & 0.189 & 0.190 & 0.194 & 0.199 & 0.207 & 0.209 & 0.214 & 0.220 & 0.224 & 0.229 & 0.231 & & \\
\hline & \multicolumn{19}{|c|}{ Day } \\
\hline & 0 & 1 & 7 & 14 & 21 & 28 & 35 & 43 & 58 & 69 & 84 & 98 & 127 & 203 & 243 & 329 & 470 & & \\
\hline & \multicolumn{19}{|c|}{ Expansion } \\
\hline 1200_2 & 0.000 & 0.045 & 0.102 & 0.126 & 0.140 & 0.146 & 0.155 & 0.154 & 0.165 & 0.170 & 0.178 & 0.187 & 0.190 & 0.197 & 0.201 & 0.207 & 0.206 & & \\
\hline 1200_2 & 0.000 & 0.044 & 0.102 & 0.126 & 0.141 & 0.146 & 0.156 & 0.160 & 0.166 & 0.170 & 0.178 & 0.179 & 0.186 & 0.197 & 0.208 & 0.207 & 0.206 & & \\
\hline \multirow[t]{4}{*}{ Average } & 0.000 & 0.044 & 0.102 & 0.126 & 0.140 & 0.146 & 0.156 & 0.157 & 0.165 & 0.170 & 0.178 & 0.183 & 0.188 & 0.197 & 0.204 & 0.207 & 0.206 & & \\
\hline & \multicolumn{19}{|c|}{ Day } \\
\hline & $\mathbf{0}$ & 1 & 3 & 7 & 14 & 21 & 28 & 35 & 42 & 50 & 65 & 76 & 91 & 105 & 134 & 210 & 250 & 336 & 477 \\
\hline & \multicolumn{19}{|c|}{ Expansion } \\
\hline 750_4 & 0.000 & 0.049 & 0.061 & 0.081 & 0.098 & 0.099 & 0.106 & 0.106 & 0.111 & 0.113 & 0.110 & 0.117 & 0.120 & 0.120 & 0.122 & 0.126 & 0.128 & 0.130 & 0.134 \\
\hline 750_4 & 0.000 & 0.053 & 0.064 & 0.086 & 0.102 & 0.105 & 0.112 & 0.113 & 0.119 & 0.119 & 0.115 & 0.121 & 0.124 & 0.124 & 0.126 & 0.130 & 0.131 & 0.134 & 0.139 \\
\hline $750 \_4$ & 0.000 & 0.050 & 0.062 & 0.082 & 0.099 & 0.103 & 0.108 & 0.106 & 0.113 & 0.114 & 0.111 & 0.117 & 0.122 & 0.120 & 0.123 & 0.124 & 0.126 & 0.130 & 0.136 \\
\hline
\end{tabular}




\begin{tabular}{|c|c|c|c|c|c|c|c|c|c|c|c|c|c|c|c|c|c|c|c|}
\hline Average & 0.000 & 0.051 & 0.062 & 0.083 & 0.100 & 0.102 & 0.109 & 0.108 & 0.114 & 0.115 & 0.112 & 0.118 & 0.122 & 0.121 & 0.124 & 0.127 & 0.129 & 0.131 & 0.136 \\
\hline & \multicolumn{19}{|c|}{ Day } \\
\hline & 0 & 1 & 6 & 13 & 20 & 26 & 33 & 41 & 49 & 64 & 75 & 90 & 104 & 133 & 209 & 249 & 335 & 476 & \\
\hline & \multicolumn{19}{|c|}{ Expansion } \\
\hline $900 \_4$ & 0.000 & 0.054 & 0.094 & 0.132 & 0.142 & 0.151 & 0.154 & 0.162 & 0.165 & 0.164 & 0.173 & 0.179 & 0.178 & 0.185 & 0.192 & 0.196 & 0.202 & 0.198 & \\
\hline 900_4 & 0.000 & 0.055 & 0.090 & 0.129 & 0.138 & 0.150 & 0.148 & 0.158 & 0.162 & 0.162 & 0.168 & 0.175 & 0.177 & 0.181 & 0.188 & 0.192 & 0.198 & 0.194 & \\
\hline $900 \_4$ & 0.000 & 0.053 & 0.092 & 0.130 & 0.141 & 0.150 & 0.153 & 0.160 & 0.163 & 0.163 & 0.172 & 0.176 & 0.176 & 0.185 & 0.187 & 0.191 & 0.197 & 0.194 & \\
\hline \multirow[t]{4}{*}{ Average } & 0.000 & 0.054 & 0.092 & 0.130 & 0.140 & 0.150 & 0.152 & 0.160 & 0.163 & 0.163 & 0.171 & 0.177 & 0.177 & 0.183 & 0.189 & 0.193 & 0.199 & 0.195 & \\
\hline & \multicolumn{19}{|c|}{ Day } \\
\hline & 0 & 1 & 4 & 11 & 18 & 26 & 41 & 52 & 67 & 81 & 110 & 168 & 226 & 312 & 454 & & & & \\
\hline & \multicolumn{19}{|c|}{ Expansion } \\
\hline 1050_4 & 0.000 & 0.063 & 0.075 & 0.110 & 0.129 & 0.142 & 0.151 & 0.159 & 0.168 & 0.170 & 0.178 & 0.190 & 0.195 & 0.203 & 0.206 & & & & \\
\hline 1050_4 & 0.000 & 0.062 & 0.075 & 0.111 & 0.130 & 0.141 & 0.152 & 0.160 & 0.170 & 0.172 & 0.178 & 0.191 & 0.196 & 0.203 & 0.207 & & & & \\
\hline \multirow[t]{4}{*}{ Average } & 0.000 & 0.063 & 0.075 & 0.110 & 0.129 & 0.141 & 0.152 & 0.160 & 0.169 & 0.171 & 0.178 & 0.191 & 0.196 & 0.203 & 0.206 & & & & \\
\hline & \multicolumn{19}{|c|}{ Day } \\
\hline & $\mathbf{0}$ & 1 & 7 & 14 & 21 & 28 & 35 & 43 & 58 & 69 & 84 & 98 & 127 & 203 & 243 & 329 & 470 & & \\
\hline & \multicolumn{19}{|c|}{ Expansion } \\
\hline 1200_4 & 0.000 & 0.047 & 0.056 & 0.061 & 0.070 & 0.072 & 0.080 & 0.073 & 0.084 & 0.088 & 0.094 & 0.095 & 0.100 & 0.110 & 0.114 & 0.122 & 0.121 & & \\
\hline 1200_4 & 0.000 & 0.025 & 0.047 & 0.060 & 0.068 & 0.070 & 0.077 & 0.080 & 0.082 & 0.085 & 0.094 & 0.094 & 0.102 & 0.109 & 0.114 & 0.120 & 0.120 & & \\
\hline Average & 0.000 & 0.036 & 0.051 & 0.060 & 0.069 & 0.071 & 0.078 & 0.076 & 0.083 & 0.086 & 0.094 & 0.095 & 0.101 & 0.110 & 0.114 & 0.121 & 0.120 & & \\
\hline
\end{tabular}


Water expansion - Class-F fly ash/45 $\mu \mathrm{m}$

\begin{tabular}{|c|c|c|c|c|c|c|c|c|c|c|c|c|c|c|c|c|c|c|c|}
\hline \multirow{2}{*}{ 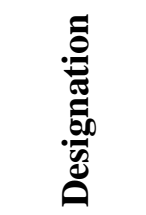 } & \multicolumn{19}{|c|}{ Day } \\
\hline & 0 & $\mathbf{1}$ & 3 & 7 & 14 & 21 & 28 & 35 & 42 & 50 & 65 & 76 & 91 & 105 & 134 & 210 & 250 & 336 & 477 \\
\hline & \multicolumn{19}{|c|}{ Expansion } \\
\hline 750_1 & 0.000 & 0.034 & 0.041 & 0.046 & 0.053 & 0.052 & 0.055 & 0.054 & 0.058 & 0.058 & 0.052 & 0.059 & 0.062 & 0.058 & 0.059 & 0.059 & 0.060 & 0.062 & 0.062 \\
\hline 750_1 & 0.000 & 0.036 & 0.040 & 0.048 & 0.055 & 0.054 & 0.058 & 0.055 & 0.059 & 0.060 & 0.055 & 0.060 & 0.061 & 0.059 & 0.061 & 0.060 & 0.062 & 0.063 & 0.066 \\
\hline 750_1 & 0.000 & 0.034 & 0.042 & 0.046 & 0.054 & 0.054 & 0.057 & 0.054 & 0.058 & 0.058 & 0.053 & 0.058 & 0.061 & 0.058 & 0.060 & 0.058 & 0.058 & 0.060 & 0.063 \\
\hline \multirow[t]{4}{*}{ Average } & 0.000 & 0.035 & 0.041 & 0.047 & 0.054 & 0.053 & 0.057 & 0.054 & 0.058 & 0.059 & 0.053 & 0.059 & 0.061 & 0.058 & 0.060 & 0.059 & 0.060 & 0.061 & 0.063 \\
\hline & \multicolumn{19}{|c|}{ Day } \\
\hline & 0 & 1 & 6 & 13 & 20 & 26 & 33 & 41 & 49 & 64 & 75 & 90 & 104 & 133 & 209 & 249 & 335 & 476 & \\
\hline & \multicolumn{19}{|c|}{ Expansion } \\
\hline 900_1 & 0.000 & 0.038 & 0.053 & 0.068 & 0.070 & 0.075 & 0.073 & 0.079 & 0.080 & 0.074 & 0.081 & 0.083 & 0.082 & 0.084 & 0.086 & 0.088 & 0.091 & 0.092 & \\
\hline 900_1 & 0.000 & $\mathbf{0 . 0 3 7}$ & 0.053 & 0.068 & 0.070 & 0.075 & 0.073 & 0.076 & 0.079 & 0.075 & 0.082 & 0.084 & 0.083 & 0.084 & 0.086 & 0.086 & 0.090 & 0.092 & \\
\hline 900_1 & 0.000 & 0.036 & 0.051 & 0.068 & 0.070 & 0.074 & 0.073 & 0.077 & 0.078 & 0.074 & 0.080 & 0.083 & 0.081 & 0.084 & 0.084 & 0.086 & 0.088 & 0.092 & \\
\hline \multirow[t]{4}{*}{ Average } & 0.000 & 0.037 & 0.052 & 0.068 & 0.070 & 0.075 & 0.073 & 0.077 & 0.079 & 0.074 & 0.081 & 0.083 & 0.082 & 0.084 & 0.086 & 0.087 & 0.090 & 0.092 & \\
\hline & \multicolumn{19}{|c|}{ Day } \\
\hline & 0 & 1 & 7 & 14 & 21 & 28 & 35 & 43 & 58 & 69 & 84 & 98 & 127 & 203 & 243 & 329 & 470 & & \\
\hline & \multicolumn{19}{|c|}{ Expansion } \\
\hline
\end{tabular}




\begin{tabular}{|c|c|c|c|c|c|c|c|c|c|c|c|c|c|c|c|c|c|c|c|}
\hline 1050_1 & 0.000 & 0.066 & 0.113 & 0.128 & 0.138 & 0.138 & 0.146 & 0.138 & 0.150 & 0.152 & 0.157 & 0.158 & 0.161 & 0.164 & 0.167 & 0.170 & 0.169 & & \\
\hline 1050_1 & 0.000 & 0.067 & 0.112 & 0.126 & 0.135 & 0.137 & 0.144 & 0.146 & 0.146 & 0.150 & 0.155 & 0.155 & 0.159 & 0.162 & 0.165 & 0.167 & 0.169 & & \\
\hline \multirow[t]{4}{*}{ Average } & 0.000 & 0.066 & 0.112 & 0.127 & 0.136 & 0.138 & 0.145 & 0.142 & 0.148 & 0.151 & 0.156 & 0.156 & 0.160 & 0.163 & 0.166 & 0.168 & 0.169 & & \\
\hline & \multicolumn{19}{|c|}{ Day } \\
\hline & $\mathbf{0}$ & 1 & 7 & 14 & 21 & 28 & 35 & 43 & 58 & 69 & 84 & 98 & 127 & 203 & 243 & 329 & 470 & & \\
\hline & \multicolumn{19}{|c|}{ Expansion } \\
\hline 1200_1 & 0.000 & 0.060 & 0.126 & 0.150 & 0.164 & 0.169 & 0.177 & 0.173 & 0.184 & 0.189 & 0.195 & 0.196 & 0.203 & 0.210 & 0.214 & 0.218 & 0.215 & & \\
\hline 1200_1 & 0.000 & 0.061 & 0.129 & 0.151 & 0.166 & 0.170 & 0.179 & 0.183 & 0.185 & 0.190 & 0.198 & 0.198 & 0.203 & 0.211 & 0.214 & 0.218 & 0.217 & & \\
\hline \multirow[t]{4}{*}{ Average } & 0.000 & 0.060 & 0.128 & 0.150 & 0.165 & 0.169 & 0.178 & 0.178 & 0.184 & 0.190 & 0.196 & 0.197 & 0.203 & 0.210 & 0.214 & 0.218 & 0.216 & & \\
\hline & \multicolumn{19}{|c|}{ Day } \\
\hline & $\mathbf{0}$ & 1 & 6 & 13 & 20 & 26 & 33 & 41 & 49 & 64 & 75 & 90 & 104 & 133 & 209 & 249 & 335 & 476 & \\
\hline & \multicolumn{19}{|c|}{ Expansion } \\
\hline 750_2 & 0.000 & 0.035 & 0.048 & 0.058 & 0.059 & 0.063 & 0.062 & 0.066 & 0.066 & 0.062 & 0.067 & 0.070 & 0.067 & 0.070 & 0.071 & 0.073 & 0.074 & 0.077 & \\
\hline 750_2 & 0.000 & 0.035 & 0.049 & 0.059 & 0.059 & 0.064 & 0.062 & 0.067 & 0.069 & 0.063 & 0.068 & 0.071 & 0.068 & 0.071 & 0.071 & 0.073 & 0.075 & 0.078 & \\
\hline 750_2 & 0.000 & 0.034 & 0.046 & 0.058 & 0.061 & 0.063 & 0.061 & 0.064 & 0.066 & 0.063 & 0.067 & 0.072 & 0.068 & 0.070 & 0.069 & 0.071 & 0.074 & 0.078 & \\
\hline \multirow[t]{4}{*}{ Average } & 0.000 & 0.035 & 0.048 & 0.058 & 0.060 & 0.063 & 0.061 & 0.066 & 0.067 & 0.063 & 0.067 & 0.071 & 0.068 & 0.070 & 0.070 & 0.072 & 0.074 & 0.078 & \\
\hline & \multicolumn{19}{|c|}{ Day } \\
\hline & 0 & 1 & 6 & 13 & 20 & 26 & 33 & 41 & 49 & 64 & 75 & 90 & 104 & 133 & 209 & 249 & 335 & 476 & \\
\hline & \multicolumn{19}{|c|}{ Expansion } \\
\hline 900_2 & 0.000 & 0.054 & 0.084 & 0.112 & 0.116 & 0.124 & 0.124 & 0.130 & 0.132 & 0.130 & 0.138 & 0.142 & 0.139 & 0.143 & 0.147 & 0.150 & 0.153 & 0.157 & \\
\hline
\end{tabular}




\begin{tabular}{|c|c|c|c|c|c|c|c|c|c|c|c|c|c|c|c|c|c|c|}
\hline 900_2 & 0.000 & 0.054 & 0.086 & 0.113 & 0.118 & 0.126 & 0.126 & 0.130 & 0.133 & 0.130 & 0.139 & 0.144 & 0.142 & 0.145 & 0.148 & 0.151 & 0.154 & 0.159 \\
\hline 900_2 & 0.000 & 0.054 & 0.084 & 0.111 & 0.118 & 0.125 & 0.124 & 0.131 & 0.132 & 0.129 & 0.136 & 0.140 & 0.140 & 0.144 & 0.146 & 0.150 & 0.153 & 0.159 \\
\hline Average & 0.000 & 0.054 & 0.085 & 0.112 & 0.117 & 0.125 & 0.125 & 0.131 & 0.132 & 0.130 & 0.138 & 0.142 & 0.140 & 0.144 & 0.147 & 0.150 & 0.153 & 0.158 \\
\hline & \multicolumn{18}{|c|}{ Day } \\
\hline & 0 & 1 & 4 & 11 & 18 & 26 & 41 & 52 & 67 & 81 & 110 & 168 & 226 & 312 & 454 & & & \\
\hline & \multicolumn{18}{|c|}{ Expansion } \\
\hline 1050_2 & 0.000 & 0.072 & 0.086 & 0.122 & 0.139 & 0.148 & 0.158 & 0.166 & 0.176 & 0.176 & 0.182 & 0.192 & 0.196 & 0.202 & 0.202 & & & \\
\hline 1050_2 & 0.000 & 0.070 & 0.083 & 0.119 & 0.136 & 0.150 & 0.156 & 0.162 & 0.168 & 0.172 & 0.177 & 0.188 & 0.192 & 0.198 & 0.199 & & & \\
\hline \multirow[t]{4}{*}{ Average } & 0.000 & 0.071 & 0.085 & 0.121 & 0.138 & 0.149 & 0.157 & 0.164 & 0.172 & 0.174 & 0.179 & 0.190 & 0.194 & 0.200 & 0.201 & & & \\
\hline & \multicolumn{18}{|c|}{ Day } \\
\hline & $\mathbf{0}$ & 1 & 7 & 14 & 21 & 28 & 35 & 43 & 58 & 69 & 84 & 98 & 127 & 203 & 243 & 329 & 470 & \\
\hline & \multicolumn{18}{|c|}{ Expansion } \\
\hline 1200_2 & 0.000 & 0.067 & 0.105 & 0.136 & 0.152 & 0.158 & 0.171 & 0.168 & 0.179 & 0.185 & 0.194 & 0.196 & 0.202 & 0.214 & 0.218 & 0.225 & 0.225 & \\
\hline 1200_2 & 0.000 & 0.066 & 0.105 & 0.135 & 0.152 & 0.158 & 0.170 & 0.174 & 0.178 & 0.183 & 0.194 & 0.196 & 0.202 & 0.213 & 0.218 & 0.225 & 0.225 & \\
\hline \multirow[t]{4}{*}{ Average } & 0.000 & 0.067 & 0.105 & 0.136 & 0.152 & 0.158 & 0.171 & 0.171 & 0.179 & 0.184 & 0.194 & 0.196 & 0.202 & 0.213 & 0.218 & 0.225 & 0.225 & \\
\hline & \multicolumn{18}{|c|}{ Day } \\
\hline & $\mathbf{0}$ & 1 & 6 & 13 & 20 & 26 & 33 & 41 & 49 & 64 & 75 & 90 & 104 & 133 & 209 & 249 & 335 & 476 \\
\hline & \multicolumn{18}{|c|}{ Expansion } \\
\hline 750_4 & 0.000 & 0.048 & 0.074 & 0.090 & 0.093 & 0.098 & 0.098 & 0.105 & 0.106 & 0.101 & 0.109 & 0.113 & 0.110 & 0.113 & 0.118 & 0.118 & 0.121 & 0.124 \\
\hline 750_4 & 0.000 & 0.049 & 0.074 & 0.090 & 0.093 & 0.099 & 0.097 & 0.104 & 0.106 & 0.101 & 0.108 & 0.111 & 0.109 & 0.111 & 0.115 & 0.117 & 0.120 & 0.125 \\
\hline
\end{tabular}




\begin{tabular}{|c|c|c|c|c|c|c|c|c|c|c|c|c|c|c|c|c|c|c|}
\hline 750_4 & 0.000 & 0.050 & 0.075 & 0.092 & 0.094 & 0.100 & 0.100 & 0.104 & 0.104 & 0.102 & 0.109 & 0.112 & 0.109 & 0.113 & 0.116 & 0.118 & 0.120 & 0.125 \\
\hline Average & 0.000 & 0.049 & 0.074 & 0.091 & 0.093 & 0.099 & 0.098 & 0.104 & 0.105 & 0.101 & 0.109 & 0.112 & 0.109 & 0.112 & 0.117 & 0.118 & 0.120 & 0.125 \\
\hline & \multicolumn{18}{|c|}{ Day } \\
\hline & 0 & 1 & 6 & 13 & 20 & 26 & 33 & 41 & 49 & 64 & 75 & 90 & 104 & 133 & 209 & 249 & 335 & 476 \\
\hline & \multicolumn{18}{|c|}{ Expansion } \\
\hline 900_4 & 0.000 & 0.054 & 0.100 & 0.139 & 0.150 & 0.159 & 0.163 & 0.170 & 0.175 & 0.173 & 0.182 & 0.187 & 0.188 & 0.194 & 0.201 & 0.202 & 0.208 & 0.214 \\
\hline 900_4 & 0.000 & 0.056 & 0.102 & 0.142 & 0.153 & 0.164 & 0.166 & 0.174 & 0.178 & 0.177 & 0.185 & 0.190 & 0.190 & 0.194 & 0.203 & 0.207 & 0.212 & 0.218 \\
\hline 900_4 & 0.000 & 0.054 & 0.100 & 0.142 & 0.152 & 0.163 & 0.165 & 0.173 & 0.176 & 0.175 & 0.184 & 0.190 & 0.190 & 0.194 & 0.202 & 0.206 & 0.210 & 0.218 \\
\hline \multirow[t]{4}{*}{ Average } & 0.000 & 0.055 & 0.101 & 0.141 & 0.152 & 0.162 & 0.165 & 0.172 & 0.176 & 0.175 & 0.183 & 0.189 & 0.189 & 0.194 & 0.202 & 0.205 & 0.210 & 0.217 \\
\hline & \multicolumn{18}{|c|}{ Day } \\
\hline & $\mathbf{0}$ & $\mathbf{1}$ & 7 & 14 & 21 & 28 & 35 & 43 & 58 & 69 & 84 & 98 & 127 & 203 & 243 & 329 & 470 & \\
\hline & \multicolumn{18}{|c|}{ Expansion } \\
\hline 1050_4 & 0.000 & 0.046 & 0.116 & 0.144 & 0.160 & 0.166 & 0.178 & 0.174 & 0.188 & 0.193 & 0.202 & 0.206 & 0.211 & 0.222 & 0.226 & 0.234 & 0.234 & \\
\hline 1050_4 & 0.000 & 0.046 & 0.115 & 0.144 & 0.159 & 0.167 & 0.178 & 0.182 & 0.187 & 0.192 & 0.203 & 0.203 & 0.212 & 0.222 & 0.227 & 0.234 & 0.234 & \\
\hline \multirow[t]{4}{*}{ Average } & 0.000 & 0.046 & 0.116 & 0.144 & 0.160 & 0.167 & 0.178 & 0.178 & 0.188 & 0.192 & 0.202 & 0.204 & 0.212 & 0.222 & 0.227 & 0.234 & 0.234 & \\
\hline & \multicolumn{18}{|c|}{ Day } \\
\hline & $\mathbf{0}$ & 1 & 7 & 14 & 21 & 28 & 35 & 43 & 58 & 69 & 84 & 98 & 127 & 203 & 243 & 329 & 470 & \\
\hline & \multicolumn{18}{|c|}{ Expansion } \\
\hline 1200_4 & 0.000 & 0.040 & 0.060 & 0.078 & 0.089 & 0.092 & 0.102 & 0.098 & 0.107 & 0.113 & 0.122 & 0.122 & 0.131 & 0.138 & 0.144 & 0.150 & 0.153 & \\
\hline 1200_4 & 0.000 & 0.042 & 0.062 & 0.079 & 0.090 & 0.095 & 0.105 & 0.108 & 0.110 & 0.115 & 0.123 & 0.123 & 0.130 & 0.141 & 0.146 & 0.153 & 0.154 & \\
\hline
\end{tabular}




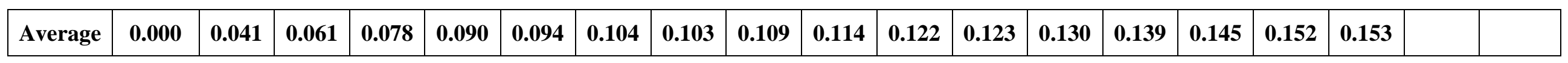

Water expansion - Class-CI fly ash/100 $\mu \mathrm{m}$

\begin{tabular}{|c|c|c|c|c|c|c|c|c|c|c|c|c|c|c|c|c|c|}
\hline \multirow{3}{*}{ 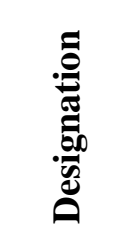 } & \multicolumn{17}{|c|}{ Day } \\
\hline & $\mathbf{0}$ & 1 & 6 & 13 & 20 & 27 & 34 & 42 & 57 & 68 & 83 & 97 & 126 & 202 & 242 & 328 & 469 \\
\hline & \multicolumn{17}{|c|}{ Expansion } \\
\hline 750_1 & 0.000 & 0.037 & $\begin{array}{c}0.04 \\
3\end{array}$ & $\begin{array}{c}\mathbf{0 . 0 4} \\
3\end{array}$ & $\begin{array}{c}0.04 \\
6\end{array}$ & $\begin{array}{c}\mathbf{0 . 0 3} \\
9\end{array}$ & $\begin{array}{c}0.04 \\
3\end{array}$ & $\begin{array}{c}0.04 \\
4\end{array}$ & $\begin{array}{c}0.04 \\
2\end{array}$ & $\begin{array}{c}0.04 \\
2\end{array}$ & $\begin{array}{c}0.04 \\
6\end{array}$ & $\begin{array}{c}0.04 \\
3\end{array}$ & $\begin{array}{c}0.04 \\
6\end{array}$ & $\begin{array}{c}0.04 \\
6\end{array}$ & $\begin{array}{c}0.04 \\
6\end{array}$ & $\begin{array}{c}0.04 \\
7\end{array}$ & 0.046 \\
\hline 750_1 & 0.000 & 0.034 & $\begin{array}{c}0.03 \\
9 \\
\end{array}$ & $\begin{array}{c}0.03 \\
8\end{array}$ & $\begin{array}{c}0.04 \\
0\end{array}$ & $\begin{array}{c}0.03 \\
6\end{array}$ & $\begin{array}{c}0.03 \\
9\end{array}$ & $\begin{array}{c}0.03 \\
1\end{array}$ & $\begin{array}{c}0.03 \\
8\end{array}$ & $\begin{array}{c}0.03 \\
8\end{array}$ & $\begin{array}{c}0.04 \\
3\end{array}$ & $\begin{array}{c}0.04 \\
0\end{array}$ & $\begin{array}{c}0.04 \\
3\end{array}$ & $\begin{array}{c}0.04 \\
4\end{array}$ & $\begin{array}{c}0.04 \\
4\end{array}$ & $\begin{array}{c}0.04 \\
7\end{array}$ & 0.046 \\
\hline \multirow[t]{4}{*}{$\begin{array}{c}\text { Averag } \\
\text { e }\end{array}$} & 0.000 & 0.035 & $\begin{array}{c}0.04 \\
1 \\
\end{array}$ & $\begin{array}{c}\mathbf{0 . 0 4} \\
\mathbf{0} \\
\end{array}$ & $\begin{array}{c}0.04 \\
3 \\
\end{array}$ & $\begin{array}{c}0.03 \\
8 \\
\end{array}$ & $\begin{array}{c}0.04 \\
1 \\
\end{array}$ & $\begin{array}{c}0.03 \\
8 \\
\end{array}$ & $\begin{array}{c}\mathbf{0 . 0 4} \\
\mathbf{0} \\
\end{array}$ & $\begin{array}{c}0.04 \\
0 \\
\end{array}$ & $\begin{array}{c}0.04 \\
5 \\
\end{array}$ & $\begin{array}{c}0.04 \\
2 \\
\end{array}$ & $\begin{array}{c}0.04 \\
5 \\
\end{array}$ & $\begin{array}{c}0.04 \\
5 \\
\end{array}$ & $\begin{array}{c}0.04 \\
5 \\
\end{array}$ & $\begin{array}{c}0.04 \\
7 \\
\end{array}$ & 0.046 \\
\hline & \multicolumn{17}{|c|}{ Day } \\
\hline & $\mathbf{0}$ & 1 & 3 & 10 & 17 & 24 & 31 & 39 & 54 & 65 & 80 & 94 & 123 & 199 & 239 & 325 & 466 \\
\hline & \multicolumn{17}{|c|}{ Expansion } \\
\hline 900_1 & 0.000 & 0.062 & $\begin{array}{c}0.08 \\
2\end{array}$ & $\begin{array}{c}0.09 \\
1 \\
\end{array}$ & $\begin{array}{c}0.09 \\
5\end{array}$ & $\begin{array}{c}0.09 \\
2\end{array}$ & $\begin{array}{c}0.09 \\
7\end{array}$ & $\begin{array}{c}0.09 \\
0\end{array}$ & $\begin{array}{c}0.09 \\
9\end{array}$ & $\begin{array}{c}0.09 \\
9\end{array}$ & $\begin{array}{c}0.10 \\
6\end{array}$ & $\begin{array}{c}0.10 \\
3\end{array}$ & $\begin{array}{c}0.10 \\
8\end{array}$ & $\begin{array}{c}0.11 \\
1\end{array}$ & $\begin{array}{c}0.11 \\
2\end{array}$ & $\begin{array}{c}0.11 \\
6\end{array}$ & 0.116 \\
\hline 900_1 & 0.000 & 0.046 & $\begin{array}{c}0.06 \\
5\end{array}$ & $\begin{array}{c}\mathbf{0 . 0 7} \\
\mathbf{3}\end{array}$ & $\begin{array}{c}0.07 \\
7\end{array}$ & $\begin{array}{c}0.07 \\
4\end{array}$ & $\begin{array}{c}\mathbf{0 . 0 7} \\
9\end{array}$ & $\begin{array}{c}\mathbf{0 . 0 7} \\
3\end{array}$ & $\begin{array}{c}\mathbf{0 . 0 8} \\
0\end{array}$ & $\begin{array}{c}0.08 \\
1\end{array}$ & $\begin{array}{c}0.08 \\
6\end{array}$ & $\begin{array}{c}0.08 \\
6\end{array}$ & $\begin{array}{c}\mathbf{0 . 0 9} \\
0\end{array}$ & $\begin{array}{c}0.09 \\
4\end{array}$ & $\begin{array}{c}0.09 \\
5\end{array}$ & $\begin{array}{c}0.09 \\
8\end{array}$ & 0.098 \\
\hline \multirow[t]{3}{*}{$\begin{array}{c}\text { Averag } \\
\text { e }\end{array}$} & 0.000 & 0.054 & $\begin{array}{c}\mathbf{0 . 0 7} \\
\mathbf{3} \\
\end{array}$ & $\begin{array}{c}0.08 \\
2 \\
\end{array}$ & $\begin{array}{c}0.08 \\
6 \\
\end{array}$ & $\begin{array}{c}0.08 \\
3 \\
\end{array}$ & $\begin{array}{c}0.08 \\
8 \\
\end{array}$ & $\begin{array}{c}0.08 \\
1\end{array}$ & $\begin{array}{c}\mathbf{0 . 0 9} \\
0 \\
\end{array}$ & $\begin{array}{c}\mathbf{0 . 0 9} \\
\mathbf{0}\end{array}$ & $\begin{array}{c}0.09 \\
6 \\
\end{array}$ & $\begin{array}{c}0.09 \\
4 \\
\end{array}$ & $\begin{array}{c}0.09 \\
9 \\
\end{array}$ & $\begin{array}{c}0.10 \\
2 \\
\end{array}$ & $\begin{array}{c}0.10 \\
4 \\
\end{array}$ & $\begin{array}{c}0.10 \\
7 \\
\end{array}$ & 0.107 \\
\hline & \multicolumn{17}{|c|}{ Day } \\
\hline & 0 & 1 & 3 & 10 & 17 & 25 & 40 & 51 & 66 & 80 & 109 & 185 & 225 & 311 & 452 & & \\
\hline
\end{tabular}

cccxviii 


\begin{tabular}{|c|c|c|c|c|c|c|c|c|c|c|c|c|c|c|c|c|c|}
\hline & \multicolumn{17}{|c|}{ Expansion } \\
\hline 1050_1 & 0.000 & 0.063 & $\begin{array}{c}0.07 \\
1\end{array}$ & $\begin{array}{c}0.08 \\
3\end{array}$ & $\begin{array}{c}0.09 \\
4\end{array}$ & $\begin{array}{c}0.09 \\
8\end{array}$ & $\begin{array}{c}0.09 \\
7\end{array}$ & $\begin{array}{c}0.10 \\
4\end{array}$ & $\begin{array}{c}0.10 \\
9\end{array}$ & $\begin{array}{c}0.10 \\
9\end{array}$ & $\begin{array}{c}0.11 \\
4\end{array}$ & $\begin{array}{c}0.12 \\
3\end{array}$ & $\begin{array}{c}0.12 \\
6\end{array}$ & $\begin{array}{c}0.12 \\
9\end{array}$ & $\begin{array}{c}0.13 \\
0\end{array}$ & & \\
\hline 1050_1 & 0.000 & 0.064 & $\begin{array}{c}0.07 \\
4\end{array}$ & $\begin{array}{c}0.10 \\
5\end{array}$ & $\begin{array}{c}0.09 \\
4\end{array}$ & $\begin{array}{c}0.09 \\
8\end{array}$ & $\begin{array}{c}0.09 \\
8\end{array}$ & $\begin{array}{c}0.10 \\
5\end{array}$ & $\begin{array}{c}0.11 \\
1\end{array}$ & $\begin{array}{c}0.11 \\
1\end{array}$ & $\begin{array}{c}0.11 \\
9\end{array}$ & $\begin{array}{c}0.12 \\
5\end{array}$ & $\begin{array}{c}0.12 \\
7\end{array}$ & $\begin{array}{c}0.13 \\
1\end{array}$ & $\begin{array}{c}0.13 \\
2 \\
\end{array}$ & & \\
\hline \multirow[t]{4}{*}{$\begin{array}{c}\text { Averag } \\
\text { e } \\
\end{array}$} & 0.000 & 0.064 & $\begin{array}{c}0.07 \\
2 \\
\end{array}$ & $\begin{array}{c}0.09 \\
4 \\
\end{array}$ & $\begin{array}{c}0.09 \\
4 \\
\end{array}$ & $\begin{array}{c}0.09 \\
8 \\
\end{array}$ & $\begin{array}{c}0.09 \\
8 \\
\end{array}$ & $\begin{array}{c}0.10 \\
4 \\
\end{array}$ & $\begin{array}{c}0.11 \\
0\end{array}$ & $\begin{array}{c}0.11 \\
0\end{array}$ & $\begin{array}{c}0.11 \\
6\end{array}$ & $\begin{array}{c}0.12 \\
4 \\
\end{array}$ & $\begin{array}{c}0.12 \\
7 \\
\end{array}$ & $\begin{array}{c}0.13 \\
0\end{array}$ & $\begin{array}{c}0.13 \\
1 \\
\end{array}$ & & \\
\hline & \multicolumn{17}{|c|}{ Day } \\
\hline & $\mathbf{0}$ & 1 & 3 & 10 & 17 & 25 & 40 & 51 & 66 & 80 & 109 & 185 & 225 & 311 & 452 & & \\
\hline & \multicolumn{17}{|c|}{ Expansion } \\
\hline 1200_1 & 0.000 & 0.086 & $\begin{array}{c}\mathbf{0 . 1 0} \\
\mathbf{0}\end{array}$ & $\begin{array}{c}0.12 \\
2\end{array}$ & $\begin{array}{c}0.13 \\
8\end{array}$ & $\begin{array}{c}0.14 \\
2\end{array}$ & $\begin{array}{c}0.14 \\
6\end{array}$ & $\begin{array}{c}0.15 \\
4\end{array}$ & $\begin{array}{c}0.16 \\
3\end{array}$ & $\begin{array}{c}0.16 \\
5\end{array}$ & $\begin{array}{c}0.17 \\
5\end{array}$ & $\begin{array}{c}0.18 \\
6\end{array}$ & $\begin{array}{c}\mathbf{0 . 1 9} \\
\mathbf{0}\end{array}$ & $\begin{array}{c}0.19 \\
8\end{array}$ & $\begin{array}{c}0.20 \\
1\end{array}$ & & \\
\hline 1200_1 & 0.000 & 0.087 & $\begin{array}{c}0.10 \\
5 \\
\end{array}$ & $\begin{array}{c}0.11 \\
6 \\
\end{array}$ & $\begin{array}{c}0.12 \\
8 \\
\end{array}$ & $\begin{array}{c}0.13 \\
1 \\
\end{array}$ & $\begin{array}{c}0.13 \\
9 \\
\end{array}$ & $\begin{array}{c}0.14 \\
4 \\
\end{array}$ & $\begin{array}{c}0.15 \\
0\end{array}$ & $\begin{array}{c}0.15 \\
9\end{array}$ & $\begin{array}{c}0.16 \\
7 \\
\end{array}$ & $\begin{array}{c}0.17 \\
7 \\
\end{array}$ & $\begin{array}{c}0.18 \\
4 \\
\end{array}$ & $\begin{array}{c}0.19 \\
0\end{array}$ & $\begin{array}{c}0.19 \\
4 \\
\end{array}$ & & \\
\hline 1200_1 & 0.000 & 0.090 & $\begin{array}{c}\mathbf{0 . 1 0} \\
3\end{array}$ & $\begin{array}{c}0.12 \\
6\end{array}$ & $\begin{array}{c}0.13 \\
8\end{array}$ & $\begin{array}{c}0.14 \\
6\end{array}$ & $\begin{array}{c}0.15 \\
0\end{array}$ & $\begin{array}{c}0.15 \\
8\end{array}$ & $\begin{array}{c}0.16 \\
6\end{array}$ & $\begin{array}{c}0.16 \\
7\end{array}$ & $\begin{array}{c}0.17 \\
6\end{array}$ & $\begin{array}{c}0.18 \\
8\end{array}$ & $\begin{array}{c}0.19 \\
2\end{array}$ & $\begin{array}{c}0.20 \\
0\end{array}$ & $\begin{array}{c}0.20 \\
2\end{array}$ & & \\
\hline \multirow[t]{4}{*}{$\begin{array}{c}\text { Averag } \\
\text { e }\end{array}$} & 0.000 & 0.088 & $\begin{array}{c}0.10 \\
3 \\
\end{array}$ & $\begin{array}{c}0.12 \\
1 \\
\end{array}$ & $\begin{array}{c}0.13 \\
4 \\
\end{array}$ & $\begin{array}{c}0.14 \\
0\end{array}$ & $\begin{array}{c}0.14 \\
5 \\
\end{array}$ & $\begin{array}{c}0.15 \\
2 \\
\end{array}$ & $\begin{array}{c}0.15 \\
9 \\
\end{array}$ & $\begin{array}{c}0.16 \\
4 \\
\end{array}$ & $\begin{array}{c}0.17 \\
3 \\
\end{array}$ & $\begin{array}{c}0.18 \\
4 \\
\end{array}$ & $\begin{array}{c}0.18 \\
9 \\
\end{array}$ & $\begin{array}{c}0.19 \\
6 \\
\end{array}$ & $\begin{array}{c}0.19 \\
9 \\
\end{array}$ & & \\
\hline & \multicolumn{17}{|c|}{ Day } \\
\hline & 0 & 1 & 6 & 13 & 20 & 27 & 34 & 42 & 57 & 68 & 83 & 97 & 126 & 202 & 242 & 328 & 469 \\
\hline & \multicolumn{17}{|c|}{ Expansion } \\
\hline 750_2 & 0.000 & 0.056 & $\begin{array}{c}0.06 \\
7\end{array}$ & $\begin{array}{c}\mathbf{0 . 0 7} \\
\mathbf{0}\end{array}$ & $\begin{array}{c}\mathbf{0 . 0 7} \\
\mathbf{3}\end{array}$ & $\begin{array}{c}\mathbf{0 . 0 7} \\
\mathbf{0}\end{array}$ & $\begin{array}{c}\mathbf{0 . 0 7} \\
2\end{array}$ & $\begin{array}{c}0.06 \\
8\end{array}$ & $\begin{array}{c}0.07 \\
4\end{array}$ & $\begin{array}{c}0.07 \\
4 \\
\end{array}$ & $\begin{array}{c}0.08 \\
2\end{array}$ & $\begin{array}{c}\mathbf{0 . 0 7} \\
9\end{array}$ & $\begin{array}{c}0.08 \\
2\end{array}$ & $\begin{array}{c}0.08 \\
4\end{array}$ & $\begin{array}{c}0.08 \\
4\end{array}$ & $\begin{array}{c}0.08 \\
6\end{array}$ & 0.086 \\
\hline 750_2 & 0.000 & 0.054 & $\begin{array}{c}0.06 \\
6\end{array}$ & $\begin{array}{c}\mathbf{0 . 0 7} \\
\mathbf{0}\end{array}$ & $\begin{array}{c}\mathbf{0 . 0 7} \\
\mathbf{3}\end{array}$ & $\begin{array}{c}\mathbf{0 . 0 7} \\
\mathbf{0}\end{array}$ & $\begin{array}{c}\mathbf{0 . 0 7} \\
5\end{array}$ & $\begin{array}{c}0.06 \\
8\end{array}$ & $\begin{array}{c}0.07 \\
5\end{array}$ & $\begin{array}{c}0.07 \\
5\end{array}$ & $\begin{array}{c}0.08 \\
1\end{array}$ & $\begin{array}{c}\mathbf{0 . 0 7} \\
8\end{array}$ & $\begin{array}{c}0.08 \\
2\end{array}$ & $\begin{array}{c}0.08 \\
5\end{array}$ & $\begin{array}{c}0.08 \\
6\end{array}$ & $\begin{array}{c}0.08 \\
8\end{array}$ & 0.088 \\
\hline \multirow[t]{2}{*}{$\begin{array}{c}\text { Averag } \\
\text { e }\end{array}$} & 0.000 & 0.055 & $\begin{array}{c}0.06 \\
6 \\
\end{array}$ & $\begin{array}{c}\mathbf{0 . 0 7} \\
\mathbf{0}\end{array}$ & $\begin{array}{c}\mathbf{0 . 0 7} \\
\mathbf{3} \\
\end{array}$ & $\begin{array}{c}\mathbf{0 . 0 7} \\
\mathbf{0}\end{array}$ & $\begin{array}{c}0.07 \\
4 \\
\end{array}$ & $\begin{array}{c}0.06 \\
8 \\
\end{array}$ & $\begin{array}{c}0.07 \\
5 \\
\end{array}$ & $\begin{array}{c}0.07 \\
5 \\
\end{array}$ & $\begin{array}{c}0.08 \\
2 \\
\end{array}$ & $\begin{array}{c}\mathbf{0 . 0 7} \\
8 \\
\end{array}$ & $\begin{array}{c}0.08 \\
2 \\
\end{array}$ & $\begin{array}{c}0.08 \\
4 \\
\end{array}$ & $\begin{array}{c}0.08 \\
5 \\
\end{array}$ & $\begin{array}{c}0.08 \\
7 \\
\end{array}$ & 0.087 \\
\hline & \multicolumn{17}{|c|}{ Day } \\
\hline
\end{tabular}




\begin{tabular}{|c|c|c|c|c|c|c|c|c|c|c|c|c|c|c|c|c|c|}
\hline & $\mathbf{0}$ & 1 & 3 & 10 & 17 & 24 & 31 & 39 & 54 & 65 & 80 & 94 & 123 & 199 & 239 & 325 & 466 \\
\hline & \multicolumn{17}{|c|}{ Expansion } \\
\hline 900_2 & 0.000 & 0.035 & $\begin{array}{c}0.05 \\
5\end{array}$ & $\begin{array}{c}0.07 \\
8\end{array}$ & $\begin{array}{c}0.09 \\
1\end{array}$ & $\begin{array}{c}0.09 \\
8\end{array}$ & $\begin{array}{c}0.10 \\
6\end{array}$ & $\begin{array}{c}0.11 \\
2\end{array}$ & $\begin{array}{c}0.11 \\
4\end{array}$ & $\begin{array}{c}0.12 \\
0\end{array}$ & $\begin{array}{c}0.12 \\
9\end{array}$ & $\begin{array}{c}0.13 \\
1\end{array}$ & $\begin{array}{c}0.13 \\
2\end{array}$ & $\begin{array}{c}0.15 \\
0\end{array}$ & $\begin{array}{c}0.15 \\
8\end{array}$ & $\begin{array}{c}0.16 \\
5\end{array}$ & 0.170 \\
\hline 900_2 & 0.000 & 0.034 & $\begin{array}{c}0.05 \\
3\end{array}$ & $\begin{array}{c}0.07 \\
6\end{array}$ & $\begin{array}{c}\mathbf{0 . 0 9} \\
0\end{array}$ & $\begin{array}{c}0.09 \\
5\end{array}$ & $\begin{array}{c}\mathbf{0 . 1 0} \\
\mathbf{3}\end{array}$ & $\begin{array}{c}0.10 \\
9\end{array}$ & $\begin{array}{c}0.11 \\
3\end{array}$ & $\begin{array}{c}0.11 \\
8\end{array}$ & $\begin{array}{c}0.12 \\
8\end{array}$ & $\begin{array}{c}0.12 \\
9\end{array}$ & $\begin{array}{c}0.13 \\
8\end{array}$ & $\begin{array}{c}0.14 \\
9\end{array}$ & $\begin{array}{c}0.15 \\
5\end{array}$ & $\begin{array}{c}0.16 \\
2\end{array}$ & 0.166 \\
\hline \multirow[t]{4}{*}{$\begin{array}{c}\text { Averag } \\
\text { e }\end{array}$} & 0.000 & 0.035 & $\begin{array}{c}0.05 \\
4 \\
\end{array}$ & $\begin{array}{c}0.07 \\
7 \\
\end{array}$ & $\begin{array}{c}0.09 \\
1 \\
\end{array}$ & $\begin{array}{c}0.09 \\
6 \\
\end{array}$ & $\begin{array}{c}0.10 \\
4 \\
\end{array}$ & $\begin{array}{c}0.11 \\
0 \\
\end{array}$ & $\begin{array}{c}0.11 \\
3 \\
\end{array}$ & $\begin{array}{c}0.11 \\
9 \\
\end{array}$ & $\begin{array}{c}0.12 \\
8 \\
\end{array}$ & $\begin{array}{c}0.13 \\
0 \\
\end{array}$ & $\begin{array}{c}0.13 \\
5 \\
\end{array}$ & $\begin{array}{c}0.15 \\
0 \\
\end{array}$ & $\begin{array}{c}0.15 \\
6 \\
\end{array}$ & $\begin{array}{c}0.16 \\
3 \\
\end{array}$ & 0.168 \\
\hline & \multicolumn{17}{|c|}{ Day } \\
\hline & $\mathbf{0}$ & 1 & 3 & 10 & 17 & 24 & 31 & 39 & 54 & 65 & 80 & 94 & 123 & 199 & 239 & 325 & 466 \\
\hline & \multicolumn{17}{|c|}{ Expansion } \\
\hline $1050 \_2$ & 0.000 & 0.083 & $\begin{array}{c}0.11 \\
8\end{array}$ & $\begin{array}{c}0.13 \\
2\end{array}$ & $\begin{array}{c}0.13 \\
8\end{array}$ & $\begin{array}{c}0.13 \\
7 \\
\end{array}$ & $\begin{array}{c}0.14 \\
2\end{array}$ & $\begin{array}{c}0.13 \\
8\end{array}$ & $\begin{array}{c}0.14 \\
6\end{array}$ & $\begin{array}{c}0.14 \\
6\end{array}$ & $\begin{array}{c}0.15 \\
4\end{array}$ & $\begin{array}{c}0.15 \\
3\end{array}$ & $\begin{array}{c}0.15 \\
8\end{array}$ & $\begin{array}{c}0.16 \\
4\end{array}$ & $\begin{array}{c}0.16 \\
6\end{array}$ & $\begin{array}{c}0.17 \\
1\end{array}$ & 0.172 \\
\hline 1050_2 & 0.000 & 0.207 & $\begin{array}{c}0.23 \\
9\end{array}$ & $\begin{array}{c}0.25 \\
4\end{array}$ & $\begin{array}{c}0.26 \\
1\end{array}$ & $\begin{array}{c}0.26 \\
0\end{array}$ & $\begin{array}{c}0.26 \\
7\end{array}$ & $\begin{array}{c}0.25 \\
9\end{array}$ & $\begin{array}{c}0.26 \\
9\end{array}$ & $\begin{array}{c}0.27 \\
1\end{array}$ & $\begin{array}{c}0.27 \\
8\end{array}$ & $\begin{array}{c}0.27 \\
6\end{array}$ & $\begin{array}{c}0.28 \\
2\end{array}$ & $\begin{array}{c}0.28 \\
9\end{array}$ & $\begin{array}{c}0.29 \\
0\end{array}$ & $\begin{array}{c}0.29 \\
5\end{array}$ & 0.298 \\
\hline \multirow[t]{4}{*}{$\begin{array}{c}\text { Averag } \\
\text { e }\end{array}$} & 0.000 & 0.145 & $\begin{array}{c}0.17 \\
8 \\
\end{array}$ & $\begin{array}{c}0.19 \\
3 \\
\end{array}$ & $\begin{array}{c}0.19 \\
9 \\
\end{array}$ & $\begin{array}{c}0.19 \\
8 \\
\end{array}$ & $\begin{array}{c}0.20 \\
4 \\
\end{array}$ & $\begin{array}{c}0.19 \\
8 \\
\end{array}$ & $\begin{array}{c}0.20 \\
8 \\
\end{array}$ & $\begin{array}{c}0.20 \\
8 \\
\end{array}$ & $\begin{array}{c}0.21 \\
6 \\
\end{array}$ & $\begin{array}{c}0.21 \\
4 \\
\end{array}$ & $\begin{array}{c}0.22 \\
0 \\
\end{array}$ & $\begin{array}{c}0.22 \\
6 \\
\end{array}$ & $\begin{array}{c}0.22 \\
8 \\
\end{array}$ & $\begin{array}{c}0.23 \\
3 \\
\end{array}$ & 0.235 \\
\hline & \multicolumn{17}{|c|}{ Day } \\
\hline & $\mathbf{0}$ & 1 & 3 & 10 & 17 & 24 & 31 & 39 & 54 & 65 & 80 & 94 & 123 & 199 & 239 & 325 & 466 \\
\hline & \multicolumn{17}{|c|}{ Expansion } \\
\hline 1200_2 & 0.000 & 0.071 & $\begin{array}{c}0.08 \\
7\end{array}$ & $\begin{array}{c}0.10 \\
3\end{array}$ & $\begin{array}{c}0.11 \\
5\end{array}$ & $\begin{array}{c}0.11 \\
8\end{array}$ & $\begin{array}{c}0.12 \\
6\end{array}$ & $\begin{array}{c}0.13 \\
1\end{array}$ & $\begin{array}{c}0.13 \\
4\end{array}$ & $\begin{array}{c}0.14 \\
2\end{array}$ & $\begin{array}{c}0.15 \\
2\end{array}$ & $\begin{array}{c}0.15 \\
3\end{array}$ & $\begin{array}{c}0.16 \\
5\end{array}$ & $\begin{array}{c}0.17 \\
5\end{array}$ & $\begin{array}{c}0.18 \\
3\end{array}$ & $\begin{array}{c}0.19 \\
0\end{array}$ & 0.196 \\
\hline 1200_2 & 0.000 & 0.076 & $\begin{array}{c}0.09 \\
1\end{array}$ & $\begin{array}{c}0.10 \\
6\end{array}$ & $\begin{array}{c}0.12 \\
0\end{array}$ & $\begin{array}{c}0.11 \\
6\end{array}$ & $\begin{array}{c}0.13 \\
1\end{array}$ & $\begin{array}{c}0.13 \\
5\end{array}$ & $\begin{array}{c}0.13 \\
8\end{array}$ & $\begin{array}{c}0.14 \\
6\end{array}$ & $\begin{array}{c}0.15 \\
4\end{array}$ & $\begin{array}{c}0.15 \\
8\end{array}$ & $\begin{array}{c}0.16 \\
6\end{array}$ & $\begin{array}{c}0.18 \\
1\end{array}$ & $\begin{array}{c}0.18 \\
8\end{array}$ & $\begin{array}{c}0.19 \\
6\end{array}$ & 0.204 \\
\hline \multirow[t]{2}{*}{$\begin{array}{c}\text { Averag } \\
\text { e }\end{array}$} & 0.000 & 0.074 & $\begin{array}{c}0.08 \\
9\end{array}$ & $\begin{array}{c}0.10 \\
4\end{array}$ & $\begin{array}{c}0.11 \\
8\end{array}$ & $\begin{array}{c}0.11 \\
7\end{array}$ & $\begin{array}{c}0.12 \\
9\end{array}$ & $\begin{array}{c}0.13 \\
3\end{array}$ & $\begin{array}{c}0.13 \\
6\end{array}$ & $\begin{array}{c}0.14 \\
4\end{array}$ & $\begin{array}{c}0.15 \\
3\end{array}$ & $\begin{array}{c}0.15 \\
5\end{array}$ & $\begin{array}{c}0.16 \\
6\end{array}$ & $\begin{array}{c}0.17 \\
8\end{array}$ & $\begin{array}{c}0.18 \\
6\end{array}$ & $\begin{array}{c}0.19 \\
3\end{array}$ & 0.200 \\
\hline & \multicolumn{17}{|c|}{ Day } \\
\hline
\end{tabular}




\begin{tabular}{|c|c|c|c|c|c|c|c|c|c|c|c|c|c|c|c|c|c|}
\hline & $\mathbf{0}$ & 1 & 6 & 13 & 20 & 27 & 34 & 42 & 57 & 68 & 83 & 97 & 126 & 202 & 242 & 328 & 469 \\
\hline & \multicolumn{17}{|c|}{ Expansion } \\
\hline 750_4 & 0.000 & 0.078 & $\begin{array}{c}0.09 \\
5\end{array}$ & $\begin{array}{c}0.10 \\
3\end{array}$ & $\begin{array}{c}0.10 \\
9\end{array}$ & $\begin{array}{c}0.10 \\
6\end{array}$ & $\begin{array}{c}0.11 \\
2\end{array}$ & $\begin{array}{c}0.10 \\
5\end{array}$ & $\begin{array}{c}0.11 \\
4\end{array}$ & $\begin{array}{c}0.11 \\
3\end{array}$ & $\begin{array}{c}0.12 \\
2\end{array}$ & $\begin{array}{c}0.12 \\
0\end{array}$ & $\begin{array}{c}0.12 \\
5\end{array}$ & $\begin{array}{c}0.13 \\
0\end{array}$ & $\begin{array}{c}0.13 \\
1\end{array}$ & $\begin{array}{c}0.13 \\
7\end{array}$ & 0.136 \\
\hline $750 \_4$ & 0.000 & 0.078 & $\begin{array}{c}0.09 \\
4\end{array}$ & $\begin{array}{c}0.10 \\
2\end{array}$ & $\begin{array}{c}0.10 \\
8\end{array}$ & $\begin{array}{c}0.10 \\
6\end{array}$ & $\begin{array}{c}0.11 \\
0\end{array}$ & $\begin{array}{c}0.10 \\
4\end{array}$ & $\begin{array}{c}0.11 \\
4\end{array}$ & $\begin{array}{c}0.11 \\
4\end{array}$ & $\begin{array}{c}0.12 \\
1\end{array}$ & $\begin{array}{c}0.12 \\
0\end{array}$ & $\begin{array}{c}0.12 \\
4\end{array}$ & $\begin{array}{c}\mathbf{0 . 1 3} \\
0\end{array}$ & $\begin{array}{c}0.13 \\
1\end{array}$ & $\begin{array}{c}0.13 \\
6\end{array}$ & 0.136 \\
\hline \multirow[t]{4}{*}{$\begin{array}{c}\text { Averag } \\
\text { e }\end{array}$} & 0.000 & 0.078 & $\begin{array}{c}0.09 \\
5\end{array}$ & $\begin{array}{c}\mathbf{0 . 1 0} \\
\mathbf{3}\end{array}$ & $\begin{array}{c}0.10 \\
8\end{array}$ & $\begin{array}{c}0.10 \\
6\end{array}$ & $\begin{array}{c}0.11 \\
1\end{array}$ & $\begin{array}{c}0.10 \\
4\end{array}$ & $\begin{array}{c}0.11 \\
4\end{array}$ & $\begin{array}{c}\mathbf{0 . 1 1} \\
\mathbf{3}\end{array}$ & $\begin{array}{c}0.12 \\
1\end{array}$ & $\begin{array}{c}0.12 \\
0\end{array}$ & $\begin{array}{c}0.12 \\
4\end{array}$ & $\begin{array}{c}0.13 \\
0\end{array}$ & $\begin{array}{c}0.13 \\
1\end{array}$ & $\begin{array}{c}0.13 \\
6\end{array}$ & 0.136 \\
\hline & \multicolumn{17}{|c|}{ Day } \\
\hline & $\mathbf{0}$ & 1 & 3 & 10 & 17 & 25 & 40 & 51 & 66 & 80 & 109 & 185 & 225 & 311 & 452 & & \\
\hline & \multicolumn{17}{|c|}{ Expansion } \\
\hline $900 \_4$ & 0.000 & 0.075 & $\begin{array}{c}0.08 \\
9\end{array}$ & $\begin{array}{c}0.11 \\
8\end{array}$ & $\begin{array}{c}0.13 \\
4\end{array}$ & $\begin{array}{c}0.14 \\
3\end{array}$ & $\begin{array}{c}0.15 \\
0\end{array}$ & $\begin{array}{c}0.16 \\
0\end{array}$ & $\begin{array}{c}0.16 \\
9\end{array}$ & $\begin{array}{c}0.17 \\
\mathbf{3}\end{array}$ & $\begin{array}{c}0.18 \\
2\end{array}$ & $\begin{array}{c}0.20 \\
2\end{array}$ & $\begin{array}{c}0.20 \\
7\end{array}$ & $\begin{array}{c}0.21 \\
7\end{array}$ & $\begin{array}{c}0.22 \\
5\end{array}$ & & \\
\hline 900_4 & 0.000 & 0.074 & $\begin{array}{c}0.08 \\
6\end{array}$ & $\begin{array}{c}0.11 \\
5\end{array}$ & $\begin{array}{c}0.13 \\
0\end{array}$ & $\begin{array}{c}0.13 \\
8\end{array}$ & $\begin{array}{c}0.14 \\
6\end{array}$ & $\begin{array}{c}0.15 \\
4\end{array}$ & $\begin{array}{c}0.16 \\
6\end{array}$ & $\begin{array}{c}0.17 \\
0\end{array}$ & $\begin{array}{c}0.18 \\
0\end{array}$ & $\begin{array}{c}0.19 \\
7\end{array}$ & $\begin{array}{c}0.20 \\
2\end{array}$ & $\begin{array}{c}0.21 \\
2\end{array}$ & $\begin{array}{c}0.21 \\
9\end{array}$ & & \\
\hline $900 \_4$ & 0.000 & 0.074 & $\begin{array}{c}0.08 \\
6 \\
\end{array}$ & $\begin{array}{c}0.11 \\
5\end{array}$ & $\begin{array}{c}0.13 \\
1\end{array}$ & $\begin{array}{c}0.14 \\
2\end{array}$ & $\begin{array}{c}0.14 \\
9\end{array}$ & $\begin{array}{c}0.15 \\
7\end{array}$ & $\begin{array}{c}0.16 \\
8\end{array}$ & $\begin{array}{c}0.17 \\
2\end{array}$ & $\begin{array}{c}0.18 \\
2\end{array}$ & $\begin{array}{c}0.20 \\
0\end{array}$ & $\begin{array}{c}0.20 \\
5\end{array}$ & $\begin{array}{c}0.21 \\
6\end{array}$ & $\begin{array}{c}0.22 \\
2\end{array}$ & & \\
\hline \multirow[t]{4}{*}{$\begin{array}{c}\text { Averag } \\
\text { e }\end{array}$} & 0.000 & 0.074 & $\begin{array}{c}0.08 \\
7 \\
\end{array}$ & $\begin{array}{c}0.11 \\
6\end{array}$ & $\begin{array}{c}0.13 \\
2 \\
\end{array}$ & $\begin{array}{c}0.14 \\
1 \\
\end{array}$ & $\begin{array}{c}0.14 \\
9\end{array}$ & $\begin{array}{c}0.15 \\
7 \\
\end{array}$ & $\begin{array}{c}0.16 \\
7 \\
\end{array}$ & $\begin{array}{c}0.17 \\
1 \\
\end{array}$ & $\begin{array}{c}0.18 \\
2 \\
\end{array}$ & $\begin{array}{c}0.19 \\
9\end{array}$ & $\begin{array}{c}0.20 \\
5\end{array}$ & $\begin{array}{c}0.21 \\
5 \\
\end{array}$ & $\begin{array}{c}0.22 \\
2 \\
\end{array}$ & & \\
\hline & \multicolumn{17}{|c|}{ Day } \\
\hline & $\mathbf{0}$ & 1 & 3 & 10 & 17 & 24 & 31 & 39 & 54 & 65 & 80 & 94 & 123 & 199 & 239 & 325 & 466 \\
\hline & \multicolumn{17}{|c|}{ Expansion } \\
\hline 1050_4 & $\mathbf{0}$ & $\begin{array}{c}0.298 \\
8\end{array}$ & $\begin{array}{c}0.35 \\
3\end{array}$ & $\begin{array}{c}0.38 \\
2\end{array}$ & $\begin{array}{c}0.39 \\
6\end{array}$ & $\begin{array}{c}0.39 \\
9\end{array}$ & $\begin{array}{c}0.40 \\
8\end{array}$ & $\begin{array}{c}0.40 \\
4\end{array}$ & $\begin{array}{c}0.41 \\
7\end{array}$ & $\begin{array}{c}0.41 \\
9\end{array}$ & $\begin{array}{c}0.43 \\
2\end{array}$ & $\begin{array}{c}0.43 \\
2\end{array}$ & $\begin{array}{c}0.44 \\
1\end{array}$ & $\begin{array}{c}0.45 \\
6\end{array}$ & $\begin{array}{c}0.46 \\
0\end{array}$ & $\begin{array}{c}0.46 \\
9\end{array}$ & 0.473 \\
\hline 1050_4 & 0.000 & 0.216 & $\begin{array}{c}0.25 \\
5\end{array}$ & $\begin{array}{c}0.27 \\
7\end{array}$ & $\begin{array}{c}0.28 \\
7\end{array}$ & $\begin{array}{c}0.28 \\
9\end{array}$ & $\begin{array}{c}0.29 \\
6\end{array}$ & $\begin{array}{c}0.29 \\
2\end{array}$ & $\begin{array}{c}0.30 \\
2\end{array}$ & $\begin{array}{c}0.30 \\
4\end{array}$ & $\begin{array}{c}0.31 \\
2\end{array}$ & $\begin{array}{c}\mathbf{0 . 3 1} \\
\mathbf{3}\end{array}$ & $\begin{array}{c}0.31 \\
9\end{array}$ & $\begin{array}{c}\mathbf{0 . 3 3} \\
\mathbf{0}\end{array}$ & $\begin{array}{c}0.33 \\
3\end{array}$ & $\begin{array}{c}\mathbf{0 . 3 3} \\
9\end{array}$ & 0.343 \\
\hline $\begin{array}{c}\text { Averag } \\
\text { e }\end{array}$ & 0.000 & 0.258 & $\begin{array}{c}0.30 \\
4\end{array}$ & $\begin{array}{c}0.33 \\
0\end{array}$ & $\begin{array}{c}0.34 \\
2\end{array}$ & $\begin{array}{c}0.34 \\
4\end{array}$ & $\begin{array}{c}0.35 \\
2\end{array}$ & $\begin{array}{c}0.34 \\
8\end{array}$ & $\begin{array}{c}0.35 \\
9\end{array}$ & $\begin{array}{c}0.36 \\
2\end{array}$ & $\begin{array}{c}0.37 \\
2\end{array}$ & $\begin{array}{c}0.37 \\
3\end{array}$ & $\begin{array}{c}0.38 \\
0\end{array}$ & $\begin{array}{c}0.39 \\
3\end{array}$ & $\begin{array}{c}0.39 \\
7\end{array}$ & $\begin{array}{c}0.40 \\
4\end{array}$ & 0.408 \\
\hline
\end{tabular}

cecxxi 


\begin{tabular}{|c|c|c|c|c|c|c|c|c|c|c|c|c|c|c|c|c|c|}
\hline & \multicolumn{17}{|c|}{ Day } \\
\hline & $\mathbf{0}$ & 1 & 3 & 10 & 17 & 24 & 31 & 39 & 54 & 65 & 80 & 94 & 123 & 199 & 239 & 325 & 466 \\
\hline & \multicolumn{17}{|c|}{ Expansion } \\
\hline 1200_4 & 0.000 & 0.022 & $\begin{array}{c}0.02 \\
9\end{array}$ & $\begin{array}{c}0.03 \\
7\end{array}$ & $\begin{array}{c}0.04 \\
2\end{array}$ & $\begin{array}{c}0.04 \\
2\end{array}$ & $\begin{array}{c}0.04 \\
6\end{array}$ & $\begin{array}{c}0.05 \\
0\end{array}$ & $\begin{array}{c}0.05 \\
0\end{array}$ & $\begin{array}{c}0.05 \\
6\end{array}$ & $\begin{array}{c}0.06 \\
2\end{array}$ & $\begin{array}{c}0.06 \\
3\end{array}$ & $\begin{array}{c}0.07 \\
1\end{array}$ & $\begin{array}{c}0.07 \\
9\end{array}$ & $\begin{array}{c}0.08 \\
6\end{array}$ & $\begin{array}{c}0.09 \\
3\end{array}$ & 0.098 \\
\hline 1200_4 & 0.000 & 0.020 & $\begin{array}{c}0.02 \\
8\end{array}$ & $\begin{array}{c}0.03 \\
5\end{array}$ & $\begin{array}{c}0.04 \\
2\end{array}$ & $\begin{array}{c}0.04 \\
2\end{array}$ & $\begin{array}{c}0.04 \\
7\end{array}$ & $\begin{array}{c}0.05 \\
2\end{array}$ & $\begin{array}{c}0.05 \\
0\end{array}$ & $\begin{array}{c}0.05 \\
7\end{array}$ & $\begin{array}{c}0.06 \\
2\end{array}$ & $\begin{array}{c}0.06 \\
2\end{array}$ & $\begin{array}{c}\mathbf{0 . 0 7} \\
\mathbf{0}\end{array}$ & $\begin{array}{c}0.07 \\
9\end{array}$ & $\begin{array}{c}0.08 \\
5\end{array}$ & $\begin{array}{c}0.09 \\
1\end{array}$ & 0.098 \\
\hline $\begin{array}{c}\text { Averag } \\
\text { e }\end{array}$ & 0.000 & 0.021 & $\begin{array}{c}0.02 \\
8\end{array}$ & $\begin{array}{c}0.03 \\
6\end{array}$ & $\begin{array}{c}0.04 \\
2\end{array}$ & $\begin{array}{c}0.04 \\
2\end{array}$ & $\begin{array}{c}0.04 \\
7\end{array}$ & $\begin{array}{c}0.05 \\
1\end{array}$ & $\begin{array}{c}\mathbf{0 . 0 5} \\
0\end{array}$ & $\begin{array}{c}0.05 \\
6\end{array}$ & $\begin{array}{c}0.06 \\
2\end{array}$ & $\begin{array}{c}0.06 \\
3\end{array}$ & $\begin{array}{c}0.07 \\
0\end{array}$ & $\begin{array}{c}0.07 \\
9\end{array}$ & $\begin{array}{c}0.08 \\
5\end{array}$ & $\begin{array}{c}0.09 \\
2\end{array}$ & 0.098 \\
\hline
\end{tabular}

Water expansion - Class-CI fly ash/45 $\mu \mathrm{m}$

\begin{tabular}{|c|c|c|c|c|c|c|c|c|c|c|c|c|c|c|c|c|c|}
\hline \multirow{3}{*}{ 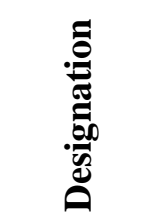 } & \multicolumn{17}{|c|}{ Day } \\
\hline & $\mathbf{0}$ & 1 & 3 & 10 & 17 & 24 & 31 & 39 & 54 & 65 & 80 & 94 & 123 & 199 & 239 & 325 & 466 \\
\hline & \multicolumn{17}{|c|}{ Expansion } \\
\hline 750_1 & 0.000 & 0.052 & 0.063 & 0.066 & 0.067 & 0.064 & 0.067 & 0.059 & 0.066 & 0.066 & 0.072 & 0.069 & 0.072 & 0.074 & 0.075 & 0.079 & 0.086 \\
\hline 750_1 & 0.000 & 0.039 & 0.048 & 0.051 & 0.051 & 0.049 & 0.051 & 0.045 & 0.051 & 0.051 & 0.055 & 0.053 & 0.055 & 0.057 & 0.057 & 0.060 & 0.066 \\
\hline \multirow[t]{4}{*}{ Average } & 0.000 & 0.046 & 0.055 & 0.058 & 0.059 & 0.057 & 0.059 & 0.052 & 0.058 & 0.058 & 0.063 & 0.061 & 0.063 & 0.066 & 0.066 & 0.070 & 0.076 \\
\hline & \multicolumn{17}{|c|}{ Day } \\
\hline & 0 & 1 & 3 & 10 & 17 & 24 & 31 & 39 & 54 & 65 & 80 & 94 & 123 & 199 & 239 & 325 & 466 \\
\hline & \multicolumn{17}{|c|}{ Expansion } \\
\hline 900_1 & 0.000 & 0.138 & 0.157 & 0.165 & 0.168 & 0.165 & 0.170 & 0.163 & 0.170 & 0.172 & 0.177 & 0.174 & 0.178 & 0.182 & 0.184 & 0.187 & 0.187 \\
\hline
\end{tabular}




\begin{tabular}{|c|c|c|c|c|c|c|c|c|c|c|c|c|c|c|c|c|c|}
\hline 900_1 & 0.000 & 0.110 & 0.128 & 0.136 & 0.139 & 0.137 & 0.142 & 0.134 & 0.142 & 0.143 & 0.150 & 0.146 & 0.150 & 0.154 & 0.158 & 0.158 & 0.158 \\
\hline Average & 0.000 & 0.124 & 0.142 & 0.150 & 0.154 & 0.151 & 0.156 & 0.149 & 0.156 & 0.158 & 0.163 & 0.160 & 0.164 & 0.168 & 0.171 & 0.173 & 0.173 \\
\hline & \multicolumn{17}{|c|}{ Day } \\
\hline & 0 & 1 & 3 & 10 & 17 & 24 & 31 & 39 & 54 & 65 & 80 & 94 & 123 & 199 & 239 & 325 & 466 \\
\hline & \multicolumn{17}{|c|}{ Expansion } \\
\hline 1050_1 & 0.000 & 0.093 & 0.106 & 0.119 & 0.129 & 0.131 & 0.135 & 0.142 & 0.140 & 0.146 & 0.154 & 0.154 & 0.154 & 0.168 & 0.174 & 0.178 & 0.180 \\
\hline 1050_1 & 0.000 & 0.092 & 0.104 & 0.117 & 0.127 & 0.129 & 0.135 & 0.140 & 0.140 & 0.147 & 0.152 & 0.154 & 0.159 & 0.167 & 0.173 & 0.178 & 0.180 \\
\hline \multirow[t]{4}{*}{ Average } & 0.000 & 0.092 & 0.105 & 0.118 & 0.128 & 0.130 & 0.135 & 0.141 & 0.140 & 0.147 & 0.153 & 0.154 & 0.156 & 0.168 & 0.173 & 0.178 & 0.180 \\
\hline & \multicolumn{17}{|c|}{ Day } \\
\hline & $\mathbf{0}$ & 1 & 3 & 10 & 17 & 24 & 31 & 39 & 54 & 65 & 80 & 94 & 123 & 199 & 239 & 325 & 466 \\
\hline & \multicolumn{17}{|c|}{ Expansion } \\
\hline 1200_1 & 0.000 & 0.108 & 0.126 & 0.144 & 0.158 & 0.162 & 0.170 & 0.176 & 0.178 & 0.186 & 0.194 & 0.196 & 0.207 & 0.215 & 0.222 & 0.227 & 0.233 \\
\hline 1200_1 & 0.000 & 0.107 & 0.125 & 0.142 & 0.158 & 0.162 & 0.172 & 0.176 & 0.178 & 0.186 & 0.193 & 0.196 & 0.195 & 0.215 & 0.222 & 0.229 & 0.234 \\
\hline Average & 0.000 & 0.108 & 0.125 & 0.143 & 0.158 & 0.162 & 0.171 & 0.176 & 0.178 & 0.186 & 0.194 & 0.196 & 0.201 & 0.215 & 0.222 & 0.228 & 0.233 \\
\hline & \multicolumn{17}{|c|}{ Day } \\
\hline & $\mathbf{0}$ & 1 & 3 & 10 & 17 & 24 & 31 & 39 & 54 & 65 & 80 & 94 & 123 & 199 & 239 & 325 & 466 \\
\hline & \multicolumn{17}{|c|}{ Expansion } \\
\hline $750 \_2$ & $\mathbf{0}$ & 0.0784 & 0.096 & 0.103 & 0.106 & 0.103 & 0.107 & 0.100 & 0.109 & 0.108 & 0.115 & 0.112 & 0.116 & 0.1192 & 0.1192 & 0.122 & 0.120 \\
\hline 750_2 & $\mathbf{0}$ & 0.0712 & 0.088 & 0.095 & 0.099 & 0.096 & 0.100 & 0.093 & 0.102 & 0.102 & 0.107 & 0.104 & 0.110 & 0.1128 & 0.1136 & 0.118 & 0.136 \\
\hline Average & 0.000 & 0.075 & 0.092 & 0.099 & 0.102 & 0.100 & 0.104 & 0.096 & 0.105 & 0.105 & 0.111 & 0.108 & 0.113 & 0.116 & 0.116 & 0.120 & 0.128 \\
\hline
\end{tabular}




\begin{tabular}{|c|c|c|c|c|c|c|c|c|c|c|c|c|c|c|c|c|c|}
\hline & \multicolumn{17}{|c|}{ Day } \\
\hline & $\mathbf{0}$ & 1 & 3 & 10 & 17 & 24 & 31 & 39 & 54 & 65 & 80 & 94 & 123 & 199 & 239 & 325 & 466 \\
\hline & \multicolumn{17}{|c|}{ Expansion } \\
\hline $900 \_2$ & 0.000 & 0.034 & 0.057 & 0.080 & 0.094 & 0.098 & 0.104 & 0.111 & 0.114 & 0.122 & 0.131 & 0.133 & 0.135 & 0.154 & 0.159 & 0.166 & 0.170 \\
\hline $900 \_2$ & 0.000 & 0.033 & 0.056 & 0.078 & 0.093 & 0.098 & 0.105 & 0.111 & 0.115 & 0.123 & 0.130 & 0.134 & 0.141 & 0.154 & 0.158 & 0.165 & 0.170 \\
\hline \multirow[t]{4}{*}{ Average } & 0.000 & 0.034 & 0.056 & 0.079 & 0.094 & 0.098 & 0.105 & 0.111 & 0.115 & 0.123 & 0.131 & 0.133 & 0.138 & 0.154 & 0.159 & 0.165 & 0.170 \\
\hline & \multicolumn{17}{|c|}{ Day } \\
\hline & 0 & 1 & 3 & 10 & 17 & 24 & 31 & 39 & 54 & 65 & 80 & 94 & 123 & 199 & 239 & 325 & 466 \\
\hline & \multicolumn{17}{|c|}{ Expansion } \\
\hline 1050_2 & 0.000 & 0.106 & 0.139 & 0.158 & 0.166 & 0.163 & 0.170 & 0.163 & 0.174 & 0.174 & 0.181 & 0.178 & 0.184 & 0.188 & 0.190 & 0.194 & 0.195 \\
\hline 1050_2 & 0.000 & 0.107 & 0.143 & 0.159 & 0.166 & 0.165 & 0.170 & 0.165 & 0.174 & 0.175 & 0.180 & 0.178 & 0.184 & 0.188 & 0.190 & 0.193 & 0.195 \\
\hline \multirow[t]{4}{*}{ Average } & 0.000 & 0.106 & 0.141 & 0.159 & 0.166 & 0.164 & 0.170 & 0.164 & 0.174 & 0.174 & 0.180 & 0.178 & 0.184 & 0.188 & 0.190 & 0.193 & 0.195 \\
\hline & \multicolumn{17}{|c|}{ Day } \\
\hline & $\mathbf{0}$ & 1 & 3 & 10 & 17 & 24 & 31 & 39 & 54 & 65 & 80 & 94 & 123 & 199 & 239 & 325 & 466 \\
\hline & \multicolumn{17}{|c|}{ Expansion } \\
\hline 1200_2 & 0.000 & 0.092 & 0.110 & 0.128 & 0.144 & 0.148 & 0.156 & 0.163 & 0.166 & 0.174 & 0.185 & 0.186 & 0.198 & 0.210 & 0.217 & 0.226 & 0.234 \\
\hline 1200_2 & 0.000 & 0.092 & 0.110 & 0.127 & 0.142 & 0.146 & 0.154 & 0.162 & 0.166 & 0.174 & 0.184 & 0.187 & 0.186 & 0.210 & 0.215 & 0.225 & 0.233 \\
\hline \multirow[t]{3}{*}{ Average } & 0.000 & 0.092 & 0.110 & 0.128 & 0.143 & 0.147 & 0.155 & 0.163 & 0.166 & 0.174 & 0.184 & 0.187 & 0.192 & 0.210 & 0.216 & 0.226 & 0.233 \\
\hline & \multicolumn{17}{|c|}{ Day } \\
\hline & 0 & 1 & 3 & 10 & 17 & 24 & 31 & 39 & 54 & 65 & 80 & 94 & 123 & 199 & 239 & 325 & 466 \\
\hline
\end{tabular}




\begin{tabular}{|c|c|c|c|c|c|c|c|c|c|c|c|c|c|c|c|c|c|}
\hline & \multicolumn{17}{|c|}{ Expansion } \\
\hline 750_4 & 0.000 & 0.228 & 0.264 & 0.281 & 0.288 & 0.285 & 0.294 & 0.284 & 0.295 & 0.297 & 0.304 & 0.303 & 0.308 & 0.313 & 0.316 & 0.320 & 0.332 \\
\hline 750_4 & 0.000 & 0.145 & 0.168 & 0.178 & 0.183 & 0.181 & 0.186 & 0.180 & 0.187 & 0.188 & 0.192 & 0.192 & 0.196 & 0.199 & 0.201 & 0.203 & 0.210 \\
\hline \multirow[t]{4}{*}{ Average } & 0.000 & 0.186 & 0.216 & 0.230 & 0.235 & 0.233 & 0.240 & 0.232 & 0.241 & 0.242 & 0.248 & 0.247 & 0.252 & 0.256 & 0.258 & 0.262 & 0.271 \\
\hline & \multicolumn{17}{|c|}{ Day } \\
\hline & $\mathbf{0}$ & 1 & 3 & 10 & 17 & 24 & 31 & 39 & 54 & 65 & 80 & 94 & 123 & 199 & 239 & 325 & 466 \\
\hline & \multicolumn{17}{|c|}{ Expansion } \\
\hline 900_4 & 0.000 & 0.064 & 0.073 & 0.082 & 0.099 & 0.104 & 0.113 & 0.118 & 0.123 & 0.131 & 0.142 & 0.145 & 0.146 & 0.169 & 0.177 & 0.186 & 0.194 \\
\hline 900_4 & 0.000 & 0.036 & 0.057 & 0.078 & 0.094 & 0.100 & 0.108 & 0.115 & 0.118 & 0.126 & 0.135 & 0.139 & 0.147 & 0.164 & 0.171 & 0.180 & 0.188 \\
\hline \multirow[t]{4}{*}{ Average } & 0.000 & 0.050 & 0.065 & 0.080 & 0.097 & 0.102 & 0.110 & 0.117 & 0.120 & 0.129 & 0.138 & 0.142 & 0.147 & 0.166 & 0.174 & 0.183 & 0.191 \\
\hline & \multicolumn{17}{|c|}{ Day } \\
\hline & $\mathbf{0}$ & 1 & 3 & 10 & 17 & 24 & 31 & 39 & 54 & 65 & 80 & 94 & 123 & 199 & 239 & 325 & 466 \\
\hline & \multicolumn{17}{|c|}{ Expansion } \\
\hline 1050_4 & 0.000 & 0.323 & 0.374 & 0.403 & 0.416 & 0.419 & 0.429 & 0.423 & 0.438 & 0.439 & 0.453 & 0.452 & 0.461 & 0.474 & 0.477 & 0.486 & 0.490 \\
\hline 1050_4 & 0.000 & 0.257 & 0.298 & 0.320 & 0.331 & 0.333 & 0.341 & 0.337 & 0.348 & 0.349 & 0.360 & 0.359 & 0.366 & 0.377 & 0.379 & 0.386 & 0.390 \\
\hline \multirow[t]{4}{*}{ Average } & 0.000 & 0.290 & 0.336 & 0.362 & 0.374 & 0.376 & 0.385 & 0.380 & 0.393 & 0.394 & 0.406 & 0.406 & 0.414 & 0.426 & 0.428 & 0.436 & 0.440 \\
\hline & \multicolumn{17}{|c|}{ Day } \\
\hline & $\mathbf{0}$ & 1 & 3 & 10 & 17 & 24 & 31 & 39 & 54 & 65 & 80 & 94 & 123 & 199 & 239 & 325 & 466 \\
\hline & \multicolumn{17}{|c|}{ Expansion } \\
\hline 1200_4 & 0.000 & 0.042 & 0.050 & 0.057 & 0.064 & 0.065 & 0.070 & 0.073 & 0.074 & 0.080 & 0.087 & 0.088 & 0.099 & 0.108 & 0.115 & 0.121 & 0.129 \\
\hline
\end{tabular}




\begin{tabular}{|c|c|c|c|c|c|c|c|c|c|c|c|c|c|c|c|c|c|}
\hline 1200_4 & 0.000 & 0.044 & 0.052 & 0.059 & 0.067 & 0.067 & 0.073 & 0.077 & 0.077 & 0.084 & 0.090 & 0.091 & 0.090 & 0.109 & 0.117 & 0.123 & 0.130 \\
\hline Average & 0.000 & 0.043 & 0.051 & 0.058 & 0.066 & 0.066 & 0.072 & 0.075 & 0.076 & 0.082 & 0.089 & 0.090 & 0.094 & 0.108 & 0.116 & 0.122 & 0.129 \\
\hline
\end{tabular}




\title{
Appendix C
}

\author{
Chapter 5 raw data
}


Autoclave expansion test $-55 \%$ cement replacement of Class-F fly ash with different MgO dosages

\begin{tabular}{|c|c|}
\hline MgO dosage (\%) & Expansion (\%) \\
\hline 0 & 0.002 \\
\hline 2 & 0.084 \\
\hline 4 & 0.152 \\
\hline 5 & 0.179 \\
\hline 6 & 0.213 \\
\hline 8 & 0.250 \\
\hline 10 & 0.298 \\
\hline
\end{tabular}

Autoclave expansion test $-55 \%$ cement replacement of Class-CI fly ash with different $\mathrm{MgO}$ dosages

\begin{tabular}{|c|c|}
\hline MgO dosage (\%) & Expansion (\%) \\
\hline 0 & 0.090 \\
\hline 2 & 0.100 \\
\hline 4 & 0.206 \\
\hline 5 & 0.156 \\
\hline 6 & 0.205 \\
\hline 8 & 0.276 \\
\hline 10 & 0.353 \\
\hline
\end{tabular}

Autoclave expansion test $-\mathbf{3 0} \%$ cement replacement of Class-F fly ash with different MgO dosages

\begin{tabular}{|c|c|}
\hline MgO dosage (\%) & Expansion (\%) \\
\hline 0 & 0.095 \\
\hline
\end{tabular}

cecxxviii 


\begin{tabular}{|c|c|}
\hline 2 & 0.117 \\
\hline 4 & 0.178 \\
\hline 5 & 0.182 \\
\hline 6 & 0.207 \\
\hline 8 & 0.262 \\
\hline 10 & 0.324 \\
\hline
\end{tabular}

Water expansion test $-55 \%$ cement replacement of Class-F fly ash with different MgO dosages

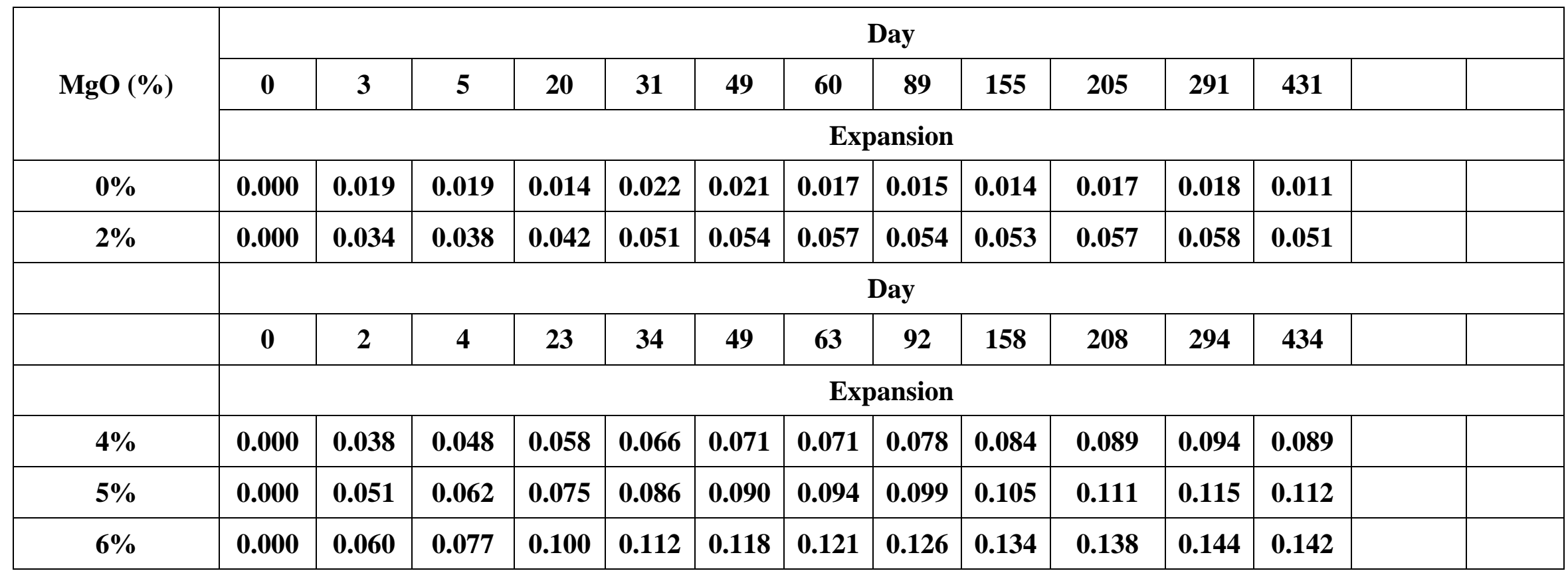




\begin{tabular}{|c|c|c|c|c|c|c|c|c|c|c|c|c|c|c|}
\hline & \multicolumn{10}{|c|}{ Day } \\
\hline & 0 & 1 & 3 & 6 & 8 & 23 & 34 & 49 & 63 & 92 & 158 & 208 & 294 & 434 \\
\hline & \multicolumn{10}{|c|}{ Expansion } \\
\hline $8 \%$ & 0.000 & 0.059 & 0.082 & 0.101 & 0.117 & 0.150 & 0.166 & 0.174 & 0.181 & 0.184 & 0.189 & 0.194 & 0.199 & 0.195 \\
\hline $10 \%$ & 0.000 & 0.082 & 0.103 & 0.134 & 0.148 & 0.176 & 0.193 & 0.202 & 0.207 & 0.208 & 0.215 & 0.221 & 0.224 & 0.221 \\
\hline
\end{tabular}

Water expansion test $-\mathbf{5 5 \%}$ cement replacement of Class-CI fly ash with different $\mathrm{MgO}$ dosages

\begin{tabular}{|c|c|c|c|c|c|c|c|c|c|c|c|c|c|c|}
\hline \multirow{3}{*}{$\operatorname{MgO}(\%)$} & \multicolumn{14}{|c|}{ Day } \\
\hline & $\mathbf{0}$ & 3 & 5 & 20 & 31 & 46 & 60 & 89 & 155 & 205 & 291 & 431 & & \\
\hline & \multicolumn{14}{|c|}{ Expansion } \\
\hline $0 \%$ & 0.000 & 0.014 & 0.014 & 0.006 & 0.018 & 0.012 & 0.010 & 0.010 & 0.013 & 0.014 & 0.015 & 0.007 & & \\
\hline \multirow[t]{4}{*}{$2 \%$} & 0.000 & 0.030 & 0.034 & $\mathbf{0 . 0 3 3}$ & 0.041 & 0.043 & 0.042 & 0.045 & 0.047 & 0.051 & 0.049 & 0.047 & & \\
\hline & \multicolumn{14}{|c|}{ Day } \\
\hline & $\mathbf{0}$ & 1 & 3 & 6 & 8 & 23 & 34 & 49 & 63 & 92 & 158 & 208 & 294 & 434 \\
\hline & \multicolumn{14}{|c|}{ Expansion } \\
\hline $4 \%$ & 0.000 & 0.048 & 0.057 & 0.073 & 0.078 & 0.090 & 0.102 & 0.108 & 0.110 & 0.110 & 0.114 & 0.118 & 0.122 & 0.117 \\
\hline $5 \%$ & 0.000 & 0.053 & 0.066 & 0.084 & 0.094 & 0.109 & 0.122 & 0.129 & 0.132 & 0.134 & 0.137 & 0.141 & 0.145 & 0.138 \\
\hline \multirow[t]{2}{*}{$6 \%$} & 0.000 & 0.065 & 0.082 & 0.106 & 0.116 & 0.138 & 0.150 & 0.160 & 0.165 & 0.166 & 0.171 & 0.176 & 0.179 & 0.176 \\
\hline & \multicolumn{14}{|c|}{ Day } \\
\hline
\end{tabular}




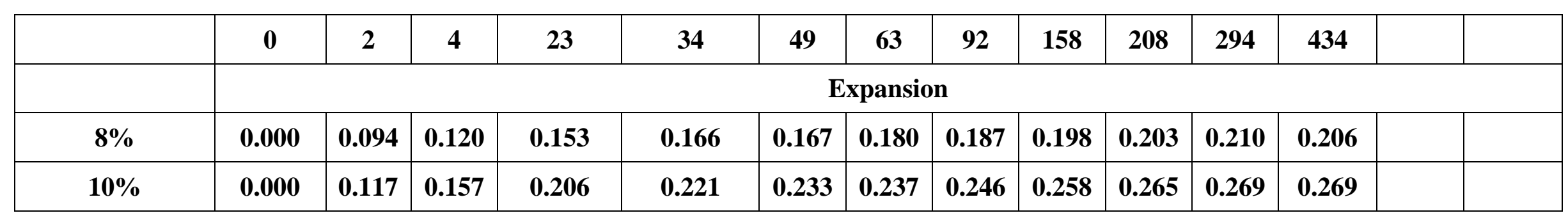

Water expansion test $-30 \%$ cement replacement of Class-F fly ash with different MgO dosages

\begin{tabular}{|c|c|c|c|c|c|c|c|c|c|c|c|}
\hline \multirow{3}{*}{$\operatorname{MgO}(\%)$} & \multicolumn{11}{|c|}{ Day } \\
\hline & $\mathbf{0}$ & 3 & 8 & 16 & 19 & 25 & 27 & 32 & 42 & 102 & 150 \\
\hline & \multicolumn{11}{|c|}{ Expansion } \\
\hline $0 \%$ & 0.000 & 0.023 & 0.031 & 0.031 & 0.030 & 0.035 & 0.032 & 0.040 & 0.038 & 0.036 & 0.028 \\
\hline $2 \%$ & 0.000 & 0.030 & 0.043 & 0.049 & 0.044 & 0.090 & 0.046 & 0.058 & 0.055 & 0.060 & 0.050 \\
\hline $4 \%$ & 0.000 & 0.037 & 0.051 & 0.058 & 0.057 & 0.066 & 0.058 & 0.072 & 0.069 & 0.080 & 0.068 \\
\hline $5 \%$ & 0.000 & 0.042 & 0.060 & 0.066 & 0.066 & 0.100 & 0.075 & 0.071 & 0.078 & 0.090 & 0.085 \\
\hline $6 \%$ & 0.000 & 0.044 & 0.062 & 0.127 & 0.071 & 0.082 & 0.076 & 0.090 & 0.090 & 0.099 & 0.095 \\
\hline $8 \%$ & 0.000 & 0.047 & 0.070 & 0.086 & 0.090 & 0.182 & 0.178 & 0.110 & 0.110 & 0.126 & 0.125 \\
\hline $10 \%$ & 0.000 & 0.065 & 0.097 & 0.114 & 0.117 & 0.207 & 0.130 & 0.146 & 0.147 & 0.170 & 0.170 \\
\hline
\end{tabular}

Comparison of water and autoclave ECC-MgO expansions as a function of MgO contents, water reading is the last reading at 150 days of expansion 


\begin{tabular}{|c|c|c|}
\hline 55\% Class-F FA with MgO $(\%)$ & water & Autoclave \\
\hline $\mathbf{0}$ & $\mathbf{0 . 0 1 4}$ & 0.002 \\
\hline 2 & 0.053 & 0.084 \\
\hline 4 & 0.084 & 0.152 \\
\hline 5 & 0.105 & 0.179 \\
\hline 6 & 0.134 & 0.213 \\
\hline 8 & 0.189 & 0.250 \\
\hline 10 & 0.215 & 0.298 \\
\hline
\end{tabular}

\begin{tabular}{|c|c|c|}
\hline 30\% Class-F FA with $\mathrm{MgO}(\%)$ & water & AC \\
\hline 0 & 0.028 & 0.095 \\
\hline 2 & 0.050 & 0.117 \\
\hline 4 & 0.068 & 0.178 \\
\hline 5 & 0.085 & 0.182 \\
\hline 6 & 0.095 & 0.207 \\
\hline 8 & 0.125 & 0.262 \\
\hline 10 & 0.170 & 0.324 \\
\hline
\end{tabular}


Effect of high volumes of MgO content on water cured ECC-MgO bar specimens with different Class-F fly ash cement replacements

\begin{tabular}{|c|c|c|c|c|c|c|c|c|c|c|c|c|c|}
\hline $\begin{array}{c}55 \% \text { Class-F } \\
\text { FA } \\
\end{array}$ & \multicolumn{13}{|c|}{ Day } \\
\hline \multirow{2}{*}{ MgO \% } & $\mathbf{0}$ & 1 & 3 & 7 & 14 & 22 & 25 & 30 & 32 & 37 & 47 & 107 & 155 \\
\hline & \multicolumn{13}{|c|}{ Expansion } \\
\hline $15 \%$ & 0.000 & 0.078 & 0.097 & 0.157 & 0.182 & 0.198 & 0.198 & 0.210 & 0.214 & 0.219 & 0.223 & 0.240 & 0.238 \\
\hline $20 \%$ & 0.000 & 0.087 & 0.151 & 0.182 & 0.212 & 0.255 & 0.235 & 0.249 & 0.254 & 0.259 & 0.262 & 0.286 & 0.285 \\
\hline $25 \%$ & 0.000 & 0.095 & 0.206 & 0.208 & 0.243 & 0.312 & 0.271 & 0.288 & 0.294 & 0.299 & 0.302 & 0.331 & 0.331 \\
\hline $30 \%$ & 0.000 & 0.117 & 0.166 & 0.258 & 0.306 & 0.349 & 0.354 & 0.374 & 0.383 & 0.392 & 0.399 & 0.443 & 0.449 \\
\hline $\begin{array}{c}30 \% \text { Class-F } \\
\text { FA } \\
\end{array}$ & \multicolumn{13}{|c|}{ Day } \\
\hline \multirow{2}{*}{ MgO \% } & $\mathbf{0}$ & 3 & 8 & 16 & 19 & 25 & 27 & 32 & 42 & 102 & 150 & 157 & \\
\hline & \multicolumn{13}{|c|}{ Expansion } \\
\hline $15 \%$ & 0.000 & 0.096 & 0.138 & 0.173 & 0.182 & 0.197 & 0.193 & 0.215 & 0.219 & 0.259 & 0.295 & 0.396 & \\
\hline $20 \%$ & 0.000 & 0.123 & 0.179 & 0.232 & 0.234 & 0.261 & 0.266 & 0.286 & 0.294 & 0.365 & 0.401 & 0.538 & \\
\hline $25 \%$ & 0.000 & 0.187 & 0.257 & 0.319 & 0.332 & 0.404 & 0.366 & 0.405 & 0.402 & 0.485 & 0.507 & 0.696 & \\
\hline $30 \%$ & 0.000 & 0.237 & 0.340 & 0.432 & 0.449 & 0.518 & 0.496 & 0.536 & 0.546 & 0.661 & 0.695 & 0.894 & \\
\hline
\end{tabular}




\section{REFERENCES}

Alampalli, S., Yannotti, A.P., 1998. In-service Performance of Integral Bridges and Joint less

Decks. Transportation Research Record; 1624(98) pp. 1-7.

Ali, M.M., Mullick, A.K., 1998. Volume Stabilisation of High MgO Cement: Effect of Curing Conditions and Fly Ash Addition. Cem. Concr. Res. 28, 1585-1594.

Amrutha, N., Narasimhan, M., Rajeeva, S., 2011. Chloride-ion impermeability of self-compacting high volume of fly ash concrete mixes. Int. J. Civ. Environ. Eng. IJCEE-IJENS 11, 29-33.

ASTM C1038-b, 2014. Standard Test Method for Expansion of Hydraulic Cement Mortar Bars Stored in Water. Am. Soc. Test. Mater. West Conshohocken, PA, USA. 1-3.

ASTM C1202, 2012. Standard Test Method for Electrical Indication of Concrete's Ability to Resist Chloride Ion Penetration. Am. Soc. Test. Mater. West Conshohocken, PA, USA. 1-8. ASTM C150, 2016. Standard Specification for Portland Cement. Am. Soc. Test. Mater. West Conshohocken, PA, USA. 1-10.

ASTM C151, 2015. Standard Test Method for Autoclave Expansion of Hydraulic Cement. Am. Soc. Test. Mater. West Conshohocken, PA, USA. 1-4.

ASTM C157, 2008. StandardTest Method for Length Change of Hardened Hydraulic-Cement Mortar and Concrete. Am. Soc. Test. Mater. West Conshohocken, PA, USA. 1-7.

ASTM C1585, 2013. Standard Test Method for Measurement of Rate of Absorption of Water by Hydraulic- Cement Concretes. Am. Soc. Test. Mater. West Conshohocken, PA, USA. 1-6.

ASTM C1679, 2014. Standard Practice for Measuring Hydration Kinetics of Hydraulic Cementitious Mixtures Using Isothermal Calorimetry. Am. Soc. Test. Mater. West Conshohocken, PA, USA. 1-15.

ASTM C39, 2016. Standard Test Method for Compressive Strength of Cylindrical Concrete Specimens. Am. Soc. Test. Mater. West Conshohocken, PA, USA. 1-7.

ASTM C490, 2011. Standard Practice for Use of Apparatus for the Determination of Length Change of Hardened Cement Paste, Mortar, and Concrete. Am. Soc. Test. Mater. West Conshohocken, PA, USA. 1-5.

ASTM C597, 2016. Standard Test Method for Pulse Velocity Through Concrete. Am. Soc. Test. 
Mater. West Conshohocken, PA, USA. 1-4.

ASTM C618, 2015. Standard Specification for Coal Fly Ash and Raw or Calcined Natural Pozzolan for Use in Concrete. Am. Soc. Test. Mater. West Conshohocken, PA, USA. 1-5.

ASTM C666, 2015. Standard Test Method for Resistance of Concrete to Rapid Freezing and Thawing. Am. Soc. Test. Mater. West Conshohocken, PA, USA. 1-7.

ASTM C78-b, 2015. Standard Test Method for Flexural Strength of Concrete (Using Simple Beam with Third-Point Loading). Am. Soc. Test. Mater. West Conshohocken, PA, USA. 1-4.

Au, A., Lam, C., Au, J., Tharmabala, B., 2013. Eliminating Deck Joints Using Debonded Link Slab: Research and Field Test in Ontario. Journal of Bridge Engineering, 18(8), pp. 768-778. J. Chem. Inf. Model.

Bhashkar, S., 2016. Self-Healing Bacterial Cementitious Concrete Composites: Development and Performance Evaluation. Civil Engineering Department, Ryerson University.

Bhaskar, S., Lachemi, M., Hossain, A., Wolfaardt, G., Kroukamp, M., 2015. An Experimental Investigation on The Strength and Durability of Self-Healing Bacterial Concrete, CSCE conference, Building on Our Growth Opportunities, May 27 - 30, 2015, Regina, SK, Canada. 10.

Bhaskar, S., Lachemi, M., Hossain, A., Wolfaardt, G., Kroukamp, M.O., 2014. Characteristcs of bacteria incorporated self- healing behaviour in concrete", 10th fib International $\mathrm{PhD}$ symposium in Civil Engineering, July 21, Quebec, Canada 19-24.

Bisaillon, A.., Rivest, M.., Malhotra, V.. M.., 1994. Performance of High Volume Fly Ash Concrete in Large Experimental Monoliths. ACI Mater. J. 91, 178-187.

Caner, A., Zia, P., 1998. Behavior and Design of Link Slabs for Jointless Bridge Decks. PCI J. 43, 68-81.

Carpinteri, A., Brighenti, R., Spagnoli, A., Vantadori, S., 2013. Tensile Cracking Behaviour of Strain-Hardening Cement-Based Composites using a Micromechanical Lattice Model. Cp2009 1151-1158.

Choi, S. w., Jang, B. s., Kim, J. h., Lee, K. m., 2014. Durability characteristics of fly ash concrete containing lightly-burnt MgO. Constr. Build. Mater. 58, 77-84.

Choi, S.W., Jang, B.S., Lee, K.M., 2013. Fundamental Properties of Fly ash Concrete Contain Lightly Burnt MgO Powder. J. Korean Soc. Civ. Eng. 33, 475-481 (In Korean). 
Christophe, C., Raoul, F., 1997. Effect of the Leaching of Calcium Hydroxide from Cement Paste on Mechanical and Physical Properties. Cem. Concr. Res. 27, 539-550.

CISC, 2010. Handbook of Steel Construction, 10th edition, Ontario, Canada: CISC.

De Muynck, W., Cox, K., Belie, N., Verstraete, W., 2008a. Bacterial carbonate precipitation as an alternative surface treatment for concrete. Constr. Build. Mater. 22, 875-885.

De Muynck, W., Debrouwer, D., De Belie, N., Verstraete, W., 2008b. Bacterial carbonate precipitation improves the durability of cementitious materials. Cem. Concr. Res. 38, 10051014.

Dry, C., 1996. Procedures Developed for Self-Repair of Polymer Matrix Composite Materials. Compos. Struct. 35, 263-269.

Dry, C., 1994. Matrix Cracking Repair and Filling Using Active and Passive Modes for Smart Timed Release of Chemicals from Fibers into Cement Matrices. Smart Mater. Struct. 3, 118123.

Dry, C.M., 2001. Three Designs for The Internal Release of Sealants, Adhesives , and Waterproofing Chemicals into Concrete to Reduce Permeability. Cem. Concr. Res. 30, 19691977.

Du, C., 2005. A Review of Magnesium Oxide in Concrete. Concr. Int. 45-50.

Edvardsen, C., 1999. Water Permeability and Autogenous Healing of Cracks in Concrete. ACI Mater. J. 96, 448-454.

Fernandez, L., Alonso, C., Andrade, C., Hidalgo, A., 2008. The Interaction of Magnesium in Hydration of C3S and CSH Formation Using 29Si MAS-NMR. J. Mater. Sci. 43, 5772-5783.

Fernandez, L., Alonso, C., Andrade, C., Hidalgo, A., 2005. The Role of Magnesium During the Hydration of C3S and C-S-H Formation. Scanning Electron Microscopy and Mid-Infrared Studies. Adv. Cem. Res. 17, 9-21.

Fischer, G., Li, V.C., 2003. Intrinsic Response Control of Moment Resisting Frames Utilizing Advanced Composite Material and Structural Elements. ACI Struct. J. 100, 166-176.

Gahtrehsamani, S., 2015. Structural Performance of Link Slabs Subjected to Monotonic and Fatigue Loading Incorporating Engineered Cementitious Composites. Civil Engineering Department, Ryerson University.

Gao, P., Lu, X., Geng, F., Li, X., Hou, J., Lin, H., Shi, N., 2008. Production of MgO-Type 336 
Expansive Agent in Dam Concrete by Use of Industrial By-Products. Build. Environ. 43, $453-457$.

Gao, P., Wu, S., Lu, X., Deng, M., Lin, P., Wu, Z., Tang, M., 2007. Soundness Evaluation of Concrete with MgO. Constr. Build. Mater. 21, 132-138.

Gao, T., Shen, L., Shen, M., Liu, L., Chen, F., 2016. Analysis of material flow and consumption in cement production process. J. Clean. Prod. 112, 553-565.

Gastal, F., Zia, P., 1989. Analysis of bridge beams with jointless decks. Proc. IASBE Symp. Lisbon.

Ghosh, S.K., 2009. Self-healing Materials: Fundamentals, Design Strategies, and Applications. WILEY-VCH Verlag GmbH \& Co., KGaA, Weinheim, pp. 291.

Gilani, A., 2001. Link slabs for Simply Supported Bridges Incorporating Engineered Cementitious Composites. MDOT SPR-54181. Structural Research Unit, MDOT, USA.

Halikia, I., Zoumpoulakis, L., Christodoulou, E., Prattis, D., 2001. Kinetic study of the thermal decomposition of calcium carbonate by isothermal methods of analysis. Eur. J. Process. Enviromental Prot. 1, 89-102.

Heide, N., Schlangen, E., 2007. Selfhealing of Early Age Cracks in Concrete. Proc. First Int. Conf. Self Heal. Mater. Noordwijk aan Zee, Netherlands, 1-12.

Helmuth, R., West, P.B., 1998. Reappraisal of the Autoclave Expansion Test. Cem. Concr. Aggregates, CCAGDP 20, 194-219.

Herbert, E., Li, V., 2013. Self-Healing of Microcracks in Engineered Cementitious Composites (ECC) Under a Natural Environment. Materials (Basel). 6, 2831-2845.

Homma, D., Mihashi, H., Nishiwaki, T., 2009. Self-Healing Capability of Fibre Reinforced Cementitious Composites. J. Adv. Concr. Technol. 7, 217-228.

Hossain, K.M.A., Anwar, M.S., 2014. STRENGTH AND DEFORMATION CHARACTERISTICS OF ECC LINK SLAB IN JOINT - FREE BRIDGE DECKS Link Slab in Joint-free Bridge Decks. Istanbul Bridg. Conf.

Hossain, K.M.A., Gahtrehsamani, S., Anwar, M.S., 2015. Flexural fatigue performance of ECC link slabs for bridge deck applications. Sustain. Bridg. Struct. (Ed. K M Mahmoud), Chapter 22, CRC Press. Taylor Fr. Group, EH Leiden, Netherlands 247-260.

Hossain, K.M.A., Lachemi, M., 2014. Engineered Concrete Systems for Structural Applications. 
MTO Report, Department of Civil Engineering, Ryerson University , p.150. 2014.

Hossain, K.M.A., Mak, C., Ametrano, D., 2012. GFRP Reinforced UHPC Composites for Sustainable Bridge Construction, in: Canadian Civil Engineer. pp. 12-15.

http://toronto.weatherstats.ca/ [WWW Document], 2016. . Weather. Toronto.

Jonkers, H.M., Schlangen, E., 2009. A two component bacteria based self-healing concrete. Concr. Repair, Rehabil. Retrofit. II 215-220.

Jonkers, H.M., Schlangen, E., 2008. Development of a bacteria-based self healing concrete. Proc. int. FIB Symp. 1, 425-430.

Joshi, R.C., 1997. Fly ash in concrete: production, properties and uses, Australia; Newark: Gordon and Breach.

Kan, A., Ramazan, D., 2009. A novel material for lightweight concrete production. Cem. Concr. Compos. 31, 489-495.

Kanda, T., Li, V.C., 1998. Multiple Cracking Sequence and Saturation in Fiber Reinforced Cementitious Composites. Concr. Res. Technol. JCI 9, 19-33.

Kim, J.S., Schlangen, E., 2011. Self-Healing in ECC Stimulated by SAP under Flexural Cyclic Load, in: In Proceedings of 3rd International Conference on Self Healing Materials, Bath, UK. pp. 3-4.

Kim, Y.Y., Fischer, G., Li, V.C., 2004. Performance of bridge deck link slabs designed with ductile engineered cementitious composite. ACI Struct. J. 101, 792-801.

Lam, C., Lai, D., Au, J., Lim, L., Young, W., Tharmabala, B., 2008. Development of Concrete Link Slabs To Eliminate Bridge Expansion Joints Over Piers. Annu. Conf. Transp. Assoc. Canada 1-20.

Langley, W.S., Carette, G.G., Malhotra, V, M., 1992. Strength Development and Temperature Rise in Large Concrete Blocks Containing High Volumes of Low- Calcium ( ASTM Class F ) Fly Ash. ACI Mater. J. 89, 362 to 368.

Lepech, M.D., Li, V.C., 2009a. Water Permeability of Engineered Cementitious Composites. Cem. Concr. Compos. 31, 744-753.

Lepech, M.D., Li, V.C., 2009b. Application of ECC for bridge deck link slabs. Mater. Struct. 42, $1185-1195$.

Lepech, M.D., Li, V.C., 2008. Large Scale Processing of Engineered Cementitious Composites. 338 
ACI Mater. J. 105, 358-366.

Li, M., Li, V.C., 2011. Cracking and Healing of Engineered Cementitious Composites under Chloride Environment. ACI Mater. J. 333-340.

Li, V.C., 2011. High-Ductility Concrete for resilient infrastructures. J. Adv. High-Performance Mater. Build. Infrastruct. community 16-21.

Li, V.C., 2003. On Engineered Cementitious Composites ( ECC ) A Review of the Material and Its Applications. J. Adv. Concr. Technol. 1, 215-230.

Li, V.C., 1997. Engineered Cementitious Composites (Ecc) - Tailored Composites through Micromechanical Modeling. Can. Soc. Civ. Eng. 1-38.

Li, V.C., 1993. From Micromechanics to Structural Engineering-The Design of Cementitious Composites for Civil Engineering Applications. JSCE J Struct. Mech. Earthq. Eng. 10, 3748.

Li, V.C., Fischer, G., Kim, Y., Lepech, M., Qian, M., Weimann, M., Wang, S., 2003. Final Report on Durable Link Slabs for Jointless Bridge Decks Based on Strain-Hardening Cementitious Composites. Adv. Civ. Eng. Mater. Res. Lab. MDOT Proj. Manag. Roger Till.

Li, V.C., Kanda, T., 1998. Engineered Cementitious Composites for structural applications. J. Mater. Civ. Eng. 10, 66-69.

Li, V.C., Lepech, M., Wang, S., Weimann, M., Keoleian, G., 2004. Development of Green ECC for Sustainable Infrastructure Systems, in: Proceedings of the International Workshop on Sustainable Development and Concrete Technology. Beijing, China, Wang, K., Ed., pp. 181192.

Li, V.C., Lim, Y.M., Chan, Y.-W., 1998. Feasibility study of a passive smart self-healing cementitious composite. Compos. Part B Eng. 29, 819-827.

Li, V.C., Mishra, D.K., Wu, H.C., 1995. Matrix Design for Pseudo-Strain-Hardening Fibre Reinforced Cementitious Composites. Mater. Struct. 28, 586-595.

Li, V.C., Wang, S., Wu, C., 2001. Tensile Strain-Hardening Behavior or Polyvinyl Alcohol Engineered Cementitious Composite (PVA-ECC). ACI Mater. J. 98, 483-492.

Li, V.C., Wu, C., Wang, S., Ogawa, A., Saito, T., 2002. Interface Tailoring for Strain- Hardening PVA-ECC. Am. Concr. Inst. Mater. J. 99, 463-472.

Lin, Z., Kanda, T., Li, V.C., 1999. On Interface Property Characterization and Performance of 339 
Fiber Reinforced Cementitious Composites. J. Concr. Sci. Eng. RILEM 1, 173-184.

Mavani, M.B., 2012. Fresh, mechanical, durability and structural performance of enginnered cementitious composite (ecc) maulin bipinchandra mavani 2012. Thesis, Ryerson University, Toronto, Ontario, Canada.

Mehta, P.K., 1985. Influence of Fly Ash Characteristics on The Strength of Portland-Fly Ash Mixtures. Cem. Concr. Res. 15, 669-674.

Mehta, P.K., 1978. History and status of performance tests for evaluation of soundness of cements. Cem. Stand. Trends, ASTM STP 663, P. K. Mehta, Ed., Am. Soc. Test. Mater. 35-60.

Mehta, P.K., Monteiro, P.J.M., 2006. Concrete: Microstructure, Properties, and Materials, 3rd edi. ed. McGraw Hill, New York.

Mehta, P.K., Pirtz, D., Komandant, G.J., 1980. Magnesium Oxide Additive for Producing Selfstress in Mass Concrete. 7th Int. Congr. Chem. Cem. III, V-6 to V-9.

Mihashi, H., Nishiwaki, T., 2012. Development of Engineered Self-Healing and Self-Repairing Concrete-State-of-the-Art Report. J. Adv. Concr. Technol. 10, 170-184.

Mindess, S., Young, J.F., Darwin, D., 2003. Concrete, 2nd edi. ed. Prentice Hall, Pearson Education, Inc. Upper Saddle River, NJ 07458, U.S.A.

Mitamura, H., Sakata, N., Shakushiro, K., Suda, K., Hiraishi, T., 2005. Application of Overlay Reinforcement Method on Steel Deck Utilizing Engineered Cementitious Composites Mihara Bridge. Bridge and Foundation Engineering, Vol. 39, No. 8, 88-91.

Mo, L., Deng, M., Tang, M., 2010. Effects of calcination condition on expansion property of MgOtype expansive agent used in cement-based materials. Cem. Concr. Res. 40, 437-446.

Mo, L., Deng, M., Tang, M., Al-Tabbaa, A., 2014. MgO expansive cement and concrete in China: Past, present and future. Cem. Concr. Res. 57, 1-12.

Mo, L., Deng, M., Wang, A., 2012. Effects of MgO-Based Expansive Additive on Compensating the Shrinkage of Cement Paste under Non-Wet Curing Conditions. Cem. Concr. Compos. 34, 377-383.

Moradpour, R., Taheri-Nassaj, E., Parhizkar, T., Ghodsian, M., 2013. The Effects of Nanoscale Expansive Agents on the Mechanical Properties of Non-Shrink Cement-Based Composites: The Influence of Nano-MgO Addition. Compos. Part B Eng. 55, 193-202.

Na, S.H., Hama, Y., Taniguchi, M., Katsura, O., Sagawa, T., Zakaria, M., 2012. Experimental 340 
Investigation on Reaction Rate and Self-healing Ability in Fly Ash Blended Cement Mixtures. J. Adv. Concr. Technol. 10, 240-253.

Nawy, E.G. (ED), 2008. Concrete Construction Engineering Handbook, 2nd edi. ed. Taylor \& Francis Group, LLC.

Neville, A.M., 1995. Properties of Concrete, 4th ed. Pearson Education Limited, Essex, England, pp. 884.

$\mathrm{Ng}$, S., Justnes, H., 2016. Influence of plasticizers on the rheology and early heat of hydration of blended cements with high content of fly ash. Cem. Concr. Compos. 65, 41-54.

Nikiforov, Y. V., Zosoulia, R.A., 1980. Crystallization of Periclase and Soundness of Cement. 7th Int. Congr. Chem. Cem. II, Paris, 1-34 to 1-36.

Nishiwaki, T., Koda, M., Yamada, M., Mihashi, H., Kikuta, T., 2012. Experimental Study on SelfHealing Capability of FRCC Using Different Types of Synthetic Fibers. J. Adv. Concr. Technol. 10, 195-206.

Özbay, E., Karahan, O., Lachemi, M., Hossain, K.M.A., Atis, C.D., 2013a. Dual effectiveness of freezing-thawing and sulfate attack on high-volume slag-incorporated ECC. Compos. Part B Eng. 45, 1384-1390.

Özbay, E., Şahmaran, M., Lachemi, M., Yücel, H.E., 2013b. Effect of microcracking on frost durability of high-volume-fly-ash- and slag-incorporated engineered cementitious composites. ACI Mater. J. 110, 259-267.

Panzera, T.H., Christoforo, A.L., Cota, F.P., Borges, P.H.R., Bowen, C.R., 2011. Ultrasonic Pulse Velocity Evaluation of Cementitious Materials. Adv. Compos. Mater. - Anal. Nat. man-made Mater. 17, 411-436.

Qian, S., Lepech, M.D., Kim, Y.Y., Li, V.C., 2009. Introduction of Transition Zone Design for Bridge Deck Link Slabs Using Ductile Concrete. ACI structural journal, 106(1), pp. 96-105.

Qian, S.Z., Zhou, J., Schlangen, E., 2010. Influence of Curing Condition and Precracking Time on the Self-Healing Behavior of Engineered Cementitious Composites. Cem. Concr. Compos. $32,686-693$.

Ranade, R., Zhang, J., Lynch, J.P., Li, V.C., 2014. Influence of micro-cracking on the composite resistivity of Engineered Cementitious Composites. Cem. Concr. Res. 58, 1-12.

Rehsi, S.S., 1983. Magnesium Oxide in Portland Cement. Adv. Cem. Technol. Pergamon Press 
467-483.

Reinhardt, H.-W., Jooss, M., 2003. Permeability and Self-Healing of Cracked Concrete as a Function of Temperature and Crack Width. Cem. Concr. Res. 33, 981-985.

Şahmaran, M., Keskin, S.B., Ozerkan, G., Yaman, I.O., 2008. Self-Healing of MechanicallyLoaded Self Consolidating Concretes with High Volumes of Fly Ash. Cem. Concr. Compos. $30,872-879$.

Şahmaran, M., Lachemi, M., Hossain, K.M.A., Li, V.C., 2009. Internal curing of engineered cementitious composites for prevention of early age autogenous shrinkage cracking. Cem. Concr. Res. 39, 893-901.

Sahmaran, M., Lachemi, M., Hossain, K.M.A., Ranade, R., Li, V.C., 2009. Influence of Aggregate Type and Size on Ductility and Mechanical Properties of Engineered Cementitious Composites. ACI Mater. J. 308-316.

Sahmaran, M., Li, V.C., 2009. Durability properties of micro-cracked ECC containing high volumes fly ash. Cem. Concr. Res. 39, 1033-1043.

Sahmaran, M., Yildirim, G., Ahmed, K., 2013. The effect of self-healing on the durability performance of micro-cracked ECC, in: Proceedings of the 8th International Conference on Fracture Mechanics of Concrete and Concrete Structures, FraMCoS 2013. pp. 1999-2008.

Schlangen, E., Heide, N., Breugel, K., 2006. Crack Healing of Early Age Cracks in Concrete. Meas. Monit. Model. Concr. Prop. Konsta-Gdoutos, M.S., Ed.; Springer Alexandroupolis, Greece 273-284.

Shehata, M.H., 2001. The Effects of Fly Ash and Silica Fume on Alkali Silica Reaction in Concrete [WWW Document].

Sherir, M.A.A., 2012. Fracture Energy, Fatigue and Creep Properties of Engineered Cementitious Composites Incorporating Fly Ash/Slag with Different Aggregates, MASc Thesis. Dep. Civ. Eng. Ryerson Univ. 120.

Sherir, M.A.A., Hossain, K.M.A., Lachemi, M., 2016. Mechanical \& Durability Properties of Engineered Cementitious Composites with Different Aggregates. CSCE, 5th Int. Mater. Spec. Conf. London, Ontario 1-10.

Sherir, M.A.A., Hossain, K.M.A., Lachemi, M., 2015a. Structural Performance of Polymer Fiber Reinforced Engineered Cementitious Composites Subjected to Static and Fatigue Flexural 
Loading. Polymers (Basel). 7, 1299-1330.

Sherir, M.A.A., Hossain, K.M.A., Lachemi, M., 2015b. Interaction of Superabsorbent Polymers and Admixtures on THe Properties of Engineered Cementitious Composites. CSCE, 4th Int. Eng. Mech. Mater. Conf. Regina, Saskatchewan 1-10.

Sherir, M.A.A., Hossain, K.M.A., Lachemi, M., 2014. Fracture Energy Characteristics of Engineered Cementitious Composites Incorporating Different Aggregates. CSCE, 4th Int. Struct. Spec. Conf. Halifax, Nov. Scotia 1-10.

Snoeck, D., Steuperaert, S., Tittelboom, K. V., Dubruel, P., De Belie, N., 2012a. Visualization of Water Penetration in Cementitious Materials with Superabsorbent Polymers by Means of Neutron Radiography. Cem. Concr. Res. 42, 1113-1121.

Snoeck, D., Tittelboom, K. V., Steuperaert, S., Dubruel, P., Belie, N.D., 2012b. Self-Healing Cementitious Materials by The Combination of Microfibres and Superabsorbent Polymers. J. Intell. Mater. Syst. Struct. 1-12.

Thomas, M.D.A., Mukherjee, P.K., Sato, J.A., Everitt, M.F., 1995. Effect of Fly Ash Composition on Thermal Cracking in Concrete. ACI Mater. J. Spec. Publ. 153, 81-98.

Tittelboom, K. V., De Belie, N., 2013. Self-Healing in Cementitious Materials-A Review. Materials (Basel). 6, 2182-2217.

Tittelboom, K. Van, Gruyaert, E., Rahier, H., Belie, N. De, 2012. Influence of Mix Composition on The Extent of Autogenous Crack Healing by Continued Hydration or Calcium Carbonate Formation. Constr. Build. Mater. 37, 349-359.

Wang, J., Van T., K., De Belie, N., Verstraete, W., 2012. Use of silica gel or polyurethane immobilized bacteria for self-healing concrete. Constr. Build. Mater. 26, 532-540.

Wang, J.Y., De Belie, N., Verstraete, W., 2012. Diatomaceous earth as a protective vehicle for bacteria applied for self-healing concrete. J. Ind. Microbiol. Biotechnol. 39, 567-577.

Wang, S., Li, V.C., 2007. Engineered Cementitious Composites with High-Volume Fly Ash. ACI Mater. J. 104, 233-241.

Weimann, M.B., Li, V.C., 2003. Hygral behavior of engineered cementitious composites (ECC). IInternational J. Restor. Build. Monum. 9, 513-534.

White, A.H., 1928. Volume Changes of Portland Cement as Affected by Chemical Composition and Aging. Proc. Am. Soc. Test. Mater. 28, Part I, 398-431. 
Wu, M., Johannesson, B., Geiker, M., 2012. A review : Self-Healing in Cementitious Materials and Engineered Cementitious Composite as a Self-Healing Material. Constr. Build. Mater. $28,571-583$.

Xian Li, F., Zhi Chen, Y., Zong Long, S., 2010. Influence of MgO Expansive Agent on Behavior of Cement Pastes and Concrete. Arab. J. Sci. Eng. 35, 125-139.

Yang, E., Li, V.C., 2006. A Micromechanical Model for Fiber Cement Optimization and Component Tailoring, in: Proceedings of 10th International Inorganic-Bonded Fiber Composites Conference. San Paulo, Brazil, pp. 1-13.

Yang, E., Yang, Y., Li, V.C., 2007. Use of High Volumes of Fly Ash to Improve ECC Mechanical Properties and Material Greenness. ACI Mater. J. 303-311.

Zhang, B., Peng, J., Zhang, L., Ju, S., 2012. Optimization of Preparation for MgO by Calcination from Basic Magnesium Carbonate using Response Surface Methodology, in: MAGNESIUM TECHNOLOGY 2012, The Minerals, Metals \& Materials Society. pp. 75-82.

Zhang, J., Maalej, M., Quek, S.T., Teo, Y.Y., 2005. Drop weight impact on hybrid-fiber ECC blast/shelter panels, in: Proceedings of Third International Conference on Construction Materials: Performance, Innovation and Structural Applications, Vancouver, Canada.

Zheng, L., Xuehua, C., Mingshu, T., 1992. Hydration and setting time of MgO-type expansive cement. Cem. Concr. Res. 22, 1-5.

Zheng, L., Xuehua, C., Mingshu, T., 1991. MgO-Type Delayed Expansive Cement. Cem. Concr. Res. 21, 1049-1057.

Zia, P., Caner, A., El-Safte, A., 1995. Jointless bridge decks research project 23241-94-4. Center for Transportation Engineering Studies, North Carolina State, pp. 1-117.

Zuk, W., 1981. "Joint less Bridges”, Research Repo rt No. FHWA/VA-81/48, Virginia Highway and Transportation Research Council. Charlottesville, Virginia, USA. 